\title{
Systematic study of the synthesis of nanostructured multiferroic films and their structural, electronic and magnetic properties
}

\author{
by
}

Pierre Couture

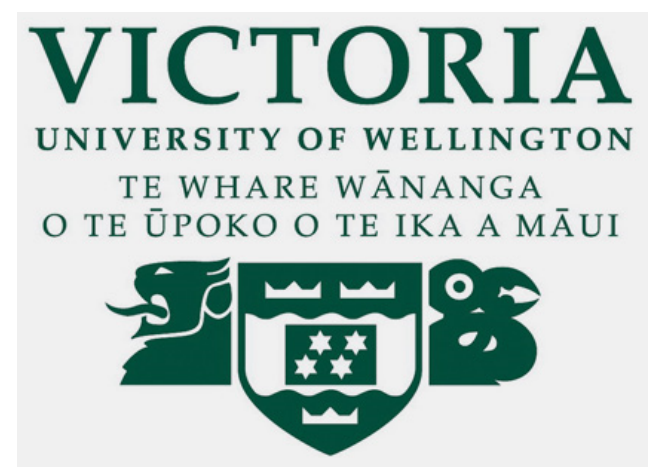

\begin{abstract}
A thesis
submitted to the Victoria University of Wellington in fulfilment of the requirements for the degree of

Doctor of Philosophy in Science, Physics.
\end{abstract}

Victoria University of Wellington

2017 



\begin{abstract}
Multiferroics are unique materials that display multiple ferroic properties (ferroelectricity, ferromagnetism and ferroelasticity) simultaneously. A number of materials containing bismuth have intrinsic multiferroic properties, including $\mathrm{BiFeO}_{3}$ and $\mathrm{BiCrO}_{3}$. Among them, $\mathrm{BiFeO}_{3}$ has attracted widespread attention because $\mathrm{BiFeO}_{3}$ was the first material to display multiferroic behaviour at ambient temperature. A weak ferromagnetism occurs only at low temperatures depending on synthesis conditions. This thesis reports the structural, magnetic and optical properties of nanostructured $\mathrm{BiFeO}_{3}$ thin films prepared by two novel approaches of ion beam sputtering and ion implantation techniques.
\end{abstract}

Nanocrystalline $\mathrm{BiFeO}_{3}$ films were prepared at ambient temperature by sputtering and thermal annealing at $500{ }^{\circ} \mathrm{C}$ in an oxygen atmosphere. The annealing resulted in the formation of multiferroic $\mathrm{BiFeO}_{3}$ phase with a reduction of iron oxide and bismuth phases. Superparamagnetism was observed and could be attributed to magnetite and maghemite nanoparticles. The magnetic properties were mainly due to magnetite and maghemite nanoparticles. The saturation magnetic moment was $60 \%$ lower after annealing, which was due to Fe in phases of iron oxide being incorporated into $\mathrm{BiFeO}_{3}$ nanoparticles. An exchange bias was observed before and after annealing. The exchange bias cannot be attributed to $\mathrm{BiFeO}_{3}$ structure. Instead, the exchange has likely arisen from magnetite and maghemite cores with spin-disordered shells. Piezoelectric responses measured by piezoelectric force microscopy confirmed the presence of $\mathrm{BiFeO}_{3}$ ferroelectric material. The Magneto-optical Kerr effect (MOKE) and optical studies were used to calculate an anomalously high Verdet constant. The MOKE and magnetic circular dichroism (MCD) displayed a significant modification in function of the wavelength. Further increasing the annealing temperature lead to an increase in iron oxide phases, while increasing the annealing duration reduced the iron oxide phases, however this increases the fraction of $\mathrm{Bi}_{2} \mathrm{Fe}_{4} \mathrm{O}_{9}$ and $\mathrm{Bi}_{2} \mathrm{O}_{3}$.

Another approach to synthesise $\mathrm{BiFeO}_{3}$ thin film was investigated by bismuth ion implantation into iron oxide thin film. An as-made iron oxide film subsequently 
implanted with bismuth and annealed showed a $6.5 \%$ reduction of the ferromagnetic phase fraction. An annealed iron oxide film subsequently implanted with bismuth and annealed show that the ferromagnetic phase was present at less than $4 \%$ while $\mathrm{Fe}_{3} \mathrm{O}_{4}$ and $\gamma-\mathrm{Fe}_{2} \mathrm{O}_{3}$ increased to $7 \%$. The coercive field is affected by annealing. However, this field is not affected by the bismuth implantation.

For the first-time, a preliminary investigation reporting the implantation of $\mathrm{Bi}$ then $\mathrm{Fe}$ then $\mathrm{O}$ into $\mathrm{SiO}_{2}: \mathrm{Si}$ was made with the aim to synthesise $\mathrm{BiFeO}_{3}$ films and magnetic nanoparticles. The implantation of $\mathrm{Fe}$ then $\mathrm{O}$ then $\mathrm{Bi}_{\text {into }} \mathrm{SiO}_{2}: \mathrm{Si}$ contained a mix of iron oxides: $\alpha-\mathrm{Fe}_{2} \mathrm{O}_{3}$ and $\mathrm{Fe}_{3} \mathrm{O}_{4}$, as confirmed by Raman spectroscopy and $\mathrm{X}$-ray diffraction, while $\gamma-\mathrm{Fe}_{2} \mathrm{O}_{3}$ was most likely also present in the film. The as-implanted sample displayed a sign of a superparamagnetic phase that was lost with annealing the sample.

Preliminary investigations of another multiferroic material, $\mathrm{BiCrO}_{3}$, were carried out. Thin films of $\mathrm{BiCrO}_{3}$ were prepared by ion beam sputtering and annealing the sample in an oxygen atmosphere which lead to $\mathrm{BiCr}_{x} \mathrm{O}_{y}$ with chromium oxides and bismuth oxide phases. Magnetic enhancement was observed when annealing above $700{ }^{\circ} \mathrm{C}$. Annealing in an oxygen atmosphere followed by an argon atmosphere created a superparamagnetic phase that was not visible under other annealing conditions. 


\section{Résumé}

Les matériaux multiferroïques sont uniques en raison de leur capacité à combiner simultanément plusieurs propriétés des matériaux ferroïques (ferroélectricité, ferromagnétisme et ferroélasticité). Plusieurs matériaux contenant du Bi ont des propriétés multiferrroïques, par exemple $\mathrm{BiFeO}_{3}$ et $\mathrm{BiCrO}_{3}$. Parmi ces matériaux, le $\mathrm{BiFeO}_{3}$ est un multiferroïque qui attire une attention particulière parce qu'il est le premier matériau dont les propriétés multiferrroïques ont été démontrées à température ambiante. Son faible ferromagnétisme se produit seulement à basse température selon la méthode de synthèse. Cette thèse présente les propriétés structurales, magnétiques et optiques de films minces nanostructurés de $\mathrm{BiFeO}_{3}$ préparés selon deux nouvelles approches : la déposition par faisceau d'ions (en anglais ion beam sputtering) et l'implantation d'ions (en anglais ion implantation).

Les films de $\mathrm{BiFeO}_{3}$ nanostructurés ont été préparés par déposition de faisceau d'ions (en anglais ion beam sputtering) à température ambiante suivie d'un recuit à $500{ }^{\circ} \mathrm{C}$ dans une atmosphère d'oxygène. Le recuit a eu pour résultat la formation de $\mathrm{BiFeO}_{3}$ multiferroïque. De plus, le recuit a réduit la quantité de sous-produits d'oxyde de fer et de bismuth. Le superparamagnétisme a été observé, mais cette propriété pourrait être attribuée aux nanoparticules de magnétite et de maghémite. Les propriétés magnétiques sont principalement causées par ces nanoparticules. La saturation du moment magnétique est $60 \%$ plus faible après le recuit, ce qui est causé par une quantité de fer sous forme de nanoparticules d'oxyde de fer formant des liens avec $\mathrm{du}$ bismuth afin de former des nanoparticules de $\mathrm{BiFeO}_{3}$. Un échange d'anisotropie (exchange bias) a été observé avant et après le recuit. L'échange d'anisotropie ne peut pas être attribué à la structure des cristaux de $\mathrm{BiFeO}_{3}$. Il est fort probable que cette propriété provienne d'une structure de noyau à base de magnétite et d'un désordre de spins d'une couche extérieure de maghémite, une structure magnétite-maghémite noyau-enveloppe (en anglais core-shell). La mesure de la réponse piézoélectrique par microscopie à force piézoélectrique confirme la présence de matériel ferroélectrique, $\mathrm{BiFeO}_{3}$. L'effet Kerr magnéto-optique (en anglais MOKE) et l'étude des propriétés 
optiques ont été dérivés pour calculer la constante de Verdet qui dans cette étude est anormalement élevée. Le MOKE et le dichroïsme circulaire magnétique (en anglais magnetic circular dichroism, MCD) varient grandement en fonction de la longueur d'onde. Augmenter la température du recuit entraine une hausse de la concentration d'oxyde de fer, alors qu'une augmentation de la durée du recuit réduit la concentration d'oxyde de fer, et augmente la concentration de $\mathrm{Bi}_{2} \mathrm{Fe}_{4} \mathrm{O}_{9}$ et de $\mathrm{Bi}_{2} \mathrm{O}_{3}$.

Une autre approche pour fabriquer un film mince de $\mathrm{BiFeO}_{3}$ a été étudiée en implantant des ions de bismuth dans un film mince d'oxyde de fer. La technique utilisée pour modifier le film est l'implantation d'ions (ion implanter). Un film tel que déposé d'oxyde de fer qui par la suite a été implanté avec des ions de bismuth puis recuit a démontré une réduction de $6.5 \%$ de la concentration du matériel ferromagnétique. Toutefois, le film d'oxyde de fer recuit puis implanté avec des ions de bismuth et recuit une deuxième fois démontre que la concentration de matériel ferromagnétique est inférieure à $4 \%$. Cette procédure a augmenté la concentration de $\mathrm{Fe}_{3} \mathrm{O}_{4}$ et $\gamma-\mathrm{Fe}_{2} \mathrm{O}_{3}$ à $7 \%$. La mesure du champ coercitif (coercive field) est affectée par le recuit, mais elle n'est pas affectée par l'implantation de bismuth.

Pour la première fois, cette étude rapporte une exploration préliminaire ayant pour objectif la synthèse de nanoparticules magnétiques et de $\mathrm{BiFeO}_{3}$ par la triple implantation : bismuth, fer et oxygène, dans un substrat de $\mathrm{SiO}_{2}$. L'implantation du fer suivie d'oxygène puis de bismuth dans le $\mathrm{SiO}_{2}$ a résulté en la formation d'un mélange d'oxyde de fer : $\alpha-\mathrm{Fe}_{2} \mathrm{O}_{3}$ et $\mathrm{Fe}_{3} \mathrm{O}_{4}$. Ce résultat a été confirmé par l'analyse de la spectroscopie Raman et la cristallographie aux rayons-X (XRD). $\gamma-\mathrm{Fe}_{2} \mathrm{O}_{3}$ est fort probablement présente dans l'échantillon. Ce dernier, tel qu'implanté, démontre des signes d'un matériau superparamagnétique non-identifié par les différentes techniques d'analyses, cependant ce matériau semble disparaitre après le recuit.

L'étude préliminaire d'un autre matériel multiferroïque, $\mathrm{BiCrO}_{3}$, a été réalisé. Le film a été préparé par déposition de faisceau d'ions puis recuit dans une atmosphère d'oxygène résultant en la formation d'un film composé de $\mathrm{BiCr}_{x} \mathrm{O}_{y}$, d'oxyde de chrome et d'oxyde de bismuth. Une augmentation du moment magnétique a été observée lorsque l'échantillon est recuit à $700{ }^{\circ} \mathrm{C}$. Un recuit dans une atmosphère d'oxygène suivi par un deuxième recuit dans une atmosphère d'argon semble créer un matériel superparamagnétique, alors que ce n'est pas le cas lorsque le film est soumis à d'autres conditions de recuit. 


\section{Acknowledgments}

I would like to express my gratitude to my PhD supervisors Dr. John V. Kennedy at GNS Science, National Isotope Centre in Gracefield, and Dr. Grant V.W. Williams at the School of Chemical and Physical Sciences, Victoria University of Wellington. I must thank them for their guidance in my work.

I gratefully acknowledge the support of my group of research. Jérôme Leveneur, Peter Murmu, Tushara Prakash, Prasanth Gupta and Konrad Suschke for their help, time and explanation that improve this work.

I would like to express my gratitude to my PhD thesis examiners for their work, comments and suggestions made to improve this thesis. I would like to thank Dr. Markwitz, GNS Science, and Dr. Strickland, Robinson Research Institute, for proofreading and revising the submitted thesis after the PhD defence. I would like to express my gratitude to Bill Trompetter, Liz Keller and Luce Gilbert for reviewing the language quality.

I must express my sincere gratitude to Dr. Shen V. Chong, Scientist at the Robinson Research Institute, Victoria University of Wellington, for all his help with the magnetometer during weekends, evening and holidays.

I would like to thank Dr. Sergey Rubanov at the Electron Microscope Unit, Melbourne University for the good quality TEM images.

I would like to thank Dr. Yoshihiko Takede at the Ion Beam Group, Quantum Beam Center, National Institute for Material Science in Sakura, Japan for the MOKE data.

I would like to thank Dr. Catalin Harnagea at the Institut National de la Recherche Scientifique, Centre-Énergie, Matériaux et Télécomunications in Varennes, Canada for the time and access to the piezoelectric force microscopy carried out within this thesis.

I would like to express my gratitude to the GNS Science staff and the Ion Beam Analysis group for their help, time and encouragement. 
I would like to thank Dr. Yves Grosdidier at the Universite de Sherbrooke, Canada, and Dr. Hugo Martel at the Université Laval, Canada, for their letter of recommendation to join this $\mathrm{PhD}$ research.

I am very thankful for the financial support of this thesis by the MacDiarmid Institute, doctoral scholarship, and by Victoria University of Wellington for the granted Victoria doctoral submission scholarship. 
À la mémoire de nos disparues.

À ceux que l'on aime et à ceux qui nous aime...

"When it is dark enough, you can see the stars" Charles Austin Beard 1927

"A man chooses, a slave obeys" Andrew Ryan 2007 "It's kind of fun to do the impossible" Walt Disney "Tomorrow is an other day to dream" Anonymus 



\section{Table of contents}

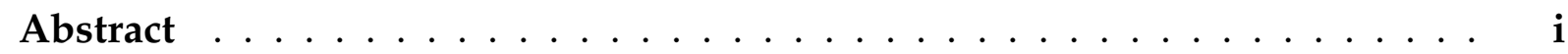

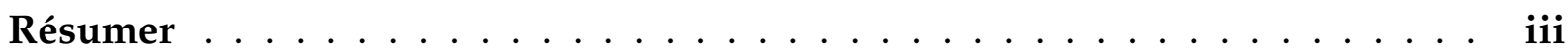

Acknowledgments ........................

Table of contents $\ldots \ldots \ldots \ldots \ldots \ldots \ldots \ldots$

Figures Index $\ldots \ldots \ldots \ldots \ldots \ldots \ldots \ldots \ldots \ldots \ldots \ldots \ldots \ldots \ldots$

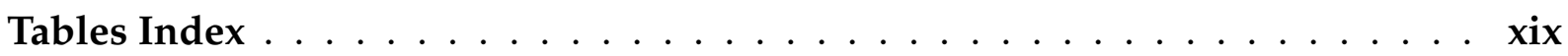

1 Introdution $\ldots \ldots \ldots \ldots \ldots \ldots \ldots$

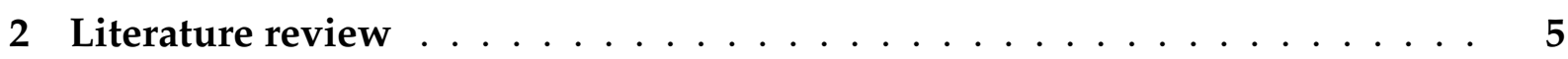

2.1 Magnetic properties . . . . . . . . . . . . . 5

2.1.1 Hamiltonian . . . . . . . . . . . . . . . . 6

2.1.2 Heisenberg 3D model . . . . . . . . . . . . . . . 7

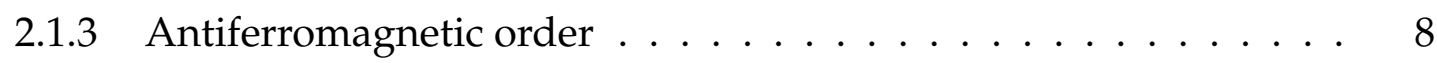

2.1.4 Superparamagnetism . . . . . . . . . . . . . . . 10

2.1.5 Blocking temperature . . . . . . . . . . . . . . . . 11

2.1.6 Exchange bias and domain wall . . . . . . . . . . . . . . . 11

2.1 .7 Spin wave . . . . . . . . . . . . . . . . . . 14

2.1 .8 Spin glass and spin disorder . . . . . . . . . . . . . . . . . . . . . . . . . . . . . . . . .

2.1 .9 Magnetic model . . . . . . . . . . . . . . . . . . 18

2.1 .10 Spin canting . . . . . . . . . . . . . . . . . . . 18

2.2 Multiferroics . . . . . . . . . . . . . . . . . . . . 19

2.2.1 Ferroelectric . . . . . . . . . . . . . . . . . . . . 19

2.2.2 Electric and magnetic coupling . . . . . . . . . . . . . . 21

2.2.3 Multiferroic model . . . . . . . . . . . . . . . 22

2.2.4 Strain, displacement and magnetic ordering . . . . . . . . . . . 24

2.3 Properties and crystal structure of $\mathrm{BiFeO} 3 \ldots \ldots$. . . . . . . . . . 26

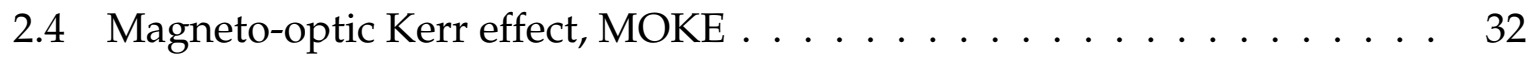

2.4 .1 Verdet constant . . . . . . . . . . . . . . 33

2.4 .2 Faraday effect . . . . . . . . . . . . . . . 34

2.5 Synthesis and preparation . . . . . . . . . . . . 35 


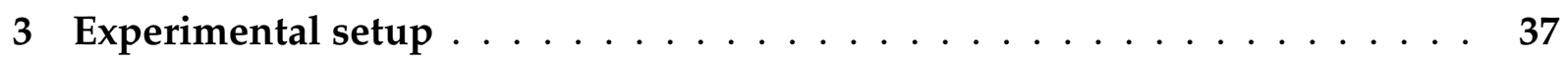

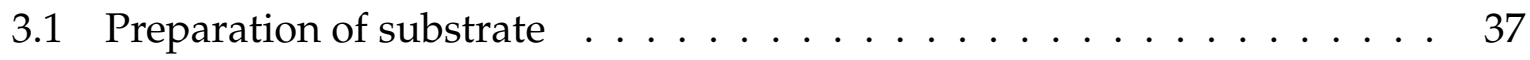

3.1 .1 Selected substrates $\ldots \ldots \ldots \ldots \ldots$

3.1 .2 Substrates treatment $\ldots \ldots \ldots \ldots \ldots$

3.2 Synthesis techniques $\ldots \ldots \ldots \ldots \ldots$

3.2 .1 Ion beam sputtering $\ldots \ldots \ldots \ldots \ldots \ldots$

3.2 .2 Ion implantation $\ldots \ldots \ldots \ldots \ldots \ldots \ldots$

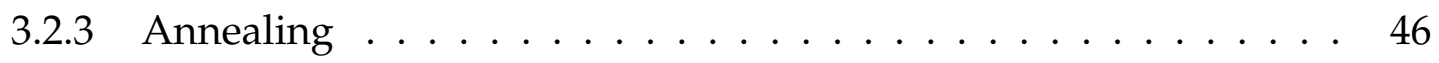

3.3 Characterisation techniques $\ldots \ldots \ldots \ldots \ldots \ldots \ldots \ldots$

3.3.1 Rutherford backscattering, RBS . . . . . . . . . . . . 50

3.3 .2 X-ray diffraction, $\mathrm{XRD} \ldots \ldots \ldots \ldots \ldots \ldots$

3.3 .3 Microscopy . . . . . . . . . . . . . . . . 60

3.3.4 Raman spectroscopy $\ldots \ldots \ldots$. . . . . . . . . . . . . 65

3.3.5 Magnetometer, magnetic moment ordering measurement . . . . 66

3.3.6 Optical light transmission and absorption . . . . . . . . . . 69

4 Evolution of the structure, surface and magnetic properties of sputtered

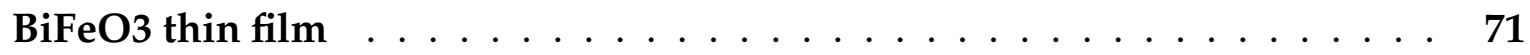

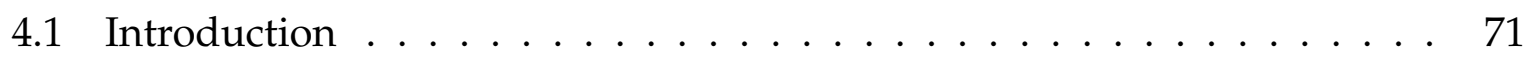

4.2 Experimental details of ion beam sputtering . . . . . . . . . 74

4.3 Synthesis and structure of BiFeO3: ion beam sputtering . . . . . . . 74

4.3.1 Rutherford backscattering, RBS . . . . . . . . . . . . 74

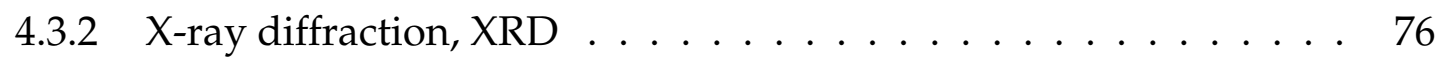

4.3.3 Atomic force microscopy, AFM . . . . . . . . . . . . 79

4.3.4 Transmission electron microscopy, TEM . . . . . . . . . 80

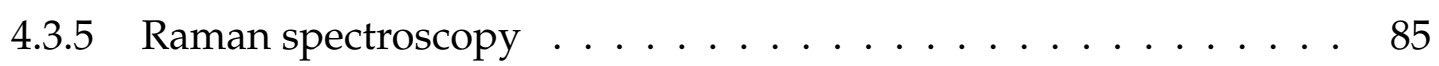

4.3.6 Optical measurements . . . . . . . . . . . . . . . 87

4.4 Magnetic properties of $\mathrm{BiFeO} 3$ at nanoscale $\ldots \ldots \ldots \ldots \ldots . \ldots . \ldots 9$

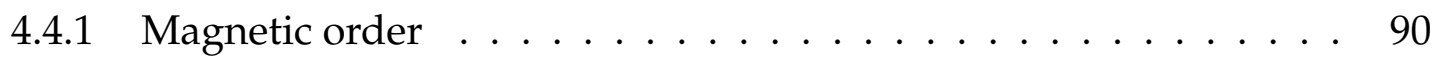

4.4 .2 Superparamagnetism . . . . . . . . . . . . . . 92

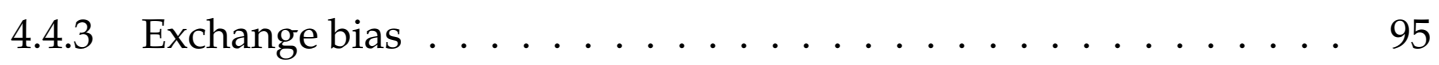

4.4.4 Magnetic force microscopy $\ldots \ldots \ldots \ldots \ldots$

4.5 Electrical properties, ferroelectricity of $\mathrm{BiFeO} 3 \ldots \ldots$. . . . . . . . 99

4.5.1 Piezoelectric force microscopy, PFM _ . . . . . . . . . . 99

4.5 .2 Kelvin probe microscopy, KPM . . . . . . . . . . . . . . 101

4.6 Magneto-optic Kerr effect, MOKE . . . . . . . . . . . . . . . . . . . . . . . . . . . . . . . . . . . . . . . . . .

4.6 .1 Verdet constant . . . . . . . . . . . . . . . . . . . . . . 105

4.6.2 Magnetic circular dichroism . . . . . . . . . . . . . . . 106 
4.6 .3 Kerr rotation . . . . . . . . . . . . . . . . . . . . . . . 108

4.7 Thin film annealed at $600{ }^{\circ} \mathrm{C} \ldots \ldots \ldots$. . . . . . . . . . . . . . 109

4.8 Thin film annealed at $500{ }^{\circ} \mathrm{C}$ for 7 hours . . . . . . . . . . . . . 113

4.9 Summary: Annealing effects . . . . . . . . . . . . . . . . 117

4.10 Conclusion . . . . . . . . . . . . . . . . . . . . . . . . 124

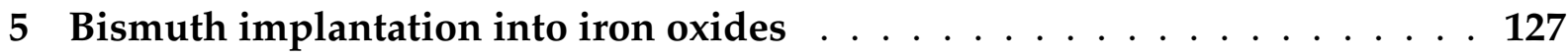

5.1 Experimental details . . . . . . . . . . . . . . . . . 128

5.2 As-made Iron oxide sputtered film, SP1 . . . . . . . . . . . . . . 130

5.3 Annealed Iron oxide sputtered film, SP2 _ . . . . . . . . . . . . 136

5.4 Simulation of Bismuth implantation . . . . . . . . . . . . . . . 139

5.5 Annealed iron oxide film implanted with $\mathrm{Bi}$ ions and annealed a second time, SP2-Bi-A . . . . . . . . . . . . . . . . . . . . . . . 142

5.6 Atomic force microscopy, AFM . . . . . . . . . . . . . . . 151

5.7 Conclusion . . . . . . . . . . . . . . . . . . . 153

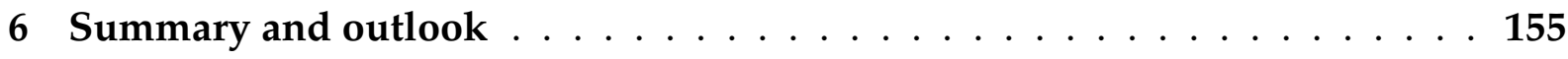

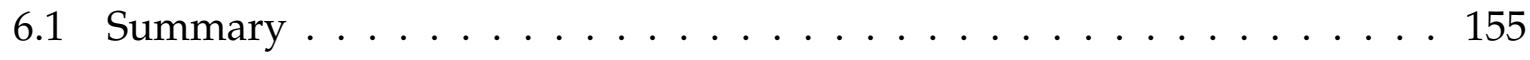

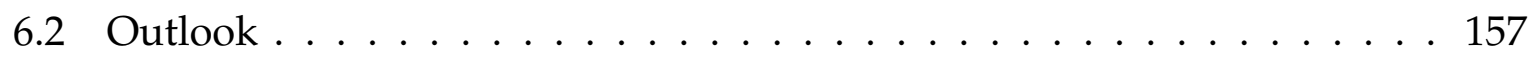

A Publication ........................... 159

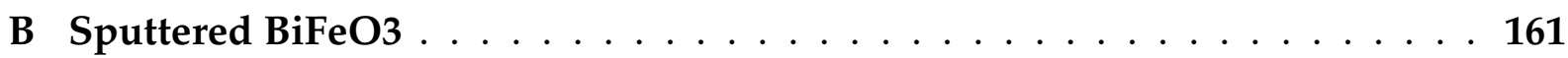

B.1 RUMP: complementary RBS analysis . . . . . . . . . . . . . 161

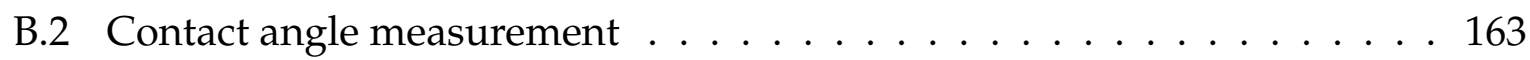

C Bismuth implanted iron oxide . . . . . . . . . . . . . 167

C.1 SP2-Bi-A: RUMP fit . . . . . . . . . . . . . . . . . . . . . . 167

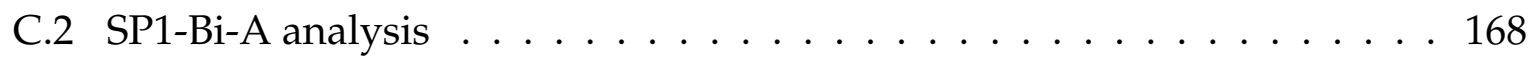

D Triple ion implantation, $\mathrm{Bi}, \mathrm{Fe}$ and $\mathrm{O}$ into $\mathrm{SiO} 2$ and $\mathrm{Si} \ldots \ldots \ldots$. . . . 177

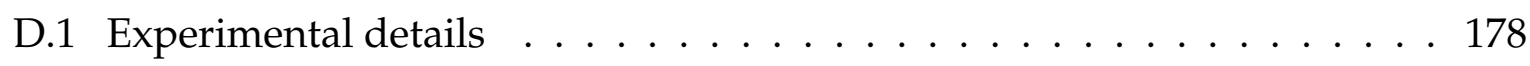

D.2 Implantation of Bi then Fe then O into SiO2:Si, IM1 . . . . . . . . . . 179

D.3 Implantation of Fe then $\mathrm{O}$ then Bi into $\mathrm{SiO2}: \mathrm{Si}$, IM2 $\ldots \ldots \ldots \ldots \ldots$

D.4 Implantation of Fe then $\mathrm{O}$ then Bi into Si, IM3 . . . . . . . . . . . . 195

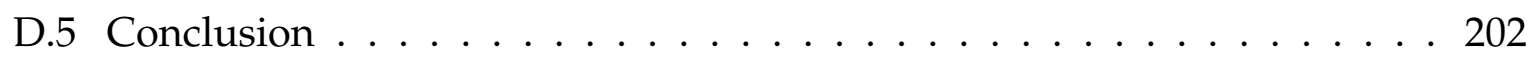

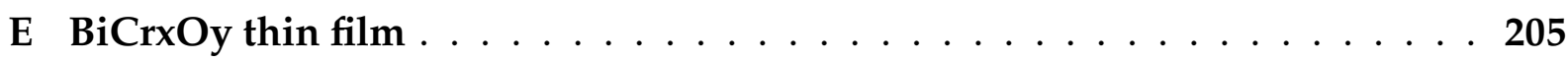

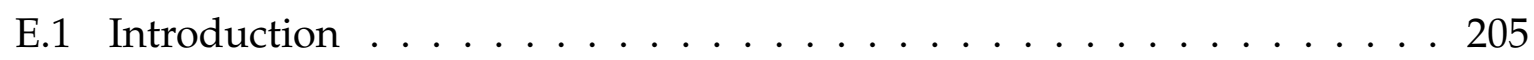

E.2 Experimental details . . . . . . . . . . . . . . . . 207

E.3 Synthesis and structure of BiCrxOy thin film _ . . . . . . . . . 207 
E.3.1 Rutherford backscattering, RBS . . . . . . . . . . . . . . . 207

E.3.2 X-Ray diffraction, XRD . . . . . . . . . . . . . . . . . . . 212

E.3.3 Transmission electron microscopy, TEM . . . . . . . . . . . . . . 214

E.3.4 Raman spectroscopy . . . . . . . . . . . . . . . . . . 216

E.3.5 Magnetic properties . . . . . . . . . . . . . . . . . . . . . . . . . . . . . . . . . . . . . . . . . . . . . . . . . . . .

E.4 Conclusion . . . . . . . . . . . . . . . . . . . . 225

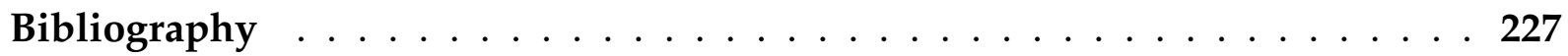




\section{Figures Index}

2.1.1 Visual representation of magnetic and electrical order . . . . . . . . 5

2.1.2 Magnetic domain modifications under an applied field . . . . . . . . 14

$2.3 .1 \mathrm{BiFeO}_{3}$ unit cell . . . . . . . . . . . . . . . . . 27

2.4 .1 MOKE modes . . . . . . . . . . . . . . . . . . . . 32

2.4 .2 Band gap structure of $\mathrm{BiFeO}_{3} \ldots \ldots \ldots \ldots \ldots$

3.2.1 Ion beam sputtering system schematic . . . . . . . . . . . . . . 40

3.2 .2 Ion implanter schematic . . . . . . . . . . . . . . . . . . . . 44

3.2.3 Theoretical simulation of Fe implantation into $\mathrm{BiFeO}_{3} \ldots \ldots$. . . . . . 46

3.2.4 Annealing furnace, GNS Science . . . . . . . . . . . . . . . 47

3.3.1 Mass resolution with an incident He ions . . . . . . . . . . . . . 52

3.3.2 Detector's angle effect on cross-section . . . . . . . . . . . . . . 53

3.3.3 Incident energy beam effect on the cross-section $\ldots \ldots \ldots 3$

3.3.4 Example of RBS spectrum to illustrate the depth scale of a thin film . 54

3.3.5 Schematic of Rutherford backscattering . . . . . . . . . . . . 55

3.3.6 Graphical representation of Bragg's Law . . . . . . . . . . . . . . . 57

3.3.7 Graphical representation of Bragg's Law, grazing angle . . . . . . . . 58

3.3.8 Atomic force microscopy, Leonard Jones potential . . . . . . . . . . 60

3.3 .9 Schematic of a SQUID sensor . . . . . . . . . . . . . . 67

3.3.10 Example of SQUID sample mounted in plastic holder . . . . . . . . . 68

4.3 .1 RBS spectra of thin film . . . . . . . . . . . . . . . 75

4.3.2 XRD, grazing angle $2^{\circ}$, from as-made and $500{ }^{\circ} \mathrm{C}$ annealed films . . . 77

4.3.3 X-ray diffraction of the $\mathrm{BiFeO}_{3}$ sputter target . . . . . . . . . . 78

4.3.4 AFM 3D topography of sputtered film . . . . . . . . . . . 80

4.3.5 Cross-sectional TEM images of an as-made film deposited on $\mathrm{SiO}_{2}: \mathrm{Si} \quad 81$

4.3.6 SAED micrograph of as-made film deposited . . . . . . . . . . . . 81

4.3.7 Cross-sectional, higher resolution, TEM images of an as-made film . $\quad 82$

4.3.8 Cross-sectional TEM images of the $500{ }^{\circ} \mathrm{C}$ annealed film deposited

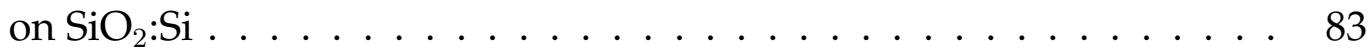

4.3.9 SAED micrograph of $500{ }^{\circ} \mathrm{C}$ annealed film deposited . . . . . . . . . . 83

4.3.10 Cross-sectional, higher resolution, TEM images of $500{ }^{\circ} \mathrm{C}$ annealed film 84

4.3.11 Raman spectra from the as-made and $500{ }^{\circ} \mathrm{C}$ annealed films . . . . . 85

4.3.12 Tauc plot of $\mathrm{BiFeO}_{3}$ annealed, $500{ }^{\circ} \mathrm{C} \ldots \ldots$. . . . . . . . . 87 
4.3.13 Optical transmission of the as-made and annealed $\mathrm{BiFeO}_{3}$ thin film . 87

4.4.1 Magnetic moment per Fe from an as-made film . . . . . . . . . . . . 90

4.4.2 Magnetic moment per Fe for as-made and $500{ }^{\circ} \mathrm{C}$ annealed $\mathrm{BiFeO}_{3}$ film at $5 \mathrm{~K} \ldots \ldots \ldots \ldots . \ldots \ldots 1$

4.4.3 ZFC-FC magnetisation at $10 \mathrm{mT}$ for an as-made film . . . . . . . . . 93

4.4.4 Magnetic moment per Fe for the $500{ }^{\circ} \mathrm{C}$ annealed film . . . . . . . . . 94

4.4.5 ZFC-FC magnetisation at $10 \mathrm{mT}$ for the $500{ }^{\circ} \mathrm{C}$ annealed film . . . . . 95

4.4.6 Exchange bias field plotted against temperature for an as-made and at $500{ }^{\circ} \mathrm{C}$ film . . . . . . . . . . . . . . . . . 96

4.4.7 Schematic representation of possible core-shell particles . . . . . . . . 97

4.4.8 Magnetic force microscope (MFM) as-made . . . . . . . . . . . . . . 98

4.4.9 Magnetic force microscope (MFM) annealed $500{ }^{\circ} \mathrm{C}, 15 \mathrm{~min}$. . . . . . 98

4.5.1 PFM of the $\mathrm{BiFeO}_{3}$ annealed at $500{ }^{\circ} \mathrm{C}$ thin film . . . . . . . . . . . 100

4.5.2 KPM, surface potential of the as-made thin film . . . . . . . . . . . . 101

4.5.3 KPM, surface potential of the $\mathrm{BiFeO}_{3}$ annealed at $500{ }^{\circ} \mathrm{C}$ thin film . . 102

4.6.1 Kerr rotation of $\mathrm{BiFeO}_{3}$ as a function of the applied field . . . . . . . . 103

4.6.2 Minimum and maximum Kerr rotation measured in function of the annealing temperature . . . . . . . . . . . . . . . . 104

4.6 .3 Verdet constant of $\mathrm{BiFeO}_{3}$. . . . . . . . . . . . . . . 105

4.6.4 $\mathrm{MCD}$ of $\mathrm{BiFeO}_{3}$ as a function of the applied field . . . . . . . . . 106

4.6.5 $\mathrm{MCD}$ of $\mathrm{BiFeO}_{3}$ as a function of the wavelength . . . . . . . . . . . 107

4.6.6 Energy value of the minimum and maximum MCD . . . . . . . . 108

4.6.7 Energy value of the minimum and maximum Kerr rotation . . . . . . 109

4.7.1 RBS spectra of the film annealed at $600{ }^{\circ} \mathrm{C}$. . . . . . . . . . . . . . 110

4.7.2 XRD, grazing angle $2^{\circ}$, from the film annealed at $600{ }^{\circ} \mathrm{C} \ldots \ldots \ldots$

4.7.3 Magnetic moment of $\mathrm{BiFeO}_{3}$ annealed at $600{ }^{\circ} \mathrm{C}$ for $15 \mathrm{~min}$. . . . . . 112

4.7.4 ZFC and FC magnetisation at $10 \mathrm{mT}$ for the $600{ }^{\circ} \mathrm{C}$ annealed film . . 112

4.7.5 Normalised saturation moment per Fe against temperature at $6 \mathrm{~T}$. . 113

4.8.1 RBS spectra for the film annealed at $500{ }^{\circ} \mathrm{C}$ for 7 hours . . . . . . . . . 114

4.8.2 XRD, grazing angle $2^{\circ}$, from film annealed at $500{ }^{\circ} \mathrm{C}$ for 7 hours . . 115

4.8.3 Magnetic moment of $\mathrm{BiFeO}_{3}$ annealed at $500{ }^{\circ} \mathrm{C}$ for 7 hours . . . . . . 116

4.8.4 Normalised saturation moment per Fe of the sample annealed at $500{ }^{\circ} \mathrm{C}$ for 7 hours . . . . . . . . . . . . . . . . . 116

4.8.5 ZFC and FC magnetisation at $10 \mathrm{mT}$ for the film annealed at $500{ }^{\circ} \mathrm{C}$, 7 hours . . . . . . . . . . . . . . . . 117

4.9.1 Crystallographic changes, grazing angle $2^{\circ}$, after oxygen-annealing at different temperatures . . . . . . . . . . . . . . . . . 118

4.9.2 Raman shift changes after oxygen-annealing at different temperatures 119 
4.9.3 Optical transmission after oxygen-annealing at different temperatures 120

4.9.4 Magnetic moment per Fe, after an oxygen-annealing at different temperatures . . . . . . . . . . . . . . . . 122

4.9.5 Magnetic moment per Fe, after oxygen-annealing at $500{ }^{\circ} \mathrm{C}$ for $15 \mathrm{~min}$ and 7 hours . . . . . . . . . . . . . . . . . . . 122

5.2.1 XRD, grazing angle $2^{\circ}$, of as-made iron oxide thin film, SP1 . . . . . 130

5.2.2 XRD, grazing angle $2^{\circ}$, of the as-made iron oxide film, SP1, pseudo Voigt . . . . . . . . . . . . . . . . . . 131

5.2.3 Raman spectroscopy of the as-made iron oxide sputtered film, SP1 . . 132

5.2.4 Magnetic moment analysis of the as-made iron oxide film, SP1 . . . . 133

5.2.5 Exchange bias of as-made iron oxide film, SP1 . . . . . . . . . . . 134

5.2.6 Temperature dependence at $6 \mathrm{~T}$ of the magnetic moment on the asmade iron oxide film, SP1 f . . . . . . . . . . . . . . . . 135

5.2.7 ZFC-FC plot for the as-made iron oxide film, SP1, at $10 \mathrm{mT}$. . . . . 135

5.3.1 XRD, grazing angle $2^{\circ}$, analysis of the iron oxide film annealed at

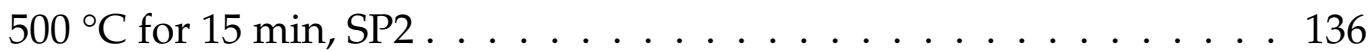

5.3.2 XRD, grazing angle $2^{\circ}$, analysis of SP2, pseudo Voigt fit . . . . . . . 136

5.3.3 Raman spectrum of the annealed iron oxide sputtered film at $500{ }^{\circ} \mathrm{C}$ for $15 \mathrm{~min}, \mathrm{SP} 2 \ldots \ldots$. . . . . . . . . . . . . . . 137

5.3.4 Magnetic moment per Fe at $5 \mathrm{~K}$ and $300 \mathrm{~K}$ for the iron oxide annealed at $500{ }^{\circ} \mathrm{C}, \mathrm{SP} 2 \ldots \ldots \ldots$. . . . . . . . . . . . . . . . . . . . . . . . . . . . . .

5.4.1 Theoretical simulation of Bi implantation into $\mathrm{Fe}_{3} \mathrm{O}_{4} \ldots \ldots$. . . . . . 139

5.4.2 Depth profile of $2 \times 10^{16}$ Bi.cm ${ }^{-2}$ into iron oxide . . . . . . . . . . . . 141

5.5 .1 RBS spectra of SP2-Bi-A . . . . . . . . . . . . . . . . 142

$5.5 .2 \quad \mathrm{XRD}$, grazing angle $5^{\circ}$, of SP2-Bi-A . . . . . . . . . . . . 144

5.5 .3 TEM image of SP2-Bi-A . . . . . . . . . . . . . . . . . . 145

5.5 .4 Raman spectrum of SP2-Bi-A . . . . . . . . . . . . . . . 147

5.5.5 Magnetic moment measurement carried out on SP2-Bi-A . . . . . . 148

5.5.6 Temperature dependence at $6 \mathrm{~T}$ of the magnetic moment per Fe on SP2-Bi-A . . . . . . . . . . . . . . . . . . . . 149

5.5.7 ZFC-FC carried out on SP2-Bi-A at $10 \mathrm{mT} \ldots \ldots . . \ldots 150$

5.5.8 Exchange bias and oercive field measurements of SP2-Bi-A . . . . . . 151

5.6.1 AFM, 3-D image of the surface of SP1 and SP2 . . . . . . . . . . 152

5.6.2 AFM, 3-D image of the surface of SP1-Bi-A and SP2-Bi-A . . . . . . 153

B.2.1 Wettability of $\mathrm{BiFeO}_{3}$ thin film . . . . . . . . . . . . 163

B.2.2 contact angle measured on $\mathrm{BiFeO}_{3}$ thin film . . . . . . . . . . . 164 
C.2.1 RBS of SP1-Bi-A . . . . . . . . . . . . . . . . . . . . . . 169

C.2.2 XRD, grazing angle $5^{\circ}$, of SP1-Bi-A . . . . . . . . . . . . 170

C.2.3 TEM image of SP1-Bi-A . . . . . . . . . . . . . . . . . . . . 171

C.2.4 Raman spectrum of SP1-Bi-A . . . . . . . . . . . . . . . 172

C.2.5 Magnetic moment per Fe for SP1-Bi-A . . . . . . . . . . . . . . 174

C.2.6 ZFC-FC of SP1-Bi-A . . . . . . . . . . . . . . . . . . . . 174

C.2.7 Temperature dependence of the magnetic moment carried out on SP1-Bi-A . . . . . . . . . . . . . . . . 175

C.2.8 Exchange bias and coercive field measurements of SP1-Bi-A . . . . 176

D.2.1 D-Trim simulation of IM1 . . . . . . . . . . . . . . . . . . 179

D.2.2 RBS analysis IM1-A . . . . . . . . . . . . . . . . . . 180

D.2.3 XRD, grazing angle of $4^{\circ}$, analysis of IM1-A . . . . . . . . . . . . 182

D.2.4 Raman spectra of IM1-A . . . . . . . . . . . . . . . . . . . 183

D.2.5 Field loop of the IM1-A . . . . . . . . . . . . . . . . . . . 184

D.2.6 ZFC-FC of IM1-A . . . . . . . . . . . . . . . . . . 185

D.3.1 D-Trim simulation of IM2 . . . . . . . . . . . . . . . 186

D.3.2 RBS of the Implanted Fe then $\mathrm{O}$ then $\mathrm{Bi} \ldots \ldots$. . . . . . . . . . . 187

D.3.3 RBS of IM2 and IM2-A . . . . . . . . . . . . . . . . . . . . . 189

D.3.4 XRD, grazing angle $5^{\circ}$ of IM2 and IM2-A . . . . . . . . . . . . 189

D.3.5 Raman spectrum of IM2 and IM2-A . . . . . . . . . . . . . . . . 190

D.3.6 Field loop of IM2 _ . . . . . . . . . . . . . . . . . . . . 191

D.3.7 Field loop of IM2-A . . . . . . . . . . . . . . . . . . . 192

D.3.8 ZFC-FC of IM2 and IM2-A . . . . . . . . . . . . . . . . . . 193

D.3.9 Temperature dependence of the saturated magnetic moment for IM2 and IM2-A . . . . . . . . . . . . . . . . . . . . . 194

D.4.1 D-Trim simulation of IM3 . . . . . . . . . . . . . . . . . . . 195

D.4.2 RBS of IM3 and IM3-A . . . . . . . . . . . . . . . . . 196

D.4.3 Raman spectrum of IM3 and IM3-A . . . . . . . . . . . . . . . . . . . . . . . . . . . . . . . . . . . . . . .

D.4.4 Field loop of IM3 and IM3-A . . . . . . . . . . . . . . . . . . . . . . . . . . . . . . . . . . . . . . . . . . . . .

D.4.5 ZFC-FC of IM3 and IM3-A . . . . . . . . . . . . . . . 201

E.1.1 $\mathrm{BiCrO}_{3}$ unit cell . . . . . . . . . . . . . . . . . . 206

E.3.1 RBS spectra of sputtered $\mathrm{BiCr}_{x} \mathrm{O}_{y}$ as-made and annealed at $500{ }^{\circ} \mathrm{C}$. . 208

E.3.2 RBS of the sputtered $\mathrm{BiCr}_{x} \mathrm{O}_{y}$ annealed at $700{ }^{\circ} \mathrm{C} \ldots \ldots . . .209$

E.3.3 XRD data of, as-made, annealed $400{ }^{\circ} \mathrm{C}$ and $500{ }^{\circ} \mathrm{C}, \mathrm{BiCr}_{x} \mathrm{O}_{y} \ldots \ldots 212$

E.3.4 XRD of $\mathrm{BiCrO}_{3}$ film annealed at $700{ }^{\circ} \mathrm{C}$ in $\mathrm{O}_{2}$ subsequently in $\mathrm{Ar}$. . . 213

E.3.5 XRD of $\mathrm{BiCrO}_{3}$ sputter target . . . . . . . . . . . . . . . . 214

E.3.6 TEM of $\mathrm{BiCr}_{x} \mathrm{O}_{y}$ as-made . . . . . . . . . . . . . . . . . 215 
E.3.7 TEM of $\mathrm{BiCr}_{x} \mathrm{O}_{y}$ annealed at $400{ }^{\circ} \mathrm{C} \ldots \ldots \ldots \ldots \ldots \ldots \ldots \ldots \ldots$

E.3.8 Raman spectrum of the $\mathrm{BiCr}_{x} \mathrm{O}_{y}$ film . . . . . . . . . . . . 216

E.3.9 Raman spectrum of the $\mathrm{BiCr}_{x} \mathrm{O}_{y}$ film annealed at $700{ }^{\circ} \mathrm{C}$ in $\mathrm{O}_{2}$ and subsequently in $\mathrm{Ar} \ldots \ldots \ldots$. . . . . . . . . . 217

E.3.10 Raman spectrum of the $\mathrm{BiCrO}_{3}$ sputter target . . . . . . . . . . 218

E.3.11 Magnetic moment of $400{ }^{\circ} \mathrm{C}$ annealed $\mathrm{BiCr}_{x} \mathrm{O}_{y}$ film . . . . . . . . . . 219

E.3.12 As-made $\mathrm{BiCr}_{x} \mathrm{O}_{y}$ thin film, magnetic moment per $\mathrm{Cr} \ldots \ldots . .219$

E.3.13 ZFC-FC, as-made $\mathrm{BiCr}_{x} \mathrm{O}_{y}$ thin film magnetic moment per $\mathrm{Cr} \ldots 220$

E.3.14 Temperature dependence of the magnetic moment per $\mathrm{Cr}$ at $6 \mathrm{~T}$ for the as-made $\mathrm{BiCr}_{x} \mathrm{O}_{y}$ film . . . . . . . . . . . . . . . . 220

E.3.15 Magnetic moment per $\mathrm{Cr}$ for the film annealed at $700{ }^{\circ} \mathrm{C}$ in oxygen . 221

E.3.16 ZFC-FC of the film annealed at $700{ }^{\circ} \mathrm{C}$ in oxygen . . . . . . . 222

E.3.17 Field loop of the film annealed at $700{ }^{\circ} \mathrm{C}$ in $\mathrm{O}_{2}$, then $\mathrm{Ar} \ldots \ldots 223$

E.3.18 ZFC-FC of the film annealed at $700{ }^{\circ} \mathrm{C}$ in $\mathrm{O}_{2}$, then $\mathrm{Ar}$. . . . . . . . . 224

E.3.19 Temperature dependence of the film annealed at $700{ }^{\circ} \mathrm{C}$ in $\mathrm{O}_{2}$, then $\mathrm{Ar} 224$ 


\section{Tables Index}

2.5.1 $\mathrm{BiFeO}_{3}$ synthesis: literature data compared with ion beam sputtering data . . . . . . . . . . . . . . . . . 36

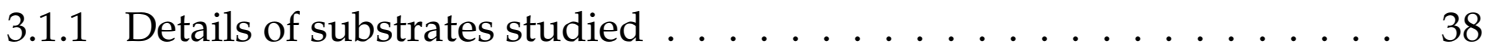

3.2.1 Ion stopping power of bismuth, iron and oxygen $\ldots \ldots \ldots \ldots$

4.9.1 Interference model parameter . . . . . . . . . . . . . . . . 120

4.9.2 Summary: Annealing effects on sputtered thin film . . . . . . . . 123

5.1.1 Sample code of the bismuth implanted iron oxides films . . . . . . . 128

5.3.1 Reported hematite Raman shift . . . . . . . . . . . . . . . . 137

B.1.1 As-made BiFeO3 RUMP fit's parameter . . . . . . . . . . . . . . . . 161

B.1.2 BiFeO3 annealed $500{ }^{\circ} \mathrm{C}, 15 \mathrm{~min}$, RUMP fit's parameter . . . . . . 162

B.1.3 BiFeO3 annealed $600{ }^{\circ} \mathrm{C}$, RUMP fit's parameter . . . . . . . . . . 162

B.1.4 BiFeO3 annealed $500{ }^{\circ} \mathrm{C}, 7$ hours, RUMP fit's parameter . . . . . . 163

C.1.1 SP2-Bi-A, RUMP fit . . . . . . . . . . . . . . . . . . . . . . 167

C.2.1 SP2-Bi-A, RUMP fit . . . . . . . . . . . . . . . . . . 168

D.1.1 Implantation order, fluence and energy of $\mathrm{Bi}, \mathrm{Fe}$ and $\mathrm{O} \quad \ldots \ldots \ldots$

D.3.1 IM2-A, RUMP fit's parameter . . . . . . . . . . . . . . . 188

E.3.1 Annealed $\mathrm{BiCr}_{x} \mathrm{O}_{y}$ at $700{ }^{\circ} \mathrm{C}$ in $\mathrm{O}_{2}$, RUMP's fit parameter . . . . . 210

E.3.2 Annealed $\mathrm{BiCr}_{x} \mathrm{O}_{y}$ at $700{ }^{\circ} \mathrm{C}$ in $\mathrm{O}_{2}$ then in Ar, RUMP's fit parameter . 211 


\section{Chapter 1}

\section{Introdution}

The $20^{\text {th }}$ century marked a revolutionary time for electronics and computing. With the first steps in computer science achieved at the beginning of the century, rapid progress enabled an increase in the number of personal computers and portable electronic devices by the end of the century. Today, tablets and smart devices are easily found in electronic stores. Research of new, smaller materials was key in making all this possible. Continued research into new and more efficient materials like multiferroics has potential to open further new technology horizons in the future.

Multiferroics are unique materials that display multiple ferroic properties (ferroelectricity, ferroelasticity and ferromagnetism) simultaneously. A more inclusive definition also includes antiferroelectricity and antiferromagnetism. Recently, the most studied multiferroic material has been bismuth ferrite, $\mathrm{BiFeO}_{3}$, which displays antiferromagnetic and ferroelectric properties at ambient temperature [Catalan and Scott, 2009]. Bismuth ferrite's ferroelectric dipole ordering and antiferromagnetic magnetic ordering are linked, allowing the magnetic ordering to alter the electrical polarisation direction while the electrical polarisation can modify the magnetic moment ordering of the material [Murakami et al., 2006]. A number of materials containing bismuth have intrinsic multiferroic properties, including $\mathrm{BiFeO}_{3}$ and $\mathrm{BiCrO}_{3}$ [Murakami et al., 2006].

The unique electrical and magnetic properties of multiferroics create numerous potential applications, such as use in spin valve devices [Dho and Blamire, 2009]. A spin valve device uses the antiferromagnetism of $\mathrm{BiFeO}_{3}$ material to control the magnetisation of a layer of ferromagnetic material via an exchange bias, leading to unidirectional anisotropy [Dho and Blamire, 2009]. $\mathrm{BiFeO}_{3}$ is also an interesting material for developing the next generation of solar panels as this material has a low band gap value $(2.67 \mathrm{eV}, 465 \mathrm{~nm})$ [Yang et al., 2010b] and excellent photovoltaic properties [Sando et al., 2013].

Despite the wide potential applications, much of the research on $\mathrm{BiFeO}_{3}$ has been focused on developing a fast, low energy and non-volatile magnetoelectric RAM 
(MeAM). MeRAM takes advantage of the $\mathrm{BiFeO}_{3}$ exchange bias, switching the magnetisation in the adjacent magnetic layer and making reading data easier [Gajek et al., 2007]. MeRAM combines the advantages of ferroelectric RAM (FeRAM), fast-reading, high-density of information and lower power usage, with the non destructive reading capability of magnetic RAM (MRAM). To identify the next generation of nano materials to build MeRAM, identifying materials with exchange biases that are able to reversibly exchange information from electrical polarisation into the magnetic domain without modifying the original data is critical.

Superparamagnetism is another interesting property to study because this state could theoretically limit the storage density available. As a function of the temperature and the size of the magnetic domain, the material can be either superparamagnetic, the magnetic domain orientation changes faster than the time required to measure those domains' orientation, or blocked in one magnetic order. Superparamagnetism is a magnetic state of a nanoscale material that is either ferromagnetic or ferrimagnetic and in which thermal energy is greater than the crystal anisotropy energy [Müller et al., 2006]. In other words, superparamagentism can be summarised as a competition between the thermal energy and the magnetocrystalline anisotropy energy of the nanoparticle. Some iron oxides are known superparamagnetic materials [Naganuma et al., 2007]. $\mathrm{BiFeO}_{3}$ is believed to display a spin glass effect, reducing the likelihood of $\mathrm{BiFeO}_{3}$ displaying a superparamagnetic state, although the iron spin canting moment could be mistakenly compared to a superparamagnetic state.

A few questions arise from bismuth-based multiferroics, such as, the systematic study of the synthesis of nanostructured multiferroic films and their structural, electronic and magnetic properties is analysis through ion beam sputtering $\mathrm{BiFeO}_{3}$ thin film and ion implantation. To investigate these topics, the nanocrystallinity, composition and the magnetic and electrical properties were studied. For the first time, the magneto-optical Kerr effect is reported on sputtered $\mathrm{BiFeO}_{3}$. The study is centre around the multiferroic properties that include the coexistence of magnetic and electric properties. Such material could serve has the basics of the next generation of faster, energy efficient memory and sensor technology. The systematic study is based on the literature review of the synthesis of $\mathrm{BiFeO}_{3}$ nanoparticle and thin film, by synthesis technic like sol-gel and pulse laser deposition. Synthesis by ion implantation was also studied, the results were compare with the literature review and the ion beam sputtered film. The motivation to use implantation technique arise from the literature confirming the formation of magnetic nanostructured material. This technique could also lead to the synthesis of heterojunction with exchange bias and potentially forming $\mathrm{BiFeO}_{3}$ nanostructures by $\mathrm{Bi}$ implantation of iron oxide thin film. A significant ad- 
vantage of doping a thin film with an ion implantation technique is to provide precise control of dopant concentration and depth.

This thesis begins with a literature review (chapter 2) summarising the history, properties and crystal structure of $\mathrm{BiFeO}_{3}$. This includes a detailed definition of multiferroic materials and the ferroic properties of $\mathrm{BiFeO}_{3}$, ferroelectricity and antiferromagnetism, the magnetic ordering of bulk material and nanoparticles. A more indepth explanation of superparamagnetic and exchange bias theory is also provided. The magneto-optical Kerr effect, Verdet constant and Faraday effect are explained.

The details of the experimental setup, including the preparation of the substrate, the sample synthesis and the characterisation techniques, are provided in chapter 3. The synthesis techniques are the ion beam sputtering, ion implantation and annealing furnace. The characterisation techniques include Rutherford backscattering spectroscopy, X-ray diffraction, microscopy (e.g. AFM, MFM, PFM), Raman spectroscopy, magnetometer (SQUID) and optical light transmission.

Chapter 4 contains the analysis of the sputtered $\mathrm{BiFeO}_{3}$ thin film. The structure, roughness, electrical properties, magnetic ordering, magneto-optical Kerr effect and optical band gap are included in this chapter. A film annealed at $600{ }^{\circ} \mathrm{C}$ for 15 minutes and a film annealed at $700{ }^{\circ} \mathrm{C}$ for 7 hours are compared to the film annealed at $500{ }^{\circ} \mathrm{C}$ for 15 minutes to study the optimisation of this synthesis technique.

Chapter 5 contains an analysis of the effect of bismuth implantation into an iron oxide film. This chapter present the structural and magnetic properties comparison of:

- as-made iron oxide film

- annealed iron oxide film

- as-made iron oxide film subsequently implanted with bismuth and annealed (presented in Appendix C.2)

- annealed iron oxide film subsequently implanted with bismuth and annealed

Chapter 6 summarises the study and provides an outline for potential future work.

The appendix contains the list of publications, complementary information for the analysis of the different films and preliminary results. The details of the RBS analysis, RUMP fit and the preliminary results of wettability of the sputtered $\mathrm{BiFeO}_{3}$ films are presented. Further details of the RUMP of the bismuth implanted iron oxide film 
are also presented. A preliminary analysis of a material synthesised by triple ion implantation $\left(\mathrm{Bi}, \mathrm{Fe}\right.$ and $\mathrm{O}$ ) into $\mathrm{SiO}_{2}: \mathrm{Si}$ and bulk $\mathrm{Si}$, with the aim to compare the resulting materials with $\mathrm{BiFeO}_{3}$ and iron phases are discussed. While ion implantation is a common technique and a double implantation has been achieved, the difficulties increase with the number of ions so the triple implantation is less frequently reported in literature. The Appendix E close with an analysis of the preliminary results of $\mathrm{BiCr}_{x} \mathrm{O}_{y}$ thin film synthesis with ion beam sputtering. The structure, phases and magnetic ordering are analysed and compared with bismuth oxide, chromium oxide and $\mathrm{BiCrO}_{3}$. 


\section{Chapter 2}

\section{Literature review}

This chapter reviews the basics of nanoparticle magnetic properties and theoretical models used to interpret the multiferroic properties. A formal introduction the state of the research of multiferroic $\mathrm{BiFeO}_{3}$ is discussed along with previously reported $\mathrm{BiFeO}_{3}$ thin film synthesis technique is presented with a discussion on the advantage and inconvenient of those techniques. The magneto-optical Kerr effect, MOKE, is discussed in this chapter.

\subsection{Magnetic properties}

Magnetic particles can align their spins in similar direction. Antiparallel; antiferromagnetic or ferrimagnetic, parallel; ferromagnetic and particles size or other material frustration sources could happen to create a spin canting, those are all possible ordering of clusters of nanoparticles, Figure 2.1.1.

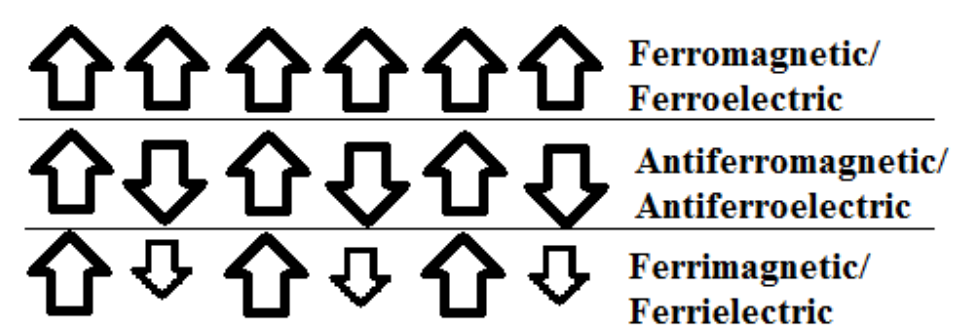

Figure 2.1.1: Visual representation of magnetic and electrical order, ferro-alignment (top), antiferro-alignment (middle) and ferri-alignment (bottom).

Magnetic nanoparticles can react to an applied magnetic field as they are often made of a magnetic metal element like iron integrated in a more complex molecule [Tadic et al., 2014]. Magnetic nanoparticle properties may change with different synthesis techniques, grain size or structure. The superparamagnetic behaviour is found in some magnetic nanoparticles [Lu et al., 2007].

There are a few types of well-studied magnetic nanoparticles, like the ferrite 
oxides, metallic particles. The iron oxides, magnetite and maghemite, are discussed with $\mathrm{BiFeO}_{3}$ in Section 2.3. They are the most studied magnetic nanoparticles, able to become superparamagnetic under the ideal condition, with grain size smaller than $\sim 130 \mathrm{~nm}$ [Lu et al., 2007]. Superparamagnetic clusters can increase the magnetic moment by manipulating individual superparamagnetic nanoparticles [Tadic et al., 2014]. After the applied magnetic field vanishes, the nanoparticles will lose their remanence like non-magnetic oxide, $\mathrm{Bi}_{2} \mathrm{O}_{3}$ [Tadic et al., 2014]. Magnetite and maghemite bonding with other molecules can improve their magnetic moment by coated silica on their surface [Kralj et al., 2010]. If other properties are required, these iron oxide materials can be coated with other elements to acquire those properties. There are several advantages to work with coated ferrites instead of metallic ions. The ferrites allow for an increasing stability, the magnetic moment depends on the cluster size or domains size and synthesis technique can modify the size of those domains and the particle's size; the particle's size may have an influence on the chemical bonding and the blocking temperature of the superparamagnetic effect [Tadic et al., 2014]. Tadic et al. [2014] displays the magnetic behaviour of Vogel-Fulcher's law and the Langevin function equation 2.1.1:

$$
M=M_{S}\left(\operatorname{coth}\left(\frac{m_{p} H}{k_{B} T}\right)-k_{B} T m_{p} H\right)+\chi H .
$$

Where $\chi$ is the high-field susceptibility, $k_{B}$ is the Boltzmann constant, $M_{S}$ is the magnetic moment at saturation, $m_{p}$ the magnetic moment of the particles. The mean magnetic particle size can be estimated from the magnetic moment, $m_{p}=\pi d^{3} M_{s} / 6$ [Tadic et al., 2014]. Goveas et al. [2015] compared the bulk and nanoparticle and confirmed that the ferromagnetism of the bulk compound originates from the superexchange between the molecules while the nanoparticles material's ferromagnetism originates from uncompensated spins at the surface.

\subsubsection{Hamiltonian}

The Hamiltonian of a 3-dimensions bulk material can be written as equation 2.1.2 [Deng et al., 2005]:

$$
\frac{H}{k_{B} T}=-K \sum_{i j}{ }^{(b)} S_{i} S_{j}-H \sum_{k}{ }^{(b)} S_{k}-K_{1} \sum_{\langle p q\rangle}{ }^{(b)} S_{p} S_{q}-H_{1} \sum_{r}{ }^{(S)} S_{r} .
$$


Where $S$ is a unit vector of number component n. $K$ parameter is the strength coupling the nearest neighbour in the bulk. $H$ and $H_{1}$ represent the induced magnetic field. $K$ and $H$ are characterising the bulk and $H_{1}$ the surface layers. The first two summations in equation 2.1.2 represent the effect of the bulk material and the other two the spin orbit interaction. In a ferromagnet both $K$ are positives.

The Hamiltonian is most of the time based on the minimum energy principle, the most stable Hamiltonian written is the one allowing to minimise the magnetic energy. This leads to interactions between near-neighbours to align themselves or be opposed at $180^{\circ}$ [Goldman, 2012]. Hamiltonians were used in this thesis to explained the absence of short magnetic range interaction in the films modified by ion implantation.

\subsubsection{Heisenberg 3D model}

The Heisenberg model is generally described as quantum of electron spin responsible for the magnetic transition a material undergoes. This is based on statistical mechanics. The Heisenberg model is an approximation solution to the random field strength system.

The Ising theory can be compared with the Heisenberg model in one dimension [Malozemoff, 1988]. While the Ising model is mainly driven by the anisotropy energy, this is not the case for the Heisenberg model [Malozemoff, 1988]. The energy per unit volume in the Heisenberg model can be written as equation 2.1.3 [Malozemoff, 1988]:

$$
W \propto-\frac{f}{l^{d / 2}}+\frac{1}{l^{2}}
$$

Where $l$ the domain size is smaller than $\delta$ the domain wall width. $\delta$ is proportional to $\sqrt{A / K} / a$ [Malozemoff, 1988].

The analysis is valid for a smooth sample, however the case where the bulk material is rough is closer to reality. The roughness is represented by the domain's centre wall to bow with an amplitude $W$ caused by an excitation at a wavelength.

The Heisenberg model, in the rough bulk, is a nonlocal energy exchange. The bowed centre of the wall domain may be well defined with a first order approximation. In this model, the electron spin located further away from the centre of distortion may 
not rotate as those spins can take part in a cancelling effect resulting in spin pressure affecting a different area of the domain wall. The rotation effect caused by the bow shape is limited to the distance $b$ from the centre of the bowed area. This model could explain the spin canting seen in $\mathrm{BiFeO}_{3}$. The derivative analysis of this case leads to equation 2.1.4:

$$
\frac{W}{L} \propto f\left(\frac{b^{\frac{4-d}{2}}}{a}\right)
$$

The Heisenberg model has a critical dimensionality of 4 to explain the physics inside the domain and domain wall magnetic energy. For a weak random field $\mathrm{f}$ and at dimensions greater than 4 , a large regular domain will be visible. This approximation reaches the infinite domain calculation as $b \rightarrow \infty$ so $W / L \rightarrow 0$. This is a limit of the Heisenberg model that can be avoided by using the Ising model, at those limits. In the case where the dimensionality is less than 4 and $\eta$ is greater than 2 and knowing the scale $b / a$ is less than $\delta$ in the Heisenberg area, in equation 2.1.4 could conclude to $f \delta^{(4-d) / 2}<4 / d$. This shows that the domain size is proportional to $W / L$ meaning if the domain size is reduced, so is the energy of the domain. Other conditions like dimensions 2 and $\eta=2$ show a moderate distortion in the domain shape while the Ising model for $\eta<1$ is to be circular, this can lead to the extrapolation of the limiting condition where the domain should be roughly circular.

The Heisenberg model for the antiferromagnetic material should be taken with caution as the analysis requires several assumptions that could be misleading. Using cylindrical domain, as required, to simplify the 3-dimension equations for the exchange of anisotropy, leads to an upper critical antiferromagnetic film thickness [Malozemoff, 1988]. While Malozemoff [1988] concludes an expansion of the domain size could create a limit to those cylinder domains, a thick antiferromagnet would not have exchange anisotropy energy. This model was used in this thesis to explained the magnetic domain seen in magnetic force microscopy.

\subsubsection{Antiferromagnetic order}

Antiferromagnetism is a magnetic ordering created by antiparallel electron dispositions of the nearest neighbours, alternating spin up and down in a regular pattern [Goldman, 2012]. The antiferromagnetic order exists at temperatures below the Néel 
temperature. This order is broken down at temperatures above the Néel temperature where materials can become paramagnetic [Néel, 1949]. Without an applied magnetic field, the antiferromagnetic material would display an absence of net magnetic moment, a magnetic moment of $0 \mu_{B}$. While applying an external magnetic field, one of the spin orientations might increase the magnetic strength, leading to a net weak magnetic moment. Like the $\alpha-\mathrm{Fe}_{2} \mathrm{O}_{3}$ hematite, the antiferromagnetic behaviour can be modified by spin canting. The magnetic ordering and the applied magnetic field may create a preferred orientation leading to a canting of some spins, some electron spins are no longer oriented at $180^{\circ}$, however they might vary a few degrees from that $180^{\circ}$ value. The canting will result in a small net magnetic moment [Goldman, 2012; Lieb et al., 1961].

Néel discovered, at low temperature, antiferromagnetic alloys follow the CurieWeiss law equation 2.1.5a [Goldman, 2012]. The Curie-Weiss law is applicable to paramagnetic material where the general formula is equation 2.1.5b [Levy, 1968]:

$$
\begin{aligned}
& \chi=\frac{\mu_{0} \mu_{B}^{2}}{3 k_{B}} N g^{2} J \frac{J+1}{T+\theta}, \\
& \chi=\frac{\mu_{0} \mu_{B}^{2}}{3 k_{B}} N g^{2} J(J+1) .
\end{aligned}
$$

Where $\theta$ is an experimentally determined constant and $\mathrm{T}_{N}$ is the Néel temperature [Goldman, 2012] and $k_{B}$ is the Boltzmann constant, $g$ is the Landé factor, $\mu_{B}$ is Bohr magneton and $J$ is the quantum angular momentum [Levy, 1968]. From the equation 2.1.5a, the magnetic susceptibility is maximum around the Néel temperature. A linear extrapolation of the relation $1 / \chi$ in function of the temperature will display a negative slope value or negative Curie point [Goldman, 2012]. This leads to postulate the neighbouring spins are antiparallel. At low temperature, the negative exchange blocks the paramagnetism seen in most magnetic material, however as the temperature increases the exchange interaction weaken and the paramagnetic behaviour is reached. This transition happens at the Néel temperature [Goldman, 2012].

The oxide materials, like the ferrites, have their magnetic ions located in the interstices, to explain the antiferromagnetic behaviour, this leads to Kramer's postulate of mechanism of metal ions exchange with oxygen, indirect exchange is named superexchange [Goldman, 2012]. In the superexchange theory, an oxygen atom creates a bond with a metallic ion where their spins align in antiparallel disposition. They leave the other oxygen electron free to pair with another metallic ion, as the two electron spins 
in the oxygen are antiparallel, this forces a preferred spin orientation on the metallic ion. The oxygen acts in a similar fashion to a chain by creating a linked to two nearest metallic ions [Goldman, 2012]. This explains the stability of the two metallic ions with antiparallel spin. Another explanation was formulated by Zener, the double exchange. In this model, the electron spin of multiple valencies are exchange, leading to $\mathrm{Fe}^{2+}-\mathrm{O}-\mathrm{Fe}^{3+}$ becomes $\mathrm{Fe}^{3+}-\mathrm{O}-\mathrm{Fe}^{2+}$ [Goldman, 2012].

Other interaction models are possible like the Ising model; the sum of the nearest pair is negative, geometrical frustration; the crystal geometry might force a magnetic ordering of the spin, the model is often a competition between a ferromagnetic and antiferromagnetic state [Wannier, 1950].

An Antiferromagnetic and a ferromagnetic material can be couple through an exchange bias mechanism. The ferromagnetic layer will align their magnetic domains with the antiferromagnetic layer; this is a property used in spin valve devices, a magnetic sensor device. The Néel temperature is still indicative of the transition from antiferromagnetic to paramagnetic, around or below that temperature the interaction between the two layers is modify and resulting in a blocking temperature. Synthetic antiferromagnetic materials are often made from layers of ferromagnetic and non-magnetic materials [Forrester and Kusmartsev, 2014].

\subsubsection{Superparamagnetism}

Superparamagnetism is a magnetic state present in some nanoscale materials that are ferro- or ferri-magnetic [Müller et al., 2006]. The small size of the material and the temperature causes the magnetic moments of some particles to randomly flip. If the magnetisation measurements are taken at intervals greater than the Néel relaxation time $\left(\tau_{N}=\tau_{0} \exp \left(K V / k_{B}\right\} T\right)$; where $\tau_{0}$ is the material's characteristic time length, $K$ is the magnetocrystalline anisotropy energy, $V$ is the volume of the sample and $T$ is the temperature), the magnetisation averages to zero under zero applied magnetic field at a temperature that is sufficiently higher than the blocking temperature. The remnant magnetisation will also relax to zero over a long enough time scale. Superparamagnetism is a competition between the thermal energy and the magnetocrystalline anisotropy energy $(K V)$ of the nanoparticle. At high temperatures, when the thermal energy exceeds $K V$, the spins will randomly flip. However at low temperatures, when $K V$ is greater than the thermal energy, the material exhibits hysteresis and remnant magnetisation [Nadeem et al., 2011]. 


\subsubsection{Blocking temperature}

The blocking temperature can be taken as an estimate of where the thermal energy and the anisotropy energy are equal. For low magnetic nanoparticle concentrations, the blocking temperature, $T_{B}$ can be written as equation 2.1.6 [DiPietro et al., 2010]:

$$
T_{B}=\frac{K V}{25 k_{B}} .
$$

Where $K$ is the magnetocrystalline anisotropy energy, $V$ is the nanoparticle volume, and $k_{B}$ is Boltzmann's constant.

Below the blocking temperature the spins are forced along the "easy" anisotropic axis. While above that temperature, the thermal energy frees the spins from their anisotropic axis and particles enter the superparamagnetic state [DiPietro et al., 2010].

\subsubsection{Exchange bias and domain wall}

To minimise the magnetostatic energy domains are formed. The macroscopic scale of the sample might show one magnetic domain under specific condition at first, however to reduce the magnetic energy the sample could be separated in domains, i.e. two ferromagnets with an orientation of $180^{\circ}$ of each other. Those ferromagnets could be split into more than two domains. Those two domains can then be split again until the system requires more energy to form new domains. After the $\mathrm{n}^{\text {th }}$ division of the domain, the total energy of the structure will be $1 / n^{\text {th }}$ the energy of the single domain structure. In general, magnetic domains contain about $10^{12}$ to $10^{15}$ atoms and their dimensions are in the order of microns $\left(10^{-6} \mathrm{~m}\right)$ [Goldman, 2012]. Impurities, non-magnetic elements, grain size and defects can also locally influence the domain structure [Goldman, 2012].

In magnetic ordered materials, magnetic moments are often measured at a low value as those materials will have a "domain formation" reducing the measured magnetic moment [McElfresh, 1994]. The minimum energy configuration in a sample is formed when the magnetic moments form magnetic domains, not necessarily when all the magnetic moments are aligned. Instead of the entire sample, those domains can have different orientations and on the macroscopic scale could even cancel each other. 
On the other side, in a magnetic domain, all the moments are aligned in one direction. The magnetic domains are not necessarily linked to the crystallographic domains, the magnetic domains are only based on the electronic spin, not the structure, although the crystal structure in some cases can enhance a preferred spin orientation like in the case of the frustrated geometry as discussed in subsection Antiferromagnetic order. The magnetic domains are only visible with magnetic imaging techniques [McElfresh, 1994]. The domain walls are the perimeter of those domains, those walls are the area where the magnetisation changes from one area to another. The irreversibility in some ferromagnetic materials is caused by the movement of those walls in the material [McElfresh, 1994]. The domain grows when an applied field provides a preference for them, the selected domain will expand the domain borders, a visual representation can be compared to a liquid spillage. To visualise a multiple domain size modification at the same time this representation could be more accurately compared to multiple liquid spillages at the same time. The territorial expansion of the preferred domain will continue until all the domains are aligned parallel to each other or this propagation could be limited by another external applied field. In the case where the entire domain forms only one domain for the entire sample, this does not mean that every spin will be aligned with the applied field. Each crystal has their preferred orientation or magnetic order that will try to follow a natural rest configuration. This phenomenon is caused by the magnetic anisotropy; the crystal minimum energy requires a specific magnetic order like antiferromagnetic or ferromagnetic. The magnetic saturation is obtained when the magnetic domain is oriented parallel to the applied magnetic field regardless of the preferred magnetic orientation [McElfresh, 1994].

The magnetostatic energy is the energy of demagnetisation and the energy required to align a magnetic pole in a specific orientation. $M_{S}$ is the saturation magnetisation and is one criterion to optimise the magnetic domain. A material of width $d$, assuming the general formula, is calculated with equation 2.1.7 [Goldman, 2012]. The equation 2.1.7 confirms previous claim, smaller width decreases the magnetostatic energy.

$$
E_{p}=\text { Constant } \cdot d \cdot M_{S}^{2}
$$

The preferred orientation comes from the angle of a spin-spin coupling between two nearest neighbour spins [Cullity and Graham, 2009]. The modification of the spinspin orientation requires energy and increases the magnetic domain with an applied magnetic field [Cullity and Graham, 2009]. In the case where the magnetic domains in 
a crystal align themselves with the crystal's structure, this alignment is called the easy direction while the hard direction is the case where the alignment does not follow one of the preferred orientations. Magnetocrystalline anisotropy energy is the difference in energy between the easy direction and the hard direction alignment or any other direction [Goldman, 2012].

If the magnetic material is stressed, the orientation of their magnetic moment will align with the direction of the stress. The magnetic moment may increase with the stress resulting in a material expansion or the material may contract while increasing the magnetic moment resulting in a material contraction with the stress. This property, magnetostriction, is caused by incomplete orbital quenching and spin-orbit [Goldman, 2012]. Stress can come from mechanical and thermal origin.

The domain wall energy, the area where the magnetisation is gradually change from the previous area to the next one, is proportional to the number of atoms contain in that layer the magnetisation must change from the initial direction to the final direction. The exchange energy in this transition layer is defined as equation 2.1.8 [Goldman, 2012]:

$$
E_{e}=\frac{k T_{C}}{a}
$$

Where $k T_{C}$ is the thermal energy at the Curie temperature and a is the distance between the atoms. So, the exchange energy decreases with the width of the wall. In the case of having an anisotropy energy, the energy of rotation required is equation 2.1.9 [Goldman, 2012]:

$$
E_{K}=k \delta
$$

$\delta$ is the thickness of the domain wall. In this case the increase layer also increases the energy. The two effects, layer thickness and energy, are opposing each other. So, the minimum energy will occur when respecting the equation 2.1.10, where $k_{a}$ is the anisotropy constant [Goldman, 2012]:

$$
E_{W}=2 \sqrt{\frac{k_{a} T_{C}}{a}}
$$


In each material, a few magnetic domain orderings can be present, from a totally random ordering of the magnetic moment to antiferromagnetic to the saturated value when the magnetic domains are reduced to only one magnetic domain in one preferred orientation. The transition from demagnetized material to saturation will be similar to Figure 2.1.2.

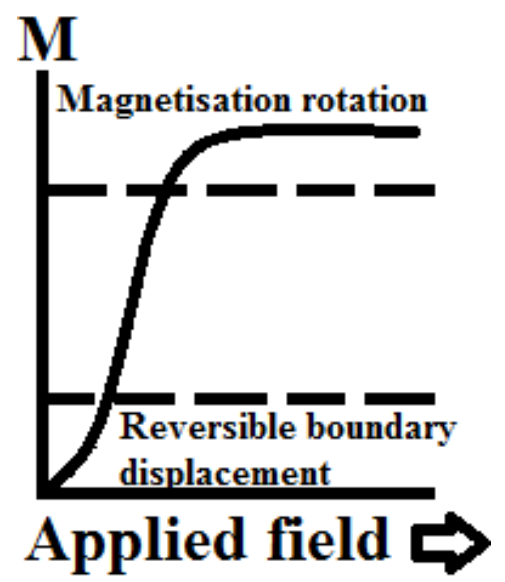

Figure 2.1.2: Magnetic domain modifications under an applied field, image based on Kittel [2005].

The Figure 2.1.2 is the magnetisation curve, where the ratio $M / H$ defines the slope of the curve as well as the susceptibility $\chi$ of the material. The lower region of the curve is called the initial susceptibility region where some domains are still able to flip between available magnetic orientations. The second area of the curve is characterised by an increasing number of irreversible domain wall movements, as the applied field increases those domains are forced in one orientation. The steep curve is also caused by a domino effect where the already aligned domains apply force to the domains that are not aligned yet. The third section, flat line, is an indication of the amount of energy required to rotate the magnetic orientation [Goldman, 2012].

\subsubsection{Spin wave}

The spin waves describe the minimum energy required to randomly flip a group of electron or nucleon spins at once. Spin waves propagation links the magnetic spin moments together. Spin waves are also known as magnons. To create a better visual representation, phonons are the structural interaction waves propagating in a material while the magnons are spin interactions propagating in a material. To solve the wave propagation situation, the Hamiltonian is written as equation 2.1.11 [Ibach and Lueth, 2009]: 


$$
H=-\sum_{i} \sum_{\delta} J_{i \delta} \cdot S_{i}-g \mu_{B} B_{0} \sum_{i} S_{i}
$$

The spin operators are represented as the Pauli spin matrices equation 2.1.12:

$$
S^{z}=\frac{1}{2}\left[\begin{array}{cc}
1 & 0 \\
0 & -1
\end{array}\right], S^{x}=\frac{1}{2}\left[\begin{array}{ll}
0 & 1 \\
1 & 0
\end{array}\right], S^{y}=\frac{1}{2}\left[\begin{array}{cc}
0 & -i \\
i & 0
\end{array}\right]
$$

A simplification in the writing can be achieved by using the spin reversal operators' equation 2.1.13:

$$
\begin{aligned}
& S^{+}=S^{x}+i \cdot S^{y}=\left[\begin{array}{ll}
0 & 1 \\
0 & 0
\end{array}\right] \\
& S^{-}=S^{x}-i \cdot S^{y}=\left[\begin{array}{ll}
0 & 0 \\
1 & 0
\end{array}\right]
\end{aligned}
$$

The operators $S^{+}$and $S^{-}$change the spin to "+" and "-". If the spin is already in the same state as the operator than the result would be zero i.e. $S^{+}|+\rangle=0$. The operator $S^{z}$ is unchanged, as described in equation 2.1.11.

The new operators can rewrite the Hamiltonian with the nearest interaction coupling $J$, equation 2.1 .12 becomes equation 2.1.14:

$$
H=-J \sum_{i} \sum_{\delta} S_{i+\delta}^{z}+\frac{1}{2}\left(S_{i}^{+} S_{i+\delta}^{-}+S_{i}^{-} S_{i+\delta}^{+}\right) .
$$

In a ferromagnet, $J$ is positive, the eigenstate would be, equation 2.1.15:

$$
|k\rangle=\frac{1}{\sqrt{N}} \sum_{j} e^{i k \cdot r_{j}}\left|\downarrow_{j}\right\rangle .
$$

In the example of the equation 2.1.15 the state $\left|\downarrow_{j}\right\rangle$ was assumed by Ibach and Lueth [2009]. In this system, the eigenvalue of $S_{i}^{z}$ and $\left(S_{i}^{x}\right)^{2}+\left(S_{i}^{y}\right)^{2}$ are conserved 
while the $S_{i}^{x}$ and $S_{i}^{y}$ are zero. This implies a precession of the spin around the $\mathrm{z}$ axis. The phase shift between the spins is determined by the vector $|k\rangle$, equation 2.1.15.

By applying the Hamiltonian to the vector $|k\rangle, E \approx E_{0}+1 / 2 J \sum_{\delta}(k \cdot r \delta)^{2}$. This implies a proportionality between the energy required to flip a spin and the $k$. The magnetisation also depends on the thermal energy as shown in the equation 2.1.16 [Ibach and Lueth, 2009]:

$$
M=M_{S}-\frac{1}{2} g \mu_{B} \frac{1}{V} \sum n(k) .
$$

Where $n(k)$ is the number of excited spin waves with the wave vector $|k\rangle$. With several assumptions, like assuming the Hamiltonian is a linear operator and assumes a supportability of wave vector, the energy could be written like a harmonic oscillator. The thermal energy on the system should follow the Bloch law where the magnetisation is of the order of $T^{3 / 2}$ [Ibach and Lueth, 2009]. The spin wave mechanism can be an important energy loss in a system.

\subsubsection{Spin glass and spin disorder}

A spin glass is a material with disordered magnetic moments, spins are not aligned in any regular pattern. The magnetic mapping of the material is amorphous like a glass structure. A spin glass material has approximately the same amount of ferromagnetic and antiferromagnetic bonds. This arrangement can lead to geometrical frustration discussed in the subsection Antiferromagnetic order, this could also lead to multiple stable geometrical configuration. The mean field theory is based on short range interaction, spin disorder that explains iron based material magnetic properties, the EdwardsAnderson model is based on the mean field theory [Binder and Young, 1986]. A critical temperature separating the spin glass behaviour and the other more stable, minimum energy system such as paramagnetic, magnetic state is observe. Above that temperature the thermal energy is providing the energy to randomly flip the spin so the system tries to recover the minimum energy alignment. Below that critical energy the material does not have enough energy to flip spins that are not aligned with the field or a relaxed state, instead the material "froze" in the current state. Cooling the material with an applied magnetic field will follow the Curie's law, equation 2.1.17 [Griffiths and College, 1999]: 


$$
M=C \frac{B}{T}
$$

Where $M$ is the magnetisation, $B$ is the magnetic field, $T$ is the temperature and $C$ is the Curie constant. When the sample reaches the critical temperature, this sample becomes a spin glass material and does not follow Curie's law, equation 2.1.17, above the threshold temperature the material behaves like a paramagnetic material while below that temperature the spin "freezes" in the material. Cooling down the material induces almost no magnetisation modification after reaching the critical temperature. A remnant magnetisation is measured when the external magnetic field is removed, allowing the spin glass spins' disposition to relax. The magnetisation decrease rapidly to the new lower value.

When the magnetisation approaches zero, losing magnetisation moment takes more time. The lost is not following an exponential decay rate anymore. This phenomenon is observed only in spin glass materials [Joy et al., 1998]. Joy et al. [1998] have recorded this phenomenon in time frames of days.

The main difference between spin glass material and other orderly magnetic materials is decay rate after removing the magnetic applied field. The spin glass may take days to reach a zero magnetisation while ferromagnetic material will maintain a preferred magnetic orientation if the material is not disturbed. A paramagnetic material has a magentic decay rate almost exponential. One model to explain the spin glass behaviour is the Edwards-Anderson model. This is a functional model at dimensions' $\mathrm{d}$ assuming interaction only with the nearest neighbours [Nishimori, 2001]. Sherrington and Kirkpatrick model is based on the symmetry and replication of spin interaction systems according to the long range Ising model for the frustrated ferromagnetic and antiferromagnetic coupling. Sherrington and Kirkpatrick model describes well the slow process involved in the spin glass material. The main comparison between this model and the Edwards-Anderson model is that Sherrington and Kirkpatrick consider every spin interaction, not only the nearest neighbours [Boettcher, 2005; Sherrington and Kirkpatrick, 1975]. The infinite-range model considers the spin interaction with a distance $r$, when $r \leq N, N$ represent the number of spin in the material and the range of the interaction leans toward infinity, $r \rightarrow \infty$. This is a generalization of the Sherrington-Kirkpatrick model. The range of spin interaction is infinite [Kirkpatrick and Sherrington, 1978]. 


\subsubsection{Magnetic model}

In a general analysis, the magnetic model in matter requires the Maxwell's equation as a reference. The interest of that is to observe the symmetry in between the electric and magnetic field [Schwinger, 1969].

Charge quantisation is another magnetic model. This model treats the magnetisation element as quanta, for a non-relativistic particle with an electric charge and a magnetic charge moving in a static body. The motion result is presented in equation 2.1.18 [Schwinger, 1969]:

$$
J=r \times p+S=L+S .
$$

Where $p$ and $S$ are the combination involved in the momentum vector $L$. The value of $S$ and $J$ provides magnetic ordering information [Levy, 1968]. This analysis is use to identify some weakly order $\mathrm{Fe}^{3+}$ and $\mathrm{Fe}^{2+}$ [Levy, 1968].

\subsubsection{Spin canting}

Antiferromagnetic material can have a small magnetic moment even at low temperatures, at $5 \mathrm{~K}$, caused by a spin canting. Spin canting is the tilting of the spin, instead of the two nearest spins being exactly antiparallel, they could be at an angle of $179^{\circ}$ or $178^{\circ}$ or even up to $160^{\circ}$. The tilting weakens the net magnetic moment in one of the two directions while the other magnetic moment direction keeps the entire value. In a perfect antiferromagnetic material, for example the up spin and down spin are both having magnetic moments equal to $1 \mu_{B}$ and they cancelled each other out at larger scale. If the up spins are titled or some of the up spins are titled so the up spins will generate a value of less than $1 \mu_{B}$ in the up direction. If the value is $0.9 \mu_{B}$, the result is a net moment of $0.1 \mu_{B}$ in the down direction, as the down spins keep their magnetic moment of $1 \mu_{B}$ [Winpenny, 2012].

The spin canting arises from two opposing forces in a material. Isotropic exchange would reduce the system energy by aligning all the spins in the antiparallel position, which is responsible for the antiferromagnet configuration. However, the spin-orbit coupling would prefer to align the spins perpendicular to each other. The 
opposition of these two effects creates a small perturbation resulting as a tilting of some spins. The strength of the perturbation varies in function of the relative strength of these effects [Winpenny, 2012].

The antiferromagnetic spin tilted effect can be observed in $\alpha-\mathrm{Fe}_{2} \mathrm{O}_{3}$ [Bhowmik and Saravanan, 2010].

\subsection{Multiferroics}

Multiferroics are materials displaying multiple ferroics properties simultaneously. Ferroics properties include ferroelectricity, ferromagnetism and ferroelasticity. A property of multiferroic materials is the ability to couple the electric and magnetic fields as described in subsection 2.2.2. Some theoretical models explain the magnetic-electric, electric-magnetic coupling and the multiferroics behaviour which are detailed in the subsection Multiferroic model. Some models study the strain and displacement of multiferroics like the oxygen octahedron in $\mathrm{BiFeO}_{3}$, subsection 2.2.4. A few materials containing bismuth have intrinsic multiferroic properties, including $\mathrm{BiFeO}_{3}$ and $\mathrm{BiCrO}_{3}$ [Murakami et al., 2006].

\subsubsection{Ferroelectric}

There are several mechanisms to explain ferroelectricity in multiferroics. Currently, the main mechanisms are related to a lone pair of electrons, the geometry of the compound, the charge ordering, magnetically driven source, and spin-spiral order. The lone pair mechanism mainly describes ferroelectricity of perovskite materials (materials with an $\mathrm{A}^{2+} \mathrm{B}^{4+} \mathrm{X}_{3}^{2-}$ stoichiometry, like $\mathrm{CaTiO}_{3}$ ) [Wenk and Bulakh, 2004]. The active lone pair between the cation " $\mathrm{A}$ " and the anion " $\mathrm{X}$ " in the s-orbital causes a hybridisation of their p-orbitals. This hybridisation changes the geometry (by modifying the centre of mass) of the atoms and creates the ferroelectric properties [Neaton et al., 2005]. In other words, the stereochemically active $\mathrm{Bi}^{3+} 6 \mathrm{~s}^{2}$ lone pair causes a $\mathrm{Bi} 6 \mathrm{p}$ orbital to have an energy close to an oxygen $2 \mathrm{p}$ orbital. This leads to Bi $6 \mathrm{p}$ and oxygen $2 \mathrm{p}$ hybridisation, which changes the geometry around the Bi atom and causes the compound to be ferroelectric. The improper geometric ferroelectric property is related to molecular geometry. This model is typically present in rare-earth manganite; where high temperatures cause changes to the structure and properties of the compounds. 
For example, $\mathrm{YMnO}_{3}$ is ferroelectric at ambient temperature and paraelectric at temperatures above $1270 \mathrm{~K}$ [Fennie and Rabe, 2005]. The phenomenon could be linked to the phonon mode, however more research is required to confirm this [Fennie and Rabe, 2005]. The ferroelectric behaviour of $\mathrm{h}-\mathrm{RMnO}_{3}$ is related to high temperature tilting of the $\mathrm{MnO}_{6}$ octahedron. Charge-ordering leading to ferroelectricity can also occur in mixed-valence compounds, or those with geometric or magnetic constraints. $\mathrm{LuFe}_{2} \mathrm{O}_{4}$ is an example of a compound featuring the mixed-valence $\mathrm{Fe}^{2+}$ and $\mathrm{Fe}^{3+}$ [Ikeda et al., 2005]. Magnetically-driven ferroelectricity occurs mainly in insulating materials and oxides. These materials require a macroscopic electrical polarisation field to induce magnetic order [Cheong and Mostovoy, 2007]. Lawes et al. [2005] developed a similar theory to Landau for explaining the impact of magnetic ordering on ferroelectric properties [Landau et al., 1984].

Heating a ferroelectric material has a similar result to heating a ferromagnet when reaching the Curie temperature. The Curie temperature happens when the thermal energy is sufficient to randomly change the electrical dipole, or magnetic spin in the case for the ferromagnet's orientation. This breaks the initial order created by the material anisotropy, causing thermal destruction of the ferroic properties. Heating the material may also lead to a change of crystal structure, the rhombohedral $\mathrm{BiFeO}_{3}$ becomes monoclinic when heated to temperatures higher than $900{ }^{\circ} \mathrm{C}$, however the new crystal structure is not necessarily ferroelectric. At least one case of ferroelectric material near the Curie temperature displaying an enhanced piezoelectric response was reported [Wylie-van Eerd et al., 2010]. The Curie-Weiss law, equation 2.2.1, represents the general dielectric constant of a ferroelectric or ferrielectric as a function of temperature:

$$
\epsilon=\epsilon_{0}+\frac{C}{T-T_{C}}
$$

Where $\epsilon$ is the dielectric constant of the material, $\epsilon_{0}$ is the permittivity of free space, $C$ is the Curie-Weiss constant, $T$ is the temperature of the thin film and $T_{C}$ is the Curie temperature; the temperature at which the dielectric constant tends to be maximum while the Curie point is defined by the temperature where a material loses a ferroelectric or ferrielectric phase and piezoelectric response [Webster and Eren, 2014]. Often the Curie point is less than $10{ }^{\circ} \mathrm{C}$ below the Curie temperature [Webster and Eren, 2014]. The ferroelectric Curie temperature of $\mathrm{BiFeO}_{3}$ is $1100 \mathrm{~K}$ [Spaldin et al., 2010]. $\mathrm{BiCrO}_{3}$ displays a much lower temperature transition, as detailed by Niitaka et al. [2004]. $\mathrm{BiCrO}_{3}$ has a dielectric transition, loss of ferroelectric behaviour, between 400 and $440 \mathrm{~K}$. This dielectric transition could be caused by a phase modification of 
$\mathrm{BiCrO}_{3}$ (Appendix E) from orthorhombic to monoclinic [Niitaka et al., 2004].

\subsubsection{Electric and magnetic coupling}

Tian et al. [2014] have confirmed the electric and magnetic coupling in metal-organic multiferroics by showing the magnetic field dependence of the dielectric constant. Below the Néel temperature, the dielectric constant seems suppressed by the applied magnetic field. This phenomenon is not seen above the transition temperature $\left(T_{N}\right)$. The electric field controlling magnetism effect is demonstrated by measuring the temperature dependence of magnetic moment with and without an applied electric field. Evidence of the influence of the electric field is shown in this experiment. Tian et al. [2014] also did a $5 \mathrm{~K}$ magnetic moment measurement as a function of the applied magnetic field, the field was limited between 0.1 to $1 \mathrm{kOe}$. The measurements were carried out with and without an applied electrical field and noticed a significant difference in the net magnetic moment. These confirm the magneto-electrical and electricalmagneto coupling.

Ederer and Spaldin [2005b] uses $\mathrm{BiFeO}_{3}$ to prove a coupling of the magnetic order and the ferroelectric configuration is possible. The Dzyaloshinskii and Moriya (DM), the antisymmetric exchange model is assumed to discuss the $\mathrm{BiFeO}_{3}$ magnetoelectric coupling [Ederer and Spaldin, 2005b]. The DM or antisymmetric exchange is based on Landau's theory [Moriya, 1960]. The antisymmetry exchange interaction comes from the spin-orbit coupling written as equation 2.2.2 [Moriya, 1960]:

$$
H_{D M}=\overrightarrow{\mathbf{D}}_{i j}\left(\overrightarrow{\mathbf{S}_{i}} \times \overrightarrow{\mathbf{S}_{j}}\right)
$$

Where $i$ and $j$ represent two neighbouring spins. The $H_{D M}$ is known as the Dzyaloshinskii-Moriya interaction term. This interaction may be the source of weak ferromagnetic or antiferromagnetic behaviour. The orientation of $\overrightarrow{\mathbf{D}}_{i j}$ follows the symmetry limitation imposition [Moriya, 1960]. In the simple case where the two neighbouring spins interact with each other by the presence of a chain link; a single ion, or ligand $\overrightarrow{\mathbf{D}}_{i j}$ is proportional to $r_{i} \times r_{j}$. Where $r_{i}$ and $r_{j}$ represent respectively the distance between the ligand and the spin $i$ and the spin $j$ [Keffer, 1962]. So, the tensor $\overrightarrow{\mathbf{D}}_{i j}$ perpendicular to the plane composed of the two spins and the bonding single ion would be zero if the trio form a line. This means to consider this phenomenon a 2-dimensions based structure is required. 
This model is important in understanding the multiferroic coupling of electric and magnetic fields. Displacing the ligand ion $\overrightarrow{\mathbf{D}}_{i j}$ by inducing magnetic ordering is possible as the materials enhance the magnetic interaction energy before the energy cost for a stable structure's stability. Under the right conditions, all the ligand ions were displaced in one specific direction creating an electric polarisation [Cheong and Mostovoy, 2007].

By neglecting the spin-orbit interaction in the DM interaction model, this results in the absence of macroscopic magnetisation caused by the magnetic moment being co-linear.

The reverse relationship, from the electric field to the magnetic field requires an intermediary; the crystal structure. To understand the impact of the structure, the weak ferroelectric behaviour is generated by the symmetry of $\mathrm{BiFeO}_{3}$. The structure is composed of the superposition of the polar displacement of all anions and cation sublattices and the antiferrodistortion originated from the oxygen octahedron rotation. The rotation of the octahedron leads to the $\mathrm{BiFeO}_{3} \mathrm{R} 3 \mathrm{c}$ space group, while the Perovskite rotation leads to the space group (Pm3m). The combination of both rotations lead to the space group R3c; the multiferroic $\mathrm{BiFeO}_{3}$ structure. Ederer and Spaldin [2005b] show the rotation of the octahedron is linked to the direction of tensor $\overrightarrow{\mathbf{D}}$, leading to the structure influencing the magnetic orientation of the crystal. $\mathrm{BiFeO}_{3}$ has the ability under an applied electric field to switch between stable crystal states (e.g. rhombohedral, monoclinic), some of those states are not ferroic while the R3c is multiferroic. With multiple possible orientations, they can even cancel each other out and explained the weak magnetic moment measured on $\mathrm{BiFeO}_{3}$ or this material's antiferromagnetic state [Ederer and Spaldin, 2005b].

\subsubsection{Multiferroic model}

Multiferroic models like the more general case of ferroic material, magnetically or electrically ordered, have domains and domain walls. The particularities of the multiferroic domains are, for the intrinsic multiferroics, to couple the electrical and magnetic ordering parameters. The coupling can modify the distribution or topography of the domains which is only possible in multiferroic materials. The coupling domains are generally homogeneous domains leading to the most significant information being in some case the average net value of the domain, not actual single value of each parameter [Heron et al., 2014]. 
An inhomogeneity of the domain in multiferroics materials may modify the multiferroic properties, creating some interaction that could weaken either the magnetic or electric order. The dimension of the domain, the width of the wall and the symmetry of the wall can all be modified by the inhomogeneity of the wall [Seidel et al., 2009]. Ferroelectric materials have a magnetoelectric interaction modified by the motion of the domain walls while multiferroic materials have a magneto-electrical coupling both magnetic and electrical ordering could be disturbed by the domain wall's motion [Hoffmann et al., 2011].

Johnson et al. [2012] found in the case of the multiferroic $\mathrm{CaMn}_{7} \mathrm{O}_{12}$ that the electric polarisation is perpendicular to the helical magnetic structure. So, the multiferroic behaviour cannot be explained with the DM model [Sergienko and Dagotto, 2006] or dynamic spin interaction. The polarisation is within the spins rotation plane [Katsura et al., 2005]. CaMn $\mathrm{O}_{12}$ allows the magnetoelectric properties by ferroaxial coupling [Johnson et al., 2012]. This explains other helical magnetic structured multiferroics. The model is based on the helical magnetic structure equation 2.2.3 [Johnson et al., 2012]:

$$
\overrightarrow{\mathbf{P}}_{z} \propto-\epsilon_{z z} \sigma \overrightarrow{\mathbf{A}}
$$

Where $\overrightarrow{\mathbf{P}}_{z}$ is the polarisation tensor, $\epsilon_{z z}$ is the dielectric constant in the free energy, $\overrightarrow{\mathbf{A}}$ is the homogeneous structural rotation axial tensor and $\sigma$ is the helical magnetic structure's chirality. $\overrightarrow{\mathbf{A}}$ is assumed constant at a specific temperature, and $\sigma=$ $r_{i j} \cdot\left(S_{i} \times S_{j}\right)$. So, $\overrightarrow{\mathbf{P}}_{z}$ is proportional to $\sigma$. In the mean-field approximation, $\overrightarrow{\mathbf{P}}_{z}$ is proportional to the temperature [Johnson et al., 2012]. In this model, the microscopic coupling spin-dipole requires more investigations due to the complexity of the system. This coupling could be caused by exchange striction with the charge ratio of $\mathrm{Mn}^{3+}$ over $\mathrm{Mn}^{4+}$ [Johnson et al., 2012].

There are a few models to explain the ferroelectric ordering in type-II multiferroics. The spin current theory [Katsura et al., 2005] and the inverse DM model [Sergienko and Dagotto, 2006; Mostovoy, 2006]; both explanations require the cycloidal magnetic structure [Ribeiro et al., 2016]. While the spin-dependent metal-ligand hybridisation [Arima, 2007] and the extended inverse-DM [Kaplan and Mahanti, 2011; Johnson et al., 2011; Johnson et al., 2012] models do not require cycloidal structure. Ribeiro et al. [2016] studied the delafossite compounds, like $\mathrm{CuFeO}_{2}$. The multiferroic behaviour is explained by spin-lattice coupling allowed in the structural symme- 
try. The spin-lattice coupling is possible by bi-quadratic symmetry and anti-symmetry. With the symmetrical arguments, the variety of models to explain type-II multiferroics and the presence of two types of polar magnetic order could explain the microscopic multiferroics behaviour [Ribeiro et al., 2016].

In the analysis of spin canting, Kaplan and Mahanti [2011] modifies the DM model by adding a few requirements. First requirement is symmetry; if the spin interaction is from spin A to spin B providing the tensor $\overrightarrow{\mathbf{D}}$ then the inverse; spin B to spin A will result in the $\overrightarrow{\mathbf{D}}$ in the opposite direction, $-\overrightarrow{\mathbf{D}}$. The other symmetrical requirement is the mirror image of the transformation; any operation will have the same result in the image coordinates. Unlike the Landau theory, the theory proposed by Kaplan and Mahanti [2011] is propagated throughout the crystal regardless of the continuous phase transition of the material. In the specific case of a 3-dimensional antiferromagnetic material, the spins will align themselves antiferromagnetically at an angle of $120^{\circ}$ caused by the spin order and the threefold axis [Kaplan and Mahanti, 2011].

\subsubsection{Strain, displacement and magnetic ordering}

A way to increase the strain on a film is to grow a magnetoelastic material on a piezoelectric substrate. The structural dimension of magnetoelastic material can be modified by applying an external magnetic field. This will introduce other strain effects which are transmitted to the piezoelectric layer and produce a polarisation in the substrate. So, the polarisation can be modified by applying a magnetic field. The interface in this scenario is responsible for the magnetoelectric coupling [Scott, 2007].

Another way to introduce strain is by doping a material, Schiemer et al. [2013] has doped $\mathrm{BiFeO}_{3}$ with calcium to study the strain and oxygen vacancies created by this ion. The Ca content causes sheer strain in the $\mathrm{BiFeO}_{3}$ rhombohedral proportional to $\cos \alpha$, where $\alpha$ is the angle of the $\mathrm{BiFeO}_{3}$ rhombohedral [Schiemer et al., 2013]. Schiemer et al. [2013] shows a reduction of the sheer strain with increasing Ca content at ambient temperature. The modification of the magnetic structure modifies the crystal structure, however the Néel temperature seems immune to those factors in Cadoped $\mathrm{BiFeO}_{3}$. The temperature maybe another factor to affect strain, between $\sim 650$ and $\sim 800 \mathrm{~K}$ the rhombohedral lattice angle varies slightly [Schiemer et al., 2013]. There is only a weak coupling of the magnetic order with the volume strain [Schiemer et al., 2013]. The strain of $\mathrm{BiFeO}_{3}$ films are not sensitive to ferroelectric polarisation or the oxygen vacancy [Ederer and Spaldin, 2005a]. The magnetic state modification from 
an antiferromagnetic ordering scheme to a weakly ferromagnetic is not linked to a macroscopic transformation of strain or other order parameters [Schiemer et al., 2013]. However, this could be caused by a local heterogeneous strain for a slightly Ca-doped $\mathrm{BiFeO}_{3}$. The doping may suppress the coupling between the gradients of magnetic order [Schiemer et al., 2013]. Oxygen vacancies gain mobility through the thermal energy. Those vacancies have a variation in their relaxation time caused by electric and stress fields [Schiemer et al., 2013].

The Curie temperature and spontaneous polarisation can be influenced by the strain effects Choi et al. [2004]. $\mathrm{BiFeO}_{3}$ is likely to have a thickness inversely proportional relation with the strain of the thin film crystal [Ederer and Spaldin, 2005a]. Other reports confirm the ferroelectric polarisation in $\mathrm{BiFeO}_{3}$ is weakly dependent on the strain in comparison to conventional ferroelectric materials. The dependence is too weak to explain the polarisation variation reported in thin film samples. Magnetisation is also weakly dependent on the strain. This dependence is caused by the ionic stability of the $\mathrm{BiFeO}_{3}$ structure, where only small variations occur with the ionic shift, while the lattice parameter undergoes a strain effect [Ederer and Spaldin, 2005a].

$\mathrm{BiFeO}_{3}$ displays a neglectable relation between the ferroelectric polarisation and the oxygen vacancies due to the high stability of the ferroelectric order in the crystal [Ederer and Spaldin, 2005a]. However, oxygen vacancies can lead to some magnetic modifications depending greatly on the position's vacancy [Ederer and Spaldin, 2005a]. The oxygen vacancies create $\mathrm{Fe}^{2+}$ ions, iron ions are known to be magnetically ordered while under an applied field. The charge differences through the different iron sites are small [Ederer and Spaldin, 2005a].

$$
\epsilon=\frac{a_{\text {hex }}}{a_{\text {hex }, 0}}-1
$$

Equation 2.2.4 is the definition of the strain in $\mathrm{BiFeO}_{3}$. Where $a_{\mathrm{hex}, 0}$ is the corresponding lattice constant for the unstrained system [Ederer and Spaldin, 2005a]. The small strain effect on the polarisation can be seen in equation 2.2.5 [Ederer and Spaldin, 2005a]:

$$
P_{S}(\epsilon)=P_{S}(\epsilon=0)+\frac{1}{V(\epsilon)} \sum_{i} Z_{i}\left(R_{i}(\epsilon)-R_{i}(\epsilon=0)\right) .
$$

Where $Z_{i}$ is the charge associated with ion $i, R_{i}(\epsilon)$ is the corresponding strain de- 
pendent ionic position, $V$ is the unit cell volume; the volume is also strain dependent, and the sum extends over all ions in the unit cell. This result is comparable to other models studying the stain-polarisation dependence.

The weak strain-ionic displacement dependence can be explained by the high Curie temperature and $\mathrm{BiFeO}_{3}$ as a ground state $\mathrm{R} 3 \mathrm{c}$ of $\sim 0.25 \mathrm{eV}$ per formula unit while the centre symmetry structure $\mathrm{R} 3 \mathrm{c}$ has a ground state of $\sim 1 \mathrm{eV}$. The stability and energy difference prevent the structural modification. The other idea to explain this stability would be to assume that the lone pair, responsible of the ferroelectric order, is inert. Generally, in Perovskite materials the ferroelectric stabilisation is caused by a charge transfer from the oxygen. The charge transfer could be reacting more to the bond length then to the charge. The oxygen octahedron could be responsible for stabilising the ferroelectric and limiting the strain induced displacement [Ederer and Spaldin, 2005a].

Ederer and Spaldin [2005a] displayed the effect of growing $\mathrm{BiFeO}_{3}$ on a substrate oriented $\langle 001\rangle$ and as they expected the $\mathrm{BiFeO}_{3}$ grown is monoclinic. With a "thin film", the orientation of $\mathrm{BiFeO}_{3}$ tends to align with the substrate, toward the $\langle 001\rangle$ direction [Ederer and Spaldin, 2005a].

The oxygen vacancies do not explain the modification of the observed magnetisation. As the DM model is involved and this model is strongly influenced by the structure. This implies that the vacancies create enough perturbation to modify the DM model significantly [Ederer and Spaldin, 2005a].

\subsection{Properties and crystal structure of $\mathrm{BiFeO3}$}

Recently, the most studied multiferroic material has been bismuth ferrite $\left(\mathrm{BiFeO}_{3}\right.$; often called BFO), as bismuth ferrite was the first material to show multiferroic behaviour at ambient temperature [Heron et al., 2014; Wang and Nan, 2006]. This material displays antiferromagnetic and ferroelectric properties at ambient temperature [Catalan and Scott, 2009]. Bismuth ferrite can reversibly transfer information from the magnetisation domain to the electrical domain [Murakami et al., 2006].

The unique electrical and magnetic properties of multiferroics creates numerous potential applications; for example, multiferroics could be use in a spin valve device [Dho and Blamire, 2009]. A spin valve device uses the antiferromagnetism of $\mathrm{BiFeO}_{3}$ 
to control the magnetisation of a layer of ferromagnetic material via an exchange bias, leading to unidirectional anisotropy [Dho and Blamire, 2009]. $\mathrm{BiFeO}_{3}$ is also an interesting material for developing the next generation of solar panels as this material has a low direct band gap (2.667(5) eV, 465 nm) [Yang et al., 2010b; Basu et al., 2008], and excellent photovoltaic properties [Sando et al., 2013]. Although, the competition is strong in this field of research.

Despite the wide potential applications, much of the research on $\mathrm{BiFeO}_{3}$ has been focused on developing fast, low energy and non-volatile magnetoelectric randomaccess memory (MeRAM). MeRAM takes advantage of the $\mathrm{BiFeO}_{3}$ exchange bias modification caused by an applied electrical field. By changing the exchange bias, the magnetisation switches in the adjacent magnetic layer, making reading data easier [Gajek et al., 2007]. MeRAM combines the advantages of ferroelectric RAM (FeRAM); fast reading, high-density information and low power, with the non-destructive reading capability of magnetic RAM (MRAM). To identify the next generation of nano-materials for MeRAM, identifying materials with exchange biases that can reversibly exchange information from electrical polarisation into the magnetisation domain without modifying the original data is critical.

$\mathrm{BiFeO}_{3}$ ferroelectricity is caused by the lone pair hybridisation, the magnetism is caused by the presence of localised electrons, mostly in the partially filled $\mathrm{d}$ or $\mathrm{f}$ shells of Bi ions which have a corresponding localised spin or magnetic moment [Khomskii, 2009]. The coexistence and interaction of the magnetic and electric ordering originates from the rotation of the Oxygen octahedral [Khomskii, 2009].

$\mathrm{BiFeO}_{3}$ is ferroelectric below $T_{c} \sim 1100 \mathrm{~K}$ and antiferromagnetic up to $T_{N} \sim 650 \mathrm{~K}$ [Wang and Nan, 2006]. The weak ferromagnetism state of $\mathrm{BiFeO}_{3}$ occurs only at low temperatures depending on how this material has been prepared. The weak ferromagnetism is also related to the antiferromagnetic properties, under certain condition the spins in the material are canting, showing behaviour similar to an antiferromagnetic material [Wang et al., 2006]. $\mathrm{BiFeO}_{3}$ has a

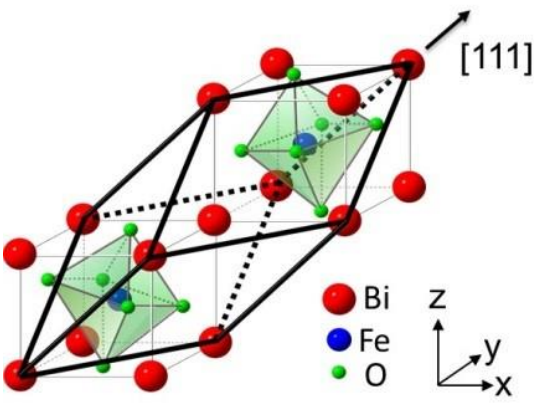

Figure 2.3.1: $\mathrm{BiFeO}_{3}$ unit cell Naganuma [2011]. distorted rhombohedral structure, Figure 2.3.1, with a crystallographic symmetry of $3 \mathrm{~m}$ [Wang et al., 2006]. The theoretical rhombohedral $\mathrm{R} 3 \mathrm{c} \mathrm{BiFeO}_{3}$ density is $8.02 \mathrm{~g} / \mathrm{cm}^{3}$ [Jain et al., 2013]. The ferroelectric properties are related to the $6 \mathrm{~s}$ lone pair of the Bismuth atom [Priya et al., 2007]. $\mathrm{BiFeO}_{3}$ has been extensively characterised. Despite the wide range of studies performed on $\mathrm{BiFeO}_{3}$, there is still a wealth of opportunities 
for further study. For example, attempting to synthesise $\mathrm{BiFeO}_{3}$ using ion implantation and sputtering, studying the doping effect of ion implantation and studying a multiferroic material at the nanoscale, are all novel research avenues. Because of the extensive work on $\mathrm{BiFeO}_{3}$, new studies are focused on comparing $\mathrm{BiFeO}_{3}$ with other Bi-containing perovskite compounds (e.g. $\mathrm{BiCrO}_{3}$ and $\mathrm{Bi}_{2} \mathrm{FeCrO}_{6}$ ).

Several issues have caused setbacks in the development of new technologies using $\mathrm{BiFeO}_{3}$, like problems with leakage current and $\mathrm{BiFeO}_{3}$ low resistivity at ambient temperature [Awan and Bhatti, 2011]. Cr-doped $\mathrm{BiFeO}_{3}$ is one of many proposed solutions to improve issues related to leakage current [Lee et al., 2007; Das et al., 2006], and $\mathrm{Nb}$-doped $\mathrm{BiFeO}_{3}$ is proposed to improve the resistivity at ambient temperature [Jun et al., 2005].

Some challenges arise with the synthesis of $\mathrm{BiFeO}_{3}$ like the crystal structure, phase purity. The rhombohedral structure is essential to form multiferroic material and secondary phases could results in stress and strain in the crystal. To address those challenges, this thesis proposed to studied a new $\mathrm{BiFeO}_{3}$ synthesis technique, an approach by ion beam technology.

$\mathrm{BiFeO}_{3}$ is known to have a magnetic colloidal spiral magnetic structure with a period of $620 \pm 20 \AA$ [Sosnowska et al., 1982]. Sosnowska et al. [1982] confirms the G-type antiferromagnetic structure is not the complete magnetic structure for $\mathrm{BiFeO}_{3}$ as the method of analysis describes a vanishing of magnetic moment above the Néel temperature. The G-structure of $\mathrm{BiFeO}_{3}$ includes a long-range modulation like the spiral structure, the experiments are based on the assumption of a pure $\mathrm{BiFeO}_{3}$ film without contamination. This requires satellite peaks associated with reciprocal lattice vectors [Sosnowska et al., 1982]. Most spiral magnetic structures come from multiple simultaneous magnetic frustration, symmetry exchange interaction. In some case, a one-dimension frustration between the nearest neighbour direction (NN) and the next-nearest neighbour (NNN) is responsible for the spiral structure [Hase et al., 2011] or the spiral magnetic structure could be explained by the competition between symmetric exchange and the Dzyaloshinskii-Moriya interaction [Hase et al., 2011]. To date, the link between the ferroelectricity and antiferromagnetism properties of $\mathrm{BiFeO}_{3}$ with the spin-spiral configuration of $\mathrm{BiFeO}_{3}$ requires further study to be established. However, $\mathrm{BiFeO}_{3}$ has a very long spiral period comparable to what can be found in metal [Sosnowska et al., 1982].

The magnetic structure of $\mathrm{BiFeO}_{3}$ depends on the internal features of the material and the synthesis technique [Sosnowska et al., 1982]. The magnetic features can be 
destroyed by adding impurities. While the magnetic structure is sensible to internal stress, the magnetic structure is stable under an external applied perturbation. The temperature, during the measurement, does not seem to cause modification in the period of the spiral. With that established, an external field may slightly modifies the spin structure but not enough to impact the spiral period or the domains' orientation.

Secondary phases can be found in $\mathrm{BiFeO}_{3}$ thin film, e.g. iron oxides. The magnetite $\mathrm{Fe}_{3} \mathrm{O}_{4}$ is ferrimagnetic displaying the spin-wave-interaction with a Curie temperature of $858 \mathrm{~K}$. Magnetite has a Verwey phase transition $\sim 122 \mathrm{~K}$ and a magnetic anomaly $\sim 250 \mathrm{~K}$ [Aragón, 1992]. $\mathrm{Fe}_{3} \mathrm{O}_{4}$ is a sensitive material to study, the metaloxygen ratio has an impact on the material properties, and a slight modification can create sufficient perturbation to modify the spin ordering and interaction [Aragón, 1992]. Magnetite is a complete inverse spinal with net magnetic moment of $4 \mu_{B}$ cause by the $\mathrm{Fe}^{2+}$ octahedron [Goldman, 2012].

The acoustic magnon of $\mathrm{Fe}_{3} \mathrm{O}_{4}$ can be written as equation 2.3.1 [Aragón, 1992]:

$$
h \omega=\frac{11}{16} J_{A B} S_{A} \frac{S_{B 1}+S_{B 2}}{S_{B 1}+S_{B 2}-S_{A}} k^{2} a^{2} .
$$

Where $\omega$ is the frequency; $k$ is the spin-wave momentum; $a$ is the cubic unit-cell parameter. $S_{A}+S_{B 2}-S_{A}$ and $S_{B 1}$ represent the spin of $\mathrm{Fe}^{3+}$ in the tetrahedral and the octahedral sites while $S_{B 2}$ represents the spin of the $\mathrm{Fe}^{2+}$ octahedron. $J_{A B}$ represents the exchange constant between tetrahedral and octahedral sites. In this situation, $S_{A}=$ $2.5, S_{B 1}=2.5, S_{B 2}=2$.

The magnetisation is theoretically given by the equation 2.3.2 [Aragón, 1992]:

$$
\begin{aligned}
M_{z}=M_{z 0} & \left(1-\frac{0.05864}{4}\left(S_{B 1}+S_{B 2}\right.\right. \\
& \left.\left.\quad-S_{A}\right)\right)\left(16\left(S_{B 1}+S_{B 2}-S_{A}\right) \frac{k_{B} T}{11} J_{A B} S_{A}\left(S_{B 1}+S_{B 2}\right)\right)^{3 / 2}
\end{aligned}
$$

Where $M_{z 0}$ is the magnetisation at $0 \mathrm{~K}$, the experimental uncertainty is around $\sim 5 \%$. This leads to the heat capacity and the magnetisation being proportional to the temperature to the power of $1.5, \mathrm{C} \sim \mathrm{T}^{3 / 2}$ and $M_{z} \sim \mathrm{T}^{3 / 2}$. The relation $\mathrm{C} \sim \mathrm{T}^{3 / 2}$ assume the stoichiometry is respected through the analysis of the sample. The restriction on 
the equation 2.3.2 could explain the anomaly at low temperature. Those equations expected a deformation, non-linearity, at a temperature $T \sim J_{A B} / k_{B}$ and $J_{A B} / k_{B} \approx 6 \mathrm{~K}$.

The Verwey transition is based on the Ising model of the nearest neighbour and lead to the transition temperature $T_{V}$ being develop as equation 2.3.3 [Aragón, 1992]:

$$
T_{V}=\operatorname{fracz} J k_{B}
$$

Where $z$ represents the number of nearest neighbours.

An approximation of the combination of the Ising model and the Heisenberg model provide the equation 2.3.4 to determine the Curie temperature of $\mathrm{Fe}_{3} \mathrm{O}_{4}$ :

$$
\begin{aligned}
& T_{C} \cong 4 \frac{\sqrt{2 J_{A B}}}{k_{B}} \sqrt{\left(S_{A} S_{B}\left(S_{A}+1\right)\left(S_{B}+1\right)\right)}, \\
& T_{C} \cong 45.2 \frac{\sqrt{J_{A B}}}{k_{B}}
\end{aligned}
$$

The Curie temperature is $\sim 850 \mathrm{~K}$ according to the equation 2.3.4. A challenge arises when measuring that order of temperature for magnetite, as a prolonged heating above ambient temperature will enhance the separation of $\mathrm{Fe}_{2} \mathrm{O}_{3}$ from $\mathrm{Fe}_{1-x} \mathrm{O}$. The result might end up measuring another oxide structure.

Heating magnetite $\left(\mathrm{Fe}_{3} \mathrm{O}_{4}\right)$ in an oxygenated environment leads to the formation of maghemite $\left(\gamma-\mathrm{Fe}_{2} \mathrm{O}_{3}\right)$, equation 2.3.5 [Cullity and Graham, 2011]:

$$
2 \mathrm{Fe}_{3} \mathrm{O}_{4}+1 / 2 \mathrm{O}_{2} \rightarrow 3 \mathrm{Fe}_{2} \mathrm{O}_{3}
$$

Maghemite is a ferrimagnetic semiconductor with a Néel temperature of $610 \mathrm{~K}$. $\gamma-\mathrm{Fe}_{2} \mathrm{O}_{3}$ is magnetically ordered with an inverse spinel structure and vacancies at the octahedral sites [Nadeem et al., 2011]. The spinel ferrite structure is composed of oxygen FCC-lattice, the cations are separated in tetrahedral, site A, and octahedral, site B. The $\mathrm{A}$ and $\mathrm{B}$ site spins are antiparallel to each other. The ion $\mathrm{Fe}^{3+}$ is found on both the $\mathrm{A}$ and $\mathrm{B}$ site. Maghemite has the required conditions to experience spin glass behaviour [Nadeem et al., 2011]. 
$\gamma-\mathrm{Fe}_{2} \mathrm{O}_{3}$ has a blocking temperature; a transition temperature where the spin no longer aligns along the anisotropy easy axis. The blocking temperate of nanoparticles varies in function of the environment and the particle interaction. Maghemite nanoparticles could form a magnetic spin glass order caused by random freezing and frustration effects or they could condense into a superspin glass state caused by random particle interactions. Maghemite is also known to be superparamagnetic [Nadeem et al., 2011]. The blocking temperature for $\gamma-\mathrm{Fe}_{2} \mathrm{O}_{3}$ is reported to be around $\sim 50 \mathrm{~K}$ [Nadeem et al., 2011]. Nadeem et al. [2011] describes spin glass freezing on the surface of maghemite material. The theoretical model is visible on the zero-field-cooled, fieldcooled (ZFC-FC) technique and this model assumes an absence of interaction below the blocking temperature. The reality is that there might still be some spin glass interactions as the field-cooled (FC) measurement requires temperatures below the blocking temperature to "freeze" the magnetic moment. To distinguish the superparamagnetic effect from superspin glass, below the blocking temperature the FC curve continuously increases while in the case of a superparamagnetic state FC is nearly flat in the case of the superspin glass state [Sasaki et al., 2005]. A slight dip in the FC measurement can be explained by spin glass and other particle interactions.

For maghemite single-barrier blocked particles follows equation 2.3.6. Where $\tau_{0}$ is the atomic time between two random spin flips, $E_{a}$ is the thermal activation energy and $k_{B}$ is the Boltzmann constant. $\tau_{0}$ and $E_{a} / k_{B}$ are parameter fit through experiment, they are expressed in Kelvin.

$$
\tau=\tau_{0} \cdot \exp \left(\frac{E_{a}}{k_{B}\left(T-T_{a}\right)}\right)
$$

The preparation of the maghemite sample might have an impact on the $T_{0}$ as the compacted sample has been reported to be $80 \mathrm{~K}$ while the powder was $46 \mathrm{~K}$ [Nadeem et al., 2011]. The spins of an individual nanoparticle surface can undergo spin glass state, like in bulk, a nanoparticle to achieve a spin glass freezing requires disorder and frustration in the material. The surface spin glass is more evident for fine nanoparticles due to an increased ratio surface to volume. 


\subsection{Magneto-optic Kerr effect, MOKE}

The magneto-optical Kerr effect (MOKE) is often compared to the Faraday effect, both experiments describe the interaction of light with a magnetic material. The Kerr effect studies the light passing through the magnetic material, while MOKE studies the light reflected from the magnetic material. A reflected light can undergo polarisation and intensity modification caused by the magnetic ordering of the interacting material. The material dielectric tensor contains off-diagonal components resulting in a permittivity anisotropy allowing a variation of the relative index, speed of light in material, in a material with different light polarisations. The equation 2.4.1 displays the relation between the speed of light in a material, $v_{p}$, and the electrical permittivity of that material $\epsilon$ and the magnetic permeability $\mu$ [Griffiths and College, 1999]:

$$
v_{p}=\frac{1}{\epsilon \mu}
$$

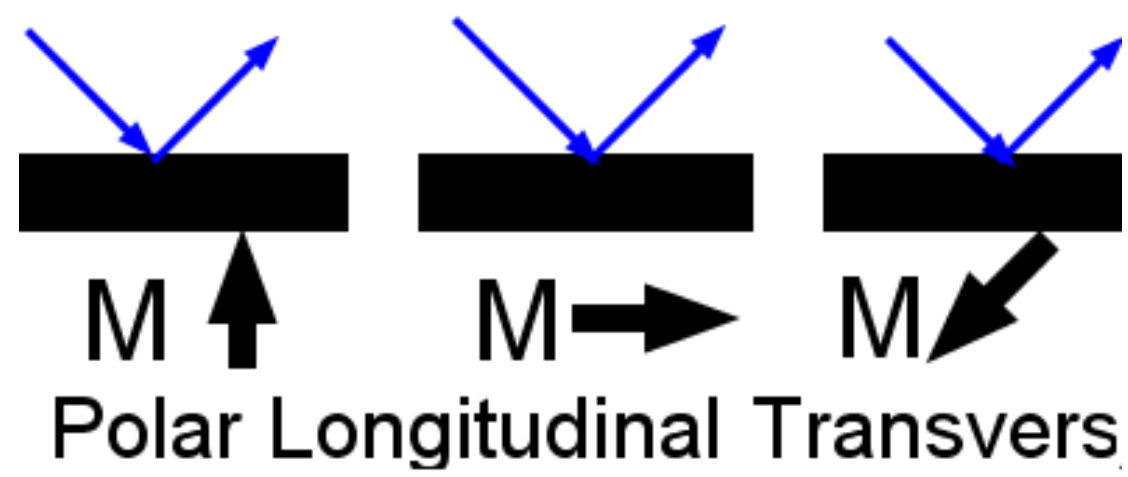

Figure 2.4.1: MOKE modes, the blue arrows represent the incident and reflected light. The black arrows display the magnetic polarisation of the material. From left to right, the schematic describes the polar, longitudinal and traversal MOKE.

The equation 2.4.1 confirms the speed of light will vary with the light orientation leading to fluctuations in the phase of polarised incident light. As seen in Figure 2.4.1, the polar MOKE happens when the sample magnetisation is perpendicular to the surface of the light reflection. This setup could be useful to analyse polar geometry. The longitudinal MOKE requires the sample's magnetisation to be parallel to the surface of reflection. Both the polar and longitudinal MOKE results from a reflected light that is not perpendicular to the incident light. Linearly polarised light would become elliptically polarised. Elliptical reflection is made of two perpendicular electrical components: Fresnel reflection coefficient, r, and Kerr coefficient, k. In general, $\mathrm{k}$ is smaller than $r$. The transverse MOKE occurs when the sample's magnetisation is perpendicular to the plane of incidence and parallel to the sample's surface. In this setup, the 
reflectivity, $r$, is measured instead of the Kerr or Fresnel coefficients. The reflectivity is modified proportionally to the perpendicular part of the sample's magnetisation. If the source of light is traveling in the same direction as the perpendicular component of the sample magnetisation then the reflected intensity would increase as the sum of the Kerr and Fresnel amplitude vectors $|r+k|^{2}$ while the antiparallel configuration between the perpendicular sample's magnetisation and incident light would result in the subtraction of the Kerr and Fresnel amplitude's vector $|r-k|^{2}$ [Weinberger, 2008]. The MOKE setup requires a laser and a polariser to modify the light source polarisation to comply with the different MOKE modes available [Kennedy et al., 2012]. A range of experimental configurations can provide an image map of the magnetic field [Weinberger, 2008].

\subsubsection{Verdet constant}

Verdet constant is constant in function of the wavelength describing the Faraday effect and the MOKE. In general, the Verdet value for most material is very small. This constant is evaluated by equation 2.4.2:

$$
V=\frac{\beta}{B d}
$$

Where $\beta$ is the Kerr rotation, $B$ is the applied magnetic field of $7.5 \mathrm{kOe}$ in this report, and $d$ is the distance the light beam travel through the material which is the thickness of the sample. Bi et al. [2008] has reported a Verdet constant at $1550 \mathrm{~nm}$ of $18 \pm 2{ }^{\circ} \mathrm{cm}^{-1} \mathrm{kOe}^{-1}, 0.31 \pm 0.03 \mathrm{rad} \mathrm{cm}^{-1} \mathrm{kOe}^{-1}$. The exception confirming the rules of a small Verdet constant come from $\mathrm{NdF}_{3}$ and $\mathrm{Pr}_{3}$, they are the compounds as of today, that displays the strongest Verdet value between 200 and $600 \mathrm{~nm}$. These compounds are difficult to store and toxic. Finding another more stable and environment friendly compound could increase potential applications. $\mathrm{PrF}_{3}$ has a Verdet constant of $-506 \mathrm{rad} \mathrm{cm}^{-1} \mathrm{kOe}^{-1}$ at $460 \mathrm{~nm}$ and $-524 \mathrm{rad} \mathrm{cm}^{-1} \mathrm{kOe}^{-1}$ at $502 \mathrm{~nm}$ [Bi et al., 2008]. $\mathrm{NdF}_{3}$ has a Verdet constant of $-297 \mathrm{rad} \mathrm{cm}^{-1} \mathrm{kOe}^{-1}$ at $460 \mathrm{~nm}$ and $-314 \mathrm{rad} \mathrm{cm}^{-1} \mathrm{kOe}^{-1}$ at $502 \mathrm{~nm}$ [Bi et al., 2008]. To place these values in perspective, $\mathrm{Fe}_{2} \mathrm{O}_{3}$ hematite has a value of $0.00175 \mathrm{rad} \mathrm{cm}^{-1} \mathrm{kOe}^{-1}$ at $400 \mathrm{~nm}$ and $472 \mathrm{~nm}$ and $0.014 \mathrm{rad} \mathrm{cm}^{-1} \mathrm{kOe}^{-1}$ at $535 \mathrm{~nm}$ [Guerreroa et al., 1997] and $\mathrm{Fe}_{3} \mathrm{O}_{4}$ has a value of $0.0026 \mathrm{rad} \mathrm{cm}^{-1} \mathrm{kOe}^{-1}$ at $400 \mathrm{~nm}$ and $472 \mathrm{~nm}$ and $0.003 \mathrm{rad} \mathrm{cm}^{-1} \mathrm{kOe}^{-1}$ at $535 \mathrm{~nm}$ [Lopez-Santiago et al., 2009]. Terbium gallium garnets are considered by some to have an "extremely" high Verdet 
constant, $\sim-1.34 \mathrm{rad} \mathrm{cm}^{-1} \mathrm{kOe}^{-1}$ [Northrop Grumman, 2016]. The band gap structure is visualise in the Figure 2.4.2.

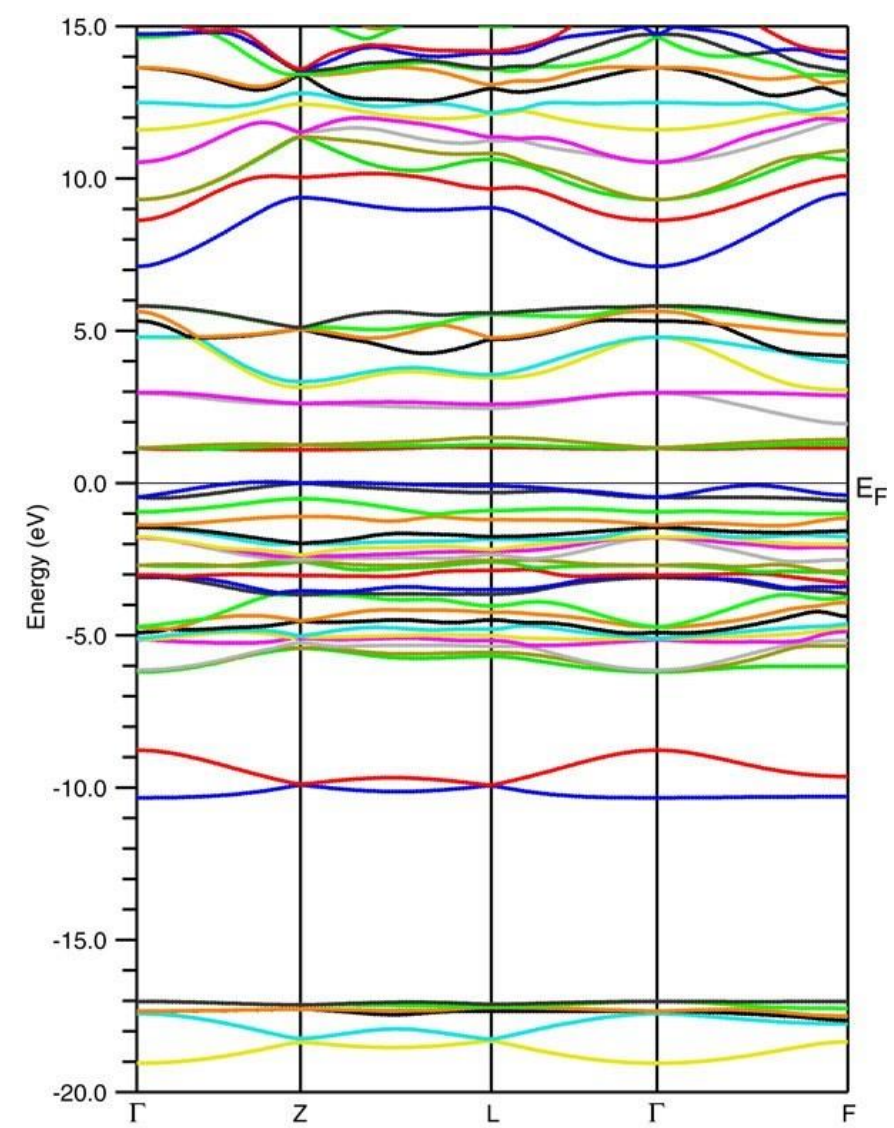

Figure 2.4.2: Band gap structure of $\mathrm{BiFeO}_{3}$. Figure reproduce from Wang et al. [2009a].

\subsubsection{Faraday effect}

The Faraday effect is an interaction of light with a magnetically ordered material. As light passes through the sample, the magnetic medium generates a rotation of the light polarisation. If the incident light is linearly polarised and composed of a left and right circular polarisation, the speed of light in the left polarisation would be different to the right and thus result in the rotation of the linear plane polarisation [Prati, 2003].

As mentioned in the previous paragraph, linear polarisation is composed of superposition of light polarised right and left circularly. The electric field's rotation follows a specific frequency and creates a circular polarisation. Within matter, this field applies a force which charges particles. The electron is most affected as the electron mass is low, resulting in a motion of the electrons that creates an internal magnetic field. If the internal field is parallel to one of the circular polarisations, this will enhance the rotation of one of the polarisation components and reduces the rotation of 
the other polarisation. This shift of the light rotation in the sample results in a new linear polarisation angle. To see if the left or right polarisation is more affected would requires a detailed analysis of the refractive index of each polarisation of the sample [Prati, 2003].

The Faraday rotation is characterised by the Verdet constant equation 2.4.2. A positive or negative Verdet constant means a left-rotation or right-rotation, respectively [Kales, 1953]. If the light passes through and is then reflected, the rotation angle double.

\subsection{Synthesis and preparation}

The main $\mathrm{BiFeO}_{3}$ nanostructure film synthesis technique reported in the literature are pulse laser deposition and sol-gel. Both deposition techniques, in the literature, are followed by an annealing at temperature above $450{ }^{\circ} \mathrm{C}$, however most report suggest $\mathrm{BiFeO}_{3}$ forms above $500{ }^{\circ} \mathrm{C}$ or $600{ }^{\circ} \mathrm{C}$ [Jia et al., 2009; Popa et al., 2007; Chen et al., 2012; Park et al., 2007; [Liu et al., 2011]; Zavaliche et al., 2006; Kumar et al., 2010; ZHANG et al., 2006]. The annealing is carried out to reduce the quantity of secondary phases, however during that process the particle grain size increases significantly. The increase grain size reduces some magnetic properties e.g. superparamagnetism. Annealing does not remove all the undesired secondary phases in the films. Sol-gel approach has the advantage to produce an important quantity of samples per recipe, while this synthesis technique has the inconvenient to require an annealing or heating for up to $24 \mathrm{~h}$ at temperature up to $1200{ }^{\circ} \mathrm{C}$, which can be a risky enterprise with some toxic chemical and acids [Qi et al., 2004].

The state of the research of $\mathrm{BiFeO}_{3}$ synthesis is summarises in the Table 2.5.1. $\mathrm{BiFeO}_{3}$ thin film synthesis by ion beam sputtering was achieved for the first time in this study. Table 2.5.1 displays a direct comparison between common synthesis $\mathrm{BiFeO}_{3}$ techniques and ion beam sputtering. 
Table 2.5.1: $\mathrm{BiFeO}_{3}$ synthesis: literature data compared with ion beam sputtering data.

\begin{tabular}{|c|c|c|c|}
\hline & Sol-gel & PLD & IBS \\
\hline Structure & Nanostructure $^{1-2}$ & Nanostructure $^{6}$ & Nanostructure \\
\hline XRD & Rhombohedral $^{2}$ & Rhombohedral $^{6}$ & $\begin{array}{l}\text { Rhombohedral, } \\
\text { R3c }\end{array}$ \\
\hline $\begin{array}{l}\text { Nanoparticle } \\
\text { size }\end{array}$ & $\begin{array}{l}26-120 \mathrm{~nm}^{1} \\
7-11 \mathrm{~nm}^{2}\end{array}$ & $\sim 100 \mathrm{~nm}^{7}$ & $\begin{array}{l}\sim 30 \mathrm{~nm} \\
\text { Average surface } \\
\text { roughness } \sim 25 \mathrm{~nm}\end{array}$ \\
\hline Band gap & $2.4-2.8 \mathrm{eV}^{3}$ & $2.68 \mathrm{eV}^{8}$ & $2.7 \mathrm{eV}$ \\
\hline $\begin{array}{l}\text { Magnetic } \\
\text { moment }\end{array}$ & $0.02-0.1 \mu_{B}{ }^{4}$ & $0.06-1 \mu_{B}{ }^{7}$ & $\begin{array}{l}0.44 \mu_{B}(15 \mathrm{~min}, \\
\text { anneal }) \\
0.097 \mu_{B} \text { (7 hours, } \\
\text { anneal) }\end{array}$ \\
\hline $\begin{array}{l}\text { Superpara- } \\
\text { magnetic }\end{array}$ & $\mathrm{T}_{B} \sim 50-200 \mathrm{~K}^{4}$ & $\mathrm{~T}_{B} \sim 225-275 \mathrm{~K}^{9}$ & $\mathrm{~T}_{B} \sim 150 \mathrm{~K}$ \\
\hline Electrical order & Ferroelectric $^{5}$ & Ferroelectric $^{6}$ & Ferroelectric \\
\hline $\begin{array}{l}\text { Secondary } \\
\text { phases }\end{array}$ & $\begin{array}{l}\mathrm{Bi}_{2} \mathrm{Fe}_{4} \mathrm{O}_{9}{ }^{1} \\
\mathrm{Bi}_{25} \mathrm{FeO}_{40}{ }^{2}\end{array}$ & $\begin{array}{l}\mathrm{Bi}_{2} \mathrm{Fe}_{4} \mathrm{O}_{9}{ }^{10} \\
\mathrm{Bi}_{2} \mathrm{O}_{2.75}{ }^{10}\end{array}$ & Iron oxides \\
\hline $\begin{array}{l}\text { Annealing } \\
\text { condition }\end{array}$ & $\begin{array}{l}450-650{ }^{\circ} \mathrm{C}^{1-5} \\
1-3 \text { hours }\end{array}$ & $\begin{array}{l}\text { Deposited } \\
\text { at } 670{ }^{\circ} \mathrm{C}^{6} \\
600{ }^{\circ} \mathrm{C}, 2 \text { hours } \\
\\
450{ }^{\circ} \mathrm{C}^{10-11}\end{array}$ & $\begin{array}{l}500^{\circ} \mathrm{C} \text { in } \mathrm{O}_{2}, \\
15 \mathrm{~min}\end{array}$ \\
\hline
\end{tabular}

\footnotetext{
${ }^{1}$ Jia et al. [2009].

${ }^{2}$ Popa et al. [2007].

${ }^{3}$ Chen et al. [2012].

${ }^{4}$ Park et al. [2007].

${ }^{5}$ Liu et al. [2011].

${ }^{6}$ Zavaliche et al. [2006].

${ }^{7}$ Wang et al. [2003].

${ }^{8}$ Kumar et al. [2010].

${ }^{9}$ Raghavender et al. [2011].

${ }^{10}$ ZHANG et al. [2006].

${ }^{11}$ secondary phases present.
} 


\section{Chapter 3}

\section{Experimental setup}

This chapter details the experimental setup, with the preparation of the substrate in Section 3.1, the different synthesis techniques in Section 3.2, and the analysis technique in Section 3.3.

\subsection{Preparation of substrate}

The selection of the substrate and the level of cleanliness are two aspects that need careful attention to synthesis nano- $\mathrm{BiFeO}_{3}$ thin film. Different substrates can result in different crystal structures due to lattice parameter and stress induced to deposited thin film which can modify the material properties [Chu et al., 2007b]. $\mathrm{BiFeO}_{3}$ has different crystal configuration showing different magnetic properties. The cleanliness of the substrate is important to improve the likelihood of quality film deposition, limiting the probability of contamination. In the following sections, detailed information is provided on the substrates selected and the cleaning procedure.

\subsubsection{Selected substrates}

The substrates used in this project are silicon dioxide films on silicon wafer, silicon $\langle 111\rangle$ and optical grade silica wafer materials. The details of these substrates are provided in Table 3.1.1. Silicon based substrates were chosen for potential applications in electronic and micro-electronics. The $\mathrm{SiO}_{2}$ layer on the substrate is form by thermally oxidising the silicon substrate. Those substrates were used to deposit thin films and implant ions for this project. The oxidised layer thickness, for practical reasons, varied between 100 and $500 \mathrm{~nm}$. The thickness variation of the amorphous layer is not influencing the thin film formation [Tang et al., 2009], however this can depend on the dry or wet etching techniques used. The $500 \mathrm{~nm} \mathrm{SiO} 2$ on $\mathrm{Si}$ layer was the main substrate used in this research. The main reason to choose a thick oxide layer was to facilitate the 
RBS analysis by displaying a clear $\mathrm{SiO}_{2}: \mathrm{Si}$ transition. The effects of the oxide layer's thickness have been studied and displayed no difference in the context of this work, structure, magnetic ordering and electric properties are similar regardless of the $\mathrm{SiO}_{2}$ thickness, for a layer thicker than $100 \mathrm{~nm}$ as expected. $\mathrm{SiO}_{2}$ :Si substrate were used for almost every analysis a part from a few exceptions detailed next.

Table 3.1.1: Details of substrates studied.

\begin{tabular}{|c|c|c|c|c|}
\hline Substrate & $\begin{array}{l}\text { Thickness of } \\
\text { the substrate, } \\
\mathrm{SiO}_{2}: \mathrm{Si}\end{array}$ & $\begin{array}{l}\text { Thickness } \\
\text { of the oxide } \\
\text { layer, } \mathrm{SiO}_{2}\end{array}$ & orientation & $\begin{array}{l}\text { Substrate } \\
\text { used in }\end{array}$ \\
\hline $\mathrm{SiO}_{2}: \mathrm{Si}$ & $260 \pm 20 \mu m$ & $500 \mathrm{~nm}$ & n.a. & $\begin{array}{l}\text { Chapter } 4,5 \text {, } \\
\text { B.2 and Ap- } \\
\text { pendix D }\end{array}$ \\
\hline $\begin{array}{l}\text { Silica (optical } \\
\text { grade quality) }\end{array}$ & $1 \pm 0.5 \mathrm{~mm}$ & n.a. & n.a. & 4.3 .6 and 4.6 \\
\hline $\mathrm{SiO}_{2}: \mathrm{Si}$ & $650 \pm 50 \mu m$ & $100 \mathrm{~nm}$ & n.a. & $\begin{array}{l}\text { 4.3.1, } 4.3 .4 \text { and } \\
\text { Appendix E }\end{array}$ \\
\hline Si & $600 \pm 50 \mu m$ & n.a. & 111 & $\begin{array}{l}\text { Chapter } 5 \text { and } \\
\text { Appendix D }\end{array}$ \\
\hline $\mathrm{SiO}_{2}: \mathrm{Si}$ & $525 \pm 25 \mu m$ & $400 \mathrm{~nm}$ & n.a. & Appendix E \\
\hline
\end{tabular}

The silica (optical grade quality) is pre-cut and sealed, so this substrate was assumed to be clean and this silica was used as is from the manufacturer. The substrate is square-shaped with dimensions of $1 \mathrm{~cm}$ by $1 \mathrm{~cm}$ and a thickness of $1 \mathrm{~mm}$. These are double side polished substrates, assumed to be perfectly uniform and ideal for optical measurement. The optical band gap, light absorption, magneto-optical Kerr effect and magnetic circular dichroism were all measured using thin films deposited on silica substrate.

Silicon substrates, without an $\mathrm{SiO}_{2}$ layer, with an orientation of $\langle 111\rangle$ were used to extend the analysis of implanted ions. The orientation of other well-studied substrates generates enough strain or lattice miss-match to modify the $\mathrm{BiFeO}_{3}$ crystal structure [Chu et al., 2007a]. 


\subsubsection{Substrates treatment}

Each substrate used to synthesise nanoparticle thin films followed a rigorous cleaning procedure. After cutting the substrate into the required shape and size, the pieces are placed in a pressurized filtered air stream to remove any debris or dust that could contaminate the products. A drop of $5 \mathrm{~mL}$ Decon90 is added to a beaker of distilled water and placed in an ultrasonic bath. The cut substrates are placed face-up at about $85^{\circ}$ relative to the surface. An angle is required to ensure that the liquid will not dry on the substrate. After the first ultrasonic bath cleaning, the beaker is clean. The substrates are placed as previously in the beaker with only distilled water for 3 cycles of ultrasonic baths. Between each cycle the beaker is cleaned with distilled water. To avoid any water contamination and potentially lengthening the time required to reach the necessary vacuum pressure for deposition or implantation, the substrates are placed in an ultrasonic bath with isopropanol for two cycles. Isopropanol tends to react with water to create another membrane that is easier to remove than water [Devi et al., 2005]. In the final step, to ensure no trace of any product remains on the substrate, each substrate is passed under compressed air. A visual inspection completed the quality test. Each ultrasonic bath is carried out at ambient temperature for 5 minutes with about one minute of relaxing time. This cleaning procedure was used for the $\mathrm{BiFeO}_{3}$ thin film and implanted film characterised by AFM, TEM and Raman spectroscopy.

Another cleaning procedure was used for the $\mathrm{BiCrO}_{3}$ thin films and the $\mathrm{BiFeO}_{3}$ thin film analysed by the SQUID. For these films, the first step after cutting the substrate is to remove debris with compressed air. The substrates are then placed in an ozone cleaning chamber, the PSD Pro Series Digital UV Ozone system. The temperature in the chamber is held at $80{ }^{\circ} \mathrm{C}$ for 15 minutes, and then a cool-down period of 1 minute is applied. Before any synthesis technique is carried out, the sample are place under compressed air treatment.

\subsection{Synthesis techniques}

Two synthesis techniques were explored, ion beam sputtering and ion implantation, which are described in the next two sections. Both techniques, in the specific conditions used, require a further step, namely, the annealing process, detailed in subsection 3.2.3, to form a crystal or to transform oxide impurities into the desired nano-material. Annealing a sample introduces energy into the system to enable crystal formation [Fujii 
et al., 1991], increase the crystal quality and increase the concentration of oxygen in the films [Johan et al., 2011]. Annealing can reduce the stress from the film by modifying the material [Fu and $\mathrm{Du}, 2003]$.

\subsubsection{Ion beam sputtering}

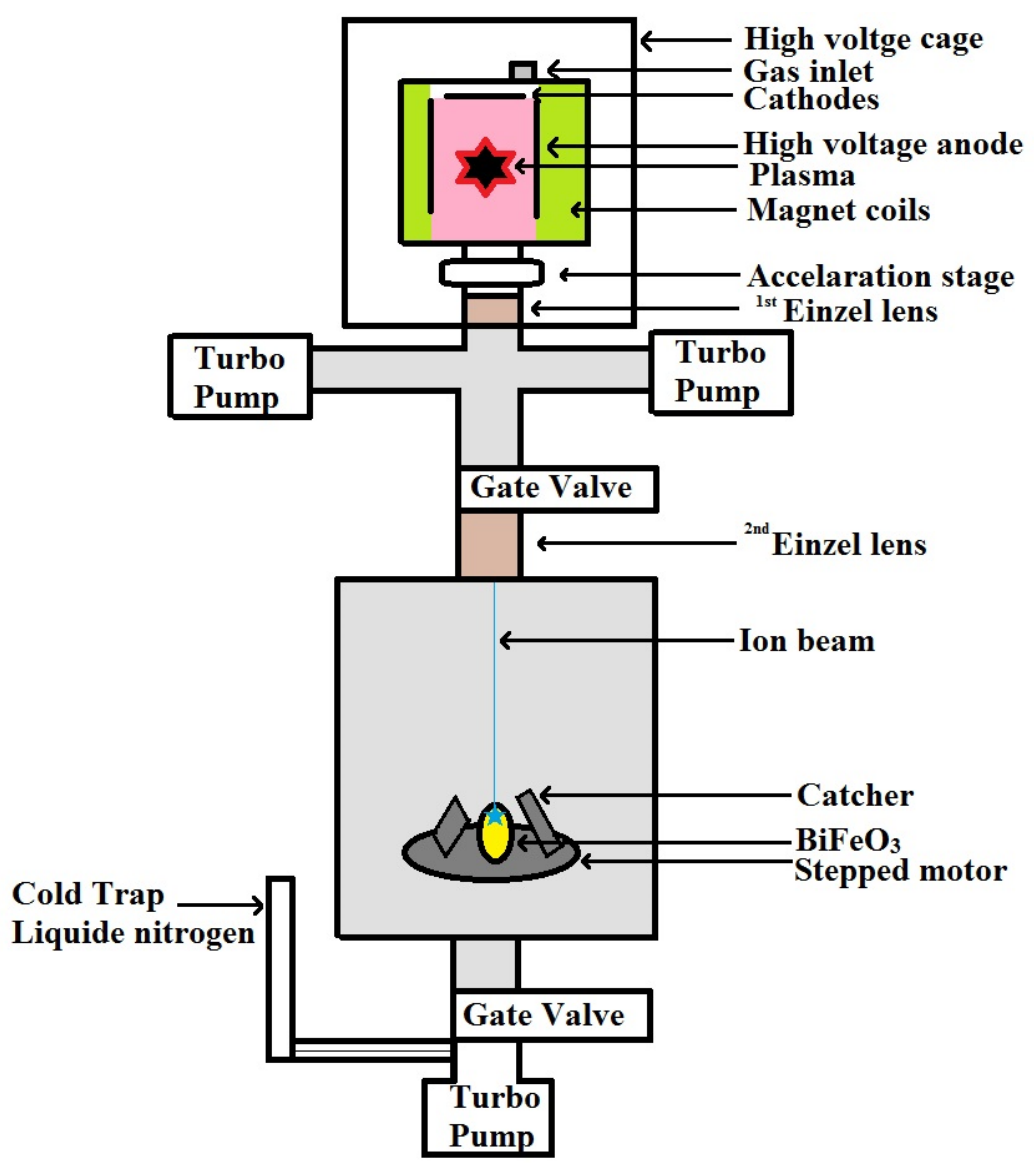

Figure 3.2.1: Ion beam sputtering system in GNS Science. Schematic based on GNS Science archive and modified to reflect the system in 2017 [Markwitz et al., 1992; Murmu et al., 2012].

The ion beam sputtering system at GNS Science is a novel technique for making thin films. A $20 \mathrm{keV} \mathrm{Ar}{ }^{+}$ion beam was used to deposit bismuth oxide, iron oxides and bismuth ferrite oxides thin films at ambient temperature using a bulk $\mathrm{BiFeO}_{3}$ and $\mathrm{Fe}_{3} \mathrm{O}_{4}$ magnetite target in a high vacuum $\left(<2 \times 10^{-6} \mathrm{kPa}\right)$. The $\mathrm{Ar}^{+}$ion beam was directed onto the sputtering target placed at $45^{\circ}$ angle to improve homogeneity. The films were deposited onto 100 and $500 \mathrm{~nm}$, thermally oxidised, silicon dioxide $\left(\mathrm{SiO}_{2}\right)$ on a Si substrate mounted on rotating catchers. Target holder and catchers were rotated in opposite direction to promote lateral uniformity of the deposited film.

Ion beam sputtering can be described in three main parts: 1) the Penning ion 
source, 2) the acceleration and focussing stage and 3) the sample chamber. The ion source is based on the Penning ion source developed by Baumann [Baumann and Bethge, 1974]. For this experiment, argon gas is used. Argon is a noble gas with high sputtering yield [Wei et al., 1995] combined with an ionising cross-section that makes Ar the most suitable gas for ion beam sputtering at $20 \mathrm{kV}$.

The ion source contains two cathodes, an anode and a solenoid magnet [Baumann and Bethge, 1974]. Positively charged ions are generated in the plasma by electron interactions. The solenoid magnet surrounding the plasma chamber induces a magnetic field causing secondary electrons to spiral in the plasma [Markwitz, A, personal communication, August 2017] and increases the probability to further ionise gas atoms [Engström et al., 2000].

The second part of the system is the acceleration stage. Positive ions are accelerated by the terminal voltage toward ground potential considering that the anode voltage is set to terminal voltage potential. The focus system has a series of two Einzel lenses to focus the beam onto the target. To increase the sputtering yield, the lens system was set to focus the beam on the target until the beam spot covers a large area of the target. A large diameter beam reduces the depth of the sputter crater and effectively uses the target.

The final part of the system is the sample chamber. The ion beam "sees" the target when this ion beam arrives in the chamber. The beam will implant argon in the target with enough energy to break molecular bonds in the near surface region, resulting in sputtering atoms and molecular fragments from the target. Since the emission profile is cone shaped, the target and the catcher rotate to allow uniform lateral deposition. The catchers hold samples in place at an angle of $60^{\circ}$ to improve the uniformity of the deposition layer. Another important aspect is the quality of the cleaning; contamination inside the surface of the chamber can increase the chance of depositing contaminants in the synthesis material. To avoid this issue, pre-sputtering is required (sputtering of 100-200 nm without samples to cover the chamber with molecules from the target). The pre-sputtering masks contaminants that could be anywhere in the chamber and limit the contamination.

To tackle contamination from oxygen, nitrogen, carbon, hydrogen and other atoms, the system has a liquid nitrogen trap. Liquid nitrogen condenses water vapour and other light elements at the cold trap inside vacuum.

The sputtering can be described as the erosion of a sample or target by an en- 
ergetic continuous outflow of particles [Nastasi et al., 2004]. Sputtering occurs when an incident ion transfers more energy to the elements near the surface than the surface binding energy of the target. The sputtering yield $Y$ is defined in equation equation 3.2.1 by the ratio of emitted atoms over the incident particles [Nastasi et al., 2004],

$$
Y=\frac{\text { number of emited atoms }}{\text { number of incident particles }}
$$

The sputtering yield mainly varies as a function of the target material, ion species and ion energy. The surface orientation may influence the sputtering cross-section, and the incident particles beam sputtering efficiency - angular dependent sputtering [Nastasi et al., 2004]. The sputtering yield can also be expressed by a material correction factor multiplied by the energy deposited on the surface [Nastasi et al., 2004]. This definition and equation 3.2.1 assume a uniform, mono-atomic sputtered target. Several tables exist to demonstrate the theoretical calculation of mid-range ion beam energy on a target of common periodic table elements [Andersen and Bay, 1974; Matsunami et al., 1987]. Different elements in compounds or alloys might have different sputtering yields resulting in a preferential sputtering. Preferential sputtering can modify the target stoichiometry and explained the stoichiometry of the deposited films [Nastasi et al., 2004]. In the case of $\mathrm{BiFeO}_{3}$, the sputtering yield is defined similarly to equation 3.2.1. However, the sputtering yield including bismuth, iron and oxygen can be written as equation 3.2.2:

$$
Y=\frac{\text { number of emited atoms, } \mathrm{Bi}, \mathrm{Fe}, \mathrm{O}}{\text { number of incident particles }}
$$

The individual sputtering yield of each element of $\mathrm{BiFeO}_{3}$ sputter target, equation 3.2.1, are different. At $20 \mathrm{keV}, \mathrm{Ar}^{+}$sputters between 0.88 and 3.84 bismuth atoms per argon ion [Deoli et al., 2014], between $2.1 \pm 0.3$ and $3.7 \pm 0.5$ iron atoms per argon ion [Viefhaus et al., 1994] and more than 2 oxygen atoms per argon ion [Seah, 1981]. Equation 3.2.3 highlights the preferential sputtering:

$$
\frac{Y_{B i}}{Y_{F e}}=r \frac{N_{B i}}{N_{F e}}
$$

where $r$ is a sputtering factor, and $N_{B i}$ and $N_{F e}$ are the surface concentrations of bismuth and iron [Nastasi et al., 2004]. The sputtering factor $r$ varies as a function 
of the preferential sputtering yield, usually between 0.5 and 2 [Nastasi et al., 2004]. The sputtering yield experimentally measured for equation 3.2.3 suggests a $r$ value between 0.5 and 0.9 [Deoli et al., 2014; Viefhaus et al., 1994].

As the bulk target is made from a mix of bismuth oxide, iron oxides and bismuth ferrite oxides, this can result in a non-stoichiometric deposited film due to localise area with more iron or bismuth available for sputtering. While sputtering allows a minimum of ion mixing, bond creation between sputtered ions and the substrate, the energy involved is too low to create bismuth ferrite only by ion beam sputtering [Zhang et al., 2006]. As experimentally observed, synthesising bismuth ferrite by ion beam sputtering requires annealing the thin film to change the crystal phase from amorphous to nanostructured [Fruth et al., 2005]. Annealing is discussed in subsection 3.2.3.

Theories exist on the formation of thin films by ion beam sputtering. Those theories indicate that a material can reach a critical thickness where the film is a more or less a uniform deposition (island formation) [Frost et al., 2004]. Annealing will improve the uniformity of the sputtered layer, shown in Chapter 4, as annealing increases the mobility of bismuth ions to form $\mathrm{BiFeO}_{3}$. The first elements to be sputtered may follow a pattern, either a series of dots, a series of pits, or ripples [Carter, 2001; Valbusa et al., 2002; Gailly et al., 2012; Feder et al., 2013]. Another theory is the continuum evolution of the nonlinear equation of Kuramoto-Sivashinsky [Castro et al., 2005]. There are theoretical surface roughness models of ion beam sputtering. A study of silicon crystal and nickel beryllium alloy is known to have a predicted roughness of $R_{a}=3.92 \pm 24.58 d$ and $\log R_{a}=1.65+2.028 \times 10^{-3} V$, where $R_{a}$ is the average roughness, $V$ is the terminal voltage $(\mathrm{keV})$ and $d$ is the theoretical pixel spacing in mm [Ali et al., 2010].

\subsubsection{Ion implantation}

The second synthesis technique used in this study is ion implantation. This synthesis technique was carried with a Penning ion source. Neon gas is used to sputter ions from the source targets. The bending magnet provides a mass selection of the ions. Ion implantation like ion beam sputtering are two ion beam synthesis technique [Wei et al., 1995].

Ion implanters can be divided into three sections to facilitate the explanation of the physics involved during ion implantation: 1) the ion source, 2) the accelerator stage and 3) the target material. The source uses neon as tracer gas. Neon is a noble gas that offer a good compromise between ionisation and sputtering yield. Cosmic 
rays create the first avalanche of electrons. The electrons travel from the anode to the cathode ionising the plasma. The solenoid magnetic field applied by the surrounding solenoid increases the travel length of the electrons by inducing a spiral trajectory. The positively charged neon ions are accelerated toward the sputter targets, the anode potential, leading to sputtering of the cathodes. Each end of the source has a sputter target. A back target (a full disc located close to the source gas) and a front target (a disc with a hole in the middle to allow the gas and the ions to pass through to the next section of the system, located at the end of the ion penning source), provide the ions of interest to be implanted. The extraction process is caused by the electrical potential between cathode and anode and the acceleration potential (the source is placed at high voltage while the other parts of the implanter at ground potential). This results in ions to be accelerated at a select potential [Markwitz and Kennedy, 2005].

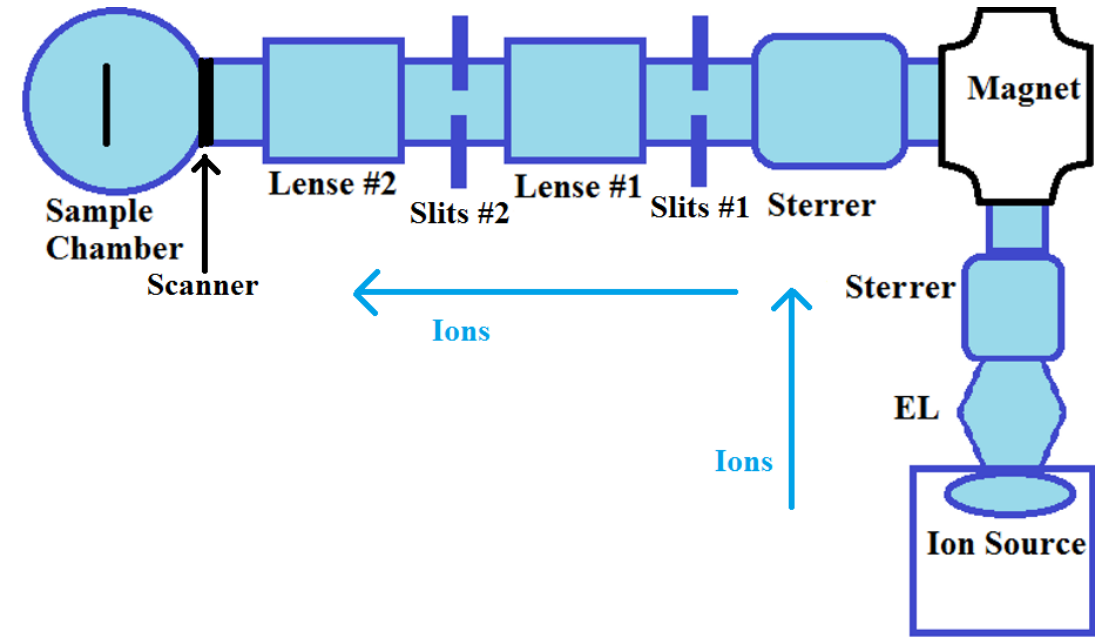

Figure 3.2.2: Ion implanter schematic, at GNS Science as of March 2017.

Bismuth ferrite implantation requires that the ions be implanted in a particular order: iron first, then bismuth. Generally, the heavier ion is implanted first to increase the stopping power of the material and ensure the lighter ions are implanted at a similar depth [Ziegler, 1998]. As bismuth has a high sputtering yield, bismuth was implanted last to reach stoichiometry. For oxygen, two approaches are possible, either implanting oxygen followed by an annealing under an oxygen atmosphere or oxidise the material by annealing this material without implanting oxygen. To implant bismuth, as the bulk $\mathrm{Bi}$ is a soft metal with a melting point of $271^{\circ} \mathrm{C}$ [Tooth et al., 2008], at atmospheric pressure, careful attention must be paid to the internal temperature of the ion source (a low anode current to minimise the internal temperature increase), the ions source is optimised (pressure) for an efficient implantation. A small change of either of these parameters makes a big difference in the bismuth yield. Also, the cathodes can melt in the source.

The extraction current of the implanter has already been described in the previ- 
ous section. When ions leave the source, they pass through a magnetic field, which is essentially a mass discrimination process. Einzel lens (Einzel lens are made of 3 electrostatic plates or tubes where the centre electrode is placed at high voltage between the 2 cathode tubes at ground potential) are place between the Penning ion source and the bending magnet. The ions are focussed by Einzel lens. After the magnet, a series of slits and quadrupole lenses steer and focus the beam towards the target. The quadrupole duplet is made of 2 electrostatic plates in horizontal and 2 electrostatic plates vertical configuration followed by 2 electrostatic plates in the vertical and 2 in the horizontal position. A scanner was used to scan the focused ion beam across the sample area. The scanner has pairs of variable " $\mathrm{X}$ " and " $\mathrm{Y}$ " electrostatic plates $( \pm 2000 \mathrm{~V})$.

Once the ions reach the target, the final stage involves the implantation of ions in the material. Eventually, ions will form layers under the surface of the substrate. The depth penetration of the ions is guided by the sum of the electronic stopping power and the nuclear stopping power of the sample being implanted [Ziegler, 1998]. The electronic stopping power leads ions to lose kinetic energy caused by inelastic collisions between the electron of the medium and the ions. The nuclear stopping power is resulting from the elastic collision between ions and atoms [Amable et al., 2017; Yang et al., 2002]. In the example of implanting $\mathrm{Bi}$ into $\mathrm{SiO}_{2}$, $\mathrm{Bi}$ ion will penetrate the sample until this ion collides with a $\mathrm{Si}$ or $\mathrm{O}$ atom in the substrate. The electronic stopping

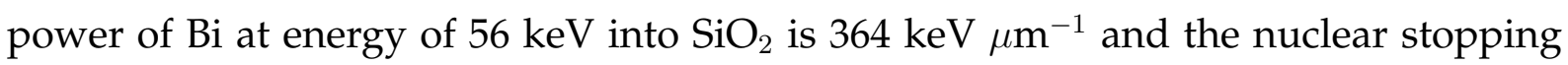

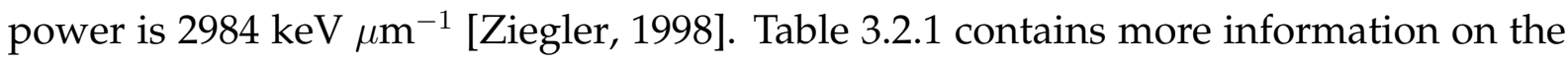
stopping power calculated by Stopping and Range of Ions in Mater, SRIM, software [Ziegler, 1998]. Bi ions will stop only once those ions have lost all their kinetic energy. During this process, a series of collisions will create disorder in the crystal (the atoms that have been hit by the ion will travel farther into the sample and hit other atoms, and at some point, those atoms should create their own space, forming a chain reaction) and damage on the surface (a change in roughness on the surface). During the implantation process, ions will interact with previously implanted atoms. As the mass of the implanted $\mathrm{Bi}$ ions is the same as the ones previously implanted, the stopping power will increase. This means the implantation depth will decrease. Also, the chance of hitting a previously implanted ion increases with time. For some ion-material systems, the sample reaches the equilibrium of implantation, each incoming ion will displace another implanted ion.

D-Trym simulation, Figure 3.2.3, shows iron implantation at $38 \mathrm{keV}$ into $\mathrm{BiFeO}_{3}$ saturate at a fluence of $8 \times 10^{16}$ at. $\mathrm{cm}^{-2}$. At a depth of $\sim 75 \mathrm{~nm}$ the concentration of implanted iron is $27 \mathrm{Fe} . \mathrm{cm}^{-2}$. Iron implantation at $38 \mathrm{keV}$ in $\mathrm{BiFeO}_{3}$ has an electronic stopping power of $82.5 \mathrm{keV} \mu \mathrm{m}^{-1}$ and a nuclear stopping power of $735.4 \mathrm{keV} \mu \mathrm{m}^{-1}$ 
assuming the $\mathrm{BiFeO}_{3}$ density, default value from SRIM, of $4.389 \mathrm{~g} \mathrm{~cm}^{-3}$, unit cell is 1000 A. Stopping power and target density of material modification, Chapter 55 and Appendix C and Appendix D, are presented in Table 3.2.1.

Table 3.2.1: Ion stopping power of bismuth, iron and oxygen [Ziegler, 1998].

\begin{tabular}{|c|c|c|c|}
\hline & $\begin{array}{l}\text { Electronic stopping } \\
\text { power }\left[\mathrm{keV} \mu \mathrm{m}^{-1}\right]\end{array}$ & $\begin{array}{l}\text { Nuclear stopping } \\
\text { power }\left[\mathrm{keV} \mu \mathrm{m}^{-1}\right]\end{array}$ & $\begin{array}{l}\text { Target density } \\
{\left[\mathrm{g} \mathrm{cm}^{-3}\right]}\end{array}$ \\
\hline $\mathrm{Bi}, 56 \mathrm{keV}$ & 364 & 2984 & $\mathrm{SiO}_{2}: 2.65$ \\
\hline $\mathrm{Fe}, 38 \mathrm{keV}$ & 131 & 1173 & $\mathrm{SiO}_{2}: 2.65$ \\
\hline O, $7.5 \mathrm{keV}$ & 838 & 2418 & $\mathrm{SiO}_{2}: 2.65$ \\
\hline $\mathrm{Bi}, 56 \mathrm{keV}$ & 450 & 4711 & $\mathrm{Fe}_{3} \mathrm{O}_{4}: 5.17$ \\
\hline
\end{tabular}

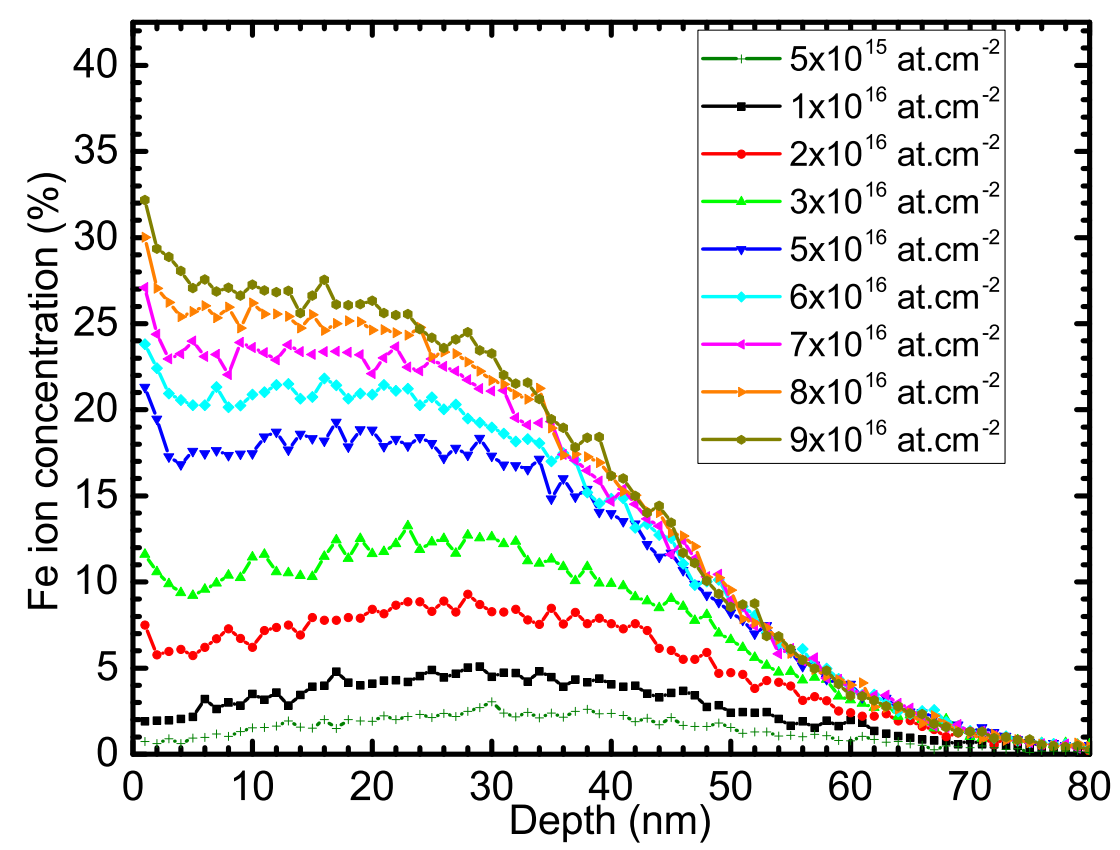

Figure 3.2.3: Theoretical simulation of Fe implantation into $\mathrm{BiFeO}_{3}$. The saturation occurs at a fluence of $8 \times 10^{16}$ at.cm $^{-2}$. At of depth $75 \mathrm{~nm}$, the concentration is 27 at.cm ${ }^{-2}$.

\subsubsection{Annealing}

GNS Science's annealing furnace is a radiative furnace allowing use of different gas with a variable flow pressure. The furnace temperature is up to $1000{ }^{\circ} \mathrm{C}$. The sputtered bismuth ferrite phase films were annealed at temperatures up to $700{ }^{\circ} \mathrm{C}$ for up to 7 hours. The oven was preheated to the set temperature before annealing and the samples were taken out of the oven once the annealing time had elapsed; the samples were let to cooldown at ambient temperature. The implanted films were annealed at temperature up to $900{ }^{\circ} \mathrm{C}$ for 2 hours. 
Annealing is required to improve the quality of the sputtered thin films as the asmade films are oxygen deficient and contain secondary phases. The system allows to purge the laboratory air and replace that air with a partial atmosphere of different gases like oxygen, nitrogen and argon. Bismuth has a melting point of $270{ }^{\circ} \mathrm{C}$ [Kindler et al., 2003]. In order to improve the $\mathrm{BiFeO}_{3}$ structure a temperature of $500{ }^{\circ} \mathrm{C}$ is required, this will be discussed in Chapter 4 . An oxygen atmosphere, $\sim 40 \mathrm{kPa}$ with a volumetric flow rate of $40 \mathrm{~mL} \cdot \mathrm{min}^{-1}$, is introduced in the tube with the sample while annealing. This is done to limit the evaporation of bismuth. Bismuth oxide, $\mathrm{Bi}_{2} \mathrm{O}_{3}$, has a melting temperature of $800{ }^{\circ} \mathrm{C}$ [Qiu et al., 2006], the oxygen pressure (as study in Chapter 4) retain more bismuth atoms in the film. Increasing the temperature allows to remove some secondary phases or to give bismuth atoms enough mobility to bond with other atoms like iron or oxygen, this can lead to the formation of the desired film. Annealing can provide sufficient energy to change the crystal structure of the thin film [Fujii et al., 1991; Johan et al., 2011] as discussed in Chapter 4, X-ray diffraction analysis.

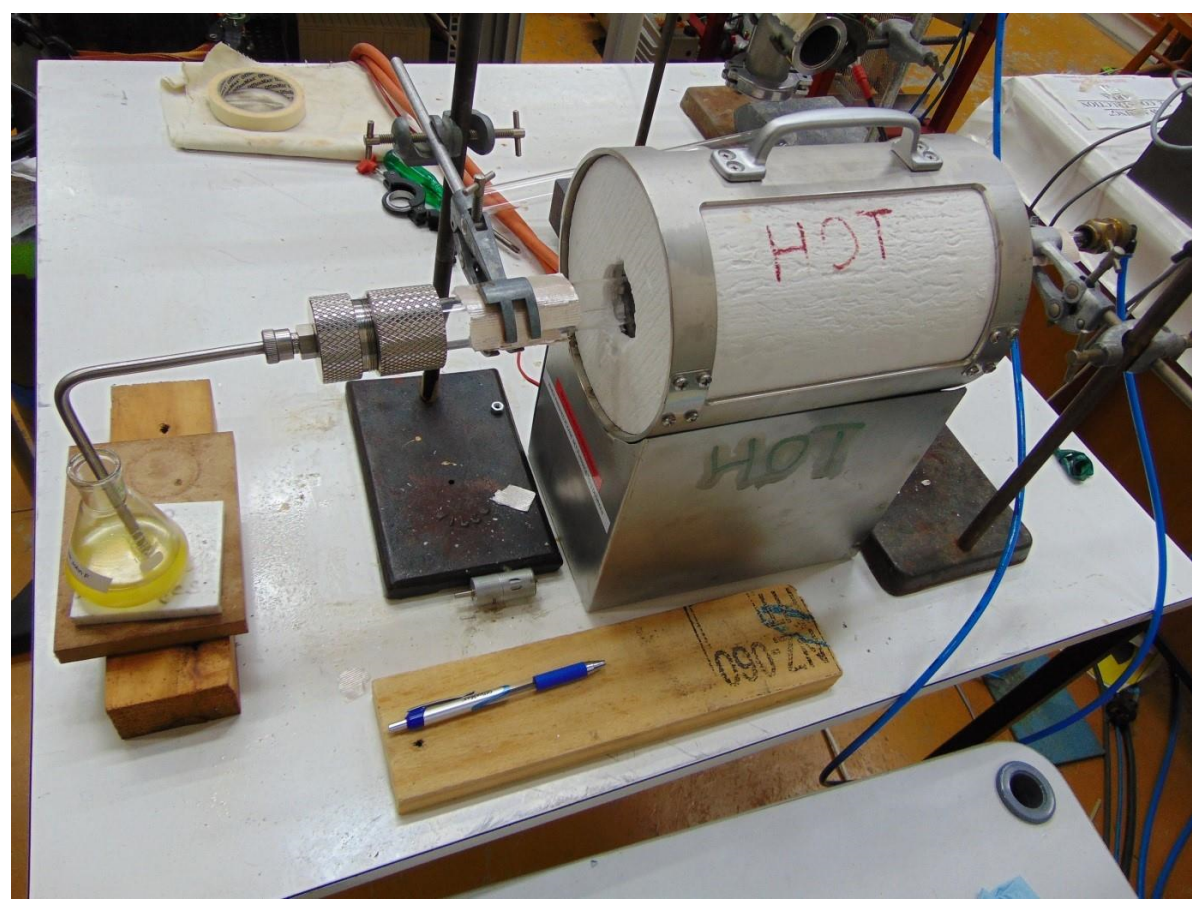

Figure 3.2.4: Annealing furnace, GNS Science. The oven is open at both extremities. The quartz tube can be connected to a gas flow regulator.

The temperature setting is controlled by a proportional-integral-derivative controller (PID). A PID controller runs a loop system that calculates the uncertainty. This will result in an overshoot and a stabilisation around the set value over time as described by equation 3.2.4: 


$$
u(t)=K_{p} e(t)+\frac{K_{p}}{T_{i}} \sum_{t} e(t)+K_{p} T_{d} \frac{d}{d t} e(t) .
$$

Where $T_{i}$ is the integral time, $T_{d}$ is the derivative time, $K_{p}$ is the gain applied, $e(t)$ is the difference between the set temperature and the measured temperature and $u(t)$ is the controller output.

The energetic atoms that are either sputtered or implanted eventually lose their energy and come to rest on a substrate or inside, becoming subject to the lattice parameter of that substrate and causing a potential mobility in the process limiting early stage of film deposition. Increasing the temperature is enhancing ions mobility [Townsend et al., 1976]. Diffusion is defined by the random walk movement of the sputtered or implanted ions [Townsend et al., 1976].

The diffusion coefficient $\mathrm{D}$ is generally assumed independent of the atoms concentration. Einstein has confirmed, in the case of an ideal diluted solution, the coefficient $\mathrm{D}$ can be written as the following:

$$
D=\mu k_{B} T
$$

where $\mu$ is the mobility of the diffusing ions, $k_{B}$ is the Boltzmann's constant and $T$ is temperature [Townsend et al., 1976]. Every element and compound has their own diffusion coefficient, so in a mixed element layer the diffusion will be directiondependent [Townsend et al., 1976]. The elements and compounds near the surface or near any defects have a greater mobility than elements in a perfect solid material [Townsend et al., 1976]. In the case of thin films, oxidising metals during the annealing allows them to chemically bond to substrate like $\mathrm{SiO}_{2}$ and increases the adhesion [Townsend et al., 1976]. To control the oxidation and make fewer modifications as acceptable to the material's properties, some have suggested creating stacks of elements. Ti will create a bond with the $\mathrm{SiO}_{2}$ substrate, while Pt will limit the oxidation and improve the electronic contact [Townsend et al., 1976]. Diffusion is also seen in non-metallic material [Myers, 1974]. Annealing $\mathrm{BiFeO}_{3}$ in oxygen improves the microstructure and the electrical properties [Prashanthi et al., 2013]. The implantation of ions might result in stress in the sample. The relaxation of that stress can result in rifts, voids and other modifications to the surface tension, modifying the ion diffusion rate compared to an unstressed thin film [Townsend et al., 1976]. Oxidation in thin films 
can occur while annealing, especially annealing in an oxygen atmosphere [Townsend et al., 1976].

The diffusion coefficient represents the probability of an atomic diffusing and was successfully compared to a modified Arrhenius equation, equation 3.2.6:

$$
D=D_{0} \exp \left(-\frac{E}{k_{B} T}\right)
$$

where $D$ is the diffusion coefficient, $D_{0}$ is the best-fit pre-exponential factor, $E$ is the energy required to activate the diffusion, $k_{B}$ is the Boltzmann's constant and $T$ is the temperature [Coogan et al., 2005]. A mixed layer of $\mathrm{Bi}_{2} \mathrm{O}_{3}-\mathrm{Fe}_{2} \mathrm{O}_{3}$ displays a similar Arrhenius diffusion type. Bismuth oxide, when annealed up to $650{ }^{\circ} \mathrm{C}$, diffuses several microns into $\mathrm{Fe}_{2} \mathrm{O}_{3}$, however iron oxide does not diffuse into $\mathrm{Bi}_{2} \mathrm{O}_{3}$ [Rojac et al., 2014]. This result tends to confirm that the formation of $\mathrm{BiFeO}_{3}$ is limited by the diffusion of bismuth toward iron oxide [Rojac et al., 2014].

Taking the natural logarithm of equation 3.2.6, the equation 3.2.6 becomes 3.2.7:

$$
\ln D=\ln D_{0}-\frac{E}{k_{B} T}
$$

The plot of the natural logarithm of the diffusion coefficient as a function of the inverse temperature, $T^{-1}$, is called the Arrhenius plot. This is used to measure the value $D_{0}$ which is the interceptor and the activation energy which is the slope times Boltzmann's constant [Würschum et al., 2003].

The thermodynamic instability of $\mathrm{BiFeO}_{3}$ was studied and confirmed by Selbach et al. [2008] to follow equation 3.2.8:

$$
\frac{12}{49} \mathrm{Bi}_{2} \mathrm{Fe}_{4} \mathrm{O}_{9}+\frac{1}{49} \mathrm{Bi}_{25} \mathrm{FeO}_{39} \leftrightarrow \mathrm{BiFeO}_{3}
$$

Both Rojac et al. [2014] and Selbach et al. [2008] confirmed a synthesis mechanism starting with bismuth oxides and iron oxide as seen in equation 3.2.9: 


$$
\frac{1}{2} \mathrm{Bi}_{2} \mathrm{O}_{3}+\frac{1}{2} \mathrm{Fe}_{2} \mathrm{O}_{3} \rightarrow \mathrm{BiFeO}_{3}
$$

Equation 3.2.9 is supported by the diffusion of bismuth oxide into iron oxide as mentioned earlier by Rojac et al. [2014].

Annealing a $\mathrm{BiFeO}_{3}$ thin film over $880{ }^{\circ} \mathrm{C}$ could result in losing an amount of bismuth ferrite in favour of another oxide phase as described in equation 3.2.10, [Rojac et al., 2014]:

$$
8 \mathrm{BiFeO}_{3} \rightarrow 2 \mathrm{Bi}_{2} \mathrm{Fe}_{4} \mathrm{O}_{9}+4 \mathrm{BiO}+\mathrm{O}_{2} .
$$

A note on the $\mathrm{BiFeO}_{3}$ decomposition equation 3.2.10, $\mathrm{BiFeO}_{3}$ and $\mathrm{Bi}_{2} \mathrm{Fe}_{4} \mathrm{O}_{9}$ are both solid particles in a thin film, however $\mathrm{Bi}_{2} \mathrm{O}_{3}$ and $\mathrm{O}_{2}$ are both in a gaseous state. The presence of $\mathrm{Bi}_{2} \mathrm{Fe}_{4} \mathrm{O}_{9}$ in sample that are annealed in unoptimised conditions (annealed at $600{ }^{\circ} \mathrm{C}$ and annealed for 7 hours) could be explain by the two thermodynamic reactions, equation 3.2.8 and equation 3.2.10.

\subsection{Characterisation techniques}

Thin film sample structures are characterised with ion beam analysis techniques, $\mathrm{X}$-ray diffraction, microscopy (e.g. AFM, MFM, PFM) and Raman spectroscopy. The magnetic moment is analysed by a superconducting quantum interference device, SQUID. Magneto-optical coupling is studied with the magneto-optical Kerr effect (MOKE), and magnetic-circular dichroism (MCD). These techniques are all discussed in this chapter.

\subsubsection{Rutherford backscattering, RBS}

Rutherford backscattering was carried out on $3 \mathrm{MeV}$ Van de Graaff generator at GNS Science. The measurements were done with $2 \mathrm{MeV}^{4} \mathrm{He}^{+}$ions with the detector placed at $165^{\circ}$ and the sample placed at normal incident. To avoid channelling issues, a rotation or tilting of the sample was induced [Wang and Nastasi, 2009]. 
Rutherford backscattering spectrometry (RBS) is an ion beam analysis characterisation technique used in fields of material science to investigate the composition and depth profile of a selected element, such as bismuth, iron and oxygen in the case of $\mathrm{BiFeO}_{3}$ thin film [Wang and Nastasi, 2009; Kennedy et al., 2007]. RBS is a technique based on Rutherford scattering of helium particles from atoms' matrix. The kinematic factor is the ratio between the scatter ion energy over the incident ion energy. This equation provides the energy for every element and many isotopes at the surface of a material. The number of counts per area units can identify the stoichiometry, composition of the film. The kinematic factor, stopping power and depth resolution are parameters evaluated by RUMP, Rutherford Universal Manipulation Program. The calculated spectrum is compared with the experiment to estimate the concentration of atoms, the density and thickness of the film.

RBS is based on the study of elastic collisions between an incident particle, in this case $2 \mathrm{MeV}^{4} \mathrm{He}^{+}$, and a stationary target, the material to be analysed. The conservation of energy and momentum in this experiment implies that the scattered particle's energy $E_{1}$ would be a fraction of the incident particle energy $E_{0}$, written as $E_{1}=K E_{0}$ [Wang and Nastasi, 2009]. where $K$ is named the kinematic factor, $K$ is equal to:

$$
K=\left(\frac{\left(M_{2}^{2}-M_{1}^{2} \sin ^{2} \theta\right)^{1 / 2}+M_{1} \cos \theta}{M_{1}+M_{2}}\right)^{2} .
$$

$\theta$ is the scattered angle with respect to the laboratory reference, which is the position of the detector, $M_{1}$ is the mass of the incident ion and $M_{2}$ is the mass of the atom in the matrix. The definition of the kinematic factor is based on the conservation of the energy and momentum before and after the collision between the incoming particle and the target material. Knowing $E_{0}$ (the energy set by our particle accelerator), $\theta$ and $M_{1}, M_{2}$ can be determined by equation 3.3.1. The Figure 3.3.1 (a) displays the effect of modifying the detector angle analytically form the equation 3.3.1. The detector angle is placed at $165^{\circ}$ for practical reason, optimisation of mass resolution and depth profile. The mass separation capability is highlighted in Figure 3.3.1 (b).

At an angle of $165^{\circ}$, the detector can separate different masses at different incident energies $E_{0}$, as seen in Figure 3.3.1 (b).

The energy resolution or the mass resolution, $\Delta E_{M_{2}}$, is defined by equation 3.3.2 [Wang and Nastasi, 2009]: 


$$
\Delta E_{i}=E_{0}\left(\frac{d K}{d M_{2}}\right) \Delta E_{M_{2}}
$$

If the difference in energy, $\Delta E_{i}$, is the minimum separable energy possible for the detector, then mass resolution can be written as:

$$
\Delta E_{M_{2}}=\partial M_{2}=\frac{\partial E}{E_{0}\left(\frac{d K}{d M_{2}}\right)} .
$$

The mass resolution is inversely proportional to the energy of the incident beam, so increasing the incident energy results in an increase mass separation, while the mass resolution is directly proportional to the energy resolution of the detector.

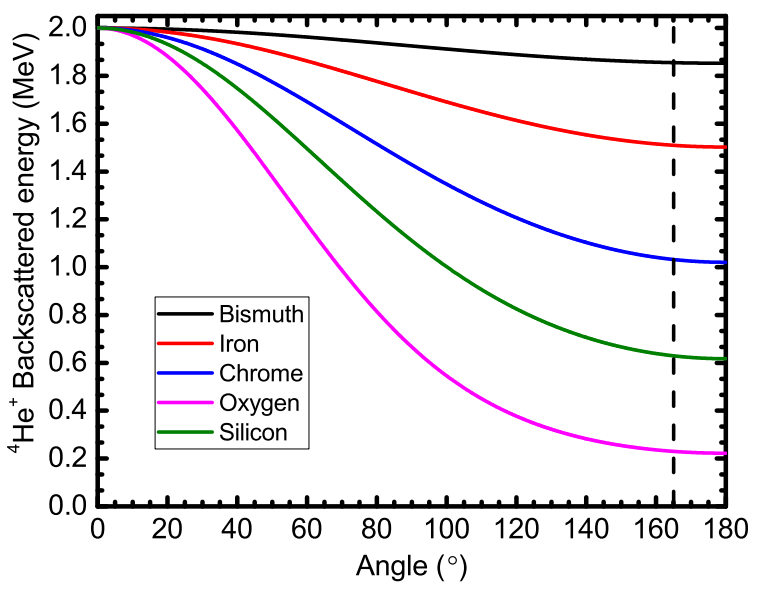

(a) Incident angle effects.

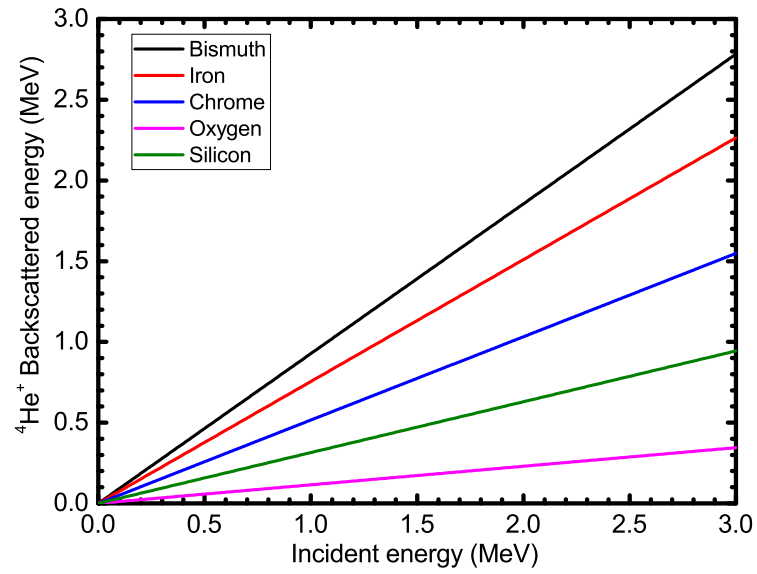

(b) Incident energy effects.

Figure 3.3.1: (a) Mass resolution with an incident ${ }^{4} \mathrm{He}^{+}$ions at $2 \mathrm{MeV}$ for a detector place between $0^{\circ}$ and $180^{\circ}$. The vertical dashed line shows the position of the detector in the laboratory. (b) Incident energy impact on the mass detection for a detector placed at $165^{\circ}$.

In the case of the Coulomb force Rutherford backscattering, the incident particle trajectory is hyperbolic and the cross-section is the probability that the trajectory will fall between an angle $\theta$ and $\theta+\Delta \theta$, angle that are visible to the detector. The crosssection can be written in the form [Nastasi et al., 2004]:

$$
\frac{d \sigma(E, \theta)}{d \Omega}=0.02073\left(\frac{Z_{1} Z_{2}}{4 E_{0}}\right)^{2}\left(\left(\sin \frac{\theta}{2}\right)^{-4}-2\left(\frac{M_{1}}{M_{2}}\right)^{2}\right)
$$




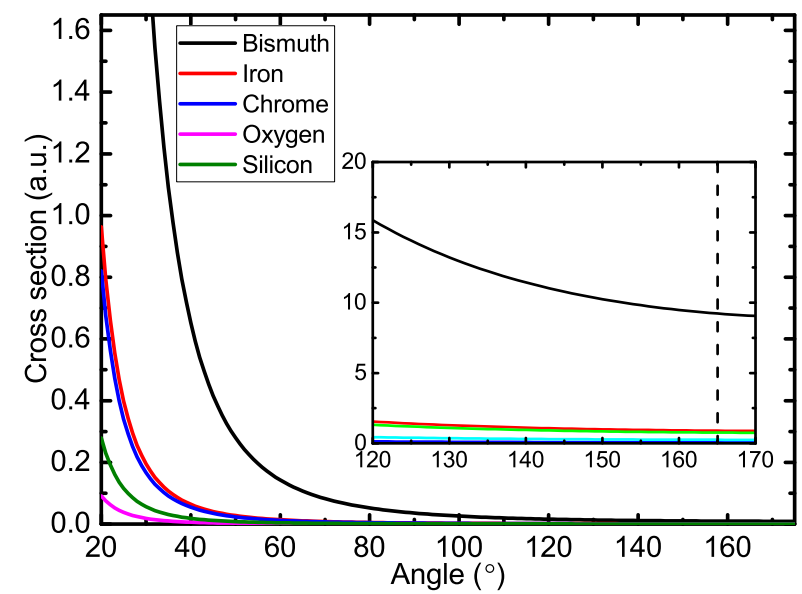

Figure 3.3.2: Detector's angle effect on cross-section for incident $2 \mathrm{MeV}^{4} \mathrm{He}^{+}$. Inset shows the cross-section between $120^{\circ}$ and $170^{\circ}$. The dash line shows the detector's position at $165^{\circ}$.

Figure 3.3.2 and Figure 3.3.3 display the impact of varying the angle $\theta$ and the incident energy $E_{0}$ on the cross-section, equation 3.3.4.

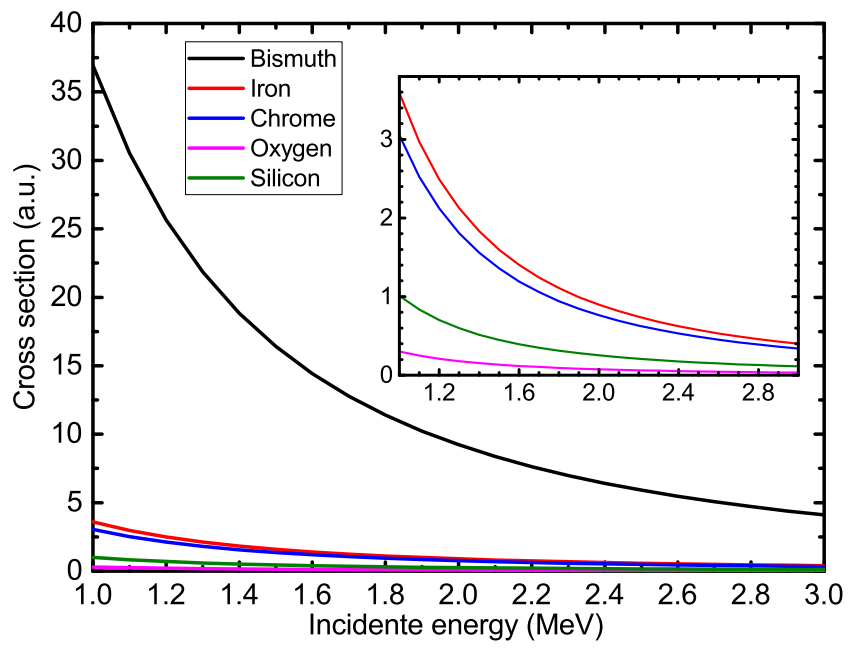

Figure 3.3.3: Incident energy beam effect on the cross-section. The inset shows the different cross-sections for $\mathrm{Fe}, \mathrm{Cr}, \mathrm{O}$ and $\mathrm{Si}$.

Backscattered particles lose more energy when they come from inside the target compared to the surface of the target. This is caused by atomic interaction; statistically, more collisions or near Coulomb interactions happen when the incident particle reaches a deeper layer of the material [Wang and Nastasi, 2009]. The energy loss is expressed with equation 3.3.5:

$$
E_{1}=E_{0}-\left.\frac{d E}{d x}\right|_{E_{0}} ^{E_{1}} x
$$


where $x$ represents the distance travelled in the target. So, a broadening of the RBS peak, illustrated in Figure 3.3.4, is linked to the thickness of the sample.

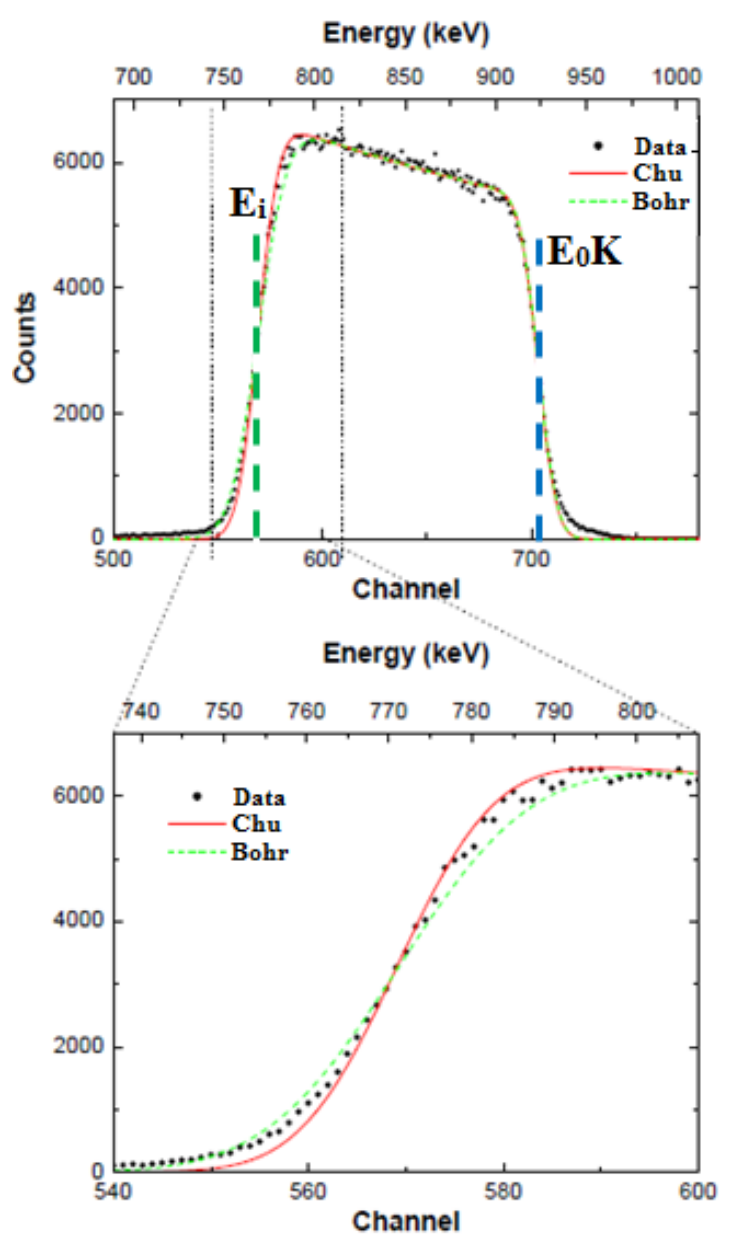

Figure 3.3.4: Example of RBS spectrum to illustrate the depth scale of Au on Si [Mayer, 1997]. Simulation of straggling effect is shown, Chu (red) and Bohr (green) models, in RBS spectra.

Figure 3.3.4 illustrate the depth scale of a thin film. The blue dashed lines represent the atoms at the surface, the surface peak $E_{0} K$. If a thin film has a negligible thickness, the surface peak and the scattered $\alpha$-particle energy, the back edge of the layer (the green short dashed line in the Figure 3.3.4, $E_{i}$ ), would be overlapping. Equation 3.3.5 provides the theoretical calculation of $E_{i}$ [Wang and Nastasi, 2009].

The loss of energy $E_{i}$ is caused by the stopping power of the matrix, equation 3.3.6 and 3.3.7:

$$
\begin{gathered}
\Delta E=K\left(\frac{d E}{d x}\right)_{\text {in }} \frac{x}{\cos \theta_{1}}+\left(\frac{d E}{d x}\right)_{\text {out }} \frac{x}{\cos \theta_{2}} \\
{[S]=K\left(\frac{d E}{d x}\right)_{\text {in }} \frac{1}{\cos \theta_{1}}+\left(\frac{d E}{d x}\right)_{\text {out }} \frac{1}{\cos \theta_{2}} .}
\end{gathered}
$$




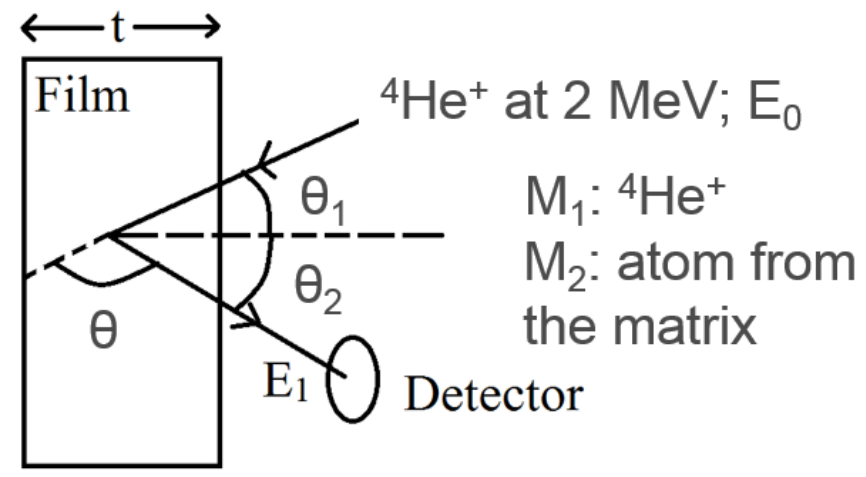

Figure 3.3.5: Schematic of ${ }^{4} \mathrm{He}^{+}$Rutherford backscattering.

As shown in Figure 3.3.5, the angle $\theta_{1}$ is the angle between the incident beam and the normal of the sample surface. $\theta_{2}$ is the angle between the scattered $\alpha$-particle, the detector position, and the normal of the sample surface.

Assuming the energy is the minimum energy detectable at a certain depth, then equation 3.3.8 is the depth resolution:

$$
x=\frac{\partial E}{[S]} .
$$

If the variation in energy in function of the depth, $d E / d x$ in equation 3.3.7, is constant then the depth resolution improves when either $\theta_{1}$ or $\theta_{2}$ increases. Tilting the sample stage would thus provide a better depth resolution [Wang and Nastasi, 2009].

The Figure 3.3.4 highlight the straggling effects. RUMP computation carried in this researched used a straggling parameter lower than 5. Straggling occurs when a beam of charged particles penetrates matter, the loss of kinematic energy during the interaction is accompanied by a spread in the beam energy. Straggling represents a statistical fluctuations of energy transfer during the Rutherford scattering [Mayer, 1997].

In the case of a thin film, the signal in the RBS spectrum for a single element is representative of the areal density of the film. For example, Figure 3.3.4 shows the Au signal of $\mathrm{Au}$ on $\mathrm{Si}$ thin films, in atoms. $\mathrm{cm}^{-2}$. The density or theoretical density allows the calculation of the thickness of the film [Wang and Nastasi, 2009].

RBS analysis requires that a few parameters be fixed, including the incident particle's mass $M_{1}$, the energy of the incident particle $E_{0}$, and the angle between the incident, backscattered beam and the normal to the surface of the target. The selected 
incident ion beam is ${ }^{4} \mathrm{He}^{+}$at $2 \mathrm{MeV}$, this energy is chosen to optimise the mass resolution and depth resolution. The angles $\theta_{1}$ and $\theta_{2}$ are fixed at $0^{\circ}$ and $15^{\circ}$ in this case, with the possibility of modifying them to improve the depth resolution as mentioned earlier, equation 3.3.7. These angles represent a good compromise to differentiate the kinetic factor and increase the cross-section for most elements. Other parameters to optimise this technique include the incident energy; the selected energy is chosen after analysing the depth profile of elements in the thin film, the mass separation. A multilayered thin film sample may increase the complexity of analysing the depth profile, the element separation could be more extensive if heavy elements are in layers below the surface. To analyse the composition, a clear separation of the peaks is required; the mass of bismuth, iron and oxygen is sufficient for a film of less than $200 \mathrm{~nm}$ to be resolved with a $2 \mathrm{MeV}^{4} \mathrm{He}^{+}$beam.

Sample charging and channelling are two phenomena that can have a significant impact on the RBS spectrum. Sample charging is possible with an electrically isolating material, one possibility in that context is an accumulation of charge on the surface sufficient to divert the ion beam inducing inaccuracy in the charge statistic [Wang and Nastasi, 2009]. Channelling, or alignment of the incident beam with one of the crystal's preferred orientations as shown in Figure 3.3.5, can happen in a crystal or a polycrystalline structure. As seen in Figure 3.3.5, the incident particle can travel farther into a deeper layer with a limited interaction with the channelled layer; resulting in a lower count of the channelled element. This would thus underestimate the concentration and stoichiometry of those elements [Wang and Nastasi, 2009]. To limit the probability of channelling, inducing a rotation or tilting the sample is required.

\subsubsection{X-ray diffraction, XRD}

X-ray diffraction (XRD) measurements were carried out using a $\mathrm{Co} \mathrm{K} \alpha$, wavelength of $0.1789 \mathrm{~nm}, \mathrm{X}$-ray source. The operating voltage was $40 \mathrm{kV}$ with a current of $35 \mathrm{~mA}$. The samples are place at grazing angle in the case of $\mathrm{BiFeO}_{3}$ thin film and implanted films.

X-ray diffraction is a non-destructive analytical technique to characterise phase composition structure of samples [Agbo et al., 1832; Ermrich and Opper, 2013]. The phase identification is possible by comparing the X-ray diffraction of an unknown sample with a series of references [Ermrich and Opper, 2013]. X-rays are produced after exiting the electronic cloud of cobalt $56,{ }^{56} \mathrm{Co}$, and a loss of electronic energy by 
Bremsstrahlung. The recombination electrons-holes between the orbital L and K result in the emission of light, this occurs in the X-ray spectrum, the other wavelength and transition are filtered out to avoid light contamination [Ermrich and Opper, 2013]. The $\mathrm{X}$-ray generator tube is filtered to prevent the emission of more energetic light particles like the $\mathrm{K} \beta$. The concept of this analytical technique is visualised in Figure 3.3.6. The $X$-ray source generates a parallel beam of $X$-ray light directed towards the sample at an angle $\theta$. If the reflected light creates a constructive wave front, then the detector placed at an angle $\theta$ will detect and convert that light into an electronic signal.

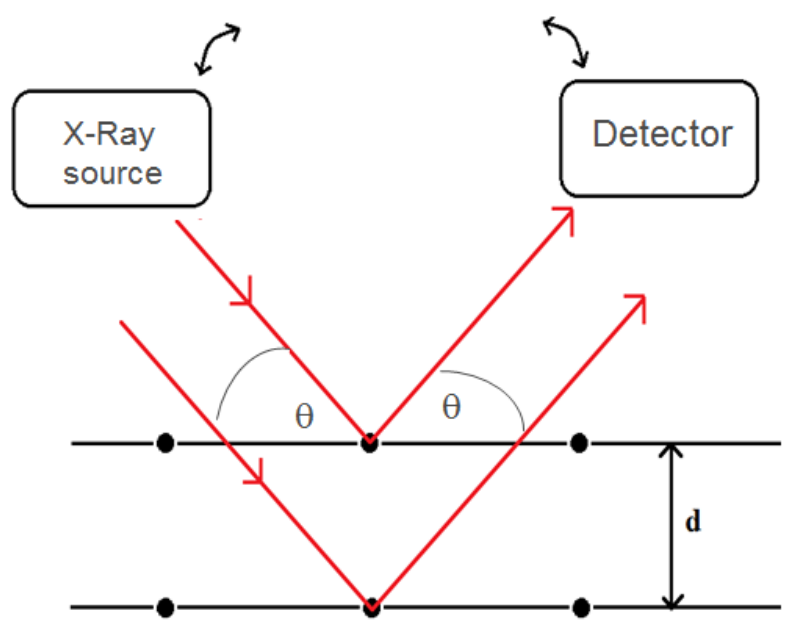

Figure 3.3.6: Graphical representation of Bragg's Law.

Bragg's law, equation 3.3.9, describes the constructive interference reflected light.

$$
2 d \sin \theta=n \lambda
$$

Where $d$ is the spacing between the two planes of the sample, $\theta$ is the incident and reflected beam angle, $\lambda$ is the wavelength of the X-ray source light and $n$ is the interference order, which is a multiple of the wavelength [Ermrich and Opper, 2013]. Constructive reflection happens in the cases of crystals or polycrystalline samples not for amorphous materials. The d-spacing comes from the cubic structure: $1 / d^{2}=\left(h^{2}+\right.$ $\left.k^{2}+l^{2}\right) / a^{2}$. The $\mathrm{d}$-spacing is the conversion from the Miller indices to the real coordinates [Ermrich and Opper, 2013]. In the case of a rhombohedral material like $\mathrm{BiFeO}_{3}$, equation 3.3.10 gives the d-spacing value [Duffy, 2009],

$$
\frac{1}{d^{2}}=\frac{\left(h^{2}+k^{2}+l^{2}\right) \sin ^{2} \alpha+2(h k+k l+h l)\left(\cos ^{2} \alpha-\cos \alpha\right)}{a^{2}\left(1-3 \cos ^{2} \alpha+2 \cos ^{3} \alpha\right)} .
$$


The reflected X-ray light should be constructive when $d$ in equation 3.3.10 satisfies the relation 3.3.9, unless the sample absorbs the incident light [Ermrich and Opper, 2013].

To optimise the signal in the diffractogram, the incident $X$-ray beam is placed at a grazing angle with the substrate for thin films. This allows the reflection from the substrate to be minimised, since the thin film is too thin to prevent light reflection from the silicon substrate [Ermrich and Opper, 2013]. The Figure 3.3.6 represents the normal incidence analysis that was used to study the bulk material and $\mathrm{BiCrO}_{3}$. While the Figure 3.3.7 represent the XRD grazing angle configuration used to analysis the $\mathrm{BiFeO}_{3}$ thin film at a grazing angle of $2^{\circ}$ and the implanted film at a grazing angle between $2^{\circ}$ and $5^{\circ}$.

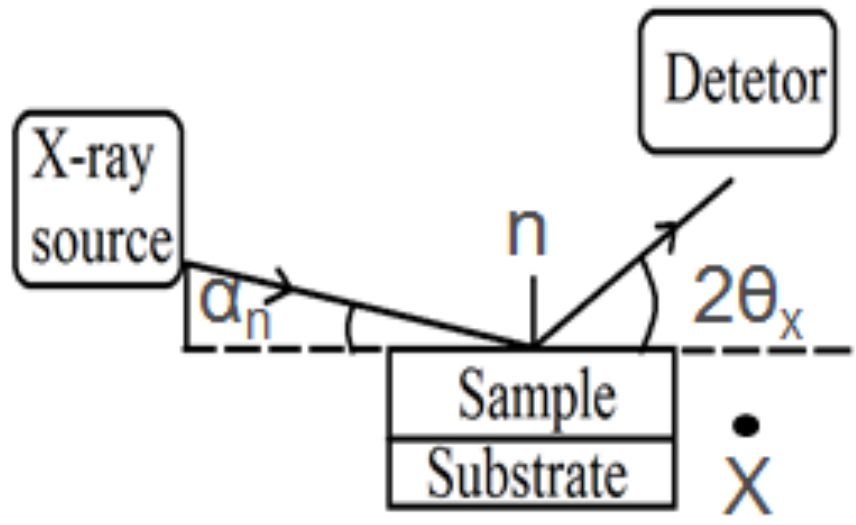

Figure 3.3.7: Graphical representation of Bragg's Law, grazing angle.

The intensity of the reflected light is proportional to the constant for the instrument, X-ray generator, (generally a value between 0.8 and 0.95 ), the absorption coefficient of the material, the polarisation factor, the Lorentz factor, and the sum of the scattering factor for each atom divided by the volume of the unit cell [Ermrich and Opper, 2013]. This explains the variability of the relative intensity measured by the detector. The absorption coefficient can explain why some combinations of Miller indices do not generate any reflected light. The limit of detection is $3 \sigma$. Increasing the time of measurement results in a better signal to noise ratio [Ermrich and Opper, 2013].

The Scherrer equation, equation 3.3.11 [Scherrer, 1918], in X-ray and crystallography is a formula that relates the size of crystallites or sub-micro particles in a solid to the diffraction pattern peak broadening [Scherrer, 1918; Patterson, 1939]:

$$
\tau=\frac{K \lambda}{\beta \cos \theta} .
$$


where $\tau$ is the mean size of the crystallites, $K$ is a dimensionless shape factor of 0.9 in this case, $\lambda$ is the $X$-ray wavelength, $\beta$ is the line broadening at full width at half maxima after removing the instrumental line broadening and $\theta$ is the diffraction and incident angle. The real broadening $\beta$ is calculated with equation 3.3.12,

$$
\beta^{2}=\beta_{\text {measure }}^{2}-\beta_{\text {instrument }}^{2}
$$

Where $\beta_{\text {measure }}$ is the broadening measure in the diffractogram and $\beta_{\text {instrument }}$ is the broadening generated by the instrument, which has a value of 0.1 in this study.

The Wilson equation, equation 3.3.13, links the peak broadening with the microstrain [Ermrich and Opper, 2013]:

$$
\epsilon=\frac{\beta_{e}}{4 \tan \theta}
$$

$\beta_{e}$ is the broadening caused by the lattice strain, $\epsilon$ is the micro-strain and $\theta$ is the diffraction angle.

Assuming the total broadening is the sum of the crystallite size broadening and the micro-strain (equation 3.3.11 and 3.3.13) and defining the constant $C$ to simplify the equation, the Williamson-Hall relation can be written as equation 3.3.14:

$$
\begin{gathered}
\beta_{\mathrm{tot}}=\beta+\beta_{e}=\frac{K \lambda}{\tau \cos \theta}+C \epsilon \tan \theta, \\
\beta_{\mathrm{tot}} \cos \theta=\frac{K \lambda}{\tau}+C \epsilon \sin \theta .
\end{gathered}
$$

The Williamson-Hall plot, $\beta \cos \theta$ as a function of $\sin \theta$ equation 3.3.14, provides the micro-strain, which is given by the slope $(C \epsilon)$, and the grain size, which is given by the interceptor $(K \lambda / \tau)$ (this is only an approximation and must be treated carefully) [Ermrich and Opper, 2013].

The depth profiling of the X-ray varies as a function of the absorption of the sample. Metallic samples tend to absorb light [Fitzpatrick et al., 2005]. This aspect is not a concern for this project, as the thin films are visible and thin. Also, by measuring the $\mathrm{X}$-ray diffraction at the grazing angle, the substrate is not visible. 
X-ray diffraction is a non-destructive analysis technique, in our case for the study of thin films, that is useful for confirming the structure and crystallography space group. By comparing the diffractogram with a series of references and Scherrer's equation, this technique provides an indication of the crystallite's size [Lacroix et al., 2011].

\subsubsection{Microscopy}

This section focuses on atomic force microscopy and other analyses techniques available with similar setups. Transmission electron microscope is also discussed in this section.

\subsubsection{Atomic force microscope, AFM}

The atomic force microscopy follows the Leonard-Jones, shown in Figure 3.3.8, potential where the repulsive force of the Coulomb interaction and the Pauli exclusion and the attraction of the Van der Waals force modify the cantilever frequency and amplitude in function of the distance between the tip and the surface of the material study.

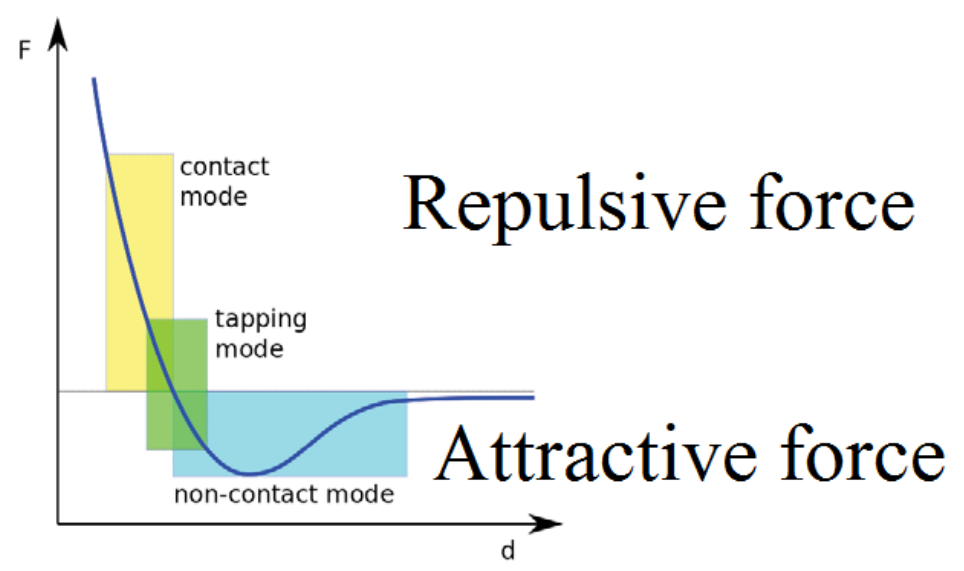

Figure 3.3.8: Atomic force microscopy, Leonard Jones potential [Renkert, 2014].

The atomic force microscope is made of a tip fixed to a cantilever; a laser is reflected off the cantilever and directed to a photo-detector whose output signal is collected and interpreted by Nanosurf software [Nanosurf, 2015]. Three modes of contact can be used to scan the surface of samples. In the contact mode or static mode, the tip touches the surface of the sample with a force of less than $10 \mathrm{nN}$ while scanning the surface. The tip will raise and lower following the particle grain size. In the tapping mode or dynamic mode, the tip oscillates at a specific frequency, each tip has a specified frequency by the manufacturer, maintained by the system. Each perturbation 
from the sample surface, like the Van der Waals force, is converted into particle height. The third mode is non-contact. In this mode, the cantilever oscillates over the surface of the sample, the tip is maintained at a distance of less than $200 \mathrm{~nm}$ from the surface of the sample, and detects the Van der Waals interaction with the surface to map the surface of the sample [Nanosurf, 2015]. The scanning speed is about 1.1 to 1.3 points per second for image of size between $10 \mu \mathrm{m}$ to $50 \mu \mathrm{m}$. The contact mode and tapping mode were used for practical reason. The non-contact mode was used to study the magnetic and electrical response from the tip.

Atomic force microscopy (AFM) is a surface scanning technique detailing the grain size, height and the average plane height. AFM can be used for force measurement, imaging and manipulation [Wiesendanger and Meyer, 2009]. The imaging is carried out by probing the force that the sample applies to the tip. This can be used to perform a 3D mapping of the sample surface. At GNS Science, the AFM is a Nanosurf FlexAFM model, and the sample images presented in this thesis have been taken in contact or tapping mode. The vertical resolution is typically in the order of $0.5 \mathrm{~nm}$ and the horizontal resolution is in the order of 1-5 nm due to the shape of the tip and the condition of operation [Nanosurf, 2015].

Every image was process with Gwyddion software to remove the background with a polynomial function. The grains were levelled to face upward and a polynomial function is applied to align the topographic scanned lines. Horizontal artefacts were subtracted. All the images were scaled with the same values and render in 3-D.

\subsubsection{Transmission electron microscopy, TEM}

Transmission electron microscopy was used to characterise the micro-structure of the film. The sample preparation used the focus ion beam, FIB, technique. This is done in place of the commonly ion milling technique. The sample was coated with $\mathrm{Pt}$ and $\mathrm{C}$ to eliminate secondary electrons. The TEM resolution is limited by the electron diffraction. Cross-sectional transmission electron microscope (TEM) images were taken using a Tecnai TF20 with an acceleration voltage of $200 \mathrm{kV}$. The measurements were carried out by Dr Serguey Rubanov's research group at the University of Melbourne, Australia. The samples were prepared using the focused ion beam lift-out methods [Giannuzzi et al., 1998]. Selected area electron diffraction (SAED) micrographs were also recorded.

Prior to TEM analysis, the samples need to be prepared. The preparation tech- 
nique used by Dr Rubanov is focused ion beam (FIB). The cross-section is milled out of the sample by an electron-transparent membrane. The sample is then placed on a carbon-coated [Giannuzzi et al., 1998] platinum substrate. FIB, Ga beam, is a precise sputtering technique, removing as little material as required from the cross-section sample. This sputtering technique can be carried out with gallium ion plasma that could modify the composition and structure of the sample by implanting gallium [Baram and Kaplan, 2008]. Polishing the sample with this method requires hours to remove a few microns.

TEM is an electron beam microscope technique [Carter and Williams, 2016; Cowley and Rees, 1958], bright field imaging in this case. The electron beam is transmitted through the material to be analysed, and the interaction of the beam with the sample provides an image with resolution in the order of a few nanometres. TEM imaging contrast can be caused by the sample partially absorbing the electron beam, $\mathrm{z}$-contrast. The absorption happens with heavier elements or with thick films [Carter and Williams, 2016; Cowley and Rees, 1958].

The theoretical image resolution is limited by the wavelength of the source, equation 3.3.15 [Fultz and Howe, 2012; Newnham and Roy, 1921]:

$$
d=\frac{\lambda}{2 n \sin \theta}
$$

where $d$ is defined as the image resolution, $\lambda$ is the wavelength of the source and $\theta$ is the angle between the electron beam source and the sample surface. The electron wavelength can be approximated by equation 3.3.16 [Goodhew, 2001]:

$$
\lambda_{e}=\frac{h}{\sqrt{2 m_{0} E\left(1+\frac{E}{2 m_{0} c^{2}}\right)}} .
$$

Where $\lambda_{e}$ is the electron wavelength, $h$ is the Planck constant, $m_{0}$ is the rest mass of an electron, $E$ is the energy of the accelerated electron and $c$ is the speed of light.

The bright field image maps an area where the contrast is modified mainly by the absorption or reflection of the electron beam [Cowley and Rees, 1958] shows the cross-section of the thin film, depth profile of bismuth, iron and oxygen. In the case of 
a high and well-defined contrast, the image allows the measurement of the molecule's dimension, assuming a flat two-dimensional film.

SAED is a technique based on a diffraction pattern of the crystal structured material. The position and the size of the aperture is adjustable to fulfil the measurement requirements. The position of the beam on the sample is important for extensive analysis of polycrystalline material; the selected area can be limited to one single crystal at a time. Comparing two crystals can provide information on their relative orientations.

SAED is projected in the Fourier plane. If the sample is placed in a low crystal index area, the crystal structures and lattice parameter can be measured by SAED. This can be difficult to achieve with amorphous or polycrystalline samples. This technique can identify certain defects, lattice matching, and interfaces [Neogy, 2012; SAED, 2016]. Diffraction of a single pure crystal will result in a series of dots. Crystals like the silicon substrate, when undisturbed, display diffraction patterns in discrete positions, constructive Bragg's diffracted areas. While a polycrystalline sample would result in diffraction of a series of ring shape [Cowley and Moodie, 1957]. The diffraction radius is dependent on the d-spacing of the crystals, while the d-spacing depends on the crystal's orientation and Miller indices. SAED analysis is limited by sample thickness, orientation, lens focus and optical aberrations [Cowley and Moodie, 1957]. Any impurities that do not displace the crystal lattice will, if visible, display a weak contrast [Hull and Bacon, 2001].

\subsubsection{Magnetic force microscopy, MFM}

The magnetic response is similar to the AFM setup. The difference between the two operating modes is that the MFM analysis requires the tip to be magnetically charged in order to analyse the magnetic response and image the results. The charged tip will induce an attractive or repulsive force, leading to a deflection of the cantilever. This technique can be used to image magnetic domain on the surface of a sample.

The non-contact mode was used to study the magnetic response from the tip. The tip is maintained at a distance of less than $200 \mathrm{~nm}$ from the surface of the sample, and detects the Van der Waals interaction with the electric charge at the surface of the sample magnetic moment [Nanosurf, 2015]. The scanning speed is about 1.1 to 1.3 points per second for image of size between $10 \mu \mathrm{m}$ to $50 \mu \mathrm{m}$. The vertical resolution is typically in the order of $0.5 \mathrm{~nm}$ and the horizontal resolution is in the order of 1-5 nm due to the shape of the tip and the condition of operation [Nanosurf, 2015]. 


\subsubsection{Piezoelectric force microscopy, PFM}

The piezoelectric response is similar to the AFM setup. The difference between the two operating modes is that the PFM analysis requires the tip to be electrically charged in order to analyse the piezoelectric response and image the results. The charged tip will induce a deformation of the sample due to the piezoelectric effect, leading to a deflection of the cantilever. This technique is ideal for imaging the ferroelectric domain on the surface of a sample. Two effects can be visible from PFM analysis, the lateral and the amplitudinal piezoelectric response. The lateral response is caused by a deflection of the cantilever as the tip is either attracted or repulsed by the charge near the surface of the sample. The amplitude is the movement up and down the cantilever, which will occur as the sample attracts or repulses the tip from the electrical charge at the surface. As a simple photo-detector can separate the two motions and provide both analyses at the same time. By optimising the applied voltage on the cantilever, the lateral and amplitudinal response can be tune to be the in-plane and out-of-plane polar components. With the charged tip in contact with the sample, the sample can be charge locally and then analyse the piezoresponse to confirm the presence of the ferroelectric domain at the surface. In this case study, the ferroelectricity was confirmed at ambient temperature with this technique. The ferroelectric property confirms the sample contain a significant amount of $\mathrm{BiFeO}_{3}$. Combining the PFM analysis with the magnetic ordering also confirms the presence of a multiferroic material.

The non-contact mode was used to study the electrical response from the tip. The tip is maintained at a distance of less than $200 \mathrm{~nm}$ from the surface of the sample, and detects the Van der Waals interaction with the electric charge at the surface of the sample piezoelectric response [Nanosurf, 2015]. The scanning speed is about 1.1 to 1.3 points per second for image of size between $10 \mu \mathrm{m}$ to $50 \mu \mathrm{m}$. The vertical resolution is typically in the order of $0.5 \mathrm{~nm}$ and the horizontal resolution is in the order of $1-5 \mathrm{~nm}$ due to the shape of the tip and the condition of operation [Nanosurf, 2015].

\subsubsection{Kelvin probe microscopy, KPM}

Kelvin probe force microscopy, KPFM or KPM, can be visualised as an electricallycharged AFM tip in tapping mode [Nanosurf, 2015]. This characterisation technique is used to analyse the local work function. The work function is the minimum energy required to strip an electron from the surface of a solid material [Kittel, 2005]. The work function is defined in equation 3.3.17: 


$$
W=-e \phi-E_{F} .
$$

Where $-e$ is the charge of electron, $\phi$ is the electrostatic potential in the vacuum near the sample surface and $E_{F}$ is the Fermi level [Kittel, 2005]. If a readable voltage from a voltmeter is defined as $V=-E_{F} / e$, then equation 3.3 .17 can be rewritten as equation 3.3.18:

$$
\phi=V-\frac{W}{e} .
$$

This technique probes the mechanical response of the cantilever to the difference in voltage between the tip and the material under study. The experimenter controls the applied voltage, $V$, on the cantilever. The electric field between the sample and the probe can be applied and maintain constant resulting in a direct comparison of the work function between the sample and the tip of the microscope. This process can be visualised as a capacitor system; the current flow will change as a function of the distance between the tip and the material given a constant applied voltage. This technique provides another point of view on the piezoresponse of the material. KPM was used for practical reason to replace and confirm the PFM results. The operation condition and the resolution are the same as the PFM measurements.

The same setup can also create local (the area of a few atoms) I-V curves or current as a function of the voltage and provide a nanoscale resistivity analysis. The measurements were carried at ambient temperature.

\subsubsection{Raman spectroscopy}

Raman spectroscopy analysis was carried at Victoria University of Wellington with the confocal LabRam, $514 \mathrm{~nm}, 1800 \mathrm{~cm}^{-1}$ grating with an objective of $100 \times$ and $50 \times$. The beam spots when using the $100 \times$ objective is in the order of a micron and the laser power was limited to less than $10 \mathrm{~mW}$ to avoid heating the samples. The Raman setup reduces the detected scatter light from air.

Raman spectroscopy is a characterisation technique to analyse the vibration and rotation of atoms in materials by using an inelastic light collision [Le Ru and Etchegoin, 
2008]. Raman spectroscopy uses a laser to excite the ions in a material, in this case a polycrystalline thin film, resulting in vibration along the degrees of freedom allowed by the crystal structure, generating a wavelength in the material called normal mode. Any unstable excited mode dissipates. The normal modes are unique characteristic. The crystal case is interesting, as the vibration can be approximated by an oscillator system with an energy of $E=(n+1 / 2) h / \lambda$. These quanta of energy represent the energy of the Raman mode and are described as phonons. The phonon is to material vibration what the photon is to light; they both describe the wavelength behaviour of a system. Raman scattering occurs when the incident light is scattered by a phonon. In the case where the light interacts with the crystal, a phonon can be created, this event is called Stokes scattering, however if the interaction results in absorption of a phonon, then this event is said to be anti-Stokes. The incident light can potentially interact with multiple phonons at the same time; these interactions are higher order. Raman scattering of first order happens only when the incident light is scattered by one phonon. Due to the higher number of interactions, higher order events result in a weaker relative intensity and broader Raman shift scattered light. The Stokes scattering probability is proportional to the available occupied low-energy vibration states and vacant high-energy vibrational states, while the probability of anti-Stokes scattering is inversely proportional to these states [Le Ru and Etchegoin, 2008]. Raman spectroscopy is instantaneous, no intermediate states are required to physically explain the phenomenon.

\subsubsection{Magnetometer, magnetic moment ordering measurement}

Magnetisation measurements were performed on a Quantum Design, Superconducting QUantum Interference Device (SQUID) at Robinson Research Institute. The measurements were performed on films sputtered and implanted on silicon dioxide and silicon. McElfresh [1994] provides details about the SQUID components and assemblage. The magnetometer is made of a superconducting solenoid, Figure 3.3.9(a), acting like a superconducting magnet and a superconducting detection coil, and a pick-up coil [McElfresh, 1994]. The SQUID is based on a Josephson junction. The detector senses the magnetic field from the sample as the sample's magnetic moment induces a small voltage in the pick-up coil [McElfresh, 1994]. The Figure 3.3.9(b) displays a gradiometer. the gradiometer is a detection coil made of a single piece of superconductor with two counterclockwise and two clockwise turning coils counterbalance any modification of the magnetic flux caused by the sample. The signal output is analysed using an iterative method. The SQUID voltage is fitted to equation 3.3.19 [McElfresh, 1994]: 


$$
\begin{gathered}
f(z)=P_{1}+P_{2}+P_{3}\left(2 \cdot\left(0.97^{2}+\left(z+P_{4}\right)^{2}\right)^{-\frac{3}{2}}-\left(0.97^{2}+\left(1.519+\left(z+P_{4}\right)^{2}\right)\right)^{-\frac{3}{2}}\right. \\
\left.-\left(0.97^{2}+\left(1.519+\left(z+P_{4}\right)^{2}\right)\right)^{-\frac{3}{2}}\right) .
\end{gathered}
$$

where $P_{1}$ is an offset constant and $P_{2}$ is the linear background of the vertical position $z$ through the coil. $P_{3}$ and $P_{4}$ are dipole components; $P_{4}$ is a shift in the function of vertical position [McElfresh, 1994]. The magnetic moment (emu) is defined by equation 3.3.20:

$$
\text { moment }=\frac{P_{3} S_{1}}{S_{2} s S_{3}}
$$

$S_{1}, S_{2}$ and $S_{3}$ are the calibration factors $\left(S_{1}=1.825, S_{2}=7512.185, S_{3}=0.9125\right)$ and $s$ is the sensitivity calibration factor.
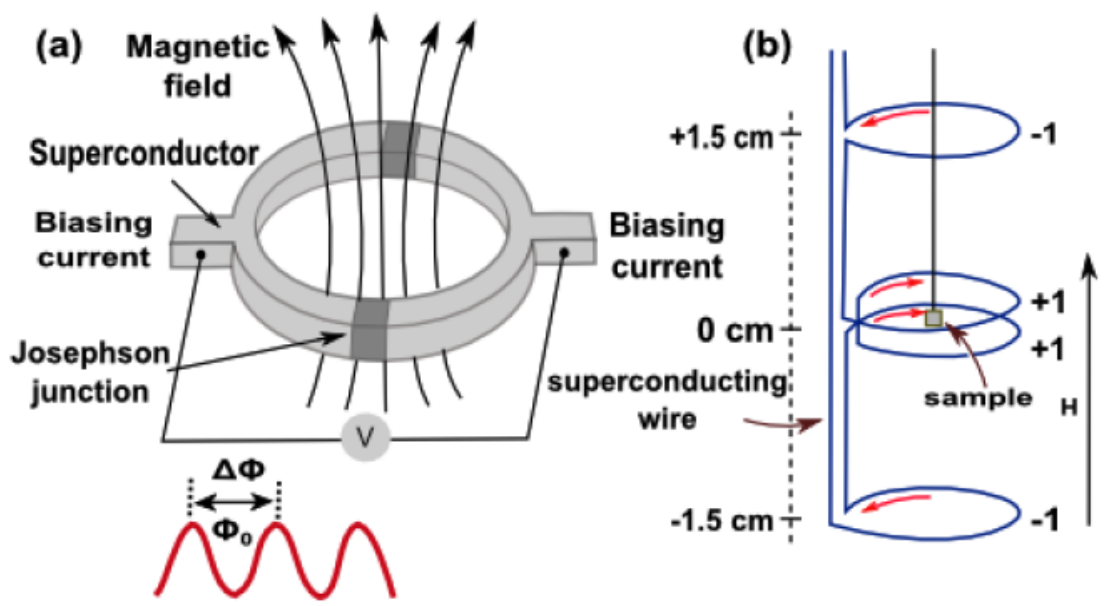

Figure 3.3.9: (a) Schematic of a SQUID sensor [Rohlf, 1994] and (b) second-order gradiometer superconducting pick-up coils [McElfresh, 1994].

The sample's holder is made of two straws as displayed in Figure 3.3.10, the straws made of plastic are used to avoid any stray magnetic moments. The sample holder is attached to a rigid rod to bring the sample to the superconducting coil. If the sample is longer than the scanned area, then the fit might not be reliable. Another potential issue is a non-uniform magnetic sample; the ideal case would then not apply [McElfresh, 1994]. Another limiting factor is the strength of the signal measured. A magnetic sample on the order of $\sim 10^{-6} \mathrm{emu}$ and lower results in poor-quality fitting, with the limit being from $10^{-8}$ to $2 \mathrm{emu}$. The vibrating sample magnetometer (VSM) is limited to $10^{-6} \mathrm{emu}$, this is two orders of magnitude less sensitive [McElfresh, 1994]. 
The magnetic moments are measured at a variable applied magnetic field, from $-6 \mathrm{~T}$ to $6 \mathrm{~T}$, and variable temperature, from $5 \mathrm{~K}$ to $340 \mathrm{~K}$. The field loop measurements were carried out at $5 \mathrm{~K}$ and $300 \mathrm{~K}$. The temperature dependence measurement was carried out at $2.5 \mathrm{~T}$ and $6 \mathrm{~T}$ while the zero-field-cooled and field-cooled (ZFC-FC) measurements were carried out at $10 \mathrm{mT}$. The zero-field-cooled (ZFC) sample is initially cooled down to $5 \mathrm{~K}$ and measured while the temperature increases to $340 \mathrm{~K}$. During the cooling stage the field is zero Tesla, while during the measurement the applied magnetic field is $10 \mathrm{mT}$. The field-cooled (FC) measurement is carried out at $10 \mathrm{mT}$ during the cooling of the sample from $340 \mathrm{~K}$ to $5 \mathrm{~K}$. This low field was chosen to analyse the hysteresis loop behaviour without the saturation moment. The sample, the straws and any tools that could be in contact with the sample were cleaned with isopropanol. The samples were handled with plastic tweezers as shown in Figure 3.3.10 to minimise any magnetic contamination [Abraham et al., 2005].

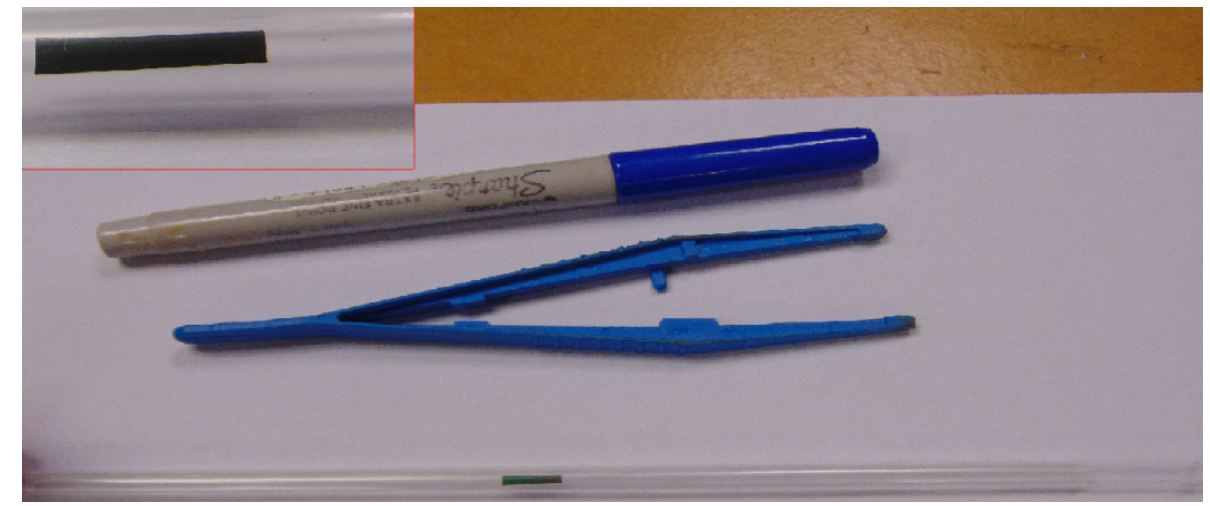

Figure 3.3.10: Example of SQUID sample mounted in plastic holder. The sample is placed between a folded plastic straw and another straw. The sample is placed at $4 \mathrm{~cm}$ from the top of the straw, optimum distance for measurement. The upper inset is an enhancement of the sample area. Samples are cut to have a width between 0.2 and $0.4 \mathrm{~cm}$ and height between 0.6 and $1 \mathrm{~cm}$ for an optimise analysis.

The software MPMS MultiVu of Quantum Design, MPMS MultiVu application Revision 1.61, Build 082 [McElfresh, 1994] converts the voltage signal to the magnetic moment in emu, as described previously. In this report, the magnetic data are plotted in units of formula unit per atom $\left(\mu_{B} \mathrm{Fe}^{-1}\right)$. The first step is to convert the magnetic field from Oersted to Tesla (10000 Oe $=1 \mathrm{~T})$. The magnetic moment measured in emu is converted to Amps $\times$ square meter: $\mathrm{m}\left[\mathrm{A} \cdot \mathrm{m}^{2}\right]=\mathrm{m}[\mathrm{emu}] 10^{-3}$ [A.m ${ }^{2}$.emu]. The magnetic susceptibility of the sample is calculated with equation 3.3.21 and provides the magnetic moment: 


$$
m_{F e}=\frac{m-\chi_{S i}\left(A \frac{B}{\mu_{0}}\right)}{\mu_{B} A n_{F e}} .
$$

$\chi_{S i}$ is the silicon susceptibility $\left(-3.26 \times 10^{-6} \mathrm{emu}\right.$, measured with the SQUID), $A$ is the sample area, $B$ is the magnetic field, $\mu_{0}$ is the permittivity of free space, and $n_{F e}$ is the Fe dose estimated from the RBS data analysis. The silicon susceptibility was measured using the SQUID. The saturation moment can be analysed from the temperature dependence given by equation 3.3.22:

$$
m_{s, F e}=m_{F e}(0)\left(1-D T^{n}\right) .
$$

where $m_{s, F e}(0)$ is the saturation moment per iron atom at $0 \mathrm{~K}, n$ is the exponent, and $D$ the temperature prefactor and is related to the spin stiffness [Bloch, 1930].

Superparamagnetism (discussed in subsection 2.1.4) occurs when the thermal energy is greater than the magnetocrystalline anisotropy energy. This magnetic state can be observed by measuring the ZFC and FC curves at low magnetic fields, and the temperature where the ZFC curve reaches a peak can be taken as an estimate of the blocking temperature $T_{B}$, equation 2.1.6 [DiPietro et al., 2010].

$\mathrm{ZFC}$ is measured during a warming up stage, at $10 \mathrm{mT}$, from $5 \mathrm{~K}$ to $330 \mathrm{~K}$. The temperature ramp up speed is $0.8 \mathrm{~K}$ per minute between $5 \mathrm{~K}$ and $15 \mathrm{~K}, 2 \mathrm{~K}$ per minute between $15 \mathrm{~K}$ and $20 \mathrm{~K}$ and $1.8 \mathrm{~K}$ per minute above $21 \mathrm{~K}$. The sample, prior to the measurements, was cool down from $330 \mathrm{~K}$ to $5 \mathrm{~K}$ without an applied magnetic field. FC was measured with and applied magnetic field of $10 \mathrm{mT}$ after the ZFC measurement with the same temperature speed. Both measurements were taken during the temperature sweep, the temperature was not stabilise to measure the magnetic moment, using the Quantum Design RSO mode. The measured values, at $0.5 \mathrm{~Hz}$, are the combination of 2 scan cycles.

\subsubsection{Optical light transmission and absorption}

The optical transmission measurement was performed at Callaghan Innovation on a split light beam transmission system. One light path passes through a blank substrate 
while the other one passes through the $\mathrm{BiFeO}_{3}$ layer deposited on the substrate. The relative transmission is provided with a spectrograph.

The semiconductor optical band gap allows for the absorption of some of the light. The absorption wavelength depends on the energy of the optical band gap. The amount of light absorbed is defined by equation 3.3.23 [Jiles, 2001]:

$$
\begin{array}{r}
I=I_{0} \exp (-\alpha x), \\
\alpha=\ln \left(\frac{I_{0}}{I}\right) \times x^{-1} .
\end{array}
$$

where $I_{0}$ is the incident light, $x$ is the thickness of the sample and $I$ is the light transmitted through the sample. This experiment can provide the optical direct band gap value from equation 3.3.24 [Jiles, 2001]:

$$
\hbar \omega \alpha=\alpha_{D} \sqrt{\hbar \omega-E_{g}}
$$

The indirect band gap can be found from equation 3.3.25 [Jiles, 2001]:

$$
\hbar \omega \alpha=\alpha_{i}\left(\hbar \omega-E_{g}\right)^{2} .
$$

where $E_{g}$ is the optical band gap energy and $\hbar \omega$ is the photon energy. A Tauc plot, which plots the absorption coefficient as a function of the photon energy, is a technique to determine the optical band gap energy [Tauc et al., 1966]. An abrupt change in the slope indicates the band gap value. To extend the Tauc plot analysis, equation 3.3.26 is necessary [Davis and Mott, 1970]:

$$
\hbar \omega \alpha=\alpha_{i}\left(\hbar \omega-E_{g}\right)^{r}
$$

The direct optical band gap $E_{g}$ is measured with an $r$ value of $1 / 2$, and the direct forbidden transition for $r$ is equal to 3/2 [Davis and Mott, 1970]. The indirect optical band gap has a $r$ value of 2 and the forbidden indirect transition of $r$ is equal to 3 [Davis and Mott, 1970]. Some authors like Jiles, 2001 neglect $\hbar \omega$ on the left side of the Tauc plot equation, equation 3.3.26. 


\section{Chapter 4}

\section{Evolution of the structure, surface and magnetic properties of sputtered BiFeO3 thin film}

This chapter details the synthesis, growth and structure of nano-polycrystalline bismuth ferrite: $\mathrm{BiFeO}_{3}$ thin film synthesized by ion beam sputtering. The magnetic properties and ordering of the films are analysed at different annealing temperatures and durations. The electrical properties are described using a piezoelectric force microscope. The magneto-optical Kerr effect (MOKE) is studied for the first time on a nano- $\mathrm{BiFeO}_{3}$ thin film between 200 and $600 \mathrm{~nm}$. The only previous work was carried out on $\mathrm{BiFeO}_{3}$ thin film at a wavelength around $1550 \mathrm{~nm}$. The analysis of bismuth ferrite is compared with iron oxide powders and thin films.

\subsection{Introduction}

Multiferroic materials, which show coupling between the electric and magnetic field, have attracted a lot of attention from researchers, as they have many potential applications [Ramesh and Spaldin, 2007; Mocherla et al., 2014] such as magnetoelectric RAM [Ortiz-Quiñonez et al., 2013; Kondo et al., 2007; Wang et al., 2013], magnetic sensors [Ortiz-Quiñonez et al., 2013], and photovoltaic cells [Yang et al., 2009]. A multiferroic interacting with light could potentially have a greater impact on future memory chip technology if the magnetic moment of the nano-material can be linked to a reflected wavelength, increasing the efficiency of such a device [Kézsmárki et al., 2011]. This property could potentially improve the integrability of optical isolators [Lopez-Santiago et al., 2009] and under specific process could be part of a quantum non-demolition dot [Puri et al., 2014]. $\mathrm{BiFeO}_{3}$ is the most studied multiferroic, as this material was the first confirmed ambient temperature multiferroic [Dho et al., 2006]. This material has interesting properties, e.g. a Curie temperature of 1103 K, and a Néel 
temperature of $643 \mathrm{~K}$ [Mocherla et al., 2014; Mazumder et al., 2007]. $\mathrm{BiFeO}_{3}$ has Gtype antiferromagnetic behaviour and has a spin canting moment of iron [Park et al., 2007]. Superparamagnetic $\mathrm{BiFeO}_{3}$ has been reported [DiPietro et al., 2010]. The $\mathrm{BiFeO}_{3}$ Verdet constant has been reported at $18 \pm 2{ }^{\circ} \mathrm{cm}^{-1} \mathrm{kOe}^{-1}$ at ambient temperature for a wavelength of $1550 \mathrm{~nm}$ [Bi et al., 2008]. The Verdet constant can be calculated from the Faraday or Kerr rotation, which provides a link between light and the magnetic moment. This knowledge could initiate a new research focus for the well-studied $\mathrm{BiFeO}_{3}$ multiferroic. The optical band gap of $\mathrm{BiFeO}_{3}$ is reported to be between 2.2 to $2.8 \mathrm{eV}$ [Chen et al., 2011; Wang et al., 2013], with most groups agreeing that the optical band bag value should be $2.5 \mathrm{eV}$.

Magnetic circular dichroism (MCD) is an experimental technique to measure the relative absorption intensity of left and right circularly polarised light while the sample is under the influence of a strong magnetic field [Stephens, 1974]. MCD is a technique, as precise as the conventional Superconducting QUantum Interference Device (SQUID) [Domingo et al., 2004], to analyse the composition of magnetic material. MCD analysis focuses on the absorption band of the material, more precisely on the Zeeman splitting and the presence of intermediate state bands between the grounded and exited bands [Stephens, 1974]. Very little work has been done on $\mathrm{BiFeO}_{3} \mathrm{MCD}$, however a few reports using soft $\mathrm{X}$-ray $\mathrm{MCD}(\mathrm{XMCD})$ on $\mathrm{BiFeO}_{3}$ are available for comparison.

The bulk compound has a small net magnetic moment when averaged over the spin cycloid period [Park et al., 2007]. The measured magnetic moment in bulk $\mathrm{BiFeO}_{3}$ can be significantly enhanced by partial substitution of different ions for Bi or Fe [Wen et al., 2016], which might be due to the suppression of the spin cycloid. An exchange bias, $\mathrm{B}_{\mathrm{ex}}$, has been reported in thin film multilayers that use $\mathrm{BiFeO}_{3}$ as a pinning layer next to a ferromagnetic layer [Wang et al., 2009b; Wu et al., 2010; Li et al., 2016] where that layer can be electrically switched [Wang et al., 2009b; Wen et al., 2016], which is particularly useful for magneto-electric RAM. $B_{\text {ex }}$ can occur when an antiferromagnetic phase is adjacent to a ferromagnetic phase and the sample is cooled to the Néel temperature in the presence of an applied magnetic field [Nogués and Schuller, 1999; Meiklejohn and Bean, 1957; Malozemoff, 1987].

Many studies on $\mathrm{BiFeO}_{3}$ nanoparticles and nanoceramics have reported that the saturation moment per $\mathrm{Fe}, m_{s, F e}$, is enhanced when the nanoparticle size is reduced [Park et al., 2007; Mazumder et al., 2007; Reddy et al., 2012; Dhir et al., 2015; Dhanalakshmi et al., 2016]. The saturation moment can reach $0.41 \mu_{B} / F e$ [Mazumder et al., 2007], where $\mu_{B}$ is the Bohr magneton. This is far greater than the bulk value of $0.02 \mu_{B} / F e$ [Park et al., 2007]. The difference could be due to the nanoparticles being 
smaller than the spin cycloid period. Under this condition, the spin cycloid structure may be reduced resulting in uncompensated surface spins that lead to a ferromagnetic shell [Park et al., 2007; Mazumder et al., 2007; Reddy et al., 2012; Dhir et al., 2015; Vijayasundaram et al., 2016]. The small nanoparticle size can also lead to enhanced magnetic, electric [Dhir et al., 2015] and multiferroic properties [Mukherjee et al., 2014]. An exchange bias has also been reported, which is absent in bulk $\mathrm{BiFeO}_{3}$ [Park et al., 2007; Mazumder et al., 2007; Dhir et al., 2015]; exchange bias either increases [Mazumder et al., 2007; Dhir et al., 2015] or decreases as the nanoparticle size is reduced [Park et al., 2007]. The exchange bias is believed to arise in $\mathrm{BiFeO}_{3}$ from a ferromagnetic shell induced in part by uncompensated surface spins [Park et al., 2007; Mazumder et al., 2007; Dhir et al., 2015]. Interestingly, there have been a few studies that do not report a significant enhancement of the saturation moment per Fe or the appearance of $B_{e x}$ [Mocherla et al., 2014; Chakrabarti et al., 2011], but a small amount of Eu doping leads to a large enhancement of the saturation moment [Chakrabarti et al., 2011]. This suggests that the appearance of an enhanced moment and exchange bias depends on the nanoparticle preparation method. For application purposes, small $\mathrm{BiFeO}_{3}$ nanoparticles in thin films might be more practical than nanoparticle powders.

$\mathrm{BiFeO}_{3}$ can also have an enhanced magnetisation when the particle sizes are small enough that they suppress the spin cycloid structure [Park et al., 2007; , DiPietro et al., 2010]. The spin cycloid structure is due to canting of the Fe moments, leading to a small net moment that rotates in space with a spiral structure with a period of $62 \mathrm{~nm}$ and results in a nearly zero moment when averaged over each period for bulk $\mathrm{BiFeO}_{3}$ [Park et al., 2007]. Superparamagnetism occurs for very small particles when the magnetocrystalline anisotropy energy is less than the thermal energy [DiPietro et al., 2010].

In this chapter, the results from structural and magnetic measurements on $\mathrm{BiFeO}_{3}$ films that were made by ion beam sputtering at ambient temperature and then annealed in an oxygen atmosphere are presented. This method leads to superparamagnetic nanocrystals and that the magnetisation is enhanced by iron oxide in the $\mathrm{BiFeO}_{3}$ film. Also presented: the results of magnetic measurements, optical band gap, MCD, MOKE and Piezoelectric force microscopy (PFM) of these $\mathrm{BiFeO}_{3}$ thin films. MOKE is a novel analysis technique on $\mathrm{BiFeO}_{3}$ with little data available from literature on thin films and comparison is therefore limited, so this report will focus on the $\mathrm{BiFeO}_{3}$ thin film and comparison with possible elements, namely bismuth oxides, iron oxides and bismuth ferrite, that could be in our thin film. 


\subsection{Experimental details of ion beam sputtering}

The films were synthesised with a targeted thickness of $100 \mathrm{~nm}$ by ion beam sputtering at ambient temperature. The details are presented in subsection 3.2.1. The samples were annealed at $300{ }^{\circ} \mathrm{C}, 400{ }^{\circ} \mathrm{C}, 500{ }^{\circ} \mathrm{C}$ and $600{ }^{\circ} \mathrm{C}$ for 15 minutes. The details of annealing are found in subsection 3.2.3. To analysis the optimisation of the synthesis, one further sample was annealed for 7 hours at $500{ }^{\circ} \mathrm{C}$. This chapter is centred on the optimised sample, annealed at $500{ }^{\circ} \mathrm{C}$ for 15 minutes. Other samples are analysed more in depth and compared at the end of this chapter.

\subsection{Synthesis and structure of BiFeO3: ion beam sputter- ing}

The synthesis of $\mathrm{BiFeO}_{3}$ is achieved by ion beam sputtering. The sputter target is made of a mix of bismuth, bismuth oxide, iron oxides, bismuth ferrite oxide and bismuth ferrite. The nano-particles in this film are grown by annealing in a furnace with an oxygen atmosphere. In the following subsections, the structure is confirmed and the potential secondary phases of the thin film are analysed.

\subsubsection{Rutherford backscattering, RBS}

RBS measured the elastic scattering of alpha particles scattered from the atoms of the films. The kinematic factor provides the expected scattered energy for every element and isotope. This is shown in Figure 4.3.1 for the $\mathrm{Bi}, \mathrm{Fe}, \mathrm{Si}$ and $\mathrm{O}$ positions. The number of counts per unit area is an indication of the stoichiometry/composition of the film. The energy variation, the difference between the energy of the backscattered particle from the surface of the film and at the interface of the substrate and the film, is caused by the electronic stopping power and allows one to determine the depth resolution and provide an accurate estimation of the layer thicknesses $\left(\right.$ at. $\left.\mathrm{cm}^{-2}\right)$. SRIM data is used to estimate the density of each layers according to the theoretical relative concentration of atoms in each layer. From the density $\left(\right.$ at. $\left.\mathrm{cm}^{-3}\right)$, an estimate of the thickness (nm) for each layer are calculated.

Figure 4.3.1 shows the RBS data from an (a) as-made film and (b) a $500{ }^{\circ} \mathrm{C}$ an- 
nealed film. The peaks from Bi and Fe can clearly be seen, as well as the Si edges and lower energy peaks from oxygen in the sputtered film and the $\mathrm{SiO}_{2}$ film. Two Si edges are evident in the RBS data, $\mathrm{SiO}_{2}$ :Si interface and bulk Si from the substrate. The Bi and Fe dose were calculated by RUMP fits [Doolittle, 1985]. The as-made film has a Bi dose of $1.55 \times 10^{17}$ at. $\mathrm{cm}^{-2}$ and a Fe dose of $1.80 \times 10^{17}$ at. $\mathrm{cm}^{-2}$. The detailed of the RUMP fit is presented in Section B.1. The RUMP fit has a surface layer, $2 \times 10^{17}$ at.cm ${ }^{-2}$, compose of $\mathrm{Bi}_{1}, \mathrm{Fe}_{1}$ and $\mathrm{O}_{4}$. The density is estimated by SRIM at $4.28 \times 10^{22}$ at.cm ${ }^{-3}$ [Ziegler, 1998], so the layer is estimated to be $47 \mathrm{~nm}$. This stoichiometry suggests a mix of bismuth oxide $\mathrm{Bi}_{2} \mathrm{O}_{3}$ and iron oxide $\mathrm{Fe}_{2} \mathrm{O}_{3}$. The second layer, $4.75 \times 10^{17}$ at.cm ${ }^{-2}$, is composed of $\mathrm{Bi}_{1}, \mathrm{Fe}_{1.3}$ and $\mathrm{O}_{1.9}$. The density is $4.41 \times 10^{22}$ at.cm ${ }^{-3}$, so the layer is $108 \mathrm{~nm}$. This stoichiometry suggests a mix of $\mathrm{Bi}$, bismuth oxide $\mathrm{Bi}_{2} \mathrm{O}_{3}$ and iron oxides e.g. $\mathrm{Fe}_{2} \mathrm{O}_{3}, \mathrm{Fe}_{3} \mathrm{O}_{4}$ and $\mathrm{FeO}$. Those phases are analysed in more details by XRD, Raman spectroscopy, TEM and magnetic moment in the following section. The two layers of bismuth ferrite oxides have a total thickness of about $160 \mathrm{~nm}$ close to the theoretical deposition of $150 \mathrm{~nm}$. The third layer is the $\mathrm{SiO}_{2}$ layer, RUMP fit estimates the thickness at $5.5 \times 10^{17}$ at.cm ${ }^{-2}$, with the SRIM density calculated at $5.18 \times 10^{22}$ at.cm ${ }^{-3}$, the thickness is $106 \mathrm{~nm}$. The manufacturer of this substrate specified an oxide layer thermally grown at $100 \pm 30 \mathrm{~nm}$. The layer below is the bulk silicon from the substrate. This analysis suggests that the deposited layer is oxygen deficient.

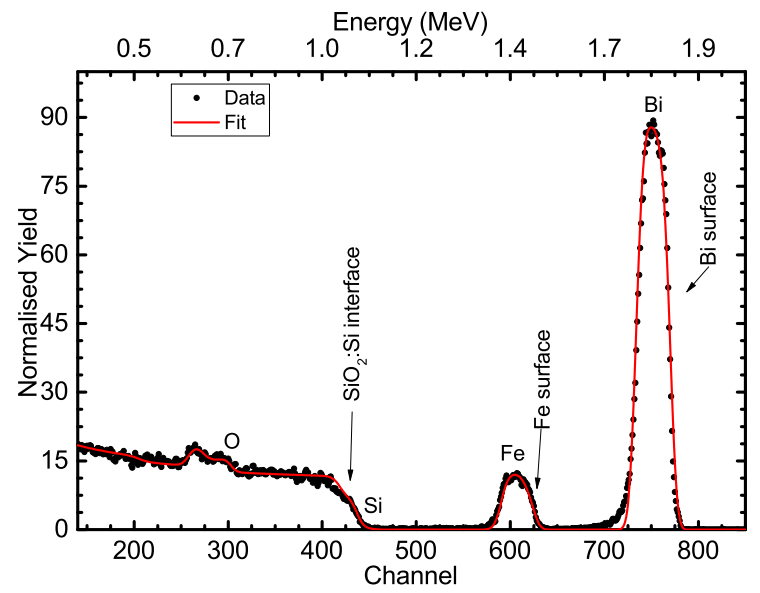

(a) as-made film.

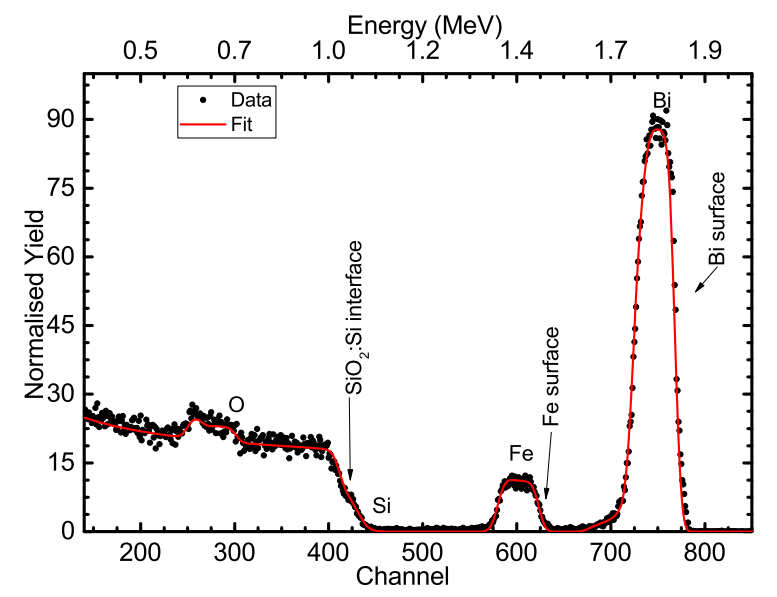

(b) $500{ }^{\circ} \mathrm{C}$ annealed film.

Figure 4.3.1: RBS spectra of thin film (circle) data along with the RUMP fit (line).

The annealed film's RUMP fit shows a film that gain oxygen. This film has a Bi dose of $1.54 \times 10^{17} \mathrm{~cm}^{-2}$ and a Fe dose of $1.80 \times 10^{17} \mathrm{~cm}^{-2}$. The comparison before and after annealing of the dose indicates that bismuth and iron have not evaporated in significant quantity. Details of the RUMP fit are presented in Section B.1. The RUMP fit has a surface layer, $7 \times 10^{22}$ at.cm ${ }^{-2}, \mathrm{Bi}_{1}, \mathrm{Fe}_{1.2}$ and $\mathrm{O}_{3.6}$. This stoichiometry suggests the presence of $\mathrm{BiFeO}_{3}$ and iron oxide $\mathrm{Fe}_{2} \mathrm{O}_{3}$. Further analysis will confirm the phases, 
magnetic measurement will discuss the concentration of iron oxide in the film. With a density of $4.40 \times 10^{22}$ at.cm ${ }^{-2}$, the thickness of that layer is $160 \mathrm{~nm}$. The second layer is, $2.4 \times 10^{22}$ at.cm ${ }^{-2}, \mathrm{Bi}_{1}, \mathrm{Fe}_{1.8}, \mathrm{O}_{4.2}, \mathrm{Si}_{1.7}$. The density is $4.81 \times 10^{22}$ at.cm ${ }^{-3}$, with a thickness of $50 \mathrm{~nm}$. The stoichiometry can be explained by a combination of $\mathrm{BiFeO}_{3}, \mathrm{SiO}_{2}$ and iron oxides. The third layer is composed of, $6 \times 10^{22}$ at.cm ${ }^{-2}, \mathrm{Bi}_{0.02}$ and $\mathrm{SiO}_{2}$, with a density of $5.03 \times 10^{22}$ at.cm ${ }^{-3}$, this layer is $119 \mathrm{~nm}$. The fourth layer is, $5 \times 10^{22}$ at.cm ${ }^{-2}$, $\mathrm{Bi}_{0.004}$ and $\mathrm{Si}_{1}$. The density is $4.9 \times 10^{22}$ at. $\mathrm{cm}^{-3}, 100 \mathrm{~nm}$. Those two layers and the following one, presented in Section B.1, have two options to explain the physics. Option 1 is bismuth diffusion through the $\mathrm{SiO}_{2}$ and bulk Si layers which is possible as the bismuth diffusion through $\mathrm{SiO}_{2}$ reported to be $\sim 10^{-14} \mathrm{~cm}^{2} \mathrm{~s}^{-1}$ at $800{ }^{\circ} \mathrm{C}$ [Büngener et al., 2003]. Option 2, the bismuth seen in small fraction in the $\mathrm{SiO}_{2}$ layer could be due to the low resolution of the detector, the background count is about 5 . There is also the possibility that both effects are seen in the spectra.

Figure 4.3.1 shows backscattered particles from bismuth, iron, oxygen and silicon. No other trace of contamination is visible. Particle-induced X-ray emission (PIXE) was also performed qualitatively while studying the RBS spectrograph. PIXE examines the $X$-ray emission resulting from the interaction of the incident beam with the sample target. Neither RBS or PIXE showed any elements that could create a contamination of the films at limits of detection of less than $100 \mathrm{ppm}$. The material phases and potential contamination sources require further investigation. That investigation will be detailed in following subsections, 4.3 through 4.6 .

\subsubsection{X-ray diffraction, XRD}

After a discussion about the composition of the film, XRD analysis provides a detailed study of the phases in the films and as established from the literature review the crystal structure is important for the film to display the proper magnetic and electric properties. The as-made film displays a $\mathrm{Bi}$ phase and a broad shoulder that could be $\mathrm{Bi}_{2} \mathrm{O}_{3}$ or $\mathrm{BiFe}_{x} \mathrm{O}_{y}$, bismuth oxide phase was also suggested by RBS analysis. The peak, $36^{\circ}$, is not clearly discernible due to these phases being from small particles. To obtain the required phase, samples were annealed at intermediate temperature of $200{ }^{\circ} \mathrm{C}, 300{ }^{\circ} \mathrm{C}$ and $400{ }^{\circ} \mathrm{C}$ (see Section 4.9). However, $\mathrm{BiFeO}_{3}$ rhombohedral phase forms only after annealing the sample at $500{ }^{\circ} \mathrm{C}$, Figure 4.3.2 (b). The peaks are broad which is measure of nanocrystalline material. A secondary phase of $\mathrm{Bi}_{2} \mathrm{O}_{3}$ is observed in the XRD, while RBS analysis suggests the presence of iron oxide secondary phase. The iron oxide secondary phase will be discussed with the magnetic measurements. 


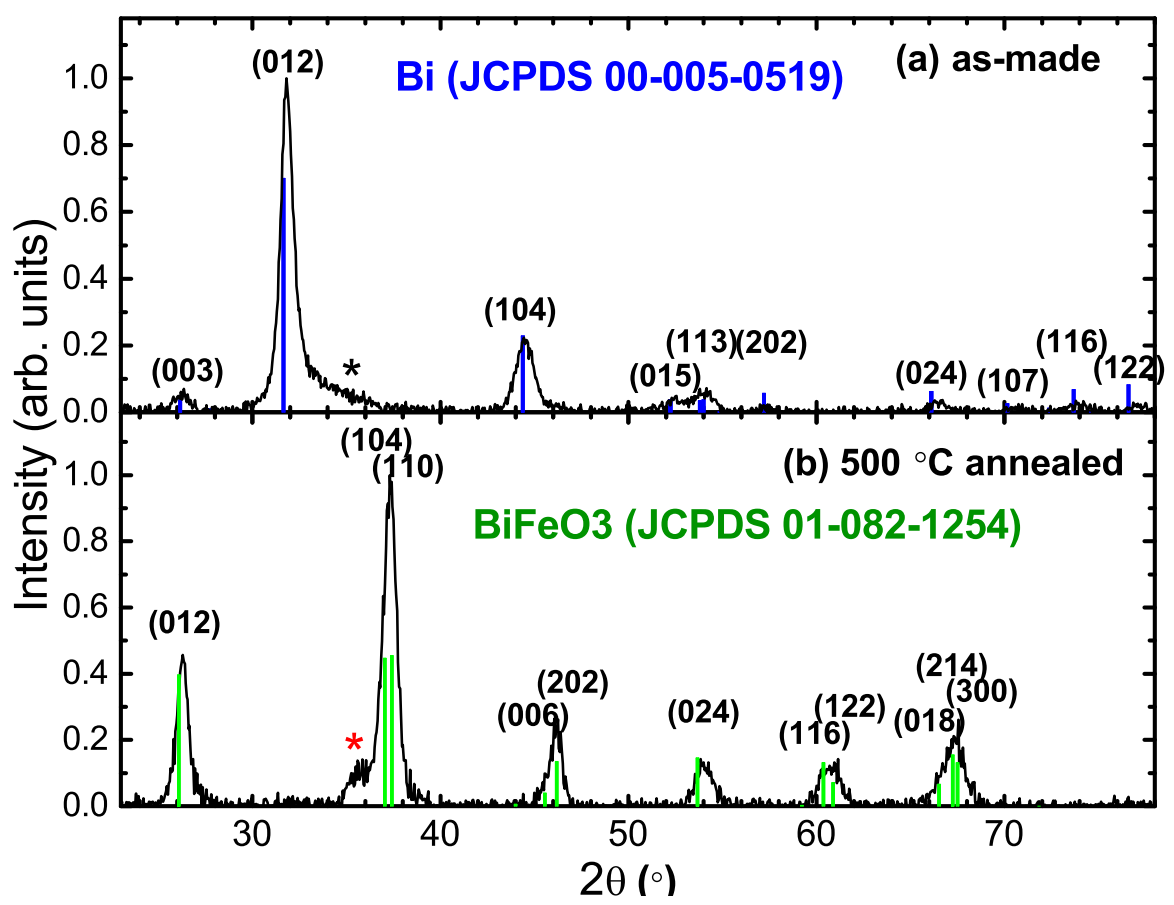

Figure 4.3.2: $\mathrm{XRD}$, grazing angle $2^{\circ}$, from (a) as-made and (b) $500{ }^{\circ} \mathrm{C}$ annealed films. The asterisks in (a), green, and (b), red, indicate an unknown $\mathrm{BiFe}_{\boldsymbol{x}} \mathrm{O}_{\boldsymbol{y}}$ and $\mathrm{Bi}_{2} \mathrm{O}_{3}$ phases. The Miller indices are shown above the peaks of (a) $\mathrm{Bi}$ and (b) $\mathrm{BiFeO}_{3}$.

The XRD data from an as-made film is plotted in Figure 4.3.2 (a). The main peaks can be assigned to rhombohedral Bi with a R-3m space group (JCPDS 00005-0519); the Miller indices are indicated in the figure. The broadening of the XRD peaks indicates that there are Bi nanocrystals, with an average diameter of $9.3 \pm 0.3 \mathrm{~nm}$ as estimated using the (104) peak and the Scherrer equation, equation 3.3.11. There is also a broad high-angle shoulder on the $\mathrm{Bi}(012)$ peak, indicated by an asterisk, that shows the presence of other phases. These phases could include $\beta-\mathrm{Bi}_{2} \mathrm{O}_{3}$ (JCPDS 01-078-1793), which usually forms at low temperatures [Salazar-Pérez et al., 2005; Steele and Lewis, 2014], and $\mathrm{Bi}_{2} \mathrm{Fe}_{4} \mathrm{O}_{9}$ (JCPDS 00-025-0090) which has his most intense peaks in the region between $33^{\circ}$ to $36^{\circ}$. The absence of a clearly discernible peak could be due to these phases having particle sizes below the limit of detection, resulting in a broadening of the XRD peaks or small X-ray form factors (i.e. the X-ray form factors are small for the elements in $\mathrm{FeOx}$ when compared with $\mathrm{Bi}$ ).

Annealing at $500{ }^{\circ} \mathrm{C}$ leads to completely different $\mathrm{X}$-rays diffraction peaks, as can be seen in Figure 4.3.2 (b). The main peaks can be assigned to rhombohedral $\mathrm{BiFeO}_{3}$ with a R3c space group (JCPDS 01-082-1254) with the Miller indices shown in the figure. The peaks are broad, indicating that the film is nanocrystalline. Using the (012) peak with the Scherrer equation, equation 3.3.11, the average particle size is estimated at $12.9 \pm 0.7 \mathrm{~nm}$. There is also a broad low-angle shoulder on the closely spaced (104) 
and (110) peaks, indicating the presence of some secondary phases. The broadening cannot be due to any of the known iron oxides, or $\mathrm{Bi}_{2} \mathrm{Fe}_{4} \mathrm{O}_{9}$. Thus, a small phase fraction of the $\mathrm{Bi}_{2} \mathrm{O}_{3}$ phase explained the feature at $36^{\circ}$. There is no evidence of iron oxide phases in the XRD data.

From the analysis of the data presented in Figure 4.3.2, the presence of iron oxides cannot be excluded. $\mathrm{A} \mathrm{Fe}_{x} \mathrm{O}_{y}$ phase could be amorphous or the crystallite sizes too small compared to the bismuth compounds to be clearly visible. This could explain some behaviour in the magnetic analysis (Section 4.4) and the superparamagnetism of $\mathrm{BiFeO}_{3}$ at the nanoscale. The presence of such a $\mathrm{Fe}_{x} \mathrm{O}_{y}$ phase is also discussed in the analysis of the Raman spectra in subsection 4.3.5.

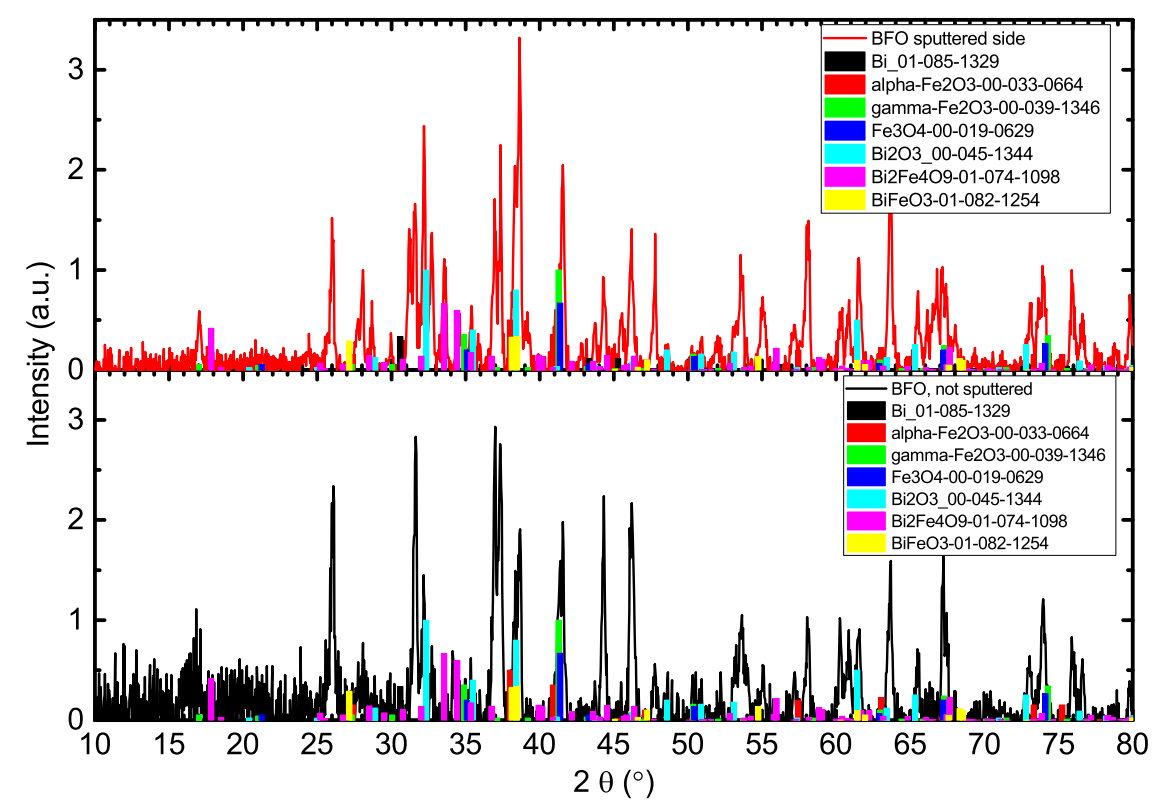

Figure 4.3.3: X-ray diffraction of the $\mathrm{BiFeO}_{3}$ sputter target, (top) is the face that was used to sputter thin films and (bottom) is the face that was not sputtered.

Figure 4.3.3 shows the XRD analysis of the sputter target used to synthesise the $\mathrm{BiFeO}_{3}$ thin film. Like the thin film, the sputter target did not show traces of any elements other than bismuth, iron and oxygen in the RBS and XRD analysis. However, the XRD analysis, Figure 4.3.3, clearly shows the presence of bismuth and $\mathrm{Bi}_{2} \mathrm{Fe}_{4} \mathrm{O}_{9}$ on both the sputtered and not sputtered face. XRD diffraction pattern is not sensitive enough to distinguish between the presence of maghemite and magnetite, both phases are possibly present on both sides of the target material. $\mathrm{BiFeO}_{3}$ is possibly present on both side, however the "not sputtered" side of the target seems to contain slightly more $\mathrm{BiFeO}_{3}$ than the sputtered side, while hematite is more present on the sputtered side than the non-sputtered side. The $\mathrm{BiFeO}_{3}$ comparison is based in part from the peak at $26^{\circ} . \mathrm{Bi}_{2} \mathrm{Fe}_{4} \mathrm{O}_{9}$ is also present on both side of the target and the quantity seems 
to be increasing with sputtering, the peak intensity increase at $34^{\circ}$ and $35^{\circ}$. The XRD analyse shows a difference in the peak intensity before and after the target was used for sputtering confirming the sputtering process modified the phases on the sputter target. The sputtering yields of bismuth, iron and oxygen are different [Nastasi et al., 2004], a $20 \mathrm{keV} \mathrm{Ar}^{+}$sputters between 0.88 and 3.84 bismuth atoms per argon ion [Deoli et al., 2014], between $2.1 \pm 0.3$ and $3.7 \pm 0.5$ iron atoms per argon ion [Viefhaus et al., 1994] and more than 2 oxygen atoms per argon ion [Seah, 1981]. Which the target heating (similar to annealing the film) could explained the modification of composition and phases in the sputter target. Before and after using the target for sputtering, this bulk material contains secondary phases. This increases the difficulty to sputter a pure $\mathrm{BiFeO}_{3}$ thin film. Most of these secondary phases can be removed by annealing the film. A secondary phases free target could increase the phase purity of the sputter film. However, deposing a phase pure film would be unlikely as the target heating from the interaction with Ar ions may results in the decomposition of $\mathrm{BiFeO}_{3}, \mathrm{BiFeO}_{3}$ has a melting temperature around $880 \mathrm{~K}$ [Rojac et al., 2014].

The XRD analysis provides insight into the ion beam sputtering synthesis of $\mathrm{BiFeO}_{3}$. The sputtering yields of bismuth, iron and oxygen compounds would be similar if the concentration of these elements were equivalent in the sputtering area of the target. The as-made film contains a significant amount of secondary phases and annealing provides ion mobility to reduce the concentration of secondary phases in the thin film, transforming some of the secondary phases into multiferroic $\mathrm{BiFeO}_{3} \mathrm{R} 3 \mathrm{C}$ film.

\subsubsection{Atomic force microscopy, AFM}

The RBS (subsection 4.3.1) and the atomic force microscopy (AFM) analyses confirm that annealing thin films up to $500{ }^{\circ} \mathrm{C}$ does not create a major composition modification; annealing rather results in phase modification as discussed in subsection 4.3.2. Atomic force microscopy shows that both the as-made and annealed film have a relatively smooth surface, as the roughness of the samples is similar. The grain size of the surface topography (compared to the smooth background) is about $30 \pm 4 \mathrm{~nm}$ in the asmade film, while the grain size in the annealed sample grew to $36 \pm 4 \mathrm{~nm}$. The increased grain size in the thin film is negligible as expected from the explanation of Murmu et al. [2012]; the mobility and in some cases the diffusivity of the ions is responsible for the growing process [Yan et al., 2008]. The increased $\mathrm{BiFeO}_{3}$ grain size also increases the leakage current [Simões et al., 2007]. This leakage current could increase the challenge 
of defining an electrical domain, so the comparison with the piezoelectric study could be of limited value.

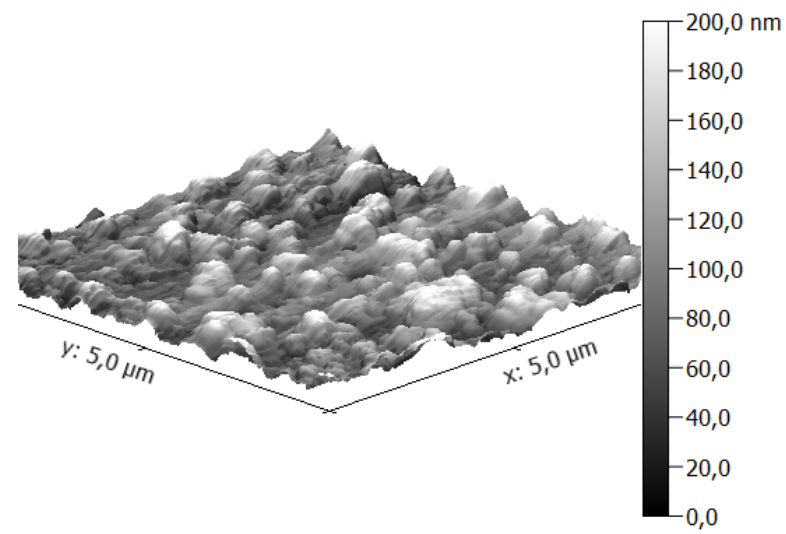

(a) as-made film.

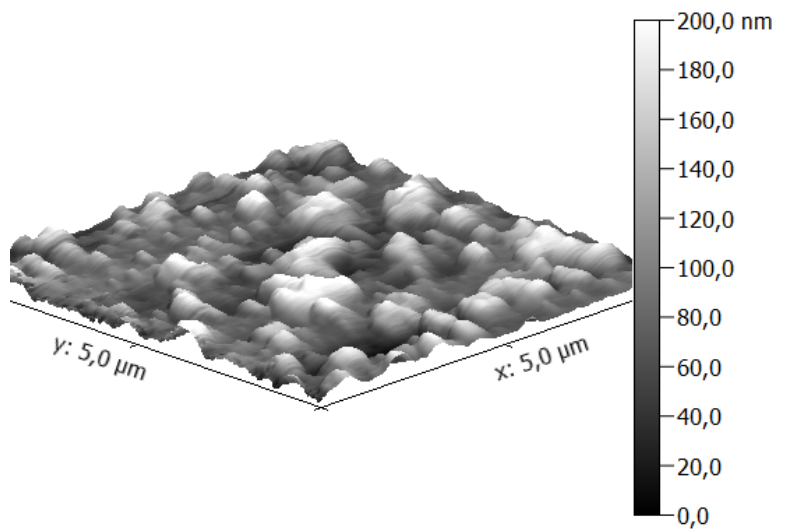

(b) $500{ }^{\circ} \mathrm{C}$ annealed film.

Figure 4.3.4: AFM 3D topography of sputtered film.

\subsubsection{Transmission electron microscopy, TEM}

Transmission electron microscopy was used to characterise the micro-structure of the film. The sample preparation used the focus ion beam, FIB, in place of the common ion milling technique. The sample was coated with $\mathrm{Pt}$ and $\mathrm{C}$ to clear the surfaces image. The TEM resolution is limited by the electron diffraction. The cross-section shows contrast regions, Figure 4.3.5, and 4.3.7, that indicate nanoscale phase separation likely to be, in the case of the as-made sample, Bi nanocrystals as seen in the XRD data and $\mathrm{BiFe}_{x} \mathrm{O}_{y}$ phases. The Figure 4.3.6 shows a SAED micrograph, discrete points form diffraction rings, which is used to estimate the d-spacing and the phase present. The as-made film shows $\mathrm{Bi}$, Magnetite $\left(\mathrm{Fe}_{3} \mathrm{O}_{4}\right)$ or maghemite $\left(\gamma-\mathrm{Fe}_{2} \mathrm{O}_{3}\right)$. From Raman spectroscopy analyse, magnetite and maghemite are present in the film. There is no clear evidence of other phases, which may be due to estimated particle size being near the limit of detection, i.e. $<5 \mathrm{~nm}$, as suggested from the XRD data.

A TEM image from an as-made film can be seen in Figure 4.3.5. There is nanostructure, which is more evident in the higher resolution image in the Figure 4.3.7. The dark and light regions indicate nanoscale phase separation that is likely to be $\mathrm{Bi}$ nanocrystals seen in the XRD data and $\mathrm{BiFe}_{x} \mathrm{O}_{y}$ phases. Figure 4.3.6 shows a SAED micrograph. There are discrete points that form rings from different phases and correspond to different crystallographic directions. However, since the orientation of each phase with respect to the incident electron beam is not known, each lattice plane cannot possibly uniquely determine. All possible orientations will form a ring and this can be 


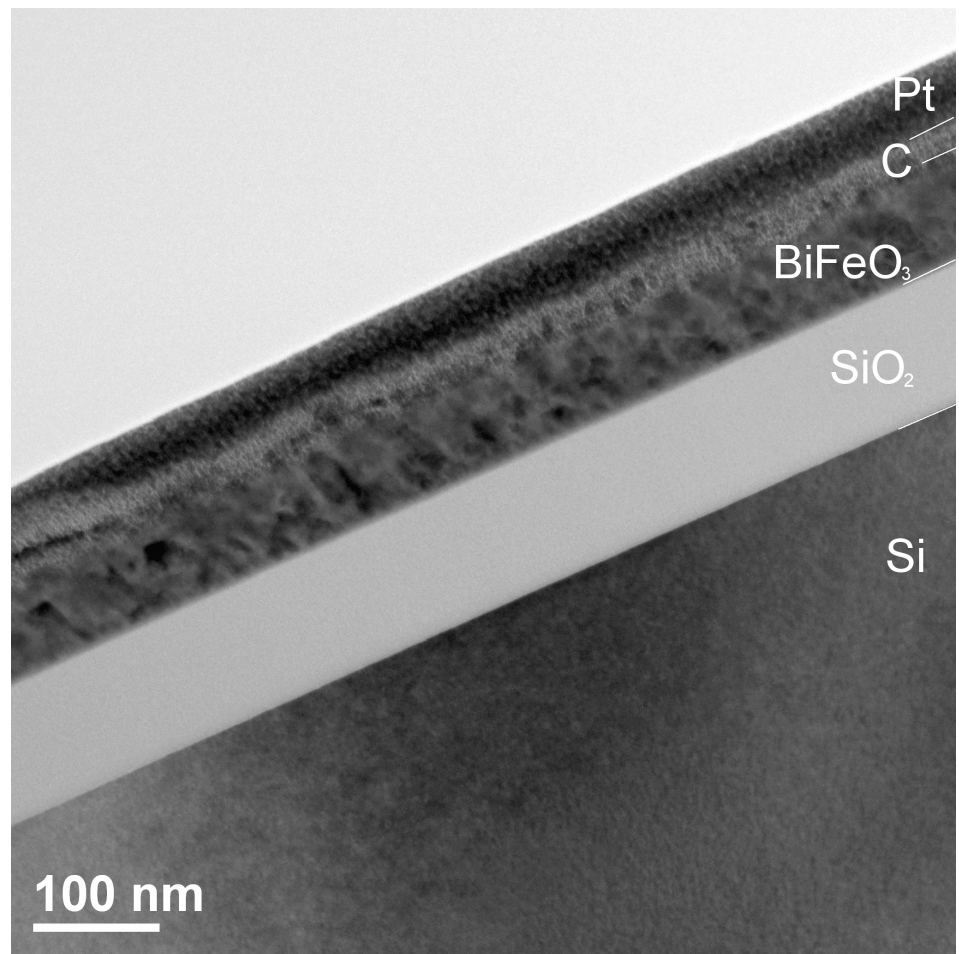

Figure 4.3.5: Cross-sectional TEM images of an as-made film deposited on $\mathrm{SiO}_{2}: \mathrm{Si}$. The film was carbon-coated then platinum-coated for the TEM measurements.

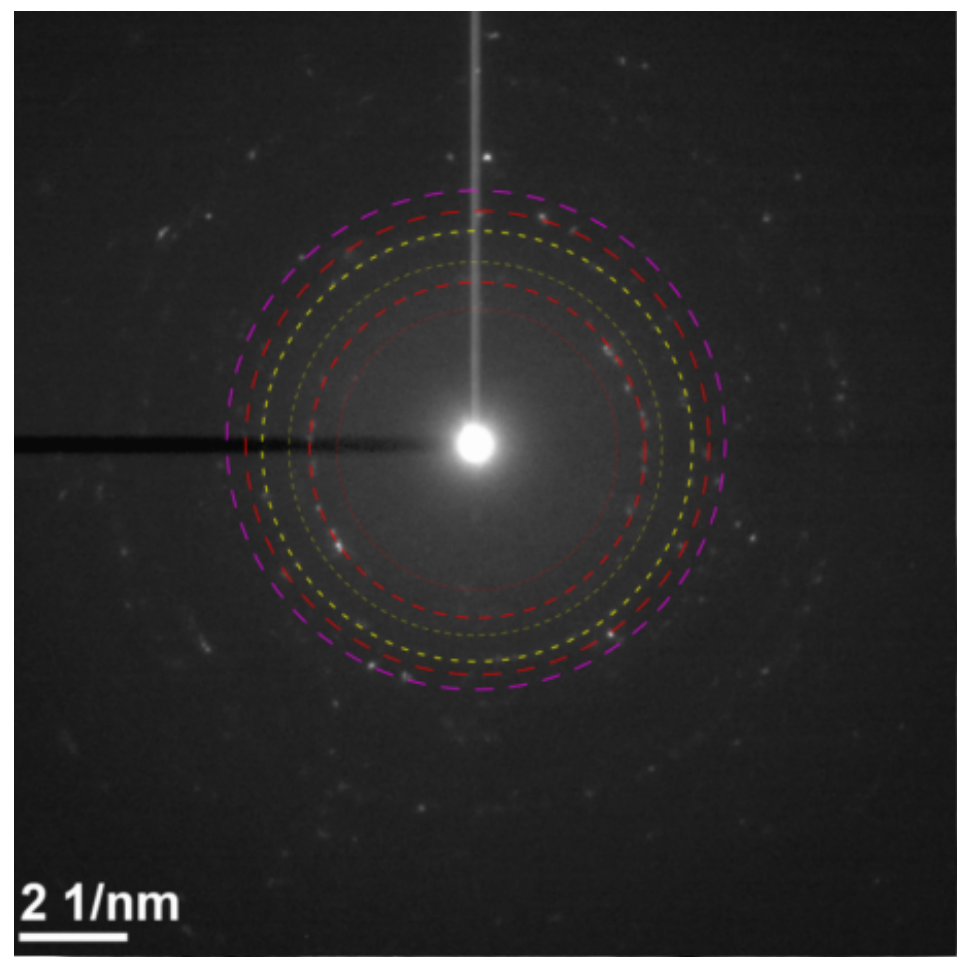

Figure 4.3.6: SAED micrograph of as-made film deposited. The circles show d-spacing from $\mathrm{Bi}$ (red), $\mathrm{Fe}_{3} \mathrm{O}_{4}$ or $\gamma-\mathrm{Fe}_{2} \mathrm{O}_{3}$ (yellow), and $\mathrm{FeO}$ (purple). 


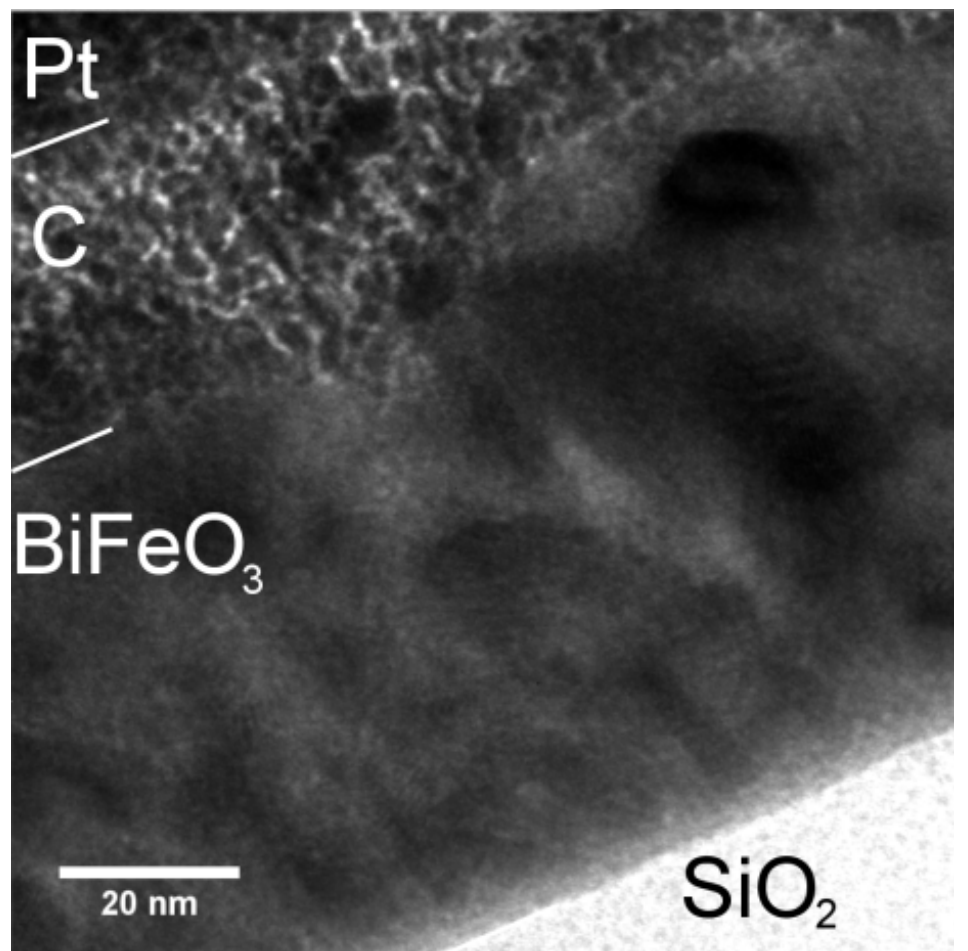

Figure 4.3.7: Cross-sectional, higher resolution, TEM images of an as-made film deposited on $\mathrm{SiO}_{2}$ :Si.

used to estimate the d-spacing and the phase. This has been done, and the corresponding rings for each phase are shown with dashed circles in Figure 4.3.6 Rhombohedral Bi can be seen and the d spacing corresponds to the (003), (012), (104) and (110) planes. Magnetite $\left(\mathrm{Fe}_{3} \mathrm{O}_{4}\right)$ or maghemite $\left(\gamma-\mathrm{Fe}_{2} \mathrm{O}_{3}\right)$ are also present and the rings are from the (220) and (311) planes. Magnetite and maghemite are not distinguishable in SAED analyse because both iron oxide phases are cubic with a Fd-3m space group and similar lattice parameters. The Raman data shows, subsection 4.3.5, that magnetite and maghemite are present. Magnetite is a ferrimagnetic metal with a Curie temperature of $858 \mathrm{~K}$ while maghemite is a ferrimagnetic semiconductor with a Néel temperature of $610 \mathrm{~K}$. There is also some Rhombohedral FeO (JCPDS 01-089-0690) with a ring from the (024) plane. FeO is an antiferromagnetic semiconductor with a Néel temperature of $198 \mathrm{~K}$. The iron oxide phases were not seen in the XRD data, which is likely due to the significantly smaller X-ray form factor of Fe in iron oxides when compared with $\mathrm{Bi}$ [Rieker et al., 1999]. There is no clear evidence for any $\mathrm{Bi}_{2} \mathrm{O}_{3}$ phases or any other phase, which could be due to their particles sizes being very small as suggested from the XRD data.

The TEM images from the $500{ }^{\circ} \mathrm{C}$ annealed film can be seen in Figure 4.3.8 and 4.3.10, where nanostructure is observed. After annealing the TEM image shows more contrast, sign of nanostructure, than the as-made TEM Figure 4.3.5 and 4.3.7. The 


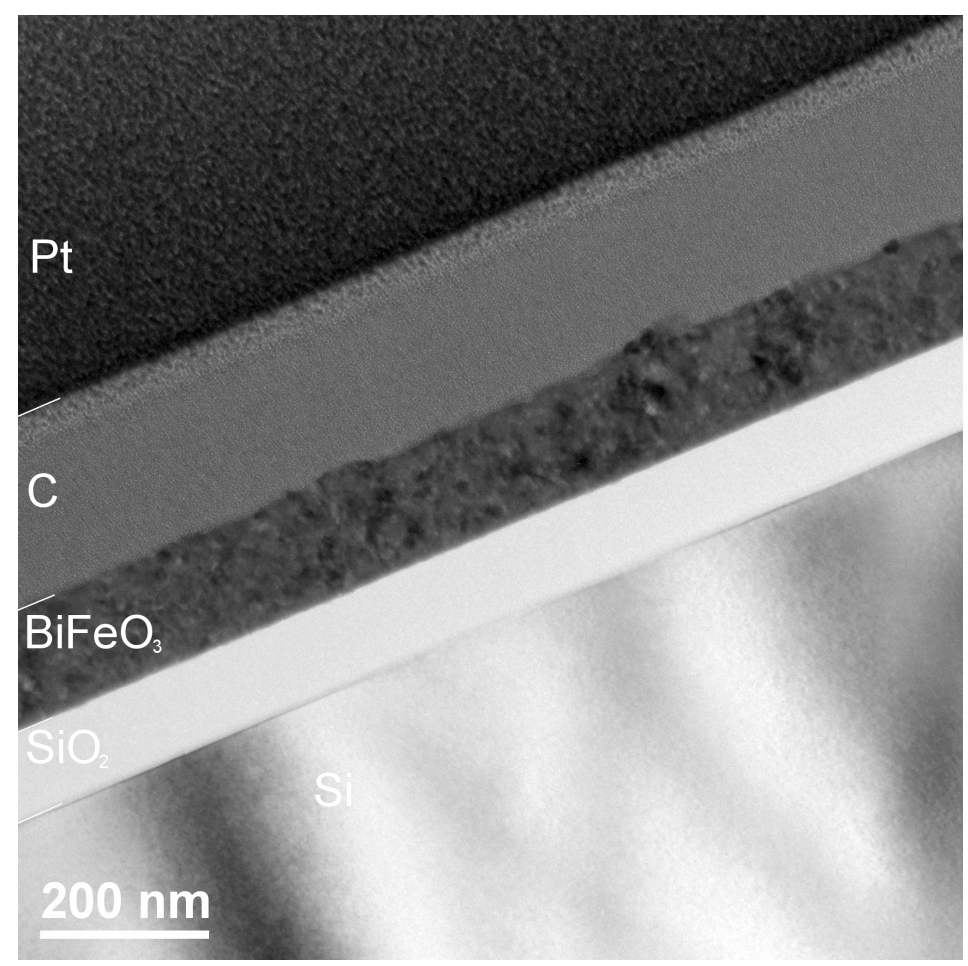

Figure 4.3.8: Cross-sectional TEM images of the $500{ }^{\circ} \mathrm{C}$ annealed film deposited on $\mathrm{SiO}_{2}: \mathrm{Si}$. The film was carbon-coated then platinum-coated for the TEM measurements.

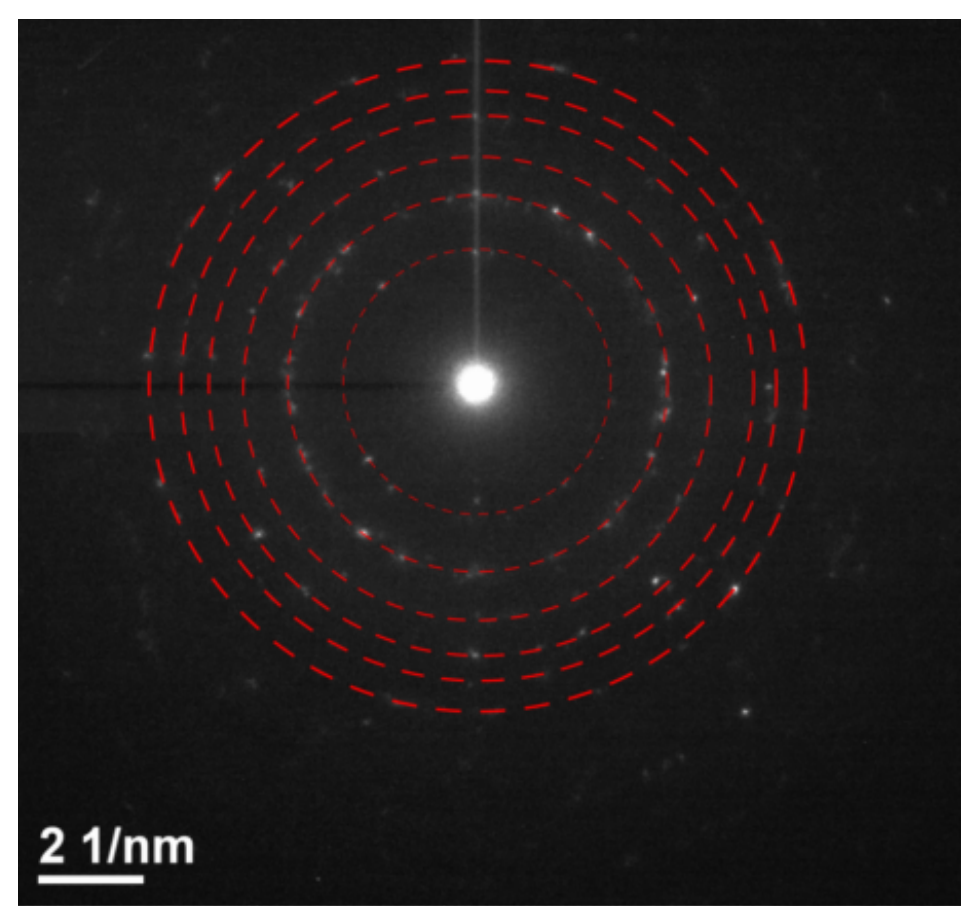

Figure 4.3.9: SAED micrograph of $500{ }^{\circ} \mathrm{C}$ annealed film deposited. The red circles show $\mathrm{BiFeO}_{3}$ d-spacing. 


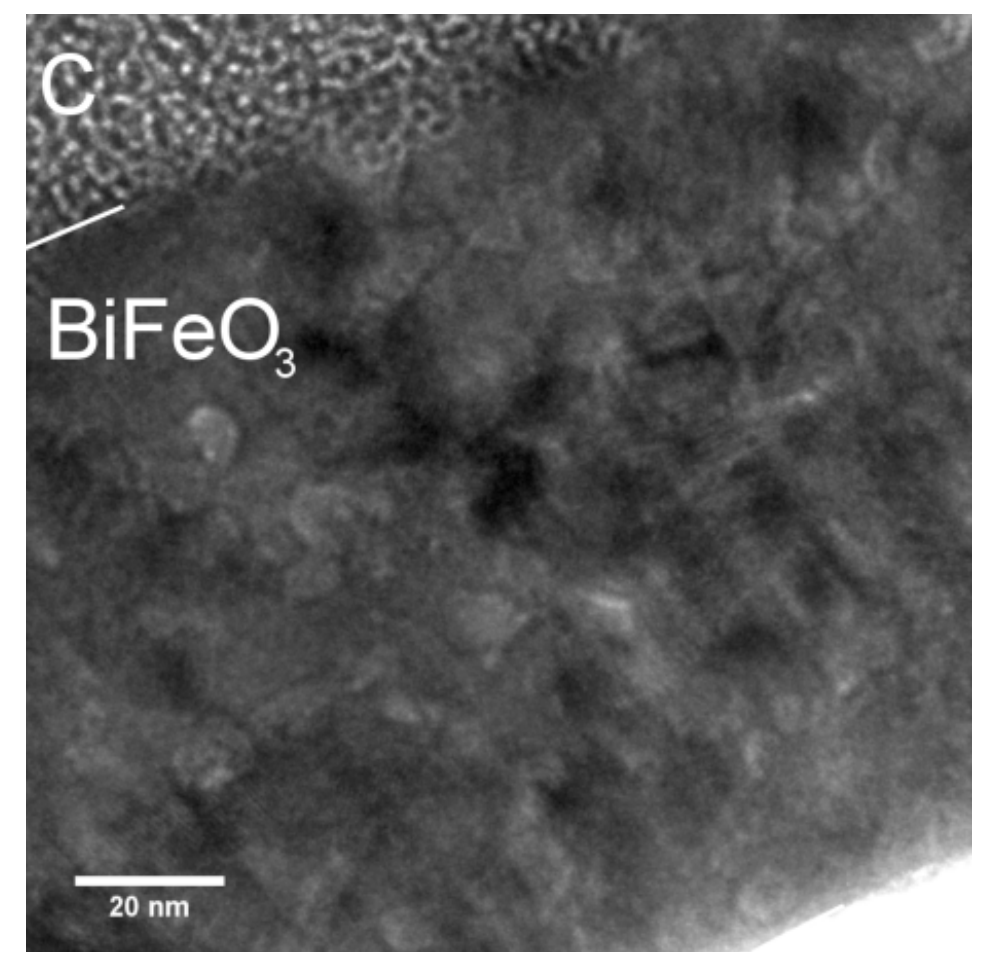

Figure 4.3.10: Cross-sectional, higher resolution, TEM images of $500{ }^{\circ} \mathrm{C}$ annealed thin film deposited on $\mathrm{SiO}_{2}$ :Si.

higher-resolution TEM image is showing a clear comparison, Figure 4.3.7 and Figure 4.3.10. A SAED micrograph is shown in Figure 4.3.9, in which there are discrete spots that indicate a small number of nanocrystals. The rings in the micrograph all correspond to planes from rhombohedral $\mathrm{BiFeO}_{3}$. There is no evidence for any iron oxide, $\mathrm{Bi}_{2} \mathrm{O}_{3}$ phases, or other phases. If they are present, then either they are amorphous, the nanocrystals are too small to be observed, or they are absent in the region selected for SAED. Other characterisations techniques (Raman spectroscopy, subsection 4.3.5, and magnetisation data, Section 4.4) show that the latter is the most likely scenario.

Cross-section of the samples is clearly visible in Figure 4.3.8 and 4.3.10. The white layer is the $\mathrm{SiO}_{2}$ from the substrate, the greyish area is the Si bulk layer in the substrate, and the dark layer is the $\mathrm{BiFeO}_{3}$. The TEM analysis does not show clearly-defined nanoparticles, however considerable nanoscale structures are shown in the XRD analysis, subsection 4.3.2, due to the nanocrystalline nature of the films. So, like the Raman spectroscopy analysis, TEM analysis suggest that iron oxides may be present in small particles. Both analysis techniques confirm that after annealing the thin film, $\mathrm{BiFeO}_{3}$ nanostructured materials are formed. 


\subsubsection{Raman spectroscopy}

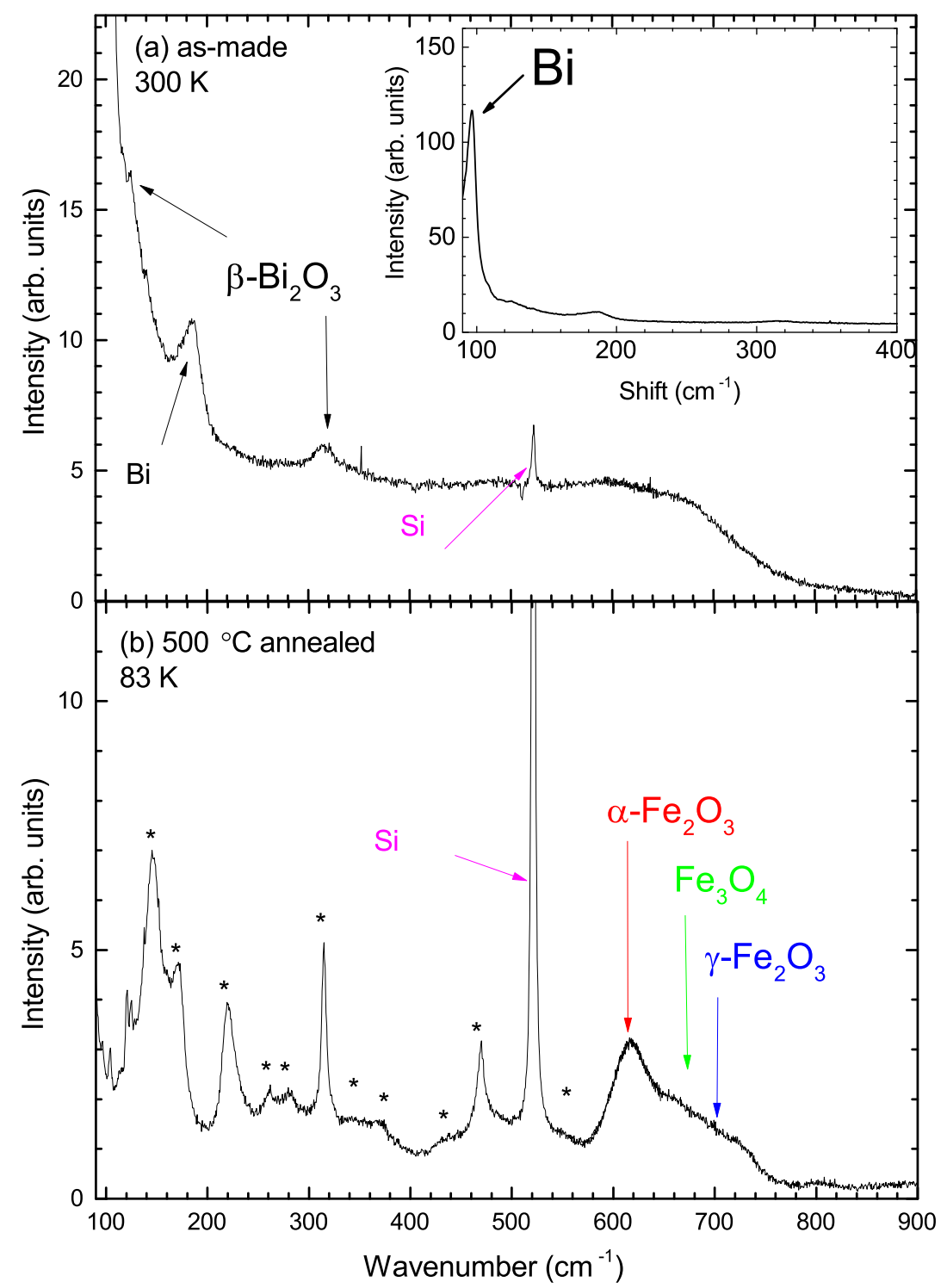

Figure 4.3.11: (a) Raman spectra from the as-made film at $300 \mathrm{~K}$. Inset: The same Raman spectra at higher intensities showing the main Bi Raman peak [Salazar-Pérez et al., 2005; Steele and Lewis, 2014]. (b) Raman spectra from the $500{ }^{\circ} \mathrm{C}$ annealed film at $83 \mathrm{~K}$. The $\mathrm{BiFeO}_{3}$ peaks are indicated with asterisks [Rout et al., 2009]. The Raman shift at $521 \mathrm{~cm}^{-1}$ is from the Si substrate.

Raman spectroscopy was used as a complementary analysis technique for structure analysis. Raman spectroscopy displays the normal mode or Raman active mode of the sample. The as-made sample displays $\mathrm{Bi}$ and $\mathrm{Bi}_{2} \mathrm{O}_{3}$ Raman modes. As XRD does not displays $\mathrm{Bi}_{2} \mathrm{O}_{3}$, this result suggests that the bismuth oxide is in a small phase fraction. 
After annealing the sample, Raman spectroscopy shows 11 Raman active modes identified to $\mathrm{BiFeO}_{3}$, this agrees with the XRD analysis. The Raman mode also shows the presence of iron oxides phases, also suggested from the RBS analysis.

Raman measurements were performed on the as-made and the $500{ }^{\circ} \mathrm{C}$ annealed films, and the results are shown in Figure 4.3.11 without any baseline subtraction. There is an intense peak at $97 \mathrm{~cm}^{-1}$ (Figure 4.3 .11 (a) inset) that can be attributed to rhombohedral Bi Salazar-Pérez et al., 2005 that is also seen in the XRD and SAED data. Four peaks can be seen at higher wavenumbers at $125 \mathrm{~cm}^{-1}, 186 \mathrm{~cm}^{-1}, 315 \mathrm{~cm}^{-1}$, and $522 \mathrm{~cm}^{-1}$ (Figure 4.3 .11 (a)). The $186 \mathrm{~cm}^{-1}$ peak is a weak second order rhombohedral Bi peak from the $97 \mathrm{~cm}^{-1}$ peak. The peaks at $125 \mathrm{~cm}^{-1}$ and $315 \mathrm{~cm}^{-1}$ can be attributed to $\beta-\mathrm{Bi}_{2} \mathrm{O}_{3}$ [Salazar-Pérez et al., 2005; Steele and Lewis, 2014; Kumari et al., 2007]. This phase is not seen in the XRD data, likely because the phase fraction is small. The $521 \mathrm{~cm}^{-1}$ peak is due to the crystalline silicon substrate. There is additional Raman scattering at higher wavenumbers extending to $\sim 800 \mathrm{~cm}^{-1}$ that cannot be due to $\mathrm{Bi}, \mathrm{Bi}_{2} \mathrm{O}_{3}$, or even $\mathrm{Bi}_{2} \mathrm{Fe}_{4} \mathrm{O}_{9}$ [Iliev et al., 2010]. The high wavenumber Raman shift is likely arising from iron oxides. For example, the $\mathrm{A}_{1 g}$ Raman modes from magnetite and maghemite occur at $672 \mathrm{~cm}^{-1}$ and $700 \mathrm{~cm}^{-1}$ in the bulk, while bulk hematite $\left(\alpha-\mathrm{Fe}_{2} \mathrm{O}_{3}\right)$ has an $\mathrm{E}_{g}$ mode at $615 \mathrm{~cm}^{-1}$ [Jubb and Allen, 2010]. Hematite is an antiferromagnetic semiconductor with a Néel temperature, $\mathrm{T}_{N}$, of $948 \mathrm{~K}$. FeO has an active move at $656 \mathrm{~cm}^{-1}$ [Jallad and Ben-Amotz, 2001].

The Raman spectrum of the $500{ }^{\circ} \mathrm{C}$ annealed film at $83 \mathrm{~K}$ has significantly more peaks than seen before annealing. The peaks marked by asterisks can be attributed to rhombohedral $\mathrm{BiFeO}_{3}$ where $4 \mathrm{~A}_{1}$ and $9 \mathrm{E}$ Raman active modes are expected [Rout et al., 2009]. The appearance of $\mathrm{BiFeO}_{3}$ in the Raman data is consistent with the XRD and SAED analysis. The Raman measurements were made at $83 \mathrm{~K}$ because the peak intensities are sufficiently enhanced at low temperatures to observe the $\mathrm{BiFeO}_{3}$ Raman active modes [Rout et al., 2009]. Measurements were also made at $300 \mathrm{~K}$ (Section 4.9) confirming that the Raman peaks moved to lower wavenumbers with a decreasing temperature, which is consistent with the expected lattice expansion as the temperature increases [Rout et al., 2009]. The crystalline silicon substrate peak at $521 \mathrm{~cm}^{-1}$ is more intense than that seen before annealing. This difference is likely because Bi is a metal, and hence there is significant laser light absorption from the metallic Bi nanocrystals before annealing. However, $\mathrm{BiFeO}_{3}$ is semiconducting with an optical band gap of $2.5 \mathrm{eV}$ [Liu et al., 2011], and hence significant optical absorption is only expected below $\sim 496 \mathrm{~nm}$, so more light can pass through the film to the crystalline Si substrate. There is also Raman shift above $\sim 570 \mathrm{~cm}^{-1}$ extending out to $\sim 800 \mathrm{~cm}^{-1}$ which is not due to $\mathrm{BiFeO}_{3}$. This likely arises from iron oxides, where the maghemite 
$\mathrm{E}_{g}$, magnetite $\mathrm{A}_{1 g}$, and hematite $\mathrm{A}_{1 g}$ peak wavenumbers seen in the bulk material are indicated in Figure 4.3.11 (b). A higher energy peak (not shown) is found at $1260 \mathrm{~cm}^{-1}$, along with a weaker peak at $1095 \mathrm{~cm}^{-1}$ and an even weaker peak at $1148 \mathrm{~cm}^{-1}$. These peaks are too low in energy to be attributed to a hematite two phonon peak that occurs at $1320 \mathrm{~cm}^{-1}$ or the two-magnon peak that occurs at a higher wavenumber [Massey et al., 1990]. They also cannot be attributed to two-phonon peaks from other iron oxide phases. The $1260 \mathrm{~cm}^{-1}$ and $1095 \mathrm{~cm}^{-1}$ peaks are close to those observed in $\mathrm{BiFeO}_{3}$ and can be assigned to $\mathrm{BiFeO}_{3}$ [Ortiz-Quiñonez et al., 2013]. The origin of the weak $1118 \mathrm{~cm}^{-1}$ peak is not clear, however iron oxide phases could explain that feature.

\subsubsection{Optical measurements}

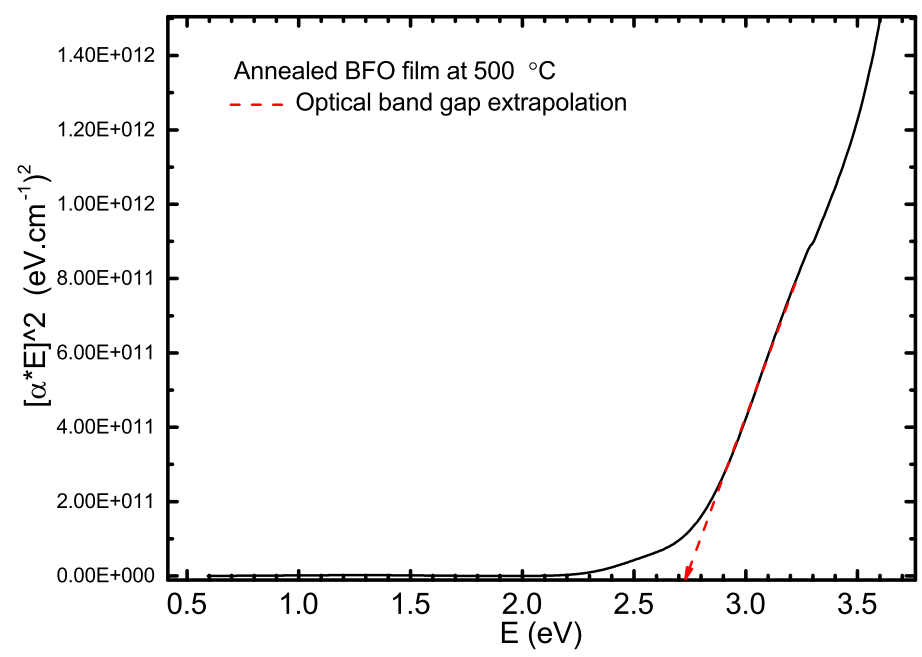

Figure 4.3.12: Tauc plot of $\mathrm{BiFeO}_{3}$ annealed, $500{ }^{\circ} \mathrm{C}$, thin film on quartz.

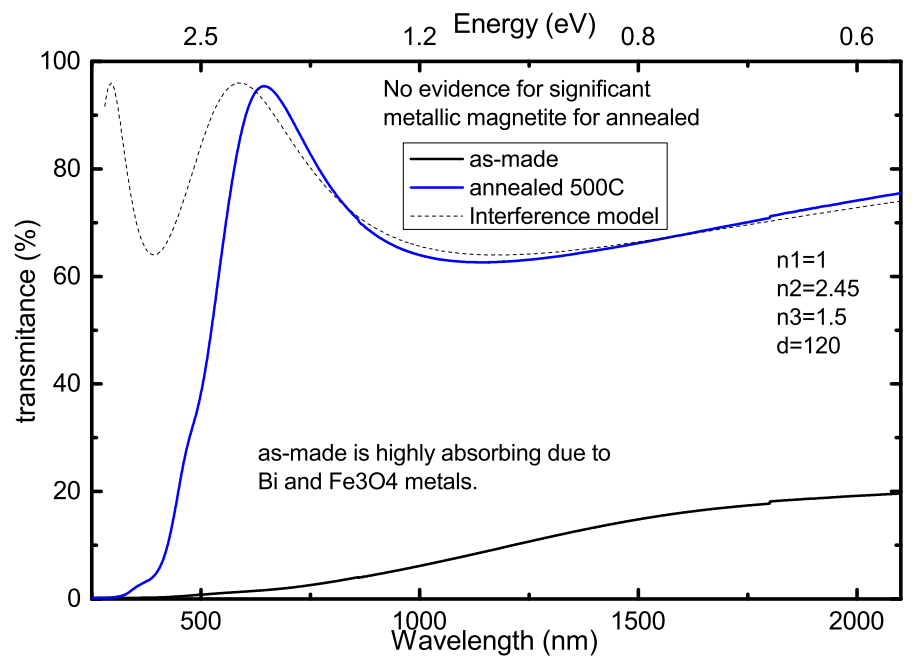

Figure 4.3.13: Optical transmission of the as-made and annealed $\mathrm{BiFeO}_{3}$ thin film. Samples sputtered on quartz. 
The optical band gap analysis confirms a phase transition after annealing, however this analyse does not confirm exact composition of the films. Figure 4.3.13 shows a transmission of the as-made and annealed $\mathrm{BiFeO}_{3}$ samples. The absorption coefficient is calculated from the measured transmission [Basu et al., 2008]. The absorption data shows, Figure 4.3.13, the as-made film absorbs nearly $98 \%$ and after annealing at $500{ }^{\circ} \mathrm{C}$, the $\mathrm{BiFeO}_{3}$ film absorbs or reflects up to $4 \%$ of the light below $700 \mathrm{~nm}$. The high absorption of light from the as-made film is consistent with the Raman spectroscopy where the laser light seems to be absorbed before reaching the silicon substrate. Raman and XRD analysis both display the presence of metal bismuth. The metallic behaviour of bismuth could be the source of the absorption. The annealed film has a much higher light transmission. The dash-line curve in the Figure 4.3.13 is an interference pattern fit on the light transmission of the annealed film. The interference is described with the equation 4.3.1:

$$
T=\frac{r_{12}^{2}+r_{23}^{2}+2 r_{12} r_{23} \cos \theta}{1+\left(r_{12} r_{23}\right)^{2}+2 r_{12} r_{23} \cos \theta} .
$$

Where,

$$
r_{12}=\frac{n_{1}-n_{2}}{n_{1}+n_{2}}, r_{23}=\frac{n_{2}-n_{3}}{n_{2}+n_{3}} \text { and } \theta=\frac{4 d \pi n_{2}}{\lambda} .
$$

$n$ is the refractive index of the material where $n_{1}$ is the refractive index of air, $n_{2}$ is linked to the sputtered film and $n_{3}$ is for the quartz, $d$ is the thickness of the film, $\lambda$ is the wavelength of the incident light. The interference fit the data at wavelength above $700 \mathrm{~nm}$, where the light absorption is minimum below that wavelength the absorption increases the inaccuracy of the fit. The fit shows a refractive index of 2.45 combined with a film thickness of $120 \mathrm{~nm}$ (equation 4.3.1).

The direct and indirect optical band gap values are extrapolated from the absorption coefficient, $(\alpha E)^{2}$ and $(\alpha E)^{1 / 2}$ as a function of the energy [Ramachandran et al., 2010; Tauc et al., 1966]. Figure 4.3.12 shows the extrapolation of the most abrupt slope, providing the value of the optical band gap, discussed in subsection 3.3.6, of the annealed sample [Basu et al., 2008]. The optical band gap value after annealing the thin film is $2.75 \mathrm{eV}$, as displayed in Figure 4.3.12. That value was confirmed with another set of samples. Tauc plot is based on the absorption coefficient; the coefficient is calculated with the absorption also converted from the transmission measurements [Tauc et al., 1966]. The annealed thin film has an optical band gap of about $2.75 \mathrm{eV}$, which agrees with previously reported $\mathrm{BiFeO}_{3}$ band gap, ranging from $2.2 \mathrm{eV}$ [Chen et al., 2011] to $2.8 \mathrm{eV}$ [Yang et al., 2010a]. A smaller band gap could be attributed to a deficiency of 
bismuth, or bismuth poor material [Ihlefeld et al., 2008]. The band gap of $\mathrm{Bi}_{2} \mathrm{O}_{3}$ is $2.8 \mathrm{eV}$ [Bian et al., 2008], hematite $\mathrm{Fe}_{2} \mathrm{O}_{3}$ is $2.1 \mathrm{eV}$ [Meng et al., 2011], maghemite $\mathrm{Fe}_{2} \mathrm{O}_{3}$ is $2.3 \mathrm{eV}$ [Chirita et al., 2009], magnetite $\mathrm{Fe}_{3} \mathrm{O}_{4}$ is $0.5 \mathrm{eV}$ [Jeng and Guo, 2002] and $\mathrm{Bi}_{2} \mathrm{Fe}_{4} \mathrm{O}_{9}$ is about $2 \mathrm{eV}$ [Liu et al., 2012]. A full study of the $\mathrm{BiFeO}_{3}$ band gap concluded that there is a direct optical band gap of $2.5 \mathrm{eV}$ [Ihlefeld et al., 2008]. Any deviation from that value is a useful tool to determine if the film is rich in either bismuth or oxygen [Pisarev et al., 2009]. The optical indirect band gap is about $1.8 \mathrm{eV}$ (not shown) which is close to the value reported by Fruth et al. [2007] of $2.0 \pm 0.1 \mathrm{eV}$.

The optical indirect band gap Tauc plot [Tauc et al., 1966], not shown, is not reliable as the transition energy is below $2 \mathrm{eV}$. Below $2 \mathrm{eV}$, there is a distribution of energy affected by light interference of the substrate and thus the extrapolation of the optical indirect band gap is difficult to estimate. Analysing the measured optical band gap confirms a structural modification happened after annealing the sample. As displayed in equation 4.3.1, the as-made film is highly absorbing while the annealed film is not. The as-made high absorption coefficient agrees with a material containing metal Bi and magnetite as discussed with XRD and Raman analysis. While, after annealing the thin film at $500{ }^{\circ} \mathrm{C}$, the light absorption coefficient is low which would agree with the XRD and Raman analysis of a film mainly compose of $\mathrm{BiFeO}_{3}$, a semiconductor with a band gap value comparable to the literature [Yang et al., 2010b]. These results, like the previous results, subsection $\mathrm{X}$-ray diffraction, do not totally exclude the presence of iron oxide, bismuth oxides or bismuth ferrite's mixed phases in the thin film. In fact, the literature value of $\mathrm{Fe}_{2} \mathrm{O}_{3}$ maghemite [Chirita et al., 2009] is just within the range of uncertainty of the measurement presented in this section. The optical analysis technique cannot be used to confirm the structure of the film; however, the phase modification is confirmed by the increase of light transmission with annealing and the modification of the optical band gap value.

\subsection{Magnetic properties of $\mathrm{BiFeO} 3$ at nanoscale}

This section focuses on the magnetic properties, subsection 4.4.1 Magnetic order, subsection 4.4.2 Superparamagnetism, subsection 4.4.3 Exchange bias and subsection 4.4.4 Magnetic force microscopy. The effect of annealing the thin film on the magnetic data is also analysed. 


\subsubsection{Magnetic order}

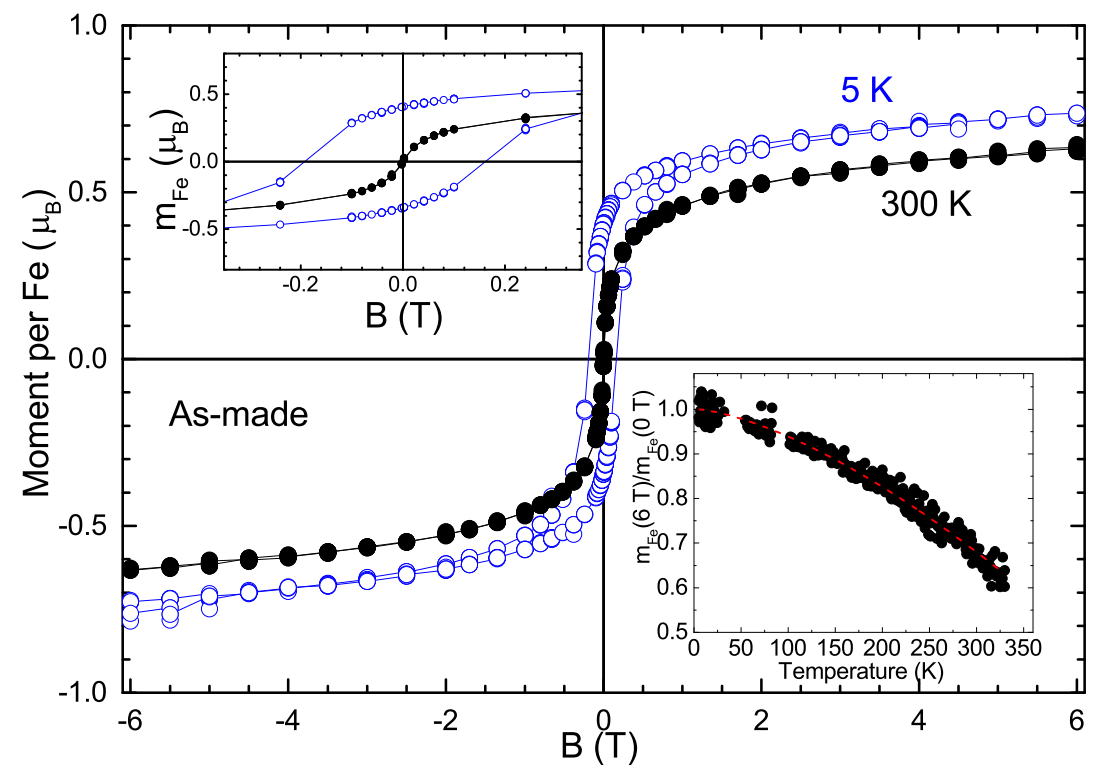

Figure 4.4.1: Magnetic moment per Fe from an as-made film at $5 \mathrm{~K}$ (open circles) and $300 \mathrm{~K}$ (filled circles) cooled in an applied magnetic field of $6 \mathrm{~T}$. Upper left inset: The same data over a smaller magnetic field range. Lower right inset: Plot of the normalised saturation moment per Fe versus temperature at $6 \mathrm{~T}$. Also shown is a fit with equation 3.3.22 (dashed curve).

Magnetic measurements were performed on an as-made film; the resultant magnetic moment per $\mathrm{Fe}, \mathrm{m}_{\mathrm{Fe}}$, is plotted in Figure 4.4 .1 at $5 \mathrm{~K}$ and $300 \mathrm{~K}$ after subtracting the diamagnetic moment from the silicon substrate using equation 3.3.21, $\chi_{\mathrm{Si}}$ is the silicon susceptibility $\left(-3.26 \times 10^{-6} \mathrm{emu}\right)$ measured with the SQUID and $\mathrm{n}_{\mathrm{Fe}}$ is the Fe fluence estimated from the RBS data. $\mathrm{m}_{F e}$ is increasing rapidly up to $\sim 0.9 \mathrm{~T}$ and then increases at a much slower rate. This is indicative of the presence of ferrimagnetic order from $\mathrm{Fe}_{3} \mathrm{O}_{4}$ and $\gamma-\mathrm{Fe}_{2} \mathrm{O}_{3}$, where the absence of complete saturation even at $6 \mathrm{~T}$ may in part be due to the appearance of a spin glass phase in the film that exists at ambient temperature. The bulk compounds have a saturation moment per $\mathrm{Fe}, \mathrm{m}_{s, \mathrm{Fe}}$, of $1.35 \mu_{B}$ for $\mathrm{Fe}_{3} \mathrm{O}_{4}$ and $1.25 \mu_{B}$ for $\gamma-\mathrm{Fe}_{2} \mathrm{O}_{3}$ [Cullity and Graham, 2009]. $\mathrm{m}_{F e}$ at $6 \mathrm{~T}$ and $5 \mathrm{~K}$ is $0.73 \mu_{B}$; this would suggest that between $\sim 54 \%$ to $\sim 58 \%$ of the available Fe is in $\mathrm{Fe}_{3} \mathrm{O}_{4}$ and $\gamma-\mathrm{Fe}_{2} \mathrm{O}_{3}$ nanoparticle form. The remaining $\mathrm{Fe}$ is in antiferromagnetic or paramagnetic $\mathrm{BiFe}_{x} \mathrm{O}_{y}$ phases that could include antiferromagnetic hematite, antiferromagnetic $\mathrm{Bi}_{2} \mathrm{Fe}_{4} \mathrm{O}_{9}$ with a Néel temperature between $237 \mathrm{~K}$ and $265 \mathrm{~K}$ [Irshad et al., 2015], and antiferromagnetic $\mathrm{FeO}\left(\mathrm{T}_{N}=198 \mathrm{~K}\right)$.

The temperature dependence of $\mathrm{m}_{F e}$ at $6 \mathrm{~T}, \mathrm{~m}_{F e}(6 \mathrm{~T})$, is plotted in the lower inset to Figure 4.4.1. This inset shows that $\mathrm{m}_{F e}(6 \mathrm{~T})$ is monotonically decreases with increasing temperature, which is due to the presence of spin-waves. In general, $\mathrm{m}_{s, F e}$ can be 
written at low temperatures as equation 3.3.22 [Bloch, 1930].

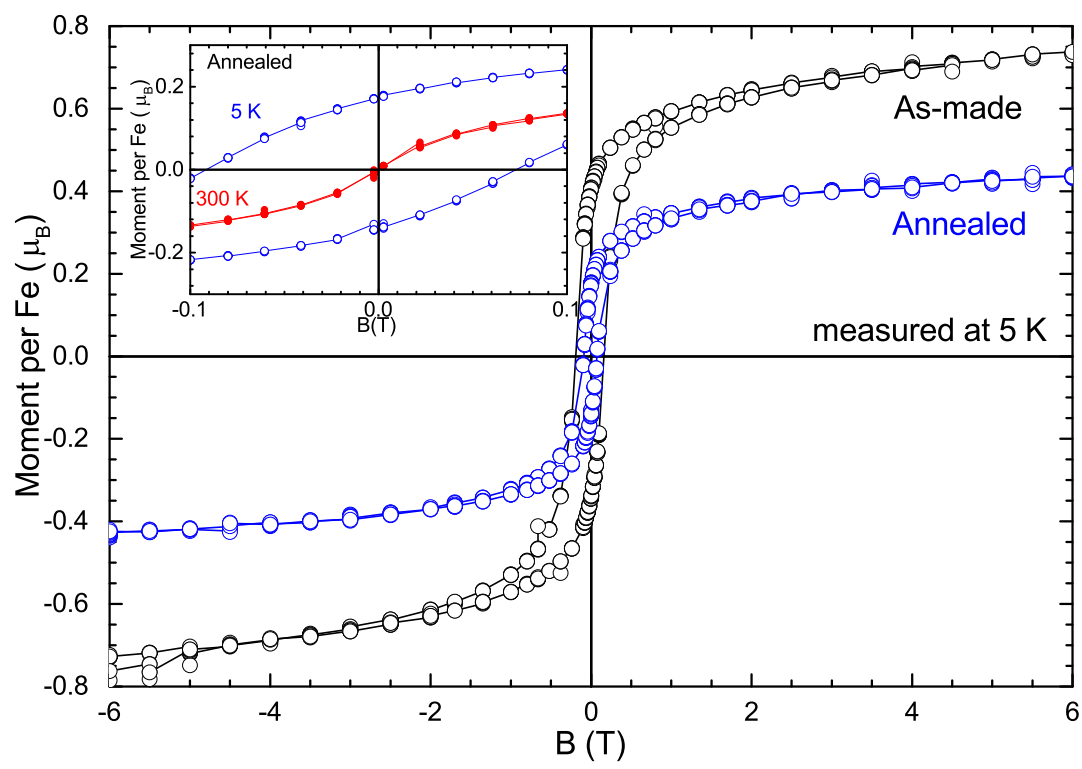

Figure 4.4.2: Magnetic moment per $\mathrm{Fe}$ for as-made and $500{ }^{\circ} \mathrm{C}$ annealed $\mathrm{BiFeO}_{3}$ film at $5 \mathrm{~K}$. Inset: magnified version of $500{ }^{\circ} \mathrm{C}$ annealed $\mathrm{BiFeO}_{3}$ film at $5 \mathrm{~K}$ and $300 \mathrm{~K}$.

When comparing the magnetic moment at $6 \mathrm{~T}$ with the expected magnetic moment of $0.02 \mu_{B} / F e$, the measured magnetic moment displays an enhance magnetic moment cause by iron oxide. The as-made film contains a significant amount of iron oxide as described by TEM and now confirm with magnetic measurements.

The inset of Figure 4.4.2 displays a magnified comparison of the annealed sample measured at ambient temperature and low temperature. The closing of the hysteresis loop at ambient temperature suggest the presence of a superparamagnetic material. When the nanoparticles size is comparable to the domain size the competition between the thermal and the anisotropy energy result in the superparamagnetic state. The magnetisation curves at $5 \mathrm{~K}$ and $300 \mathrm{~K}$ are similar to those of the as-made film, suggesting a common magnetic phase in both film. That magnetic phase could be magnetite or maghemite. The comparison between the as-made and annealed film, Figure 4.4.2, shows a reduction of about $60 \%$ of the magnetic moment in a high applied magnetic field, this suggest some $\mathrm{Fe}$ in magnetite and maghemite become $\mathrm{BiFeO}_{3}$ after annealing. This conclusion is consistent with other analysis (e.g. Raman spectroscopy and XRD). 


\subsubsection{Superparamagnetism}

This analysis requires the equation 3.3.22. In this case, $\mathrm{m}_{s, F e}(0)$ is the saturation moment per Fe at $0 \mathrm{~K}, n$ is the exponent, and $D$ the temperature prefactor and related to the spin stiffness. For a bulk 3D Heisenberg ferromagnet, $n$ equal 3/2 [Bloch, 1930; Martinez et al., 1996]. This can change for small nanoparticles, where the finite particle size cuts off the long wavelength spin-waves and can lead to an $n$ of 2 [Hendriksen et al., 1993]. Values much less than $3 / 2$ can occur in thin film composites containing magnetic nanoparticles due to the different spin-wave boundary conditions and spin-wave propagation between nanoparticles [Cojocaru et al., 2014]. An $n$ value of $3 / 2$ is not expected over the full temperature range up to $300 \mathrm{~K}$ for bulk magnetite or maghemite, where the use of two or three magnetic sublattices is required and can lead to a different $n$ over an extended temperature range [Srivastava and Aiyar, 1987; Aragón, 1992]. At low temperature, the bulk magnetite has been reported with $n=3 / 2$ for [Aragón, 1992; Prakash et al., 2016b]. In magnetite powders made by an arc-discharge method, above the Verwey transition temperature of $120 \mathrm{~K}, n$ is 2.26 [Prakash et al., 2016b] and in a single crystal magnetite has a value of 3 [Aragón, 1992]. The Verwey transition is not observed in the $\mathrm{m}_{s, F e}(\mathrm{~T})$ data for small magnetite nanoparticles. $n$ values of 3/2 [Martinez et al., 1996; Goya et al., 2003] and 2 [Ortega et al., 2010] have been reported up to $300 \mathrm{~K} . \mathrm{m}_{F e}(6 \mathrm{~T})$ data was fitted in the lower inset in Figure 4.4.1 to equation 3.3.22, the fit was carried out with $n=3 / 2$ and $D=6.2 \times 10^{-5} \mathrm{~K}^{-3 / 2}$. These values are comparable to those reported for $\mathrm{Fe}_{3} \mathrm{O}_{4}$ nanoparticles, where $D=3.3 \times 10^{-5} \mathrm{~K}^{-3 / 2}$ has been reported for $4 \mathrm{~nm}$ diameter $\mathrm{Fe}_{3} \mathrm{O}_{4}$ nanoparticles [Goya et al., 2003] and $D=2.8 \times 10^{-5} \mathrm{~K}^{-3 / 2}$ for $10^{-15} \mathrm{~nm}$ diameter $\gamma$ $\mathrm{Fe}_{2} \mathrm{O}_{3}$ nanoparticles [Martinez et al., 1996]. This is significantly larger than the bulk magnetite value of $D=4.4 \times 10^{-6} \mathrm{~K}^{-3 / 2}$ [Prakash et al., 2016b]. There is also no evidence of the Verwey transition, which indicates that the magnetite and maghemite are in nanoparticle form.

The upper inset of Figure 4.4.1 displays a large hysteresis loop at $5 \mathrm{~K}$ that becomes negligible at $300 \mathrm{~K}$ for an as-made film. This suggests that the iron oxide nanoparticles are superparamagnetic. Superparamagnetism occurs when the thermal energy is greater than the magnetocrystalline anisotropy energy. This can be observed by measuring zero-field-cooled (ZFC) and field-cooled (FC) curves at low magnetic fields. The temperature where the ZFC curve reaches a peak can be taken as an estimate of the blocking temperature, $\mathrm{T}_{B}$. For low magnetic nanoparticle concentrations, $\mathrm{T}_{B}$ can be written as equation 2.1.6 [DiPietro et al., 2010]. The ZFC peak temperature is known to be higher in magnetite nanoparticles in compact powders when compared with mag- 
netite nanoparticles in solution, which has been attributed to dipolar interactions between the magnetite nanoparticles [Vargas et al., 2005; Nadeem et al., 2011].

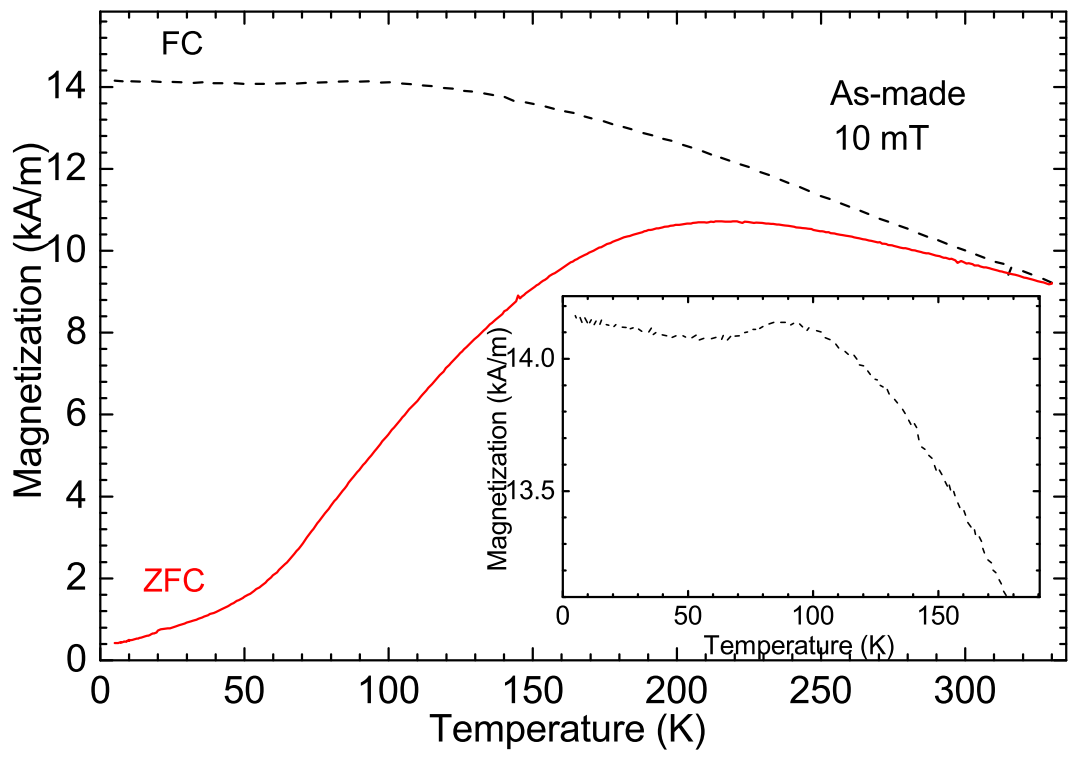

Figure 4.4.3: Plot of the ZFC (solid curve) and FC magnetisation (dashed curve) at $10 \mathrm{mT}$ for an as-made film. Inset shows the FC curve over a smaller temperature range.

Figure 4.4.3 shows the ZFC and FC magnetisation measurement from an as-made film of a magnetic moment in an applied magnetic field of $10 \mathrm{mT}$ as a function of temperature. There is a peak at $216 \mathrm{~K}$ in the ZFC data that indicates the presence of superparamagnetic magnetite and maghemite nanoparticles. Hysteresis is evident up to the highest measured temperature, which likely arises from a range of nanoparticle sizes. Superparamagnetism also affects the magnetic field dependence of the magnetisation above the blocking temperature. $\mathrm{m}_{F e}(\mathrm{~B})$ can be modelled for a narrow magnetite nanoparticle distribution in compact superparamagnetic systems using a Langevin function and a fictitious temperature, $\mathrm{T}^{*}$, to account for dipolar interactions [Vargas et al., 2005; Nadeem et al., 2011]. The Figure 4.4.3 shows that the $\mathrm{m}_{F e}(\mathrm{~B})$ data at $300 \mathrm{~K}$ does not fit the Langevin function very well. This is partly due to the wide range of magnetite and maghemite nanoparticle sizes. $\mathrm{m}_{\mathrm{Fe}}(\mathrm{B})$ at high magnetic fields increases at a faster rate than expected from the Langevin function, which may be due to a spin glass component in the film.

The inset to Figure 4.4.3 shows that the FC magnetisation does not follow the expected dependence on temperature for superparamagnetic nanoparticles, for which a monotonic increase in the magnetisation with decreasing temperature should be expected. The FC curve has a small peak at $90 \mathrm{~K}$ that indicates the existence of another magnetically-ordered phase. The initial decrease with decreasing temperature below the $90 \mathrm{~K}$ peak is indicative of an antiferromagnetic phase. The origin of this phase is 
not clear, however this cannot arise from the known antiferromagnetic phases, since their Néel temperatures are all greater than $198 \mathrm{~K}$.

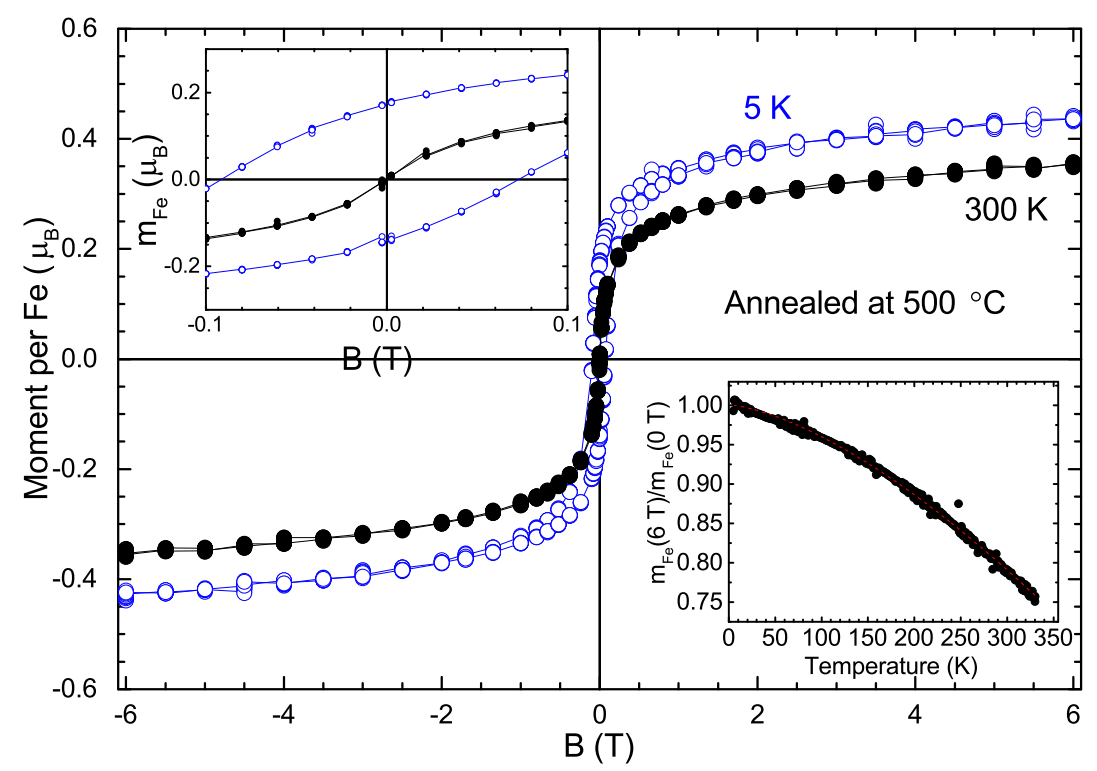

Figure 4.4.4: Magnetic moment per Fe for the $500{ }^{\circ} \mathrm{C}$ annealed film at $5 \mathrm{~K}$ (open circles) and $300 \mathrm{~K}$ (filled circles) cooled in an applied magnetic field of $6 \mathrm{~T}$. Upper right inset: The same plot over a smaller field range. Lower left inset: plot of the normalised saturation moment per Fe versus temperature at $6 \mathrm{~T}$. Also shown is a fit to equation 3.3.22 (dashed curve).

The magnetic moment per Fe is plotted in Figure 4.4.4 for the $500{ }^{\circ} \mathrm{C}$ annealed film after subtracting the Si susceptibility using equation 3.3.21. The magnetisation curves at $5 \mathrm{~K}$ and $300 \mathrm{~K}$ are similar to those of the as-made film plotted in Figure 4.4.1. The magnetisation rapidly increases to $\sim 0.9 \mathrm{~T}$ and then increases at a slower rate. Furthermore, there is negligible hysteresis at $300 \mathrm{~K}$, as can be seen in the inset of Figure 4.4.4, which is indicative of superparamagnetism. This indicates that the magnetic data is primarily from magnetite and maghemite. $\mathrm{m}_{F e}$ at $6 \mathrm{~T}$ and $5 \mathrm{~K}$ is $0.44 \mu_{B}$, which is $60 \%$ of that found in an as-made film and shows that some of the Fe from magnetite and maghemite is now in the $\mathrm{BiFeO}_{3}$ nanoparticles. $\mathrm{m}_{\mathrm{Fe}}$ at $6 \mathrm{~T}$ is plotted in the lower inset to Figure 4.4.4, where the data was fitted to equation 3.3.22 with $n=3 / 2$ and $D=6.2 \times 10^{-5} \mathrm{~K}^{-3 / 2}$. The value of $D$ is only slightly higher than that found before annealing and is consistent with the presence of magnetite and maghemite nanoparticles.

Figure 4.4.5 shows that the low field ZFC and FC magnetisation data for the $500{ }^{\circ} \mathrm{C}$ film are similar to those from the as-made film, however the peak in the ZFC data is slightly lower at $160 \mathrm{~K}$. The appearance of hysteresis up to the highest temperature indicates that there is a distribution in the magnetic nanoparticle sizes. $\mathrm{m}_{\mathrm{Fe}}(\mathrm{B})$ data at $300 \mathrm{~K}$ does not fit the Langevin function. However, the magnetic field depen- 
dence of $\mathrm{m}_{\mathrm{Fe}}(\mathrm{B})$ at $300 \mathrm{~K}$ is the same before and after annealing as can be seen in the inset to Figure 4.4.5. This shows that the magnetite and maghemite nanoparticle size distributions are similar and confirming that $\mathrm{BiFeO}_{3}$ does not significantly contribute to the magnetic properties after annealing. $\mathrm{BiFeO}_{3}$ is known to have a small magnetic moment. There is no evidence for the low temperature peak in the FC data that was seen before annealing, which indicates that this antiferromagnetic phase has disappeared after annealing. There is only a small increase in the FC magnetisation gradient below $\sim 60 \mathrm{~K}$, which is also present before annealing.

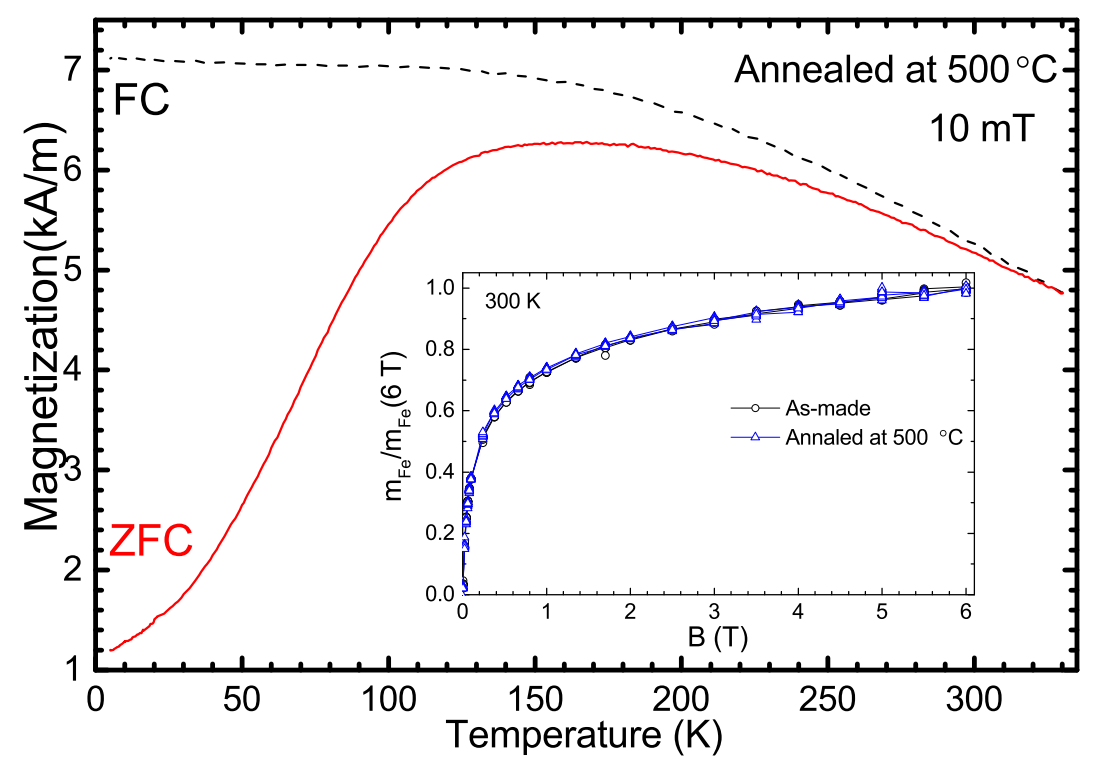

Figure 4.4.5: Plot of the ZFC (solid curve) and FC (dashed curve) magnetisation at $10 \mathrm{mT}$ for the $500{ }^{\circ} \mathrm{C}$ annealed film. Inset: Plot of the moment per Fe normalised to the value at $6 \mathrm{~T}$ versus the magnetic field for an as-made film (open circles) and the film annealed at $500{ }^{\circ} \mathrm{C}$ (open triangles).

\subsubsection{Exchange bias}

As Raman spectroscopy and the magnetic moment suggest the presence of a significant amount of iron oxide, the Bloch model was used to fit the temperature dependence of the magnetic moment at saturation. The fit corresponds to a ferromagnet that could be either magnetite or maghemite.

Those phases have been reported to generate an exchange bias. The magnetisation curves at low temperature shows an asymmetry, a magnetic moment shift towards the negative applied field is observed. These measurements were made after cooling the sample, from $330 \mathrm{~K}$, in an applied magnetic field of $6 \mathrm{~T}$. The exchange bias occurs when a ferromagnetic and antiferromagnetic interface pin some spin under the influ- 
ence of an applied field resulting in magnetic spin alignment resistance. While the exchange bias seen in the annealed sample reaches high temperature, there is still a quest for ambient temperature exchange bias material [Martin et al., 2007]. The exchange bias might be caused by a spin-disorder shell around the maghemite and magnetite.

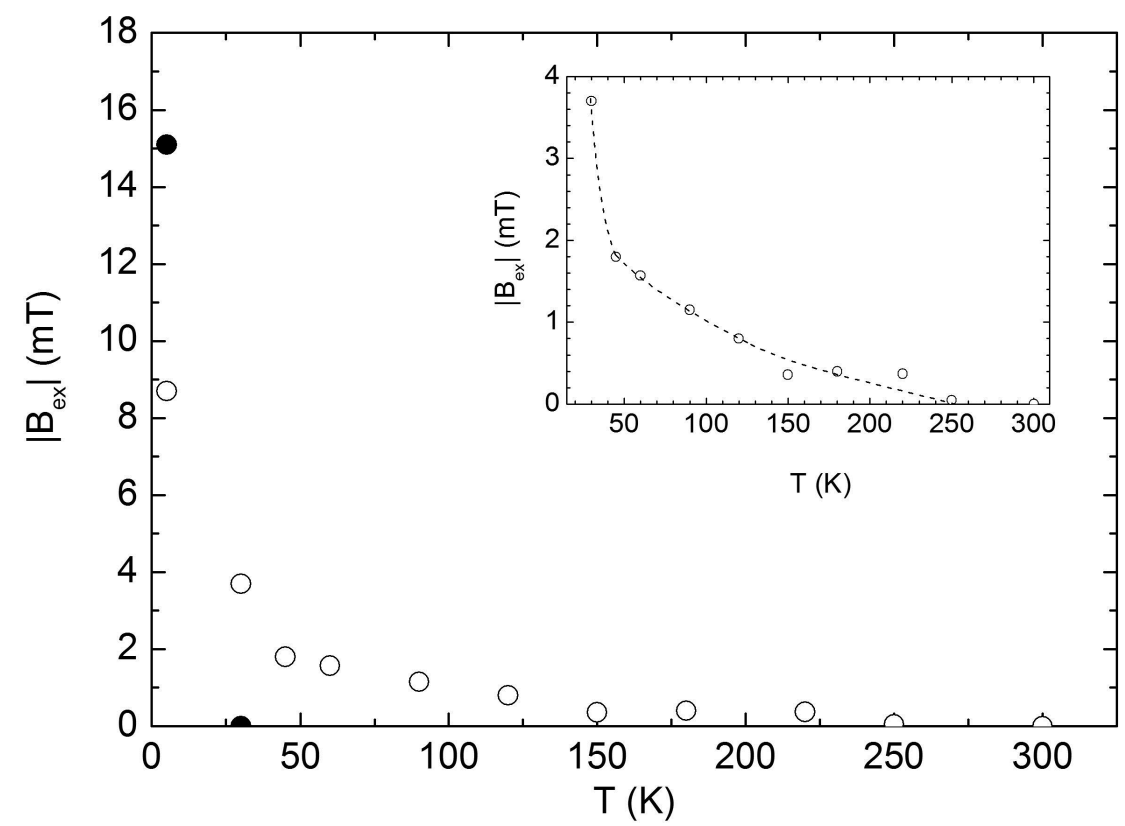

Figure 4.4.6: The magnitude of the exchange bias, $\left|B_{e x}\right|$, plotted against temperature for an as-made film (filled circles) and after annealing at $500{ }^{\circ} \mathrm{C}$ (open circles). Inset shows a plot of $\left|B_{e x}\right|$ for the annealed film. The dashed curve is a visual guide.

The upper right insets of Figure 4.4.1 and 4.4.4 displays asymetric magnetisation curves with a shift in the curves towards negative $B$ values. These measurements were made while cooling in an applied magnetic field of $+6 \mathrm{~T}$. Cooling in an applied magnetic field of $-6 \mathrm{~T}$ produces a shift to positive $B$ (not shown). This is indicative of a negative exchange bias [Nogués and Schuller, 1999; Meiklejohn and Bean, 1957; Malozemoff, 1987]. Figure 4.4.6 shows $\left|B_{e x}\right|$ obtained from the $\mathrm{m}_{F e}(\mathrm{~B})$ data plotted against temperature, where $\left|B_{e x}\right|=\left(B_{c}^{+}+B_{c}^{-}\right) / 2$ and $B_{c}^{+}$is the value of the magnetic field where $\mathrm{m}_{F e}(\mathrm{~B})=0$ for positive $B$ and $B_{c}^{-}$is the coercive field where $\mathrm{m}_{F e}(\mathrm{~B})=0$ for negative $B$. At the low temperatures, $\left|B_{e x}\right|$ has the highest value. $\left|B_{e x}\right|$ rapidly decreases with temperature for an as-made film and completely disappears by $30 \mathrm{~K} .\left|B_{e x}\right|$ is lower at $5 \mathrm{~K}$ after $500{ }^{\circ} \mathrm{C}$ annealing, however at higher temperatures $\left|B_{e x}\right|$ is larger after annealing and only disappears above $250 \mathrm{~K}$.

The core-shell structure can be visualised in Figure 4.4.7 [Hayes et al., 2014]. The first on the left represents the case of superparamagnetic, core $\mathrm{Fe}_{3} \mathrm{O}_{4}$ in blue and shell $\gamma-\mathrm{Fe}_{2} \mathrm{O}_{3}$ in green. The other representations are other possible magnetite-maghemite core-shell configuration [Hayes et al., 2014]. The core-shell follows a similar magnetic domain wall as described in the literature review [Hayes et al., 2014]. 


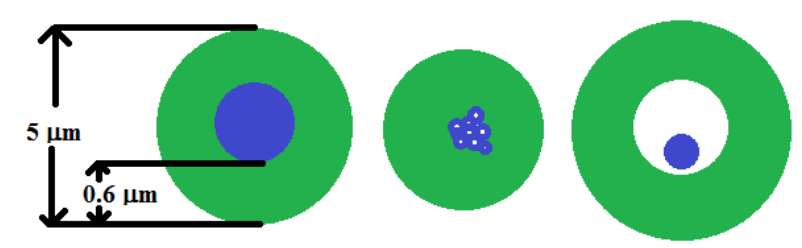

Figure 4.4.7: Schematic representation of possible core-shell particles [Hayes et al., 2014].

The appearance of an exchange bias after deposition and only before annealing shows that the exchange bias cannot be attributed to $\mathrm{BiFeO}_{3}$ with a ferromagnetic or spin glass shell, which has been reported in $\mathrm{BiFeO}_{3}$ nanoparticles [Park et al., 2007; Mazumder et al., 2007; Dhir et al., 2015]. The exchange bias, therefore, likely arises from the iron oxide phases. Exchange biases are known to occur in nanoparticles that have ferromagnetic cores and antiferromagnetic shells where $\left|B_{e x}\right|$ is zero above the Curie or Néel temperatures, depending on which is the lowest [Nogués and Schuller, 1999]. In this case, $\left|B_{e x}\right|$ is zero at $30 \mathrm{~K}$, which is far below the magnetite and maghemite Currie temperatures, the Néel temperatures from the expected antiferromagnetic phases and the blocking temperature (subsection 4.4.2). $\left|B_{\text {ex }}\right|=0 \mathrm{mT}, 30 \mathrm{~K}$ is also below the antiferromagnetic ordering temperature $(90 \mathrm{~K})$ of the unknown phase that causes a peak in the FC magnetisation (see Figure 4.4.3, inset). The exchange bias likely arises from a spin-disordered shell around the magnetite and maghemite nanoparticles. This is known to occur in other ferrite nanoparticles [Mumtaz et al., 2007; Kodama et al., 1996]. In this case study, the spin freezing temperature in the shell region is less than $60 \mathrm{~K}$.

The temperature at which $\left|B_{e x}\right|$ reaches zero after $500{ }^{\circ} \mathrm{C}$ annealing $(\sim 250 \mathrm{~K})$ is far above the antiferromagnetic ordering temperature $(90 \mathrm{~K})$ of the unknown phase that is seen before but not after annealing. This can be explained by the possible quantity of magnetite and maghemite nanoparticles that have spin-disordered shells with spin freezing temperatures that extend up to temperatures far above those seen before annealing. In this scenario, $\left|B_{e x}\right|$ should be zero above the blocking temperature. As mentioned earlier, the ZFC data (Figure 4.4.7) has a peak at $160 \mathrm{~K}$, indicating that there are superparamagnetic nanoparticles. However, the appearance of hysteresis up to $330 \mathrm{~K}$ shows that there are some nanoparticles with blocking temperatures up to at least $330 \mathrm{~K}$. Thus, the disappearance of $\left|B_{\text {ex }}\right|$ above $250 \mathrm{~K}$ is consistent with most of the nanoparticles studied that display an exchange bias and having blocking temperatures below $\sim 250 \mathrm{~K}$. 


\subsubsection{Magnetic force microscopy}

MFM images display the topography in 3D and shown in Figure 4.4.8 and 4.4.9. The colour represent the magnetic field measurements. The millivolt scale represents the feedback of the magnetic field on the microscope tip. The colour yellow represent a region of zero net magnetic moment, red is an upward net magnetic moment and blue is downward net magnetic moment.

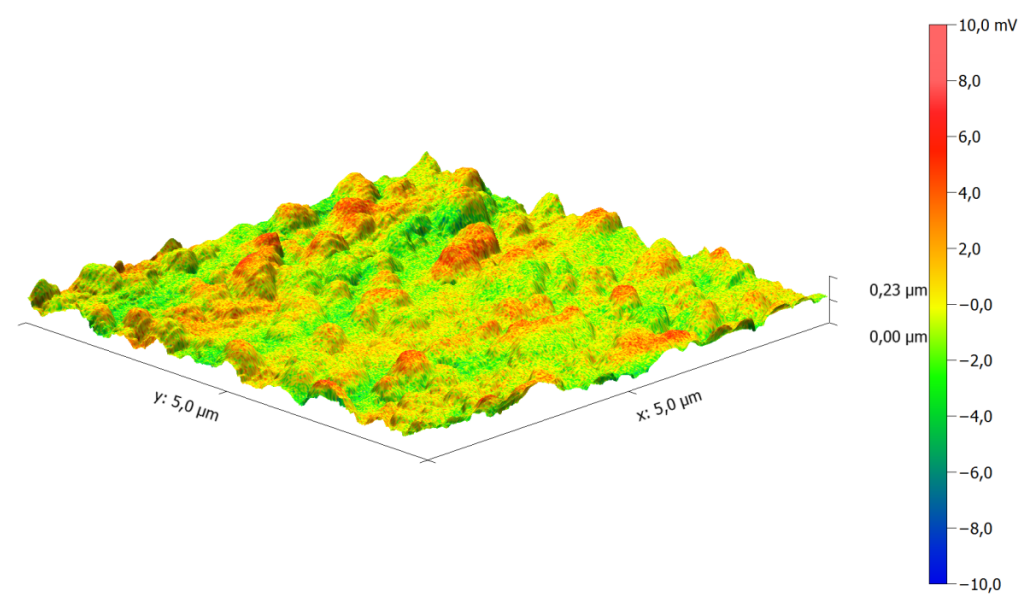

Figure 4.4.8: Magnetic force microscope (MFM) as-made.

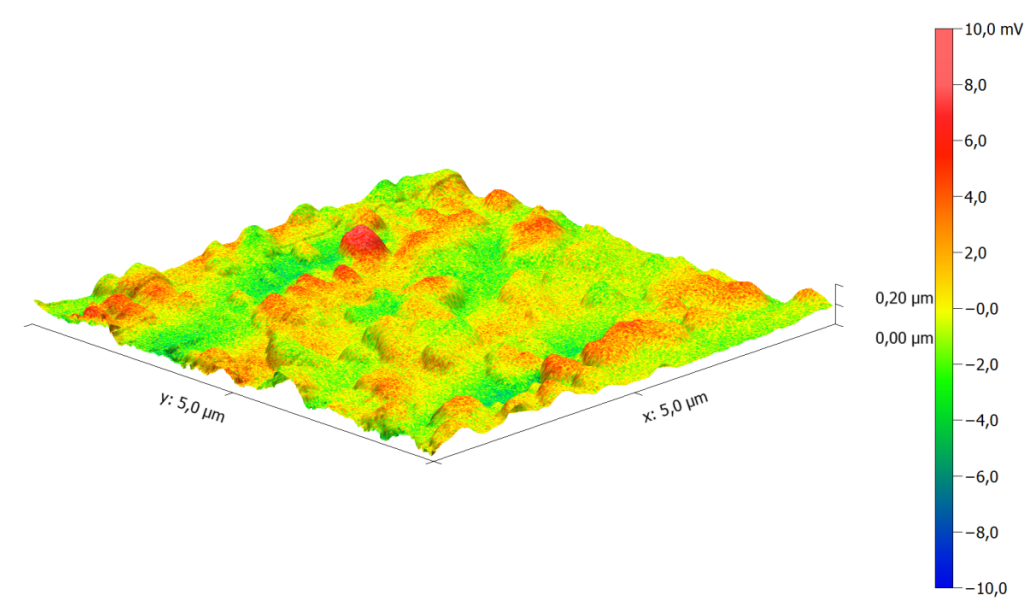

Figure 4.4.9: Magnetic force microscope (MFM) annealed $500{ }^{\circ} \mathrm{C}, 15 \mathrm{~min}$.

Magnetic force microscopy (MFM) is an available mode of operation at the AFM at GNS Science. The AFM tip is magnetized to operate in MFM mode. AFM mode is a topography measurement performed by scanning the tip on the surface of the thin film in tapping mode. After the topography of the sample is determined, the tip will remain at a distance of $200 \mathrm{~nm}$ above the film. The tip scans the surface of the sample and reacts to the dipole moment of the sample, either by torsion or a displacement in the z-axis. The combination of the AFM image with the magnetic response is shown in Figure 4.4.8 and 4.4.9. The topographic features are from the AFM image taken on the forward scan, while the colour scheme indicates the magnetic response on the 
backward scan. The colours show the different polarisation orientations, with red indicating a spin polarisation in the opposite direction to the spin polarisation coloured in blue. MFM images conclusively shows magnetic moment domains, the colour scales are not caused by the tip touching the surface. The image displays the actual magnetic moment of the sample.

The MFM images are consistent, with a phase transition through the annealing temperature. The as-made thin film displays a surface layer containing a high magnetic moment compared to the annealed samples. MFM images also show a large variation in the moment, from $-5^{\circ}$ to $+5^{\circ}$ and amplitude of $-10 \mathrm{mV}$ to $10 \mathrm{mV}$. This could be caused by the significant amount of $\mathrm{Fe}_{3} \mathrm{O}_{4}$ and $\gamma-\mathrm{Fe}_{2} \mathrm{O}_{3}$, about $\sim 58 \%$, as discussed in subsection 4.4.1 Magnetic order. The annealed $\mathrm{BiFeO}_{3} \mathrm{MFM}$ is more homogeneous (Figure 4.4.9) than the as-made film (Figure 4.4.8). This is consistent with a decrease in iron oxides, as discussed in subsection 4.4.1 Magnetic order, and an increase in $\mathrm{BiFeO}_{3}$.

The grain size and topography were already explained in subsection 4.3.3 Atomic force microscope, AFM. In summary, the as-made and annealed samples have a similar grain size and roughness, while the magnetic moment displays a small decrease with annealing, from the as-made $\sim 5^{\circ}$ to annealed $\sim 3^{\circ}$ and $1.3 \mathrm{mV}$ to $0.2 \mathrm{mV}$. This is consistent with a phase modification from mainly iron oxide to mainly $\mathrm{BiFeO}_{3}$. In both cases, the larger magnetic moments are coloured in red, annealing does not change the morphology significantly.

\subsection{Electrical properties, ferroelectricity of $\mathrm{BiFeO3}$}

This section focuses on the electrical properties, subsection 4.5.1 Piezoelectric force microscopy, PFM and subsection 4.5.2 Kelvin probe microscopy, KPM, of the ion beam sputtered $\mathrm{BiFeO}_{3}$. The effect of annealing the thin film on the electrical data is analysed.

\subsubsection{Piezoelectric force microscopy, PFM}

Piezoelectric force microscopy was carried at ambient temperature with a $15 \mathrm{~V}$ applied bias. Shown in Figure 4.5.1 (a) is the AFM topography, without applying a bias. The other two images show, in Figure 4.5.1 (b) and (c), the piezoelectric response of the film with the applied bias. The contrast displays the ferroelectric ordering at ambient temperature. From the list of phases described in the structure analysis in this film, only 
$\mathrm{BiFeO}_{3}$ is ferroelectric thus confirming the presence of the proper phase of multiferroic $\mathrm{BiFeO}_{3}$.
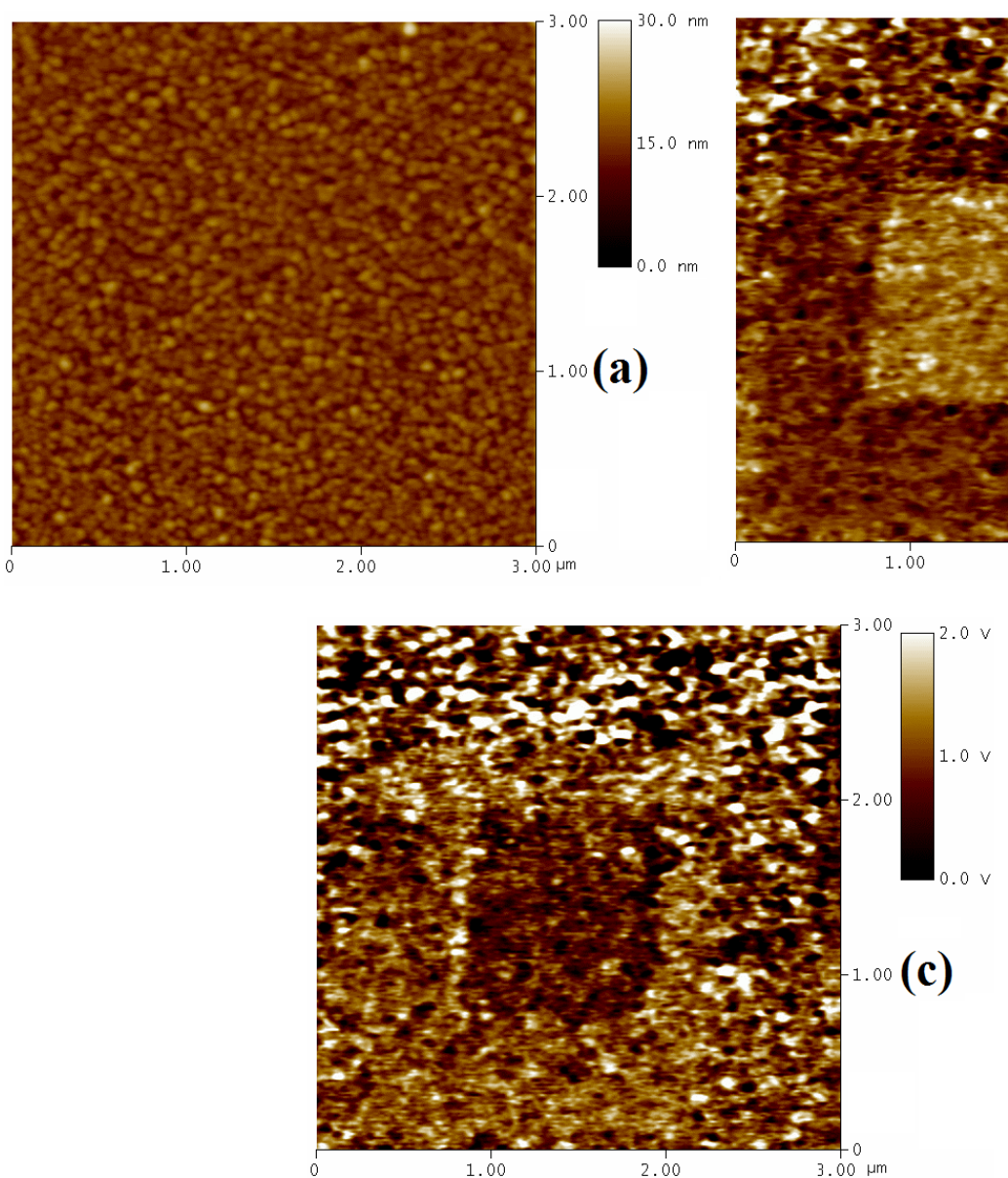

Figure 4.5.1: $\mathrm{PFM}$ of the $\mathrm{BiFeO}_{3}$ annealed at $500{ }^{\circ} \mathrm{C}$ thin film, (a) topography, AFM (b) outplane PFM (c) in-plane PFM.

PFM provides evidence that the annealing process creates an electrical dipole ordered phase after annealing at temperature over $400{ }^{\circ} \mathrm{C}$ and that the films become ferroelectric. Figure 4.5.1 displays the PFM mapping of the annealed sample. The asmade samples do not show a clear pattern (square), which is an indication that material is not ferroelectric [Zhao et al., 2006]. This agrees with the KPFM analysis. The PFM study of the annealed $\mathrm{BiFeO}_{3}$ is not as conclusive as the Kelvin probe force microscopy (KPFM), subsection 4.5.2, study. The $2 \mu \mathrm{m}$ square is the zone that serve to be written and an increase contrast, dark colour, are visible after applying the external field. From Figures 4.5.1, the electrical polarisation, domain on the surface of the thin film, can be estimated to be close to $0.1 \mu \mathrm{m}$. This is surprising, as the grain size of the annealed $\mathrm{BiFeO}_{3}$ nanoparticles are about 3 to 4 times bigger than the polarisation domain. This can be explained by the fact that $\mathrm{BiFeO}_{3}$ has a few preferred domain orientations [Chu et al., 2007b]. Contrary to research carried out by Chu et al. [2007b], the orientation based on the PFM image was not define because the film synthesised by ion beam 
sputtering is polycrystalline and theirs, (Chu's synthesized film) was crystalline [Chu et al., 2007b]. Figure 4.5.1 (c) agrees with experiments detailing the self-polarisation of $\mathrm{BiFeO}_{3}$ [Chu et al., 2006].

The PFM image confirms that a phase transition occurred after annealing at a temperature above $\sim 400{ }^{\circ} \mathrm{C}$, as below that temperature no electrical order is seen, while above that the film is ferroelectric. $\mathrm{BiFeO}_{3}$ is a known ferroelectric material [Kumar et al., 2000]. Iron oxides are ferromagnetic, but not ferroelectric. The characterisation technique provides evidence that annealing is a necessary step in the synthesis of $\mathrm{BiFeO}_{3}$. This proves that the piezoelectric force response comes from $\mathrm{BiFeO}_{3}$, since the as-made film dominated by iron oxides does not show a piezoelectric response, while the annealed film made mainly of $\mathrm{BiFeO}_{3}$ has a strong response.

Figure 4.5.1 (b) and (c) displays the ferroelectric behaviour of the annealed $\mathrm{BiFeO}_{3}$ thin film. The topography shows that the sample's surface is smooth, $\sim 30 \mathrm{~nm}$. The in-plane and out-plane images display the electrical behaviour of the sample after a square shape on the sample has been electrically charged.

\subsubsection{Kelvin probe microscopy, KPM}

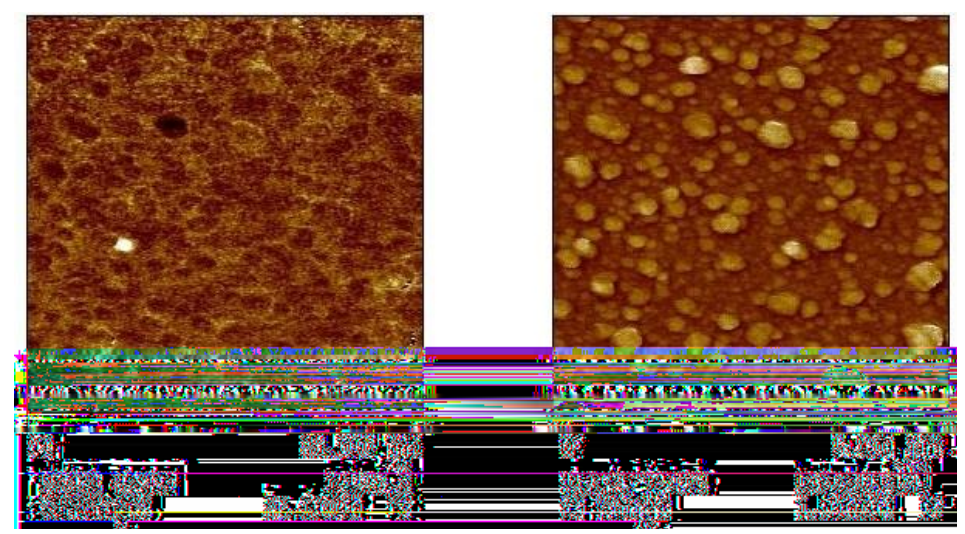

Figure 4.5.2: KPM, surface potential of the as-made thin film measured before (left) and after (right) inducing an external piezoelectric field.

The Kelvin probe microscopy, KPM, was measured at ambient temperature and this technique was used to confirm the PFM results. KPM was for practical reason tested to potentially replace in the future the PFM analysis. The Kelvin probe microscopy data clearly show the change of phase occurring around $\sim 400{ }^{\circ} \mathrm{C}$, below this temperature, the sample is not electric dipole ordered, but above $\sim 400{ }^{\circ} \mathrm{C}$, the ordering is ferroelectric. Figure 4.5.2 displays the Kelvin probe microscopy (KPM) and surface potential microscopy images of the as-made film before applying an external electri- 


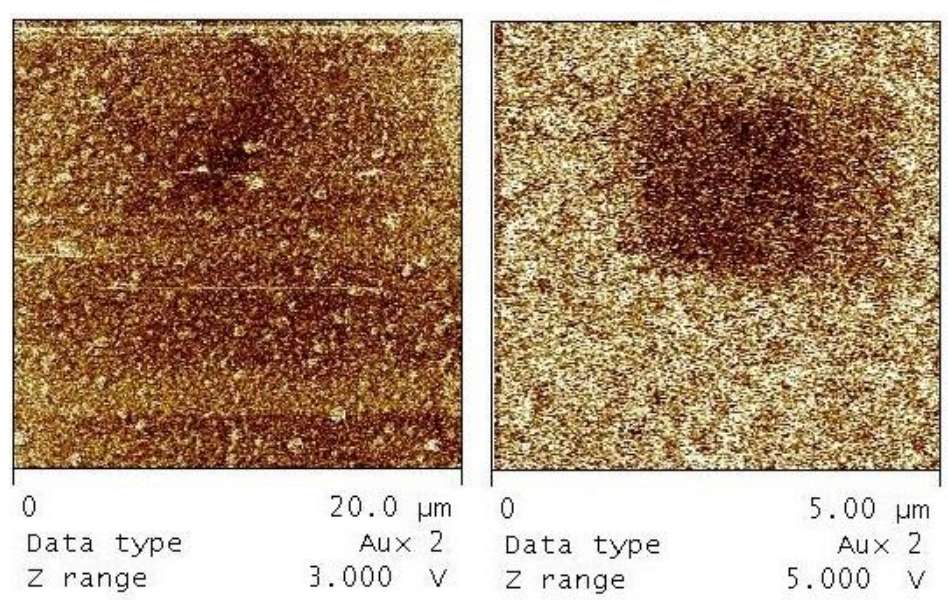

Figure 4.5.3: KPM, surface potential of the $\mathrm{BiFeO}_{3}$ annealed at $500{ }^{\circ} \mathrm{C}$ thin film measured before (left) and after (right) applying an external piezoelectric field.

cal field (Figure 4.5.2) and after applying an external field of $5 \mathrm{~V}$ (Figure 4.5.2 right). The same exercise is carried out on the annealed thin film before (Figure 4.5.3 left) and after (Figure 4.5.3 right) applying an external field. As the as-made sample is known to be an amorphous mix of bismuth oxide, iron oxide and bismuth ferrite oxide, with no clear orientation or structure (see Section 4.3 and 4.4), the comparison of the image before and after applying $5 \mathrm{~V}$, both images only show the topology of the film and they provide no information on the electrical dipole ordering, i.e. the sample has no electric ordering. No potential electrical pattern emerges from these figures, as the compound can locally behave like a ferroic material, however the surface, as a whole, is not. On the other hand, the annealed sample displays a clear piezoelectric response from the applied field. Once again, the image taken before applying the external field is very similar to the previous AFM image, however after applying an external field, the piezoelectric response is modified. The square shape in the image was created on the sample surface by polarising a small area of the sample. This is consistent with ferroelectric behaviour in the annealed sample [Chen et al., 2001]. In previous analysis (magnetic moment), the film antiferromagnetic properties at ambient temperature was demonstrated. With this proof of ferroelectricity, the ferroelectricity property confirms that this film is multiferroic at ambient temperature and that a significant amount of $\mathrm{BiFeO}_{3}$ is in the film, as all the other secondary phases have not been reported to be multiferroic at ambient temperature. This is promising for the development of the future Magnetoelectric RAM (MeRAM) (a device that would use the exchange bias of the material) by retaining the polarisation over time. The MeRAM device requires a ferroelectric and antiferromagnetic material to optimise the potential. The square is not perfect, with a slightly blurry edge; the edge effects could be caused by the leakage current of the sample. The leakage current could be caused by the size of the nanoparticles in the film, as explained previously with the AFM image. Improving the leakage 
current is a focus of the $\mathrm{BiFeO}_{3}$ study and is one of the reasons for doping $\mathrm{BiFeO}_{3}[\mathrm{Kim}$ et al., 2006b].

\subsection{Magneto-optic Kerr effect, MOKE}

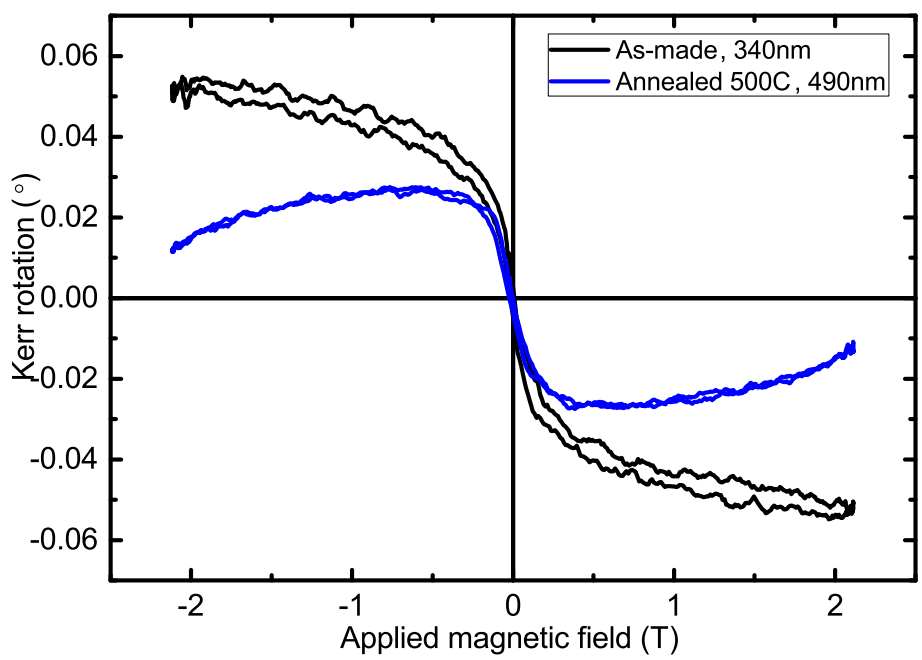

Figure 4.6.1: Kerr rotation of $\mathrm{BiFeO}_{3}$ as a function of the applied field from -2 to $2 \mathrm{~T}$. The asmade film is measured at $340 \mathrm{~nm}$, annealed $500{ }^{\circ} \mathrm{C}$ film is measured at $490 \mathrm{~nm}$.

MOKE suggests a phase transition with the annealing process, similar to XRD analysis. Figure 4.6.1 displays the Kerr rotation as a function of the applied field. The Kerr rotation is similar at every annealing temperature, with very close values between $-2 \mathrm{~T}$ and $2 \mathrm{~T}$. The as-made thin film displays a different behaviour when compared with the annealed films. While the film annealed at $500{ }^{\circ} \mathrm{C}$, although comparable to the other annealed film, is displaying something slightly different. The Kerr rotation loop seems as though a slight rotation is visible, mainly in the range of $-1.5 \mathrm{~T}$ to $1.5 \mathrm{~T}$. The rotation could be caused by an enhanced interference effect of the different phase or from the interface film-substrate.

The effect of the phase transition is seen in the magneto-optical Kerr rotation, however the limited data and the complexity of $\mathrm{BiFeO}_{3}$ and iron oxides do not allow us to identify a specific magnetic behaviour with a specific element of the molecule. The magneto-optical Kerr rotation measurements were carried out at different wavelengths. The as-made film was measured at $340 \mathrm{~nm}, 3.65 \mathrm{eV}$. This energy, when compared to the density of state of potential components, could be linked to $\mathrm{Bi}_{2} \mathrm{O}_{3} ; \mathrm{Bi}_{6 p}$ and $\mathrm{O}_{2 p}$ [Zhang et al., 2006], $\mathrm{Fe}_{3} \mathrm{O}_{4}$ magnetite; $\mathrm{Fe}_{B}^{2+}$ spin up and $\mathrm{Fe}_{A}^{3+}$ spin down [Chen et al., 2004]; and $\mathrm{BiFeO}_{3} ; \mathrm{Bi}_{1 p}, \mathrm{O}_{1 p}$ and $\mathrm{O}_{2 p}$ [Ramachandran et al., 2010]. The $\mathrm{BiFeO}_{3}$ film annealed at $500{ }^{\circ} \mathrm{C}$ was measured at $490 \mathrm{~nm}$ and $2.53 \mathrm{eV}$, which could be linked to 
$\alpha-\mathrm{Fe}_{2} \mathrm{O}_{3} ; \mathrm{O}_{2 p}$ with a hybridisation of $\mathrm{Fe}_{3 d}$ to $\mathrm{Fe}_{3 d}$ and $\mathrm{Fe}_{3 d}$ to $\mathrm{O}_{2 p}$ [Canepa et al., 2011], $\mathrm{Bi}_{2} \mathrm{O}_{3} ; \mathrm{Bi}_{6 p}$ and $\mathrm{O}_{2 p}$ [Zhang et al., 2006], $\mathrm{Fe}_{3} \mathrm{O}_{4}$ magnetite; $\mathrm{Fe}_{B}^{2+}$ spin down [Chen et al., 2004]; and $\mathrm{BiFeO}_{3} ; \mathrm{Fe}_{2 d}, \mathrm{O}_{1 p}$ and $\mathrm{O}_{2 p}$ [Ramachandran et al., 2010]. These energies have a known $\mathrm{BiFeO}_{3}$ hybridisation that occurs between oxygen and iron, $\mathrm{O}_{2 p}$ to $\mathrm{Fe}_{3 d}$ and iron-iron, $\mathrm{Fe}_{3 d}$ to $\mathrm{Fe}_{3 d}$ [Ramachandran et al., 2010]. $\mathrm{Bi}_{2} \mathrm{O}_{3}$ is not strongly magnetically order, so this bismuth oxide can be neglected in the MOKE analysis. With all that in mind the as-made film is more likely to have a larger quantity of $\mathrm{Fe}_{3} \mathrm{O}_{4}$ than $\mathrm{BiFeO}_{3}$. The film annealed at $500{ }^{\circ} \mathrm{C}$ is complex; both iron oxides, maghemite and magnetite, are found in the film. This film also contains $\mathrm{BiFeO}_{3} \mathrm{Fe}_{2 d}, \mathrm{O}_{1 p}$ and $\mathrm{O}_{2 p}$. With the current data available, element based analysis is not possible to be done. A quick comparison of the Faraday rotation of $\alpha-\mathrm{Fe}_{2} \mathrm{O}_{3}$ seems to contain enough difference to limit the possibility of seeing the $\alpha-\mathrm{Fe}_{2} \mathrm{O}_{3}$ magneto-optical Kerr effect, and $\mathrm{Fe}_{3} \mathrm{O}_{4}$ is unlikely to be present after annealing at $500{ }^{\circ} \mathrm{C}$, which leaves the possibility that oxygen reduces the signal from iron.

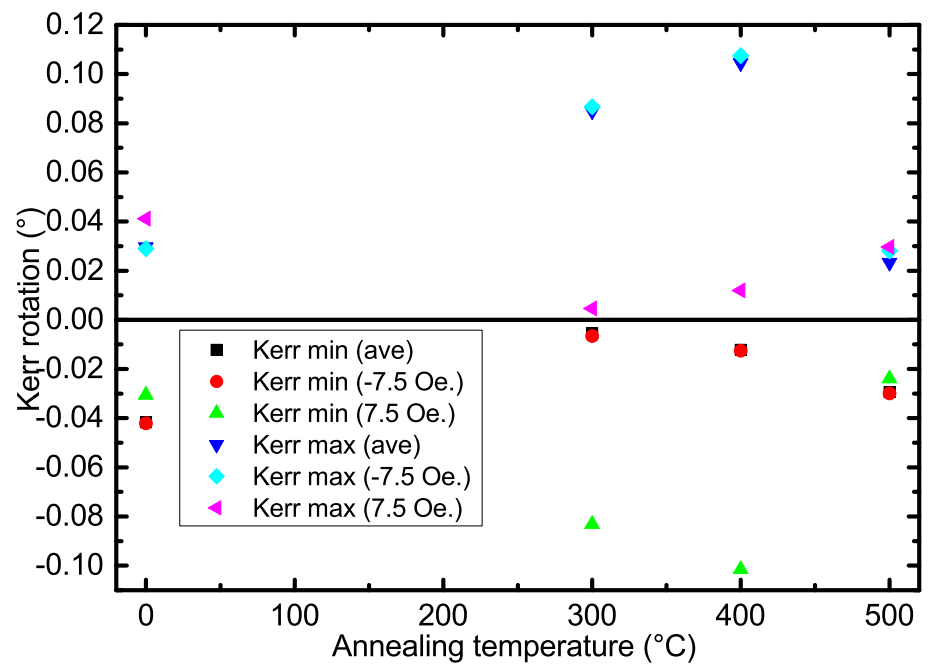

Figure 4.6.2: Minimum and maximum Kerr rotation measured in function of the annealing temperature. The difference between the positive and negative applied field at $300{ }^{\circ} \mathrm{C}$ and $400{ }^{\circ} \mathrm{C}$ is probably caused by the substrate.

Comparing the magneto-optical Kerr rotation under the opposite applied field shows a potential signal from the substrate in the films annealed at $300{ }^{\circ} \mathrm{C}$ and $400{ }^{\circ} \mathrm{C}$. The absolute value is thickness-dependent and prevents us from drawing any other conclusions. Figure 4.6.2 displays the minimum and maximum Kerr rotation measured after annealing at different temperatures. Taking the absolute value of the minimum and the maximum Kerr rotation, Figure 4.6.2 should display matching values at every annealing temperature. The maximum and minimum Kerr rotations are similar under an applied field of 7.5 Oe and -7.5 Oe for the as-made and $500{ }^{\circ} \mathrm{C}$ annealed samples. The annealed samples at $300{ }^{\circ} \mathrm{C}$ and $400{ }^{\circ} \mathrm{C}$ show an important difference in Kerr rotation under an applied field of $7.5 \mathrm{Oe}$ and $-7.5 \mathrm{Oe}$, which could be caused by the 
substrate. Comparing the analysis with other studies is difficult, as the results can be dependent on thickness, i.e. a film of $645 \mathrm{~nm} \gamma-\mathrm{Fe}_{2} \mathrm{O}_{3}$ saturates at double the value of a similar film of $1550 \mathrm{~nm}$ [Tepper et al., 2003].

\subsubsection{Verdet constant}

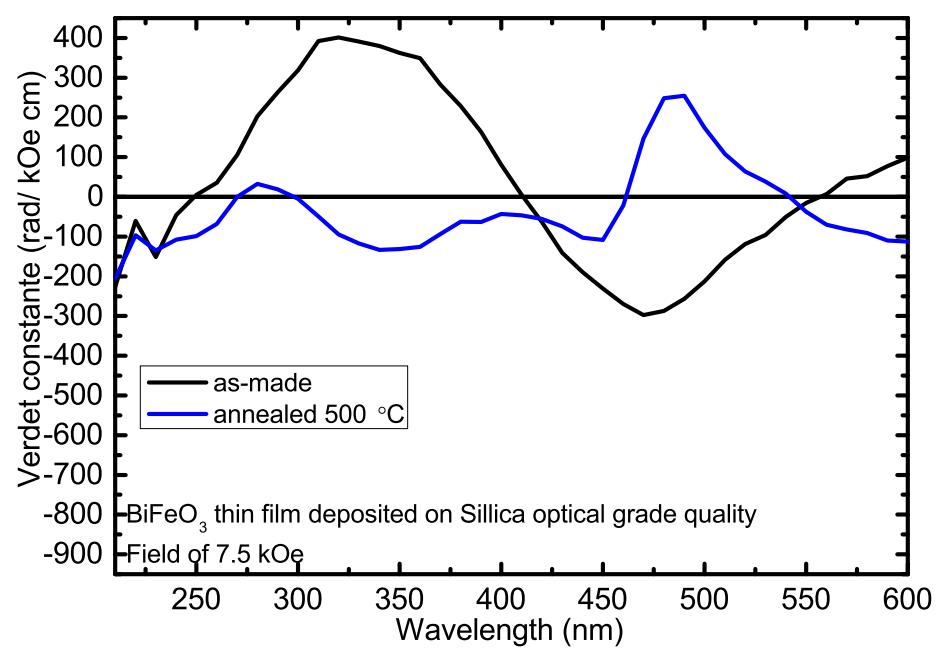

Figure 4.6.3: Verdet constant of $\mathrm{BiFeO}_{3}$ after annealing at $500{ }^{\circ} \mathrm{C}$.

The annealed $\mathrm{BiFeO}_{3}$ thin film has a very high Verdet constant at wavelengths between 200 and $600 \mathrm{~nm}$, as Figure 4.6.3 highlights. Figure 4.6.3 shows the Verdet constant of as-made and annealed $\mathrm{BiFeO}_{3}$ thin films in function of the wavelength. The Verdet constant is calculated from equation 2.4.2 [Bi et al., 2008].

In this case the magnetic field $B$ was applied at $7.5 \mathrm{kOe}$. The distance $d$ that the light beam travels in the material is the thickness of the sample, $\sim 150 \mathrm{~nm}$. This is the first time this calculation has been done on $\mathrm{BiFeO}_{3}$ thin films in the range from 200 to $600 \mathrm{~nm}$. Previous work on this topic revealed a Verdet constant at $1550 \mathrm{~nm}$ of $18 \pm 2^{\circ} . \mathrm{cm}^{-1} \mathrm{kOe}^{-1}, 0.3 \mathrm{rad} \mathrm{cm}^{-1} \mathrm{kOe}^{-1}$ [Bi et al., 2008]. This Verdet constant is of the same order of magnitude as the sputtered $\mathrm{BiFeO}_{3}$ film annealed at $500{ }^{\circ} \mathrm{C}$ for $15 \mathrm{~min}$ at a wavelength of $490 \mathrm{~nm}, 35^{\circ} \mathrm{cm}^{-1} \mathrm{kOe}^{-1}, 0.61 \mathrm{rad} \mathrm{cm}^{-1} \mathrm{kOe}^{-1}$. However, the Figure 4.6.3 highlights significant different value of Verdet constant at each wavelength, making such a comparison challenging. Nonetheless, in the wavelengths between 200 and $600 \mathrm{~nm}$, only two materials have been predicted and confirmed to have a Verdet constant greater than $\mathrm{BiFeO}_{3}$, reported here. Those materials are $\mathrm{NdF}_{3}$ and $\mathrm{PrF}_{3}$ which have a Verdet constant almost twice as significant as $\mathrm{BiFeO}_{3}$ [Leycuras et al., 1984]. Maghemite $\mathrm{Fe}_{2} \mathrm{O}_{3}$ [Guerreroa et al., 1997] and magnetite $\mathrm{Fe}_{3} \mathrm{O}_{4}$ [Lopez-Santiago et al., 2009] have a negligible Verdet constant compared to the $\mathrm{BiFeO}_{3}$ reported here. Further work is required to compare $\mathrm{Bi}_{2} \mathrm{O}_{3}$ with the sputtered $\mathrm{BiFeO}_{3}$ thin film. 


\subsubsection{Magnetic circular dichroism}

The optical magnetic circular dichroism (MCD) analysis agrees with a phase transition during annealing. Figure 4.6.4 shows the optical MCD as a function of the applied magnetic field. The as-made film displays the largest value, while the film annealed at $300{ }^{\circ} \mathrm{C}$ displays the smallest value at $20 \mathrm{kOe}$. The MCD of the films annealed at $400{ }^{\circ} \mathrm{C}$ and $500{ }^{\circ} \mathrm{C}$ have a similar absolute value. The difference between the two films seems to be only the direction of the applied external field. The MCD maximum is at $-20 \mathrm{kOe}$ for the film annealed at $500{ }^{\circ} \mathrm{C}$ and $20 \mathrm{kOe}$ in the case of the film annealed at $400{ }^{\circ} \mathrm{C}$. The apparent hysteresis loop in the $\mathrm{BiFeO}_{3}$ annealed at $500{ }^{\circ} \mathrm{C}$ is also a specific behaviour to this film, as this film is the only one showing that feature. The MCD hysteresis loop, -0.013 to $0.01 \mathrm{~T}$ is similar to the magnetic moment hysteresis loop at $5 \mathrm{~K}$, however the MCD values are not of the same order of magnitude, at $5 \mathrm{~K}, \mathrm{MCD}$ hysteresis is between $-0.17 \mathrm{~T}$ to $0.17 \mathrm{~T}$.

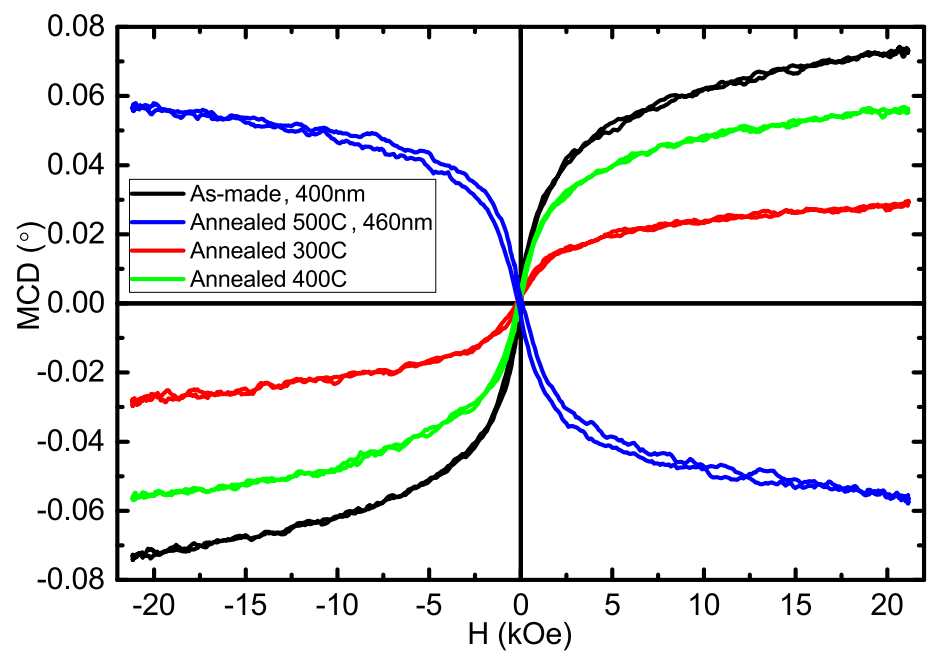

Figure 4.6.4: $\mathrm{MCD}$ of $\mathrm{BiFeO}_{3}$ as a function of the applied field.

The MCD analysis suggests the as-made film's main contributor is $\mathrm{Fe}_{3} \mathrm{O}_{4}$ magnetite. The annealed film has multiple, more complex contributors, given that there is a phase transition with the annealing process. Figure 4.6 .5 displays the optical magnetic circular dichroism measured at different wavelengths. The as-made film displays a maximum MCD around $400 \mathrm{~nm}$, compared to and $320 \mathrm{~nm}$ after annealing at $500{ }^{\circ} \mathrm{C}$. The MCD behaviour is very similar for the films annealed at $300{ }^{\circ} \mathrm{C}$ and $400{ }^{\circ} \mathrm{C}$; they both display a hybrid wavelength response that is between the response of the as-made film and the $\mathrm{BiFeO}_{3}$ annealed at $500{ }^{\circ} \mathrm{C}$. This figure highlights the wavelength dependency of the MCD phenomena. The MCD also shows that the thin film phases are different through the different annealing temperatures. The as-made thin film MCD rotation seems to be dominated by $\mathrm{Fe}_{3} \mathrm{O}_{4}$, with a central broad MCD peak near $440 \mathrm{~nm}$ 
and a negative value after $500 \mathrm{~nm}$ [Zayat et al., 2003]. The absence of a positive peak at $530 \mathrm{~nm}$ could suggest a limitation on the influence of $\alpha-\mathrm{Fe}_{2} \mathrm{O}_{3}$ in the MCD [Zayat et al., 2003]. The $\mathrm{BiFeO}_{3}$ film annealed at $500{ }^{\circ} \mathrm{C}$ displays unique behaviour compared to the other thin films. This signal should mainly come from the $\mathrm{BiFeO}_{3}$ molecules. Because of the purity of the samples, the MCD signal can include the effects of secondary phases like traces of iron oxides, however from previous analysis the secondary phases would be expected only in small concentration and therefor the main MCD contribution should come from $\mathrm{BiFeO}_{3}$.

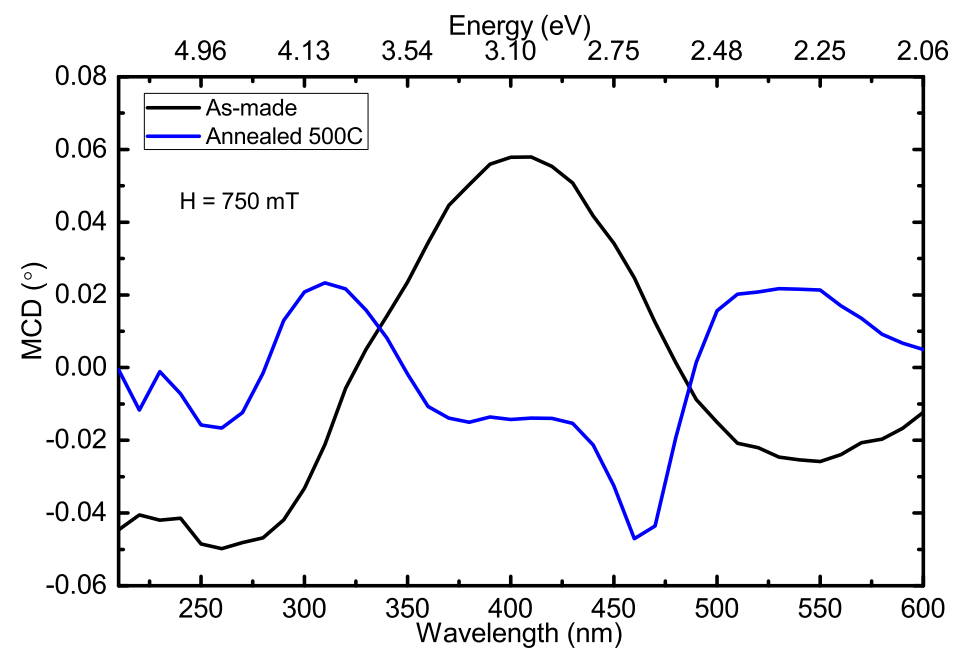

Figure 4.6.5: $\mathrm{MCD}$ of $\mathrm{BiFeO}_{3}$ as a function of the wavelength for an applied field of $750 \mathrm{mT}$.

The energy at which the extreme MCD is found shows potentially multiple MCD contributors in every film and a modification of those contributors through annealing at different temperatures. Figure 4.6.6 shows the energy at which the optical magnetic circular dichroism is at a minimum and maximum as a function of the annealing temperature. These values are compared with the energy of the band gap. Under an applied field of $7.5 \mathrm{kOe}$ or $-7.5 \mathrm{kOe}$, the MCD minimum has the same energy value. The MCD maximum, however, has different values for the film as-made, annealed at $300{ }^{\circ} \mathrm{C}$ and at $400{ }^{\circ} \mathrm{C}$. Once annealed at $500{ }^{\circ} \mathrm{C}$, the maximum is found at $4 \mathrm{eV}$ under either the positive or negative field. Following from the previous analysis of the most likely components in the film, the as-made film minimum at $4.77 \mathrm{eV}$ can be linked to $\mathrm{Bi}_{2} \mathrm{O}_{3}: \mathrm{Bi}_{6 p}$ and $\mathrm{O}_{2 p}$ [Zhang et al., 2006] and $\mathrm{Fe}_{3} \mathrm{O}_{4}: \mathrm{Fe}_{B 5 t 2}^{3+}$ and $\mathrm{Fe}_{A 5 E}^{3+}$ [Chen et al., 2004]. The maximum at $5.91 \mathrm{eV}$ includes these elements plus $\mathrm{Fe}_{3} \mathrm{O}_{4}: \mathrm{Fe}_{\text {Beg }}^{2+}$ and $\mathrm{Fe}_{B e g}^{3+}$ [Chen et al., 2004]. The maxima of the films annealed at $300{ }^{\circ} \mathrm{C}$ and $400{ }^{\circ} \mathrm{C}$ are also found at $5.91 \mathrm{eV}$, like the as-made film, and includes the same list of elements. Both the annealed $300{ }^{\circ} \mathrm{C}$ and the annealed $400{ }^{\circ} \mathrm{C}$ film have their minimum at $5.64 \mathrm{eV}$, with possible contributions coming from $\mathrm{Bi}_{2} \mathrm{O}_{3}: \mathrm{Bi}_{6 p}$ and $\mathrm{O}_{2 p}$ [Zhang et al., 2006]; and $\mathrm{Fe}_{3} \mathrm{O}_{4}$ : $\mathrm{Fe}_{B e g}^{2+}, \mathrm{Fe}_{B 5 t 2}^{3+}$ and $\mathrm{Fe}_{\text {Beg }}^{3+}$ [Chen et al., 2004]. For the $\mathrm{BiFeO}_{3}$ annealed at $500{ }^{\circ} \mathrm{C}$, the minimum MCD is close to the optical band gap value. The minimum is at $2.7 \mathrm{eV}$, which can 
be linked to $\mathrm{BiFeO}_{3}: \mathrm{Bi}_{1 p}$ [Ramachandran et al., 2010] and $\mathrm{Fe}_{2 d} ; \alpha-\mathrm{Fe}_{2} \mathrm{O}_{3}: \mathrm{Fe}_{3 d}$ and $\mathrm{O}_{2 p}$ [Canepa et al., 2011]. The maximum of $4 \mathrm{eV}$ can be linked to $\mathrm{BiFeO}_{3}: \mathrm{Bi}_{1 p}$ [Ramachandran et al., 2010]. The as-made film and the films annealed at $300{ }^{\circ} \mathrm{C}$ and $400{ }^{\circ} \mathrm{C}$ could have multiple MCD contributors, and perhaps even the substrate could be a contributor. This would be expected, as these films are a mix of iron oxide and bismuth oxide. In the case of the as-made film, as shown in Figure 4.6.5, due to the high absorption coefficient of the substrate, the MCD contribution from the substrate is not expected to be displayed. A detailed analysis of the light absorption of the as-made film has shown an absorption efficiency as high as $95 \%$ at wavelengths below $750 \mathrm{~nm}$. This confirms that the results displayed are coming from the thin film, not the substrate. The film annealed at $500{ }^{\circ} \mathrm{C}$ seems likely to have just one major MCD contributor; the Figure 4.6.6 does not show multiple maxima or minima like the films made at other annealing temperatures. As the structural analysis suggests there might be some iron oxide secondary phases, those secondary phases could contribute to the MCD. However, at this stage, if there are other MCD contributors, they cannot be clearly identified with the MCD analysis of the film annealed at $500{ }^{\circ} \mathrm{C}$.

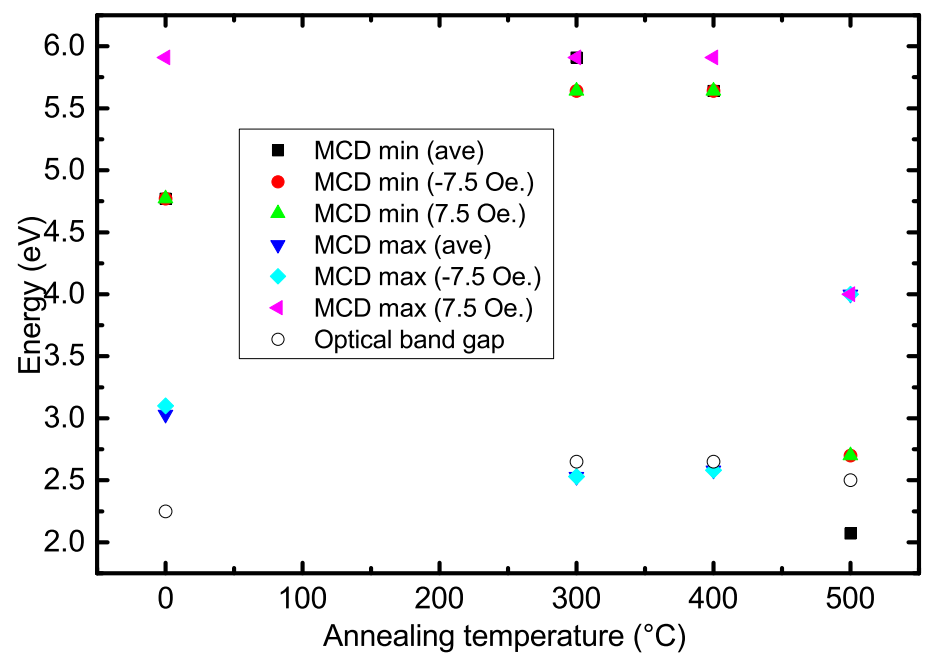

Figure 4.6.6: Energy value of the minimum and maximum MCD in function of the annealing temperature of the film.

\subsubsection{Kerr rotation}

The energy at which the extreme magneto-optical (MO) Kerr rotation is found shows potentially multiple Kerr rotation contributors in every film and a modification of those contributors through annealing at different temperatures. Figure 4.6.7 shows the energy at which the Kerr rotation is at a minimum and maximum as a function of the annealing temperature. Also in this graph, the optical band gap is compared to the 
energy of the maximum and minimum Kerr rotation. The maximum with an applied field of 7.5 Oe occurs at the same energy of the minimum with an applied field of $7.5 \mathrm{Oe}$, and vice versa. This figure highlights the modification of other contributors to the MO Kerr effect. The precise contributor is challenging to pinpoint. The increased quantity and crystallinity of the sample have a major impact on the modification of the MO Kerr effect. The minimum energy after annealing at $500{ }^{\circ} \mathrm{C}$ is the same as the band gap, which could mean a transition between $\mathrm{Fe}_{3 d}$ and $\mathrm{O}_{2 p}$ [Wang et al., 2009a] was created by the incident laser excitation light, assuming the contribution is from $\mathrm{BiFeO}_{3}$ only.

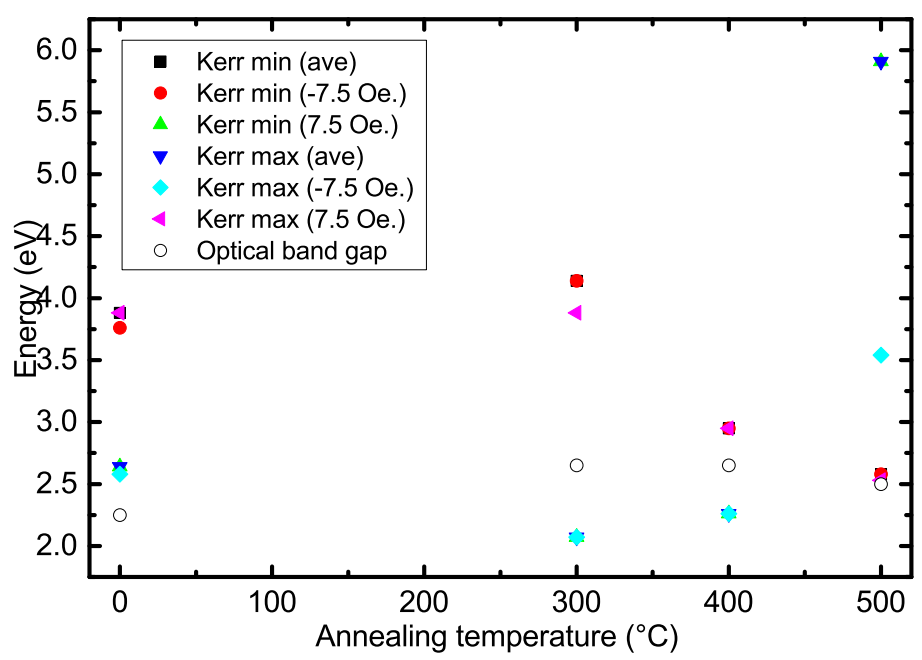

Figure 4.6.7: Energy value of the minimum and maximum Kerr rotation.

\subsection{Thin film annealed at $600^{\circ} \mathrm{C}$}

Study (Section 4.3 to 4.6 ) concluded that the $\mathrm{BiFeO}_{3}$ thin film annealed at $500{ }^{\circ} \mathrm{C}$ was the minimum annealing temperature needed to create good quality $\mathrm{BiFeO}_{3}$ thin film. The XRD, subsection 4.3.2, and the magnetic, Section 4.4, data have shown potential presence of iron oxides secondary phases. In order to test the optimisation of the synthesis, another film was annealed at $600{ }^{\circ} \mathrm{C}$. The RBS analysis in Figure 4.7.1 shows bismuth, iron and oxygen in the film and the silicon from the substrate. A good RUMP fit is achieved by assuming a material architecture of 10 layers, the details are presented in Section B.1. The surface layer, $1.75 \times 10^{17}$ at.cm ${ }^{-2}$, is composed of $\mathrm{Bi}_{1}, \mathrm{Fe}_{2}$ and $\mathrm{O}_{5}$. That stoichiometry suggests $\mathrm{BiFeO}_{3}$, and iron oxides phase could be present. The density of that layer was estimated with SRIM data to $4.91 \times 10^{2}$ at. $\mathrm{cm}^{-3}$ [Ziegler, 1998]. The thickness of the surface layer is estimated at $36 \mathrm{~nm}$. The second layer, $7 \times 10^{17}$ at.cm $^{-2}$, is composed of $\mathrm{Bi}_{1} \mathrm{Fe}_{1} \mathrm{O}_{3}$. A detailed analysis of the phases will be discussed with XRD and the magnetic moment. This suggests the atoms are not bonding to form $\mathrm{BiFeO}_{3}$. 
The density is $4.22 \times 10^{2}$ at.cm ${ }^{-3}$ and the thickness is $166 \mathrm{~nm}$. The third layer does not contain iron; however, bismuth is present. Due to the significant difference and the depth at which bismuth is found, RBS confirms a bismuth diffusion. Bismuth diffusion was expected from the RBS analysis of the film annealed at $500{ }^{\circ} \mathrm{C}$ (Figure 4.3.1 (b)). This layer, $4 \times 10^{17}$ at.cm ${ }^{-2}$, contains $\mathrm{Bi}_{0.09}$ and $\mathrm{SiO}_{2}$. The density of this layer is $4.62 \times 10^{2}$ at. $\mathrm{cm}^{-3}$, thickness of $87 \mathrm{~nm}$. The bismuth concentration decreases within the following 3 layers in $\mathrm{SiO}_{2}$ (detailed are presented in Section B.1). The layer number 7 in the RUMP fit, $7.5 \times 10^{17}$ at.cm ${ }^{-2}$ contains $\mathrm{Bi}_{0.009}, \mathrm{O}_{0.5}$ and $\mathrm{Si}_{1.1}$. Assuming the fit is describing the physics and the detector is of good quality, this suggests bismuth diffused through the $\mathrm{SiO}_{2}$ into the $\mathrm{Si}$ bulk. The oxygen in that layer could have been diffused toward the bulk silicon either by thermal annealing (substrate $\mathrm{SiO}_{2}$ is grown by thermal annealing), by the mobility of bismuth atoms or a combination of the two phenomena. This layer density was calculated to $4.95 \times 10^{2}$ at.cm ${ }^{-3}$, thickness of $151 \mathrm{~nm}$. Layer 9, $3.5 \times 10^{18}$ at. $\mathrm{cm}^{-2}$, contains $\mathrm{Bi}_{0.001}$ and $\mathrm{Si}_{1}$. Bismuth atoms at that depth are caused by diffusion of bismuth, the diffusion of bismuth into silicon has also been observed at annealing temperature about $580{ }^{\circ} \mathrm{C}$ [Fan et al., 1990]. The bismuth tail in Figure 4.7.1 could also be caused by the quality of the detector and the signal to noise ratio. Layer 9 density was estimated with SRIM data to $4.96 \times 10^{2}$ at. $\mathrm{cm}^{-2}$ and a thickness of $706 \mathrm{~nm}$. The tenth layer is bulk silicon from the substrate. There are no other elements visible, which is confirmed by the qualitative PIXE analysis.

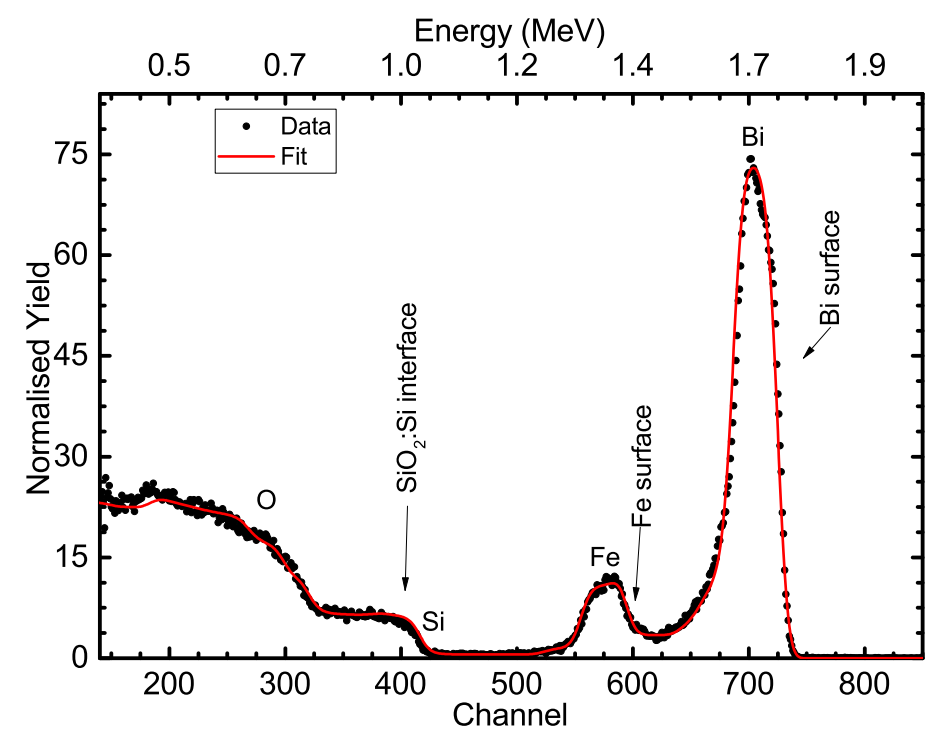

Figure 4.7.1: RBS spectra of the film annealed at $600{ }^{\circ} \mathrm{C}$ (circle) with the RUMP fit (line).

The XRD analysis of this annealed thin film at $600{ }^{\circ} \mathrm{C}$ is displayed in Figure 4.7.2. The XRD has a lot of noise to the signal and this noise increase the difficulty to identify the phases in the material. As some major phase modification happened between the film annealed at $500^{\circ} \mathrm{C}$ compare to the film annealed at $600{ }^{\circ} \mathrm{C}, \mathrm{BiFeO}_{3}$ can start decomposing at that temperature. Bismuth can diffuses as suggested by the RBS analysis and 
form other phases in layer underneath the surface. As seen in the Figure 4.7.2, the film annealed at $600{ }^{\circ} \mathrm{C}$ contain $\mathrm{Fe}_{3} \mathrm{O}_{4}, \alpha-\mathrm{Fe}_{2} \mathrm{O}_{3}, \gamma-\mathrm{Fe}_{2} \mathrm{O}_{3}$ and $\mathrm{BiFeO}_{3}$. No trace of $\mathrm{Bi}_{2} \mathrm{Fe}_{4} \mathrm{O}_{9}$ was found. However as discussed in the subsection 4.3.2, the film annealed at $500{ }^{\circ} \mathrm{C}$ only $\mathrm{BiFeO}_{3}$ was identified. So, annealing at $600{ }^{\circ} \mathrm{C}$ might be sufficient to decompose some of the $\mathrm{BiFeO}_{3}$ into iron oxides or allows for a greater quantity of iron oxides to form. The broadening of XRD peaks increase meaning the nano-crystal is becoming smaller. A volume reduction while annealing at higher temperature can be counter intuitive, however this could be possible in the case where $\mathrm{BiFeO}_{3}$ decompose [Tan et al., 2005]. The other option to explain the broadening is $\mathrm{BiFeO}_{3}$ becomes amorphous. The film annealed at $500{ }^{\circ} \mathrm{C}, \mathrm{BiFeO}_{3}$ dominated the XRD spectrum while three iron oxides phases were either amorphous or too small compare to the bismuth particle size to be seen. In the present case, iron oxides phases are dominating while $\mathrm{BiFeO}_{3}$ phases could have decompose. The XRD spectrum is not sufficiently conclusive to compare the particle size of each compounds. XRD simply shows a general phase modification above $600{ }^{\circ} \mathrm{C}$, however the new phases are not moving towards the formation of $\mathrm{BiFeO}_{3}$.

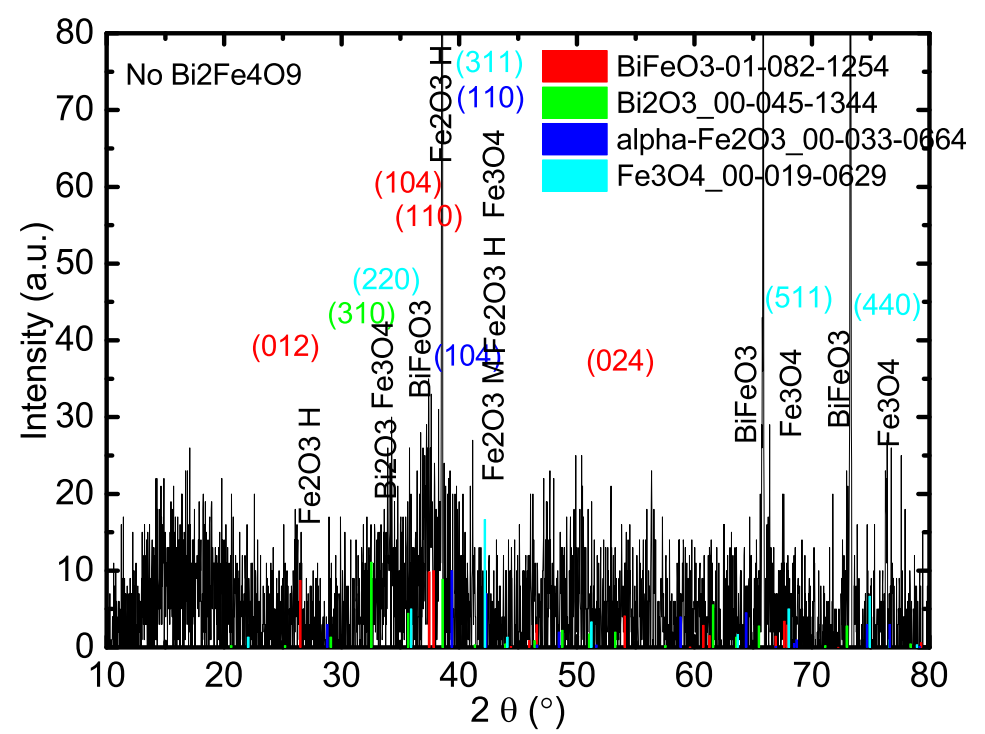

Figure 4.7.2: XRD, grazing angle $2^{\circ}$, from the film annealed at $600{ }^{\circ} \mathrm{C}$.

The magnetic data agrees with the XRD analysis. Both low temperature and ambient temperature field loop measurements show an increase in magnetic saturation moment at 6 T, Figure 4.7.3. This increase is consistent with an increase of iron oxides in the thin film. The hysteresis loop at $5 \mathrm{~K}$ is half the value after annealing at $600{ }^{\circ} \mathrm{C}$ compared to $500{ }^{\circ} \mathrm{C}$. The new film might have lost a certain number of superparamagnetic particles, which seems to be the case. From the XRD analysis, iron oxides are taking the place of the $\mathrm{BiFeO}_{3}$. The magnetic data can indicate that about half of the iron particles are magnetic and could form a mix of $\mathrm{Fe}_{3} \mathrm{O}_{4}$ and $\gamma-\mathrm{Fe}_{2} \mathrm{O}_{3}$. This confirms that annealing temperature above $500{ }^{\circ} \mathrm{C}$ results in an increase in iron oxides in the $\mathrm{BiFeO}_{3}$ film, and annealing at $500{ }^{\circ} \mathrm{C}$ is the optimum annealing temperature to synthe- 
sis the largest amount of $\mathrm{BiFeO}_{3}$.

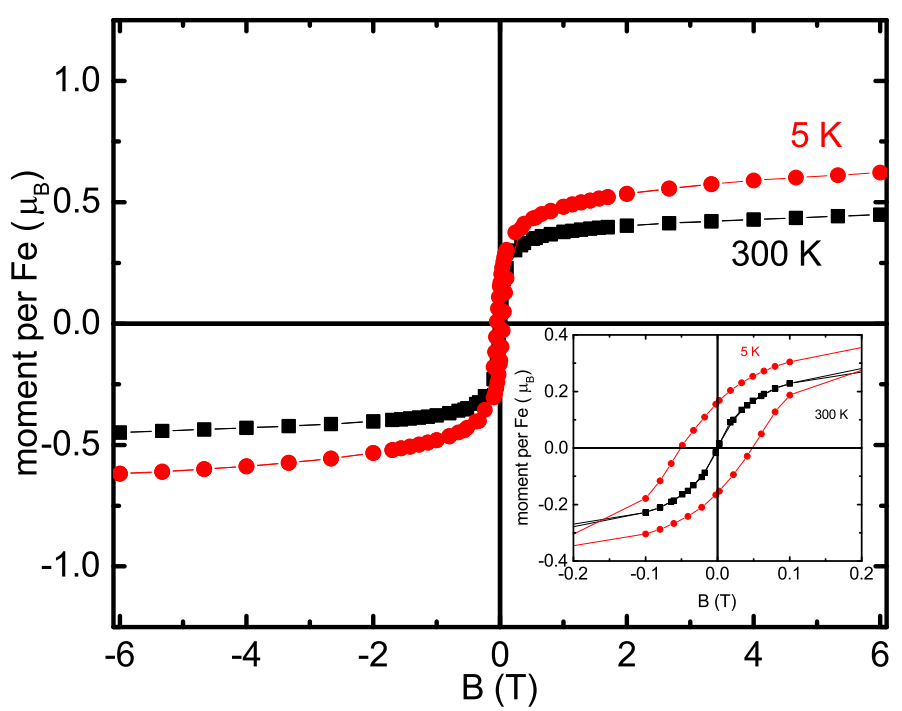

Figure 4.7.3: Magnetic moment at $300 \mathrm{~K}$ and $5 \mathrm{~K}$ of $\mathrm{BiFeO}_{3}$ annealed at $600{ }^{\circ} \mathrm{C}$ for $15 \mathrm{~min}$.

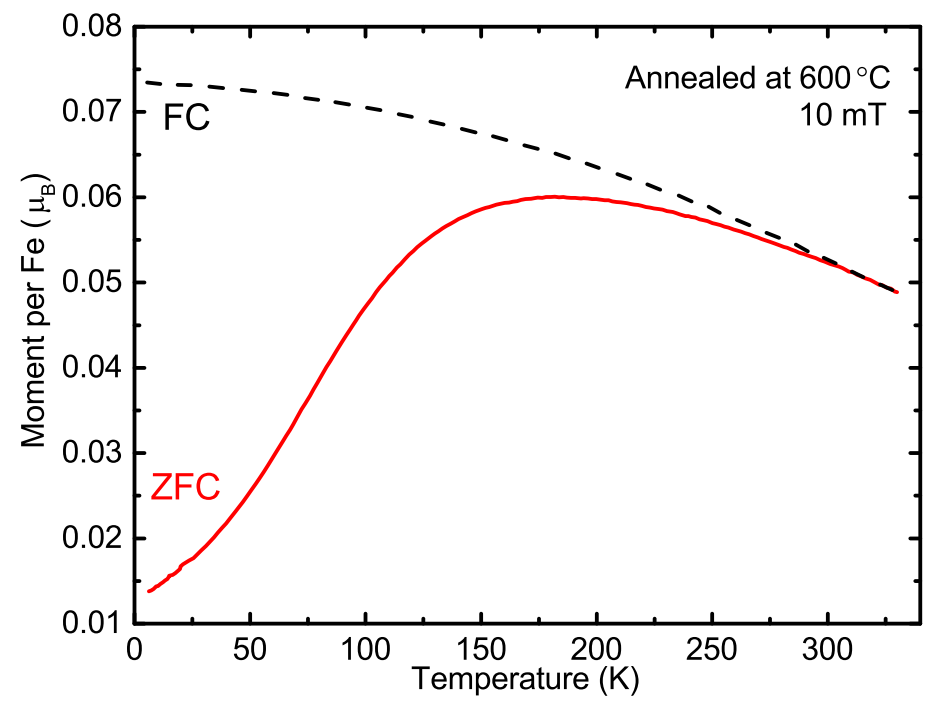

Figure 4.7.4: Plot of the ZFC (solid curve) and FC (dashed curve) magnetisation at $10 \mathrm{mT}$ for the $600^{\circ} \mathrm{C}$ annealed film.

The zero-field-cooled, field-cooled (ZFC-FC), Figure 4.7.4, displays the comparison ZFC and FC after annealed the film at $600{ }^{\circ} \mathrm{C}$. The hysteresis seems to close near $260{ }^{\circ} \mathrm{C}$, this is a lower temperature compared to the blocking temperature of the of the film annealed at $500{ }^{\circ} \mathrm{C}$. The blocking temperature of the film annealed at $500{ }^{\circ} \mathrm{C}$ is not as well define as the blocking temperature of the film annealed at $600{ }^{\circ} \mathrm{C}$. If the anisotropy constant is assumed constant between the two annealed $\left(600{ }^{\circ} \mathrm{C}\right.$, and $500{ }^{\circ} \mathrm{C}$ ) films, this would suggest that the grain size of the particles in both films are responsible for the modification of the blocking temperature. The Figure 4.7.4 has a more define ZFC transition, this could also indicate a size distribution less variable after annealing at $600{ }^{\circ} \mathrm{C}$, in other words, the film seems more uniform. 


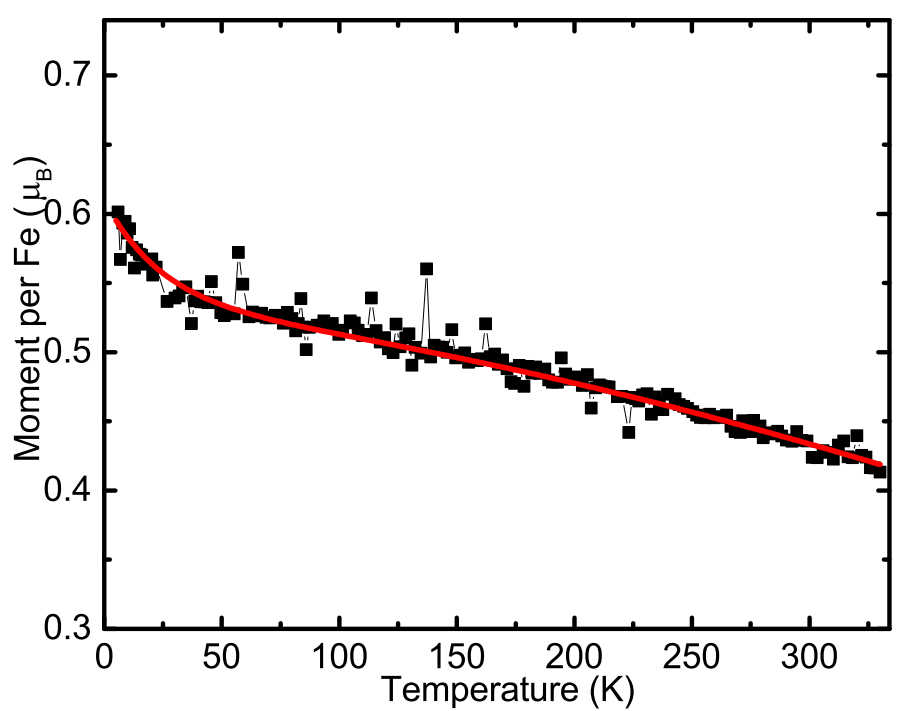

Figure 4.7.5: Plot of the normalised saturation moment per Fe against temperature at $6 \mathrm{~T}$.

The temperature dependence of $\mathrm{m}_{\mathrm{Fe}}$ at $6 \mathrm{~T}, \mathrm{~m}_{\mathrm{Fe}}(6 \mathrm{~T})$, is plotted in the Figure 4.7.5. The magnetic temperature dependence is fitted with the equation 3.3.22 with an additional term to compensate for the spin glass phenomenological [Prakash et al., 2016b]. Equation 3.3.22 can be written as equation 4.7.1 [Prakash et al., 2016b]:

$$
m_{i}(T)=m_{c}(0)\left[1-D T^{3 / 2}\right]+m_{s}(0) \exp ^{-T / T_{f}}
$$

Where $m_{i}$ is the magnetic moment of the ions, $m_{c}$ is the magnetic moment contribution from the core material, $m_{s}$ is the magnetic moment from the shell, $T_{f}$ is the spin freezing temperature and $D$ is spin stiffness and $T$ is the temperature [Prakash et al., 2016b]. In the Figure 4.7.5, the fit was achieved with $m_{c}=0.53 \mu_{B} / F e, D=$ $3.5 \times 10^{-5} \mathrm{~K}^{-3 / 2}, m_{s}=0.08 \mu_{B} / F e$ and $T_{f}=25 \mathrm{~K} . D$ is greater than the Fe bulk value of $3 \times 10^{-6} \mathrm{~K}^{-3 / 2}$ [Prakash et al., 2016a]. The magnetic data confirms the XRD analysis, increasing the annealing temperature up to $600{ }^{\circ} \mathrm{C}$ increase the formation of iron oxide.

\subsection{Thin film annealed at $500{ }^{\circ} \mathrm{C}$ for 7 hours}

The sample was annealed at $500{ }^{\circ} \mathrm{C}$ for 7 hours in an oxygen atmosphere; to compare the effect of different annealing times. The previous samples were annealed for $15 \mathrm{~min}-$ utes. The film was synthesized on $\mathrm{SiO}_{2}$ with an oxide layer about $\sim 500 \mathrm{~nm}$ thick; on silicon bulk. The RBS, Figure 4.8.1, shows backscattering particles of bismuth, iron and oxygen and the surface edge of silicon from the substrate. RUMP fit is achieved by 
assuming a material with about 11 layers, the details are presented in Section B.1. The surface layer, $1.75 \times 10^{17}$ at.cm ${ }^{-2}$, is composed of $\mathrm{Bi}_{1}, \mathrm{Fe}_{1}$ and $\mathrm{O}_{3}$. That stoichiometry suggests $\mathrm{BiFeO}_{3}$ could be present, further analysis of phases by XRD and the magnetic moment will be discussed and suggest that $\mathrm{BiFeO}_{3}$ could be present, however secondary phases are present in greater quantity. The density of that layer was estimated with SRIM software at $4.22 \times 10^{22}$ at.cm ${ }^{-3}$ [Ziegler, 1998]. The thickness of the surface layer is $160 \mathrm{~nm}$. The second layer, $1 \times 10^{17}$ at.cm ${ }^{-2}$, is compose of $\mathrm{Bi}_{1}, \mathrm{Fe}_{1}$ and $\mathrm{O}_{2.7}$. This could also have $\mathrm{BiFeO}_{3}$ or a phase with oxygen deficiency. The density is $4.21 \times 10^{22}$ at. $\mathrm{cm}^{-3}$, a thickness of $24 \mathrm{~nm}$. The second layer contains a deficiency of iron, $\mathrm{Bi}_{1}, \mathrm{Fe}_{0.8}$ and $\mathrm{O}_{2.7}$, this could suggest a possible bismuth oxide phase, details are presented in Section B.1. Layer 5 displays bismuth diffusion into $\mathrm{SiO}_{2}, \mathrm{Bi}_{0.08}$. The density estimated with SRIM is $4.67 \times 10^{22}$ at. $\mathrm{cm}^{-3}$ and the thickness of that layer is $86 \mathrm{~nm}$. As for the RBS analysis of the sample annealed at $600{ }^{\circ} \mathrm{C}$, the Figure 4.8.1 shows a possible diffusion of bismuth through the $\mathrm{SiO}_{2}$ layer and the bulk $\mathrm{Si}$, both diffusion have been reported in the literature [Büngener et al., 2003; Fan et al., 1990]. There are no other elements visible, which is confirmed by the qualitative PIXE analysis.

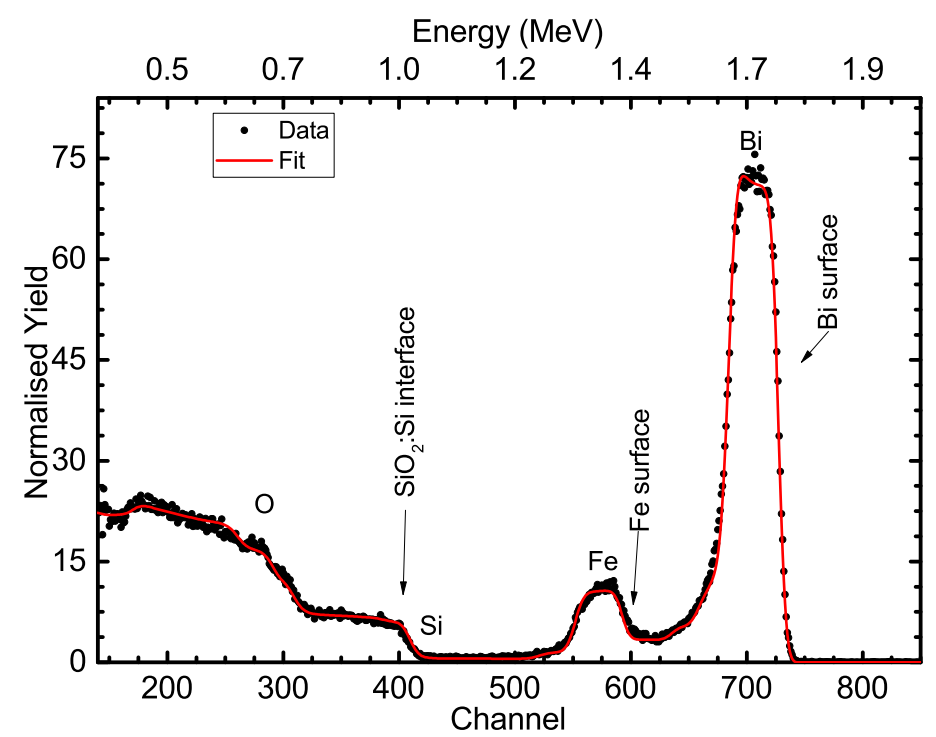

Figure 4.8.1: RBS spectra for the film annealed at $500{ }^{\circ} \mathrm{C}$ for 7 hours (circle) with the RUMP fit (line).

The XRD spectrograph, Figure 4.8.2, displays the presence of $\mathrm{BiFeO}_{3}$ and possibly other amorphous phases such as $\mathrm{Bi}_{2} \mathrm{Fe}_{4} \mathrm{O}_{9}$ and $\mathrm{Bi}_{2} \mathrm{O}_{3}$. Interestingly, there is no trace of bismuth, $\mathrm{Fe}_{3} \mathrm{O}_{4}, \alpha-\mathrm{Fe}_{2} \mathrm{O}_{3}$ or $\gamma-\mathrm{Fe}_{2} \mathrm{O}_{3}$. This means that all of the bismuth particles from the as-made film are either evaporated or have undergone a phase transition. The iron oxides could be amorphous, but that is less likely as the sample annealed at $500{ }^{\circ} \mathrm{C}$ for 15 min shows the presence of magnetic enhancement from the oxides. Iron is likely to be in $\mathrm{BiFeO}_{3}$ and $\mathrm{Bi}_{2} \mathrm{Fe}_{4} \mathrm{O}_{9}$ phases, however some small iron oxides particles might still be present. The magnetic data quantify the iron oxides concentration in the sample. 


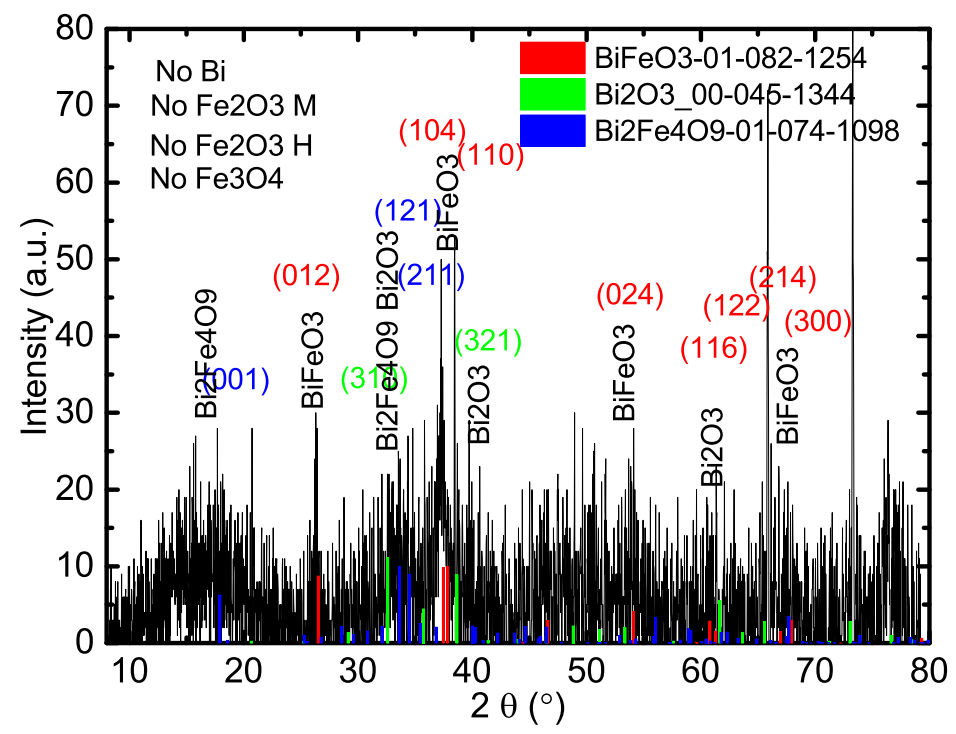

Figure 4.8.2: $\mathrm{XRD}$, grazing angle $2^{\circ}$, from film annealed at $500{ }^{\circ} \mathrm{C}$ for 7 hours.

The increase level of noise in the XRD data, Figure 4.8.2 compared to Figure 4.3.2, might arise from the sample size. The first set of sample were bigger than the latter annealed sample, that could decrease the diffraction or there might be less $\mathrm{BiFeO}_{3}$ in that sample than there is in the sample annealed at $500{ }^{\circ} \mathrm{C}$ for $15 \mathrm{~min}$. The longer annealing might have decomposed or evaporate an amount of $\mathrm{BiFeO}_{3}$.

The field loop at $5 \mathrm{~K}$ and $300 \mathrm{~K}$ has a similar magnetic saturation moment (Figure 4.8.3): at $300 \mathrm{~K}, m_{s}$ is $0.097 \mu_{B} / F e$ and at $5 \mathrm{~K}, m_{s}$ is $0.15 \mu_{B} / F e$. These values are within the range of experimental uncertainty. There is no significant magnetic moment enhancement with the temperature of measurement. The magnetic moment at saturation, $6 \mathrm{~T}$, displays the same behaviour. Comparing the field loop and the temperature dependence at saturation, the value of the saturation magnetic moment is the same at both ambient temperature and low temperature. This could indicate the absence of a spin disorder interaction [Haas, 1968]. The inset shows a hysteresis loop at low temperature that disappeared at ambient temperature. This effect can be explained by a superparamagnetic material [Chen et al., 1995]. The magnetic moment at saturation at $5 \mathrm{~K}$ is about $\sim 0.15 \mu_{B} / \mathrm{Fe}$. Knowing the magnetic moment of $\mathrm{BiFeO}_{3}$ and iron oxide (subsection 4.4.1), if neglecting the presence of weak magnetic secondary phase, the concentration of the magnetic iron is in $\mathrm{BiFeO}_{3}$ can be estimate between $78 \%$ to $80 \%$. The XRD and magnetic data do not show any trace of iron oxide. $\alpha-\mathrm{Fe}_{2} \mathrm{O}_{3}$ can still be present, however that phase cannot be identified because hematite is not magnetic and the form factor of bismuth could hide the iron oxide in the XRD analysis.

Looking at the inset of Figure 4.8.3, no exchange bias is visible at low temperature. This could mean that there is only one main magnetic domain order. The other domain 
could be too small to create an exchange bias. To draw any conclusions on this subject, further work would be required, i.e. performing the exchange bias analysis at several low temperatures like this study was done on the $\mathrm{BiFeO}_{3}$ sample annealed at $500{ }^{\circ} \mathrm{C}$ for $15 \mathrm{~min}$.

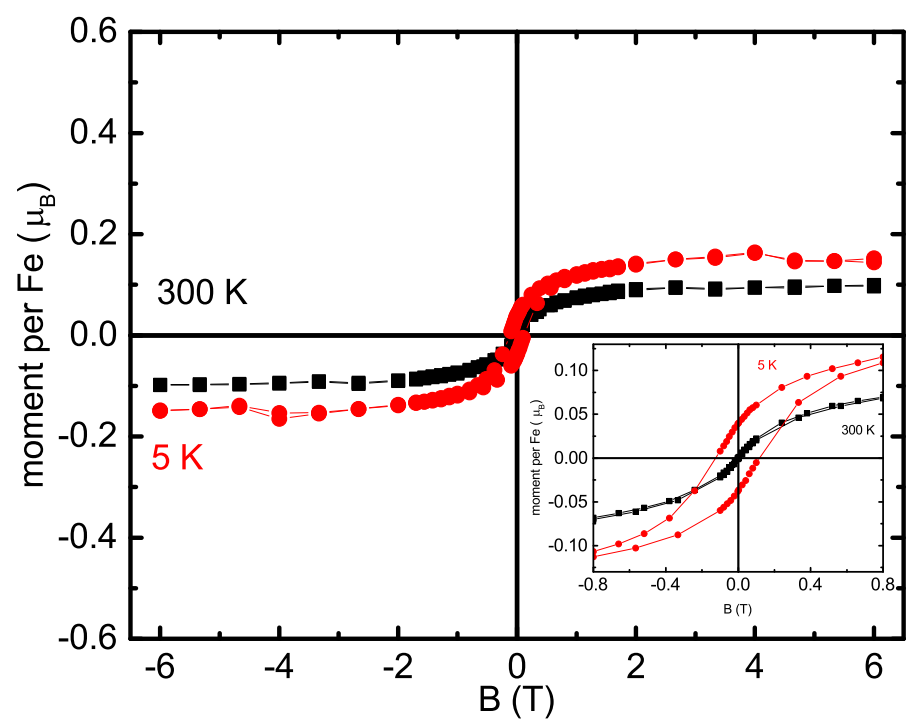

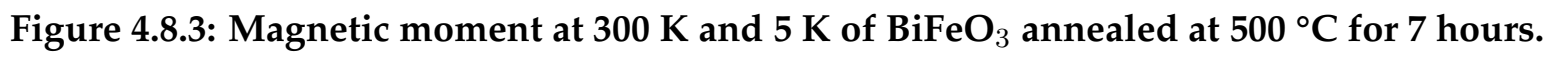

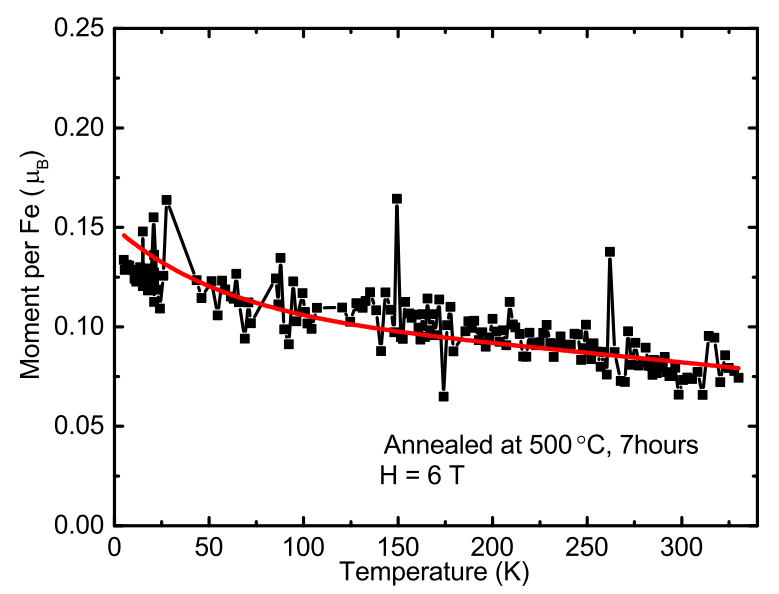

Figure 4.8.4: Plot of the normalised saturation moment per Fe versus temperature at $6 \mathrm{~T}$ of the sample annealed at $500{ }^{\circ} \mathrm{C}$ for 7 hours.

The temperature dependence at $6 \mathrm{~T}$, Figure 4.8.4, provides a limited information caused by the important noise level in the data. The magnetic moment is not significantly enhanced with temperature. In the Figure 4.8.4, the fit was achieved with the equation 4.7 .1 with value of $m_{c}=0.1 \mu_{B / F e}, D=3.5 \times 10^{-5} \mathrm{~K}^{-3 / 2}, m_{s}=0.05 \mu_{B} / F e$ and $T_{f}=60 \mathrm{~K} . D$ is greater than the Fe bulk value of $3 \times 10^{-6} \mathrm{~K}^{-3 / 2}$ [Prakash et al., 2016a], however the $D$ value extrapolated from the sample annealed at $500{ }^{\circ} \mathrm{C}$ for 7 hours is comparable to the $D$ value from the sample annealed at $600{ }^{\circ} \mathrm{C}$ for $15 \mathrm{~min}$. The freezing temperature is $60 \mathrm{~K}$ compared to $25 \mathrm{~K}$ with the film annealed at $600{ }^{\circ} \mathrm{C}$, the noise issue makes a direct comparison between the two samples difficult. Both $m_{s}$ and $m_{c}$ are 
smaller in the sample annealed for 7 hours compare to the sample annealed at $600{ }^{\circ} \mathrm{C}$, the noise level could explain the different value of $m_{c}$ but not the value of $m_{s}$. The reduce magnetic moment would agree with the XRD analysis displaying more $\mathrm{BiFe}_{x} \mathrm{O}_{y}$ phases and less iron oxide phases.

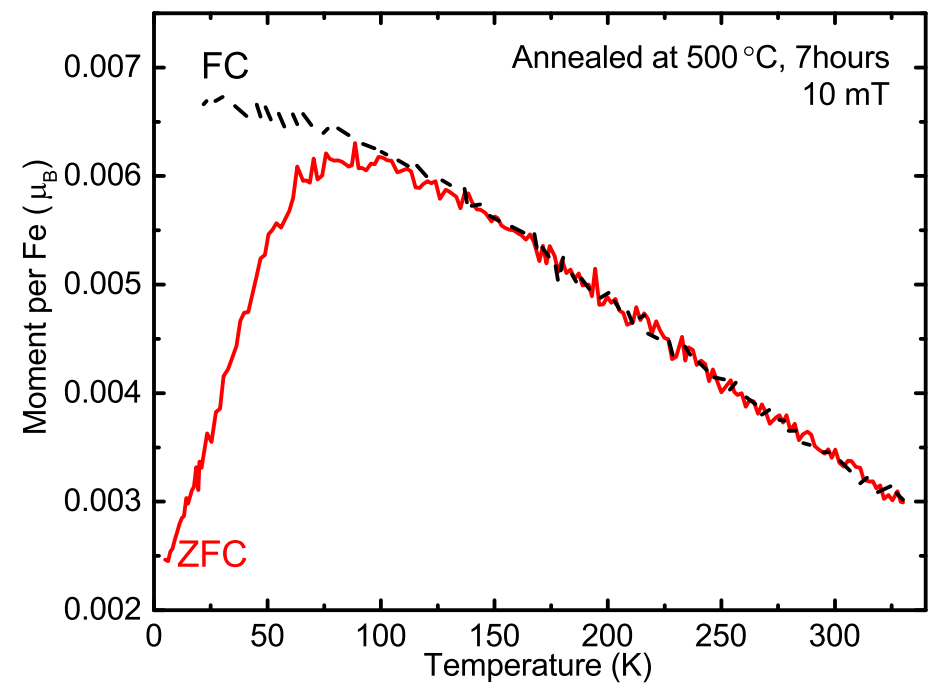

Figure 4.8.5: ZFC (solid curve) and FC (dashed curve) magnetisation at $10 \mathrm{mT}$ for the film annealed at $500{ }^{\circ} \mathrm{C}, 7$ hours. Blocking temperature is between $70 \mathrm{~K}$ and $110 \mathrm{~K}$.

The ZFC-FC, Figure 4.8.5, displays a narrow range of magnetic domain size. The ZFC and FC merge perfectly between $100 \mathrm{~K}$ and $150 \mathrm{~K}$. If the only magnetic material in the sample is assumed to be $\mathrm{BiFeO}_{3}$, then from the blocking temperature equation 2.1.6, the grain size can be estimated to be between $\sim 5 \mathrm{~nm}$ and $\sim 9 \mathrm{~nm}$. The anisotropy constant for the calculation is $5.6 \times 10^{4} \mathrm{~J} \mathrm{~m}^{-3}$ [Antic et al., 2013]. From the Figure 4.8.5, the presence of $\mathrm{Bi}_{2} \mathrm{Fe}_{4} \mathrm{O}_{9}$ must be limited as $\mathrm{Bi}_{2} \mathrm{Fe}_{4} \mathrm{O}_{9}$ is an antiferromagnet with a Néel temperature between 235 and 250 K [Ressouche et al., 2009; Du et al., 2011].

\subsection{Summary: Annealing effects}

This section regroups data from the $\mathrm{BiFeO}_{3}$ deposited film to compare the effects, compositions and magnetic moment, of annealing in an oxygen atmosphere at different temperatures. The annealing leads to the formation of $\mathrm{BiFeO}_{3}$. The annealing time effects on the thin film are also discussed in this section.

Figure 4.9.1 displays the crystallographic modifications occurring under different annealing temperature, leading to the formation of $\mathrm{BiFeO}_{3}$ when annealed at $500{ }^{\circ} \mathrm{C}$. The as-made film displays $\mathrm{Bi}$ phase and possibly $\mathrm{Bi}_{2} \mathrm{O}_{3}$ or $\mathrm{BiFe}_{x} \mathrm{O}_{y}$ phases. Due to the 

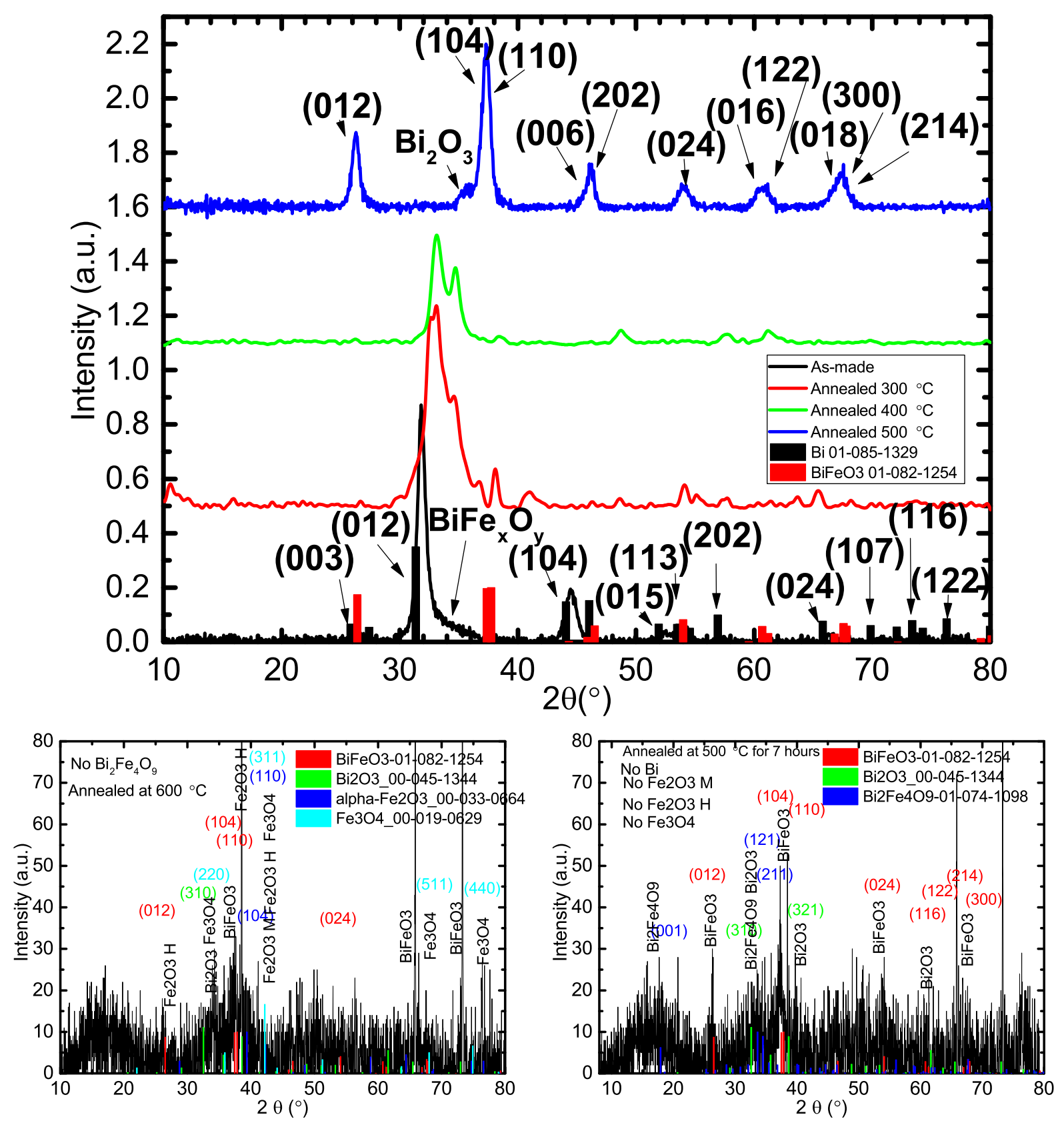

Figure 4.9.1: Crystallographic changes, grazing angle $2^{\circ}$, after 15 min oxygen-annealing at different temperatures leading to $\mathrm{BiFeO}_{3}$. XRD experimental data (lines) and peak position and intensity of the main features of various $\mathrm{Bi}_{x} \mathrm{Fe}_{y} \mathrm{O}_{z}$ compounds (bars). 
small particles of those possible phases, XRD does not allow to clearly identify those phases. To obtain the required phase of $\mathrm{BiFeO}_{3}$, the sample was annealed at intermediate temperature of $200{ }^{\circ} \mathrm{C}, 300{ }^{\circ} \mathrm{C}$ and $400{ }^{\circ} \mathrm{C}$, however the $\mathrm{BiFeO}_{3}$ rhombohedral phase forms only after annealing the sample at $500{ }^{\circ} \mathrm{C}$ as shown in Figure 4.9.1. Crystallography analysis of the intermediary temperature displays iron oxides and bismuth oxides phases. As mentioned in subsection 4.3.2, the as-made film has iron oxides phases that are assumed to be amorphous. The figure 4.9.1 shows those amorphous phases becoming nanostructured while annealing the thin film at intermediary temperature. This figure shows that only when annealed at $500{ }^{\circ} \mathrm{C}, \mathrm{BiFeO}_{3}$ forms and is nanostructured. Annealing at $600{ }^{\circ} \mathrm{C}$ or for 7 hours results in the loss of the $\mathrm{BiFeO}_{3}$ phase. This suggest the optimal annealing conditions are at $500{ }^{\circ} \mathrm{C}$, for $15 \mathrm{~min}$ in an oxygen atmosphere.

A significant signal to noise ratio difference is seen in the XRD between the sample annealed at $500{ }^{\circ} \mathrm{C}$ for $15 \mathrm{~min}$ and the samples annealed at $600{ }^{\circ} \mathrm{C}$ for $15 \mathrm{~min}$ and at $500{ }^{\circ} \mathrm{C}$ for 7 hours. The difference is explained by the different phases present and the loss of nanostructure. In the case of the film annealed at $500{ }^{\circ} \mathrm{C}$ for $15 \mathrm{~min}$, the film is mainly composed of $\mathrm{BiFeO}_{3}$, magnetite and maghemite. In the other two materials, a mix of phases of iron oxide, bismuth oxide, $\mathrm{Bi}_{2} \mathrm{Fe}_{4} \mathrm{O}_{9}$ are present. The RBS analysis provides another important information to explain the difference: the film annealed at $500{ }^{\circ} \mathrm{C}$ for $15 \mathrm{~min}$ is fitted with less than 6 layers with the $\mathrm{BiFeO}_{3}$ layer divided in two sublayers of about $160 \mathrm{~nm}$ and the other sublayer is about $50 \mathrm{~nm}$. The other two films, as describe by the RBS analysis, are compose of more than 10 layers with 2 or $3 \mathrm{BiFeO}_{3}$ sublayers. The film annealed for a longer time shows the presence of more phases in the XRD which agrees with the RBS.

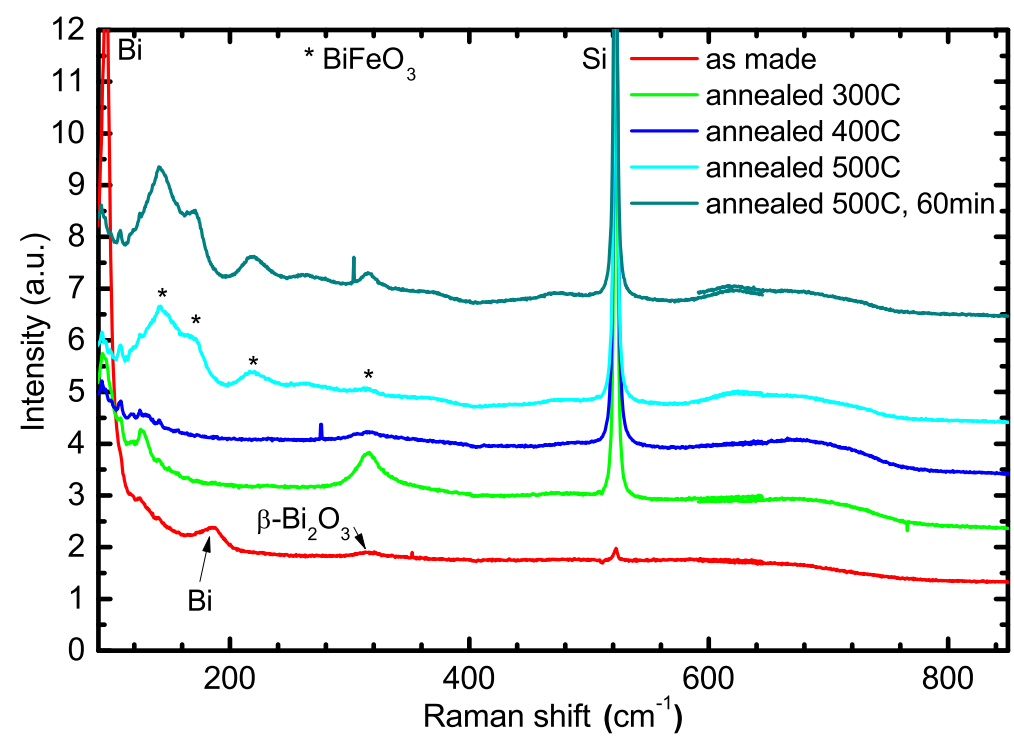

Figure 4.9.2: Raman shift changes after oxygen-annealing at different temperatures leading to $\mathrm{BiFeO}_{3}$. The annealing was carried over $15 \mathrm{~min}$ and $60 \mathrm{~min}$ (dark cyan). 
Figure 4.9.2 displays the Raman shift modifications occurring under different annealing temperatures, leading to the formation of $\mathrm{BiFeO}_{3}$ when annealed at $500{ }^{\circ} \mathrm{C}$. Like the XRD analysis the as-made film displays a Bi phase and possibly a $\mathrm{Bi}_{2} \mathrm{O}_{3}$ phase. The films annealed at intermediate temperature are showing an intermediate composition between the bismuth phase of the as-made film and the $\mathrm{BiFeO}_{3}$ phase of the film annealed at $500{ }^{\circ} \mathrm{C}$. The Raman shift above $650 \mathrm{~cm}^{-1}$ displays a secondary phase of iron oxide present in every film annealed and presented in the Figure 4.9.2. After annealing the sample for a longer time period, some $\mathrm{BiFeO}_{3}$ Raman modes are active, however the XRD analysis shows that other phases form. Those phases could be difficult to see in Raman spectroscopy when carried at ambient temperature. The thermal energy, ambient temperature, explains the study of $\mathrm{BiFeO}_{3}$ thin film below $90 \mathrm{~K}$ in the subsection 4.3.5. In the Figure 4.3.11 (b) 11 of the 13 Raman active modes of $\mathrm{BiFeO}_{3}$ are seen while at ambient temperature, in Figure 4.9.2, only 4 Raman mode of $\mathrm{BiFeO}_{3}$ are seen. The conclusion drawn from the Raman analysis is similar to the XRD analysis, the optimal annealing conditions are at $500{ }^{\circ} \mathrm{C}$ in an oxygen atmosphere.

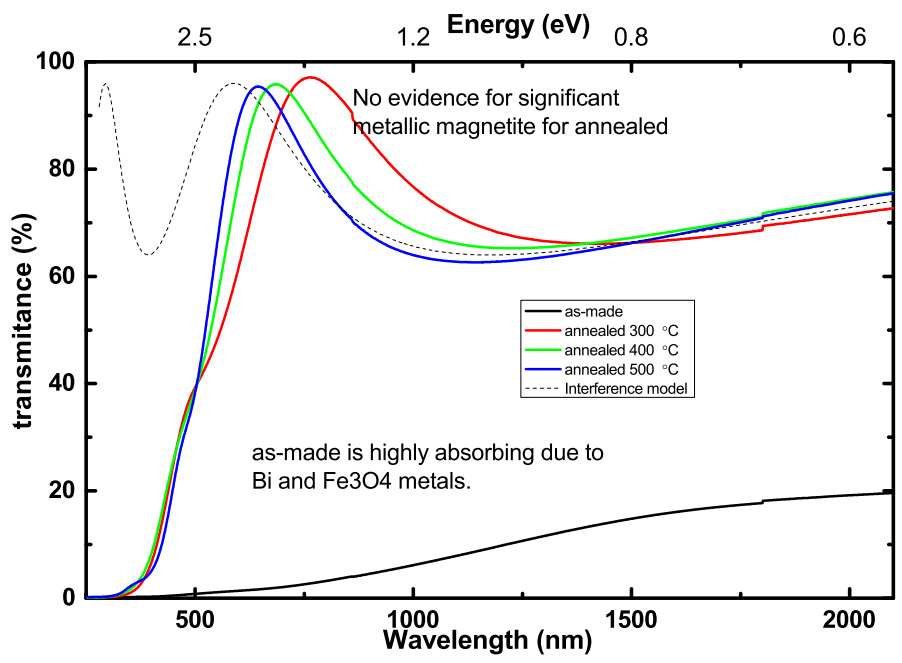

Figure 4.9.3: Optical transmission after 15 min oxygen-annealing at different temperatures. Dash line shows the light interference pattern fitted on the sample annealed at $500{ }^{\circ} \mathrm{C} . n_{1}, n_{2}$ and $n_{3}$ are the refractive index of air, the thin film and quartz (substrate). $d$ is the thickness of the film in $\mathrm{nm}$.

Table 4.9.1: Interference model parameter for samples annealed at temperature up to $500{ }^{\circ} \mathrm{C}$.

\begin{tabular}{|l|l|l|l|l|l|}
\hline Annealing & $\boldsymbol{n}_{\mathbf{1}}$ & $\boldsymbol{n}_{\mathbf{2}}$ & $\boldsymbol{n}_{\mathbf{3}}$ & $\begin{array}{l}\text { Thickness } \\
{[\mathbf{n m}]}\end{array}$ & $\begin{array}{l}\text { Band gap } \\
{[\mathbf{e V}]}\end{array}$ \\
\hline as-made & n.a. & n.a. & n.a. & n.a. & 2.25 \\
\hline $\mathbf{3 0 0}{ }^{\circ} \mathbf{C}$ & 1 & 2.37 & 1.5 & 155 & 2.4 \\
\hline $\mathbf{4 0 0}{ }^{\circ} \mathbf{C}$ & 1 & 2.40 & 1.5 & 135 & 2.55 \\
\hline $\mathbf{5 0 0}{ }^{\circ} \mathbf{C}$ & 1 & 2.45 & 1.5 & 120 & 2.75 \\
\hline
\end{tabular}


The as-made thin film significant light absorption, more than $80 \%$, could be explain by the composition of the film; mix of iron oxide and bismuth ferrite phases. This film contains metallic material with a great light absorption coefficient. This agrees with the XRD analysis in subsection 4.3.2. After annealing the thin films, nanostructures are form as discussed in subsection 4.3.2 (XRD analysis). The annealed films contain less metallic material and more semi-conductor materials (e.g. $\mathrm{Bi}_{2} \mathrm{O}_{3}$, hematite) which have smaller light absorption coefficient. After annealing at $500{ }^{\circ} \mathrm{C}$, the thin film is mainly composed of $\mathrm{BiFeO}_{3}$. While the films annealed at $300{ }^{\circ} \mathrm{C}$ and $400{ }^{\circ} \mathrm{C}$ have an important quantity of iron oxide and bismuth oxide. Those mix phases modify the refractive index as shown in Figure 4.9.3 and they also modify the thickness of the film and thus the interference pattern is modified. The interference was fitted with equation 4.3.1. The refractive index, thickness and optical band gap of samples annealed at $300{ }^{\circ} \mathrm{C}, 400{ }^{\circ} \mathrm{C}$ and $500{ }^{\circ} \mathrm{C}$ are presented in Table 4.9.1. The refractive indexes after annealing suggests a phase transition toward $\mathrm{BiFeO}_{3}$. The absorbance of the film is derived from the transmittance. The optical band gap of those films was estimated with the Tauc plot method. The band gap value is $2.25 \mathrm{eV}$ for the as-made sample. The optical band gap analysis also shows a transition leading to the formation of $\mathrm{BiFeO}_{3}$ through different annealing temperature. As the band gap of bismuth oxide and iron oxide are close to $\mathrm{BiFeO}_{3}$, the phases could be determined by other characteristic techniques like XRD and Raman spectroscopy.

When comparing the magnetic moment at $6 \mathrm{~T}$ with the expected magnetic moment of $0.02 \mu_{B} / \mathrm{Fe}$ for $\mathrm{BiFeO}_{3}$, the measured magnetic moment displays an enhance magnetic moment cause by iron oxide. The as-made film contains a significant amount of iron oxide as described by TEM and this is now confirmed with magnetic measurements. Increasing the annealing temperature to $500{ }^{\circ} \mathrm{C}$ reduces the magnetic moment at $6 \mathrm{~T}$. This suggests an oxidation of the magnetic iron oxide phases to form hematite phase and $\mathrm{BiFeO}_{3}$. Annealing the sample beyond $500{ }^{\circ} \mathrm{C}$ seems to increase the magnetic moment, suggesting an increasing amount of iron oxide phases form from the decomposition of $\mathrm{BiFeO}_{3}$. The decomposition of $\mathrm{BiFeO}_{3}$ that was synthesised by chemical deposition has been reported after annealing at temperature around $880 \mathrm{~K}\left(\sim 600{ }^{\circ} \mathrm{C}\right)$ [Rojac et al., 2014]. XRD analysis displays an increase of iron oxides phases as suggested by the magnetic moment analysis.

The inset of Figure 4.9.4 displays a magnified comparison of the annealed sample measured at $5 \mathrm{~K}$. The magnetisation curves at $5 \mathrm{~K}$ for the annealed samples are similar to the magnetisation curve of the as-made film, suggesting a common magnetic phase is present in the thin films, that phase could be a mix of magnetite and maghemite. As discuss in the subsection 4.4.1, the comparison of the as-made film with the annealed 
film at $500{ }^{\circ} \mathrm{C}$ shows a reduction of about $60 \%$ of the magnetic moment, this suggest some $\mathrm{Fe}$ in magnetite and maghemite phase bond to form $\mathrm{BiFeO}_{3}$ after annealing.

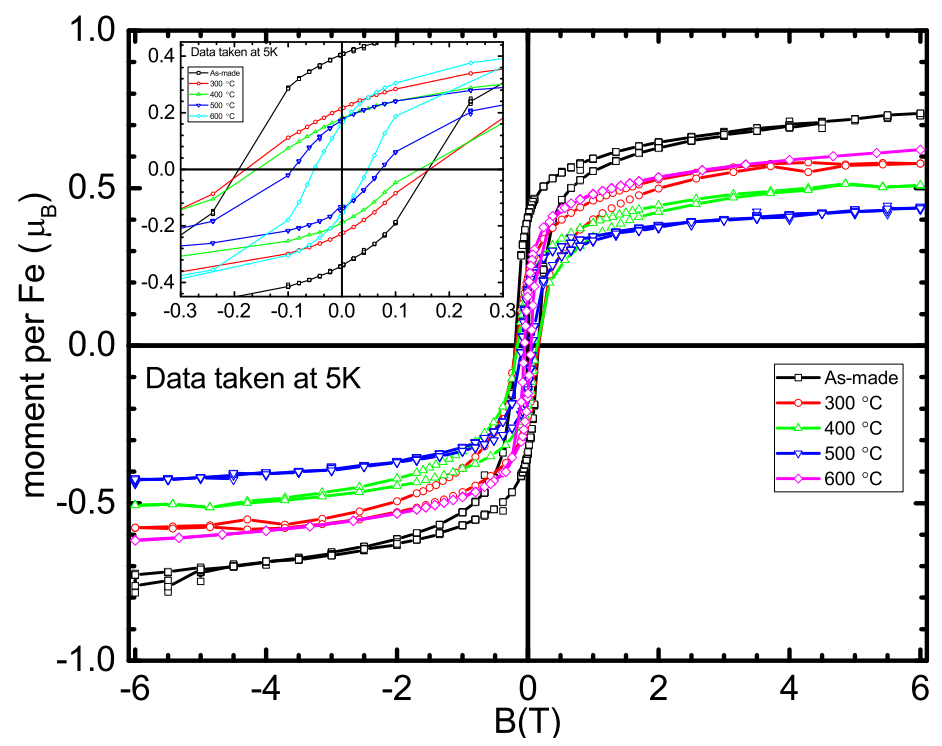

Figure 4.9.4: Magnetic moment per Fe, after 15 min oxygen-annealing at temperatures up to $600{ }^{\circ} \mathrm{C}$, measured at $5 \mathrm{~K}$. Inset: shows the magnetic moment per Fe between $-0.3 \mathrm{~T}$ and $0.3 \mathrm{~T}$.

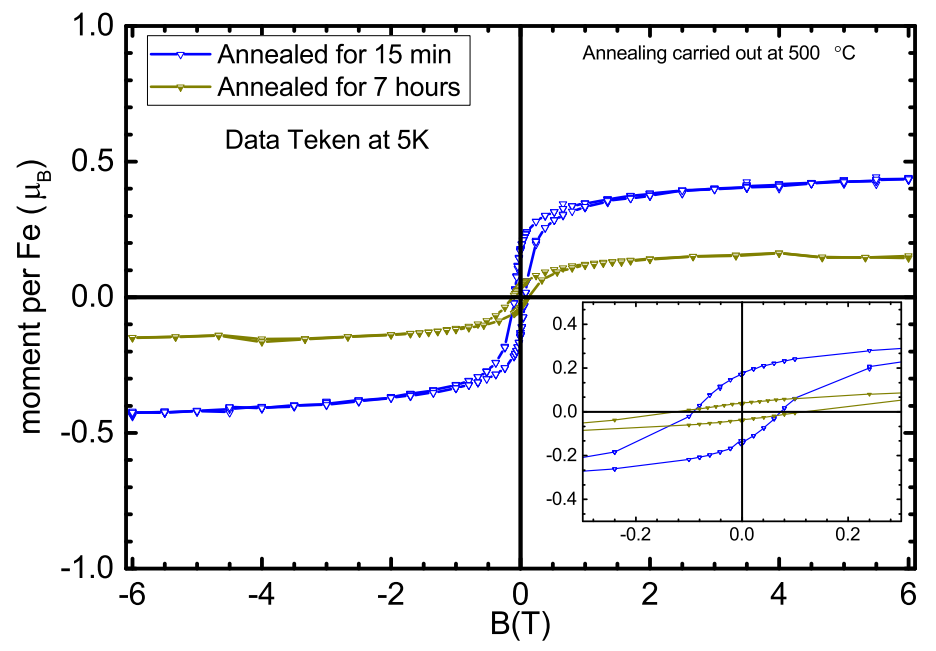

Figure 4.9.5: Magnetic moment per Fe, after oxygen-annealing at $500{ }^{\circ} \mathrm{C}$ for $15 \mathrm{~min}$ (blue open triangle) and 7 hours (green half fill triangle), measured at $5 \mathrm{~K}$. Inset: shows the magnetic moment per Fe between $-0.3 \mathrm{~T}$ and $0.3 \mathrm{~T}$.

Figure 4.9.5 provides a clear comparison between annealing thin film at $500{ }^{\circ} \mathrm{C}$ for $15 \mathrm{~min}$ and for 7 hours. The annealing time of 7 hours was for practical reasons the longest annealing time possible. Increasing the annealing time reduces the magnetic moment at $6 \mathrm{~T}$. The reduce time also reduces the magnetic hysteresis loop at low temperature, $5 \mathrm{~K}$. The reduced magnetic moment suggests more iron oxide phase has been transformed into other phases like $\mathrm{BiFeO}_{3}$ and $\mathrm{Bi}_{2} \mathrm{Fe}_{4} \mathrm{O}_{9}$ (from XRD analysis those are 
the phases that are forming). Bismuth oxide is also seen by XRD analysis and is a phase that reduces the magnetic moment. Although this analysis does not find any trace of hematite or $\mathrm{Bi}_{25} \mathrm{FeO}_{39}$, those two phases are theoretically present in the chemical synthesis of $\mathrm{BiFeO}_{3}$, subsection 3.2.3. Both phases are likely to be present, in small quantity or in an amorphous form, in this film and thus reducing the magnetic moment. The oxidation of magnetite and maghemite results in hematite [Cullity and Graham, 2009] which has a small magnetic moment. The inset displaying the magnetic moment between $-0.3 \mathrm{~T}$ and $0.3 \mathrm{~T}$, displays the magnetic hysteresis is smaller after annealing the sample for 7 hours, which suggest a smaller ferro/ferri-magnetic phase in the film, this confirms the reduction of magnetite and maghemite in the film.

Table 4.9.2: Summary: Annealing effects on sputtered thin film.

\begin{tabular}{|c|c|c|c|c|c|c|}
\hline Annealed & As-made & $300^{\circ} \mathrm{C}$ & $400^{\circ} \mathrm{C}$ & $500^{\circ} \mathrm{C}$ & $600^{\circ} \mathrm{C}$ & $\begin{array}{c}500{ }^{\circ} \mathrm{C} \\
7 \mathrm{~h}\end{array}$ \\
\hline $\mathrm{BiFeO}_{3}$ & Not seen & Not seen & $\begin{array}{l}\text { Could be } \\
\text { forming }\end{array}$ & R3c & $\begin{array}{c}\text { Could be } \\
\text { present }\end{array}$ & $\mathrm{R} 3 \mathrm{c}$ \\
\hline $\begin{array}{l}\text { Secondary } \\
\text { phase }\end{array}$ & $\begin{array}{c}\mathrm{Bi}, \mathrm{Bi}_{2} \mathrm{O}_{3}, \\
\mathrm{Fe}_{x} \mathrm{O}_{y}\end{array}$ & $\begin{array}{c}\mathrm{Bi}, \mathrm{Bi}_{2} \mathrm{O}_{3}, \\
\mathrm{Fe}_{x} \mathrm{O}_{y}\end{array}$ & $\mathrm{Fe}_{x} \mathrm{O}_{y}$ & $\mathrm{Fe}_{x} \mathrm{O}_{y}$ & $\mathrm{Fe}_{x} \mathrm{O}_{y}$ & $\begin{array}{c}\mathrm{Fe}_{x} \mathrm{O}_{y} \\
\mathrm{Bi}_{2} \mathrm{O}_{3} \\
\mathrm{Bi}_{2} \mathrm{Fe}_{4} \mathrm{O}_{9}\end{array}$ \\
\hline Structure & Nano & Nano & Nano & Nano & $\begin{array}{c}\text { Amorphous } \\
\text { phases } \\
\text { could be } \\
\text { present }\end{array}$ & $\begin{array}{c}\text { Amorphous } \\
\text { phases } \\
\text { could be } \\
\text { present }\end{array}$ \\
\hline $\begin{array}{l}\text { Electric } \\
\text { ordering }\end{array}$ & No order & n.a. & n.a. & Ferro & n.a. & n.a. \\
\hline $\begin{array}{l}\text { Magnetic } \\
\text { moment }\end{array}$ & $<0.73 \mu_{B}$ & $<0.6 \mu_{B}$ & $<0.5 \mu_{B}$ & $<0.44 \mu_{B}$ & $<0.62 \mu_{B}$ & $\sim 0.097 \mu_{B}$ \\
\hline $\begin{array}{l}\text { Blocking } \\
\text { temperature }\end{array}$ & High $^{1}$ & $<115 \mathrm{~K}$ & $<140 \mathrm{~K}$ & $<160 \mathrm{~K}$ & $<260 \mathrm{~K}$ & $<150 \mathrm{~K}$ \\
\hline $\begin{array}{l}\text { Exchange } \\
\text { bias }\end{array}$ & $<30 \mathrm{~K}$ & n.a. & n.a. & $<250 \mathrm{~K}$ & n.a. & Not seen \\
\hline
\end{tabular}

The Table 4.9.2 highlights the main modifications the thin films undergo after being annealed in different conditions. This table shows that to form $\mathrm{BiFeO}_{3}$ phase an annealing at $500{ }^{\circ} \mathrm{C}$ must be carried on with an oxygen atmosphere. Annealing at higher temperature results in the decomposition of $\mathrm{BiFeO}_{3}$ and annealing for a long time reduces the ferro/ferri-magnetic phase without proof of reducing the concentration of secondary phases. For an application perspective, the secondary phases of the

\footnotetext{
${ }^{1}$ The high blocking temperature is likely caused by the range of particle size in the film.
} 
$\mathrm{BiFeO}_{3}$ thin film annealed at $500{ }^{\circ} \mathrm{C}$ for 15 min have more interesting magnetic properties then the secondary phases resulting from a longer annealing time. Also, both the film annealed at $600{ }^{\circ} \mathrm{C}$ and the film annealed for 7 hours resulted in the loss of nanostructure and thus reducing the potential for device applications.

\subsection{Conclusion}

In conclusion, multiferroic nanocrystalline $\mathrm{BiFeO}_{3}$ films have been made by ambient temperature sputtering and thermal annealing at $500{ }^{\circ} \mathrm{C}$ in an oxygen atmosphere. The magnetic properties are primarily due to a secondary phase of iron oxides that induced an exchange bias. The Ferroelectricity is confirmed by the piezoelectric response which also confirms the proper phase of $\mathrm{BiFeO}_{3}$ is formed. XRD measurements before annealing show the presence of nanocrystalline $\mathrm{Bi}$ and other unidentified $\mathrm{BiFe}_{x} \mathrm{O}_{y}$ phases. SAED shows that there is also some magnetite or maghemite as well as some $\mathrm{FeO}$. The Raman data are consistent with the presence of $\mathrm{Bi}$ and $\beta-\mathrm{Bi}_{2} \mathrm{O}_{3}$ as well as magnetite, maghemite, and hematite. Annealing in an oxygen atmosphere at $500{ }^{\circ} \mathrm{C}$ resulted in the formation of multiferroic $\mathrm{BiFeO}_{3}$ nanoparticles. Raman measurements showed that there was also magnetite, maghemite, and hematite. Magnetic measurements on an as-made film showed that there was a large moment per Fe at $6 \mathrm{~T}$. This is due to magnetite and maghemite where between $54 \%$ to $58 \%$ of the Fe is in these two phases. There was evidence for superparamagnetism from the two iron oxide phases. The $500{ }^{\circ} \mathrm{C}$ annealed film also showed a large moment per Fe at $6 \mathrm{~T}$. The magnetic moment was $60 \%$ of the value found before annealing because some of the Fe in the magnetite and maghemite appears in the $\mathrm{BiFeO}_{3}$ nanoparticles. Superparamagnetism also occurs with a slightly lower blocking temperature, and the temperature dependence of the high field moment per Fe is comparable to that found before annealing. The similarity of the magnetic properties before and after annealing shows that the magnetic data in the $500{ }^{\circ} \mathrm{C}$ annealed film is dominated by magnetite and maghemite and any contribution from nanocrystalline $\mathrm{BiFeO}_{3}$ is small. An exchange bias was observed before annealing, where $\left|B_{e x}\right|$ rapidly decreases with increasing temperature. The exchange bias is likely due to a spin-disordered region in the shell of the magnetite and maghemite nanoparticles. The exchange bias in the $500{ }^{\circ} \mathrm{C}$ annealed film is slightly lower at $5 \mathrm{~K}$. However, the annealed film displays an exchange bias up to at $250 \mathrm{~K}$ while the as-made film exchange bias is visible only below $30 \mathrm{~K}$. This can be due to different spin disorder regions in the shell where the spin freezing temperature extends up to higher temperatures and $\left|B_{e x}\right|$ disappears for those magnetite and maghemite 
nanoparticles with blocking temperatures below $250 \mathrm{~K}$. The Magneto-Optical Kerr Effect (MOKE) confirms the great optical properties seen in the optical measurement, the band gap, with one of the highest Verdet constants reported. This could suggest possible applications such as a magneto-optical memory device. MOKE and MCD analysis confirm the presence of a small ferromagnetic material contribution as expected from the iron oxide analysis carried out by XRD and Raman spectroscopy.

Increasing the annealing temperature above $500{ }^{\circ} \mathrm{C}$ increases the iron oxides impurities. The XRD analysis displays $\mathrm{Fe}_{3} \mathrm{O}_{4}, \alpha-\mathrm{Fe}_{2} \mathrm{O}_{3}$ and $\gamma-\mathrm{Fe}_{2} \mathrm{O}_{3}$ at the transition temperature below $400{ }^{\circ} \mathrm{C}$, which disappear at $500{ }^{\circ} \mathrm{C}$ and reappear above $500{ }^{\circ} \mathrm{C}$. The magnetic moment is enhanced by the magnetite and maghemite. The quantity of particles is less than that in the as-made thin film.

The effect of a longer annealing time on the film, at $500{ }^{\circ} \mathrm{C}$, seems to remove the iron oxide, however this increases the concentration of $\mathrm{Bi}_{2} \mathrm{Fe}_{4} \mathrm{O}_{9}$ and $\mathrm{Bi}_{2} \mathrm{O}_{3}$ secondary phases. XRD shows an increased quantity of $\mathrm{Bi}_{2} \mathrm{Fe}_{4} \mathrm{O}_{9}$ and $\mathrm{Bi}_{2} \mathrm{O}_{3}$. Both were negligible after any annealing condition. In the end, the magnetic moment mainly comes from $\mathrm{BiFeO}_{3}$. There is no magnetic enhancement visible; the superparamagnetic effect is visible. All of this seems to confirm that the iron oxides, $\mathrm{Fe}_{3} \mathrm{O}_{4}$ and $\gamma-\mathrm{Fe}_{2} \mathrm{O}_{3}$, are enabling or at least allowing the $\mathrm{BiFeO}_{3}$ to be multiferroic while enhancing the magnetic moment. $\mathrm{As} \mathrm{BiFeO}_{3}$ is an antiferromagnet, the magnetic ordering seen at $500{ }^{\circ} \mathrm{C}$ mainly comes from the iron oxide, while the ferroelectric behaviour comes from the $\mathrm{BiFeO}_{3}$, resulting in a multiferroic material.

Synthesising a pure single crystalline $\mathrm{BiFeO}_{3}$ thin film by ion beam sputtering is complex and requires further analysis to achieve that result. So far, no results displayed a single crystalline film. This could be caused by the secondary phases and the substrate. The lattice parameters should be studied as this influence the $\mathrm{BiFeO}_{3}$ crystal structure and was studied for other synthesising techniques. Those techniques generally require depositing $\mathrm{BiFeO}_{3}$ on a stack of layers. This process could be done by ion beam sputtering, or $\mathrm{BiFeO}_{3}$ could be deposited by ion beam sputtering on a stack. The issue is the uniformity of the deposition. The film as-made shows a mix of iron oxides and bismuth oxides, and the annealing provides enough mobility to the bismuth oxide to bond with iron oxide and even diffused in the $\mathrm{SiO}_{2}$ layer. This process is slightly random, as the exact deposition is not uniform. Annealing below $500{ }^{\circ} \mathrm{C}$ or above $500{ }^{\circ} \mathrm{C}$ enhance the formation iron oxides, and increasing the annealing time forms a secondary phase of $\mathrm{Bi}_{2} \mathrm{Fe}_{4} \mathrm{O}_{9}$.

Although, nano-structured $\mathrm{BiFeO}_{3}$ was successfully achieved. In future work, the 
optimisation of annealing temperature and time could improve the film quality. The iron oxide present in the film enhances the magnetic saturation moment, which is advantageous for building application devices. Sputtering $\mathrm{BiFeO}_{3}$ while the substrate is above ambient temperature should be explored as a parameter to optimise the $\mathrm{BiFeO}_{3}$ crystal synthesis. 


\section{Chapter 5}

\section{Bismuth implantation into iron oxides}

This chapter explores a new approach, bismuth implantation into iron oxide film, in order to search for modifications in iron oxide nanocrystals and the magnetic moment created by bismuth ions. This approach was also used to explore the possibility of synthesising $\mathrm{BiFeO}_{3}$ polycrystalline material by implanting iron oxide thin film with bismuth followed by annealing in an oxygen atmosphere. This technique requires sputtering iron oxides, $\mathrm{Fe}_{x} \mathrm{O}_{y}$, followed by ion implantation of bismuth and subsequent annealing in an oxygen atmosphere. This chapter focuses on the synthesis and structure of the resulting film before and after bismuth implantation. The magnetic moment ordering described with the SQUID provides more information on the mixedphase thin film obtained from this synthesis technique. The aim of this chapter is to study the resultant phases and determine the quantity of bismuth ferrite nanoparticles and other synthesized phases. Also, as of now, an ambient temperature exchange coupled system that is electrically tunable has not been demonstrated [Martin et al., 2007]. As the $\mathrm{BiFeO}_{3}$ thin film displays an exchange bias up to $250 \mathrm{~K}$, due to magnetite and maghemite, then bismuth implantation could enlighten us toward the synthesis of a heterojunction exchange bias.

The ion implantation synthesis technique has an advantage over other synthesis techniques like an increase precision of materials modification and the depth of the implanted bismuth ions. The $\mathrm{BiFeO}_{3}$, iron oxide and bismuth contributions to the magnetic ordering are studied, with a focus on iron and iron oxide concentrations. Bismuth doping can provide information on $\mathrm{BiFeO}_{3}$ synthesis mechanisms, as $\mathrm{BiFeO}_{3}$ is known to be synthesized in chemical vapour deposition by the bonding of $\mathrm{Bi}_{2} \mathrm{O}_{3}$ and iron oxide [Selbach et al., 2008]. The Selbach et al. [2008] synthesis mechanism would require the implanted bismuth to form $\mathrm{Bi}_{2} \mathrm{O}_{3}$, which will in turn form $\mathrm{BiFeO}_{3}$ when bismuth oxide bonds with iron oxides. Bismuth doping of magnetite is reported, for the first time, in this study. This study shows the effect of implanting bismuth in a mix phase iron oxide material and in a hematite rich film. In this chapter, the structure and potential secondary phases of iron oxide thin film and the bismuth-implanted iron oxide film are analysed. 


\subsection{Experimental details}

Table 5.1.1: Sample code of the bismuth implanted iron oxides films.

\begin{tabular}{|c|c|c|c|c|c|}
\hline Code & Sputtering & Annealing & $\begin{array}{l}\text { Implanted } \\
\text { ion }\end{array}$ & Annealing & Section \\
\hline SP1 & $\mathrm{Fe}_{x} \mathrm{O}_{y}$ & n.a. & n.a. & n.a. & 5.2 \\
\hline SP2 & $\mathrm{Fe}_{x} \mathrm{O}_{y}$ & $\begin{array}{l}500{ }^{\circ} \mathrm{C}, \\
15 \text { min in } \\
\mathrm{O}_{2}\end{array}$ & n.a. & n.a. & 5.3 \\
\hline SP1-Bi & $\mathrm{Fe}_{x} \mathrm{O}_{y}$ & n.a. & $\begin{array}{l}\text { Bi } 28 \mathrm{keV} \\
3 \times 10^{16} \\
\text { at.cm } \\
-2\end{array}$ & n.a. & n.a. \\
\hline SP2-Bi & $\mathrm{Fe}_{x} \mathrm{O}_{y}$ & $\begin{array}{l}500{ }^{\circ} \mathrm{C}, \\
15 \text { min in } \\
\mathrm{O}_{2}\end{array}$ & $\begin{array}{l}\text { Bi } 28 \mathrm{keV} \\
3 \times 10^{16} \\
\text { at.cm } \\
-2\end{array}$ & n.a. & n.a. \\
\hline SP1-Bi & $\mathrm{Fe}_{x} \mathrm{O}_{y}$ & n.a. & $\begin{array}{l}\text { Bi } 28 \mathrm{keV} \\
3 \times 10^{16} \\
\text { at.cm } \\
-2\end{array}$ & $\begin{array}{l}500^{\circ} \mathrm{C}, \\
15 \text { min in } \\
\mathrm{O}_{2}\end{array}$ & C.2 \\
\hline SP2-Bi & $\mathrm{Fe}_{x} \mathrm{O}_{y}$ & $\begin{array}{l}500{ }^{\circ} \mathrm{C}, \\
15 \mathrm{~min} \text { in } \\
\mathrm{O}_{2}\end{array}$ & $\begin{array}{l}\text { Bi } 28 \mathrm{keV} \\
3 \times 10^{16} \\
\text { at.cm }{ }^{-2}\end{array}$ & $\begin{array}{l}500^{\circ} \mathrm{C}, \\
15 \text { min in } \\
\mathrm{O}_{2}\end{array}$ & 5.5 \\
\hline
\end{tabular}

Two samples were synthesised with a specific protocol and studied to analysis the phase formation. Sample 1, SP1, is the as-deposited by ion beam sputtering iron oxide film. Sample 2, SP2, is another iron oxide film deposited by ion beam sputtering, however this sample was subsequently annealed (see Table 5.1.1 for more details). A piece of both iron oxide films was used as control and stored in a desiccator to limit oxidation caused by the interaction with the environment (air). The other pieces were implanted with bismuth ions using the ion implanter, the ion implanter was discussed in the subsection 3.2.2, then they were both subsequently annealed. The sample SP1Bi-A was made from the SP1 sample that was subsequently implanted with bismuth followed by an annealing at $500{ }^{\circ} \mathrm{C}$ for $15 \mathrm{~min}$ in an oxygen atmosphere. The sample SP2-Bi-A was made from the SP2 sample, iron oxide annealed film, that was subsequently implanted with bismuth ions followed by an annealing at $500{ }^{\circ} \mathrm{C}$ for $15 \mathrm{~min}$ in an oxygen atmosphere. Table 5.1.1 details the synthesising of these implanted films. SP1 and SP2 samples are control sample in order to compare the effect of bismuth ions implanted in the samples SP1-Bi-A and SP2-Bi-A. SP1-Bi and SP2-Bi samples are not 
analysis here for practical reason, as the conditions of implantation are synthesising, in this case with these specific conditions of synthesis discussed in Table 5.1.1, an amorphous material and thus imposing some limitation on the films characterisation (e.g. Raman spectroscopy and XRD may not be suited to analysis the phase form in those samples). The annealing effect before and after ion implantation has been studied on other materials [Murmu et al., 2012]. Murmu et al. [2012] clearly identify the annealing effect has an important parameter to study; implanted bismuth ions could be bonding more easily with the iron oxide film after annealing the sample. To disclose more information on the annealing effect, the analysis of the sample SP1-Bi-A is presented in Section C.1. This annex is provided as an optimisation's study in the attempt to form $\mathrm{BiFeO}_{3}$ from the synthesis of bismuth ions implanted into an iron oxide film. The synthesis of the sample SP1-Bi-A leads to a material with less potential to form $\mathrm{BiFeO}_{3}$ and a material with less device application potential then the sample SP2-Bi-A. Those are the main reason explaining that the study of the sample SP1-Bi-A is only presented in Section C.1, while the sample SP2-Bi-A is presented in this chapter.

The first step in the synthesis consists of sputtering iron oxide on silicon dioxide substrate at ambient temperature, using a $\mathrm{Fe}_{3} \mathrm{O}_{4}$ magnetite sputtered target made at GNS Science with a pressed pallet and a commercial $\mathrm{Fe}_{3} \mathrm{O}_{4}$ magnetite powder. The powder was made by Skyspring Nanomaterials, Inc., with grain size between 20$30 \mathrm{~nm}$, and pure at $98 \%$. As magnetite is easy to oxidize, the target was stored in a desiccator to limit the aging effect. Ion beam sputtering was described in subsection 3.2.1, and sputtering synthesis was detailed in Chapters 3 and 4. From the film monitor thickness, the iron oxide film is estimated to be close to $100 \mathrm{~nm}$. RBS analysis later in this chapter will provide a more reliable value for the thickness. The iron oxide sputtering was done on $\mathrm{SiO}_{2}(500 \mathrm{~nm})$ onto Si bulk. Every anneal was carried out at $500{ }^{\circ} \mathrm{C}$ for 15 minutes. The ion implantation of bismuth was carried out at a dose of $3 \times 10^{16}$ at. $\mathrm{cm}^{-2}$ into the iron oxide film, SP1 and SP2. The bismuth implantation was done by ion implantation at GNS Science using the technique described in subsection 3.2.2. Bismuth ions were implanted with an energy of $28 \mathrm{keV}$ and a selected fluence of about $3 \times 10^{16}$ at.cm ${ }^{-2}$. Double charged ions, $\mathrm{Bi}^{2+}$, were used to increase the bismuth depth penetration inside the iron oxide film for TEM analysis. For simplification, this second set of sample were implanted at an energy of $56 \mathrm{keV}$ for each $\mathrm{Bi}^{+}$. The bismuth implantation depth is theoretically less than $50 \mathrm{~nm}$, which will be detailed in Section 5.4 Simulation of bismuth implantation. 


\subsection{As-made Iron oxide sputtered film, SP1}

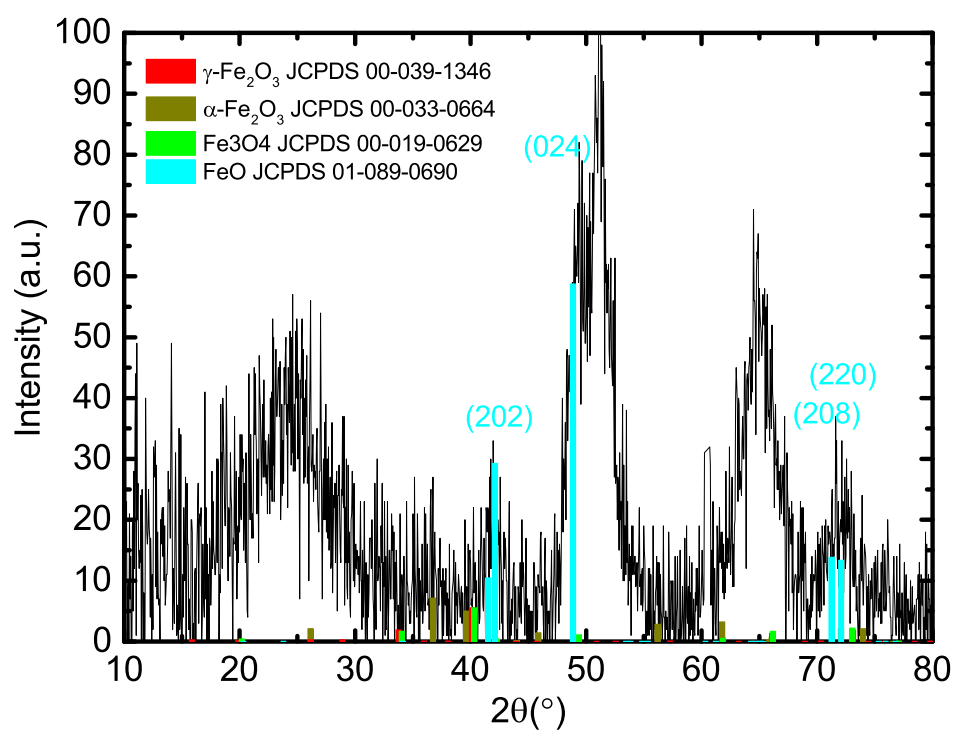

Figure 5.2.1: $X R D$, grazing angle $2^{\circ}$, of as-made iron oxide thin film, SP1. From the XRD, the film seems to contain an amorphous material (broad peak) and FeO (JCPDS 01-089-0690). Also shown are XRD reference patterns for magnetite (JCPDS 00019-0629) and maghemite (JCPDS 00-039-1346).

XRD analysis of the as-made ion beam sputtered iron oxide thin film, SP1, is shown in Figure 5.2.1. Figure 5.2.1 also shows the possible presence of FeO (JCPDS 01089-0690) rhombohedral R-3, whose reference pattern matches the XRD peaks. Other iron oxides are not clearly identifiable. This iron oxide phases (SP1) will be compared with other samples, SP2 and SP2-Bi-A and with $\alpha$ - $\mathrm{Fe}_{2} \mathrm{O}_{3}$ hematite, $\gamma-\mathrm{Fe}_{2} \mathrm{O}_{3}$ maghemite and $\mathrm{Fe}_{3} \mathrm{O}_{4}$ magnetite. However, none of those iron oxide phases are clearly visible in the XRD analysis. The broad peak at $25^{\circ}$ might be indicative of an amorphous phase, however this phase is difficult to identify. From the literature, iron oxide deposition at ambient temperature is known to contain an amount of amorphous material [Machala et al., 2007; Tang et al., 2006]. Electronic transport measurement might be able to provide a more definitive explanation for the broad features. The sharp peak at $52^{\circ}$ is not identified, however that feature is not from iron. By eliminating the possibilities, the $52^{\circ}$ peak is likely an iron oxide phase signal. Three iron oxides $\left(\alpha-\mathrm{Fe}_{2} \mathrm{O}_{3}, \gamma-\mathrm{Fe}_{2} \mathrm{O}_{3}\right.$ and $\mathrm{Fe}_{3} \mathrm{O}_{4}$ ) could be present, but the particles would have to be small enough to broaden the XRD peaks, and they would be difficult to analyse. FeO is thus the only identified material from the XRD analysis.

Figure 5.2.2 shows a magnified scale centred around the $50^{\circ}$ peak of the XRD analysis that is shown in Figure 5.2.1. Also shown in Figure 5.2.2 is the JCPDS 01-0890690 (FeO rhombohedral R-3) and pseudo Voigt function of two peaks, fitted using. 


$$
I=I_{0}+A\left(\mu \frac{2}{\pi}\right)\left(\frac{w}{\left(4\left(x-x_{c}\right)\right)^{2}+w^{2}}\right)+(1-\mu) \frac{\sqrt{4 \ln 2}}{\sqrt{\pi} \cdot w} e^{-\frac{4 \ln 2}{w^{2}}\left(x-x_{c}\right)^{2}} .
$$

where the pseudo Voigt function include, in the first part, a Lorentz profile and, in the second part, a Gaussian distribution, $w$ is the full width half maximum, $x_{c}$ is the position of the centre of the distribution, $A$ is a pre-factor, $I_{0}$ is the background intensity and $\mu$ is the off centre line shift between the Gaussian distribution and Lorentzian profile.

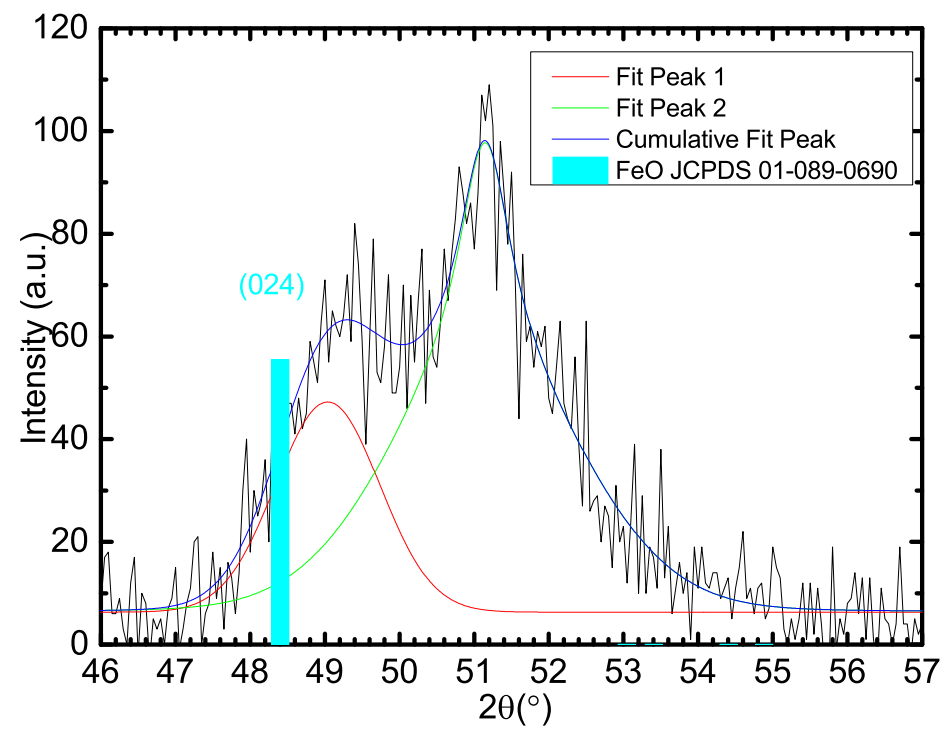

Figure 5.2.2: XRD, grazing angle $2^{\circ}$, of the as-made iron oxide film, SP1, with a magnified scale centred around the $50^{\circ}$ peak shown in Figure 5.2.1. Also, shown in this figure is a pseudo Voigt function fit of the merged peak at $49^{\circ}$ and $51^{\circ}$, the cumulative peak fit and the JCPDS pattern 01-089-0690 (FeO rhombohedral R-3).

There are two peaks $49^{\circ}$ and $51^{\circ}$. The $49^{\circ}$ peak has $\mu=0$ and $\mathrm{W}_{G}=1.6 \pm 0.1^{\circ}$. The $49^{\circ}$ is likely to be due to $\mathrm{FeO}$ where the small shift from the reference pattern angle is likely to be due to strain effects. The $\mu$ value of zero for the FeO (024) peak indicates that fit is a pure Gaussian peak. The $51^{\circ}$ peak has $\mu=0.296$ and $\mathrm{W}_{G}=3 \pm 0.3^{\circ} . \mu$ and $\mathrm{W}_{G}$ are different for this peak and indicate that source of that signal comes from another phase that cannot be identified. From Scherer's equation, the FeO particle size is estimated to be $9 \pm 1 \mathrm{~nm}$. The second peak has an estimated size of $5 \pm 1 \mathrm{~nm}$ obtained from Scherer's equation.

The presence of magnetite is expected, as the target is made of magnetite, however that phase was not identified by XRD analysis. This could be either because magnetite was deposited at ambient temperature and formed an amorphous material [Machala et al., 2007; Tang et al., 2006], or a difference between the sputtering yields 
of iron and oxygen resulted in a sputtered material containing a phase other than the target material. The greater sputtering yield of iron compared to that of oxygen could explain the presence of $\mathrm{FeO}$, which was clearly identified in the XRD analysis. The fact that other phases were not identified might suggest that those phases could be present in smaller fractions compared to $\mathrm{FeO}$, or they could be amorphous. The amorphous material indicated by the broad peak in the XRD analysis suggests that other iron oxide materials might be present in the film, if present they will need to be identified by other characterisation techniques.

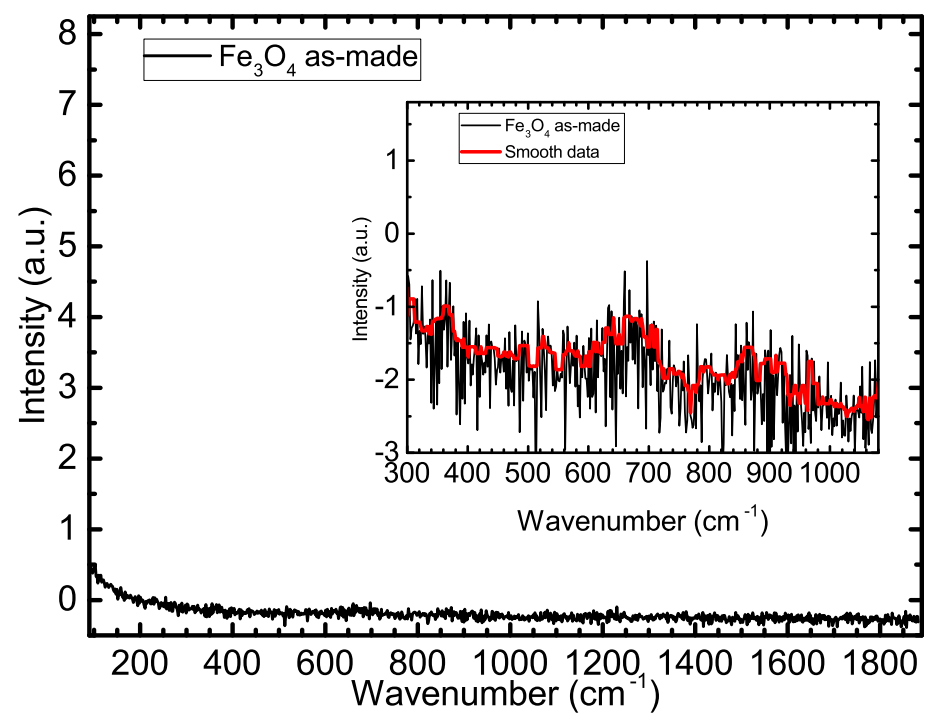

Figure 5.2.3: Raman spectroscopy of the as-made iron oxide sputtered film, SP1. The inset displays a magnified scale in the area 300 to $1100 \mathrm{~cm}^{-1}$ with the Raman signal averaged in red.

The Raman spectrum of the as-made iron oxide thin film is displayed in Figure 5.2.3. Also, shown in the inset is a magnified scale centred around $700 \mathrm{~cm}^{-1}$ with the average signal (red line) overlaid. The as-made ion beam sputtered iron oxide film only displays a signal from an amorphous material. Raman spectroscopy analysis indicate the absence of a well-defined structure. There is a broad low intensity feature extending from $\sim 600 \mathrm{~cm}^{-1}$ to $\sim 800 \mathrm{~cm}^{-1}$. This is in the range where Raman peaks are expected from FeO. FeO has a Raman mode at $650 \mathrm{~cm}^{-1}$ [De Faria et al., 1997]. The Raman shift between $\sim 600 \mathrm{~cm}^{-1}$ to $\sim 800 \mathrm{~cm}^{-1}$ is also the region where Raman peaks have been reported for magnetite, maghemite, and hematite. The absence of a well-defined Raman peak from FeO may be due to the small particles size that can lead to broadening the Raman peak. The broad low intensity peak may also have a contribution from very small or highly disordered $\mathrm{FeO}_{x}$ phases.

A silicon line at $521 \mathrm{~cm}^{-1}$ is invisible, this means the laser light is absorbed before reaching the substrate. A high absorbance could indicate the presence of a material 
with metallic behaviour or a material with a band gap of less than $2.4 \mathrm{eV}(514 \mathrm{~nm})$. The $\mathrm{FeO}$ band gap is known to be below $2.2 \mathrm{eV}$. However, in a non-stoichiometric configuration, FeO band gap can be lower than $1 \mathrm{eV}$ [Jain et al., 2013; Schrettle et al., 2012, Ashraf et al., 2014].

Figure 5.2.3, inset, displays the Raman spectrum of a broad and low-intensity peak, and the average of the Raman scattering overlaid in red. The amplitude of the low-intensity peak is close to the noise level, which increases the uncertainty of the existence of the peak. The peak at $672 \mathrm{~cm}^{-1}$ (from $\sim 600 \mathrm{~cm}^{-1}$ to $780 \mathrm{~cm}^{-1}$ ) is a signature of iron oxide phase material. The broad peak could indicate a small nanoparticle or amorphous material, however this could also be cause by traces of $\mathrm{FeO}$, as $\mathrm{FeO}$ has a strong peak at $650 \mathrm{~cm}^{-1}$ [De Faria et al., 1997]. The low intensity can indicate a material with a small band gap like $\mathrm{FeO}$ (below $2.2 \mathrm{eV}$ and in a non-stoichiometric configuration below $1 \mathrm{eV}$ [Jain et al., 2013; Schrettle et al., 2012, Ashraf et al., 2014]) or $\mathrm{Fe}_{3} \mathrm{O}_{4} . \mathrm{Fe}_{3} \mathrm{O}_{4}$, as mentioned in Chapter 4, has a reported band gap of less than $0.5 \mathrm{eV}$ [Jeng and Guo, 2002]. Materials with small band gap values can absorb laser light, limiting the Raman scattering of the material and the silicon substrate.

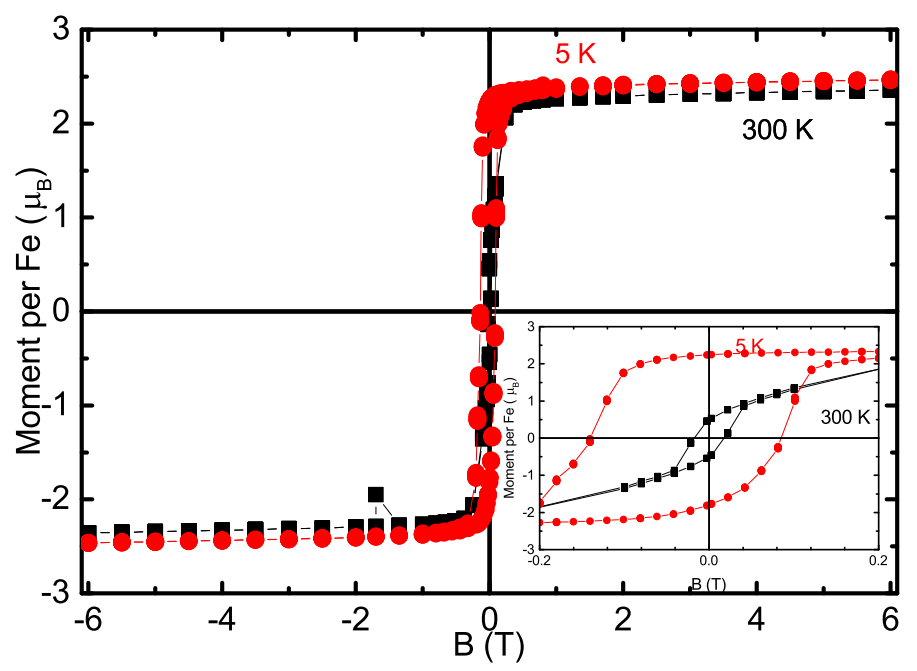

Figure 5.2.4: Magnetic moment of the as-made iron oxide film, SP1. This figure shows the field loop measurement at $5 \mathrm{~K}$ and $300 \mathrm{~K}$. The inset shows that magnetic data over a small magnetic field range where an exchange bias is evident at $5 \mathrm{~K}$.

Figure 5.2.4 displays the magnetic moment per Fe at $300 \mathrm{~K}$ and $5 \mathrm{~K}$ of the as-made iron oxide material after subtracting the diamagnetic signal from the silicon substrate using equation 3.3.21. The $\mathrm{FeO}_{x}$ film thickness was estimated to be $100 \mathrm{~nm}$, which is the film thickness measured after Bi implantation and annealing using the RBS data discussed later. The saturation moment is $2.46 \mu_{B} / \mathrm{Fe}$ and $2.34 \mu_{B} / \mathrm{Fe}$ at $5 \mathrm{~K}$ and $300 \mathrm{~K}$, respectively. The moment is only slightly lower at $300 \mathrm{~K}$ and indicates that the film is ferromagnetic with a Curie temperature far above ambient temperature. The satura- 
tion moment is far greater than that expected from known $\mathrm{FeO}_{x}$ phases. This cannot be due to $\mathrm{FeO}$ since that iron oxide is antiferromagnetic with a Néel temperature of 198 K [Dimitrov et al., 1999]. Magnetite and maghemite are ferrimagnetic with high Curie temperatures and a saturation moments of only $1.35 \mu_{B}$ for $\mathrm{Fe}_{3} \mathrm{O}_{4}$ and $1.25 \mu_{B}$ for $\gamma-\mathrm{Fe}_{2} \mathrm{O}_{3}$ [Cullity and Graham, 2011]. However, there is no clear evidence, from Raman spectroscopy and XRD, for these phases although they could be present in particle sizes smaller than the limit of detection. Hematite is antiferromagnetic. Bulk Fe is known to be ferromagnetic with a high Curie temperature and a saturation moment of $2.2 \mu_{B} / F e$. If the magnetic response was from Fe then this would imply that nearly all the film is Fe, which would appear to be unlikely. Thus, the source of the large magnetic signal is identified as a new unknown phase. This new unknown phase could be a mix of different iron oxide $\left(\alpha-\mathrm{Fe}_{2} \mathrm{O}_{3}, \gamma-\mathrm{Fe}_{2} \mathrm{O}_{3}, \mathrm{Fe}_{3} \mathrm{O}_{4}, \mathrm{FeO}\right)$ with iron.

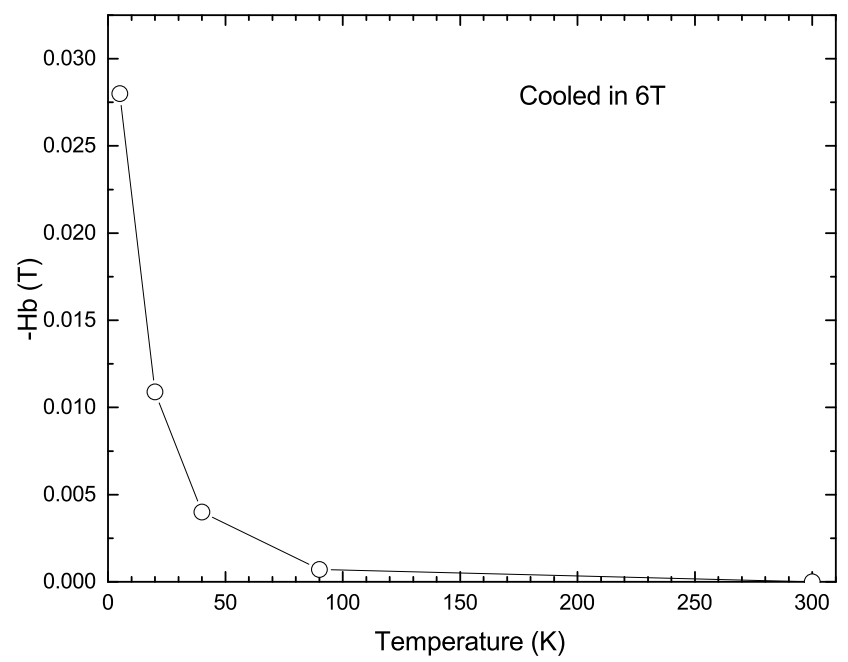

Figure 5.2.5: Exchange bias of as-made iron oxide film, SP1. The exchange bias is obtained by cooling the sample in a $6 \mathrm{~T}$ applied magnetic field.

The inset to Figure 5.2.4 clearly shows that the hysteresis is offset at $5 \mathrm{~K}$. This indicates the presence of an exchange bias. The resulting exchange bias is plotted in Figure 5.2.5 at different temperatures. The data presented in Figure 5.2.5 suggest that the exchange bias disappears above $\sim 100 \mathrm{~K}$. The appearance of an exchange bias indicates that there is a ferromagnetic phase in contact with an antiferromagnetic phase in parts of the film.

Figure 5.2.6 displays the temperature-dependent response of the as-made iron oxide thin film while under a $6 \mathrm{~T}$ applied magnetic field. Also shown is a fit to Block's $3 / 2$ equation 3.3 .21 where $D=8.45 \times 10^{-6} \mathrm{~K}^{-3 / 2}$. The value $D$ is slightly larger than expected for bulk Fe or $\mathrm{Fe}_{3} \mathrm{O}_{4}$.

The Figure 5.2.7 displays the ZFC-FC curve for the as-made iron oxide film, SP1, 


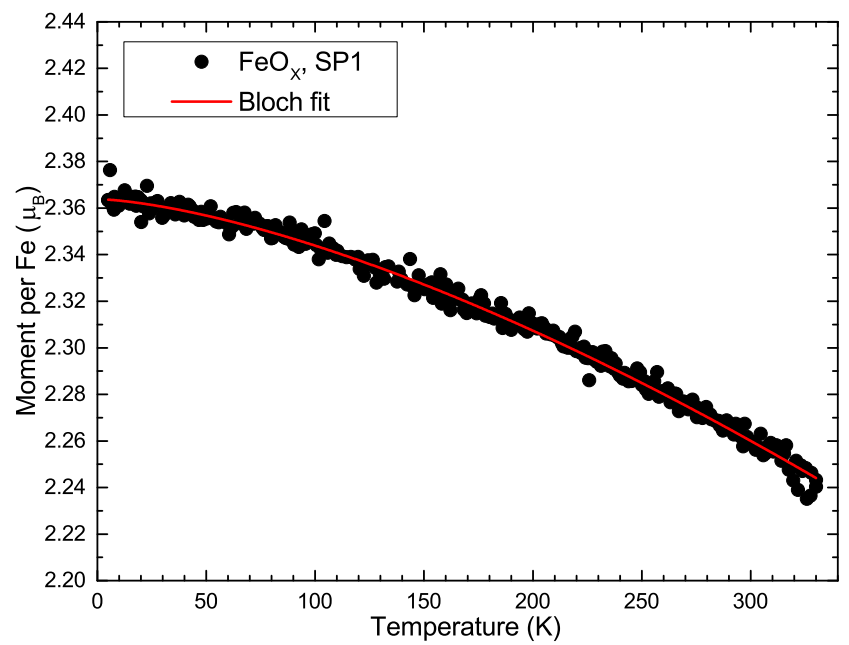

Figure 5.2.6: Temperature dependence at $6 \mathrm{~T}$ of the magnetic moment on the as-made iron oxide film, SP1. Also, shown in this figure is the Bloch $3 / 2$ fit (red).

at $10 \mathrm{mT}$. The superparamagnetism effect could explain the ZFC-FC splitting. However, a gap between ZFC and FC is visible at high temperature which is less likely to be explain by superparamagnetism. There is hysteresis and a separation of the curves at lower temperatures where the ZFC curve has a peak at $120 \mathrm{~K}$. This suggests that there is an antiferromagnetic phase with a Néel temperature of $\sim 120 \mathrm{~K}$. That phase may come from FeO where bulk FeO has a Néel temperature of 198 K. The lower Néel temperature may be because the particle sizes are small. The Néel temperature is close to the temperature where the exchange bias seen in Figure 5.2.5 disappears and hence the phase leading to this antiferromagnetic transition is likely to also be the antiferromagnetic material that is in contact with the unknown ferromagnetic phase in parts of the film.

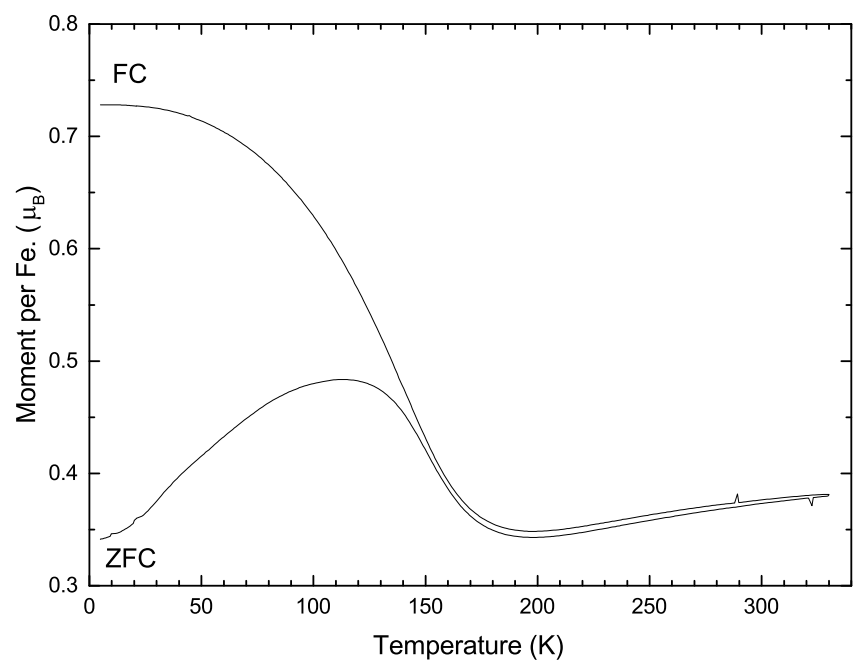

Figure 5.2.7: ZFC-FC plot for the as-made iron oxide film, SP1, at $10 \mathrm{mT}$. 


\subsection{Annealed Iron oxide sputtered film, SP2}

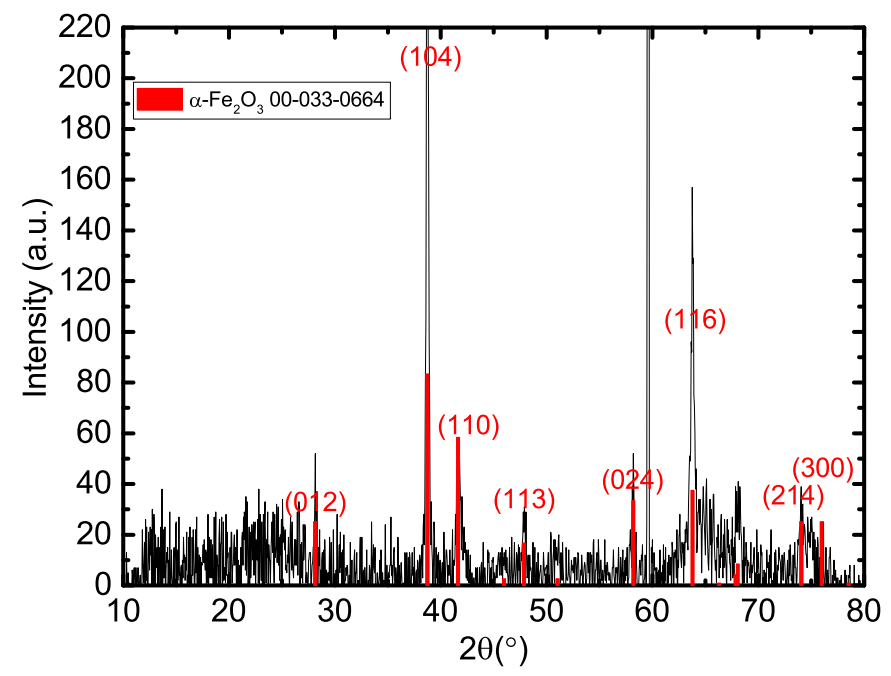

Figure 5.3.1: XRD, grazing angle $2^{\circ}$, analysis of the iron oxide film annealed at $500{ }^{\circ} \mathrm{C}$ for $15 \mathrm{~min}, \mathrm{SP2}$. Also shown is hematite reference pattern (JCDPS 00-033-0664).

XRD analysis of the iron oxide film annealed (SP2) at $500{ }^{\circ} \mathrm{C}$ in an oxygen atmosphere is shown in Figure 5.3.1; also shown is JCPDS 00-033-0664 (hematite). No other phases are clearly identifiable by XRD. Broad peaks are also present, which could indicate the presence of small nanoparticles or amorphous material. Figure 5.3.2 shows a pseudo-Voigt fit to the hematite (104) peak where the full width half maximum is $0.26^{\circ}$ and $\mu=0.39$. From Scherrer's equation and an instrumental broadening of 0.1 the average nanoparticle size is estimated to be $45 \mathrm{~nm}$. The size estimation from the peak (110) is approximately $45 \mathrm{~nm}$. There is no evidence for other $\mathrm{FeO}_{x}$ phases such as magnetite and maghemite. If they are present, then their relative phase fractions are low.

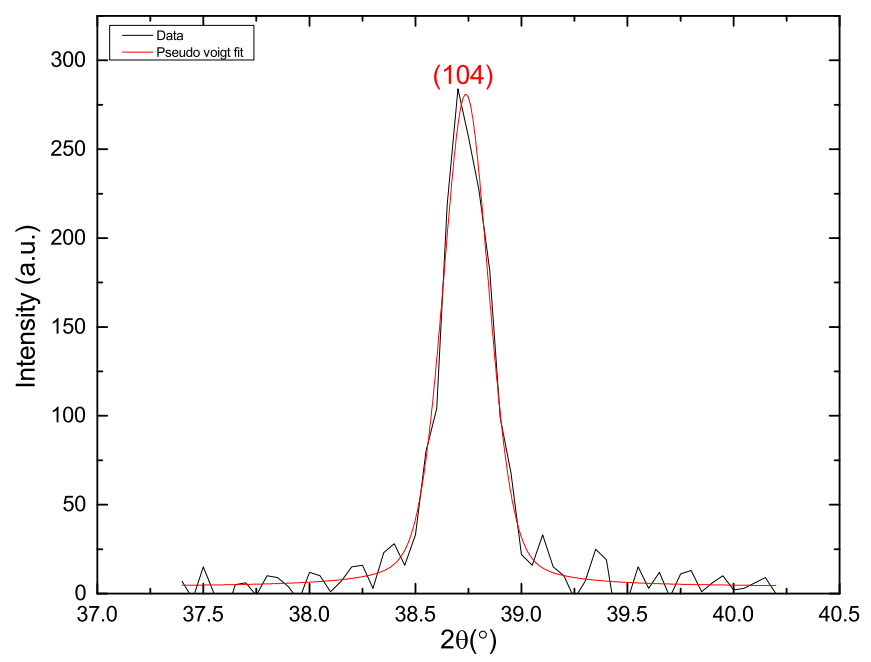

Figure 5.3.2: XRD, grazing angle $2^{\circ}$, analysis of SP2, magnified around the hematite peak (104). Also shown is a fit to a pseudo Voigt fit. 


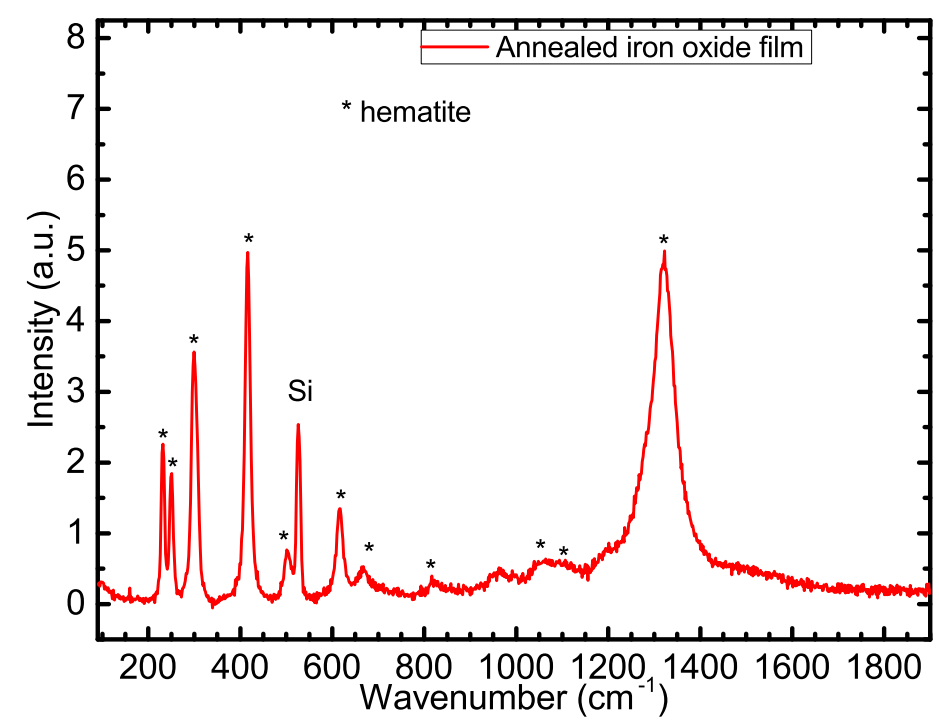

Figure 5.3.3: Raman spectrum of the annealed iron oxide sputtered film at $500{ }^{\circ} \mathrm{C}$ for $15 \mathrm{~min}$, SP2. The expected $\alpha-\mathrm{Fe}_{2} \mathrm{O}_{3}$ peaks are shown by stars and the silicon substrate at $521 \mathrm{~cm}^{-1}$ is also seen.

The Raman spectrum of the annealed iron oxide film is displayed in Figure 5.3.3. The Raman spectrum clearly identifies $\alpha-\mathrm{Fe}_{2} \mathrm{O}_{3}$ hematite. The reported hematite peaks are listed in Table 5.3.1. No other phases are identified; the Raman spectroscopy result is similar to the XRD analysis. If other phases are present, they must be at low concentrations. Maghemite and magnetite, two magnetic iron oxide phases, are not clearly seen in the Raman spectrum, however they could be present in low quantities or be amorphous. The Raman shift at $670 \mathrm{~cm}^{-1}$ is reported to be a hematite Raman forbidden mode and an infrared active mode.

Table 5.3.1: Reported hematite Raman shift.

\begin{tabular}{|c|c|}
\hline \multicolumn{2}{|c|}{$\begin{array}{c}\text { Hematite Raman shift }\left(\mathrm{cm}^{-1}\right) \\
\text { [Jubb and Allen, 2010] }\end{array}$} \\
\hline 229 & $\mathrm{~A}_{i g}$ \\
\hline 249 & $\mathrm{E}_{g}$ \\
\hline 295 & $\mathrm{E}_{g}$ \\
\hline 302 & $\mathrm{E}_{g}$ \\
\hline 414 & $\mathrm{E}_{g}$ \\
\hline 500 & $\mathrm{~A}_{1 g}$ \\
\hline 615 & $\mathrm{E}_{g}$ \\
\hline 600 & LO, IR-active $\mathrm{E}_{u}$ \\
& [Shim and Duffy, 2002] \\
\hline 809 & [Martin et al., 1977] \\
\hline
\end{tabular}


The Si substrate peak can clearly be seen with a Raman shift at $521 \mathrm{~cm}^{-1}$. The silicon substrate displays a possible second order peak between $950 \mathrm{~cm}^{-1}$ to $1000 \mathrm{~cm}^{-1}$. The absorption coefficient is modified by annealing the film; the film absorbed less light when annealed. The iron oxide mixed phases become mainly hematite when annealed, and the material has a larger band gap, about $2.1 \mathrm{eV}$ [Meng et al., 2011], which could explain the decrease in the absorption. The presence of hematite is also confirmed by XRD (Figure 5.3.1).

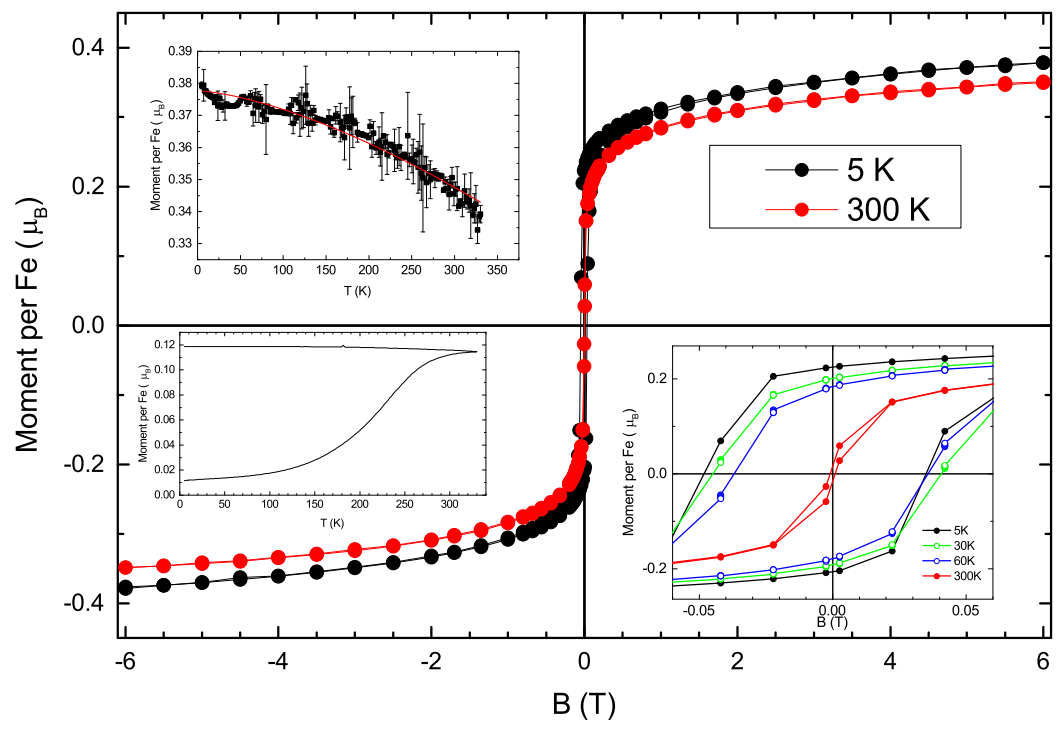

Figure 5.3.4: Magnetic moment per Fe at $5 \mathrm{~K}$ and $300 \mathrm{~K}$ for the iron oxide annealed at $500{ }^{\circ} \mathrm{C}$, SP2. The lower left inset shows the moment over a smaller magnetic field range as well as data at $30 \mathrm{~K}$ and $60 \mathrm{~K}$. The upper right inset shows that magnetic moment per Fe at $6 \mathrm{~T}$ (symbols) and a Bloch fit to the data (solid curve). The lower left inset shows the ZFC-FC data taken with an applied magnetic field of $10 \mathrm{mT}$.

Figure 5.3.4 displays the magnetic moment per Fe after annealing at $500{ }^{\circ} \mathrm{C}, \mathrm{SP} 2$. The saturation magnetic moment is only slightly lower at $300 \mathrm{~K}$ and indicates that the magnetic ordering temperature is far above ambient temperature. The saturation moment at $5 \mathrm{~K}$ is $0.38 \mu_{B} / \mathrm{Fe}$. This value is $15 \%$ of the saturated moment seen before annealing. The moment also begins to saturate at a similar magnetic field and this suggests that the moment is predominately due to $15 \%$ of the magnetic phase seen before annealing.

The lower left inset to Figure 5.3.4 shows the moment per Fe over a smaller magnetic field range where an exchange bias is evident. Also shown is data at $30 \mathrm{~K}$ and $60 \mathrm{~K}$ where their measurements were taken after cooling in an applied magnetic field of $6 \mathrm{~T}$. The origin of the exchange bias is likely to have a similar origin to the as-made film (SP1). This will be discussed in relation to the Bi implanted films in Section 5.5. 
The moment at $6 \mathrm{~T}$ is plotted as a function of temperature in the upper right inset in Figure 5.3.4. Also shown is a fit to the Bloch $3 / 2$ function (equation 3.3.22). The fitted $D$ is found to be $15 \times 10^{-6} \mathrm{~K}^{-1.5}$ which is $\sim 2$ times that seen before annealing (SP1).

The ZFC-FC curves are shown in Figure 5.3.4 (lower left inset). There is a hysteresis up to the highest measured temperature and indicates that there is no evidence for superparamagnetism. The antiferromagnetic phase seen at lower temperature in the iron oxide film before annealing, SP1, was not identified in the annealed film SP2.

\subsection{Simulation of Bismuth implantation}

Theoretical simulation of bismuth implantation was carried out with the D-Trim software. The simulation parameters are selected to represent the implantation taking place in the laboratory with a unit cell of $10000 \AA$. A first set of samples implanted with single charged bismuth, $\mathrm{Bi}^{+}$, were used for RBS, XRD, Raman and magnetic characterisation. In order to increase the contrast, the TEM images were analysed with the second set of samples based on implantation of double charged bismuth, $\mathrm{Bi}^{2+}$. Doubling the ions charge for the same applied potential double the implantation energy, and thus this should result in a deeper ion implantation and a modification in the concentration of ions. The comparison is theoretically simulated and shown in Figure 5.4.1.

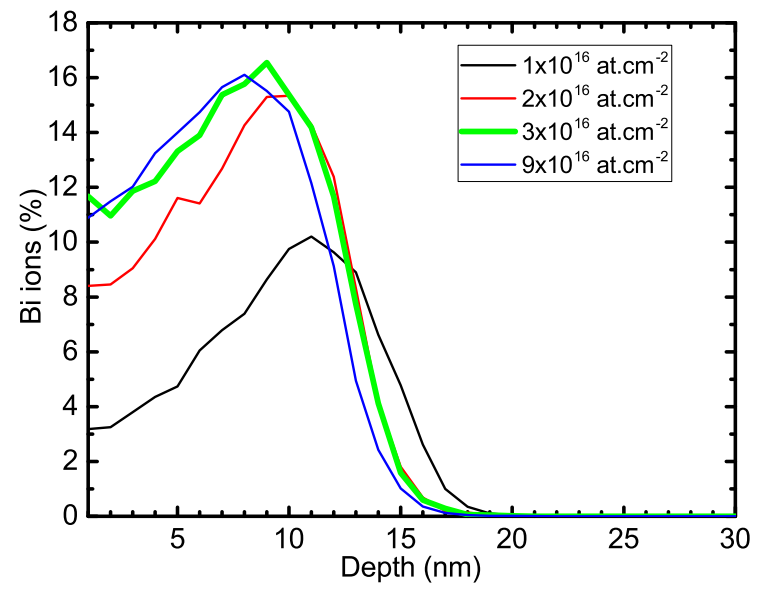

(a) Implantation of Bi at $28 \mathrm{keV}$.

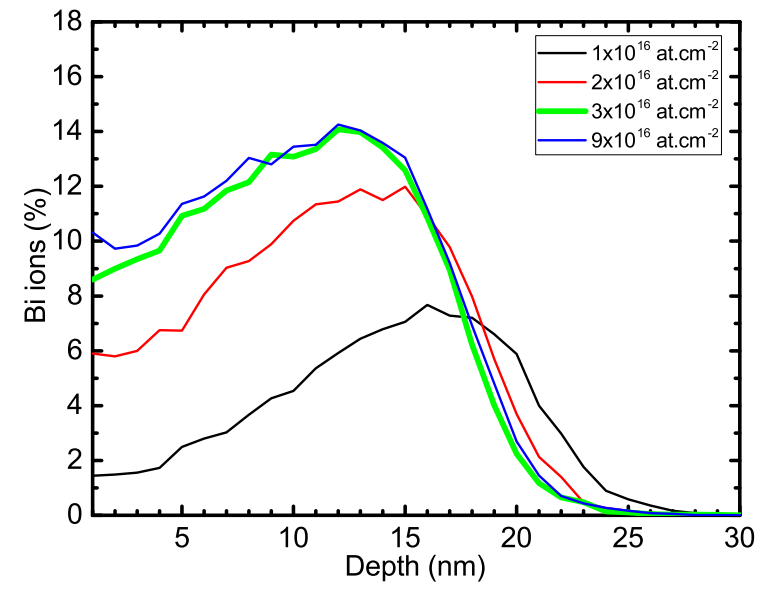

(b) Implantation of Bi at $56 \mathrm{keV}$.

Figure 5.4.1: Theoretical simulation of $\mathrm{Bi}$ implantation into $\mathrm{Fe}_{3} \mathrm{O}_{4}$. The saturation occurs at a fluence of $3 \times 10^{16}$ at.cm ${ }^{-2}$ (green line). (a) At a depth of $17 \mathrm{~nm}$, the concentration is 27 at.cm ${ }^{-2}$ and (b) at a depth of $25 \mathrm{~nm}$, the concentration is $10 \mathrm{at.cm}^{-2}$.

To synthesis this material, an iron oxide film is deposited by ion beam sputter- 
ing. The $\mathrm{Bi}$ is implanted at $28 \mathrm{keV}$ (56 keV for the samples analysed with TEM). The projected range is $14 \mathrm{~nm}$. From the Dyn-TRIM simulation, the Bi implantation resulted in the sputtering of a significant amount of O, Figure 5.4.2. Dynamic TRIM calculates the ions range profile, composition of the target, stopping power and the preferential sputtering yield for a dynamically varying target.

D-Trim is a software program that simulates the theoretical implantation of a selected ion species into a selected targeted material. In this case, the implantation of bismuth ions into iron oxide is modelled at at $28 \mathrm{keV}$ and $56 \mathrm{keV}$. This implantation energy was selected for practical reasons. The simulations are based on the assumption that the target material is a pure $\mathrm{Fe}_{3} \mathrm{O}_{4}$ magnetite film. The presence of $\alpha-\mathrm{Fe}_{2} \mathrm{O}_{3}$ hematite and $\gamma-\mathrm{Fe}_{2} \mathrm{O}_{3}$ maghemite would modify the general density of the target and thus modify the stopping range power [Sigmund, 2004]. The stopping power is based on series of interaction between the implanted ions and the atoms' electrons from the target material; a denser material has a higher stopping power [Sigmund, 2004]. The energy of implantation is another simulated parameter. Increasing the energy increases the kinematic energy of the incident ions and leads to implanting ions deeper under the target material surface [Rivière and Myhra, 2009]. After a certain number of events, or ions implanted, a charge effect could occur, resulting in an increased stopping power of the incident ions [Sigmund, 2004]. Implantation destroys the crystallographic configuration, creating disorder in the material, leading to more interstitial vacancies being occupied and modifying the ions range [Sigmund, 2004]. During the implantation, the series of inelastic collisions can provide the energy necessary to break some of the bonds between the target's molecules, gaining mobility to either diffuse towards the inner layers or outward (sputtering) [Sigmund, 2004]. Newly implanted ions may also be sputtered out [Sigmund, 2004]. Implantation saturation occurs when incident ions sputter out other ions of the same type, i.e. each implanted ion sputters another ion outside the material [Sigmund, 2004]. From the D-Trim simulation, bismuth implantation saturation is theoretically reached at a fluence of $3 \times 10^{16}$ at.cm ${ }^{-2}$ [Sigmund, 2004]. Implanting heavy elements in a light material modifies the density of the target, creating an energy barrier, and results in a shallower implantation even when the accelerated energy is constant.

The D-trim simulations of bismuth implantation into $\mathrm{Fe}_{3} \mathrm{O}_{4}$ are displayed in Figure 5.4.1 (a), Bi at $28 \mathrm{keV}$, and (b) Bi at $56 \mathrm{keV}$. Figure 5.4.1 displays different bismuth implantation fluences, in an effort to increase the contrast from implanted bismuth from the sputtered film, from $1 \times 10^{16}$ at. $\mathrm{cm}^{-2}$ to $9 \times 10^{16}$ at. $\mathrm{cm}^{-2}$. From this figure, the implantation saturation is reached when implanting at a fluence of $3 \times 10^{16}$ at.cm ${ }^{-2}$ (green line), which is the dose selected in the laboratory. The same saturation level 
exists when doubling the implanted energy, Figure 5.4.1.

The simulations were first optimised as a function of the incident energy of single charged bismuth ion, $\mathrm{Bi}^{+}$(Figure 5.4.1 (a)), and double charged ions, Bi2 ${ }^{+}$(Figure 5.4.1 (b)). The double charged ions have twice the energy compared to single charged ions with the same acceleration potential, meaning $\mathrm{Bi}^{2+}$ implanted at an incident potential of $28 \mathrm{kV}$ is equivalent to $\mathrm{Bi}^{+}$implanted at $56 \mathrm{kV}$ [Sigmund, 2004]. The first set of implantations was used to analyse the surface, so the implantations were purposefully shallow and limited to about $\sim 25 \mathrm{~nm}$. The optimal energies to compare with other synthesis techniques discussed previously in Chapter $4 \mathrm{BiFeO}_{3}$ thin film at nanoscale and other material synthesis by ion implantation presented in Appendix D are $28 \mathrm{keV}$ and $56 \mathrm{keV}$. Fluence optimisation is based on the fluence at saturation and is theoretically determined with D-Trim for a specific energy and target. This optimisation is shown in Figure 5.4.1. The bismuth saturation fluence is about $3 \times 10^{16} \mathrm{at}_{\mathrm{cm}} \mathrm{cm}^{-2}$. RBS analysis on several implanted samples confirms the accuracy of the theoretical model of D-Trim [Sigmund, 2004]. In Figure 5.4.1, the area under the curve represents the dose of bismuth implanted into the target material. The effect mentioned earlier in which increasing the fluence creates an energy barrier and increases the stopping power range is visible in these figures, as the implantation depth becomes shallower as the fluence increases.

These simulations will guide future work on bismuth-implanted iron oxide material, as the next set of samples made will have an iron oxide sputtered film thickness limited to less than $50 \mathrm{~nm}$ in order to match the implantation depth profile of the double charged ions. This has potential to enhance bismuth-implanted properties that might be hidden in the current analysis.

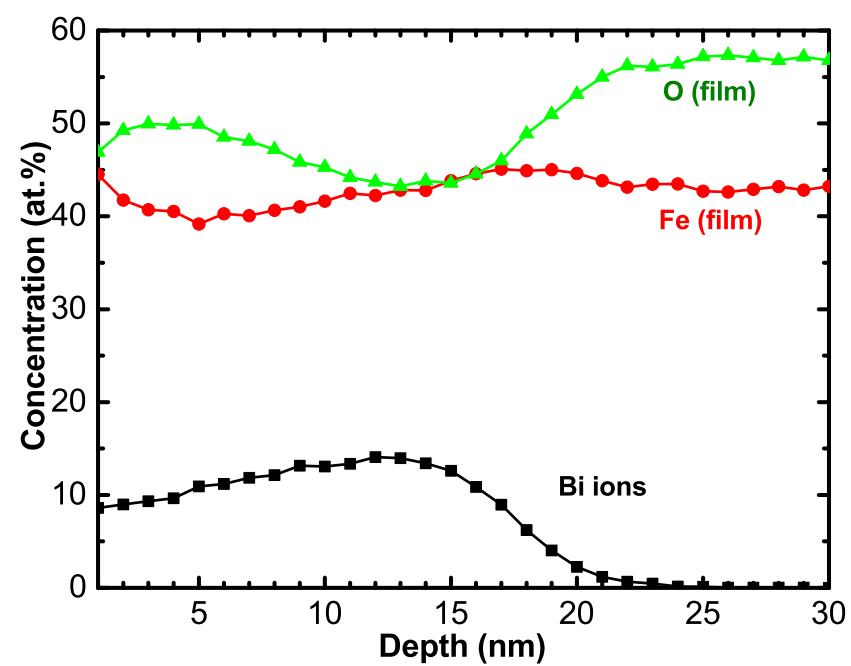

Figure 5.4.2: Depth profile of $2 \times 10^{16} \mathrm{Bi}^{-\mathrm{cm}^{-2}}$ into iron oxide calculated with D-TRIM. 
The Figure 5.4.2 shows the theoretical effect of implanting Bismuth into iron oxide film. In this simulation, the iron oxide layer was assumed to be made of magnetite, $\mathrm{Fe}_{3} \mathrm{O}_{4}$, density of $5.15 \mathrm{~g} \mathrm{~cm}^{-3}$ [Siratori and Kino, 1980]. Implanting bismuth show a preferential sputtering of oxygen.

\subsection{Annealed iron oxide film implanted with Bi ions and annealed a second time, SP2-Bi-A}

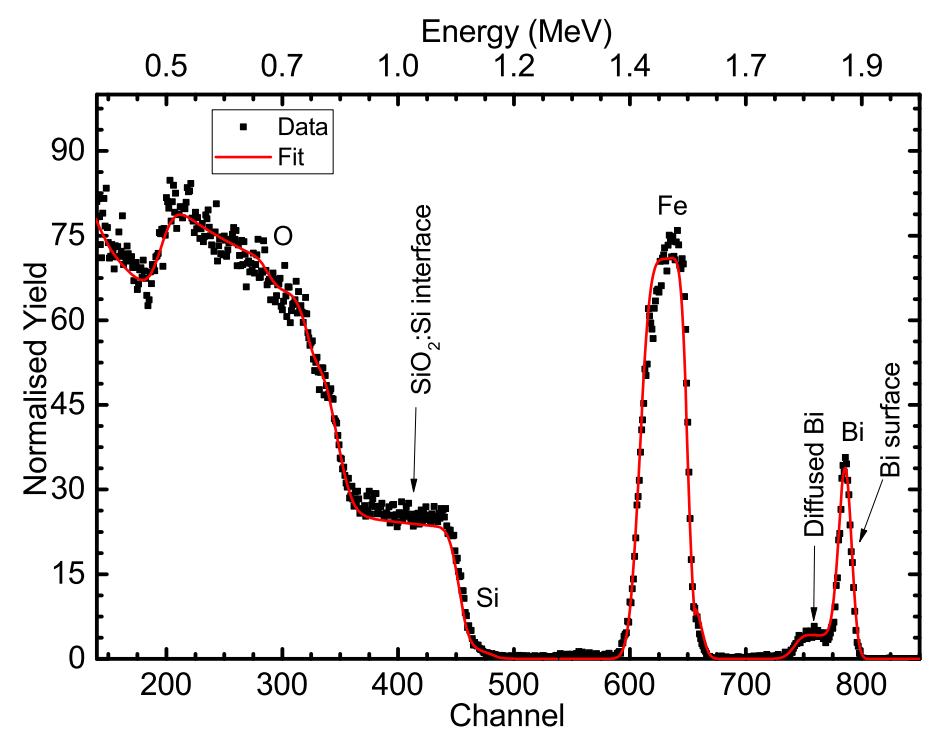

Figure 5.5.1: RBS of iron oxide film that was annealed at $500{ }^{\circ} \mathrm{C}$ for $15 \mathrm{~min}$ then implanted with bismuth and annealed at $500{ }^{\circ} \mathrm{C}$ for $15 \mathrm{~min}$, SP2-Bi-A (circle). Also, shown is the RUMP fit. Bismuth is diffused toward the inner layer of the film.

Figure 5.5.1 displays the annealed iron oxide film subsequently implanted with bismuth and annealed a second time SP2-Bi-A. The RUMP simulation is made with an assumption of a material with 6 layers, the details are provided in Table C.1.1. The surface layer is composed of $\mathrm{Fe}_{2.5}, \mathrm{O}_{2}$ and $\mathrm{Bi}_{1.7}$ with an estimated thickness of $42 \mathrm{~nm}\left(1.9 \times 10^{17}\right.$ at. $\mathrm{cm}^{-2}, 4.48 \times 10^{22}$ at. $\left.\mathrm{cm}^{-3}\right)$. This stoichiometry could be explained with phases of $\mathrm{Bi}, \mathrm{FeO}$ and $\mathrm{Fe}$. The second layer is made of $\mathrm{Fe}_{2}, \mathrm{O}_{3}, \mathrm{Si}_{0.1}$ and $\mathrm{Bi}_{0.012}$ with a thickness of $108 \mathrm{~nm}\left(8 \times 10^{17}\right.$ at. $\mathrm{cm}^{-2}, 7.43 \times 10^{22}$ at. $\left.\mathrm{cm}^{-3}\right)$. This stoichiometry can be explained with phases of $\mathrm{Fe}_{2} \mathrm{O}_{3}$ and atoms of bismuth diffusion into silicon. The third layer is composed of $\mathrm{Fe}_{0.1}, \mathrm{Si}_{1} \mathrm{O}_{2}$ and $\mathrm{Bi}_{0.012}$ with an estimated thickness of $19 \mathrm{~nm}\left(1 \times 10^{17}\right.$ at. $\mathrm{cm}^{-2}, 5.37 \times 10^{22}$ at. $\left.\mathrm{cm}^{-3}\right)$. The stoichiometry can be explained by the diffusion of bismuth and iron into $\mathrm{SiO}_{2}$. The fourth layer is $\mathrm{SiO}_{2}$ with an estimated thickness of $636 \mathrm{~nm}\left(3.3 \times 10^{18}\right.$ at. $\mathrm{cm}^{-2}, 5.18 \times 10^{22}$ at.cm $\left.{ }^{-3}\right)$. The layers' underneath are showing the bulk silicon, detailed are provided in Table C.1.1. The bismuth concen- 
tration, estimated with the RUMP simulation, is about $0.9 \times 1016$ at.cm ${ }^{-2}$ and iron is estimated at $4 \times 10^{17}$ at. $\mathrm{cm}^{-2}$. From this technique, the oxygen from the substrate can't be distinguish from the oxygen in the film.

The Bi dose is significantly less than expected from the theoretical implantation conditions which is $\sim 64 \%$ of the dose measured from the as-made (SP1) sample which is more than the dose of $\mathrm{Bi}$ atoms after annealing the film. This increase loss may be due to enhanced Bi diffusion that leads to a greater Bi loss during the annealing. This is supported by the theoretical modelling that shows a significant Bi diffusion within the film. The diffusion is not seen in sample SP1-Bi-A (Section C.2). A possible explanation for this increased diffusivity could be that nanocrystalline iron oxide has formed after the initial annealing (SP2), and that the Bi diffusivity is higher in this iron oxide film when compared with the unknown phases that exist before annealing (SP1).

The loss of $\mathrm{Bi}$ after annealing was not seen in Chapter 4 for $\mathrm{BiFeO}_{3}$ thin films made by sputtering and annealing. The $\mathrm{BiFeO}_{3}$ film retained most of the bismuth when annealing in an oxygen atmosphere. This suggest that Bi ions form weaker bonds when they are implanted into iron oxide then when they are sputtered with iron and oxygen. The two samples are different material and the diffusivity of Bi may suggest that different phases are formed on the different samples (sputtered $\mathrm{BiFeO}_{3}$ and $\mathrm{SP} 2-$ Bi-A).

The XRD analysis of SP2-Bi-A is shown in Figure 5.5.2 (a). Hematite (JCPDS 00033-0664) can be clearly seen in the XRD data indicating that this phase is still present. The XRD intensities are weaker in the case of the SP2-Bi-A sample then the sample SP1 and SP2, which suggests that the hematite fraction has reduced after Bi implantation and annealing. Figure 5.5.2 (b) shows a hematite (104) peak and a pseudo Voigt fit. For this fit $\mu$ is 1 , which indicates a Lorentzian peak. The full width half maximum is the same as that found in the annealed $\mathrm{FeO}_{x}$ film (SP2) and indicates that the average nanoparticle sizes are the same.

Figure 5.5.2 (a) displays sign of signal from magnetite and/or maghemite. Both magnetite and maghemite have similar lattice parameters and similar X-ray diffraction peaks. Figure 5.5 .2 (c) shows an expanded XRD plot centred at $50^{\circ}$. That figure is displaying the magnetite (400) peak as well as the hematite (224) peak. There is also another peak that is likely to be the FeO (024) peak. The FeO peak is shifted due to strain. Also, shown in the figure is three pseudo Voigt fits. The magnetite peak has a full width half maximum of $0.94^{\circ}$ and a Scherrer analysis implies an average nanoparticles size of $11 \pm 4 \mathrm{~nm}$. The $\mu$ parameter is 0 that indicates a Gaussian peak. 


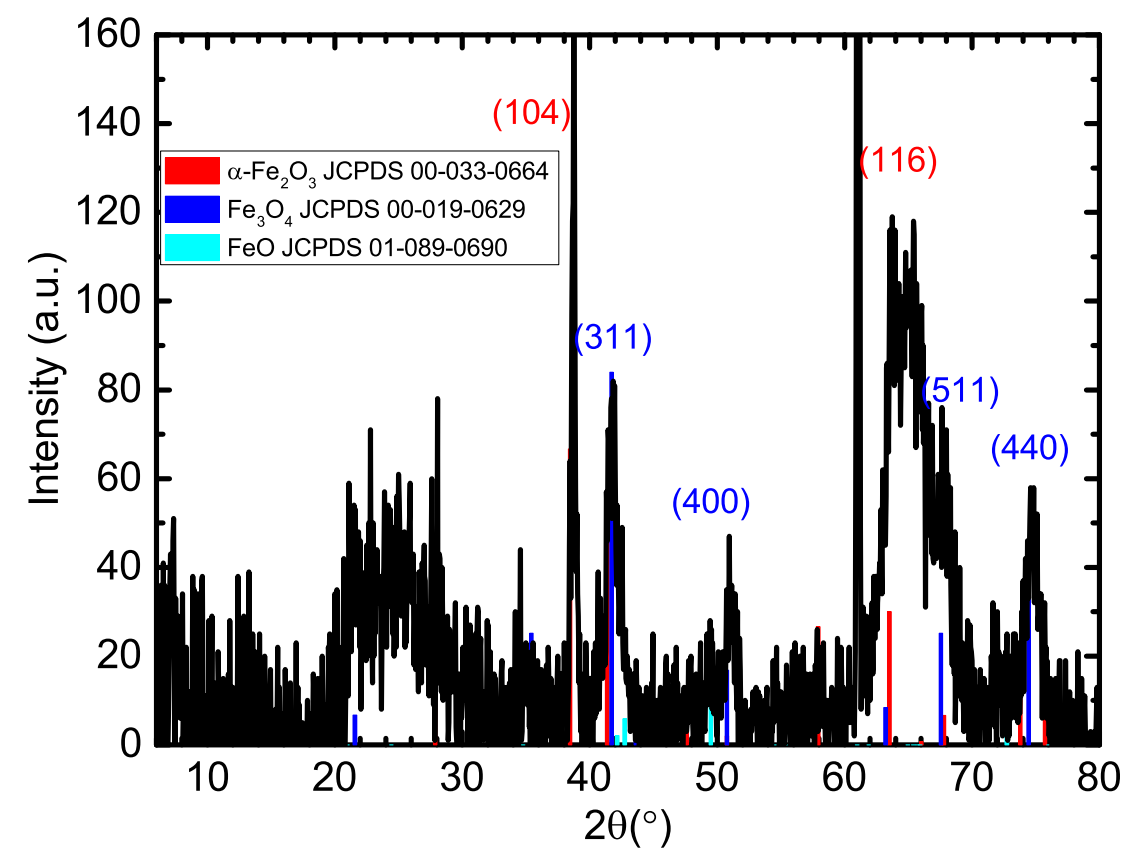

(a) XRD, grazing angle $5^{\circ}$, of SP2-Bi-A on $\mathrm{SiO}_{2}$.

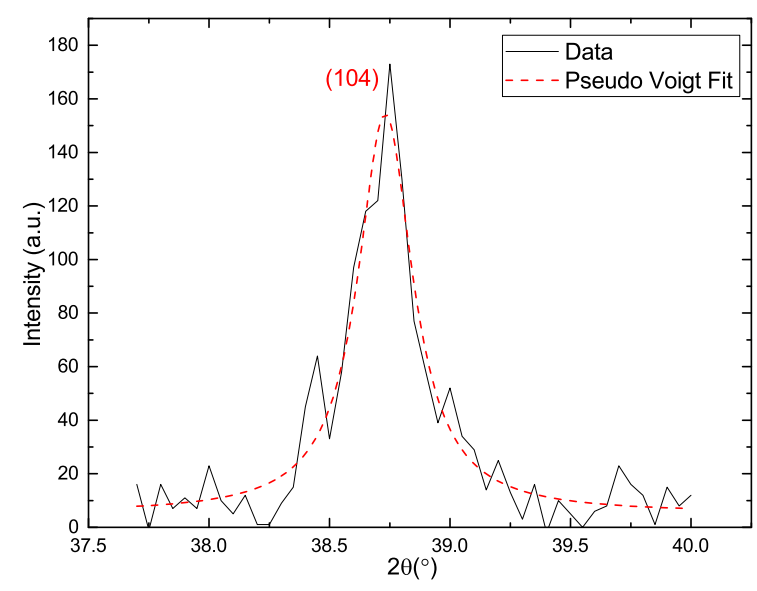

(b) Pseudo Voigt fit centred at $38.7^{\circ}$.

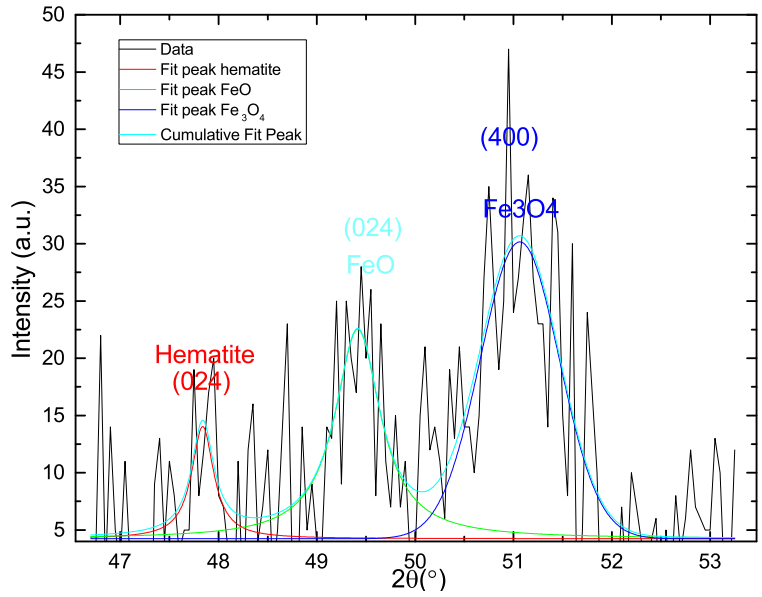

(c) Voigt fit centred at $47.8^{\circ}, 49.4^{\circ}, 51^{\circ}$.

Figure 5.5.2: $\mathrm{XRD}$, grazing angle $5^{\circ}$, of $\mathrm{SP2}-\mathrm{Bi}-\mathrm{A}$ on $\mathrm{SiO}_{2}$. Also, seen in this figure are JCPDS 00-033-0664 (hematite), JCPDS 00-019-0629 (magnetite) and JCPDS 01-089-0690 (FeO). (b) Magnified around the hematite (104) peak. Also shown is a pseudo Voigt fit centred at $38.7^{\circ}$ with a full width half maximum of $0.27^{\circ}$. (c) Magnified around $50^{\circ}$. Also seen is the hematite peak (024) and Voigt fit function centred at $47.8^{\circ}$ with a full width half maximum of $0.27^{\circ}$, $\mathrm{FeO}$ peak (024) with a Voigt function centred at $49.4^{\circ}$ and a full width half maximum of $0.56^{\circ}$ and $\mathrm{Fe}_{3} \mathrm{O}_{4}$ with a Voigt function centred at $51^{\circ}$ and a full width half maximum of $0.94^{\circ}$. 
The hematite peak could be fitted with the same full width half maximum seen for the (104) peak. The peak in the middle had a full width half maximum of $0.56^{\circ}$ and average particle size was $25 \pm 5 \mathrm{~nm}$ as estimated from the Scherrer equation. The $\mu$ parameter is 1 that indicates a Lorentzian peak. Thus, the XRD analysis shows that there are some small magnetite and $\mathrm{FeO}$ nanoparticles. The hematite nanoparticle size has not changed after implanting $\mathrm{Bi}$ and this could because the remaining hematite phase is located further into the film as seen in the TEM data discussed below, Figure 5.5.3.

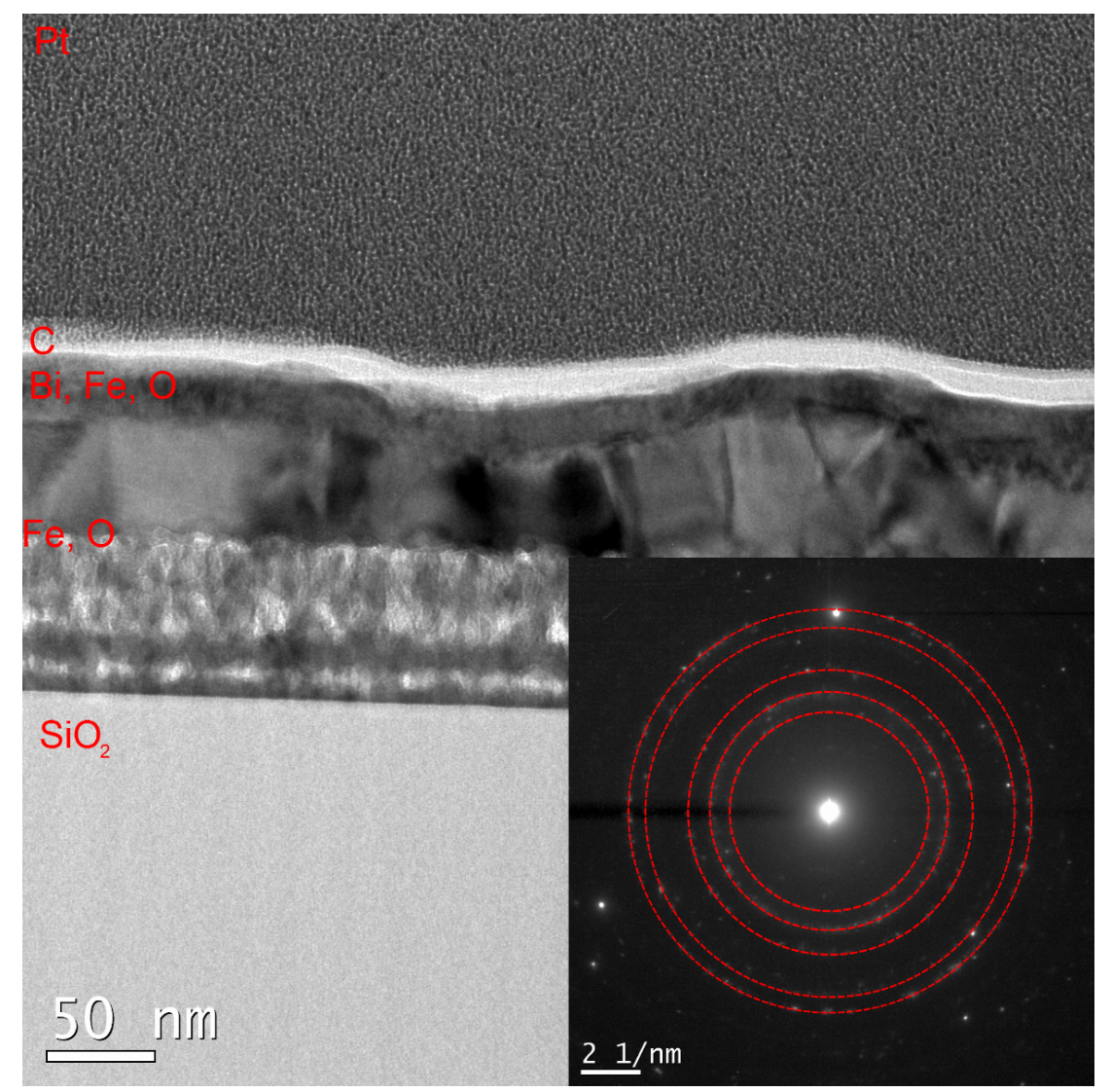

Figure 5.5.3: TEM image of SP2-Bi-A. Also, shown in this figure are the $\mathrm{SiO}_{2}$ substrate, $130 \mathrm{~nm}$ of iron oxide deposited film (label Fe, O), and $25 \mathrm{~nm}$ of Bi implanted into iron oxide (label $\mathrm{Bi}, \mathrm{Fe}, \mathrm{O}$ ). $\mathrm{A}$ carbon and platinum layer, not part of the sample, were deposited for TEM analysis. Inset shows the SAED analysis. The d-spacing, red rings, are matching magnetite or maghemite.

Figure 5.5.3 shows the TEM image of SP2-Bi-A. Also, shown in this figure are the $\mathrm{SiO}_{2}$ substrate, $130 \mathrm{~nm}$ of iron oxide deposited film, and $25 \mathrm{~nm}$ of bismuth implanted into iron oxide. There is also a carbon and a platinum layer, which are not part of the sample. The coating was applied as the preparation for TEM analysis. The bottom layer of the iron oxide shows more order and structure then the TEM of SP1-Bi-A (Section C.2), suggesting that a polycrystalline material might have formed. The top layer of the iron oxide is more uniform, indistinguishable apart from a few dark columns. This might suggest that area of the film is highly distorted or amorphous. 
The implanted bismuth ions seem to be more concentrated in the top $30 \mathrm{~nm}$ layer, with possible diffusion into the $60 \mathrm{~nm}$ area. The sample analysed by TEM is different than the sample used for the other analyses in this chapter, that can be explained a few differences. The TEM analysis is made on a sample implanted with Bi at $56 \mathrm{keV}$. The D-Trim simulation predicted an implantation depth of $25 \mathrm{~nm}$ and the RBS analysis does show bismuth diffusion toward the inner layers. The diffusion could explain the $60 \mathrm{~nm}$ layer containing bismuth.

Also, shown in Figure 5.5.3 is the SAED analysis, with 5 red rings enhancing the diffraction contrast. The SAED rings are almost complete, however the contrast is still faint. The 5 red rings displayed on the SAED come from magnetite or maghemite. The concentration of bismuth might be insufficient to be seen in this analysis. The 5 rings are linked to the 5 most intense XRD diffractions of magnetite and maghemite. The most intense XRD peaks of hematite are not visible in the d-spacing. Those rings are unlikely diffracted from a hematite phase.

Also, shown in the inset of Figure 5.5.3 are a few white spots not linked to any ring, which might suggest other material was formed. Both implanted bismuth films (SP1-Bi-A and SP2-Bi-A) have a similar SAED image. Both materials show diffraction rings that could be linked to $\mathrm{Fe}_{3} \mathrm{O}_{4}$ or $\gamma-\mathrm{Fe}_{2} \mathrm{O}_{3}$, and both display white spots that could indicate another crystalline material is present in the film. Further comparison between the TEM images shows that both films are very similar, with a bismuth-implanted region and an iron oxide layer having one half well-structured and the other half almost amorphous. The similarities are unexpected because RBS analysis clearly shows a bismuth diffusion in the sample SP2-Bi-A, however this diffusion is not seen in the sample SP1-Bi-A. This occurs while both TEM images do not show bismuth diffusion in the films.

The XRD displays different iron oxide as this was possible to suspect from the RBS analysis, while the TEM analysis suggest that the iron oxides phases are mainly magnetite and maghemite. The SAED diffraction peaks show the presence nanocrystalline phases.

The Raman spectrum, Figure 5.5.4, of SP2-Bi-A mainly displays hematite. Also, shown in this figure are the as-made iron oxide film (SP1) and the annealed iron oxide film (SP2) for comparison. The Raman spectrum displays $\alpha-\mathrm{Fe}_{2} \mathrm{O}_{3}$ hematite that is also seen in the XRD data. The inset shows that the peak widths do not change. The hematite Raman intensities are lower after the bismuth implantation and the annealing process that might be expected from the XRD analysis. The XRD data is inconclusive 
on the presence of a bismuth phase, however Raman spectroscopy confirms the presence of $\mathrm{Bi}$ in the sample. The limit of detection and the low concentration of bismuth could explain the absence of $\mathrm{Bi}$ XRD diffraction peak in Figure 5.5.2. While magnetite is visible in the XRD and SAED analysis, the Raman spectrum exclude the presence of magnetite, this suggest either $\mathrm{Fe}_{3} \mathrm{O}_{4}$ is in small concentration. As the Raman shift at $\sim 705 \mathrm{~cm}^{-1}$ is not seen in Figure 5.5.4, the Raman spectrum analysis suggest that maghemite is present in this film or the concentration of maghemite is smaller than the concentration of magnetite. Raman spectrum confirm the presence of bismuth phase, however $\mathrm{BiFeO}_{3}$ phases does not form unless the concentration of that phase is below the limit of detection.

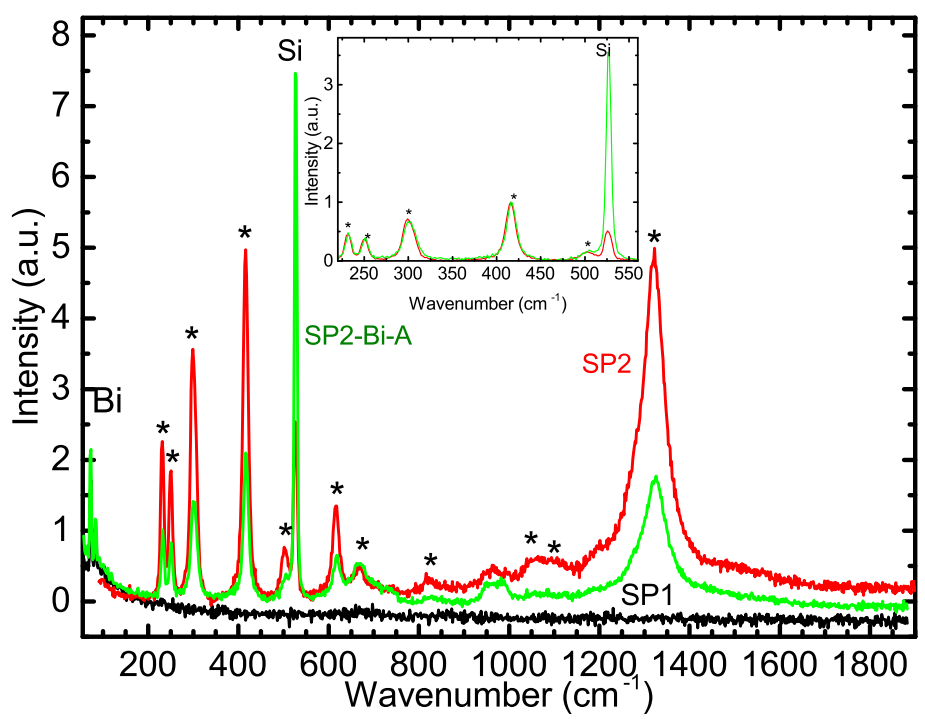

Figure 5.5.4: Raman spectrum of SP2-Bi-A. The bismuth-implanted film (green) is compared with SP1 (black) and SP2 (red). The Raman spectrum shows hematite (stars) and silicon substrate at $521 \mathrm{~cm}^{-1}$. Inset shows a comparison of normalised SP2 and SP2-Bi-A, normalisation based on the hematite $414 \mathrm{~cm}^{-1}$ Raman shift.

The silicon peak after bismuth implantation (SP2-Bi-A) is more intense than the silicon peak before implanting bismuth and annealing, as-made film (SP1). This suggests that the absorption coefficient of the film is lower after $\mathrm{Bi}$ implantation followed by an annealing (SP2-Bi-A). This is not expected since metallic Bi is seen in the Raman data, SP2-Bi-A Figure 5.5.4. This could be caused by the Bi metal fraction being too low to affect the absorption coefficient. The absorption changes are due to a change in the magnetite fraction as there is more maghemite after the Bi implanting and annealing.

The Raman active mode before (SP1 and SP2) and after implanting Bi (SP2-Bi-A) are from hematite and $\mathrm{Bi}$ is also observable after the implantation. Other iron oxides were expected from the XRD analysis. The absence of other iron oxide signal can be explained by the quantity of hematite being more significant than the concentration 
of magnetite and maghemite. The measurement of magnetic properties will provide more information on the phase fraction of magnetite and maghemite.

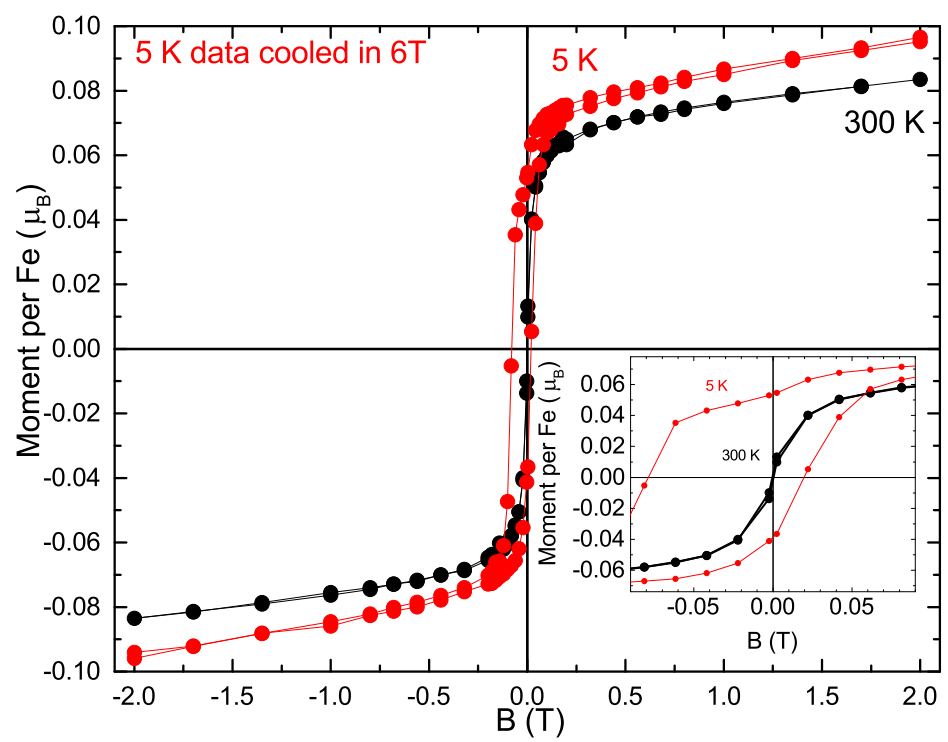

Figure 5.5.5: Magnetic moment measurement carried out on SP2-Bi-A. The field loop shows an enhanced magnetic moment at saturation at low temperature. The inset shows a hysteresis loop at low temperature (red) that is not clearly visible at ambient temperature (black). The field loop is plotted from $-2 \mathrm{~T}$ to $2 \mathrm{~T}$. The $5 \mathrm{~K}$ temperature measurement was taken after cooling the sample in a $6 \mathrm{~T}$ applied field, resulting in an exchange bias as seen in the inset.

The magnetic moment of SP2-Bi-A is shown in Figure 5.5.5. This film has a magnetic moment of $0.096 \mu_{B} / F e$ at $6 \mathrm{~T}$ and the saturation point does not seem to be reached. The magnetic moment is higher than the reported value for antiferromagnetic $\mathrm{BiFeO}_{3}$ which suggests that the film does not have a significant $\mathrm{BiFeO}_{3}$ fraction. This is consistent with the XRD and Raman analysis where $\mathrm{BiFeO}_{3}$ is not observed. The $6 \mathrm{~T}$ moment is $26 \%$ of that found in the $500{ }^{\circ} \mathrm{C}$ annealed film. This value is $4 \%$ of that seen in the as-made $\mathrm{FeO}_{x}$ film (SP1). This could suggest that the magnetic moment is due to a very small fraction of the ferromagnetic phase seen in the as-made film (SP1). The inset shows that there is still an exchange bias that will be discussed with the Figure 5.5.8 (a). The SP2-Bi-A film shows ferrimagnetic magnetite and/or maghemite in the XRD and SAED data. Thus, they could also be contributing to the moment. In that case, there would be $\sim 7 \%$ of magnetite and/or maghemite. The moment does not saturate even at $6 \mathrm{~T}$ that could suggest an antiferromagnetic phase and/or the present of a disordered magnetic phase.

Figure 5.5.6 displays the temperature-dependent response under the influence of a $6 \mathrm{~T}$ applied magnetic field of SP2-Bi-A. The moment per Fe has a low temperature upturn that was not seen in the other films. This could arise from unordered Fe moments 
for this reason the saturation moment was fitted to $m_{F e}=m_{b} *\left(1-D \cdot x^{n}\right)+C_{0} /((x-\theta))$ where the first term is Bloch's $3 / 2$ function and the second term is the Curie-Weiss equation. This gives a good fit to the data with $D=8 \times 10^{-5} \mathrm{~K}^{-1.5}, C_{0}=0.075 \mathrm{~K}$, and $\theta=-7 \pm 2 \mathrm{~K}$.

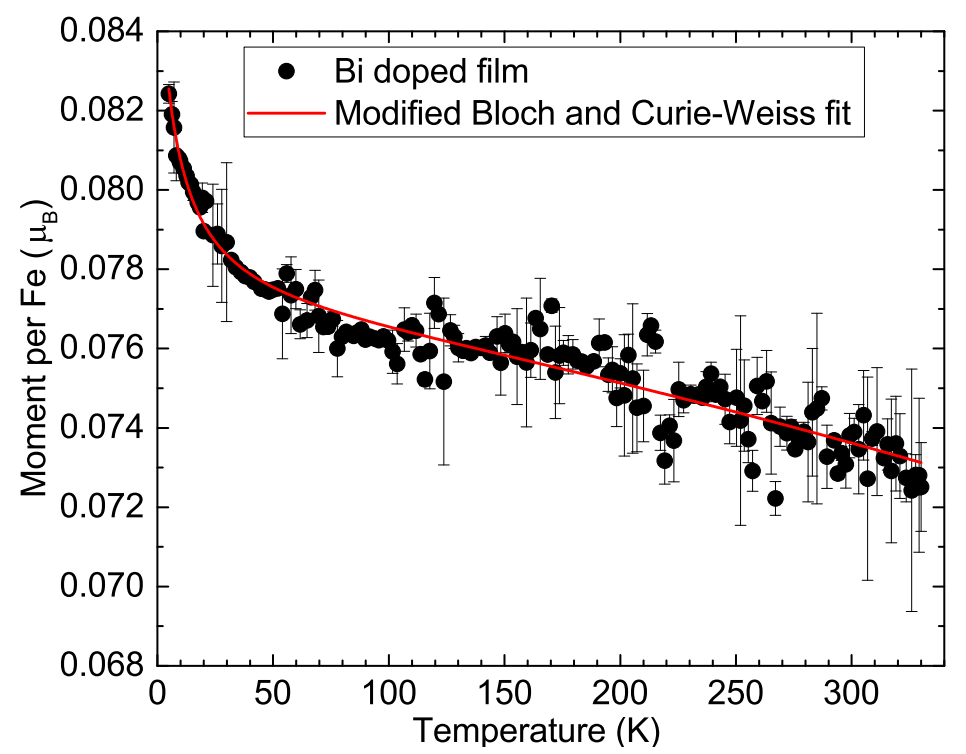

Figure 5.5.6: Temperature dependence at $6 \mathrm{~T}$ of the magnetic moment per Fe on SP2-Bi-A.

Also shown is a fit to the modified Bloch function (solid curve).

The fitted $D$ is about half the value seen in the $500{ }^{\circ} \mathrm{C}$ annealed $\mathrm{FeO}_{x}$ film (SP2). However, The $D$ value of SP2-Bi-A is very close to the value seen in the as-made film (SP1). This may suggest that magnetic moment is due to a small fraction of the unknown ferromagnetic phase that occurs in the as-made film (SP1).

The negative Curie-Weiss temperature suggests that there is an antiferromagnetic exchange between the unordered Fe moments. The Curie-Weiss function can be written as:

$$
\frac{m}{N \mu_{B}}=\frac{P_{\text {eff }}^{2} \mu_{B} B}{3 k_{B}(T-\theta)} .
$$

where $m$ is the measured magnetic moment, $N$ is the number of Fe atoms, $\mu_{B}$ is the Bohr magneton, $P_{\text {eff }}$ is the effective moment per Fe, $B$ is the applied magnetic field of $6 \mathrm{~T}, k_{B}$ is the Boltzmann constant, $T$ is the varying temperature and $\theta$ is the Curie temperature. Thus, $C_{0}=P_{\text {eff }}^{2} \times \mu_{B} \times B /\left(3 k_{B}\right)$ and hence $P_{\text {eff }}^{2}=0.86 \times\left(C_{0}\right)^{0.5}$. Using the measured $C_{0}$ then the effective moment per Fe is $0.24 \mu_{B}$. This is far less than expected for $\mathrm{Fe}^{2+}, \mathrm{Fe}^{3+}$, or metallic Fe. The theoretical effective moment for different Fe spin configurations can be calculated from $P_{\text {eff }}=\mathrm{g}(\mathrm{S}(\mathrm{S}+1)) 1 / 2$. $\mathrm{Fe}^{2+}$ in different spin 
configurations can have $\mathrm{S}=2$ or $1 / 2$ or 0 so $P_{\text {eff }}$ would be $4.90 \mu_{B}, 1.73 \mu_{B}$ or $0 \mu_{B}$. Different $\mathrm{Fe}^{3+}$ spin configurations with $\mathrm{S}=5 / 2$ or $1 / 2$ or $3 / 2$ then $P_{\text {eff }}$ of $5.91 \mu_{B}, 1.73 \mu_{B}$ or $3.87 \mu_{B}$. If the magnetic moment was from magnetite that has one $\mathrm{Fe}^{2+}$ for every $\mathrm{Fe}^{3+}$ then the effective moment should be greater than $1.41 \mu_{B}$ if all the Fe moments were magnetically ordered, which is far greater than the measured value. A similar analysis for maghemite would reach the same conclusion. Thus, a low effective moment for Fe is consistent with significant antiferromagnetic order from phases that include hematite seen in the XRD and Raman data.

Figure 5.5.7 displays the ZFC-FC at $10 \mathrm{mT}$ of SP2-Bi-A. There is a hysteresis up to the highest measured temperature and this indicates that there is no evidence for superparamagnetism. Magnetite nanoparticles are seen in the XRD and SAED data. However, the average particle sizes, estimated with the XRD analysis and the Scherrer's equation (equation 3.3.11) are too large for a blocking temperature to occur below ambient temperature (equation 2.1.6). There is no evidence for the low temperature antiferromagnetic phase seen in the as-made sample (SP1), however the annealed $\mathrm{FeO}_{x}$ film (SP2) does show traces of that phase.

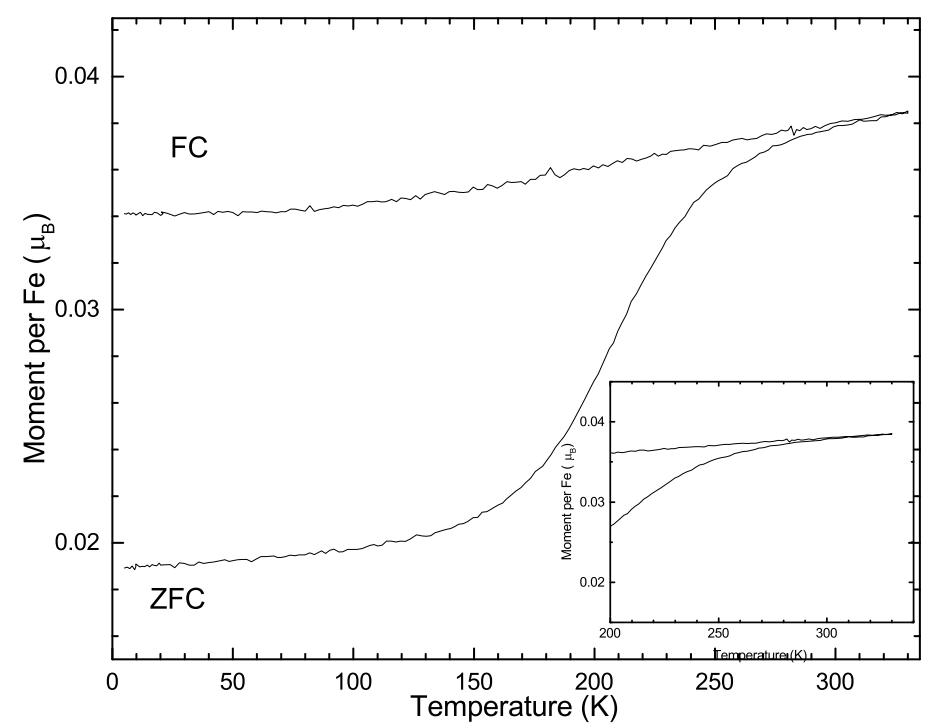

Figure 5.5.7: ZFC-FC carried out on SP2-Bi-A at $10 \mathrm{mT}$. Also, shown in the inset of this figure is a magnified scale of the FC measurement near ambient temperature.

The Figure 5.5.8 (a) displays the exchange bias of SP2-Bi-A (circles). The annealing is done under an $\mathrm{O}_{2}$ atmosphere. The exchange bias measurement was taken after cooling the sample in a $6 \mathrm{~T}$ applied field from $330 \mathrm{~K}$. The exchange bias disappears above $\sim 100 \mathrm{~K}$. This can also be seen for the as-made $\mathrm{FeO}_{x}$ film (SP1) where the data points follow a similar curve. This suggests that the exchange bias is from the same ferromagnetic and antiferromagnetic phases in both films. Also shown is the exchange bias for the $500{ }^{\circ} \mathrm{C}$ annealed film (SP2). The exchange bias of SP2 is weaker than the 
exchange bias of SP2-Bi-A, however both have $\left|B_{e x}\right| \sim 0$ at $\sim 100 \mathrm{~K}$.

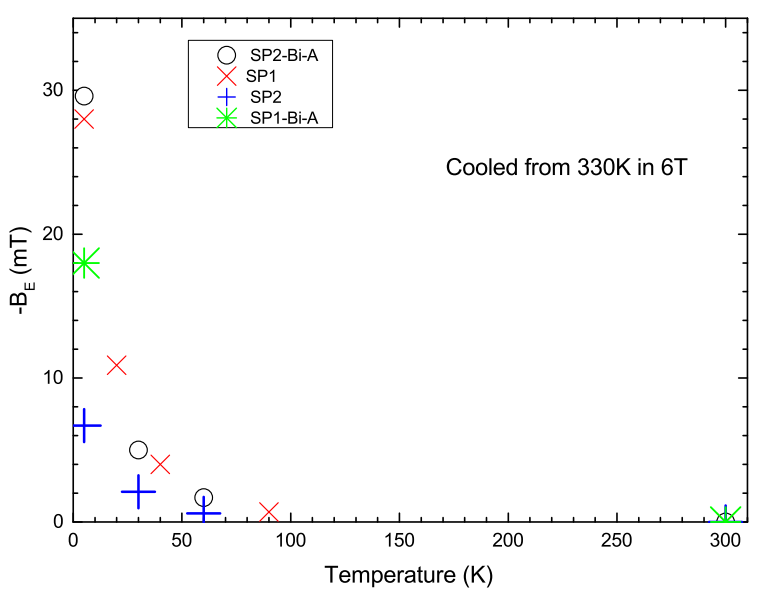

(a) Exchange bias of SP2-Bi-A (circles).

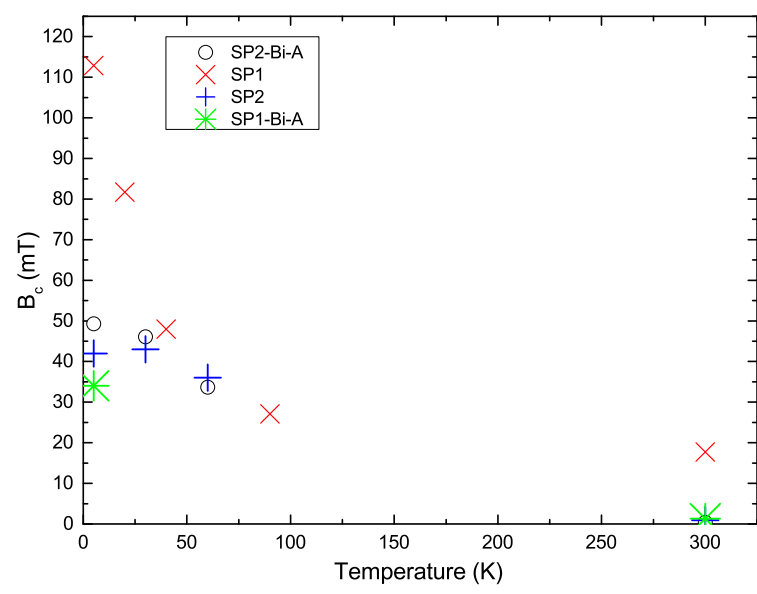

(b) Coercive field of SP2-Bi-A (circles).

Figure 5.5.8: Exchange bias and coercive field measurements were taken after cooling from $330 \mathrm{~K}$, the sample in a $6 \mathrm{~T}$ applied field. Also, shown in this figure is the exchange bias and oercive field of the annealed $\mathrm{FeO}_{x}$ film (SP2) (plus symbols), as-made iron oxide film (SP1) (crosses), and SP1-Bi-A (green symbols).

The Figure 5.5.8 (b) displays the coercive field of SP2-Bi-A (circles). The annealing was done under an $\mathrm{O}_{2}$ atmosphere. The coercive field measurements were taken after cooling from $330 \mathrm{~K}$ in a $6 \mathrm{~T}$ applied field. Also shown are the coercive fields from the other films. The temperature dependence of the coercive fields is similar for both $\mathrm{Bi}$ implanted films and the $5 \mathrm{~K}$ and $300 \mathrm{~K}$ data for the annealed $\mathrm{FeO}_{x}$ film (SP2) are also similar. The coercive field is small at $300 \mathrm{~K}$. This can be compared with the as-made $\mathrm{FeO}_{x}$ film (SP1) where the coercive field decreases with increasing temperature and nearly plateaued above $\sim 100 \mathrm{~K}$. This suggests that annealing has the biggest effect on the coercive field and the additional implantation of bismuth does not significantly alter the coercive field.

\subsection{Atomic force microscopy, AFM}

AFM analysis displays the deposition of iron oxides (SP1) as regular and smooth, and the average grain size is estimated to be $4 \mathrm{~nm}$ by Gwyddion software [Nečas and Klapetek, 2012]. The structure is similar to narrow islands. While annealing the iron oxide films (SP2) increases the particle grain size, with an average grain size of $11 \mathrm{~nm}$, annealing provides a degree of mobility to the molecules to form islands with enhanced topography. This seems to lead to an outward diffusion or growth of the iron oxide islands cluster. The mobility or oxidation could be responsible for the new 
surface that grew out of the surrounding material. When compared with Raman and XRD analysis, the SP1 film contains an amorphous material deduced to be iron oxide phases, while after annealing (SP2) the main iron oxide present seems to be $\alpha-\mathrm{Fe}_{2} \mathrm{O}_{3}$ with a possible mix of $\gamma-\mathrm{Fe}_{2} \mathrm{O}_{3}$ and $\mathrm{Fe}_{3} \mathrm{O}_{4}$. The mix of iron oxides could lead to the uneven surface seen in the AFM imaging.

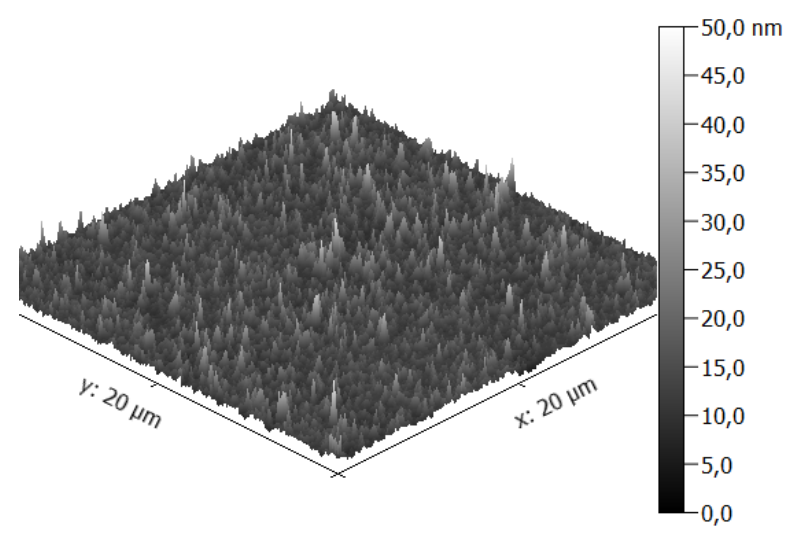

(a) SP1, average grain size $\sim 4 \mathrm{~nm}$.

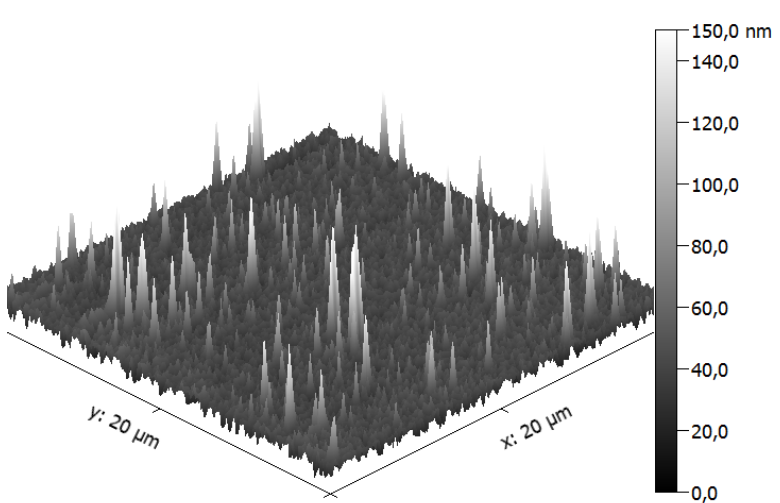

(b) SP2, average grain size $\sim 11 \mathrm{~nm}$.

Figure 5.6.1: AFM, 3-D image of the surface of SP1 and SP2.

Implanting bismuth into an as-made iron oxide film (SP1-Bi-A) increases the damage at the surface of the material; more features are visible after the implantation, however the particles' grain size is similar to SP1, with an average grain size of $3 \mathrm{~nm}$. This could be indicative of molecular bonds breaking under the incident kinetic bismuth interaction with the iron oxide film. From Raman and XRD analysis, the sample mainly contains iron oxides. The bismuth implantation does not seem to have a significant effect on the structure. The differences in topography between the as-made iron oxide (SP1) and the bismuth implanted in as-made iron oxide (SP1-Bi-A) are very small (Figure 5.6.2 (a)).

Annealing before and after implanting bismuth in an iron oxide film (SP2-Bi-A) results in a smooth film, with an average grain size of $8 \mathrm{~nm}$. The material was able to recover from the surface damage by increasing the surface particles' grain size. The biggest change is the loss of the longitudinal growth that was present in the annealed iron oxide film (SP2). The different composition analysis (e.g. XRD and Raman spectroscopy) performed on this film (SP2-Bi-A) confirms that $\mathrm{Fe}_{3} \mathrm{O}_{4}$ is in the film and that $\alpha-\mathrm{Fe}_{2} \mathrm{O}_{3}$ is also present. The AFM analysis highlights the importance of annealing and the order of implantation and annealing. Comparing the SP1-Bi-A sample and the SP2-Bi-A sample, shows a significant modification to the roughness, topography and a difference in grain size. Under the AFM analysis, the as-made iron oxide film (SP1) is similar to SP1-Bi-A, while the annealed iron oxide film (SP2) is similar to SP2-Bi-A. 
In the last comparison, the annealed iron oxide film is smoother than the bismuthimplanted film. This comparison could suggest that the double-annealed film (SP2-BiA) contains a quantity of $\mathrm{Fe}_{3} \mathrm{O}_{4}$, while the film annealed only once (SP1-Bi-A) contains more $\alpha-\mathrm{Fe}_{2} \mathrm{O}_{3}$. This is seen in the magnetic order figures. The bismuth implantation seems to have stopped the growth of iron oxides islands. In this sample with bismuth (SP2-Bi-A), the features seem more uniform and closer to the surface when compared to the annealed iron oxide film (SP2) without implantation.

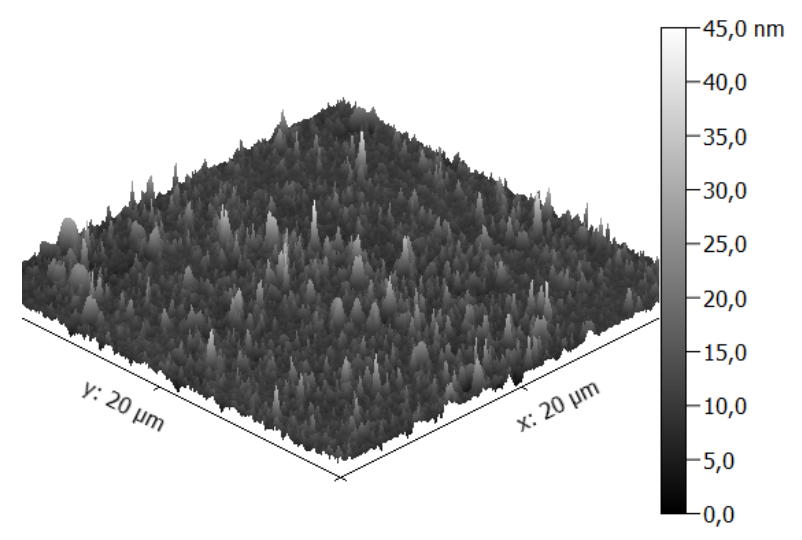

(a) SP1-Bi-A, average grain size $\sim 3 \mathrm{~nm}$.

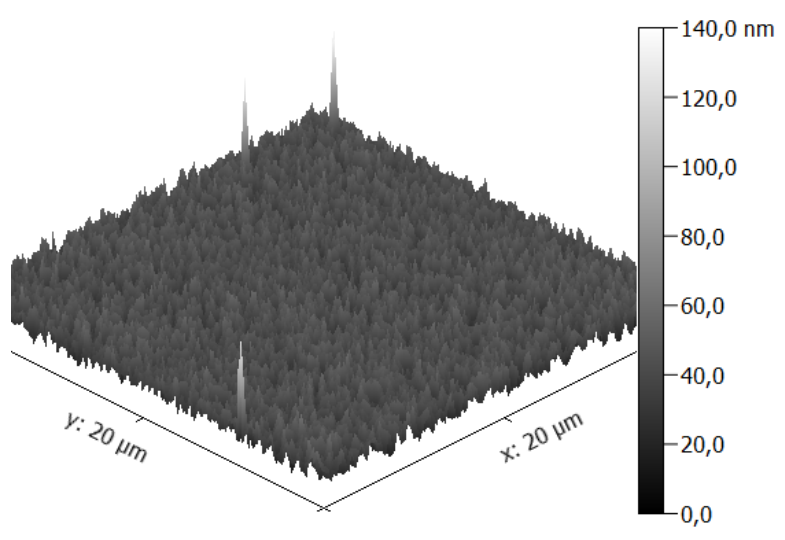

(b) SP2-Bi-A, average grain size $\sim 8 \mathrm{~nm}$. Figure 5.6.2: AFM, 3-D image of the surface of SP1-Bi-A and SP2-Bi-A.

\subsection{Conclusion}

In conclusion, implanting bismuth ions into iron oxide thin film modifies the magnetic moment, the topography and the surface roughness. Implanting bismuth changes the iron oxide phases in the film and the crystal formation. Although no $\mathrm{BiFeO}_{3}$ was seen after implanting bismuth into iron oxide thin film, further work is required to synthesise a multiferroic material. Interesting magnetic and structural modifications were seen after implanting bismuth ions. The iron oxide sputtered as-made film (SP1) seems to contain a small, $5 \mathrm{~nm}$, unknown phase with FeO, $9 \mathrm{~nm}$. This unknown phase displays a strong magnetic-ordered. This new phase could be in a metallic form. The exchange bias confirms that film contains a ferromagnetic and antiferromagnetic interface, the antiferromagnetic phase is likely to be $\mathrm{FeO}$ and the ferromagnetic phase could be this unidentified phase with a possible contribution from magnetite. Magnetite is expected and the temperature dependence also suggest an amount of $\mathrm{Fe}_{3} \mathrm{O}_{4}$ is present in the film.

Annealing the iron oxide film leads to a material mainly composed of $\alpha-\mathrm{Fe}_{2} \mathrm{O}_{3}$, 
however other iron oxides could still be present in the film. If $\mathrm{Fe}_{3} \mathrm{O}_{4}$ and $\gamma-\mathrm{Fe}_{2} \mathrm{O}_{3}$ are present, they must be in low quantities and small in size or amorphous to not be seen in Raman and XRD analysis. The annealing significantly reduced the magnetic moment, $15 \%$ less, suggesting that the highly magnetic phase in the as-made iron oxide film (SP1) is reduced with annealing (SP2). An exchange bias is seen after annealing where the source is likely to be from the same phase that were found in the as-made iron oxide film.

SP2-Bi-A clearly shows the presence of hematite phase although that phase's concentration was reduced after implanting bismuth and followed by an annealing. Magnetite and maghemite, possibly $7 \%$, are also present in this film. A very small fraction of the ferromagnetic phase found in the as-made iron oxide film (SP1), maybe $4 \%$ or less, could still be present in SP2-Bi-A.

Comparing SP2-Bi-A with SP1 and SP2 magnetic moment suggest that the same antiferromagnetic and ferromagnetic phases are present in each film. The comparison between SP2-Bi-A, SP1 and SP2 confirms that the coercive field is influence by the annealing of the films, however the bismuth implantation does not have a significant effect on the coercive field. The implanted bismuth ions have a greater diffusivity than the bismuth in the $\mathrm{BiFeO}_{3}$ sputtered film. Implanting bismuth in the annealed iron oxide further increases the diffusivity of bismuth after the second annealing. So, the magnetic ordering is mainly caused by iron oxides, a ferromagnetic and antiferromagnetic ordering is discussed and the iron oxide phases induce the exchange bias, which confirms the exchange bias analysis of the $\mathrm{BiFeO}_{3}$ sputtered thin film which could come from iron oxide phases present in the sputtered film.

For future work, some parameters should be study further, like the annealing temperature and duration, to understand the formation of the different phases. With the aim of synthesising $\mathrm{BiFeO}_{3}$, increasing the implantation energy or implanting iron ions into a bismuth oxide film could be of interest. In order to focus on the synthesis of $\mathrm{BiFeO}_{3}$, implanting bismuth into a thinner iron oxide film would also be of interest. 


\section{Chapter 6}

\section{Summary and outlook}

This chapter summarises the work carried out and provides an outlook for future research that can extend the analysis and potentially lead to new magneto-electric, magneto-optical or magneto-electric-optical devices, such as magneto-electric random access memory (MeRAM) and other micro-chips for computers.

\subsection{Summary}

This thesis is about the study of the synthesis of nanostructured multiferroic films and their structural, electronic and magnetic properties. A multiferroic $\mathrm{BiFeO}_{3}$ thin film was successfully synthesised by ion beam sputtering and subsequent annealing at $500{ }^{\circ} \mathrm{C}$ for 15 minutes in an oxygen atmosphere. The magnetic properties are primarily due to secondary phases of iron oxides that induced an exchange bias and spin glass. Ferroelectricity is confirmed by the piezoelectric response which also confirms the proper phase of $\mathrm{BiFeO}_{3}$ is formed. Another approach to synthesise $\mathrm{BiFeO}_{3}$ thin film was attempted by implanting bismuth ion implantation into iron oxide thin film. The synthesis resulted in the formation of a mix of ferromagnetic and antiferromagnetic materials, however there was no evidence that $\mathrm{BiFeO}_{3}$ was formed.

To conclude the key findings of the two different ion beam synthesis techniques. The ion beam sputtering of $\mathrm{BiFeO}_{3}$ successfully synthesis multiferroic nanocrystalline $\mathrm{BiFeO}_{3}$ films. The magnetic properties are primarily due to iron oxides which induced an exchange bias. The ferroelectricity is confirmed by piezoelectric response and this ferroelectric property also confirms the proper phase of $\mathrm{BiFeO}_{3}$ is synthesis after an annealing at $500{ }^{\circ} \mathrm{C}$. The bismuth implanted film mainly contains iron oxide which causes the exchange bias.

$\mathrm{BiFeO}_{3}$, rhombohedral, is a multiferroic material with potential application in small scale electronic, opto-electronic applications. The films synthesis by ion beam 
sputtering displayed an exchange bias up to $250 \mathrm{~K}$. Ambient temperature exchange bias material has potential applications as there is still a quest to achieved high temperature exchange bias. Triple ion implantation was also carried out and the preliminary results suggest those films to mainly contains iron oxide even though no exchange bias was observed.

Chapter 4, ion beam sputtering of $\mathrm{BiFeO}_{3}$, showed that this material is multiferroic at ambient temperature. As described in that chapter, the $\mathrm{BiFeO}_{3}$ thin film contains some iron oxide secondary phases, so synthesising a pure monocrystalline material would require more work and optimisation of the synthesis technique. One possibility to reduce the impurities is to increase the temperature of the substrate during the ion beam sputtering of $\mathrm{BiFeO}_{3}$. Due to the quantity of iron oxide in the film, the ferro-electrical properties of $\mathrm{BiFeO}_{3}$ in a superparamagnetic state was not studied. $\mathrm{BiFeO}_{3}$ displayed multiferroic properties and an enhance magnetic response generated by some iron oxide phases. The Magneto-Optical Kerr Effect (MOKE) confirms the great optical properties with one of the highest Verdet constants reported. The concentration of $\mathrm{BiFeO}_{3}$ is also tuned by the annealing temperature while increasing the annealing duration result in the formation of $\mathrm{Bi}_{2} \mathrm{Fe}_{4} \mathrm{O}_{9}$. After discussing in detail the presence of secondary phases in $\mathrm{BiFeO}_{3}$, the main secondary phases were confirmed to be iron oxides. The effect of iron oxides on the film is to enhance the magnetic moment at saturation and to change the magnetic ordering of the film. Some iron oxides are weakly ferromagnetic, ferrimagnetic and antiferromagnetic. These properties can be seen through magnetic analyses. $\mathrm{BiFeO}_{3}$ is ferroelectric and antiferromagnetic, resulting in an ambient temperature intrinsic multiferroic. The iron oxide fraction is up to $40 \%$ of the films. The electrical ordering comes from $\mathrm{BiFeO}_{3}$ nanoparticles, while the magnetic ordering comes from a mix of $\mathrm{BiFeO}_{3}$ and iron oxides phases. Increasing the iron content of a multiferroic or piezoelectric material has already been studied to build an extrinsic multiferroic material that displays a stronger magnetic moment. In the end, nano-structured $\mathrm{BiFeO}_{3}$ was successfully achieved.

The synthesis of $\mathrm{BiFeO}_{3}$ phases by bismuth implantation of iron oxide, Chapter 5 , led to an interesting study of the limited effect of bismuth on iron oxide. The iron-oxygen bond resists the implantation of bismuth at high energies at a depth of up to $50 \mathrm{~nm}$. The films are almost entirely made of iron oxides. The as-made iron oxide film is a mix of iron oxide phases, not clearly identified, and FeO. The main iron oxide present after annealing this film is hematite, however magnetite, maghemite, and $\mathrm{FeO}$ might also be present. The annealing reduces the fraction of the magnetic phases from the as-made iron oxide film. After bismuth implantation, non-magnetic materials like bismuth or bismuth oxide are also present. The ferromagnetic phase is reduced when 
the as-made iron oxide is implanted with bismuth and annealed. That phase is further reduced when the annealed iron oxide film is implanted with bismuth and annealed. The implantation of bismuth in an iron oxide film does not significantly modify the coercive field while annealing the film does. The bismuth implantation result in an increase bismuth ions diffusivity when compared to a sputter thin film. The diffusivity is even greater if the iron oxide film is annealed before implanting bismuth ions. Bismuth implantation seems to stop the growth of iron oxide phases.

\subsection{Outlook}

This section will outline possible future projects, which would add further information and improve the scientific knowledge of the presented results. Further measurements that may include leakage current, magneto-electric coupling for potential application devices and confirms a multiferroic model. The study of elements doping and material modifications dependency on $\mathrm{BiFeO}_{3}$ thin film can increase the knowledge of the structural, magnetic and electrical properties and their influence.

To improve the ion beam sputtering synthesis of $\mathrm{BiFeO}_{3}$, Chapter 4 , several possibilities are available like investigating the nanostructured film, investigating the synthesis a secondary phase free thin film and synthesis a monocrystalline thin film.

The Investigation of the nanostructured film could rely on Core-shell study as iron oxide phases are present an exchange bias could be explained with magnetitemaghemite core-shell structure. Core-shell interaction can be seen with SEM, detailing the nanoparticles in high resolution. The iron oxide present in the film enhances the magnetic saturation moment, which is advantageous for building application devices. The nanostructure can be studied by modifying significantly the structure with doping ions, e.g. Xe, Fe, Cr. Chromium doping is known to reduce the leakage current, however the interaction model is not clearly identified. As iron oxide phases are responsible for the magnetic moment, increasing the amount of iron would be interesting to analyse the magnetic response and ferroelectricity property. For application device, enhancing the magnetic moment in a multiferroic material is of high interest. The magnetic moment and the piezoelectric coefficient dependency to the thickness of the film is another subject to be further studied.

The investigation of a secondary phase free thin film should be search to further understand the multiferroic model and confirming the existence of intrinsic multifer- 
roic. This research might focus on the optimisation of annealing temperature, annealing duration and the sputter target quality that could limit the secondary phase in the deposited film.

The investigation of the synthesis of a monocrystalline thin film would require to selected the substrate or stack of contact layer that limit strain and stress on the deposited film. Depositing thinner film on a smaller area would increase the probability to form a monocrystalline film. An optimisation of the cleaning and storage procedure would be necessary to reduce contamination that could generate disorder. An optimisation of the synthesis technique could be attempted, however ion beam sputtering is not known to deposit monocrystalline film, ripple effects have been reported. To increase the probability of deposing a monocrystalline film, an optimisation of the technique and conditions could be studied like increasing the pressure or the substrate temperature during sputtering, also the annealing could be done with a slower temperature ramp up and ramp down to limit the thermal stress.

The interest of bismuth implanted iron oxide thin film came from the possibility to form $\mathrm{BiFeO}_{3}$ and magnetic nanoparticles. The results suggest that the formation of $\mathrm{BiFeO}_{3}$ could be unlikely with this approach, an optimisation of this procedure is still required to completely dismiss the possibility of forming $\mathrm{BiFeO}_{3}$ nanoparticles. Although, the results show the formation of magnetic nanoparticles which can be interesting for applications. The optimisation suggested are the implantation energy (increase the implantation energy might increase the quantity of bismuth bonding with iron oxide), implantation order (iron doping of bismuth oxide could be a more promising doping technique as iron creates bond with oxygen easily), annealing temperature and duration by doing intermediary condition this could lead to an understanding of the formation and atoms binding. The effect at the surface of the film could be interesting to study, that study could be done by reducing the bismuth implantation energy. 


\section{Appendix A}

\section{Publication}

List of publications arising from the thesis work:

- Couture, P.; Leveneur, J.; Williams, G.V.M.; Kennedy, J.V.; Murmu, P.P.; Chong, S.V.; Rubanov, S. An optical, magneto-optical Kerr effect, and piezoelectric force microscopy study of multiferroic $\mathrm{BiFeO}_{3}$ synthesised by ion beam sputtering (in preparation)

- Couture P, Williams GV, Kennedy J, Leveneur J, Murmu PP, Chong SV, Rubanov S. Nanocrystalline multiferroic $\mathrm{BiFeO}_{3}$ thin films made by room temperature sputtering and thermal annealing, and formation of an iron oxideinduced exchange bias. Journal of Alloys and Compounds. 2017 Feb 25;695:30618.

- Couture P, Williams GV, Kennedy J, Leveneur J, Murmu PP, Chong SV, Rubanov S. Multiferroic nanocrystalline $\mathrm{BiFeO}_{3}$ and $\mathrm{BiCrO}_{3}$ thin films prepared by ion beam sputtering. International Journal of Nanotechnology. 2017;14(16):56-65.

List of conference presentations arising from the thesis work:

- Couture, P.; Williams, G.V.M.; Kennedy, J.V.; Leveneur, J.; Murmu, P.P.; Chong, S.V.; Rubanov, S. Structural, magnetic, and optical study of ion beam sputtered multiferroic $\mathrm{BiFeO}_{3}$ nanocrystalline thin films. p. 36 IN: NZIP 2015 Conference, 6-8 July 2015, University of Waikato, Hamilton: Conference handbook. New Zealand Institute of Physics [July 2015]

- Couture, P.; Kennedy, J.V.; Leveneur, J.; Williams, G.V.M. Characterisation of multiferroic $\mathrm{Bi}_{2} \mathrm{FeCrO}_{6}, \mathrm{BiFeO}_{3}$ thin films made by ion beam sputtering. p. 334 IN: AMN7: Advanced Materials \& Nanotechnology, February 8-12, 2015, Nelson, New Zealand: [program and abstracts]. Wellington, NZ: MacDiarmid Institute for Advanced Materials and Nanotechnology [February 2015] 
- Couture, P.; Kennedy, J.V.; Williams, G.V.M.; Leveneur, J.; Murmu, P.P.; Chong, S.V.Structural and magnetic properties of nanostructured multiferroic thin films. MacDiarmid student and PostDoc symposium, 24-25 November 2014, University of Auckland, Auckland

- Couture, P.; Kennedy, J.V.; Leveneur, J.; Williams, G.V.M. Preparation and characterisation of multiferroic polycrystalline thin films by ion beam sputtering. P. 13 IN: 59th Annual Magnetism and Magnetic Materials conference, November 3-7 2014, Honolulu, Hawaii: Conference handbook, Joint MMM-Intermag Conference [November 2014]

- Kennedy, J.V.; Couture, P.; Leveneur, J.; Williams, G.V.M. Preparation and characterisation of ion beam sputtered $\mathrm{BiFeO}_{3}$ thin films. p. 24-25 IN: The 11th AsiaPacific Conference on Materials Processing (APCMP2014), 6-9 July 2014. University of Auckland [July 2014] 


\section{Appendix B}

\section{Sputtered BiFeO3}

Complementary analysis, information of the $\mathrm{BiFeO}_{3}$ sputtered films are presented in this annex. The details of the RUMP fit are provided in the tables of Section B.1 and for the first time in literature the contact angle measurement of $\mathrm{BiFeO}_{3}$ thin is presented in Section B.2.

\section{B.1 RUMP: complementary RBS analysis}

The tables in this section present the details of $\mathrm{BiFeO}_{3}$ sputtered films' RUMP fit. Thickness in at. $\mathrm{cm}^{-2}$ and stoichiometry are estimated from the RUMP fit. The layer's density in at. $\mathrm{cm}^{-3}$ is estimated with the software SRIM. The film thickness in nanometre is calculated from the thickness in at. $\mathrm{cm}^{-2}$ and the density. As-made and annealed film at $500{ }^{\circ} \mathrm{C}$ for 15 minutes RUMP fits stoichiometry includes hydrogen to simulate the channelling effect of the substrate.

Table B.1.1: As-made $\mathrm{BiFeO}_{3}$ RUMP fit's parameter.

\begin{tabular}{|c|c|ccccc|c|c|}
\hline layer & $\left.\begin{array}{c}\text { Thickness RUMP } \\
(\text { at.cm }\end{array}{ }^{-2}\right)$ & $\mathrm{Bi}$ & $\mathrm{Fe}$ & $\mathrm{O}$ & $\mathrm{Si}$ & $\mathrm{H}$ & $\begin{array}{c}\text { Density SRIM } \\
\left(\mathrm{at.cm}^{-3}\right)\end{array}$ & $\begin{array}{c}\text { Thickness } \\
(\mathrm{nm})\end{array}$ \\
\hline 1 & $2 \times 10^{17}$ & 1 & 1 & 4 & 0 & 0 & $4.28 \times 10^{22}$ & 47 \\
2 & $4.75 \times 10^{17}$ & 1 & 1.3 & 1.9 & 0 & 0 & $4.41 \times 10^{22}$ & 108 \\
3 & $5.5 \times 10^{17}$ & 0 & 0 & 2 & 1 & 0 & $5.18 \times 10^{22}$ & 106 \\
4 & $4.5 \times 10^{17}$ & 0 & 0 & 0 & 1 & 3.5 & $4.90 \times 10^{22}$ & 92 \\
5 & $7.5 \times 10^{18}$ & 0 & 0 & 0 & 1 & 2.5 & $4.92 \times 10^{22}$ & 1525 \\
6 & $5 \times 10^{18}$ & 0 & 0 & 0 & 1 & 2 & $4.93 \times 10^{22}$ & 1014 \\
7 & $5 \times 10^{18}$ & 0 & 0 & 0 & 1 & 1.5 & $4.94 \times 10^{22}$ & 1012 \\
8 & $5 \times 10^{18}$ & 0 & 0 & 0 & 1 & 1 & $4.95 \times 10^{22}$ & 1010 \\
\hline
\end{tabular}


Table B.1.2: $\mathrm{BiFeO}_{3}$ annealed $500{ }^{\circ} \mathrm{C}, 15 \mathrm{~min}$, RUMP fit's parameter.

\begin{tabular}{|c|c|ccccc|c|c|}
\hline layer & $\begin{array}{c}\text { Thickness RUMP } \\
(\text { at.cm }\end{array}$ & Bi $)$ & Fe & O & Si & H & $\begin{array}{c}\text { Density SRIM } \\
\left(\text { at.cm }^{-3}\right)\end{array}$ & $\begin{array}{c}\text { Thickness } \\
(\mathrm{nm})\end{array}$ \\
\hline 1 & $7 \times 10^{17}$ & 1 & 1.2 & 3.6 & 0 & 0 & $4.40 \times 10^{22}$ & 159 \\
2 & $2.4 \times 10^{17}$ & 1 & 1.8 & 4.2 & 1.7 & 0 & $4.81 \times 10^{22}$ & 50 \\
3 & $6 \times 10^{17}$ & 0.02 & 0 & 2 & 1 & 0.6 & $5.03 \times 10^{22}$ & 119 \\
4 & $5 \times 10^{17}$ & 0.004 & 0 & 0 & 1 & 0.6 & $4.90 \times 10^{22}$ & 102 \\
5 & $5 \times 10^{17}$ & 0.002 & 0 & 0 & 1 & 0.6 & $4.93 \times 10^{22}$ & 101 \\
6 & $1 \times 10^{19}$ & 0 & 0 & 0 & 1 & 0.6 & $4.96 \times 10^{22}$ & 2015 \\
\hline
\end{tabular}

Table B.1.3: $\mathrm{BiFeO}_{3}$ annealed $600{ }^{\circ} \mathrm{C}$, RUMP fit's parameter.

\begin{tabular}{|c|c|ccccc|c|c|}
\hline layer & $\begin{array}{c}\text { Thickness RUMP } \\
(\text { at.cm }\end{array}$ & Bi & Fe & O & Si & H & $\begin{array}{c}\text { Density SRIM } \\
\left(\text { at.cm }^{-3}\right)\end{array}$ & $\begin{array}{c}\text { Thickness } \\
(\mathrm{nm})\end{array}$ \\
\hline 1 & $1.75 \times 10^{17}$ & 1 & 2 & 5 & 0 & 0 & $4.91 \times 10^{22}$ & 36 \\
2 & $7 \times 10^{17}$ & 1 & 1.0 & 3.0 & 0 & 0 & $4.22 \times 10^{22}$ & 166 \\
3 & $4 \times 10^{17}$ & 0.09 & 0 & 2 & 1 & 0 & $4.62 \times 10^{22}$ & 87 \\
4 & $5 \times 10^{17}$ & 0.055 & 0 & 2 & 1 & 0 & $4.81 \times 10^{22}$ & 104 \\
5 & $5 \times 10^{17}$ & 0.03 & 0 & 2 & 1 & 0 & $4.96 \times 10^{22}$ & 101 \\
6 & $1.9 \times 10^{18}$ & 0.018 & 0 & 2 & 1.1 & 0 & $5.04 \times 10^{22}$ & 377 \\
7 & $7.5 \times 10^{17}$ & 0.009 & 0 & 0.5 & 1.1 & 0 & $4.95 \times 10^{22}$ & 151 \\
8 & $7.5 \times 10^{17}$ & 0.004 & 0 & 0.2 & 1.1 & 0 & $4.96 \times 10^{22}$ & 151 \\
9 & $3.5 \times 10^{18}$ & 0.001 & 0 & 0 & 1 & 0 & $4.96 \times 10^{22}$ & 706 \\
10 & $8.89 \times 10^{19}$ & 0 & 0 & 0 & 1 & 0 & $4.98 \times 10^{22}$ & 17860 \\
\hline
\end{tabular}


Table B.1.4: $\mathrm{BiFeO}_{3}$ annealed $500{ }^{\circ} \mathrm{C}, 7$ hours, $\mathrm{RUMP}$ fit's parameter.

\begin{tabular}{|c|c|c|c|c|c|c|c|c|}
\hline layer & $\begin{array}{c}\text { Thickness RUMP } \\
\left(\text { at. } \mathrm{cm}^{-2}\right)\end{array}$ & $\mathrm{Bi}$ & $\mathrm{Fe}$ & $\mathrm{O}$ & Si & $\mathrm{H}$ & $\begin{array}{l}\text { Density SRIM } \\
\quad(\text { at.cm } \\
-3)\end{array}$ & $\begin{array}{c}\text { Thickness } \\
\text { (nm) }\end{array}$ \\
\hline 1 & $6.75 \times 10^{17}$ & 1 & 1 & 3 & 0 & 0 & $4.22 \times 10^{22}$ & 160 \\
\hline 2 & $1 \times 10^{17}$ & 1 & 1 & 2.7 & 0 & 0 & $4.21 \times 10^{22}$ & 24 \\
\hline 3 & $1.25 \times 10^{17}$ & 1 & 0.8 & 2.7 & 0 & 0 & $4.05 \times 10^{22}$ & 31 \\
\hline 4 & $4 \times 10^{17}$ & 0.08 & 0 & 2 & 1 & 0 & $4.67 \times 10^{22}$ & 86 \\
\hline 5 & $4 \times 10^{17}$ & 0.06 & 0 & 2 & 1 & 0 & $4.78 \times 10^{22}$ & 84 \\
\hline 6 & $7 \times 10^{17}$ & 0.03 & 0 & 2 & 1.1 & 0 & $4.96 \times 10^{22}$ & 141 \\
\hline 7 & $1.9 \times 10^{18}$ & 0.018 & 0 & 2 & 1.1 & 0 & $5.04 \times 10^{22}$ & 377 \\
\hline 8 & $6 \times 10^{17}$ & 0.009 & 0 & 0.5 & 1.1 & 0 & $4.95 \times 10^{22}$ & 121 \\
\hline 9 & $7.5 \times 10^{17}$ & 0.004 & 0 & 0.2 & 1.1 & 0 & $4.96 \times 10^{22}$ & 151 \\
\hline 10 & $5 \times 10^{18}$ & 0.001 & 0 & 0 & 1 & 0 & $4.96 \times 10^{22}$ & 1008 \\
\hline 11 & $8.89 \times 10^{19}$ & 0 & 0 & 0 & 1 & 0 & $4.98 \times 10^{22}$ & 17860 \\
\hline
\end{tabular}

\section{B.2 Contact angle measurement}

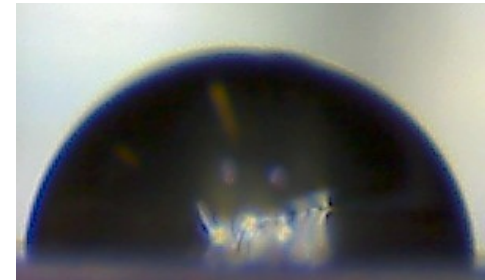

(a) Water droplet on as-made film,

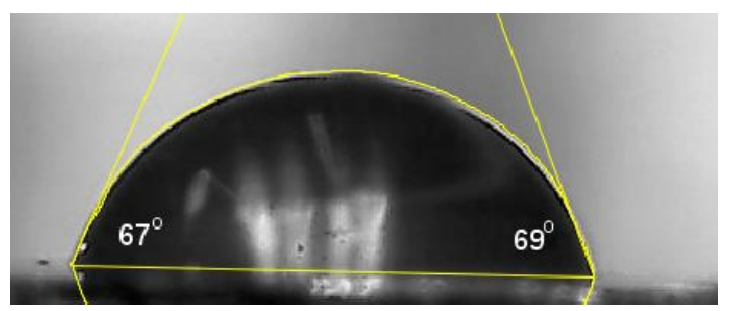

(b) on $\mathrm{BiFeO}_{3}$ annealed $500{ }^{\circ} \mathrm{C}$ for $15 \mathrm{~min}$.

Figure B.2.1: Wettability of $\mathrm{BiFeO}_{3}$ thin film.

The contact angle measurement of $\mathrm{BiFeO}_{3}$ thin film is reported for the first time. This measurement provides information on wetting properties of the films and potential applications. The technique is based on angle measurement between the tangent of droplets of water and the surface of the material (see Figure B.2.1 (a)). The contact angle on a lotus leaf is around $162^{\circ}$, allowing the droplet to easily roll off the leaf [Ensikat et al., 2011]. A droplet on a common glass surface would have a contact angle of less than $50^{\circ}$ [Chi et al., 2004]. The smaller angles results in a surface more hydrophilic. Modifying the surface of the material by, for example, implanting ions can modify the contact angle [Han et al., 1997]. The static drop method provides information about the equilibrium contact angle [Della Volpe et al., 2006]. The contact angle measurement or wettability of $\mathrm{BiFeO}_{3}$ was carried out, in this case, by dropping a static deionized water droplet, $\sim 2 \mu \mathrm{L}$, on different $\mathrm{BiFeO}_{3}$ thin films. Once the droplet equilibrium is achieved 
the contact angle is measured by taking a picture (Figure B.2.1 (a)). The photo, Figure B.2.1 (a), taken at GNS Science is an example of droplet on a $\mathrm{BiFeO}_{3}$ thin film annealed at $500{ }^{\circ} \mathrm{C}$ for 15 minutes. The analysis is carried out with the software ImageJ, ImageJ 1.50a [Schneider et al., 2012] and the complementary module Drop Analysis-Drop snake. This module highlights the edge of the droplet and measures the angle between the tangent of the sample surface and the droplet surface.

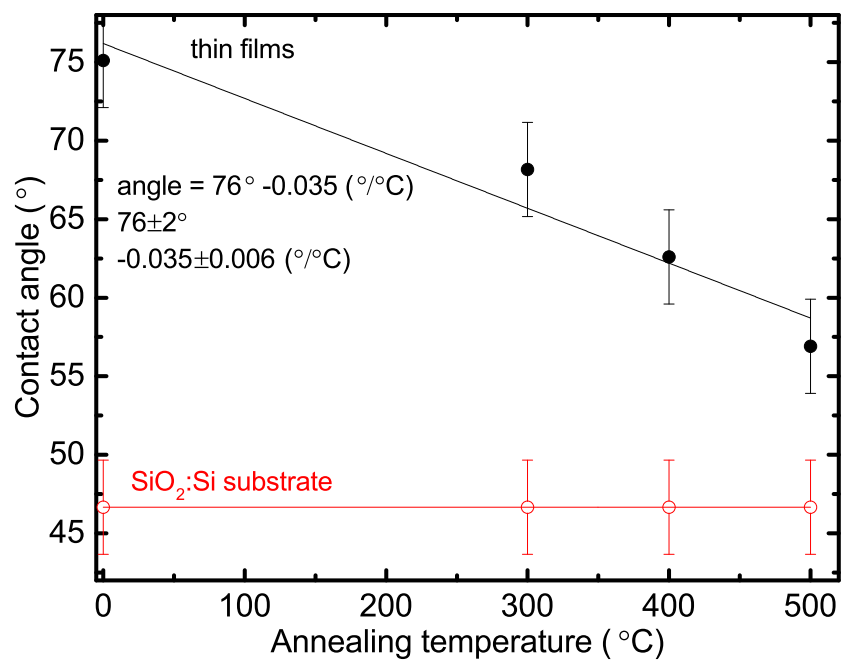

Figure B.2.2: Water droplet contact angle measured on $\mathrm{BiFeO}_{3}$ thin film (black) in function of the annealing temperature and contact angle measured on the substrate (red).

The as-made thin film has an average contact angle of about $\sim 75 \pm 3^{\circ}$. Annealing the thin films at $500{ }^{\circ} \mathrm{C}$ reduces the contact angle to $\sim 57 \pm 3^{\circ}$. This value is different from the $\sim 82^{\circ}$ found for nano-powder synthesis by chemical solution [Bajpai et al., 2014]. Comparing the two results is difficult because the synthesis technique could cause some differences in the chemical bonding of the surface. Also, the grain size of the particle, porosity, secondary phases and underlying substrate could change the hydrophobic properties measured. This is further proven as after a chemical treatment of their $\mathrm{BiFeO}_{3}$ powder, Bajpai et al., 2014 measured a contact angle of $\sim 57^{\circ}$. Further study on this topic are required to provide a conclusive expected contact angle for $\mathrm{BiFeO}_{3}$ thin film.

The results show a trend, increasing the annealing temperature resulted in decreasing the contact angle, Figure B.2.2. A rougher surface is known to increase the wettability of ceramics [Webster et al., 2001], however AFM analysis of the films (see subsection 4.3.3) confirms the roughness of the films are similar, so the film roughness does not explain the different contact angle measured through different annealing temperature. The as-made film roughness is $\sim 30 \mathrm{~nm}$ and after annealing at $500{ }^{\circ} \mathrm{C}$, the film roughness is $\sim 27 \mathrm{~nm}$. 
The contact angle variation might be indicative of phase modification through annealing temperature as suggested by XRD and Raman analysis. Intermediates annealing temperature of $300{ }^{\circ} \mathrm{C}$ and $400{ }^{\circ} \mathrm{C}$ samples displayed intermediate phases formation between the as-made film and $500{ }^{\circ} \mathrm{C}$ annealed film (Figure 4.9.1). The as-made film is mainly composed of bismuth phase has a contact angle of $\sim 75^{\circ}$, while after annealing the film is mainly compose of iron oxides and $\mathrm{BiFeO}_{3}$ phases and the contact angle decreases to a value of $\sim 57^{\circ}$. Increasing the quantity of bismuth in material has been reporting to increase the contact angle [Lee et al., 2001]. One possibility to explain the decrease of wettability could be caused by bismuth mobility and bonding with iron oxide during the annealing of the films.

An increased grain size and oxidation of the films due to annealing could also increase the contact angle [Ostrovskaya et al., 2007]. Annealing $\mathrm{BiFeO}_{3}$ in oxygen can oxidise the film (Figure B.1.1 and B.1.2) and slightly increase the grain size (subsection 4.3.2 and 4.3.3), however this resulted in a decrease of the contact angle. This can be surprising as oxidising a material could increase the contact angle [Wang and $\mathrm{Wu}$, 1994], however $\mathrm{Fe}_{2} \mathrm{O}_{3}$ is known to be superhydrophilic, contact angle is less than $10^{\circ}$ [Kulal et al., 2011]. $\mathrm{Fe}_{3} \mathrm{O}_{4}$ has been reported to have contact angle up to $157^{\circ}$ [Cheng et al., 2008]. This would suggest the as-made film has either a small amount of $\mathrm{Fe}_{2} \mathrm{O}_{3}$ or a big quantity of $\mathrm{Fe}_{3} \mathrm{O}_{4}$ and the annealed films would either have a big quantity of hematite or a small quantity of $\mathrm{Fe}_{3} \mathrm{O}_{4}$. This is consistent with the magnetic moment measurement and the XRD analysis. 


\section{Appendix C}

\section{Bismuth implanted iron oxide}

Complementary analysis, information of the bismuth implanted iron oxide sputtered films are presented in this appendix. The details of the RUMP fit are provided in the tables of Section C.1 and the analysis of the sample SP1-Bi-A is presented in Section C.2. The sample code was explained in Table 5.1.1.

\section{C.1 SP2-Bi-A: RUMP fit}

The details of SP2-Bi-A film's RUMP fit are presented in the Table C.1.1. Thickness in at. $\mathrm{cm}^{-2}$ and stoichiometry are estimated from the RUMP fit. The layer's density in at. $\mathrm{cm}^{-3}$ is estimated with the software SRIM. The film thickness in nanometre is calculated from the thickness in at. $\mathrm{cm}^{-2}$ and the density. The stoichiometry includes hydrogen to simulate the channelling effect of the substrate.

Table C.1.1: SP2-Bi-A, RUMP fit.

\begin{tabular}{|c|c|ccccc|c|c|}
\hline layer & $\begin{array}{c}\text { Thickness RUMP } \\
\left(\text { at.cm }^{-2}\right)\end{array}$ & $\mathrm{Bi}$ & $\mathrm{Fe}$ & $\mathrm{O}$ & $\mathrm{Si}$ & $\mathrm{H}$ & $\begin{array}{c}\text { Density SRIM } \\
\left(\mathrm{at.cm}^{-3}\right)\end{array}$ & $\begin{array}{c}\text { Thickness } \\
(\mathrm{nm})\end{array}$ \\
\hline 1 & $1.9 \times 10^{17}$ & 1.7 & 2.5 & 2 & 0 & 0 & $4.48 \times 10^{22}$ & 42 \\
2 & $8 \times 10^{17}$ & 0.012 & 2.0 & 3.0 & 0.1 & 0 & $7.43 \times 10^{22}$ & 108 \\
3 & $1 \times 10^{17}$ & 0.012 & 0.1 & 2 & 1 & 0 & $5.37 \times 10^{22}$ & 19 \\
4 & $3.3 \times 10^{18}$ & 0 & 0 & 2 & 1 & 0 & $5.18 \times 10^{22}$ & 636 \\
5 & $1 \times 10^{19}$ & 0 & 0 & 0 & 1 & 1.2 & $4.95 \times 10^{22}$ & 2021 \\
6 & $8 \times 10^{19}$ & 0 & 0 & 0 & 1 & 0.58 & $4.96 \times 10^{22}$ & 16121 \\
\hline
\end{tabular}




\section{C.2 SP1-Bi-A analysis}

The RBS analysis of SP1-Bi-A is presented in Figure C.2.1, displaying backscattered iron, oxygen, silicon and bismuth. The details of the RUMP's fit are presented in Table C.1.1. The RUMP simulation is made with the assumption of a 6 layers' material. The surface layer is composed of $\mathrm{Fe}_{2}, \mathrm{O}_{2.5}$, and $\mathrm{Bi}_{0.13}$ with a thickness estimated to be $55 \mathrm{~nm}\left(3.8 \times 10^{17}\right.$ at.cm ${ }^{-2}, 6.93 \times 10^{22}$ at. $\left.\mathrm{cm}^{-3}\right)$. This stoichiometry could be explained by bismuth atoms implanted in an iron oxide thin film. The possible phases to explain this stoichiometry are $\mathrm{FeO}$ and $\mathrm{Bi}_{2} \mathrm{O}_{3}$. $\mathrm{Fe}_{2} \mathrm{O}_{3}$ and $\mathrm{Fe}_{3} \mathrm{O}_{4}$ are two iron oxides expected from this synthesis as the deposited iron oxide film was sputtered from a magnetite target, however the stoichiometry of the surface layer is oxygen deficient to form those phases. Further analysis, e.g. XRD, Raman spectroscopy, will discuss in more details the phases form in SP1-Bi-A. The second layer is composed of $\mathrm{Fe}_{2}, \mathrm{O}_{3}$ and $\mathrm{Bi}_{0.01}$ with a thickness of about $40 \mathrm{~nm}\left(3 \times 10^{17}\right.$ at. $\mathrm{cm}^{-2}, 7.48 \times 10^{22}$ at. $\left.\mathrm{cm}^{-3}\right)$. This stoichiometry is explained by a $\mathrm{Fe}_{2} \mathrm{O}_{3}$ film that could be either hematite or magnetite, Raman spectroscopy and magnetic measurements will provide more information on the phase, with a small fraction of bismuth. That bismuth signal could be due to a small diffusion or the detector's resolution could be the cause of that signal. The third layer is made of $\mathrm{SiO}_{2}$ with a possible sign of iron diffusion or a limitation of the detector sensitivity. The thickness of the third layer is estimated $\left(1 \times 10^{17}\right.$ at.cm $^{-2}, 5.46 \times 10^{22}$ at.cm $\left.{ }^{-3}\right)$ around $18 \mathrm{~nm}$. The fourth layer is $\mathrm{SiO}_{2}$. The thickness of this layer is estimated at $637 \mathrm{~nm}\left(3.3 \times 10^{18}\right.$ at. $\mathrm{cm}^{-2}, 5.18 \times 10^{22}$ at. $\left.\mathrm{cm}^{-3}\right)$, equivalent to the analysis of SP2-Bi-A's substrate. The layers' underneath are bulk Si.

Table C.2.1: SP2-Bi-A, RUMP fit.

\begin{tabular}{|c|c|ccccc|c|c|}
\hline layer & $\begin{array}{c}\text { Thickness RUMP } \\
\left(\text { at.cm }^{-2}\right)\end{array}$ & $\mathrm{Bi}$ & $\mathrm{Fe}$ & $\mathrm{O}$ & $\mathrm{Si}$ & $\mathrm{H}$ & $\begin{array}{c}\text { Density SRIM } \\
\left(\mathrm{at.cm}^{-3}\right)\end{array}$ & $\begin{array}{c}\text { Thickness } \\
(\mathrm{nm})\end{array}$ \\
\hline 1 & $3.8 \times 10^{17}$ & 0.13 & 2 & 2.5 & 0 & 0 & $6.93 \times 10^{22}$ & 55 \\
2 & $3.0 \times 10^{17}$ & 0.01 & 2.0 & 3.0 & 0 & 0 & $7.48 \times 10^{22}$ & 40 \\
3 & $1 \times 10^{17}$ & 0 & 0.1 & 2 & 1 & 0 & $5.46 \times 10^{22}$ & 18 \\
4 & $3.3 \times 10^{18}$ & 0 & 0 & 2 & 1 & 0 & $5.18 \times 10^{22}$ & 637 \\
5 & $1 \times 10^{19}$ & 0 & 0 & 0 & 1 & 1.2 & $4.95 \times 10^{22}$ & 2021 \\
6 & $8 \times 10^{19}$ & 0 & 0 & 0 & 0 & 0.58 & $4.96 \times 10^{22}$ & 16121 \\
\hline
\end{tabular}

The bismuth concentration determined by the RUMP fit is about $1.4 \times 10^{16} \mathrm{at}_{\mathrm{cm}} \mathrm{cm}^{-2}$ and iron is estimated at $4.2 \times 10^{17}$ at. $\mathrm{cm}^{-2}$. From this technique, the oxygen from the substrate can't be distinguished from the oxygen in the film. The bismuth dose is significantly less than expected as the bismuth selected fluence was about $3 \times 10^{16}$ at.cm ${ }^{-2}$. 
The difference can be explained by a few possible phenomena, such as a loss of bismuth after annealing the film, resulting in bismuth evaporation. Another possible explanation is could be link to the electronic of the implanter that can induce an overestimation of the actual implanted fluence [Townsend et al., 2006]; issues with the electronic scanner during the implantation could result in a non-uniform implantation throughout the surface of the film and increase the ion concentration locally in some regions, while other regions contain less implanted ions. In the case of an insulating material, the sample surface can accumulate charge and deflect the incident ion beam, reducing the implanted dose in the film [Townsend et al., 2006]. The uncertainty generated by the graphical approach of the RUMP simulation to estimate the dose could induce a small difference of $0.05 \times 10^{16}$ at. $\mathrm{cm}^{-2}$ as the difference between the uncertainty and the value is in an order of magnitude of 100, the RUMP uncertainty is negligible.

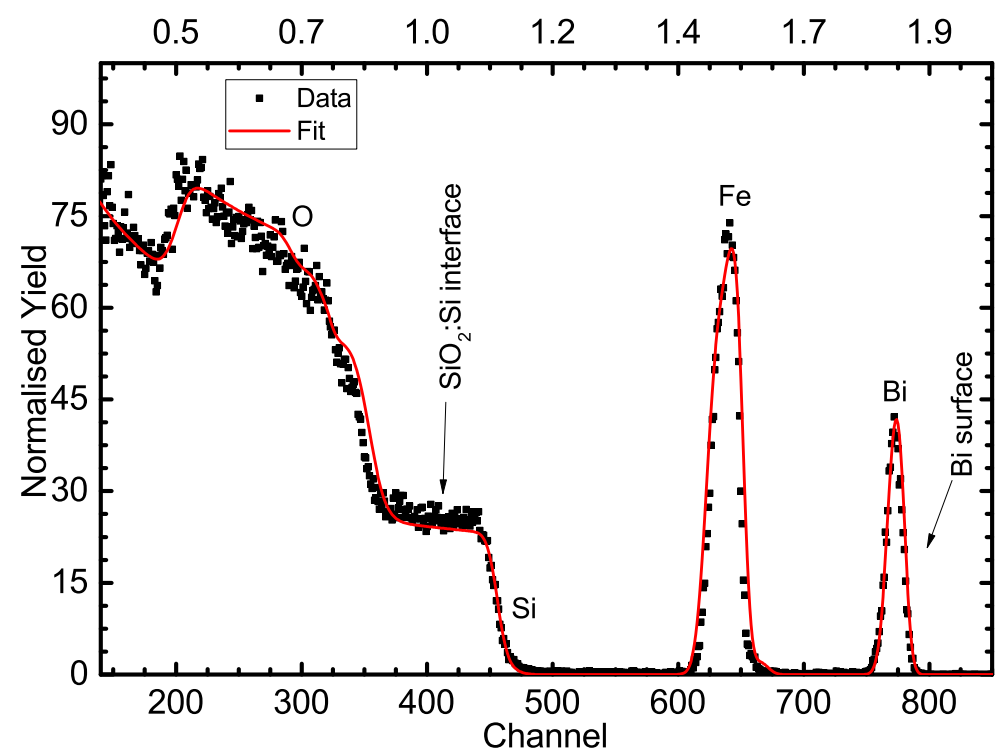

Figure C.2.1: RBS of SP1-Bi-A (circle). Synthesis details are provided in Table 5.1.1. Also, shown in this figure is RUMP fit (red line).

The RBS analysis allows to conclude that SP2-Bi-A's film has bismuth diffusing toward the inner layers while the SP1-Bi-A film does not conclusively show diffusion. A possible explanation for this increased diffusivity could be that nanocrystalline iron oxide has formed after the initial annealing (SP2-Bi-A), and that the Bi diffusivity is higher in this iron oxide when compared with the unknown phases that exist before annealing (SP1).

The XRD diffraction of SP1-Bi-A is shown in Figure C.2.2 (a). The XRD analysis does not provide clear evidence of known iron oxide phases, as the peaks are broad, which indicates small particles or amorphous phases. FeO, magnetite and maghemite could be present as small particles or could be amorphous, as they are not clearly iden- 
tified by XRD. Like the as-made iron oxide film (SP1), the XRD of SP1-Bi-A displays an amorphous material, both XRD analysis display similarities in the phases and amorphous or small particles. As the as-made iron oxide film (SP1) contains phases identified as FeO, SP1-Bi-A can also contain FeO. Both films display a similar peak at $\sim 65^{\circ}$. The other peaks are broader in the XRD of SP1-Bi-A than SP1, this is an indication of a more amorphous phase present in SP1-Bi-A. The XRD peaks broadening could come from a combination of multiple phases. In the case, the XRD broadening increases the imprecision of the grain size calculation, Scherrer's equation, to become unreliable. XRD analysis does not clearly identify the phases in the sample SP1-Bi-A.

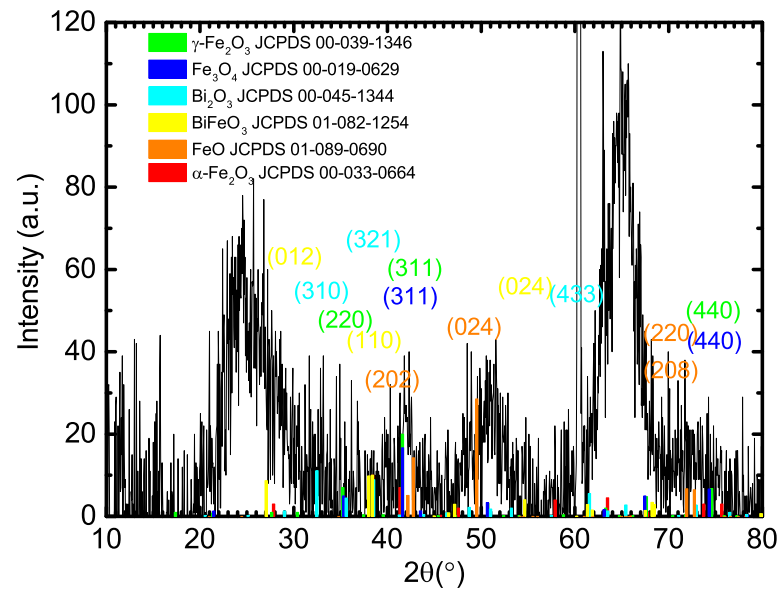

(a) XRD, grazing angle $5^{\circ}$, of SP1-Bi-A

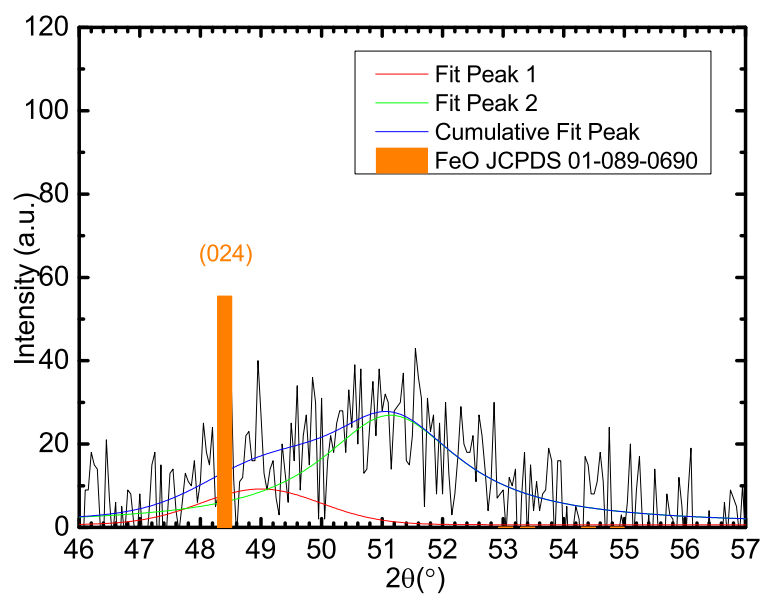

(b) Pseudo Voigt function at $49^{\circ}$ and $51^{\circ}$.

Figure C.2.2: XRD, grazing angle $5^{\circ}$, of SP1-Bi-A. (a) displays JCPDS 00-039-1346 $\left(\gamma-\mathrm{Fe}_{2} \mathrm{O}_{3}\right)$, JCPDS 00-019-0629 $\left(\mathrm{Fe}_{3} \mathrm{O}_{4}\right)$, JCPDS 00-045-1344 $\left(\mathrm{Bi}_{2} \mathrm{O}_{3}\right)$, JCPDS 01-074-1098 $\left(\mathrm{Bi}_{2} \mathrm{Fe}_{4} \mathrm{O}_{9}\right)$, JCPDS 01-082-0690 $\left(\mathrm{BiFeO}_{3}\right)$, JCPDS 00-033-0664 (hematite) and JCPDS 01-089-0690 (FeO). (b) two pseudo Voigt function fits at $49^{\circ}$ and $51^{\circ}$, the cumulative peak fit and the JCPDS pattern 01-089-0690 (FeO).

Figure C.2.2 (b) shows the magnified XRD analysis of SP1-Bi-A centred in the $50^{\circ}$ region. Also, shown in this figure is the JCDPS 01-089-1254 (FeO) and the pseudo Voigt fit function, described by equation 5.2.1. From the Voigt function, peak 1 at $49^{\circ}$ has $\mu=0$ and $W_{G}=2.3 \pm 0.8^{\circ} . W_{G}$ is the same as the $W_{G}=1.6 \pm 0.1^{\circ}$ found in the as-made $\mathrm{FeO}_{x}$ film (SP1) within the experimental uncertainty and hence there has been no significant change in the average $\mathrm{FeO}$ nanoparticle size. Peak 2 at $51^{\circ}$ represents an unidentified particle. The fit has $\mu=1$ and $W_{G}=2.8 \pm 0.6^{\circ}$, which is the same values as found before annealing (SP1). Thus, Bi implantation followed by annealing at $500{ }^{\circ} \mathrm{C}$ has not significantly changed the FeO particle size.

Figure C.2.3 displays the TEM image of SP1-Bi-A. The surface layer is platinum and the second layer from the top is carbon. The sample was coated with these materials to eliminate secondary electron. The bottom layer displays the silicon dioxide 
substrate. Between the carbon and the $\mathrm{SiO}_{2}$ layers is a layer made of iron oxide and a layer made of bismuth-implanted iron oxide. From the TEM image, the iron oxide layer thickness, from the $\mathrm{C}$ layer to the $\mathrm{SiO}_{2}$ layer, is about $140 \mathrm{~nm}$. The bottom layer of the iron oxide shows more order and structure, suggesting that part of the layer might have formed a polycrystalline material. The top layer of the iron oxide is more uniform, indistinguishable apart from a few dark columns. This might suggest that area of the film is highly distorted or amorphous.

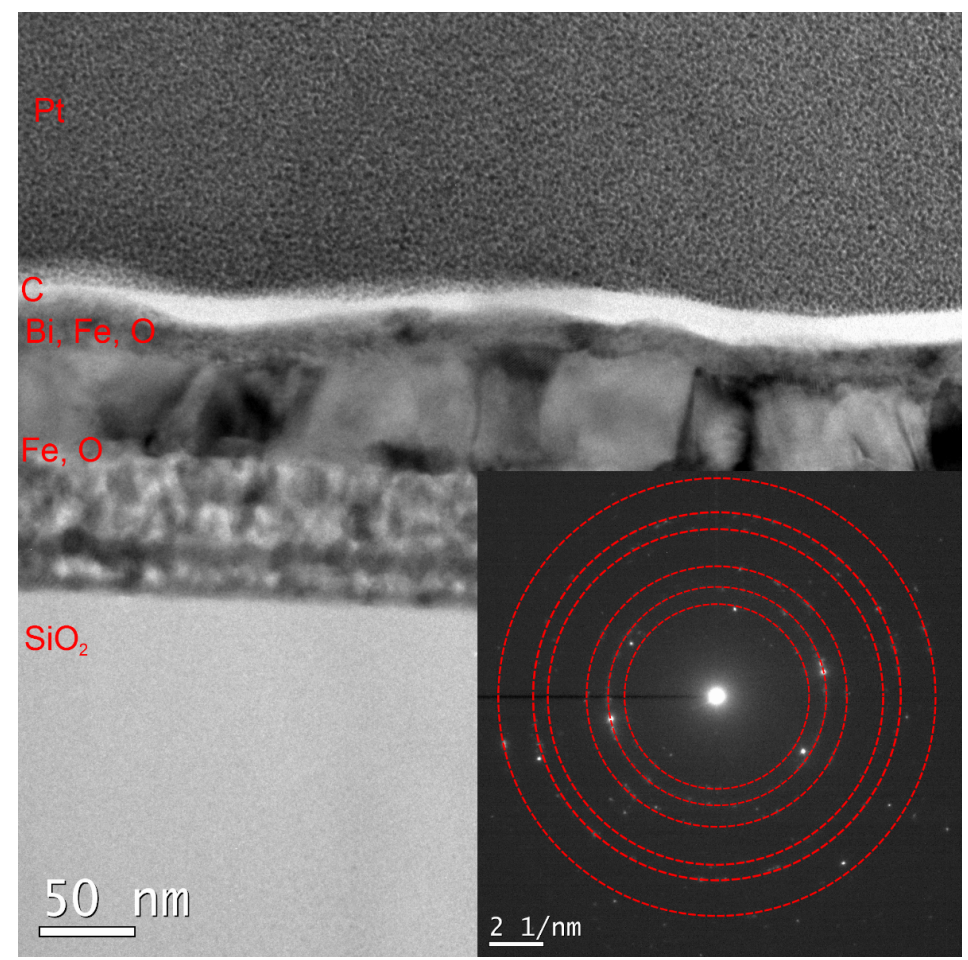

Figure C.2.3: TEM image of SP1-Bi-A. Also, shown in this figure is the $\mathrm{SiO}_{2}$ substrate, $140 \mathrm{~nm}$ of iron oxide deposited film (label Fe, $\mathrm{O}$ ), and $20 \mathrm{~nm}$ bismuth implanted into iron oxide (labelled $\mathrm{Bi}, \mathrm{Fe}, \mathrm{O}$ ). A carbon and a platinum layer, not part of the sample, were added for TEM analysis. The inset shows SAED. Also, shown are 6 rings highlighting the diffraction of either magnetite or maghemite.

The implanted bismuth ions seem to be more concentrated in the top $20 \mathrm{~nm}$ layer, with possible diffusion to a depth of $60 \mathrm{~nm}$. The sample analysed by TEM is different than the sample used for the other analyses of this chapter, this is important to note as that could have explained the different thickness of material. The TEM analysis is made on a sample implanted with Bi at $56 \mathrm{keV}$. The D-Trim simulation predicted an implantation depth of $25 \mathrm{~nm}$, and the RBS analysis does not show bismuth diffusion. This would not explain the $60 \mathrm{~nm}$ layer containing bismuth. A simpler explanation would be that a small fraction of bismuth has diffused towards the inner layer, RBS analysis (Table C.2.1) show this possibility of less than $2 \%$ concentration of bismuth in deeper layer. Such a small fraction of bismuth might be challenging to be seen with 
TEM, so the contrast area seen in Figure C.2.3 might come from different iron oxide phases.

Also, shown in this figure inset is the SAED and $6 \mathrm{~d}$-spacing ring (red), highlighting the diffraction rings in the image that are linked to $\mathrm{Fe}_{3} \mathrm{O}_{4}$. This material is expected as a powder of magnetite was press to form the sputter target. The smallest ring has a d-spacing of $2.98 \AA$ with Miller indices of (220), the second ring has d-spacing of $2.54 \AA$ (311), the third ring has a d-spacing of $2.11 \AA$ (400), the fourth ring has d-spacing of $1.64 \AA$ (511), the fifth ring has a d-spacing of $1.51 \AA$ (440) and the largest ring has a d-spacing of $1.27 \AA$ (533). The d-spacing of magnetite and maghemite are too close to one another to be distinguished by this method. SAED shows the possible presence of magnetite or maghemite. The magnetic measurement might provide more information on this topic. The iron oxide material might be in amorphous or highly disordered form, which would explain the XRD analysis. From the SAED, some definite spots are seen that are not part of any of the 6 red rings and that could indicate the presence of other particles.

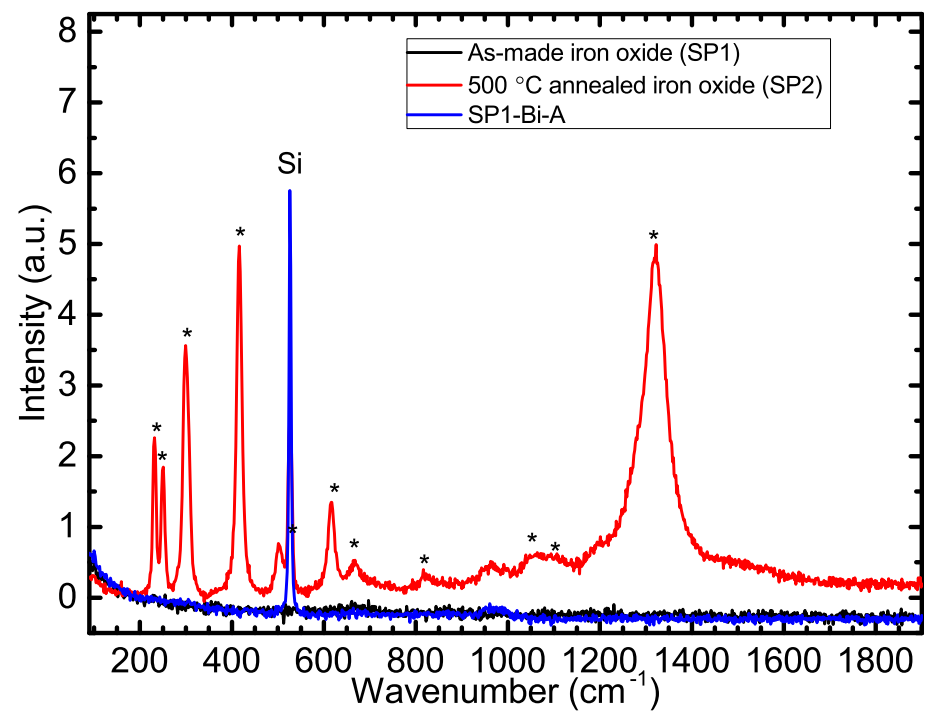

Figure C.2.4: Raman spectrum of SP1-Bi-A. The bismuth-implanted film (blue) is compared with the as-made iron oxide, SP1, (black) and annealed iron oxide film, SP2, (red). Also, shown in this figure are hematite Raman scattering (stars) and the silicon substrate at $521 \mathrm{~cm}^{-1}$.

Figure C.2.4 displays the Raman spectrum of SP1-Bi-A. This figure also shows the as-made iron oxide film (SP1) and the annealed iron oxide film (SP2). There is no evidence for any clear Raman active modes in the sample SP1-Bi-A that could be attributed to iron oxides phase. SP1-Bi-A shows the silicon substrate, peak $521 \mathrm{~cm}^{-1}$ and an amorphous material (Figure C.2.4). The Raman spectrum of the as-made iron oxide film (SP1) and SP1-Bi-A are similar. Both films are amorphous, however the 
bismuth-implanted film displays a significant Si peak at $521 \mathrm{~cm}^{-1}$. This indicates a structural modification that could be caused by the bismuth implantation or the second annealing at $500{ }^{\circ} \mathrm{C}$ for 15 minutes. A material with a greater band gap or a material with fewer metal phases would modify the light absorption coefficient. The absorption coefficient of SP1-Bi-A must be smaller than the absorption coefficient of the as-made iron oxide film (SP1). This may in part be due to a smaller FeO fraction that has a band gap below the laser energy. The implantation and annealing of Bi can have resulted in a highly disordered $\mathrm{FeO}_{x}$ component that has a large band gap. The modification of the absorption coefficient cannot be due to hematite since this would be expected to be observed in the Raman data as shown in Figure C.2.4 for the annealed $\mathrm{FeO}_{x}$ film (SP2).

The decreased absorption coefficient suggests that if metallic Bi nanoparticles are present, they are present in low fractions since high Bi nanoparticle fractions would result in an increased absorption as seen in Chapter 4 for the $\mathrm{BiFeO}_{3}$ films made by sputtering (Figure 4.3.2 (a)). There is also no evidence for bismuth or bismuth oxides in the Raman spectroscopy data (Figure C.2.4). Either the bismuth does not create bonds with the iron oxide film or the amount of bismuth bonding with the thin film is too limited to be seen in XRD and Raman spectroscopy. As bismuth is not displayed in the Raman spectrum, but Bi is seen in RBS analysis, this suggests that phases of $\mathrm{BiFe}_{x} \mathrm{O}_{y}$ could be in the film. However, those phases are very unlikely to have form. The presence of those phases could only be explained if they were either with smallsized particles or being amorphous.

Magnetic measurements were carried on SP1-Bi-A, as shown in Figure C.2.5. The resultant magnetic moment per Fe, $m_{F e}$, is plotted in Figure C. 2.5 at $5 \mathrm{~K}$ and $300 \mathrm{~K}$ after subtracting the diamagnetic moment from the silicon substrate using equation 3.3.21. The high field magnetic moment is $0.16 \mu_{B} / F e$ and the magnetic moment is only slightly lower at $300 \mathrm{~K}$ indicating that the magnetic ordering temperature is far above ambient temperature. The moment at high field is too large to come from the antiferromagnetic $\mathrm{BiFeO}_{3}$. The $6 \mathrm{~T}$ moment is $\sim 6.5 \%$ of the value seen in the as-made $\mathrm{FeO}_{x}$ (SP1) film and hence the magnetic moment could be due to a small fraction of the initial unidentified ferromagnetic phase present in SP1. Ferrimagnetic $\mathrm{Fe}_{3} \mathrm{O}_{4}$ and/or ferromagnetic $\gamma-\mathrm{Fe}_{2} \mathrm{O}_{3}$ could possibly present in that film, those phases have a saturation moments of $1.35 \mu_{B}$ for $\mathrm{Fe}_{3} \mathrm{O}_{4}$ and $1.25 \mu_{B}$ for $\gamma-\mathrm{Fe}_{2} \mathrm{O}_{3}$ [Cullity and Graham, 2011]. If this was the case, then those phases would need to be very small nanoparticles since they are not seen in the XRD data although magnetite and/or maghemite are seen in the SAED data. If they are present then their phase fractions would need to be $12 \%$ or $13 \%$, respectively. The moment has not saturated even up to the highest measured magnetic field. This may be due to the presence of a spin glass from disordered Fe 
moments. Another explaination could be attributated to fraction of antiferromagnetic $\mathrm{FeO}_{x}$ phases. The inset shows that there is also a low temperature exchange bias that will be discussed with Figure C.2.8 (a).

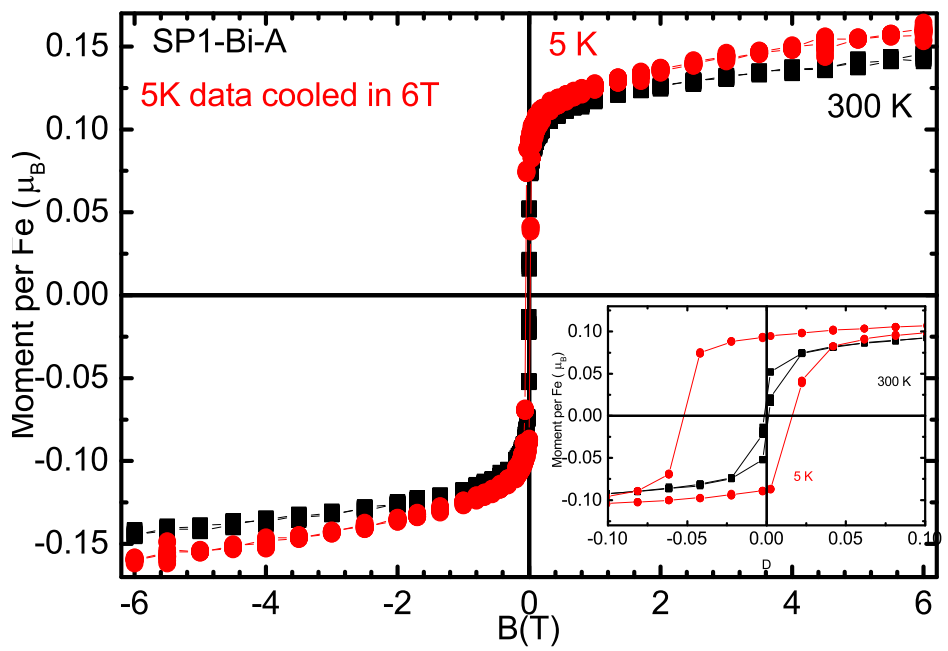

Figure C.2.5: Magnetic moment per Fe for SP1-Bi-A. The inset shows the magnetic moment measurement magnified at the centre of the field loop. The $5 \mathrm{~K}$ temperature measurement was taken after cooling the sample in a $6 \mathrm{~T}$ applied field resulting in an exchange bias as seen in the inset.

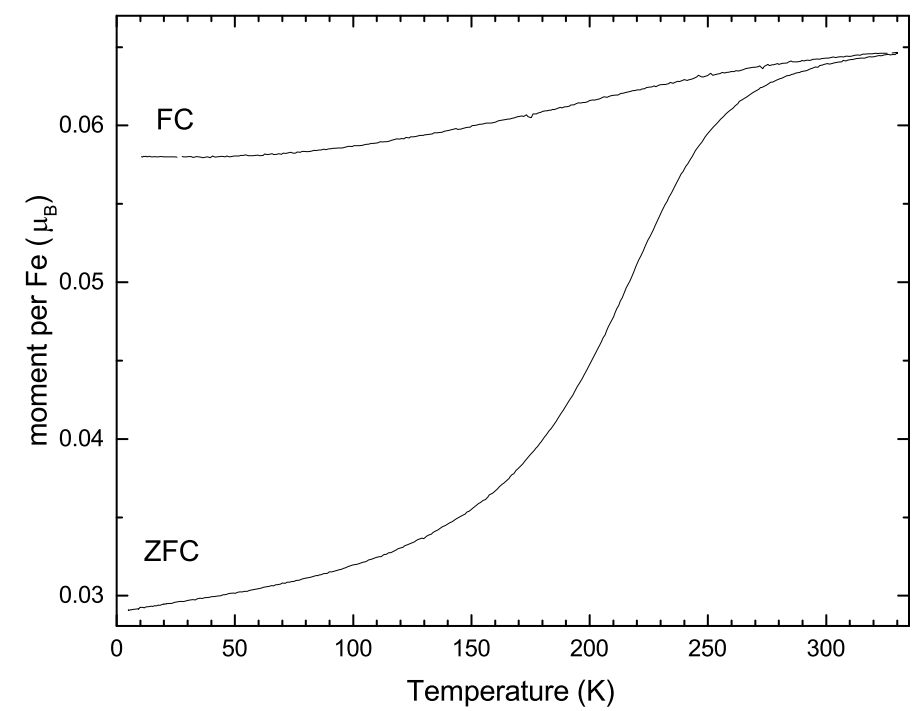

Figure C.2.6: ZFC-FC of SP1-Bi-A.

Figure C.2.6 displays the ZFC-FC of SP1-Bi-A. There is no evidence for superparamagnetism since the hysteresis is observed up to $330 \mathrm{~K}$. There is also no evidence for the low temperature antiferromagnetic signal seen in Figure C.2.6 for the as-made film (SP1). This may be because the fraction of this phase has been reduced by implanting bismuth and annealing the film. The SAED data shows the presence of magnetite and/or maghemite that are no seen in the XRD data. If these phases were present at high fractions, then they would be expected to be superparamagnetic. Using the block- 
ing temperature formula and the anisotropy energy of the magnetite and maghemite, the magnetite or maghemite nanoparticle sizes, if present in SP1-Bi-A, would be less than $\sim 10 \mathrm{~nm}$. If the nanoparticles were this large or larger, they should be seen in the XRD data. Their absence may be due to a low phase fraction.

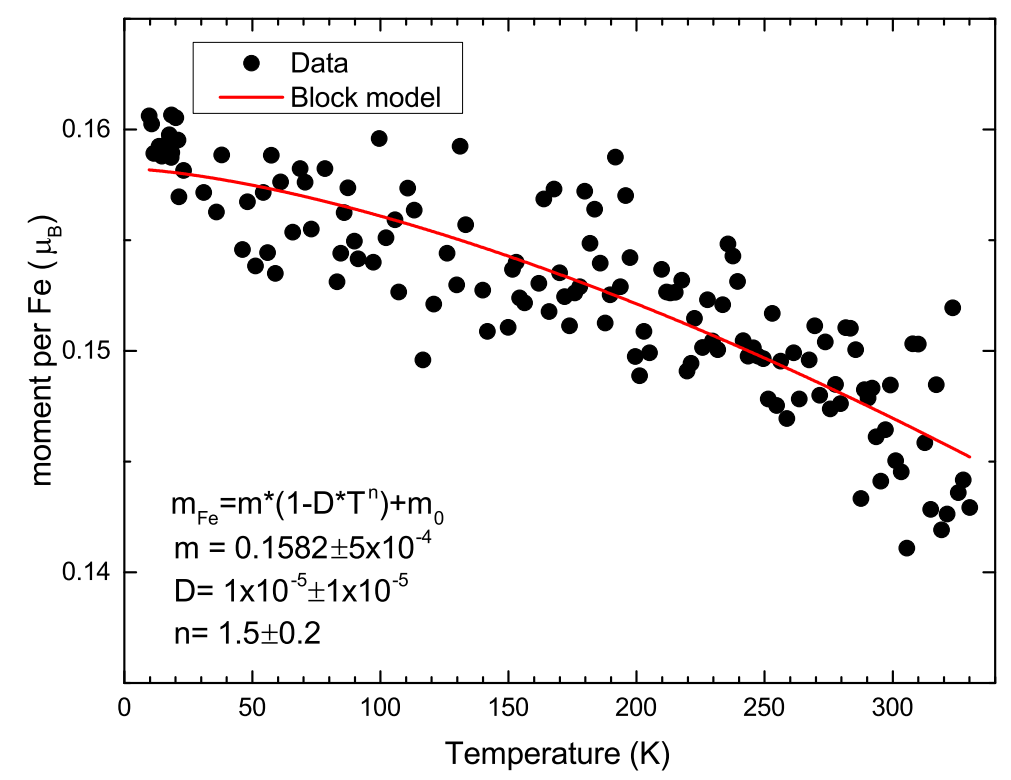

Figure C.2.7: Temperature dependence of the magnetic moment carried out on SP1-Bi-A with an applied field of 6 T. The data contain noise and reduce the quality of the Block 3/2 fit (red).

The temperature dependence of $m_{F e}$ at $6 \mathrm{~T}, m_{F e}(6 \mathrm{~T})$, is plotted in Figure C.2.7. This figure shows that $m_{F e}(6 \mathrm{~T})$ monotonically decreases with increasing temperature, which is due to the spin-waves effect. Also, shown in this Figure is a fit using the Block $3 / 2$ function. The model has a value of $D=1.3 \times 10^{-5} \mathrm{~K}^{-3 / 2}$. This $D$ value is slightly larger than the value seen in the analysis of SP1 and slightly less than the value calculated for SP2. This suggests that the magnetic phases are similar the magnetic phases in SP1. This is consistent with the magnetic data being dominated by the magnetic phase seen in as-made iron oxide rather than $\mathrm{BiFeO}_{3}$. The slightly higher value may be due to changes in the magnon dispersion and different magnon dispersions could have occurred after implanting and annealing as the TEM data clearly shows an inhomogeneous structure.

Figure C.2.8 (a) displays the measured exchange bias on SP1-Bi-A after cooling from $330 \mathrm{~K}$ to $5 \mathrm{~K}$ in an applied magnetic field of $6 \mathrm{~T}$. Also, shown in this figure is the exchange bias for the as-made film, SP1. There is no exchange bias at $300 \mathrm{~K}$. The exchange bias at $5 \mathrm{~K}$ with the bismuth film is less than the exchange bias of the as-made film (SP1). The appearance of an exchange bias in SP1-Bi-A suggests that the exchange bias, in both SP1 and SP1-Bi-A, could arise from the same magnetic phases although 
there is no evidence, from the ZFC-FC curves at low temperature, of the presence of the antiferromagnetic phase seen in SP1 (Figure 5.2.7). The exchange bias may also be due to a small magnetite or maghemite phase fraction in contact with an antiferromagnetic phase (e.g. FeO).

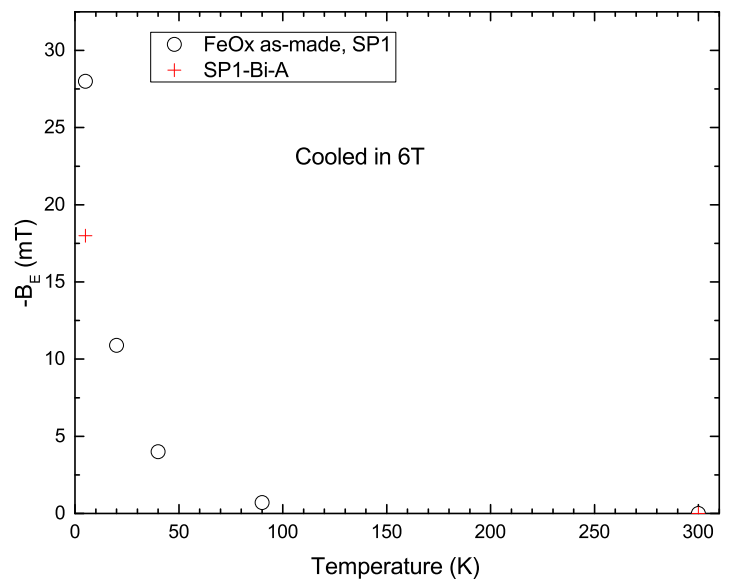

(a) Exchange bias of SP1-Bi-A (circles).

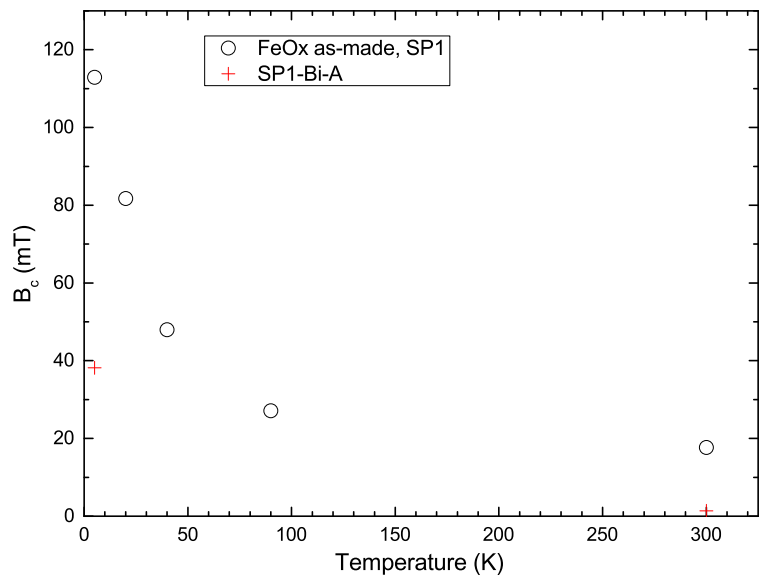

(b) Coercive field of SP1-Bi-A (circles).

Figure C.2.8: Exchange bias and coercive field of SP1-Bi-A (red cross) compared with SP1 (open circle).

Figure C.2.8 (b) displays the coercive field of SP1-Bi-A after cooling from $330 \mathrm{~K}$ to $5 \mathrm{~K}$ in an applied magnetic field of $6 \mathrm{~T}$. The coercive field at $5 \mathrm{~K}$ with the bismuth film is less than the coercive field of the as-made film (SP1) and there is a large reduction in the coercive field at $300 \mathrm{~K}$. This suggests that the Bi implantation and annealing process has modified some magnetic properties of the film. This can occur, for example, by changing the magnetic domain wall pinning energy and density. Following analysis shows that most of the changes in the coercive field occur after annealing and the $\mathrm{Bi}$ implantation does not significantly alter the coercive field.

So, SP1-Bi-A contains FeO with possibly some maghemite or magnetite. The implantation followed by annealing does not result in a significant size modification of the FeO particles. The TEM analysis shows a partly highly disordered iron oxide film, due to the bismuth implantation, and the other part of the iron oxide film is nanostructured. The magnetic ordering is about $6.5 \%$ of the as-made iron oxide film (SP1), suggesting that the magnetic moment of SP1-Bi-A is cause by a small fraction (the fraction is smaller in SP1-Bi-A then in SP1) of the unknown phase. The reduction of this unknown phase could be due to the bismuth implantation. The implantation may also have the potential to increase the maghemite or magnetite phase fraction. 


\section{Appendix D}

\section{Triple ion implantation, $B i, F e$ and $O$ into $\mathrm{SiO} 2$ and $\mathrm{Si}$}

This chapter is discussed the triple implantation of bismuth then iron then oxygen into $\mathrm{SiO}_{2}$ :Si with the motivation to study the phases form before and after annealing. Ion implantation is a synthesis technique known to form magnetic nanostructured material [Shi et al., 1996] and this technique could provide more information on the Bi-Fe interaction. The triple implantation is a new approach to attempt to synthesise $\mathrm{BiFeO}_{3}$ nanoparticles. The synthesis and structure of the nanoparticles are analysed and compared with iron oxides and bismuth oxide. The main objective of this chapter is to optimise the synthesis of nanoparticles, with the aim to create a pure material. The impact of iron oxide impurities on the material's magnetic ordering is analysed with the SQUID.

Ion implantation is a synthesis technique that provides an accurate quantity of each desired element in the material [Meldrum et al., 2001]. This technique can reduce the magnetic moment enhancement and the leakage current [Xiao et al., 2007]. Thermal annealing of nanoparticles synthesized by ion implantation is an approach to create a thin film material [Meldrum et al., 2001], however synthesising a material without secondary phases remains a challenge.

In this chapter, the results from structural and magnetic measurements on bismuth then iron then oxygen implanted films (Section D.2) at ambient temperature and films made by ion implantation of iron then oxygen then bismuth (Section D.3 and D.4) followed by annealing in an oxygen atmosphere are presented. This method leads to the formation of iron oxide nanoparticles and a possible trace amount of nano$\mathrm{BiFeO}_{3}$ particles. The magnetisation is enhanced in the $\mathrm{BiFeO}_{3}$ nanocrystals. Magnetic measurements of bismuth-implanted iron oxides (Chapter 5) are also presented with a comparison with $\mathrm{BiFeO}_{3}$ nanoparticles and iron oxide thin films and nanoparticles. 


\section{D.1 Experimental details}

The implantations were carried out at ambient temperature in $\left(\leq 3 \times 10^{-6} \mathrm{kPa}\right)$ using a bulk bismuth and iron target. The oxygen ions source came from an oxygen pure gas at $99.5 \%$. The ion beam was directed toward a substrate placed perpendiculars to the incident ion beam. The ions, first two set of samples, were implanted into a $500 \mathrm{~nm}$ layer of thermally-oxidized silicon dioxide $\left(\mathrm{SiO}_{2}\right)$ on a $\mathrm{Si}(100)$ substrate. The last set of sample was implanted into a bulk Si (111) p-type doped with Boron substrate, to study the effects of the oxygen in the substrate.

Table D.1.1: Implantation order, fluence and energy of Bi, Fe and O.

\begin{tabular}{|c|c|c|c|c|c|c|}
\hline Name & $1^{s t}$ ion & $2^{n d}$ ion & $3^{r d}$ ion & Substrate & Annealing & Section \\
\hline IM1 & $\begin{array}{l}\text { Bi } 56 \mathrm{keV} \\
3 \times 10^{16} \\
\text { at.cm } \\
-2\end{array}$ & $\begin{array}{l}\text { Fe } 38 \mathrm{keV} \\
4 \times 10^{16} \\
\text { at.cm }{ }^{-2}\end{array}$ & $\begin{array}{l}\text { O } 7.5 \mathrm{keV} \\
6 \times 10^{16} \\
\text { at.cm } \\
-2\end{array}$ & $\mathrm{SiO}_{2}: \mathrm{Si}$ & $\begin{array}{l}\text { As- } \\
\text { implanted }\end{array}$ & D.2 \\
\hline IM1-A & $\begin{array}{l}\text { Bi } 56 \mathrm{keV} \\
3 \times 10^{16} \\
\text { at.cm } \\
-2\end{array}$ & $\begin{array}{l}\text { Fe } 38 \mathrm{keV} \\
4 \times 10^{16} \\
\text { at.cm }{ }^{-2}\end{array}$ & $\begin{array}{l}\mathrm{O} 7.5 \mathrm{keV} \\
6 \times 10^{16} \\
\text { at.cm } \\
-2\end{array}$ & $\mathrm{SiO}_{2}: \mathrm{Si}$ & $\begin{array}{l}800{ }^{\circ} \mathrm{C}, \\
120 \mathrm{~min}\end{array}$ & D.2 \\
\hline IM2 & $\begin{array}{l}\text { Fe } 38 \mathrm{keV} \\
2 \times 10^{16} \\
\text { at.cm } \\
-2\end{array}$ & $\begin{array}{l}\mathrm{O} 7.5 \mathrm{keV} \\
6 \times 10^{16} \\
\text { at.cm }{ }^{-2}\end{array}$ & $\begin{array}{l}\text { Bi } 56 \mathrm{keV} \\
2 \times 10^{16} \\
\text { at.cm } \\
-2\end{array}$ & $\mathrm{SiO}_{2}: \mathrm{Si}$ & $\begin{array}{l}\text { As- } \\
\text { implanted }\end{array}$ & D.3 \\
\hline IM2-A & $\begin{array}{l}\text { Fe } 38 \mathrm{keV} \\
2 \times 10^{16} \\
\text { at.cm }{ }^{-2}\end{array}$ & $\begin{array}{l}\mathrm{O} 7.5 \mathrm{keV} \\
6 \times 10^{16} \\
\text { at.cm }{ }^{-2}\end{array}$ & $\begin{array}{l}\text { Bi } 56 \mathrm{keV} \\
2 \times 10^{16} \\
\text { at.cm }{ }^{-2}\end{array}$ & $\mathrm{SiO}_{2}: \mathrm{Si}$ & $\begin{array}{l}800^{\circ} \mathrm{C}, \\
120 \mathrm{~min}\end{array}$ & D.3 \\
\hline IM3 & $\begin{array}{l}\text { Fe } 38 \mathrm{keV} \\
2 \times 10^{16} \\
\text { at.cm }{ }^{-2}\end{array}$ & $\begin{array}{l}\text { O } 7.5 \mathrm{keV} \\
6 \times 10^{16} \\
\text { at.cm }{ }^{-2}\end{array}$ & $\begin{array}{l}\text { Bi } 56 \mathrm{keV} \\
2 \times 10^{16} \\
\text { at.cm } \\
\end{array}$ & $\mathrm{Si}$ & $\begin{array}{l}\text { As- } \\
\text { implanted }\end{array}$ & D.4 \\
\hline IM3-A & $\begin{array}{l}\text { Fe } 38 \mathrm{keV} \\
2 \times 10^{16} \\
\text { at.cm }{ }^{-2}\end{array}$ & $\begin{array}{l}\mathrm{O} 7.5 \mathrm{keV} \\
6 \times 10^{16} \\
\text { at.cm } \\
-2\end{array}$ & $\begin{array}{l}\text { Bi } 56 \mathrm{keV} \\
2 \times 10^{16} \\
\text { at.cm } \\
-2\end{array}$ & $\mathrm{Si}$ & $\begin{array}{l}800^{\circ} \mathrm{C}, \\
120 \mathrm{~min}\end{array}$ & D.4 \\
\hline
\end{tabular}

The triple ion implantation is made in a sequence of bismuth, $56 \mathrm{keV}$ Bi with a fluence of $3 \times 10^{16}$ at.cm ${ }^{-2}$, then iron, Fe at $38 \mathrm{keV}$ with a fluence of $4 \times 10^{16}$ at.cm ${ }^{-2}$, then oxygen, $\mathrm{O}$ at $7.5 \mathrm{keV}$ at fluence of $6 \times 10^{16}$ at.cm ${ }^{-2}$ (IM1 and IM1-A). The first set of sample is discussed in Section D.2. Due to an important loss of bismuth in this process, the sample IM1 and IM1-A are not studied in detail. They were used as an exploring approach to the synthesis of nanostructured material by triple implantation. Instead of 
an extended study, the synthesis procedure was modified by changing the order of implanted ions. The modification was made to study the effect of bismuth implantation in the implanted iron and oxygen material. The second sequence of triple implantation is made of iron, Fe at $38 \mathrm{keV}$ with a fluence of $2 \times 10^{16} \mathrm{at}^{-\mathrm{cm}^{-2}}$, then oxygen, $\mathrm{O}$ at $7.5 \mathrm{keV}$ at fluence of $6 \times 10^{16} \mathrm{at}_{\mathrm{cm}}{ }^{-2}$, then bismuth, $56 \mathrm{keV}$ Bi with a fluence of $2 \times 10^{16} \mathrm{at}_{\mathrm{cm}}{ }^{-2}$, (IM2 and IM2-A). The sample is presented in Section D.3. The third set of sample was made to study the effects of the oxygen in the substrate. IM3 and IM3-A were made by implanting ions into a bulk silicon substrate, Section D.4. The order of implantation was $\mathrm{Fe}$ at $38 \mathrm{keV}$ with a fluence of $2 \times 10^{16}$ at.cm ${ }^{-2}$, then oxygen, $\mathrm{O}$ at $7.5 \mathrm{keV}$ at fluence of $6 \times 10^{16}$ at.cm ${ }^{-2}$, then bismuth, $56 \mathrm{keV}$ Bi with a fluence of $2 \times 10^{16} \mathrm{at.cm}^{-2}$.

The nanoparticles are grown in an annealing furnace with an oxygen atmosphere for up to 120 minutes at temperatures up to $800{ }^{\circ} \mathrm{C}$. The films made by ion implantation are compared to the $\mathrm{BiFeO}_{3}$ thin film made by ion beam sputtering, described in Chapter 4 . They are also compared with iron oxide thin film and nanoparticle powder. The study is centred on the structure and the secondary phases are analysed.

The ion implantation and annealing were carried out at GNS Science National Isotope Centre in Lower Hutt, New Zealand.

\section{D.2 Implantation of Bi then Fe then O into SiO2:Si, IM1}

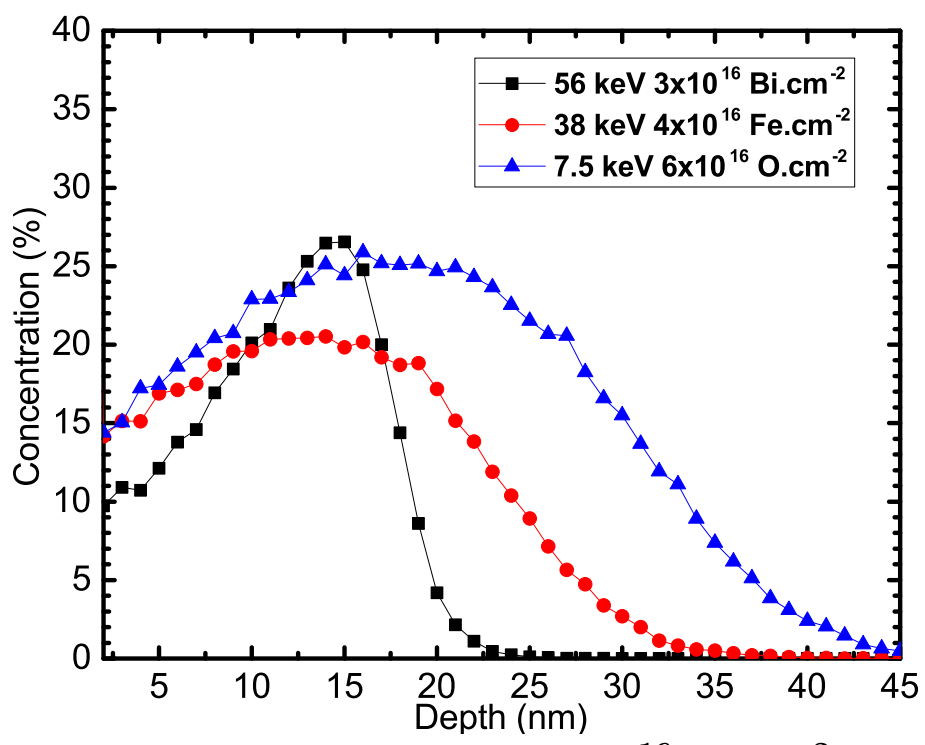

Figure D.2.1: D-Trim simulation of IM1, $56 \mathrm{keV} 3 \times 10^{16}$ Bi.cm $^{-2}$ (black), $38 \mathrm{keV} 4 \times 10^{16}$ Fe.cm ${ }^{-2}$ (red) and $7.5 \mathrm{keV} 6 \times 10^{16}{\mathrm{O} . \mathrm{cm}^{-2}}^{-2}$ (blue) into $\mathrm{SiO}_{2}: \mathrm{Si}$.

The Figure D.2.1 displays the D-Trim simulation of bismuth then iron then oxy- 
gen implanted into $\mathrm{SiO}_{2}: \mathrm{Si}$. Bismuth implantation is done at an equivalent energy of $56 \mathrm{keV}$ with a fluence of $3 \times 10^{16}$ at. $\mathrm{cm}^{-2}$. The theoretical depth profile of bismuth is limited to less than $25 \mathrm{~nm}$ with a maximal concentration of $\sim 27 \%$. Iron is the second implanted ion simulated with D-Trim. The iron implantation was done at an equivalent energy of $38 \mathrm{keV}$ with a fluence of $4 \times 10^{16} \mathrm{at}_{\mathrm{cm}}{ }^{-2}$. The theoretical depth profile of iron is about $35 \mathrm{~nm}$ with a maximal concentration of $20 \%$. In Figure D.2.1, D-Trim simulation is only showing the simulated implanted oxygen, the oxygen from the substrate is not shown. Oxygen is the third implanted ion simulated with D-Trim. The oxygen implantation was done at an equivalent energy of $7.5 \mathrm{keV}$ with a fluence of $6 \times 10^{16}$ at.cm $^{-2}$. The oxygen theoretical depth profile is about $45 \mathrm{~nm}$ with a maximal concentration of $\sim 26 \%$. The bismuth and iron is depth profile are similar and the implanted oxygen ions is about 2.8 time more important than the iron, bismuth. The silicon concentration, from the substrate, varies between $25 \%$ and $34 \%$ in the implanted region while the oxygen from the substrate is present in a concentration between $46 \%$ and $50 \%$. In the unimplanted region the ratio $\mathrm{Si}_{1} \mathrm{O}_{2}$ is respected.

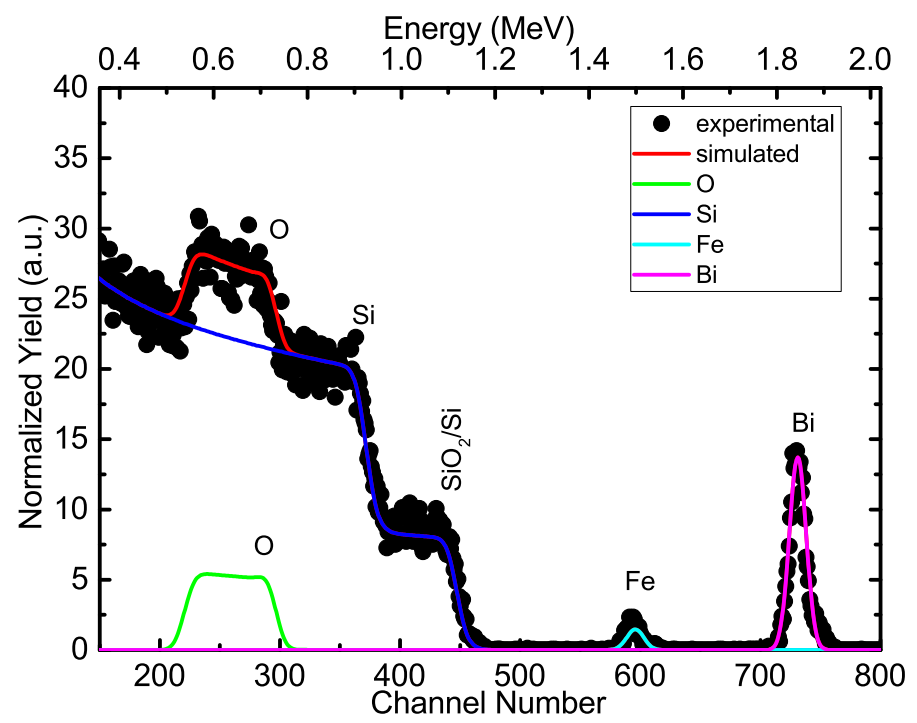

Figure D.2.2: RBS analysis IM1-A. Also, shown in this figure is the RUMP simulation (red), 3 layers' material, of Bi (purple), Fe (cyan) and O (green). The silicon (blue) substrate was also shown by RUMP simulation.

The RBS analysis is shown in Figure D.2.2, shows the particles of bismuth, iron and oxygen from the implanted thin film and silicon particles from the substrate. Also, shown in this figure is the RUMP simulation of the film made from the assumption of a 3 layers' material. The surface layer is made of $\mathrm{Bi}_{1}, \mathrm{Fe}_{1.1}$ and $\mathrm{O}_{3.5}\left(2.23 \times 10^{17}\right.$ at.cm $\left.{ }^{-2}\right)$, the density estimated with SRIM is $4.32 \times 10^{22}$ at. $\mathrm{cm}^{-3}$, with an estimated thickness of $52 \mathrm{~nm}$. That stoichiometry could suggest a mix phase layer of $\mathrm{BiFeO}_{3}, \mathrm{Bi}_{2} \mathrm{Fe}_{4} \mathrm{O}_{9}$ and $\mathrm{Fe}_{2} \mathrm{O}_{3}$. The second layer is the $\mathrm{SiO}_{2}$, density estimated by SRIM of $5.18 \times 10^{22}$ at.cm ${ }^{-3}$, (substrate) with an estimated thickness of $508 \mathrm{~nm}$, the manufacturer estimated the $\mathrm{SiO}_{2}$ 
thickness at $500 \mathrm{~nm}$, and the third layer is bulk silicon. The dose estimated by RUMP is $1.25 \times 10^{16}$ at.cm ${ }^{-2}$ of bismuth, $1.4 \times 10^{16}$ at. $\mathrm{cm}^{-2}$ of iron and $1.6 \times 10^{18}$ at.cm ${ }^{-2}$ of oxygen. The oxygen estimation is not reliable because this characterisation technique has not the precision required to separate the oxygen from the substrate and implanted oxygen ions.

The sputtered $\mathrm{BiFeO}_{3}$ film after annealing at $500{ }^{\circ} \mathrm{C}$ for 15 minutes, described in Chapter 4, shows 2 sublayers of $\mathrm{BiFeO}_{3}$ and diffusion of $\mathrm{Bi}$ into $\mathrm{SiO}_{2}$ and $\mathrm{Si}$ bulk. IM1A's RUMP fit only has 1 layer of $\mathrm{BiFeO}_{3.5}$. Bismuth does not appear to have diffused in the substrate after the sample was annealed at $800{ }^{\circ} \mathrm{C}$ for 120 minutes.

After annealing, the implanted film thickness was $\sim 52 \mathrm{~nm}$. The as-implanted material, $\left(1.15 \times 10^{17}\right.$ at.cm ${ }^{-2}, \mathrm{Bi}_{1} \mathrm{Fe}_{1} \mathrm{O}_{3.5}, 4.25 \times 10^{22}$ at.cm $\left.{ }^{-2}\right)$ has thickness of $\sim 27 \mathrm{~nm}$. The as-implanted material thickness, $27 \mathrm{~nm}$, is close to the theoretical $25 \mathrm{~nm}$ simulated by D-Trim. The difference in thickness before and after annealing suggests a diffusion toward the surface of the film or an uneven implantation across the sample.

From the RBS analysis, ions seem to have been sputtered out of the film during the triple implantation, the dose was $1.25 \times 10^{16}$ at. $\mathrm{cm}^{-2}$ for bismuth instead of the selected dose of $3 \times 10^{16}$ at. $\mathrm{cm}^{-2}$. The iron dose was $1.4 \times 10^{16} \mathrm{at}_{\mathrm{cm}}^{-2}$ instead of the selected dose of $4 \times 10^{16}$ at.cm ${ }^{-2}$. This confirms that the consecutive implantations have sputtered a significant amount of bismuth and iron. In order to preserve more bismuth in the material, the order of implantation has been modified in the subsequent implanted film. The important loss of material is another justification for the lighter analysis of this material.

The Figure D.2.3 displays the XRD analysis of IM1-A. Also, shown in this figure is the JCPDS 00-039-1346 $\left(\gamma-\mathrm{Fe}_{2} \mathrm{O}_{3}\right)$ and JCPDS 01-075-1544 $\left(\mathrm{SiO}_{2}\right)$. The XRD displayed a crystallise form of $\mathrm{SiO}_{2}$ after annealing the sample no other clear phases are distinguishable. Some broad feature around $20^{\circ}, 65^{\circ}$ and $75^{\circ}$, may suggest the sample contains an amorphous material. The peak at $42^{\circ}$ could be a signal of $\mathrm{SiO}_{2}$, magnetite or maghemite. The lattice parameter of magnetite and maghemite are too close to be clearly identify in XRD. The diffraction intensity peak clearly shows the sample is mainly composed of crystallise $\mathrm{SiO}_{2}$, if other phases are present they must be in small concentration. A small fraction of iron oxide could be present as the RBS analysis display a concentration of iron less than $1.4 \times 10^{16}$ at.cm ${ }^{-2}$. While the D-trim simulation estimated the iron concentration around $20 \%$, the silicon concentration is estimated at $30 \%$ with an oxygen concentration of more than $50 \%$. Iron oxide phases could be in the sample even with the XRD no-detection of iron oxide phases, as RBS analysis confirms 
the presence of a small amount of iron in the sample. Iron oxides phases could be either of small size or amorphous. The magnetic analysis may provide more information on that composition.

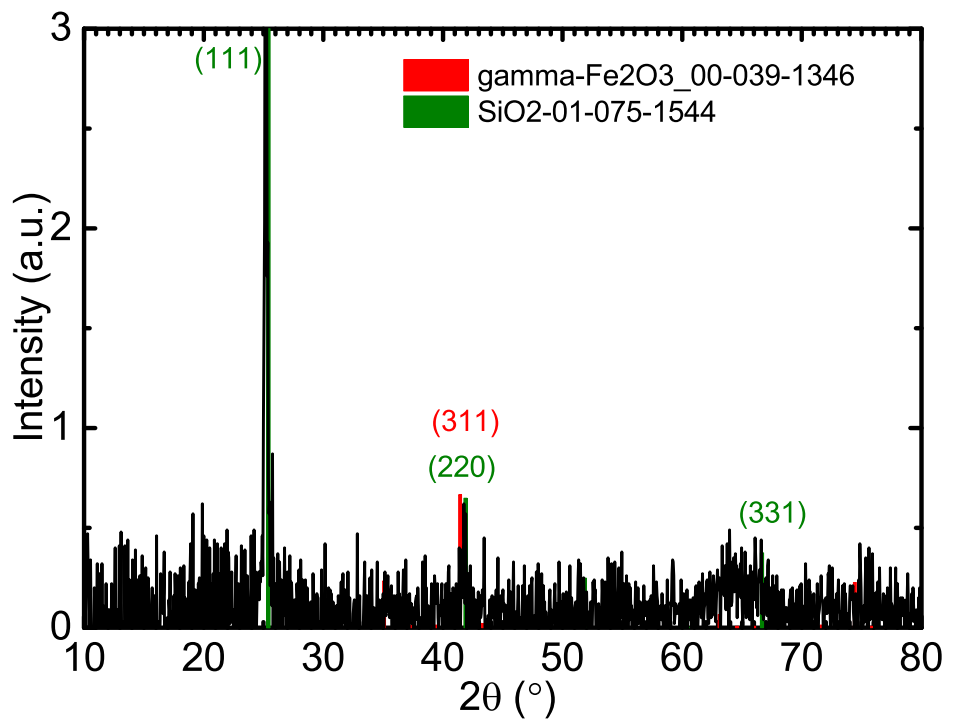

Figure D.2.3: XRD, grazing angle of $4^{\circ}$, analysis of IM1-A. Also, shown in this figure is the JCPDS 00-039-1346 $\left(\gamma-\mathrm{Fe}_{2} \mathrm{O}_{3}\right)$ and JCPDS 01-075-1544 $\left(\mathrm{SiO}_{2}\right)$. The film seems to contain an amorphous material.

JCPDS 01-075-1544 $\left(\mathrm{SiO}_{2}\right)$ peak (111), (220) and (331) are shown in Figure D.2.3. $\mathrm{SiO}_{2}$ particles size can be estimated around $\sim 60 \mathrm{~nm}$ with the Scherrer's equation and the peak (111) at $25^{\circ}$. From the peak (220), assuming the $\gamma-\mathrm{Fe}_{2} \mathrm{O}_{3}$ is negligible, the $\mathrm{SiO}_{2}$ grain size is estimated at $80 \mathrm{~nm}$. This value is less reliable because the peak (311) is at the same position and this peak is surrounded by a more important level of noise then the peak (111). The estimated grain size of from the peak (331) is not reliable as the feature centre at $65^{\circ}$, may have multiple diffraction line from maghemite or magnetite and the peak (331) is located on the edge of that feature. Any other phase than the $\mathrm{SiO}_{2}$ are not clearly identify by XRD.

The Figure D.2.4 displays the Raman spectroscopy of IM1-A (purple). Also, shown in this figure is Raman spectroscopy of the substrate (black) and annealed sputtered iron oxide thin film (Section 5.3, SP2) (blue). Also shown is the hematite $\left(^{*}\right)$ peak, magnetite (!), maghemite $(+), \beta-\mathrm{FeSi}_{2}(\Lambda)$ and two unknown feature close to the noise level $(\downarrow)$. SP2 highlights some hematite Raman active modes.

The Raman spectrum clearly shows the presence of $\alpha-\mathrm{Fe}_{2} \mathrm{O}_{3}$ hematite in IM1A. Hematite was not seen in the XRD analysis. The presence of $\mathrm{Fe}_{3} \mathrm{O}_{4}$ magnetite is not conclusive, as the feature at $\sim 650 \mathrm{~cm}^{-1}$ could come from $\alpha-\mathrm{Fe}_{2} \mathrm{O}_{3}$ hematite, $\mathrm{Fe}_{3} \mathrm{O}_{4}$ magnetite or $\gamma-\mathrm{Fe}_{2} \mathrm{O}_{3}$ maghemite. $\gamma-\mathrm{Fe}_{2} \mathrm{O}_{3}$ maghemite could also be present; broad shoul- 
der at $\sim 680 \mathrm{~cm}^{-1}$ is visible. Maghemite is more likely present $\gamma-\mathrm{Fe}_{2} \mathrm{O}_{3}$ maghemite than $\mathrm{Fe}_{3} \mathrm{O}_{4}$ magnetite, as the stoichiometry estimated from RUMP suggest $\mathrm{Fe}_{2} \mathrm{O}_{3}$. Magnetic moment measurements might be able to provide better information on their relative concentrations in this film. $\beta-F e S i_{2}$ [Yoshitake et al., 2001] is the principal candidate to have a Raman active mode at $\sim 300 \mathrm{~cm}^{-1}$. This peak is close to the peak at $289 \mathrm{~cm}^{-1}$, hematite $\alpha-\mathrm{Fe}_{2} \mathrm{O}_{3}$, and the peak at $302 \mathrm{~cm}^{-1}$, substrate. Both the substrate and hematite $\alpha-\mathrm{Fe}_{2} \mathrm{O}_{3}$ could be contributing to this feature.

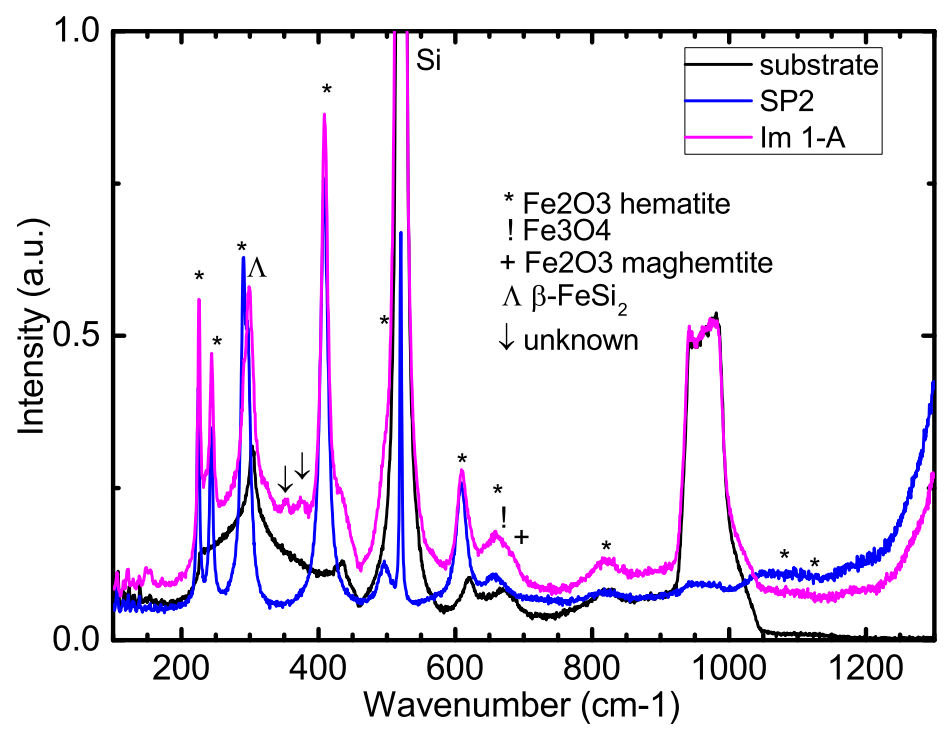

Figure D.2.4: Raman spectra of IM1-A (purple). Also, shown in this figure is Raman spectroscopy of the substrate (black) and annealed sputtered iron oxide thin film, SP2 (blue), hematite $(*)$, magnetite (!), maghemite $(+), \beta-F e S i_{2}(\Lambda)$ and two unknown feature close to the noise level $(\downarrow)$.

The broadening shoulder at $435 \mathrm{~cm}^{-1}$ could from FeSi [Nyhus et al., 1995], however as there is no strong evidence of FeSi phase, the broadening could be caused by the silicon substrate. FeSi is discarded at for this analysis as a strong Raman peak would be expected around $180 \mathrm{~cm}^{-1}$ [Nyhus et al., 1995]. Two other unidentified peaks are present. They are in the area where the silicon substrate displays Raman scattering. However, the silicon substrate is unlikely. These peaks could be from a phase of $\mathrm{Fe}_{x} \mathrm{Si}_{y}$.

Magnetic measurements were performed on an as-implanted film, and the resulting magnetic moment per Fe, $m_{F e}$, is plotted in Figure D.2.5 at $5 \mathrm{~K}$ and $300 \mathrm{~K}$. The diamagnetic moment was subtracted from the silicon substrate using equation 3.3.21. From Figure D.2.5, $m_{F e}$ increases rapidly up to $\sim 0.9 \mathrm{~T}$ and then increases at a much slower rate. This is indicative of ferrimagnetic material such as $\mathrm{Fe}_{3} \mathrm{O}_{4}$ and $\gamma-\mathrm{Fe}_{2} \mathrm{O}_{3}$. The absence of a complete saturation even at $6 \mathrm{~T}$ may be due in part to a spin glass phase or at least some free moment that exists at ambient temperature in the film. The bulk compounds have a saturation moment per $\mathrm{Fe}, m_{s, F e}$, of $1.35 \mu_{B}$ for $\mathrm{Fe}_{3} \mathrm{O}_{4}$ and 
$1.25 \mu_{B}$ for $\gamma-\mathrm{Fe}_{2} \mathrm{O}_{3}$ [Cullity and Graham, 2009]. $m_{F e}$ at $6 \mathrm{~T}$ and $5 \mathrm{~K}$ is $0.73 \mu_{B}$, the magnetic moment ratio suggests that between $\sim 54 \%$ to $\sim 58 \%$ of the available Fe is in the form of $\mathrm{Fe}_{3} \mathrm{O}_{4}$ and $\gamma-\mathrm{Fe}_{2} \mathrm{O}_{3}$ nanoparticles. This calculation is made with the assumption that $\mathrm{Fe}_{3} \mathrm{O}_{4}$ and $\gamma-\mathrm{Fe}_{2} \mathrm{O}_{3}$ are the only significant magnetically-ordered materials in the film. As the low temperature measurement does not seem to saturate, an estimation on the ambient temperature saturation of $0.046 \mu_{B} / F e$ would suggest less than $5 \%$ of the magnetic iron is from magnetite or maghemite. This $5 \%$ estimate is more reasonable then the $50 \%$ previously state as XRD and Raman analysis do not clearly identify those phases. A large concentration of iron oxide in the film could only be explained by a small particle size or an amorphous phase. The small iron fraction is more likely as the conclusion from the RBS analysis was the presence of a small fraction of Fe.

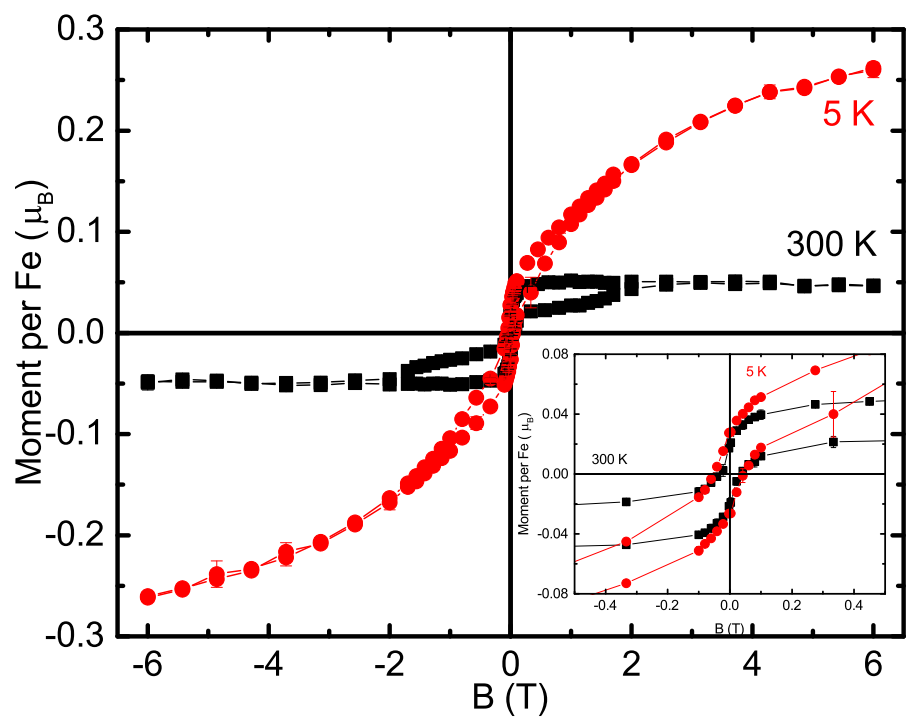

Figure D.2.5: Field loop, ambient temperature (black) and $5 \mathrm{~K}$ (red), of the IM1-A. Inset shows an expand scale near $0 \mathrm{~T}$.

The remaining $\mathrm{Fe}$ atoms are in antiferromagnetic or paramagnetic $\mathrm{BiFe}_{x} \mathrm{O}_{y}$ phases that could include antiferromagnetic $\alpha-\mathrm{Fe}_{2} \mathrm{O}_{3}$, antiferromagnetic $\mathrm{Bi}_{2} \mathrm{Fe}_{4} \mathrm{O}_{9}$ (with a Néel temperature between $237 \mathrm{~K}$ and $265 \mathrm{~K}$ [Irshad et al., 2015]), and antiferromagnetic FeO $\left(\mathrm{T}_{N}=198 \mathrm{~K}\right)$. The $\mathrm{Fe}_{x} \mathrm{Si}_{y}$ phases could also be present in the film as Raman spectroscopy might suggest the presence of $\beta-\mathrm{FeSi}_{2}$. FeSi is known to be antiferromagnetic with a Curie temperature between - $107 \mathrm{~K}$ and -149 K [Watanabe et al., 1963], the magnetic moment is reported near $0 \mu_{B} / \mathrm{Fe}$ [Berling et al., 1999]. $\alpha-\mathrm{FeSi}_{2}$ is metallic, while annealing the film at temperature above $\sim 660 \mathrm{~K}, \alpha-\mathrm{FeSi}_{2}$ become $\beta$-FeSi $i_{2}$. In some case, $\beta$-FeSi phase is semiconductor. If that material is iron rich then a ferromagnetic ordering can arise [Chen et al., 2007]. $\beta$-FeSi ${ }_{2}$ Curie temperature was measured at $100 \mathrm{~K}$ [Arushanov et al., 2004] and has a magnetic moment below $0.02 \mu_{B} / F e$ [Aigner et al., 2013]. While $\mathrm{Fe}_{3} \mathrm{O}_{4}$ magnetite and $\gamma-\mathrm{Fe}_{2} \mathrm{O}_{3}$ maghemite are both ferrimagnetic. Maghemite can often be confused with a weak ferromagnetic ordering material. As both the $300 \mathrm{~K}$ and $5 \mathrm{~K}$ 
field loop display a hysteresis loop, then IM1-A must contain a ferromagnetic material, unless the magnetic moment come from the superparamagnetic $\mathrm{Fe}_{3} \mathrm{O}_{4}$ [Dedkov et al., 2002] or $\gamma-\mathrm{Fe}_{2} \mathrm{O}_{3}$ [Nadeem et al., 2011].

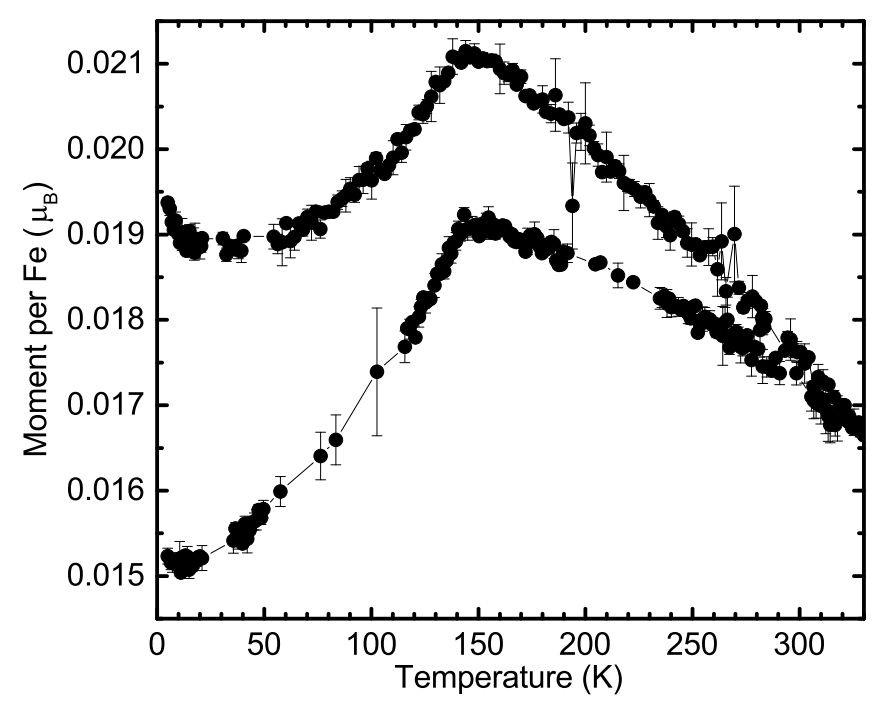

Figure D.2.6: ZFC-FC of IM1-A. The ZFC and FC curves do not seem to merge at ambient temperature. A transition temperature near $140 \mathrm{~K}$ is visible.

The Figure D.2.6 displays the ZFC-FC of the IM1-A. This figure clearly shows the ZFC and FC hysteresis remain open at high temperature. This indicates the absence of a blocking temperature or superparamagnetic state in the film. This agrees with the magnetic moment measurement: both the low-temperature and ambient temperature measurement displayed a hysteresis loop, which would indicate a ferromagnetic phase is present in IM1-A. Comparing the Figure D.2.6 with the literature, IM1-A seems to be made of a mix of $\alpha-\mathrm{Fe}_{2} \mathrm{O}_{3}, \gamma-\mathrm{Fe}_{2} \mathrm{O}_{3}$ and $\mathrm{Fe}_{3} \mathrm{O}_{4}$ [Wu et al., 2015]. From Wu et al. [2015], the ZFC displays a mix of spherical $\alpha-\mathrm{Fe}_{2} \mathrm{O}_{3}$ and square $\mathrm{Fe}_{3} \mathrm{O}_{4}$, particles. If the inflexion point is between $\sim 146 \mathrm{~K}$ and $\sim 190 \mathrm{~K}$, then the grain size would be between $\sim 6 \mathrm{~nm}$ for the spherical particles [Manukyan et al., 2014] and $\sim 11 \mathrm{~nm}$ for the cubic particles [Coey and Khalafalla, 1972].

The analysis of IM1-A is limited to the results presented in this section, due to a significant loss of Bi ions either during the implantation or annealing process. This is limiting the possible study of IM1-A. Instead of continuing this analysis, the decision was taken to study a new set of sample with greater potential by modifying the synthesis procedure. The investigation was centred on the effect of implanting bismuth, iron and oxygen ions. The following section will be the study of iron then oxygen then bismuth implanted ions into $\mathrm{SiO}_{2}: \mathrm{Si}$ and $\mathrm{Si}$.

Although, the choice was made to move on to more significant samples, in future 
work, study the temperature dependence and exchange bias of IM1-A could be of interest. The analysis of the absence of saturation at low temperature would be another interesting study. An in-depth analysis of composition and phase analysis would be required to understand the source of the magnetic moment.

\section{D.3 Implantation of Fe then O then Bi into SiO2:Si, IM2}

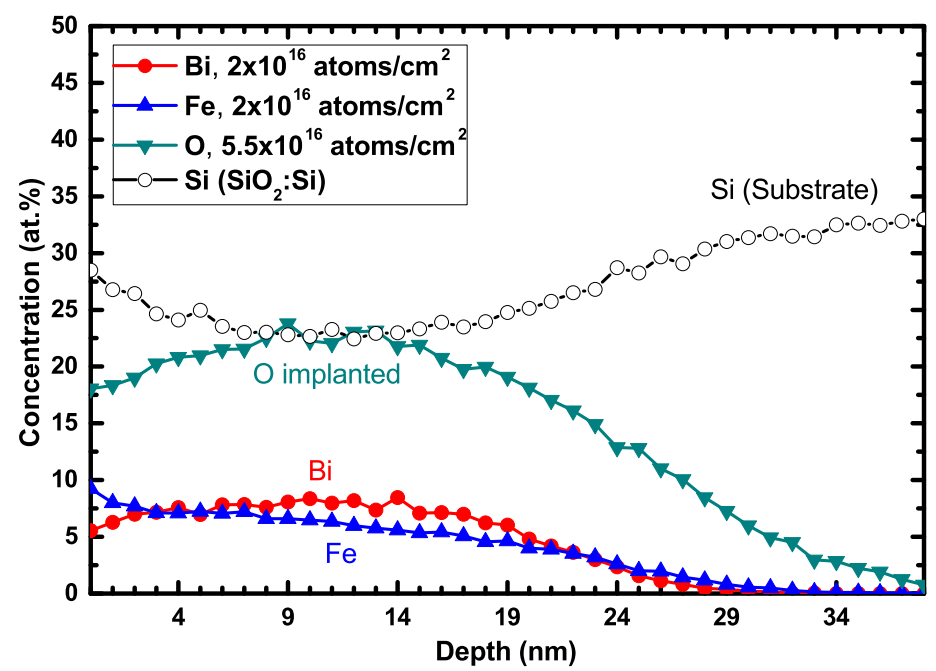

Figure D.3.1: D-Trim simulation of IM2, $56 \mathrm{keV} 2 \times 10^{16} \mathrm{Bi.cm}^{-2}$ (red), $38 \mathrm{keV} 2 \times 10^{16}$

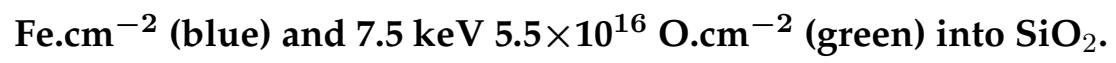

The Figure D.3.1 displays the D-Trim simulation [Biersack et al., 1991] of IM2.

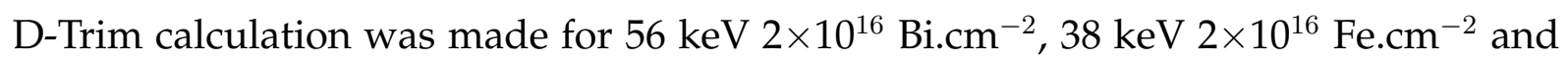
$7.5 \mathrm{keV} 5.5 \times 10^{16} \mathrm{O} . \mathrm{cm}^{-2}$ into $\mathrm{SiO}_{2}$. Bismuth and iron depth profile are similar. The theoretical depth profile of bismuth and iron is about $30 \mathrm{~nm}$ while the implanted oxygen reaches $40 \mathrm{~nm}$. The bismuth concentration is theoretically estimated at $9 \%$ and $7 \%$ for iron. The implanted oxygen has an estimated potential concentration of $24 \%$. This concentration is about 2.8 time more important than the iron and bismuth concentration. Oxygen from the substrate is not shown in the Figure D.3.1. The oxygen from the substrate is estimated with D-trim simulation around $60 \%$ in the implanted layer and $66 \%$ in the $\mathrm{SiO}_{2}$ layer, silicon concentration is estimated around $33 \%$ through the $\mathrm{SiO}_{2}$ layer.

The Figure D.3.2 (a) displays the RBS of IM2 as-implanted into $\mathrm{SiO}_{2}$ :Si. Also, shown in this figure is the RUMP fit [Doolittle, 1985] with the assumption of a 3 layers' material. The surface layer is composed of $\mathrm{Si}_{1}, \mathrm{O}_{2.3}, \mathrm{Bi}_{0.3}$ and $\mathrm{Fe}_{0.3}\left(2 \times 10^{17}\right.$ at.cm ${ }^{-2}$ and a density of $4.55 \times 1022$ at.cm ${ }^{-3}$ ) with an estimated thickness of $44 \mathrm{~nm}$. This stoichiometry could suggest a surface layer of $\mathrm{SiO}_{2}, \mathrm{FeO}$ and $\mathrm{Bi}$. The estimated depth 
profile from D-Trim was $\sim 30 \mathrm{~nm}$. This is less than the $44 \pm 2 \mathrm{~nm}$ estimated with RUMP fit. The difference could be cause either by an overestimation of the density of $\mathrm{SiO}_{2}$ in D-Trim implantation simulation which would increase the stopping power and reduce the depth profile of the implanted ions or the condition of implantation resulted in greater potential of acceleration. An unlikely, as diffusion is not seen in RUMP fit, explanation could be ion diffusion caused by the temperature increasing during the implantation. A combination of both effects could also explain the difference of depth profile. The second layer is the $\mathrm{SiO}_{2}\left(2.7 \times 10^{18}\right.$ at.cm ${ }^{-2}, 5.18 \times 1022$ at.cm $\left.{ }^{-3}\right) 521 \mathrm{~nm}$. The manufacturer specification for the $\mathrm{SiO}_{2}$ layer is $500 \pm 30 \mathrm{~nm}$. The third layer is the bulk Si.

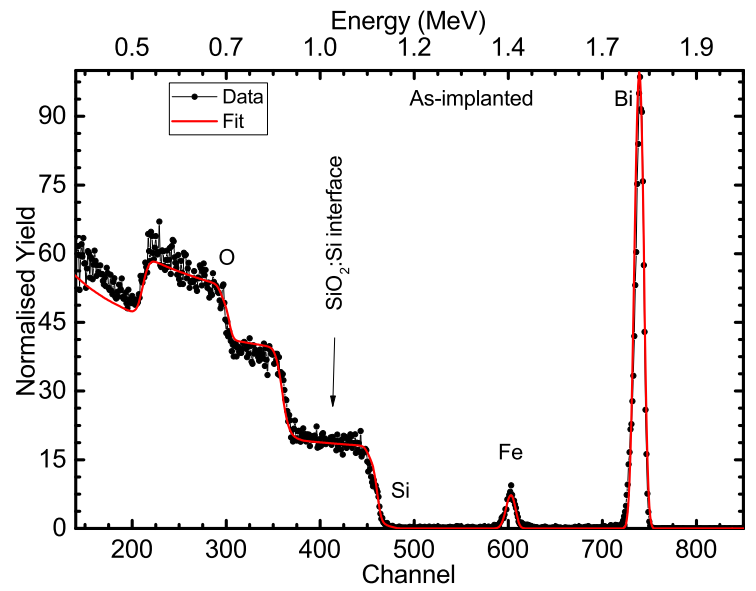

(a) RBS of IM2

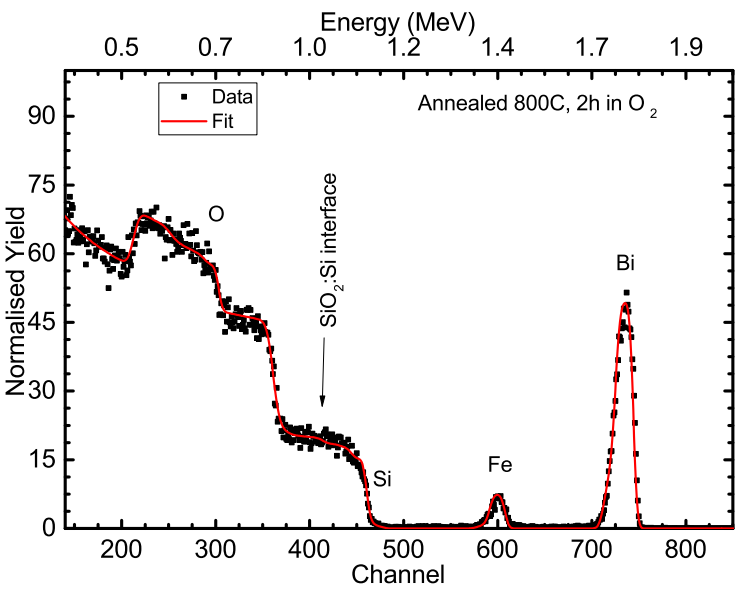

(b) RBS of IM2-A.

Figure D.3.2: RBS of implanted Fe $2 \times 10^{16}$ at.cm ${ }^{-2}$ then $O$ at $6 \times 10^{16}$ at.cm ${ }^{-2}$ then $B i 2 \times 10^{16}$ at.cm ${ }^{-2}$. Also, shown is the RUMP fit (red).

From the RUMP fit, the bismuth and the iron dose calculated at $2 \times 10^{16}{\mathrm{Bi} . \mathrm{cm}^{-2}}^{-2}$ and $1.98 \times 10^{16} \mathrm{Fe} . \mathrm{cm}^{-2}$. The selected fluence for both ions were $2 \times 10^{16} \mathrm{at}^{\mathrm{cm}}{ }^{-2}$, so IM2 retain $\mathrm{Bi}$ and Fe during the implantation. The difference between the D-Trim simulation iron fluence and the RUMP fit dose could be due to sputtering occurring while implanting oxygen followed by bismuth. The experimental setup limits the accuracy's estimation of the implanted oxygen dose as the oxygen from the substrate and implanted are not distinguishable. The total, substrate and implanted ions, oxygen dose is estimate at $1 \times 10^{19}$ at. $\mathrm{cm}^{-2}$.

Figure D.3.2 (b) displays the RBS of the IM2-A, annealed at $800{ }^{\circ} \mathrm{C}$ for $120 \mathrm{~min}$ utes. Also, shown in this figure is the RUMP fit with 7 layers (details are presented in Table D.3.1). The surface layer is composed of $\mathrm{Bi}_{0.2}, \mathrm{Fe}_{0.3}, \mathrm{Si}_{2}$ and $\mathrm{O}_{4}\left(5 \times 10^{17}\right.$ at.cm ${ }^{-2}$ and estimated density with SRIM of $4.94 \times 1022$ at.cm ${ }^{-3}$ ) with a thickness of $101 \mathrm{~nm}$. This stoichiometry would suggest a $\mathrm{SiO}_{2}$ layer with $\mathrm{Fe}$ and $\mathrm{Bi}$. The second layer is $\mathrm{SiO}_{2}$ with $\mathrm{Bi}_{0.07}$ and $\mathrm{Fe}_{0.01}\left(1 \times 10^{17}\right.$ at.cm ${ }^{-2}$ and estimated density with SRIM of 
$4.75 \times 10^{22}$ at. $\mathrm{cm}^{-3}$ ) with a thickness of $21 \mathrm{~nm}$. The third layer contains $\mathrm{Bi}_{0.02}$ and $\mathrm{Fe}_{0.01}$ in $\mathrm{SiO}_{2}$. The thickness is estimated at $69 \mathrm{~nm}$ (Table D.3.1). Annealing the implanted sample resulted in diffusion of $\mathrm{Bi}$ and $\mathrm{Fe}$, before annealing the $\mathrm{Bi}$ and $\mathrm{Fe}$ depth profile was about $42 \mathrm{~nm}$, while after annealing the ions depth profile is $191 \mathrm{~nm}$. The dose of $\mathrm{Bi}$ is $1.93 \times 10^{16}$ at.cm ${ }^{-2}$ compare to $2 \times 10^{16}$ at.cm ${ }^{-2}$ before annealing, not a significant amount of bismuth was evaporated. The Fe dose is the same before and after annealing, $1.98 \times 10^{16}$ at.cm ${ }^{-2}$. Layer 4 is composed of $\mathrm{SiO}_{2}$, the thickness estimated, Table D.3.1, is $125 \mathrm{~nm}$. Layer 5 is the last layer composed of oxygen, the layers' underneath are the bulk silicon substrate. After annealing, the $\mathrm{SiO}_{2}$ thickness is estimated at $665 \mathrm{~nm}$. This thickness suggests $\mathrm{O}$ diffusion or $\mathrm{SiO}_{2}$ layer growth during the annealing process. Oxygen diffusion could be caused by thermal annealing, the manufacturer used this technique to produce their commercial $\mathrm{SiO}_{2}: \mathrm{Si}$ substrate, or, less likely, the bismuth diffusion could carry oxygen deeper in the film. The oxygen dose is estimate at $1.4 \times 10^{19}$ at.cm ${ }^{-2}$, if this value was reliable, this would mean an increase dose of $0.4 \times 10^{19}$ at.cm ${ }^{-2}$ only due to an annealing at $800{ }^{\circ} \mathrm{C}$ for 120 minutes in an oxygen atmosphere.

Table D.3.1: IM2-A, RUMP fit's parameter.

\begin{tabular}{|c|c|ccccc|c|c|}
\hline layer & $\begin{array}{c}\text { Thickness RUMP } \\
(\text { at.cm }\end{array}$ & Bi $)$ & Fe & O & Si & H & $\begin{array}{c}\text { Density SRIM } \\
\left(\text { at.cm }^{-3}\right)\end{array}$ & $\begin{array}{c}\text { Thickness } \\
(\mathrm{nm})\end{array}$ \\
\hline 1 & $5 \times 10^{17}$ & 0.2 & 0.3 & 4 & 2 & 0 & $4.94 \times 10^{22}$ & 101 \\
2 & $1 \times 10^{17}$ & 0.07 & 0.01 & 2.0 & 1 & 0 & $4.75 \times 10^{22}$ & 21 \\
3 & $3.5 \times 10^{17}$ & 0.02 & 0.01 & 2 & 1 & 0 & $5.06 \times 10^{22}$ & 69 \\
4 & $6.5 \times 10^{17}$ & 0 & 0 & 2 & 1 & 0 & $5.18 \times 10^{22}$ & 125 \\
5 & $1.8 \times 10^{18}$ & 0 & 0 & 1.8 & 1 & 0 & $5.17 \times 10^{22}$ & 348 \\
6 & $3.8 \times 10^{18}$ & 0 & 0 & 0 & 1 & 0.4 & $4.97 \times 10^{22}$ & 765 \\
7 & $1 \times 10^{19}$ & 0 & 0 & 0 & 1 & 0 & $4.98 \times 10^{22}$ & 2009 \\
\hline
\end{tabular}

From the RBS analysis (Figure D.3.3), diffusion of bismuth after annealing is highlighted, no evaporation was reported. Bismuth diffusion is expected in $\mathrm{SiO}_{2}$, $\sim 10^{-14} \mathrm{~cm}^{2} \mathrm{~s}^{-1}$ at $800{ }^{\circ} \mathrm{C}$ [Büngener et al., 2003]. The RUMP fit, Table D.3.1, include traces of iron in layers 2 and 3, however the superposition of the RBS spectrum, Figure D.3.3, suggest iron is not diffused. So, the iron traces, instead of being a sign of diffusion could mean the detector quality was deteriorating.

The Figure D.3.4 displays the XRD of IM2-A. Also, shown in this figure are the JCPDS 01-074-0201 (Si), JCPDS 00-039-1346 ( $\left.\gamma-\mathrm{Fe}_{2} \mathrm{O}_{3}\right)$, JCPDS 00-033-0664 $\left(\alpha-\mathrm{Fe}_{2} \mathrm{O}_{3}\right)$ and JCPDS 00-019-0629 $\left(\mathrm{Fe}_{3} \mathrm{O}_{4}\right)$. The XRD does not displays any clear phase. This does not imply that iron oxide phases or iron-silicon phases are not in the material. Those 


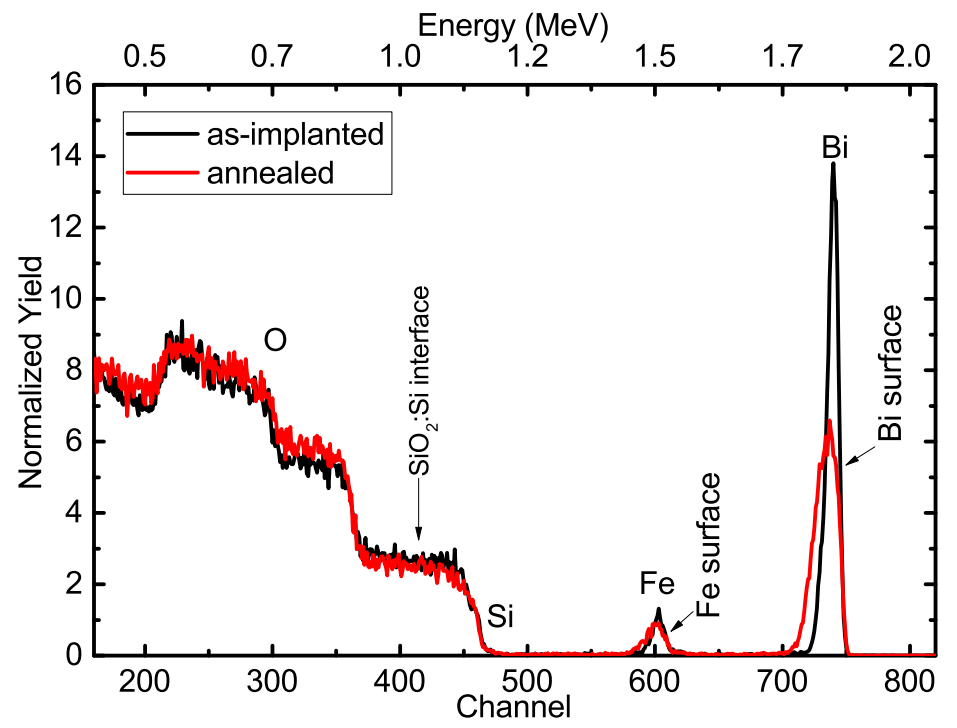

Figure D.3.3: RBS of IM2 (black) and IM2-A (red).

phases could be amorphous or in too small concentration to be seen in XRD, as RUMP fit suggest layers of $\mathrm{SiO}_{2}$ with less than 0.3 iron and bismuth ions. A broad peak centre at $30^{\circ}$ is visible and indicate the presence of an amorphous material that could be $\mathrm{SiO}_{2}$. The broad feature and the presence of the peak (101) and (002) with potential contribution of hematite, maghemite or magnetite prevent any accurate size particle estimation with the Scherrer's equation [Patterson, 1939; Scherrer, 1918]. Raman spectroscopy and magnetic moment may provide more information on the sample.

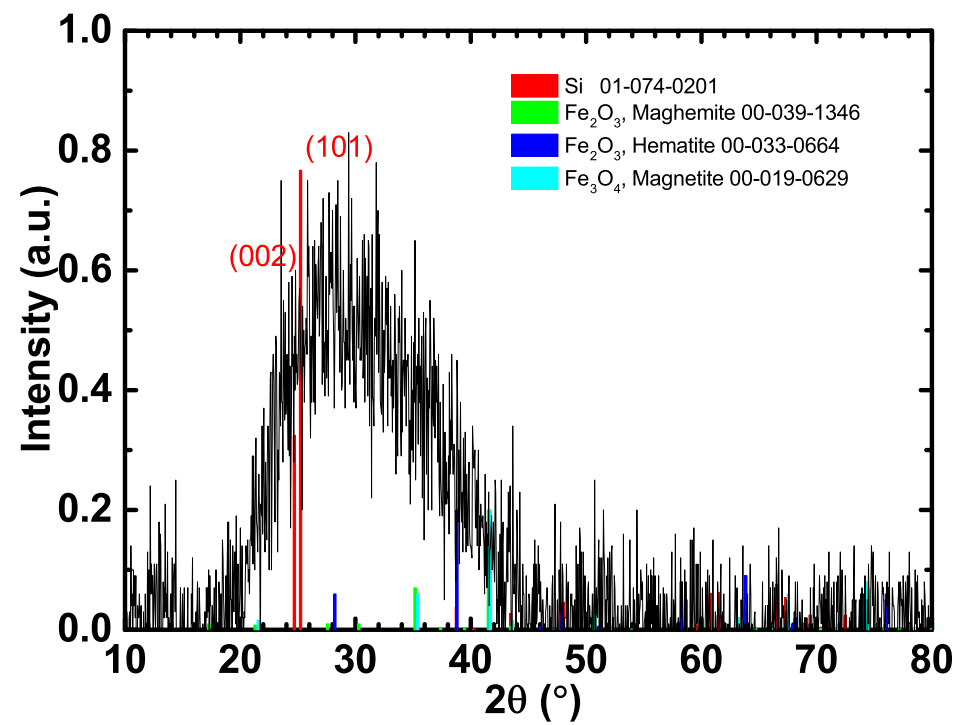

Figure D.3.4: XRD, grazing angle $5^{\circ}$, IM2-A. Also, shown in this figure are the JCPDS 01074-0201 (Si), JCPDS 00-039-1346 $\left(\gamma-\mathrm{Fe}_{2} \mathrm{O}_{3}\right)$, JCPDS 00-033-0664 $\left(\alpha-\mathrm{Fe}_{2} \mathrm{O}_{3}\right)$ and JCPDS 00-019-0629 $\left(\mathrm{Fe}_{3} \mathrm{O}_{4}\right)$.

Figure D.3.5 displays the Raman spectroscopy of IM2 and IM2-A. Also shown is the bismuth Raman scattered peak around $90 \mathrm{~cm}^{-1}$, hematite $\left.{ }^{*}\right)$ and magnetite (!). The as-implanted Raman spectroscopy (blue) shows a peak at $\sim 90 \mathrm{~cm}^{-1}$ which is a Raman 
active mode of bismuth. The rest of the spectrum of IM2 overlaps with the Raman spectrum of the silicon dioxide substrate. No other components can be seen in the Raman spectrum for the as-implanted sample, only bismuth and the $\mathrm{SiO}_{2}$ substrate. $\mathrm{SiO}_{2}$ and Bi were phases deduced from the RBS analysis, Figure D.3.2 (a). The missing phase in the Raman spectroscopy is $\mathrm{FeO}$. FeO phase, if present must be in small quantity or amorphous as IM2 does not have a Raman active mode for FeO.

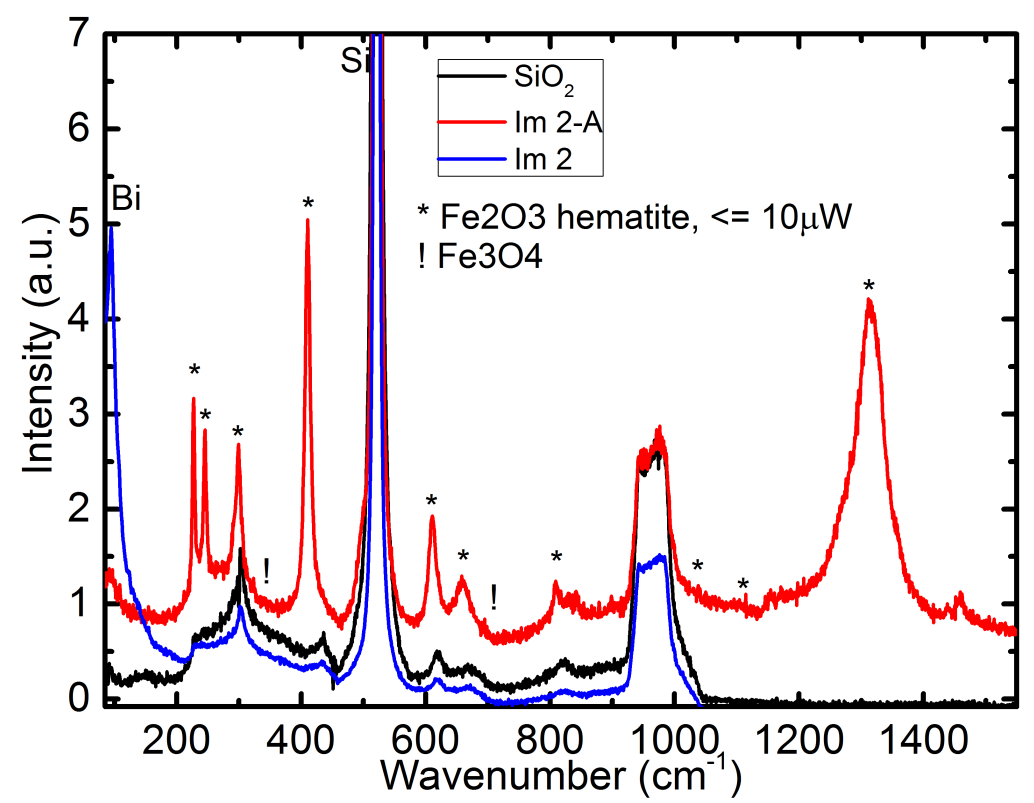

Figure D.3.5: Raman spectrum of IM2 (blue), IM2-A (red) and $\mathrm{SiO}_{2}$ substrate (black). Also, shown in this figure is bismuth Raman scattered peak $\sim 90 \mathrm{~cm}^{-1}$, hematite $\left(^{*}\right)$ and magnetite (!).

After annealed the sample for 120 minutes, the Raman spectroscopy no longer shows the bismuth peak around $\sim 90 \mathrm{~cm}^{-1}$ [Salazar-Pérez et al., 2005; Kumari et al., 2007; Steele and Lewis, 2014]. The new Raman active mode after annealing confirms that annealing IM2 modifies the phases in the film. IM2-A shows 10 Raman actives mode of $\alpha-\mathrm{Fe}_{2} \mathrm{O}_{3}$ hematite [Jubb and Allen, 2010]. There might also be a broad shoulder around $700 \mathrm{~cm}^{-1}$; if this is real, the Raman shift would indicate the presence of $\mathrm{Fe}_{3} \mathrm{O}_{4}$ magnetite [Jubb and Allen, 2010]. So, annealing might form hematite or increase the particles size to be seen in Raman spectroscopy and magnetite might also form or grow. Magnetite and maghemite presence will be further analysis with the magnetic moment analysis.

Both IM2 and IM2-A do not show any sign of FeSi [Nyhus et al., 1995; Ponosov et al., 2016] or $\beta-\mathrm{FeSi}_{2}$ [Yoshitake et al., 2001]. The only magnetic phases present in this analysis are iron oxides.

FeSi and $\beta-\mathrm{FeSi}_{2}$ may have been present in IM1-A, further analysis of that sample 
would be required to confirm that, however those phases are not present either in IM2 or IM2-A. The Raman spectroscopy is extremely different between the IM1-A and IM2A. This suggests that changing the implantation order resulted in significant different phases or IM2-A, Figure D.3.5, the hematite phase is in sufficient concentration to hide the other phases in Raman scattering. Further analysis would be required to confirm, if IM1 and IM2 are significantly different and the source of the difference.

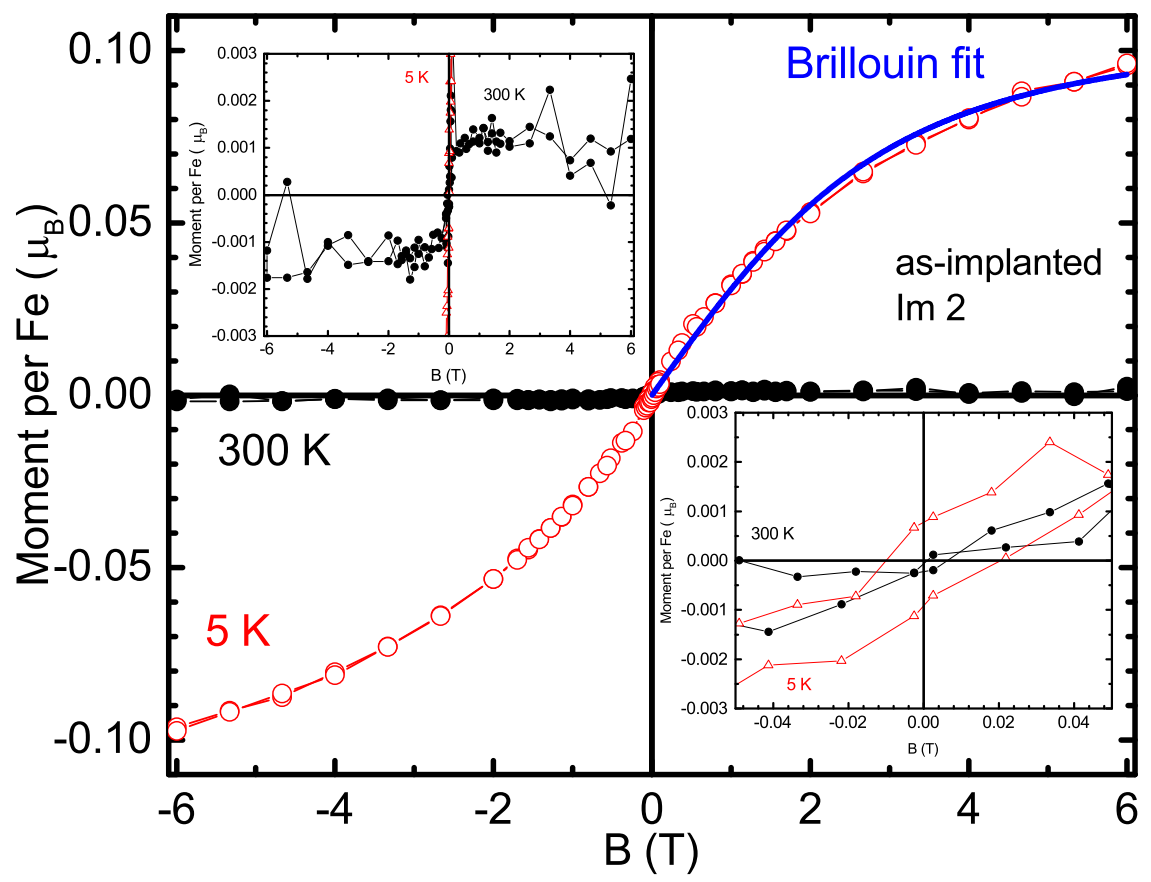

Figure D.3.6: Field loop, ambient temperature (black) and 5 K (red), of IM2. Inset shows an expand scale near $0 \mathrm{~T}$. Also, show in this figure is the Brillouin function (blue line) with $\mathrm{J}=2.5 \pm 0.1$.

The Figure D.3.6 displays the Field loop, ambient temperature (black) and $5 \mathrm{~K}$ (red), of IM2. At $300 \mathrm{~K}$, the field loop saturates at $0.01 \mu_{B} / \mathrm{Fe}$ while the low temperature field loop does not saturate. Also, shown in this figure is an inset with an expand scale near $0 \mathrm{~T}$. This inset shows a hysteresis loop at low temperature that close at ambient temperature.

The as-implanted sample has a weak magnetic signal at ambient temperature that could come from hematite. The weak magnetic signal shows that not all the iron moments are order which is consistent with the antiferromagnetic [Wu et al., 2015] phase displayed in the Raman spectroscopy [Jubb and Allen, 2010], Figure D.3.5. A very small fraction of maghemite or magnetite could explain the increase moment at low temperature and the apparent absence of saturation [Wu et al., 2015].

The Figure D.3.6 displays the Brillouin function, blue line, fitted to the magnetic 
moment of the as-implanted film, IM2. The Brillouin model fit in this figure is estimated at $\mathrm{J}=2.46$ which is close to the $\mathrm{J}=5 / 2$. This model Brillouin function is consistent to $\mathrm{Fe}^{3+}$ in high concentration [Kittel, 2005; Van Diepen and Popma, 1978].

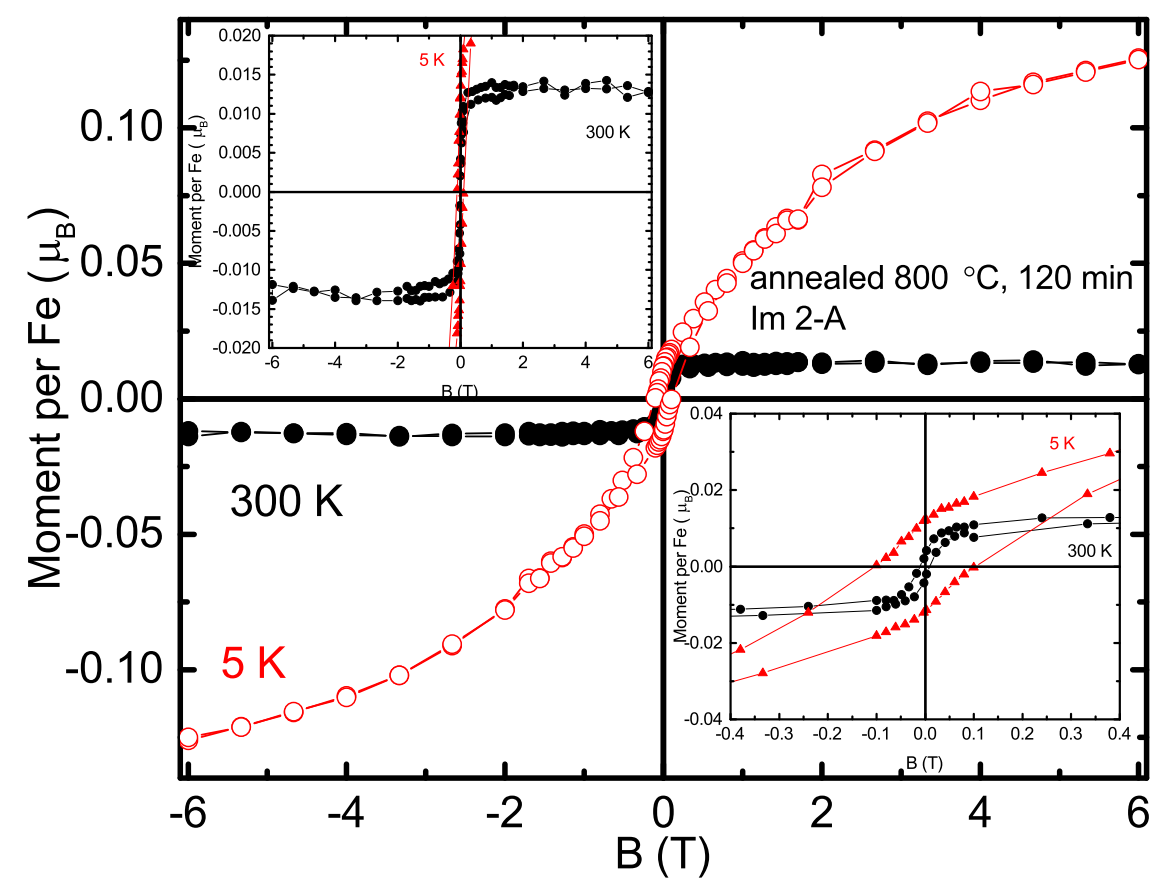

Figure D.3.7: Field loop, $300 \mathrm{~K}$ (black) and $5 \mathrm{~K}$ (red), of IM2-A. Inset shows an expand scale near $0 \mathrm{~T}$.

The Figure D.3.7 displays the Field loop, ambient temperature (black) and $5 \mathrm{~K}$ (red), of IM2-A. At $300 \mathrm{~K}$, the field loop saturates at $0.012 \mu_{B} / \mathrm{Fe}$ while the low temperature field loop does not saturate. Also, shown in this figure is an inset with an expand scale near $0 \mathrm{~T}$. This inset shows a hysteresis loop at low temperature that reduce at ambient temperature.

After annealing at $800{ }^{\circ} \mathrm{C}$ for 120 minutes, the magnetic moment is slightly enhanced, suggesting an increase in the amount of iron oxides [Cullity and Graham, 2009; Aragón, 1992; Nadeem et al., 2011]. IM2-A shows a clear ferromagnetic behaviour, displayed by a hysteresis loop at ambient temperature and low temperature. This could suggest that ferromagnetic phases of $\mathrm{Fe}_{3} \mathrm{O}_{4}$ and $\gamma-\mathrm{Fe}_{2} \mathrm{O}_{3}$ [Aragón, 1992; Nadeem et al., 2011] are present in the film. Assuming the magnetic ordering of Fe comes from $\mathrm{Fe}_{3} \mathrm{O}_{4}$ and $\gamma-\mathrm{Fe}_{2} \mathrm{O}_{3}$, those phases would compose less than $9 \%$ of the film; from the Raman analysis, the remaining components would be other iron oxide phases, a significant amount of the iron in the material is not magnetically order. The fact that the hysteresis is still visible at ambient temperature confirms the Curie temperature of IM2-A is above $300 \mathrm{~K}$. 
The Figure D.3.8 (a) displays the Zero-field-cooled (red) and field-cooled (black (ZFC-FC) of IM2. The magnetic field was set to $0.01 \mathrm{~T}$. The ZFC-FC hysteresis close between $60 \mathrm{~K}$ and $90 \mathrm{~K}$. The as-implanted film shows a blocking temperature of $63 \mathrm{~K}$. This blocking temperature indicates nanoparticles smaller than $7 \mathrm{~nm}$ in diameter assuming a superparamagnetic state coming from maghemite or magnetite [Nadeem et al., 2011; Dedkov et al., 2002]. The estimation is based on the blocking temperature, $T_{B}$ [DiPietro et al., 2010], equation 2.1.6. The small moment seen in the Figure D.3.8 (a) could be caused by a small concentration of free moment in the material.

The Figure D.3.8 (b) displays the zero-field-cooled (red) and field-cooled (black (ZFC-FC) of IM2-A. The magnetic field was set to $0.01 \mathrm{~T}$. The hysteresis of the ZFC-FC does not close at high temperature. The ZFC-FC splitting at high temperature suggest a ferromagnetic material, this can be expected with a material containing mix phases of iron oxide like $\mathrm{Fe}_{3} \mathrm{O}_{4}$ or $\gamma-\mathrm{Fe}_{2} \mathrm{O}_{3}$ [Aragón, 1992; Nadeem et al., 2011].

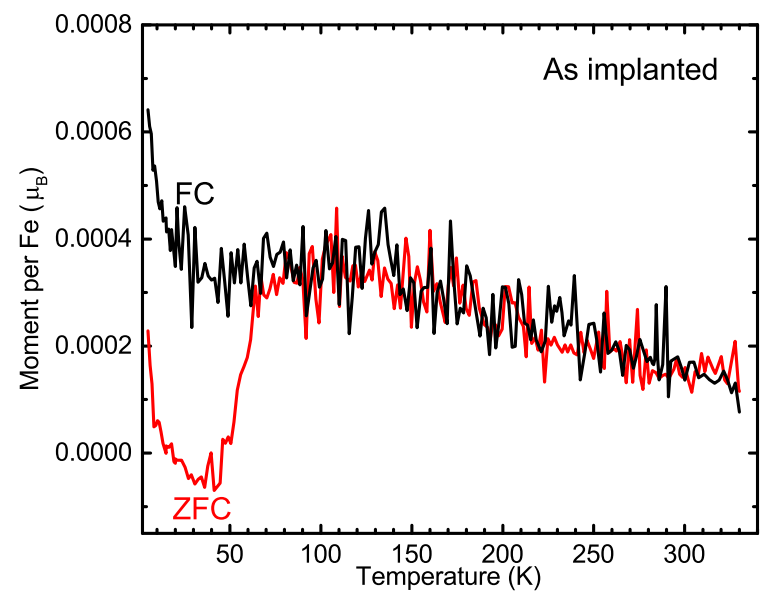

(a) ZFC-FC of IM2.

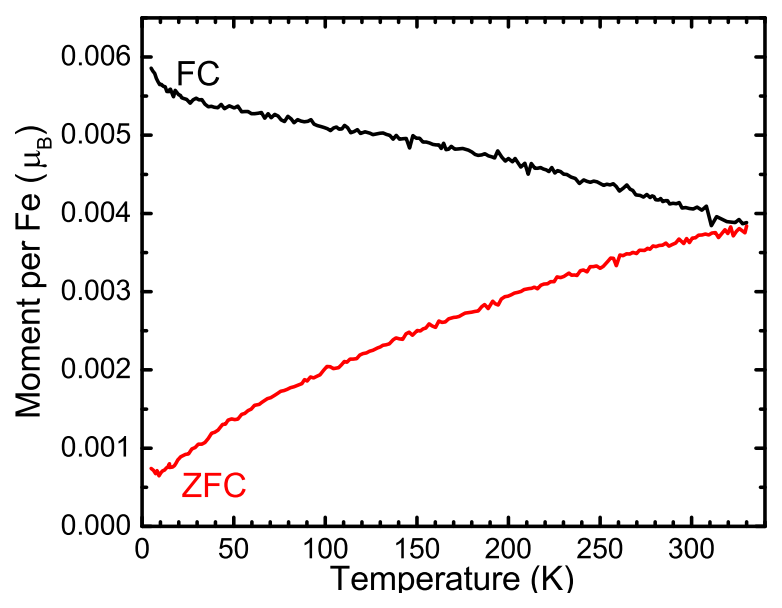

(b) ZFC-FC of IM2-A.

Figure D.3.8: Zero-field-cooled (red) and field-cooled (black (ZFC-FC) of (a) IM2, hysteresis close between $60 \mathrm{~K}$ and $90 \mathrm{~K}$ and (b) IM2-A, the hysteresis of the ZFC-FC does not close at high temperature. The magnetic field was set to $0.01 \mathrm{~T}$.

The Figure D.3.9 (a) displays the temperature dependence of the saturated magnetic moment under an applied magnetic field at $6 \mathrm{~T}$ for the sample IM2. Also, shown in this figure is a Curie-Weiss law fit (red) with a Curie-Weiss temperature near $0 \mathrm{~K}$, $(4.35 \pm 4.98 \mathrm{~K})$.

From equation D.3.1 [Hall and Hook, 1991] and the $C_{0}$ value of $1 \mu_{B}$ extracted from the Figure D.3.9 (a), the effective moment can be estimated: 


$$
\frac{m}{N \mu_{B}}=\frac{P_{\mathrm{eff}}^{2} \mu_{B} B}{3 k_{B}(T-\theta)}
$$

Where $m$ is the magnetic moment, $N$ the number of atoms, $\mu_{B}$ is the Bohr magneton, $P_{\text {eff }}$ is the effective moment, $B$ is the applied magnetic field of $6 \mathrm{~T}, k_{B}$ is the Boltzmann constant, $T$ is the varying temperature and $\theta$ is the Curie temperature. From the theory $P_{\text {eff }}=\mathrm{g}(\mathrm{S}(\mathrm{S}+1))^{1 / 2}\left[\right.$ Levy, 1968] and $\mathrm{S}_{\mathrm{Fe}^{2+}}$ can be 2, 1/2 or 0 and $\mathrm{S}_{\mathrm{Fe}^{3+}}$ can be 5/2, $1 / 2$ or $3 / 2$. Assuming each $\mathrm{Fe}^{2+}$ has an equivalent number of $\mathrm{Fe}^{3+}$ then in a simple model $P_{\text {eff }}=(1 / 3)^{1 / 2} \times\left(\mathrm{P}_{F e^{2+}}^{2}+4 \mathrm{P}_{F e^{3+}}^{2}\right)^{1 / 2}$. Assuming $\mathrm{P}_{F e^{2+}}=0$ and $\mathrm{P}_{F e^{3+}}=1.73$, both values are the smallest energy for $\mathrm{Fe}^{2+}$ and $\mathrm{Fe}^{3+}$ then $P_{\text {eff }}>1.41 . C_{0}=P_{\text {eff }}^{2} \times \mu_{B} \times \mathrm{B} /\left(3 k_{B}\right)$ and hence $P_{\text {eff }}=0.86 \times\left(C_{0}\right)^{0.5}$, so $P_{\text {eff }}=0.8 \pm 0.2 \mu_{B}$. This is less than expected for $\mathrm{Fe}^{2+}$ or $\mathrm{Fe}^{3+}$. A low effective moment for $\mathrm{Fe}$ is consistent with significant antiferromagnetic order.

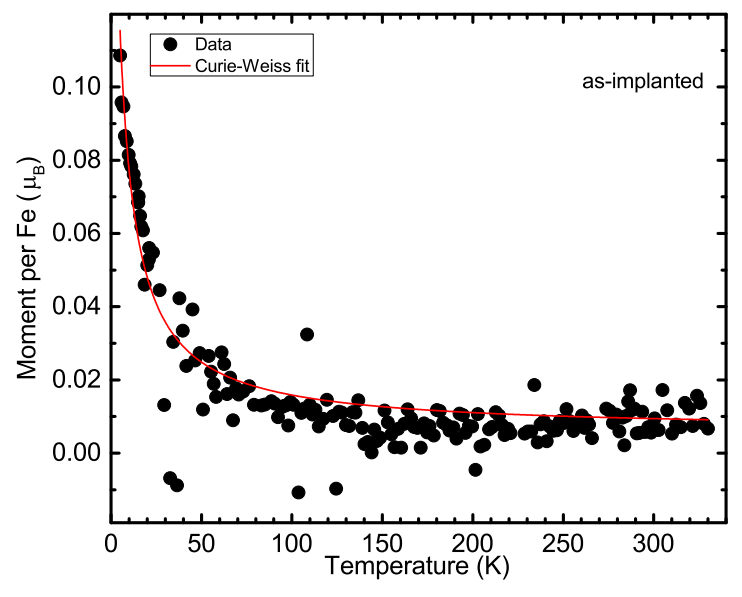

(a) IM2, $\mathrm{T}_{C}=-4.35 \mathrm{~K}, C_{0}=\mathbf{1} \pm \mathbf{0 . 4} \mu_{B}$.

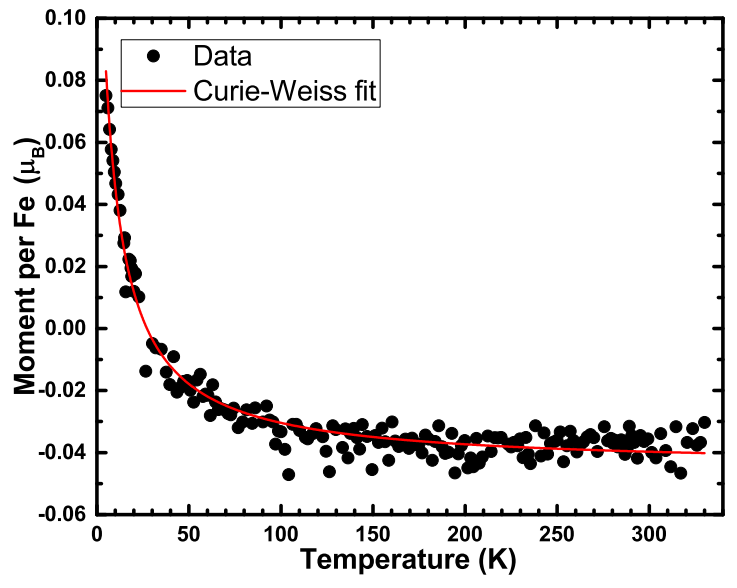

(b) IM2-A $\mathrm{T}_{C}=-7 \mathrm{~K}$.

Figure D.3.9: Temperature dependence of the saturated magnetic moment under an applied magnetic field at 6 T for (a) IM2 and (b) IM2-A. Also, shown in this figure is a Curie-Weiss law fit (red).

The Figure D.3.9 (b) displays the temperature dependence of the saturated magnetic moment under an applied magnetic field of $6 \mathrm{~T}$ for sample IM2-A. Also, shown in this figure is a Curie-Weiss law fit (red) with a Curie Weiss temperature of $-7 \mathrm{~K}$. The temperature dependence at $6 \mathrm{~T}$ follows the Curie-Weiss law with $T_{N}=-7 \mathrm{~K}$, which confirm no long range magnetic interaction are visible meaning the material may display an antiferromagnetic ordering. Both the as-implanted, IM2, and annealed, IM2-A, film follows the Curie-Weiss law. In the case of the IM2-A, the Curie Weiss temperature according to Curie Weiss's law is $T_{N}=-7 \mathrm{~K}$ instead of nearly $0 \mathrm{~K}$ for the sample IM2. The fit is better after annealing the sample, meaning the Curie-Weiss law describes the tem- 
perature dependence of this material very well, while the as-implanted sample might also have some contribution from spin disorder to explained the limited fit quality.

From the Curie-Weiss fit in Figure D.3.9 (b) and the equation D.3.1, the effective moment is estimated at $1.1 \pm 0.1 \mu_{B}$. Assuming $P_{\mathrm{Fe}^{2+}}=0$ and $P_{\mathrm{Fe}^{3+}}=1.73$, both values are the smallest energy for $\mathrm{Fe}^{2+}$ and $\mathrm{Fe}^{3+}$ then $P_{\text {eff }}>1.41$. So, the iron must be in an antiferromagnetic state like hematite phase seen in Raman spectroscopy with a small ferromagnetic contribution from maghemite and magnetite. The effective moment before annealing, $0.8 \pm 0.2 \mu_{B}$, is close to the effective moment after annealing the sample, $1.1 \pm 0.1 \mu_{B}$, so a similar phase might be present in both films. The Curie-Weiss fit indicates that an un-ordered moment is present and that un-ordered moment could originate from the bismuth ions implanted after iron oxide had form.

The as-implanted film displays an exchange bias at $5 \mathrm{~K}$ of less than $0.5 \mathrm{mT}$, while the annealed film does not seem to display any exchange bias. The almost inexistent exchange bias suggests [Nogués and Schuller, 1999] that if the films contains a ferromagnetic and antiferromagnetic phase, they are not in contact or other ions may reduce the bias.

\section{D.4 Implantation of Fe then $\mathrm{O}$ then $\mathrm{Bi}$ into $\mathrm{Si}$, IM3}

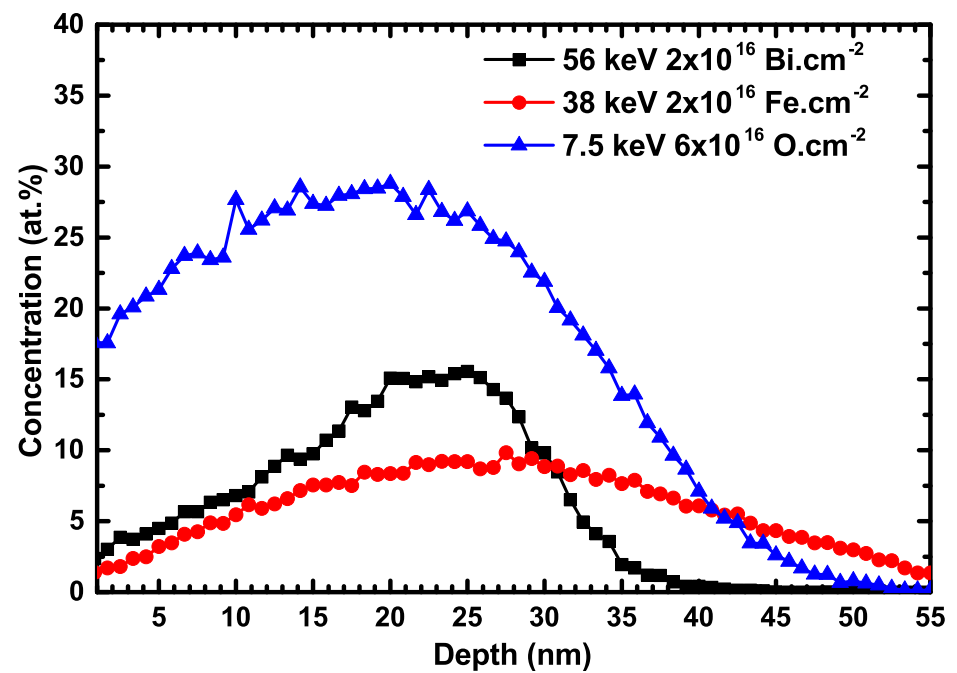

Figure D.4.1: D-Trim simulation of IM3, $56 \mathrm{keV} 2 \times 10^{16}$ Bi.cm $^{-2}$ (black), $38 \mathrm{keV} 2 \times 10^{16}$

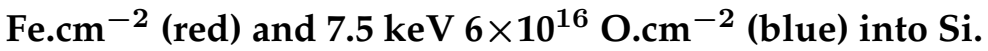

Study the effect oxygen in the substrate would be interesting and could be done by comparing the ions implantated into $\mathrm{SiO}_{2}: \mathrm{Si}$ with an implantation into bulk silicon. This could increase the knowledge the interaction of the substrate oxygen with the im- 
planted iron and bismuth ions. The results in this section are presented as preliminary results, XRD and TEM analysis were not carried on this set of sample as of now. The XRD and TEM analysis of this set of sample would be interesting to clearly identify the phases present. Preliminary results suggest the formation of another phase, FeSi or $\beta-\mathrm{FeSi}_{2}$ then those seen in IM1 and IM2.

The Figure D.4.1 displays the D-Trim simulation of implantation Fe then $\mathrm{O}$ then Bi into Si bulk, IM3. D-Trim calculation for $56 \mathrm{keV} 2 \times 10^{16}$ Bi.cm ${ }^{-2}$ (black), $38 \mathrm{keV}$ $2 \times 10^{16} \mathrm{Fe} . \mathrm{cm}^{-2}$ (red) and $7.5 \mathrm{keV} 6 \times 10^{16}$ O.cm ${ }^{-2}$ (blue) into Si. Also, shown in this figure is the bismuth depth profile estimated at $40 \mathrm{~nm}$ with a concentration of $15 \%$. Iron has a depth profile $55 \mathrm{~nm}$ with a concentration of approximately $10 \%$. The implanted oxygen depth profile is estimated at $50 \mathrm{~nm}$ with a concentration of $29 \%$.

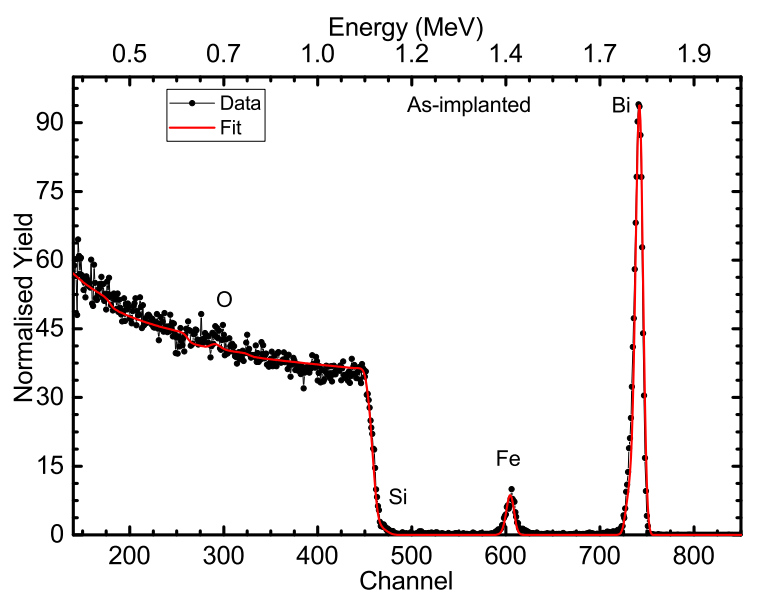

(a) IM3, as-implanted.

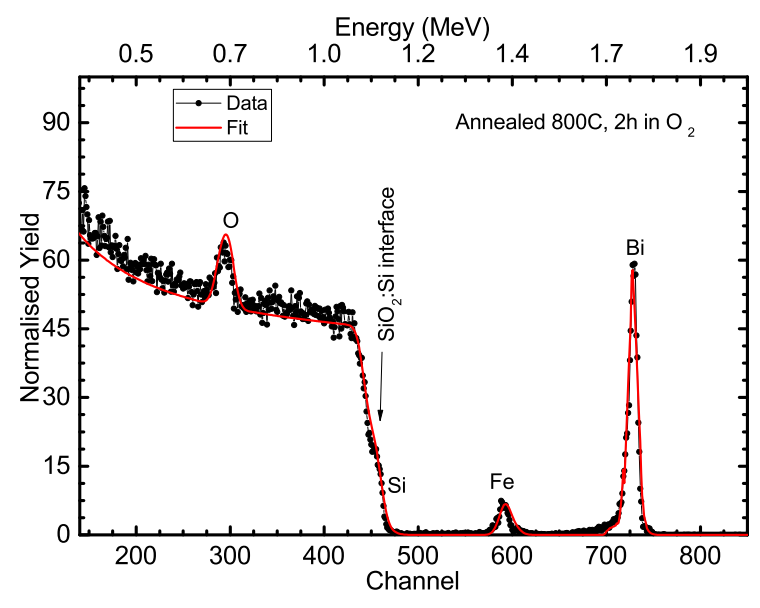

(b) IM3-A, annealed at $800{ }^{\circ} \mathrm{C}, 120 \mathrm{~min}$.

Figure D.4.2: RBS of implanted Fe $2 \times 10^{16}$ at.cm ${ }^{-2}$ then $O$ at $6 \times 10^{16}$ at.cm ${ }^{-2}$ then $B i 2 \times 10^{16}$ at.cm ${ }^{-2}$ into bulk Si. Also, shown is the RUMP fit.

The Figure D.4.2 (a) displays the RBS of the Implanted Fe $2 \times 10^{16} \mathrm{at.cm}^{-2}$ then $\mathrm{O}$ at $6 \times 10^{16}$ at.cm ${ }^{-2}$ then Bi $2 \times 10^{16}$ at.cm ${ }^{-2}$. IM3 is the as-implanted into bulk Si. Also, shown in this figure is the RUMP fit with the assumption of a 3 layers' material. The surface layer is composed of $\mathrm{Bi}_{0.3}, \mathrm{Fe}_{0.3}, \mathrm{O}_{0.1}$ and $\mathrm{Si}_{1}\left(1 \times 10^{17}\right.$ at.cm ${ }^{-2}, 4.28 \times 10^{22}$ at.cm $\left.{ }^{-3}\right)$ with a thickness of $23 \pm 3 \mathrm{~nm}$. That stoichiometry could suggest bismuth and iron bonded with the silicon substrate, however, this is difficult to achieve. The second layer is made of $\mathrm{Bi}_{0.1}, \mathrm{Fe}_{0.1}, \mathrm{O}_{0.1}$ and $\mathrm{Si}_{1}\left(2 \times 10^{17}\right.$ at.cm ${ }^{-2}, 4.57 \times 10^{22}$ at.cm $\left.{ }^{-3}\right)$ with a thickness of $44 \pm 2 \mathrm{~nm}$ and the third layer is the bulk Si. The stoichiometry of the second layer could suggest the formation of FeO, FeSi and Bi. From the RUMP fit, the bismuth dose is calculated at $2 \times 10^{16}$ at.cm ${ }^{-2}$ and the iron dose is calculated at $2 \times 10^{16}$ at.cm ${ }^{-2}$. The oxygen intensity peak is weak which increases the uncertainty of that measurement, however the total oxygen concentration can be estimated at $5 \times 10^{16}$ at.cm $^{-2}$. A native oxide layer is always expected in silicon substrate, however the native layer and 
the implanted oxygen ions layer are indistinguishable from each other. The total dose calculated with RUMP fit would include the native oxygen and the implanted oxygen ions. The RBS analysis is not sufficiently sensitive to light element like oxygen to provide an accurate concentration in the sample IM3.

The iron and bismuth dose are, $2 \times 10^{16}$ at. $\mathrm{cm}^{-2}$, which is the selected implanted fluence of $2 \times 10^{16}$ at.cm ${ }^{-2}$ (D-Trim simulation). The theoretical depth profile was estimated by D-Trim at $40 \mathrm{~nm}$ for bismuth and $55 \mathrm{~nm}$ for iron. RUMP fit derive an estimated depth profile of iron and bismuth around $67 \pm 5 \mathrm{~nm}$. The difference could come from the D-Trim assumption that the substrate is made of pure silicon while the silicon substrate used is p-type doping with boron. The boron concentration could be sufficient to modify the silicon density and thus modifying the stopping range power. The other option would be a self-annealing treatment during the implantation, both are unlikely the cause of that difference in depth profile.

The oxygen fluence was theoretically predicted by D-Trim to be $6 \times 10^{16}$ at.cm ${ }^{-2}$, however the RUMP simulation calculates a dose of $5 \times 10^{16} \mathrm{at}_{\mathrm{cm}}^{-2}$. The difference could arise from the weak oxygen signal increasing the uncertainty of the estimation or an uneven oxygen implantation. The Figure D.4.2 (b) displays the RBS of the Implanted Fe $2 \times 10^{16}$ at.cm ${ }^{-2}$ then $\mathrm{O}$ at $6 \times 10^{16}$ at.cm ${ }^{-2}$ then $\mathrm{Bi} 2 \times 10^{16}$ at.cm $^{-2}$, implanted into Si then annealed at $800{ }^{\circ} \mathrm{C}$ for 120 minutes, IM3-A. Also, shown in this figure is the RUMP fit based on the assumption of a 4 layers' material. The surface layer is composed of $\mathrm{Si}_{1} \mathrm{O}_{2}, \mathrm{Bi}_{0.001}$ and $\mathrm{Fe}_{0.03}\left(4.4 \times 10^{17}\right.$ at.cm ${ }^{-2}, 5.27 \times 10^{22}$ at.cm $\left.{ }^{-3}\right)$ with a thickness of $84 \mathrm{~nm}$. This stoichiometry suggests bismuth and iron doping of $\mathrm{SiO}_{2}$. The second layer is composed of $\mathrm{Si}_{1} \mathrm{O}_{2}, \mathrm{Bi}_{0.3}$ and $\mathrm{Fe}_{0.3}\left(1.8 \times 10^{17}\right.$ at.cm ${ }^{-2}, 4.52 \times 10^{22}$ at.cm $\left.{ }^{-3}\right)$ with a thickness of $40 \mathrm{~nm}$. This stoichiometry suggests bismuth and iron doping of $\mathrm{SiO}_{2}$. The third layer is made of $\mathrm{SiO}_{2}$ with $\mathrm{Bi}_{0.01}\left(0.8 \times 10^{17}\right.$ at.cm ${ }^{-2}, 5.11 \times 10^{22}$ at.cm $\left.{ }^{-3}\right) 16 \mathrm{~nm}$ and the fourth layer is the bulk Si. From the RUMP fit, bismuth, iron and oxygen dose are calculated at $0.9 \times 10^{16}$ Bi.cm ${ }^{-2}, 1.28 \times 10^{16}$ Fe.cm ${ }^{-2}$ and $30 \times 10^{16}$ O.cm ${ }^{-2}$.

After the sample is annealed for 2 hours in an oxygen atmosphere, the oxidation of the sample is significant, when comparing the as-implanted film oxygen dose $\left(5 \times 10^{16} \mathrm{O} . \mathrm{cm}-2\right)$ to the annealed oxygen dose $\left(30 \times 10^{16} \mathrm{O} . \mathrm{cm}^{-2}\right)$. This would imply that a significant amount of oxygen from the annealing atmosphere was incorporated into the film. The mobility of bismuth atoms is likely the cause of that increasing amount of oxygen, which allows bismuth to bond with oxygen at the surface of the material [Gujar et al., 2005]. The bismuth oxide material has a reduced mobility, and the oxygen stays in the material, in the best case scenario, to form $\mathrm{BiFeO}_{3}$ nanoparticles. The ratio of bismuth to iron is 1 to 1.4 after annealing. The bismuth concentration drops 
from $2 \times 10^{16}$ at.cm ${ }^{-2}$, before annealing, to $1.27 \times 10^{16}$ at.cm ${ }^{-2}$ after. The iron content is also reduced: the as-implanted concentration of iron is $2 \times 10^{16}$ at.cm ${ }^{-2}$, however after annealing, the concentration becomes $1.93 \times 10^{16}$ at.cm ${ }^{-2}$. This indicates evaporation of bismuth ions during the annealing with the evaporation of iron is minimal. The other option to explain the difference would be if the implantation was uneven. The evaporation of bismuth could suggest that this synthesis leads to the formation of Bi phases with weak bond to other atoms.

The other effect annealing has on the film is diffusion the as-implanted film contains bismuth and iron only in the top $\sim 67 \mathrm{~nm}$ while the annealed sample contains bismuth at depth of almost $123 \mathrm{~nm}$. Another modification is the formation of a $139 \mathrm{~nm}$ silicon dioxide layer.

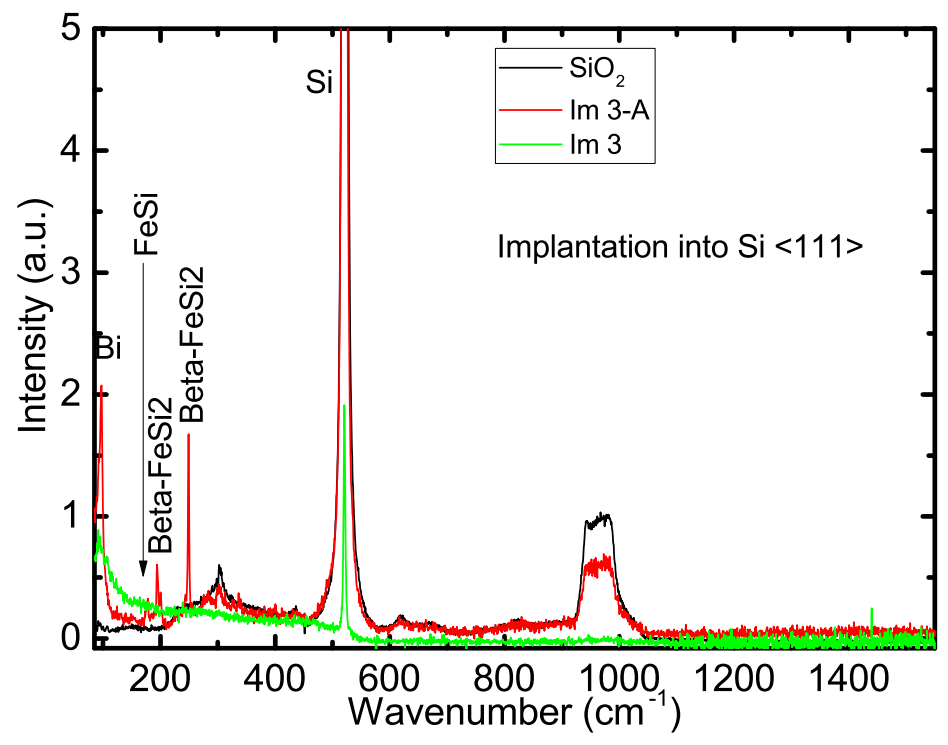

Figure D.4.3: Raman spectrum of implanted Fe then O then Bi into Si. Also, shown is the comparison between IM3-A (red), IM3 (green) and a $\mathrm{SiO}_{2}$ substrate reference (black), bismuth Raman scattered peak around $90 \mathrm{~cm}^{-1}, \beta-\mathrm{FeSi}_{2}$ and $\mathrm{FeSi}$.

The Figure D.4.3 displays the Raman spectroscopy of the sample implanted with Fe then $\mathrm{O}$ then $\mathrm{Bi}$ into $\mathrm{Si}$. Also shown is the bismuth Raman scattered peak around $90 \mathrm{~cm}^{-1}, \beta-\mathrm{FeSi}_{2}$ and FeSi $\left(180 \mathrm{~cm}^{-1}\right.$ [Ponosov et al., 2016]). The Raman spectroscopy was carried out with a laser $514 \mathrm{~nm}$ with a laser power of less than $10 \mu \mathrm{W}$ and with an objective of $100 \times$.

The as-implanted Raman spectrum displays a broad peak at $90 \mathrm{~cm}^{-1}$ that could be bismuth. Bi could be in small particles. The silicon peak is seen at $521 \mathrm{~cm}^{-1}$, however the intensity is reduced suggesting the material may be absorbing a significant part of the light. There is a broad feature around $300 \mathrm{~cm}^{-1}$, similar to the $\mathrm{SiO}_{2}$ reference that may suggest the formation of a silicon dioxide by implanting oxygen ions or this could 
also be due to a small amount of native oxide layer cover the bulk silicon substrate. The rest of the Raman spectrum seems to indicate an amorphous material.

After annealing the film, the Raman spectroscopy shows a bismuth active mode at $90 \mathrm{~cm}^{-1}$ and some Raman active move indicates a bonding between iron and silicon, $\beta-\mathrm{FeSi}_{2}$ and $\mathrm{FeSi}$. The rest of the Raman spectrum behaves exactly like the silicon dioxide blank substrate. This is interesting, as the substrate is a bulk silicon (111) wafer. This could imply that the oxygen implanted and added during annealing formed a silicon dioxide layer. The increase silicon peak intensity after annealing compared to the as-implanted material could come from a loss of ions, bismuth and iron, as discussed in the RBS analysis; the evaporation of those ions would increase the fraction of silicon and increase intensity of the Raman shift. The increase intensity could also indicate a nanostructured material was form, like $\mathrm{SiO}_{2}$. The sharpe peaks seen after annealing the sample suggest that $\beta-\mathrm{FeSi}_{2}$ and $\mathrm{FeSi}$ are large particles.

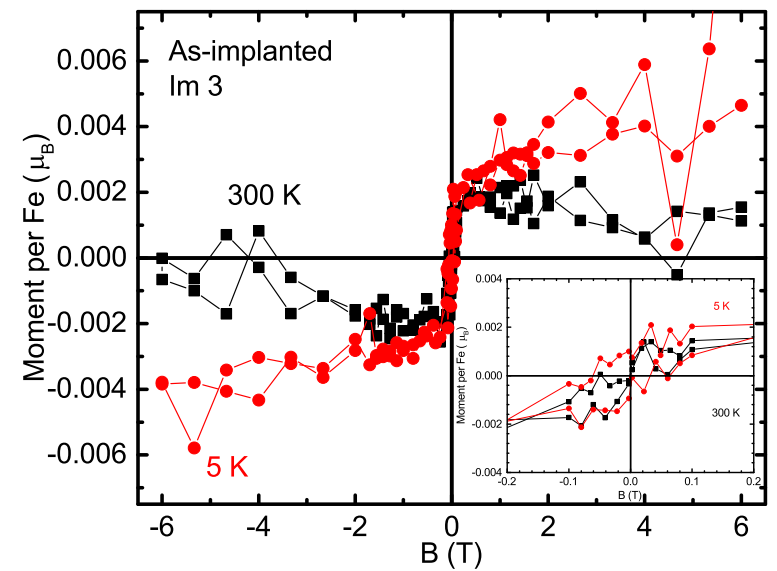

(a) IM3, as-implanted.

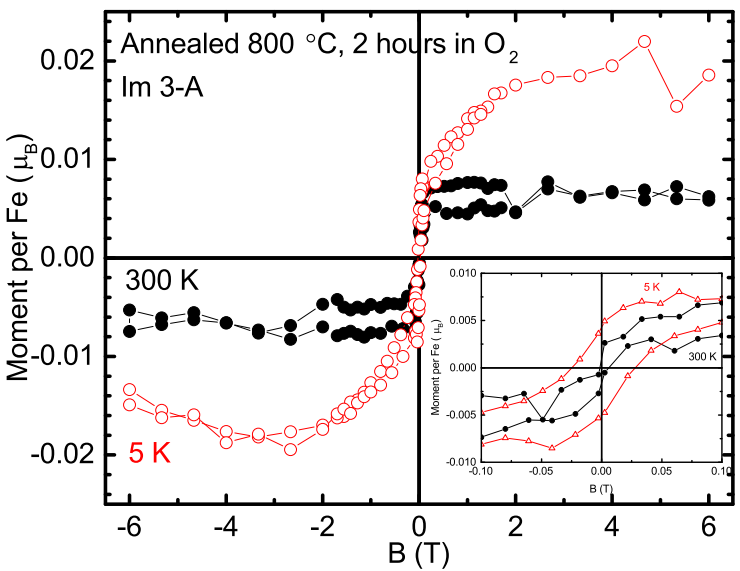

(b) IM3-A, annealed $800^{\circ} \mathrm{C}, 120 \mathrm{~min}$.

Figure D.4.4: Field loop, $300 \mathrm{~K}$ (black) and $5 \mathrm{~K}$ (red) of (a) IM3. The ambient temperature field loop saturates at $0.002 \mu_{B} / \mathrm{Fe}$ and the low temperature field loop saturate at $0.003 \mu_{B} / F e$. (b) IM3-A, the ambient temperature field loop saturates at $0.006 \mu_{B} / \mathrm{Fe}$ and the low temperature field loop saturate at $0.019 \mu_{B} / \mathrm{Fe}$. Inset: expand scale near $0 \mathrm{~T}$ showing a hysteresis loop at low temperature and ambient temperature.

The Figure D.4.4 (a) displays the field loop, ambient temperature (black) and $5 \mathrm{~K}$ (red), of IM3. The ambient temperature field loop saturates at $0.002 \mu_{B} / F e$ and the low temperature field loop saturate at $0.003 \mu_{B} / F e$. Also, shown in this figure is an inset with an expand scale near $0 \mathrm{~T}$. This inset shows a hysteresis loop at low temperature and ambient temperature.

The as-implanted sample has a weak magnetic signal at ambient temperature that 
could come from hematite. A very small fraction of maghemite or magnetite could explain the increase moment at low temperature and the apparent absence of saturation.

The Figure D.4.4 (a) inset displays a hysteresis loop at low and ambient temperature, indicative of a ferromagnetic or ferrimagnetic material. The $\beta-\mathrm{FeSi}_{2}$ saturation magnetic moment at $2 \mathrm{~K}$ was reported to be $0.3 \mu_{B} / F e$ Liang et al. [2006]. In the as-implanted film, according to Raman analysis on the annealed film, only $\beta$-FeSi ${ }_{2}$ is present with non-magnetic bismuth. Iron oxide could be present in an amorphous phase. Knowing that magnetite and maghemite have a magnetic saturation moment up to $1.35 \mu_{B} / \mathrm{Fe}$ [Cullity and Graham, 2009] then $\mathrm{Fe}_{3} \mathrm{O}_{4}$ and $\gamma-\mathrm{Fe}_{2} \mathrm{O}_{3}$ can be neglected in this analysis. A quick estimate would suggest that those iron oxide materials would be responsible for less than $1 \%$ of the Fe magnetic ordering. $\alpha-\mathrm{Fe}_{2} \mathrm{O}_{3}$ and the other iron oxide material previously analysed have a weak magnetic moment, limiting the ability to analyse them with this technique. However, the Raman spectra does not clearly show hematite which would indicate that hematite phase fraction is possibly present in a small amount or that phase is in an amorphous state. This means that the magnetic Fe might come from less than $2 \%$ of $\beta-\mathrm{FeSi}_{2}$. $\beta$-FeSi ${ }_{2}$ has been reported to be weakly ferromagnetic [Hattori et al., 2007], as seen in the Figure D.4.4 (a), the as-implanted film maybe weakly ferromagnetic.

The Figure D.4.4 (b) displays the Field loop, $300 \mathrm{~K}$ (black) and $5 \mathrm{~K}$ (red), of IM3-A. The ambient temperature field loop saturates at $0.006 \mu_{B} / F e$ and the low temperature field loop saturate at $0.019 \mu_{B} / F e$. Also, shown in this figure is an inset with an expand scale near $0 \mathrm{~T}$. The inset shows a hysteresis loop at low temperature that reduce at ambient temperature.

Annealing the implanted film leads to an enhanced magnetic moment at saturation, probably caused by an increase in iron-silicon or iron oxide formation. At ambient temperature, the magnetic moment of the as-implanted sample is $0.003 \mu_{B} / \mathrm{Fe}$ and after annealing this value double to become $0.006 \mu_{B} / F e$. As the Raman analysis shows, the only source of potential magnetic material is $\beta-\mathrm{FeSi}_{2}$, iron oxide is not clearly identified. So, assuming the only magnetic material present in the film is the iron-silicon, the magnetic Fe would compose less than $7 \%$ of the film in $\beta-\mathrm{FeSi}_{2}$. The insert, Figure D.4.4 (b), displays a hysteresis loop at low temperature that disappears at ambient temperature. With an enhanced magnetic saturation moment at low temperature compared to ambient temperature, a superparamagnetism material might be present.

The Figure D.4.5 (a) displays the Zero-field-cooled (red) and field-cooled (black (ZFC-FC) of IM3. The magnetic field was set to $10 \mathrm{mT}$. The ZFC-FC hysteresis close 
between $70 \mathrm{~K}$ and $125 \mathrm{~K}$. Due to a weak magnetic moment measured, the noise level is significant which increases the uncertainty of the blocking temperature.

The as-implanted film shows a weak magnetic response suggesting that the fraction of superparamagnetic material maybe small. The amount of potential magnetic iron was estimated to be less than $2 \%$ when analysing the field loop measurements. Assuming the ZFC and FC splitting point occurs between $70 \mathrm{~K}$ and $125 \mathrm{~K}$, this would imply $\beta$-FeSi ${ }_{2}$ nanoparticles would be larger than $60 \mathrm{~nm}$ in diameter [Chen et al., 2007]. A large particle size is consistent with the Raman analysis.

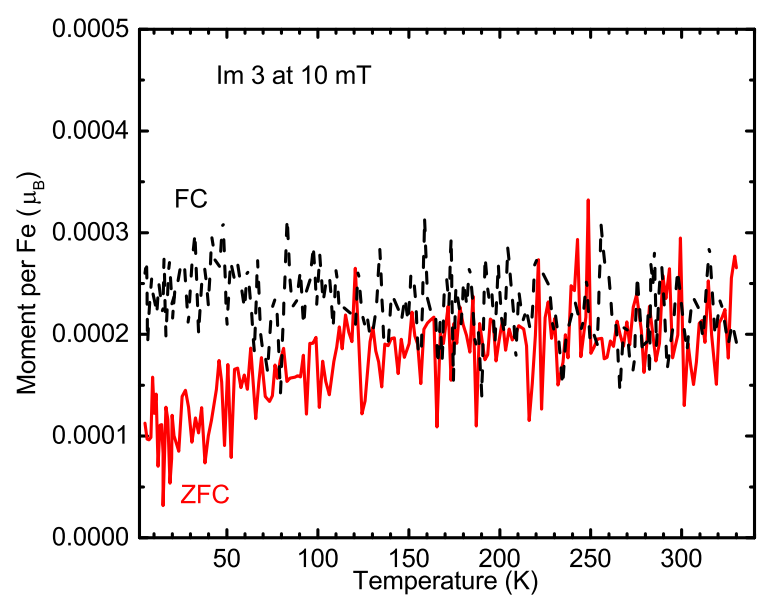

(a) IM3, as-implanted.

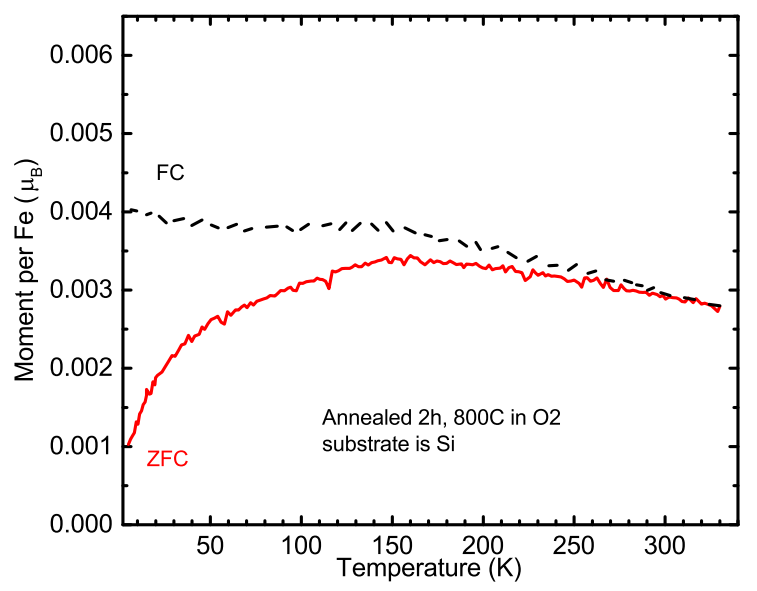

(b) IM3-A, annealed $800{ }^{\circ} \mathrm{C}, 120 \mathrm{~min}$.

Figure D.4.5: ZFC (red) and FC (black) of (a) IM3. The magnetic field was set to $0.01 \mathrm{~T}$. The ZFC-FC hysteresis close between $70 \mathrm{~K}$ and $125 \mathrm{~K}$. Due to a weak magnetic moment measured, the noise level is significant which increases the uncertainty of the blocking temperature. (b) IM3-A, the magnetic field was set to $0.01 \mathrm{~T}$. The ZFC-FC hysteresis might close near $300 \mathrm{~K}$. There might be a ferromagnetic contribution as the ZFC and FC merge close to ambient temperature.

The Figure D.4.5 (b) displays the ZFC (red) and FC (black) of IM3-A. The magnetic field was set to $10 \mathrm{mT}$. The ZFC-FC hysteresis might close near $300 \mathrm{~K}$. There might be a ferromagnetic contribution as the ZFC and FC merge close to ambient temperature, however no clear merge is reported.

Superparamagnetism could be debated in the as-implanted sample because of a weak magnetic signal, the annealed sample may show a superparamagnetic effect, ZFC-FC split and merge. If the magnetic moment is assumed to originate in the formation of iron-silicon and the blocking temperature is around $300 \mathrm{~K}$ then the nanoparticles would be larger than $65 \mathrm{~nm}$ [Chen et al., 2007]. The sharp peak from the Raman spectrum confirms the large particle size is present. The imprecision of the blocking temperature, a transition in the ZFC is seen around $150 \mathrm{~K}$, this could arise from a size 
distribution of $\beta-\mathrm{FeSi}_{2}$.

The temperature dependence is not a reliable measurement for the as-implanted and annealed sample as the silicon substrate seems to dominate the magnetic signal at saturation. The small number of implanted ions compared to the silicon substrate could explain the weak magnetic contribution of those implanted species.

\section{D.5 Conclusion}

The new approach to synthesis $\mathrm{BiFeO}_{3}$ by ion implantation technique needs further optimisation, however synthesising $\mathrm{BiFeO}_{3}$ thin film using this approach seems challenging. Nonetheless this material opens an interesting study on the particle formation, iron oxide and the effects of bismuth. Implanting bismuth first leads to a significant sputtering of implanted ions. Annealing the implanted bismuth then iron the oxygen forms a crystalize $\mathrm{SiO}_{2}$ layer with iron oxide present in small fraction compare to the $\mathrm{SiO}_{2}$. While implanting iron first leads to the formation of either iron oxide if implanted into $\mathrm{SiO}_{2}$ substrate or iron-silicon if implanted into a bulk silicon substrate, the evaporation of ions during the annealing process is negligible. The bismuth implantation as the last implanted ion seems to result in a highly disorder layer containing bismuth ions.

The results in this section are presented as preliminary results, XRD and TEM analysis were not carried on this set of sample as of now. The first set of samples, IM1, implantation of $\mathrm{Bi}$ then $\mathrm{Fe}$ then $\mathrm{O}$ into $\mathrm{SiO}_{2}: \mathrm{Si}$, has a crystalize $\mathrm{SiO}_{2}$ layer and IM1 may contain a small fraction of mix phases of iron oxides: $\alpha-\mathrm{Fe}_{2} \mathrm{O}_{3}, \gamma-\mathrm{Fe}_{2} \mathrm{O}_{3}, \mathrm{Fe}_{3} \mathrm{O}_{4}$, and $\beta-\mathrm{FeSi}_{2}$. There is no evidence of superparamagnetic effect in the annealed film. Due to a significant number of ions evaporation during the annealing process, this sample was used as an exploratory research for nanostructured material synthesis by triple implantation. For future work, investigating that crystallise $\mathrm{SiO}_{2}$ layer with TEM and modifying the annealing conditions to study the effect on phase formation and ions evaporation could be of interest.

The second set of samples, IM2, implantation of Fe then $\mathrm{O}$ then $\mathrm{Bi}$ into $\mathrm{SiO}_{2}$ :Si. This film when annealed does not lose a significant amount of implanted ions by evaporation. IM2 contains a mix phase of iron oxides; $\alpha-\mathrm{Fe}_{2} \mathrm{O}_{3}$ and $\mathrm{Fe}_{3} \mathrm{O}_{4}$. Bismuth is seen in the as-implanted sample, however that element is not clearly identified after annealing suggesting that bismuth may be in a highly disorder layer. The other annealing 
effect is to increase the quantity of iron oxide in the material. Annealing the sample seems to change the magnetic phase from a superparamagnetic, with a low effective moment, phase to a ferromagnetic phase. There is no evidence of a ferromagnetic and antiferromagnetic interface in this film.

In a future work, a TEM analysis of those samples could lead to a better understanding of the phases formed and analyse the concentration of bismuth ions. Implanting bismuth then oxygen then iron could increase the chance of creating iron-oxygenbismuth bonds. Modifying the annealing procedure, higher temperature and duration, to analysis the phases and implanting bismuth then oxygen then iron. Implanting at

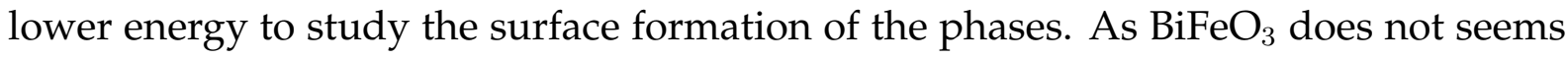
to be forming while implanting ions into silicon based substrate, use other substrates (e.g. $\mathrm{SrTiO}_{3}, \mathrm{LaAlO}_{3}$ ) could be suggested to form $\mathrm{BiFeO}_{3}$.

The third set of sample, IM3, implantation Fe then O then Bi into Si (111) does show a significant number of implanted ions loss. The film shows the preliminary results of the formation of iron-silicon phases and bismuth. The magnetic moment in IM3 is extremely weak compared to the implantation made into $\mathrm{SiO}_{2}: \mathrm{Si}$, IM2. This could mean a smaller iron oxide concentration is in the sample implanted into bulk silicon, IM3. Below $100 \mathrm{~K}$, there is no evidence of ferromagnetic phase. In future work, the study of FeSi with a TEM analysis to understand the formation of that material and the magnetic ordering would be interesting to undertake.

The comparison between implantation into pure bulk silicon and silicon dioxide could imply that the implanted iron ions interact with the substrate and form a stable compound, stable enough to avoid any other bonding even under the influence of the high kinetic energy of heavy elements like bismuth. When iron is implanted in silicon dioxide, the sample seems to contain a significant amount of iron oxide, while implanting iron into pure silicon seems to form $\beta$-FeSi ${ }_{2}$. To fully study this effect, future work would require the study of implantation of bismuth, iron and oxygen into pure silicon (111) bulk wafer or a silicon with another orientation such as (100) and different doing type. The same orientation as the silicon dioxide substrate studied in this analysis could be used to determine if the crystal bonding is subject to the substrate's crystal orientation or if this is only a question of molecular bonding. Modifying the implantation order may also provide some explanation on the phases formation.

In future work optimisation of the triple ion implantation and the electrical properties should be investigated. The optimisation analysis should include different energy, dose and substrate. As some substrate may react less with iron, this could im- 
prove the bismuth-oxygen-iron bonding. Modifying the order of implantation should be study, bismuth then oxygen then iron. This technique might have to be placed aside for the moment, as implanting bismuth requires a long period of time and special attention to preserve the target, and annealing the material results in the loss of implanted ions.

Investigating the electrical properties of the implanted films should be done for potential application device. As the magnetic analysis only shows iron oxide or ironsilicon. The creation of bonds with bismuth might have an effect, or the electrical moment might suggest the formation of an isolated island of $\mathrm{BiFeO}_{3}$ or other phases. 


\section{Appendix E}

\section{BiCrxOy thin film}

This chapter is very similar to Chapter 4 (sputtered $\mathrm{BiFeO}_{3}$ thin film), however the film studied in this chapter is the preliminary results at attempting to synthesis $\mathrm{BiCrO}_{3}$ by ion beam sputtering. The sputtered film resulted in $\mathrm{BiCr}_{x} \mathrm{O}_{y}$ phases. The purpose of this chapter is to analyse the synthesis of $\mathrm{BiCr}_{x} \mathrm{O}_{y}$ thin film and compare that study with the synthesis of $\mathrm{BiFeO}_{3}$ by ion beam sputtering.

The detailed of the synthesis, growth and structure of nano-polycrystalline bismuth chromate; $\mathrm{BiCr}_{x} \mathrm{O}_{y}$ thin film by ion beam sputtering is discussed. The magnetic properties and ordering of the films are analysed with different annealing temperatures and duration of annealing. The analysis of bismuth chromite is compared with chromium oxide powders and thin films.

\section{E.1 Introduction}

$\mathrm{BiCrO}_{3}(\mathrm{BCO})$ (Figure E.1.1) is a poorly-understood material that requires further study because first-principle calculations have predicted this material to be multiferroic [Hill et al., 2002]. Murakami et al. [2006] reported that $\mathrm{BiCrO}_{3}$ is ferroelectric at ambient temperature. $\mathrm{BiCrO}_{3}$ has a G-type antiferromagnet with a Néel temperature of $110-120 \mathrm{~K}$ [Darie et al., 2010; Kim et al., 2006a]. This material displays a poorly understood spinreorientation at low temperatures [Darie et al., 2010], suggesting that any multiferroic behaviour is observed at temperatures below $\sim 110 \mathrm{~K} . \mathrm{BiCrO}_{3}$ also displays very small ferromagnetism ordering. This result in $\mathrm{BiCrO}_{3}$ being a less likely suitable material for potential device applications [Geprägs et al., 2007]. A wide range of measurements still needs to be performed to identify whether this compound can be a useful multiferroic. Different preparation methods (ion implantation and sputtering), the effects of doping and annealing could provide important information on the magnetic and electronic properties of this compound. The study of $\mathrm{BiCrO}_{3}$ was carried out to further study the effect of the crystal structure and the link with multiferroics properties. Chal- 
lenges arise while synthesising $\mathrm{BiCrO}_{3}$ [Goujon et al., 2008], this study is an attempt at synthesising a secondary phase free thin film, with ion beam technology.

There has been considerable recent interest in multiferroic compounds that display a coupling between the structural, magnetic, and electric order parameters [Ramesh and Spaldin, 2007; Mocherla et al., 2014] because of their potential applications, including ferroelectric RAM [Ortiz-Quiñonez et al., 2013; Kondo et al., 2007], magnetic sensors [[Ortiz-Quiñonez et al., 2013]], and photovoltaic cells [Yang et al., 2009]. Most research has focussed on $\mathrm{BiFeO}_{3}$ because bismuth ferrite is multiferroic at ambient temperature, the ferroelectric Curie temperature is $1103 \mathrm{~K} . \mathrm{BiFeO}_{3}$ exhibits G-type antiferromagnetism with a Néel temperature

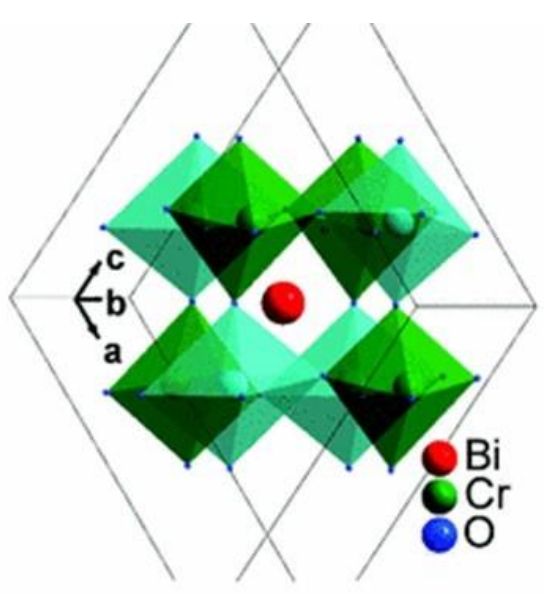

Figure E.1.1: $\mathrm{BiCrO}_{3}$ unit cell [Colin et al., 2012]. of $643 \mathrm{~K}$ [Mocherla et al., 2014; Mazumder et al., 2007]. There is interest in exploring other multiferroic compounds, including $\mathrm{BiCrO}_{3} . \mathrm{BiCrO}_{3}$ was predicted to be multiferroic with G-type antiferromagnetism and possibly with antiferroelectric order [Hill et al., 2002]. Subsequently, $\mathrm{BiCrO}_{3}$ shown evidence of multiferroic properties with antiferroelectric ordering up to the structural transition at $410 \mathrm{~K}$ [Kim et al., 2006a]. $\mathrm{BiCrO}_{3}$ has G-type antiferromagnetic ordering at $\sim 110 \mathrm{~K}$ with a small tilt of the magnetic moments as the temperature is lowered [Darie et al., 2010]. The study of nanocrystalline multiferroics is particularly interesting because of the potential applications, which include nano-scale ferroelectric RAM, and because nanocrystals can have strain effects and uncompensated surface spins that lead to an enhanced magnetisation [Park et al., 2007].

In this chapter, the preliminary results from structural and magnetic measurements on $\mathrm{BiCr}_{x} \mathrm{O}_{y}$ films synthesized by ion beam sputtering at ambient temperature and then annealed in an oxygen atmosphere are presented. In one specific case, the thin film was annealed in oxygen followed by another annealing in argon. This method leads to the formation of a thin film with a mix of bismuth chromate oxides and chromium oxides phases. 


\section{E.2 Experimental details}

A $20 \mathrm{keV} \mathrm{Ar}{ }^{+}$ion beam was used to sputter $\mathrm{BiCr}_{x} \mathrm{O}_{y}$ thin films at ambient temperature using a bulk $\mathrm{BiCrO}_{3}$ target in a high vacuum $\left(<2 \times 10^{-6} \mathrm{kPa}\right)$ [Murmu et al., 2012]. $\mathrm{The} \mathrm{Ar}^{+}$ion beam was directed to the sputtering target placed at a $45^{\circ}$ angle. The films were deposited onto $100 \mathrm{~nm}$ of thermally oxidised silicon dioxide $\left(\mathrm{SiO}_{2}\right)$ on a Si $\langle 100\rangle$ substrate mounted on rotating catchers. The target holder and catchers were rotated in opposite directions to ensure uniformity of the deposited film. The $\mathrm{BiCr}_{x} \mathrm{O}_{y}$ samples were annealed in oxygen atmosphere for 15 minutes at temperatures up to $700{ }^{\circ} \mathrm{C}$. The samples structure was imaged using cross-sectional transmission electron microscopy (XTEM) and SAED micrographs using a FEI TECNAI TF20 field emission gun transmission electron microscope operating at $200 \mathrm{kV}$. XRD measurements were carried out using a Co K $\alpha$ X-ray source with a wavelength of $0.1789 \mathrm{~nm}$. The operating voltage was $40 \mathrm{kV}$ with a current of $35 \mathrm{~mA}$. The samples were placed at a grazing angle, $2^{\circ}$. Magnetic measurements were carried out using a SQUID magnetometer in a magnetic measurements property system from Quantum Design.

\section{E.3 Synthesis and structure of BiCrxOy thin film}

The synthesis of $\mathrm{BiCr}_{x} \mathrm{O}_{y}$ is achieved by ion beam sputtering. The sputter target is made of a mix of bismuth, bismuth oxide, chromium oxides, bismuth chromium oxide and bismuth chromium. The nano-particles are grown in an annealing furnace with an oxygen atmosphere. In one case, the thin film was annealed in oxygen followed by an annealing in argon. In this section, the structure and the analyse of potential secondary phases in the film are studied.

\section{E.3.1 Rutherford backscattering, RBS}

The RBS analysis, shown in Figure E.3.1 (a), shows the backscattering particles of bismuth, chromium and oxygen from the thin film and silicon from the substrate. Also, shown in this figure the RUMP fit of the as-made thin film. The fit is made of 3 layers. The surface layer $\left(5.5 \times 10^{17}\right.$ at.cm $\left.{ }^{-2}\right)$ is composed of $\mathrm{Bi}_{1} \mathrm{Cr}_{1} \mathrm{O}_{1.5}\left(4.04 \times 10^{22}\right.$ at.cm $\left.{ }^{-3}\right)$ with a thickness of about $136 \pm 2 \mathrm{~nm}$. This stoichiometry could be explained by mix phases of $\mathrm{Bi}_{2} \mathrm{O}_{3}$ and $\mathrm{Cr}_{2} \mathrm{O}_{3}$. The second layer is $\mathrm{SiO}_{2}\left(5.0 \times 10^{17}\right.$ at.cm ${ }^{-2}$ with a density 
of $5.18 \times 10^{22}$ at.cm ${ }^{-3}$ ) with a thickness of $97 \mathrm{~nm}$ which is close to manufacturer provided thickness of $100 \mathrm{~nm}$. The third layer is the bulk silicon. From the RUMP fit, bismuth dose is estimated to be $1.4 \times 10^{17}$ at.cm ${ }^{-2}, \mathrm{Cr}$ is $1.4 \times 10^{17} \mathrm{at}_{\mathrm{cm}}^{-2}$. The oxygen value is not reliable as the contribution of oxygen in the substrate and the oxygen in the sputtered film are indistinguishable with this characterisation technique. However, the RUMP fit estimate the total oxygen dose around $4.3 \times 10^{17}$ at.cm ${ }^{-2}$. No contamination from other element is found; all the elements seen by RBS are expected.

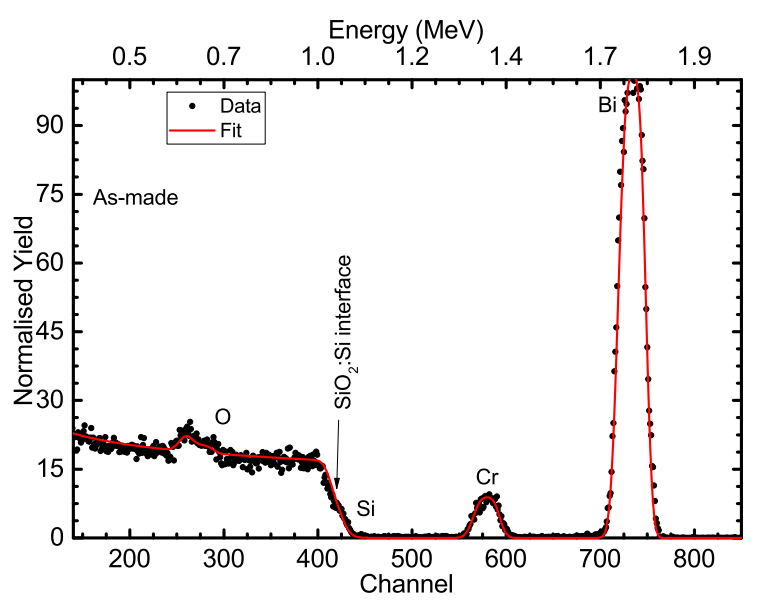

(a) As-made.

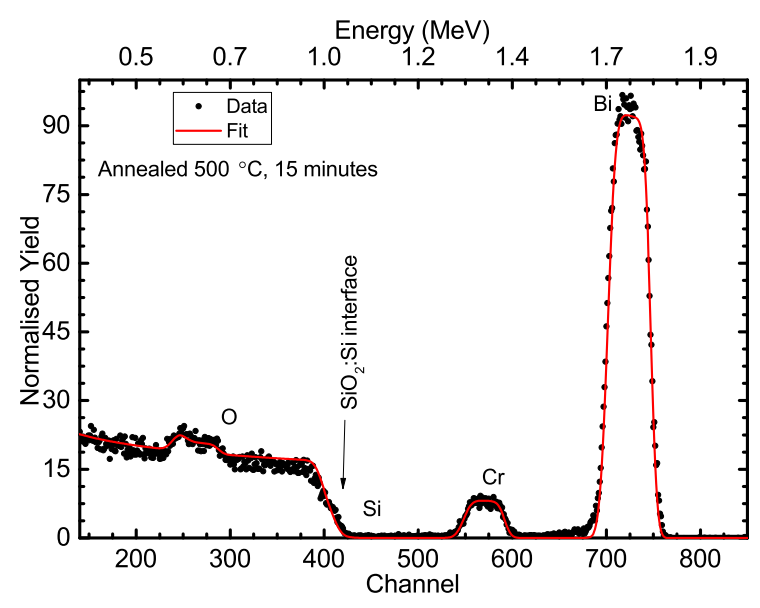

(b) Annealed at $500{ }^{\circ} \mathrm{C}$ for $15 \mathrm{~min}$.

Figure E.3.1: RBS spectra of sputtered $\mathrm{BiCr}_{x} \mathrm{O}_{y}$ (a) as-made and (b) annealed at $500{ }^{\circ} \mathrm{C}$ for 15 min in an $\mathrm{O}_{2}$ atmosphere. Also in this figure is shown a RUMP fit.

The RBS analysis of the annealed thin film is shown in Figure E.3.1 (b). Also, shown in this figure is the RUMP fit of the thin film. The simulation is based on the assumption of material with 3 layers; the surface layer is composed of $\mathrm{Bi}_{1} \mathrm{Cr}_{1} \mathrm{O}_{2.2}$ $\left(6 \times 10^{17}\right.$ at.cm ${ }^{-2}, 4.09 \times 10^{22}$ at.cm $\left.{ }^{-3}\right)$ with an estimated thickness of $147 \pm 2 \mathrm{~nm}$. This stoichiometry could be explained with mix phases of $\mathrm{Bi}_{2} \mathrm{O}_{3}, \mathrm{Cr}_{2} \mathrm{O}_{3}$ and $\mathrm{CrO}$. The second layer is $\mathrm{SiO}_{2}\left(5.0 \times 10^{17}\right.$ at.cm ${ }^{-2}$ and $5.18 \times 10^{22}$ at.cm $\left.{ }^{-3}\right)$ with a thickness of $97 \mathrm{~nm}$ which is close to manufacturer provided thickness of $100 \mathrm{~nm}$. The third layer is the bulk silicon. A full study of the annealing effect is already described in the Chapter 4 (sputtered $\mathrm{BiFeO}_{3}$ thin film). The annealing process takes place in an oxygen atmosphere that oxidizes the thin film, increases the quantity of oxygen in the surface layer, however the stoichiometry is still oxygen-deficient. As the stoichiometry is not reach, other phases in these materials, like chromium oxides and bismuth oxides, have possibly form in the films. The effects of those phases will be discussed in more detail in the subsection E.3.2, XRD. The RUMP fit provide an estimated dose for bismuth of $1.6 \times 10^{17}$ at.cm ${ }^{-2}$, for chromium of $1.6 \times 10^{17}$ at. $\mathrm{cm}^{-2}$ and for oxygen of $9.9 \times 10^{17} \mathrm{at}_{\mathrm{cm}}^{-2}$ (oxygen dose includes the oxygen in the substrate and the oxygen in the sputtered film). 
As the $\mathrm{BiCr}_{x} \mathrm{O}_{y}$ thin film annealed at $500{ }^{\circ} \mathrm{C}$ does not reach the required stoichiometry of $1: 1: 3$, a film annealed at $700{ }^{\circ} \mathrm{C}$ is analysed. The sample was cut into 3 pieces. The first piece was preserved as is, without annealing. The second piece was annealed in an oxygen atmosphere. The third piece was first annealed in an oxygen atmosphere, which was quickly purged and replaced with an argon atmosphere. As previously, the as-made piece shows the backscattered particles of bismuth, chromium, oxygen and the silicon from the $\mathrm{SiO}_{2}$ substrate. The $\mathrm{SiO}_{2}$ layer is about $108 \mathrm{~nm}\left(5.6 \times 10^{17}\right.$ at.cm ${ }^{-2}, 5.18 \times 10^{22}$ at.cm $\left.{ }^{-3}\right)$ thick and the sputtered thin film, $\mathrm{Bi}_{1} \mathrm{Cr}_{1.1} \mathrm{O}_{1.5},\left(5.8 \times 10^{17}\right.$ at.cm ${ }^{-2}, 4.12 \times 10^{22}$ at.cm $\left.{ }^{-3}\right)$ is about $140 \mathrm{~nm}$ thick, this analysis is comparable to the analysis of E.3.1 (a).

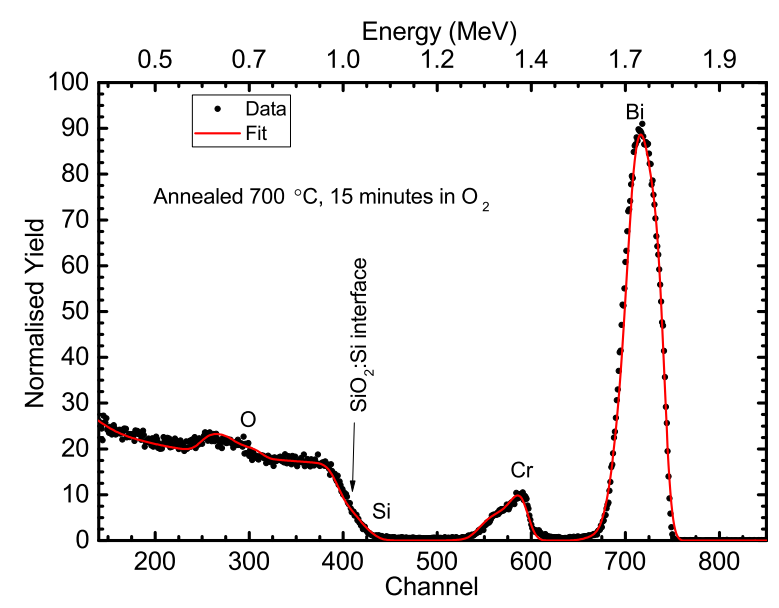

(a) Annealed in $\mathrm{O}_{2}$.

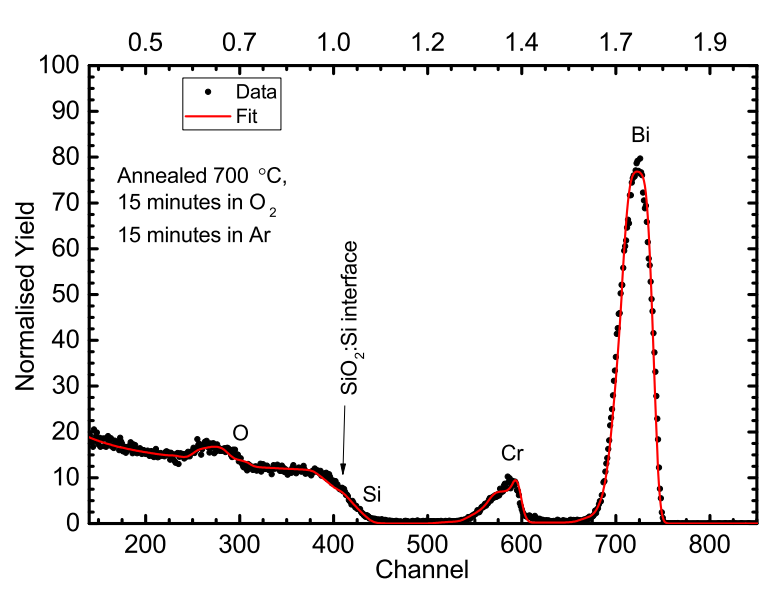

(b) Annealed in $\mathrm{O}_{2}$ followed by Ar.

Figure E.3.2: $\mathrm{RBS}$ of the sputtered $\mathrm{BiCr}_{x} \mathrm{O}_{y}$ annealed at $700{ }^{\circ} \mathrm{C}$ (a) $15 \mathrm{~min}$ in oxygen atmosphere (b) $15 \mathrm{~min}$ in an oxygen atmosphere followed by another $15 \mathrm{~min}$ in an argon atmosphere. Also, shown in this figure, the RUMP fit (red).

The Figure E.3.2 (a) displays the RBS of the sputtered $\mathrm{BiCr}_{x} \mathrm{O}_{y}$ after annealing at $700{ }^{\circ} \mathrm{C}$ in oxygen atmosphere for 15 minutes. Also in this figure is shown a RUMP fit made with the assumption of a 6 layers' material, the details of the RUMP fit are presented in Table E.3.1. The surface layer is $\mathrm{Bi}_{1} \mathrm{Cr}_{1.7} \mathrm{O}_{2.5}\left(3.8 \times 10^{17}\right.$ at.cm ${ }^{-2}$ with a density of $4.57 \times 10^{22}$ at.cm ${ }^{-3}$ ) with an estimated thickness of $83 \mathrm{~nm}$. This stoichiometry could be explained with phases of $\mathrm{Bi}_{2} \mathrm{O}_{3}, \mathrm{Cr}_{2} \mathrm{O}_{3}, \mathrm{CrO}, \mathrm{CrO}_{2}$ and $\mathrm{BiCr}_{x} \mathrm{O}_{y}$. The second layer is composed of $\left(4.2 \times 10^{17}\right.$ at.cm ${ }^{-2}, 3.96 \times 10^{22}$ at.cm $\left.{ }^{-2}\right) \mathrm{Bi}_{1} \mathrm{Cr}_{0.8} \mathrm{O}_{2.4}$ with a thickness of $106 \mathrm{~nm}$. The same phases mention for the surface layer could explain the stoichiometry of the second layer. The third layer is composed of $\left(5 \times 10^{17} \mathrm{at}_{\mathrm{cm}}{ }^{-2}, 4.65 \times 10^{22} \mathrm{at}_{\mathrm{cm}} \mathrm{cm}^{-2}\right)$ $\mathrm{Bi}_{0.1}, \mathrm{Cr}_{0.2}, \mathrm{O}_{2.5}, \mathrm{Si}_{1}$ with a thickness of $108 \mathrm{~nm}$. This stoichiometry could be explained by the diffusion of $\mathrm{Bi}$ and $\mathrm{Cr}$ into an oxygen $\mathrm{SiO}_{2}$ layer, $\mathrm{Bi}_{2} \mathrm{O}_{3}, \mathrm{Cr}_{2} \mathrm{O}_{3}, \mathrm{CrO}$. The layer 4 is showing more diffusion of bismuth and chromium, details are presented in Table E.3.1. The interest of the layers 3 and 4 is the growth of $\mathrm{SiO}_{2}$ layer through thermal 
annealing. The as-made sample had $\mathrm{SiO}_{2}$ layer of about $108 \mathrm{~nm}$, annealing this sample at $700{ }^{\circ} \mathrm{C}$ in oxygen for 15 minutes increase the thickness of $\mathrm{SiO}_{2}$ to about $263 \mathrm{~nm}$.

RUMP fit estimates the bismuth dose at $2 \times 10^{17}$ at.cm ${ }^{-2}$, the chromium dose is estimated at $2 \times 10^{17}$ at.cm ${ }^{-2}$ and $4.8 \times 10^{18}$ at.cm ${ }^{-2}$ for oxygen. The dose before and after annealing are similar suggesting a negligible number of ions might have been loss by evaporation. Annealing in an oxygen atmosphere increases the fluence of oxygen in the film while the as-made sample had an estimate of $2.2 \times 10^{17} \mathrm{at}_{\mathrm{cm}}^{-2}$ after annealing the fluence is estimated at $4.8 \times 10^{18}$ at. $\mathrm{cm}^{-2}$ (the oxygen dose includes the oxygen from the substrate and the sputtered film, this value may not be accurate due the RBS experimental sensitivity to oxygen).

Table E.3.1: Annealed $\mathrm{BiCr}_{x} \mathrm{O}_{y}$ at $700{ }^{\circ} \mathrm{C}$ in $\mathrm{O}_{2}$ for 15 minutes, $\mathrm{RUMP}$ 's fit parameter.

\begin{tabular}{|c|c|ccccc|c|c|}
\hline layer & $\begin{array}{c}\text { Thickness RUMP } \\
(\text { at.cm }\end{array}$ & Bi $)$ & Fe & O & Si & H & $\begin{array}{c}\text { Density SRIM } \\
\left(\text { at.cm }^{-3}\right)\end{array}$ & $\begin{array}{c}\text { Thickness } \\
(\mathrm{nm})\end{array}$ \\
\hline 1 & $3.8 \times 10^{17}$ & 1 & 1.7 & 2.5 & 0 & 0 & $4.57 \times 10^{22}$ & 83 \\
2 & $4.2 \times 10^{17}$ & 1 & 0.8 & 2.4 & 0 & 0 & $3.96 \times 10^{22}$ & 106 \\
3 & $5 \times 10^{17}$ & 0.2 & 0.2 & 2.5 & 1 & 0 & $4.65 \times 10^{22}$ & 108 \\
4 & $8 \times 10^{17}$ & 0.006 & 0.01 & 2 & 1 & 0 & $5.16 \times 10^{22}$ & 155 \\
5 & $8 \times 10^{18}$ & 0 & 0 & 0 & 1 & 0.2 & $4.97 \times 10^{22}$ & 1609 \\
6 & $1 \times 10^{19}$ & 0 & 0 & 0 & 1 & & $4.98 \times 10^{22}$ & 2009 \\
\hline
\end{tabular}

Out of concern, a uniformity test was previously carried out in which the thickness of 10 spots of radius less than $1 \mathrm{~mm}$ were measured on a few samples of the same size. The thickness of the material was the same within the uncertainty, in the worstcase scenario, about a $10 \mathrm{~nm}$ thickness difference was noted. The diffusion reported after annealing the sample at $700{ }^{\circ} \mathrm{C}$ for 15 minutes is ten time greater than the variation the thickness seen in one sample. The thickness is determined by the simulation software RUMP [Doolittle, 1985].

The E.3.2 (b) displays the RBS spectra of sputtered $\mathrm{BiCr}_{x} \mathrm{O}_{y}$ annealed at $700{ }^{\circ} \mathrm{C}$ for $15 \mathrm{~min}$ in an oxygen atmosphere followed by another $15 \mathrm{~min}$ in an argon atmosphere. Also, shown in this figure is the RUMP fit based on the assumption of an 8 layers' material, the details are presented in Table E.3.2. The surface layer is composed of $\mathrm{Bi}_{0.5}$, $\mathrm{Cr}_{2.4}$ and $\mathrm{C}_{1}\left(1.1 \times 10^{17}\right.$ at.cm ${ }^{-3}, 6.1 \times 10^{22}$ at.cm $\left.{ }^{-3}\right)$ with a thickness estimated at $18 \mathrm{~nm}$. This stoichiometry could be explained either by the diffusion or evaporation of $\mathrm{Bi}$. The second layer is composed of $\mathrm{Bi}_{1}, \mathrm{Cr}_{1}$ and $\mathrm{O}_{1.5}\left(5.4 \times 10^{17}\right.$ at.cm ${ }^{-2}, 4.04 \times 10^{22}$ at.cm $\left.{ }^{-3}\right)$ with a thickness estimated at $134 \mathrm{~nm}$. This stoichiometry could be explained with mix phases of $\mathrm{Bi}_{2} \mathrm{O}_{3}$ and $\mathrm{Cr}_{2} \mathrm{O}_{3}$. The layer 3 is unexpected, the concentration of oxygen is 
more important than anticipated. By deduction, the third layer should an $\mathrm{SiO}_{2}$ layer with possible diffusion of bismuth and chromium, however the layer 3 is composed of $\mathrm{Bi}_{0.6}, \mathrm{Cr}_{0.6}, \mathrm{Si}_{1}$ and $\mathrm{O}_{3}\left(3.3 \times 10^{17}\right.$ at.cm ${ }^{-2}, 4.35 \times 10^{22}$ at.cm $\left.{ }^{-3}\right)$ with a thickness estimated at $76 \mathrm{~nm}$. This stoichiometry could be explained with $\mathrm{SiO}_{2}$ and mix phases of $\mathrm{Bi}_{2} \mathrm{O}_{3}$, $\mathrm{Cr}_{2} \mathrm{O}_{3}, \mathrm{CrO}$ with a slight possibility of $\mathrm{CrO}_{2}$. The layers' underneath show the diffusion of bismuth and chromium in the substrate, details are presented in Table E.3.2.

The RUMP simulation provides an estimated fluence of bismuth $2 \times 10^{17}$ at.cm $^{-2}$, chromium $2 \times 10^{17}$ at.cm ${ }^{-2}$ and oxygen, oxygen fluence include both the oxygen in the substrate and in the sputtered film, $8 \times 10^{18}$ at. $\mathrm{cm}^{-2}$.

Table E.3.2: Annealed $\mathrm{BiCr}_{x} \mathrm{O}_{y}, 700{ }^{\circ} \mathrm{C}$ in $\mathrm{O}_{2}$ for $15 \mathrm{~min}$ then $\mathrm{Ar}$, RUMP fit parameter.

\begin{tabular}{|c|c|cccccc|c|c|}
\hline layer & $\begin{array}{c}\text { Thickness } \\
\text { RUMP } \\
\left(\text { at.cm }^{-2}\right)\end{array}$ & $\mathrm{Bi}$ & $\mathrm{Fe}$ & $\mathrm{O}$ & $\mathrm{Si}$ & $\mathrm{H}$ & $\mathrm{C}$ & $\begin{array}{c}\text { Density } \\
\text { SRIM } \\
\left(\text { at.cm }^{-3}\right)\end{array}$ & $\begin{array}{c}\text { Thickness } \\
(\mathrm{nm})\end{array}$ \\
\hline 1 & $1.1 \times 10^{17}$ & 0.5 & 2.4 & 0 & 0 & 0 & 1 & $6.10 \times 10^{22}$ & 18 \\
2 & $5.4 \times 10^{17}$ & 2 & 2 & 3 & 0 & 0 & 0 & $4.04 \times 10^{22}$ & 134 \\
3 & $3.3 \times 10^{17}$ & 0.6 & 0.6 & 3 & 1 & 0 & 0 & $4.47 \times 10^{22}$ & 76 \\
4 & $4 \times 10^{17}$ & 0.05 & 0.15 & 2.2 & 1 & 0 & 0 & $5.19 \times 10^{22}$ & 77 \\
5 & $6 \times 10^{17}$ & 0.006 & 0.02 & 1 & 1 & 0 & 0 & $5.13 \times 10^{22}$ & 117 \\
6 & $8 \times 10^{17}$ & 0.001 & 0.01 & 0 & 1 & 2 & 0 & $4.98 \times 10^{22}$ & 161 \\
7 & $4 \times 10^{18}$ & 0.001 & 0 & 0 & 1 & 0.7 & 0 & $4.94 \times 10^{22}$ & 809 \\
8 & $1 \times 10^{20}$ & 0 & 0 & 0 & 1 & 0.3 & 0 & $4.97 \times 10^{22}$ & 20122 \\
\hline
\end{tabular}

The piece annealed in oxygen and subsequently in argon has the same bismuth and chromium fluence as the other two pieces, $2 \times 10^{17}$ at.cm ${ }^{-2}$. This annealed sample does not reach the required stoichiometry either. Annealing in oxygen atmosphere increases the oxygen content in the film while the second annealing, in argon, seems to be increasing the diffusion of oxygen. What is interesting in this case is the sputtered film loses oxygen while a silicon dioxide layer grows. This can be explained by be fact that the annealing furnace is not completely purge of oxygen and the oxygen left might be incorporated in the sample. Even though RBS, $2 \mathrm{MeV}^{4} \mathrm{He}^{+}$beam, is not the best technique to characterise the oxygen content in a material the increase of oxygen is noticeable as described in the RUMP fit; $\mathrm{SiO}_{3}$ with trace of bismuth and chromium. Annealing in an oxygen atmosphere increases the oxygen content in the surface layer, however the second annealing in argon seems to remove some of that oxygen or perhaps the oxygen is just diffused deeper into the $\mathrm{SiO}_{2}$ layer.

The thickness of the third piece is more complex to analyse because of the diffu- 
sion of bismuth, chromium and oxygen toward the inner layer. A new interface grew out of the final annealing stage. Bismuth and chromium can be found at depth up to $422 \mathrm{~nm}$ after annealing the film in an oxygen atmosphere for 15 minutes and subsequently annealing in argon for 15 minutes while a single annealing in oxygen for 15 minutes resulted in bismuth and chromium to be present in the top $297 \mathrm{~nm}$ and the as-made film have them only in the top $120 \mathrm{~nm}$. The diffusion is clearly seen with the RUMP fit.

\section{E.3.2 X-Ray diffraction, XRD}
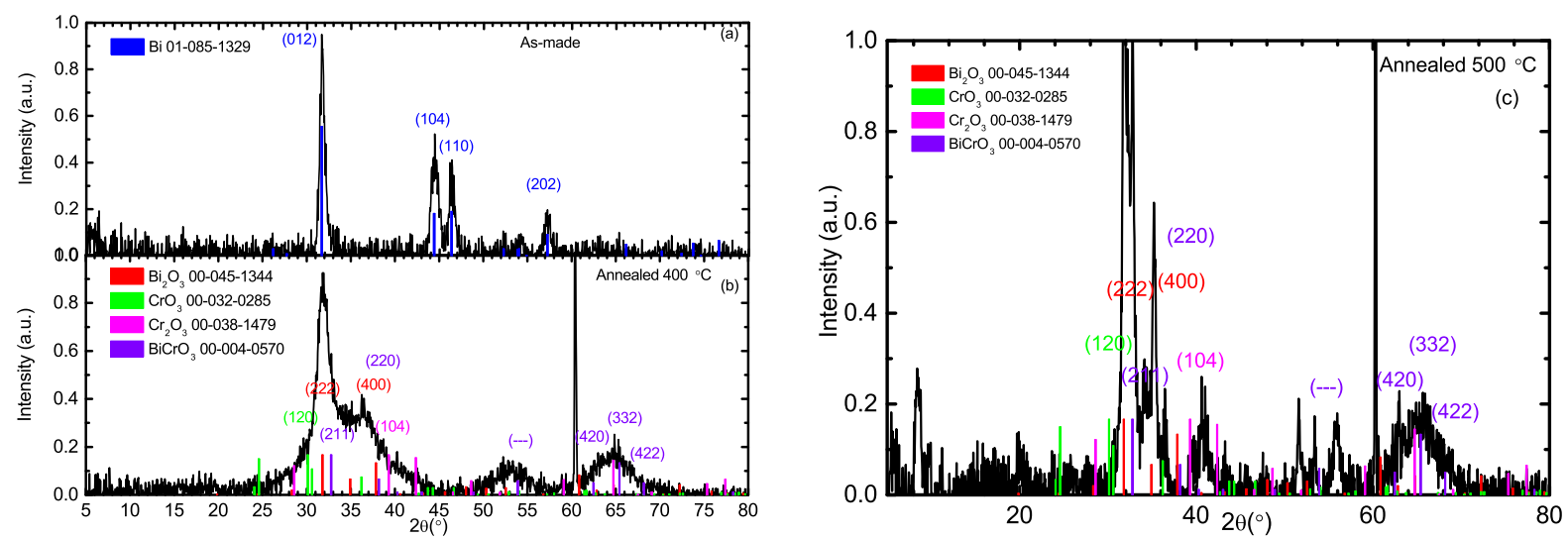

Figure E.3.3: XRD data for the(a) as-made film and (b) film annealed in oxygen at $400{ }^{\circ} \mathrm{C}$ and (c) annealed in oxygen at $500{ }^{\circ} \mathrm{C}$. Also shown are the reference patterns for (a) $\mathrm{Bi}$ (JCPDS 01-085-1329) and (b) $\mathrm{Bi}_{2} \mathrm{O}_{3}$ (JCPDS 00-22-0515), $\mathrm{Bi}_{1.78} \mathrm{Cr}_{0.62} \mathrm{O}_{12+x}$ (JCPDS 00-050-0373), orthorhombic $\mathrm{CrO}_{3}$ (JCPDS 00-032-0285), and rhombohedral $\mathrm{Cr}_{2} \mathrm{O}_{3}$ (JCPDS 00-038-1479).

The XRD analysis of the as-made thin film (Figure E.3.3 (a)) shows only nanobismuth particles (JCPDF 01-085-1329) [Ishiguro et al., 1983]. As the RBS analysis (subsection E.3.1) confirms the presence of chromium and oxygen this can be explained either by chromium oxide phases being amorphous or the atomic form factor of the chromium oxides is too small compared to bismuth to be seen in the X-ray analysis. Using Scherer's equation on the peak (012), the nano-bismuth size is estimated at around $\sim 16 \pm 0.3 \mathrm{~nm}$. There is no evidence for $\mathrm{CrO}_{x}$ phases and hence either $\mathrm{CrO}_{x}$ is amorphous or the crystallite size is small.

Annealing of the $\mathrm{BiCr}_{x} \mathrm{O}_{y}$ film to $400{ }^{\circ} \mathrm{C}$ in oxygen leads to a significant change in the XRD data as can be seen in Figure E.3.3 (b). From that figure, Bi is clearly no longer present. The peaks are broad an indication that there are small nanoparticles, however the $\mathrm{BiCr}_{x} \mathrm{O}_{y}$ phase is not clearly identify in Figure E.3.3. There is no evidence for the 
known monoclinic or orthorhombic $\mathrm{BiCrO}_{3}$ phases. The JCPDS database does include a reference pattern for what was believed to be tetragonal $\mathrm{BiCrO}_{3}$ (JCPDS 00-004-5070). However, the reference pattern quality mark is listed as blank, which indicates that the phase assignment cannot be trusted. In fact, the JCPDS 00-004-5070 reference pattern is close to that of tetragonal $\mathrm{Bi}_{7.38} \mathrm{Cr}_{0.62} \mathrm{O}_{12+x}$ (JCPDS 00-004-5070) that has the highest quality mark, which is star. The Figure E.3.3 shows that the XRD data could be close to that for tetragonal $\mathrm{Bi}_{7.38} \mathrm{Cr}_{0.62} \mathrm{O}_{12+x}$ as well as tetragonal $\mathrm{Bi}_{2} \mathrm{O}_{3}$ (JCPDS 00-0220515). $\mathrm{CrO}_{3}$ and $\mathrm{Cr}_{2} \mathrm{O}_{3}$ could also be present, however they are not clearly seen in this figure which may be due to the low atomic form factor of $\mathrm{Cr}$ when compared with Bi. Annealing the $\mathrm{BiCr}_{x} \mathrm{O}_{y}$ thin film up to $500{ }^{\circ} \mathrm{C}$ did not improve the film quality or stoichiometry. Annealing at $500{ }^{\circ} \mathrm{C}$, mainly change the nanostructure of the film, the phases are the same as Figure E.3.3 (c) show the same X-ray diffraction as the sample annealed at $400{ }^{\circ} \mathrm{C}$ (Figure E.3.3 (b)) although the broadening of the peaks are reduced when increasing the annealing temperature.

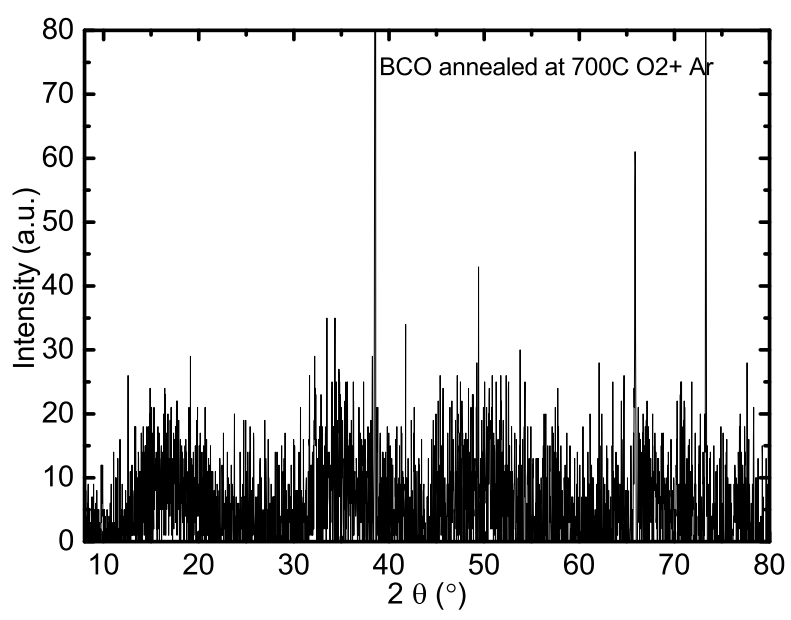

Figure E.3.4: $\mathrm{XRD}$ data of $\mathrm{BiCrO}_{3}$ film annealed at $700{ }^{\circ} \mathrm{C}$ in $\mathrm{O}_{2}$ and subsequently in Ar.

The XRD data of $\mathrm{BiCrO}_{3}$ suggest an increase difficulty to synthesise this thin film compared to $\mathrm{BiFeO}_{3}$. A chemical synthesis approach is known to require pressure of a few atmospheres and a higher temperature [Belik et al., 2007]. In an attempt to improve the synthesis of $\mathrm{BiCrO}_{3}$, the film was annealed for 15 minutes in an oxygen atmosphere and then for another 15 minutes in an argon atmosphere. Figure E.3.4 shows the resultant XRD data. The film appears to be amorphous and there are no clearly identifiable phases. If nanoparticles are present, then they would have to have small sizes or a small phase fraction.

The Figure E.3.5 displays the X-ray diffraction data for the $\mathrm{BiCrO}_{3}$ sputter target, (top) the face that was used to sputter thin films and (bottom) the face that was not sputtered. To avoid contamination, the area that was not protected by the target holder during the sputtering was masked with Teflon tape. Also, shown in this figure is the 
JCPDS 01-085-1329 (Bi), JCPDS 00-045-1344 ( $\left.\mathrm{Bi}_{2} \mathrm{O}_{3}\right)$ and JCPDS 00-038-1479 $\left(\mathrm{Cr}_{2} \mathrm{O}_{3}\right)$.

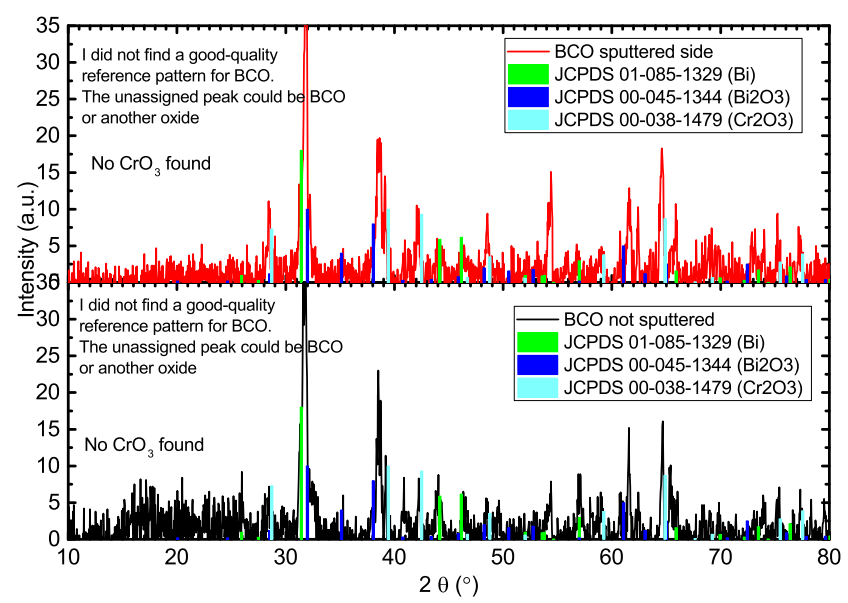

Figure E.3.5: X-ray diffraction of the $\mathrm{BiCrO}_{3}$ sputter target, (top) the face that was used to sputter thin films and (bottom) the face that was not sputtered. To avoid contamination, the area that was not protected by the target holder during the sputtering was masked with Teflon tape. Also, shown in this figure is the JCPDS 01-085-1329 (Bi), JCPDS 00-045-1344 ( $\left.\mathrm{Bi}_{2} \mathrm{O}_{3}\right)$ and JCPDS 00-038-1479 $\left(\mathrm{Cr}_{2} \mathrm{O}_{3}\right)$.

From the XRD analysis, the sputter target used in the ion beam sputtering technique is made of bismuth, bismuth oxide, $\mathrm{Cr}_{2} \mathrm{O}_{3}$ and monoclinic $\mathrm{BiCrO}_{3}$. $\mathrm{CrO}$ and $\mathrm{CrO}_{3}$ were not found; this is expected as these two oxides are not stable [Jain et al., 2013; Bergerhoff et al., 1983]. CrO tends to decompose around $300{ }^{\circ} \mathrm{C}$ into $\mathrm{Cr}_{2} \mathrm{O}_{3}$ and chromium metal, while $\mathrm{CrO}_{3}$ boils and decomposes around $\sim 250{ }^{\circ} \mathrm{C}$ into $\mathrm{Cr}_{5} \mathrm{O}_{12}$ and $\mathrm{O}_{2}$. The sputtering does not modify the target phases; XRD was performed on the sputter target before and after the first sputtering. Although the phases remain unchanged, the fraction of those phases might be modified as seen in the Figure E.3.5, some XRD peak intensity are modified. The ion beam sputtering technique sputters ions of bismuth, bismuth oxides, and $\mathrm{Cr}_{2} \mathrm{O}_{3}$. The as-made thin films seems to have only bismuth. As explained before (subsection 4.3.2, $\mathrm{XRD}$ of $\mathrm{BiFeO}_{3}$ sputtered thin film), this could be due to the large form factor of bismuth compared to chromium, or the chromium oxides could be amorphous.

\section{E.3.3 Transmission electron microscopy, TEM}

Figures E.3.6 and E.3.7 show the TEM pictures of the as-made and annealed $\mathrm{BiCr}_{x} \mathrm{O}_{y}$ films. The white layer is the $\mathrm{SiO}_{2}$ film and the dark layer is the $\mathrm{BiCr}_{x} \mathrm{O}_{y}$ sputtered film. The measured film thickness was $123 \mathrm{~nm}$ for both as-made and annealed $\mathrm{BiCr}_{x} \mathrm{O}_{y}$. The film does not show clearly-defined nanoparticles, however considerable nanoscale structures are visible. 


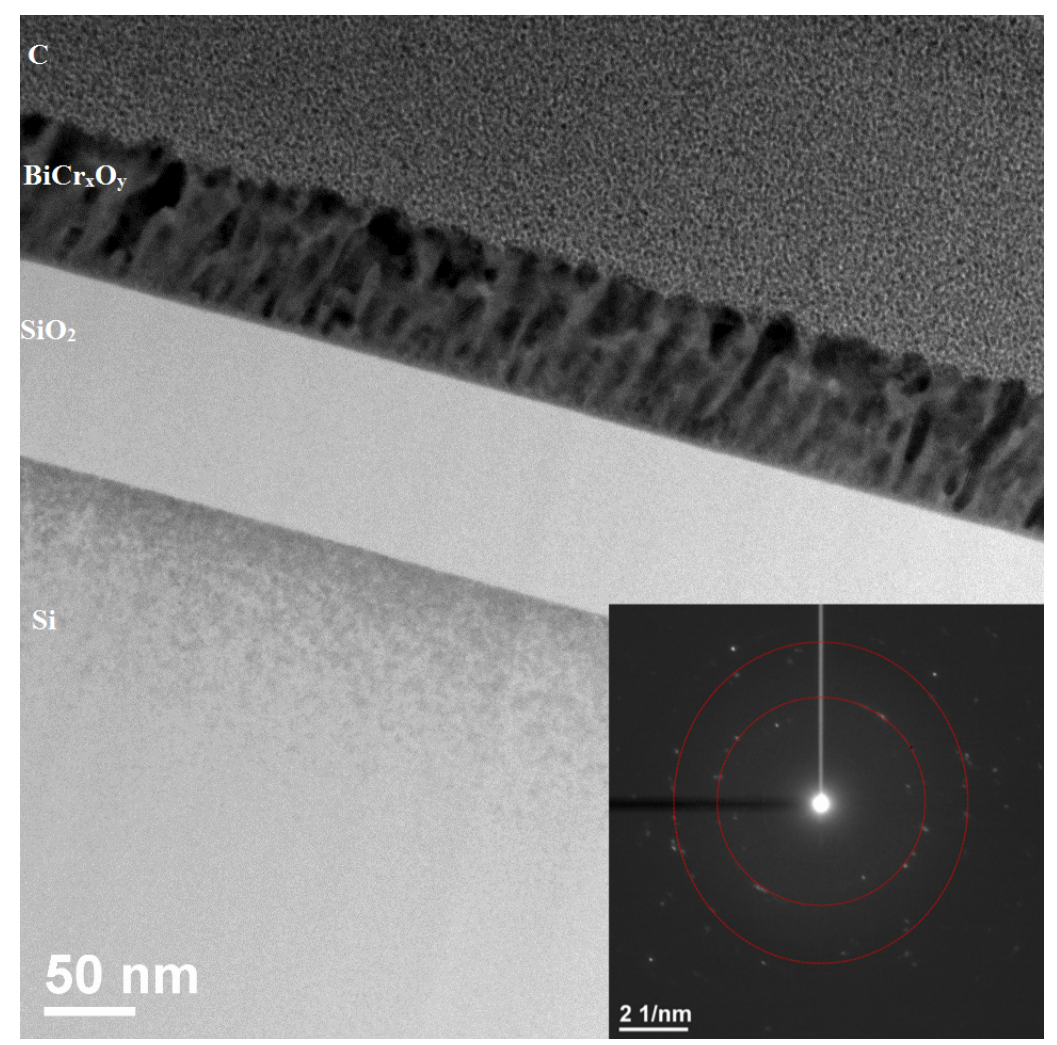

Figure E.3.6: TEM of $\mathrm{BiCr}_{x} \mathrm{O}_{y}$ as-made. Inset: shows SAED, the red circles are representative of the presence of bismuth with a d-spacing of $3.28 \AA$ (012) and $2.27 \AA$ (110).

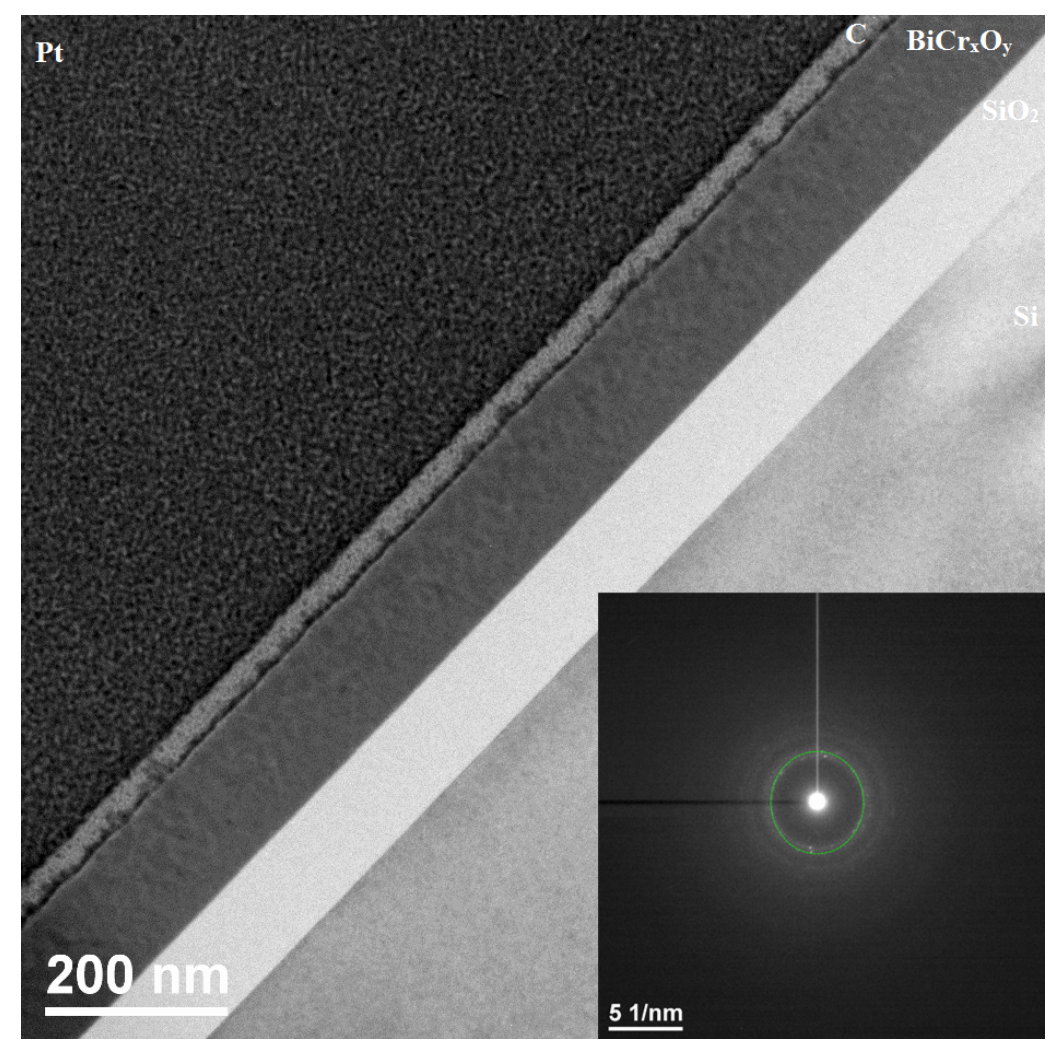

Figure E.3.7: TEM of $\operatorname{BiCr}_{x} \mathrm{O}_{y}$ annealed at $400{ }^{\circ} \mathrm{C}$. Inset: shows SAED, the green circle is close to the d-spacing of bismuth oxide, which is $3.25 \AA$ (312). 
The as-made film shows some nanostructure features; a darker contrast means more electrons are present. The contrast in the TEM cross-section could indicate a more or less uniform film or an amorphous region. The Figure E.3.7 seems to support the latter option. The cross-section does not identify which elements are in this film. The SAED inset displays two red rings, with a d-spacing measurement comparable to bismuth. This would suggest, as previously mentioned, that bismuth is visible in the material and the chromium oxides are amorphous or the atomic form factor is too low when compared with Bi.

The $\mathrm{BiCr}_{x} \mathrm{O}_{y}$ thin film annealed at $400{ }^{\circ} \mathrm{C}$ is more uniform than the as-made thin film, the contrast is limited. The green ring in the SAED inset suggests the presence of $\mathrm{Bi}_{2} \mathrm{O}_{3}$.

\section{E.3.4 Raman spectroscopy}

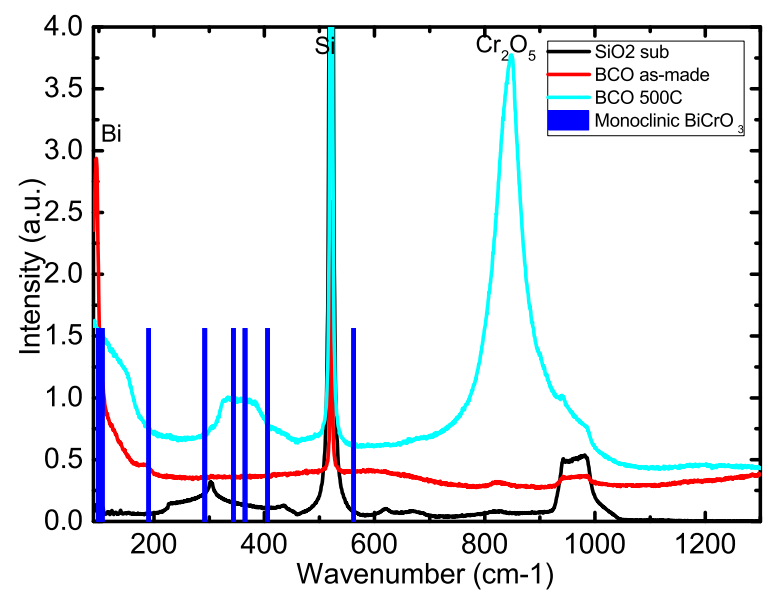

(a) As-made and annealed $500{ }^{\circ} \mathrm{C}$.

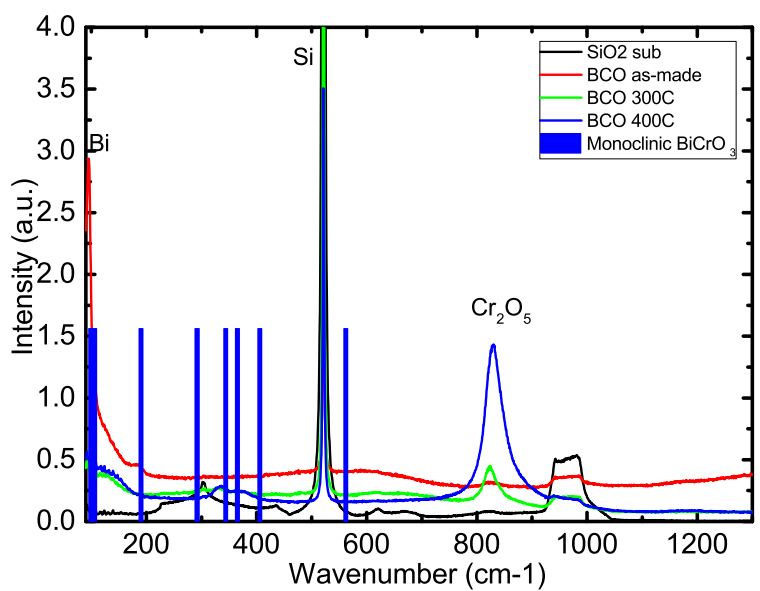

(b) Annealed below $600{ }^{\circ} \mathrm{C}$.

Figure E.3.8: Raman spectrum of the $\mathrm{BiCr}_{x} \mathrm{O}_{y}$ film with annealing at different temperatures, as-made (red), annealed at $500{ }^{\circ} \mathrm{C}$ for $15 \mathrm{~min}$ (cyan), $300{ }^{\circ} \mathrm{C}$ (green) and $400{ }^{\circ} \mathrm{C}$ (blue). Also shown, the $\mathrm{SiO}_{2}: \mathrm{Si}$ substrate is in black.

The as-made $\mathrm{BiCr}_{x} \mathrm{O}_{y}$ thin film Raman spectrum (Figure E.3.8 (a)) shows a bismuth peak at about $\sim 95 \mathrm{~cm}^{-1}$ and $\sim 186 \mathrm{~cm}^{-1}$. Similar Bi peaks were seen in the Raman spectra from the $\mathrm{BiFeO}_{3}$ as-made thin film (subsection 4.3.5 Raman spectroscopy). There is no evidence for $\mathrm{BiCrO}_{3}$ in the Raman data. A small broad feature can be seen in the $600 \mathrm{~cm}^{-1}$ region. This broad feature may be due to small or amorphous chromium oxides. There is no evidence for bismuth oxide phases that are seen in the as-made $\mathrm{BiCr}_{x} \mathrm{O}_{y}$ film. If there is a small amount of bismuth oxide, then the phase fraction is small. 
There is no evidence for $\mathrm{BiCrO}_{3}$ in the Raman data for the film annealed at $500{ }^{\circ} \mathrm{C}$ in oxygen (Figure E.3.8 (a)). There is a broad feature $\sim 300 \mathrm{~cm}^{-1}$ that cannot be attributed to any known $\mathrm{BiCr}_{x} \mathrm{O}_{y}$ phases. The peak at $\sim 845 \mathrm{~cm}^{-1}$ is likely to be due to chromium oxides [Constable et al., 2000].

The Raman data for the thin film annealed at $300{ }^{\circ} \mathrm{C}$ is shown in Figure E.3.8 (b). There is no evidence for $\mathrm{BiCrO}_{3}$ in the Raman data and the Bi peaks have disappeared. The main feature is $\sim 845 \mathrm{~cm}^{-1}$ that is likely to be from chromium oxide phases. This peak is larger in the film annealed at $400{ }^{\circ} \mathrm{C}$ is shown in Figure E.3.8 (b). There are also additional peaks at $335 \mathrm{~cm}^{-1}$ that $8375 \mathrm{~cm}^{-1}$ that may come from chromium oxide phases or from bismuth oxide phases.

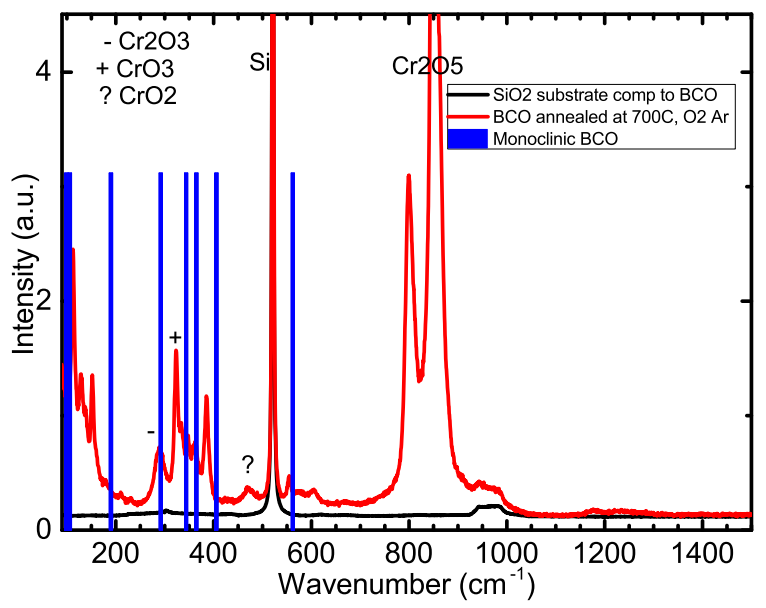

Figure E.3.9: Raman spectrum of the $\mathrm{BiCr}_{x} \mathrm{O}_{y}$ film annealed at $700{ }^{\circ} \mathrm{C}$ in $\mathrm{O}_{2}$ then in $\mathrm{Ar}$.

Annealing the $\mathrm{BiCr}_{x} \mathrm{O}_{y}$ thin film in oxygen followed by argon at $700{ }^{\circ} \mathrm{C}$ (Figure E.3.9) leads to additional Raman peaks not seen in the previous Raman data. The sharper peaks may be due to a range or chromium oxides that include $\mathrm{Cr}_{2} \mathrm{O}_{3}, \mathrm{CrO}_{3}$, $\mathrm{CrO}_{2}$ and $\mathrm{Cr}_{2} \mathrm{O}_{5}$. However, there is no evidence for $\mathrm{BiCrO}_{3}$.

The Raman spectrum of the $\mathrm{BiCrO}_{3}$ sputter target, Figure E.3.10, shows the presence of bismuth, $\mathrm{Cr}_{2} \mathrm{O}_{3}, \mathrm{CrO}_{2}$ and $\mathrm{CrO}_{3}$. The Raman spectrum of the $\mathrm{BiCrO}_{3}$ sputter target has a relatively low-intensity bismuth peak. When comparing the $\mathrm{BiCrO}_{3}$ and the $\mathrm{BiFeO}_{3}$ sputter target, such a low intensity of bismuth in the Raman spectrum of the $\mathrm{BiCrO}_{3}$ sputter target is unexpected. The $\mathrm{BiFeO}_{3}$ sputter target displays many bismuth peaks, while the $\mathrm{BiCrO}_{3}$ sputter target has a bismuth feature that is barely visible. This could be explained by two situations. The first option is that the sputter target is not homogenous. The other situation is less likely and implies that the bismuth is in an amorphous state. This is less likely because bismuth in the $\mathrm{BiFeO}_{3}$ sputter target is clearly visible which suggest a structured bismuth phase and both targets are synthesised by a similar technique in the same laboratory. Also, the XRD analysis of the 
$\mathrm{BiCrO}_{3}$ sputter target shows bismuth content. The RBS analysis shows that bismuth and chromium in similar concentration.

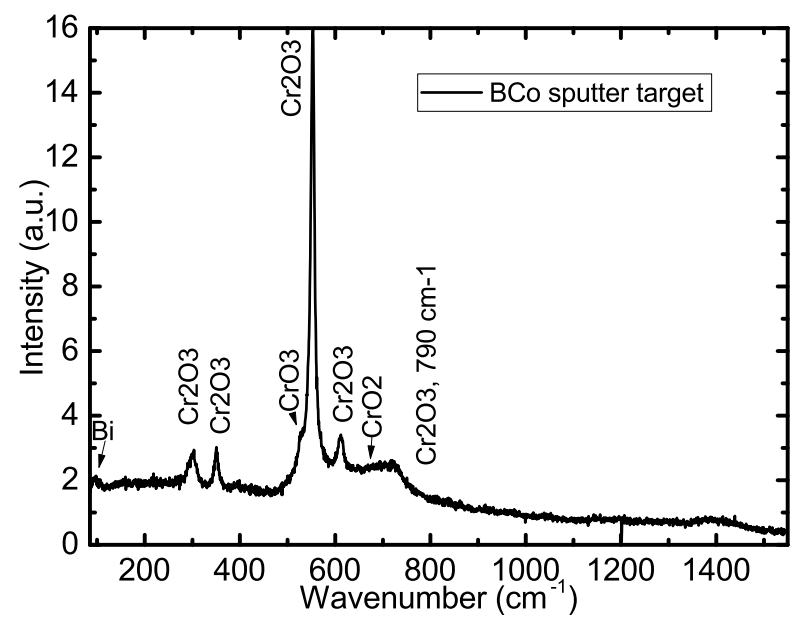

Figure E.3.10: Raman spectrum of the $\mathrm{BiCrO}_{3}$ sputter target.

No trace of $\mathrm{Cr}_{2} \mathrm{O}_{5}$ can be found in the $\mathrm{BiCrO}_{3}$ sputter target and the as-made thin film, however after annealing above $300{ }^{\circ} \mathrm{C}$, a quantity of nano- $\mathrm{Cr}_{2} \mathrm{O}_{5}$ is detected by Raman spectroscopy. Increasing the annealing temperature seems to increase the quantity of $\mathrm{Cr}_{2} \mathrm{O}_{5}$ in the thin films. $\mathrm{CrO}_{2}, \mathrm{CrO}_{3}$ and $\mathrm{Cr}_{2} \mathrm{O}_{3}$ are found in the sputter target and the sputtered films. Raman spectroscopy analysis confirms the difficulties to synthesis $\mathrm{BiCrO}_{3}$ thin film, as the main phases form are chromium oxide including the unstable $\mathrm{CrO}$ and $\mathrm{CrO}_{3}$, annealing after deposition is not sufficient to form $\mathrm{BiCrO}_{3}$. Chromium oxide is nanostructured in the thin film as demonstrated with TEM and Raman spectroscopy. Knowing the phases present in this film, the magnetic moment measurements will be centred on the study of $\mathrm{Cr}_{2} \mathrm{O}_{5}, \mathrm{CrO}_{3}, \mathrm{CrO}_{2}$ that were not expected from the RBS analysis and $\mathrm{Cr}_{2} \mathrm{O}_{3}, \mathrm{CrO}$ that were expected from the RBS analysis.

\section{E.3.5 Magnetic properties}

The Figure E.3.11 shows the moment per formula unit for the annealed $\mathrm{BiCr}_{x} \mathrm{O}_{y}$ film at $5 \mathrm{~K}$ after subtracting the diamagnetic background from the substrate. $m_{s}$ is very small, $\sim 0.02 \pm 0.01 \mu_{B}$. The low moment may be due to the presence of an antiferromagnetic phase (i.e. $\mathrm{Cr}_{2} \mathrm{O}_{3}$ with a Néel temperature of $307 \mathrm{~K}$ [Alexander et al., 2007]) or a small fraction of a ferromagnetic chromium oxide phase (i.e. $\mathrm{CrO}_{2}$ with a Curie temperature of $391 \mathrm{~K})$.

The magnetic data for the as-made thin film is plotted in Figure E.3.12 at $5 \mathrm{~K}$ and $300 \mathrm{~K}$. The saturation moment at $5 \mathrm{~K}$ is $0.04 \mu_{B} / C r$, and the magnetic moment is 
significantly less at $300 \mathrm{~K}$. The low temperature moment at high field is only slightly greater than that seen after annealing which suggests that the same magnetic phases are the same before and after annealing. The magnetic moment of both films arises from the same magnetic phases of chromium oxide.

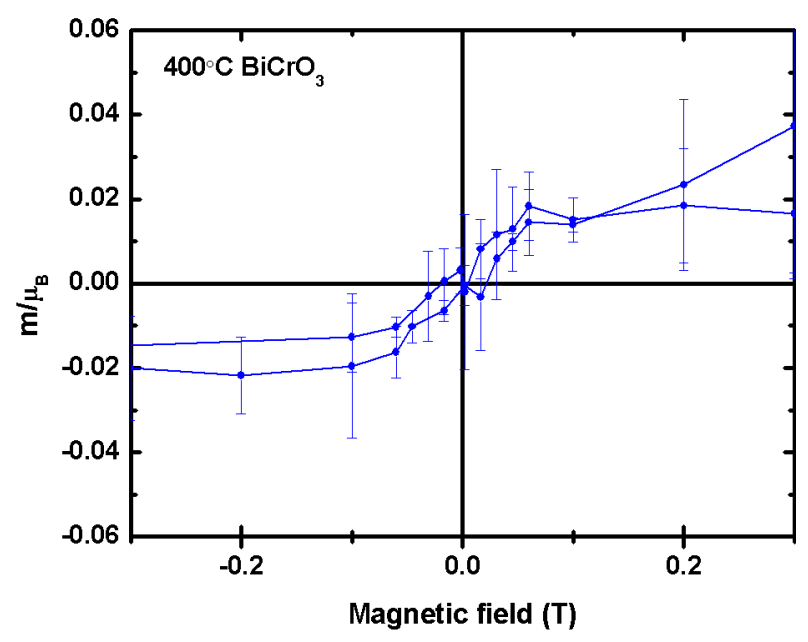

Figure E.3.11: Magnetic moment per formula unit versus the applied magnetic field at $5 \mathrm{~K}$ for the $400{ }^{\circ} \mathrm{C}$ annealed $\mathrm{BiCr}_{x} \mathrm{O}_{y}$ film.

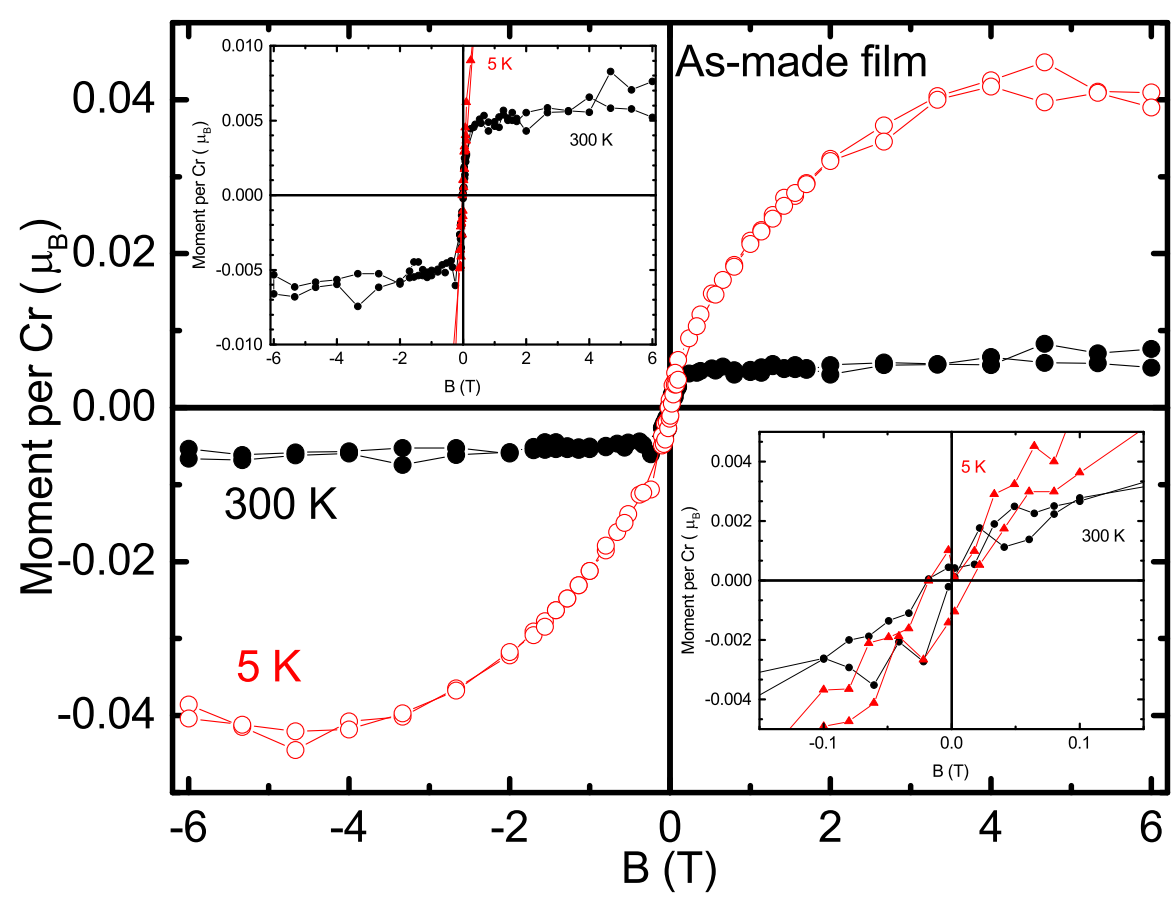

Figure E.3.12: As-made $\mathrm{BiCr}_{x} \mathrm{O}_{y}$ thin film, magnetic moment per $\mathrm{Cr}$. Inset: expand scale near $0 \mathrm{~T}$.

The ZFC-FC, Figure E.3.13, for the as-made film does not show any splitting, so this sample is not superparamagnetic or the particles sizes are very small to create a ZFC-FC splitting, equation 2.1.6. There is no evidence for antiferromagnetic $\mathrm{Cr}_{2} \mathrm{O}_{5}$ has a Néel temperature of $\sim 125 \mathrm{~K}$ [Alexander et al., 2007] or antiferromagnetic $\mathrm{Cr}_{2} \mathrm{O}_{3}$. This 
may be because their particle sizes are small or that they are present as a low volume fraction.

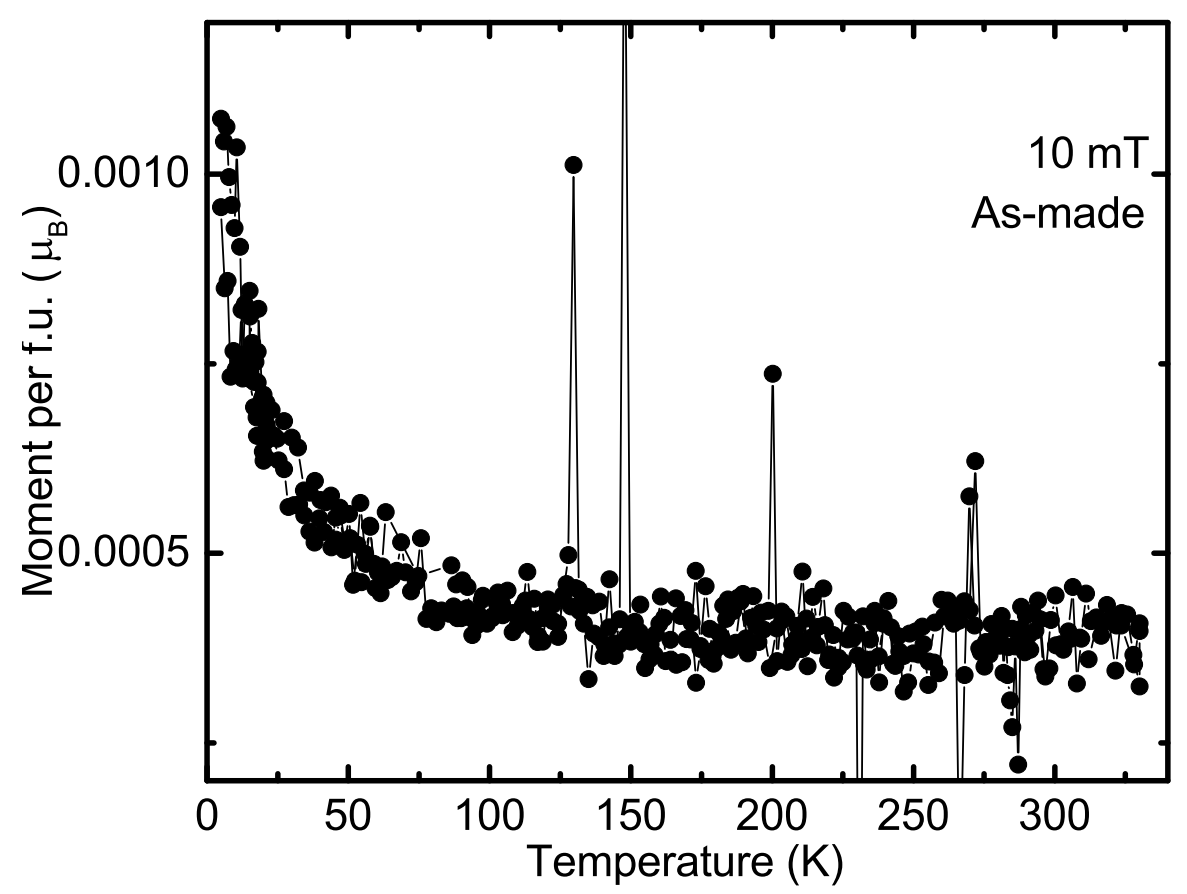

Figure E.3.13: $\mathrm{ZFC}-\mathrm{FC}$, as-made $\mathrm{BiCr}_{x} \mathrm{O}_{y}$ thin film magnetic moment per $\mathrm{Cr}$. At $10 \mathrm{mT}$, the ZFC and FC are completely superposed.

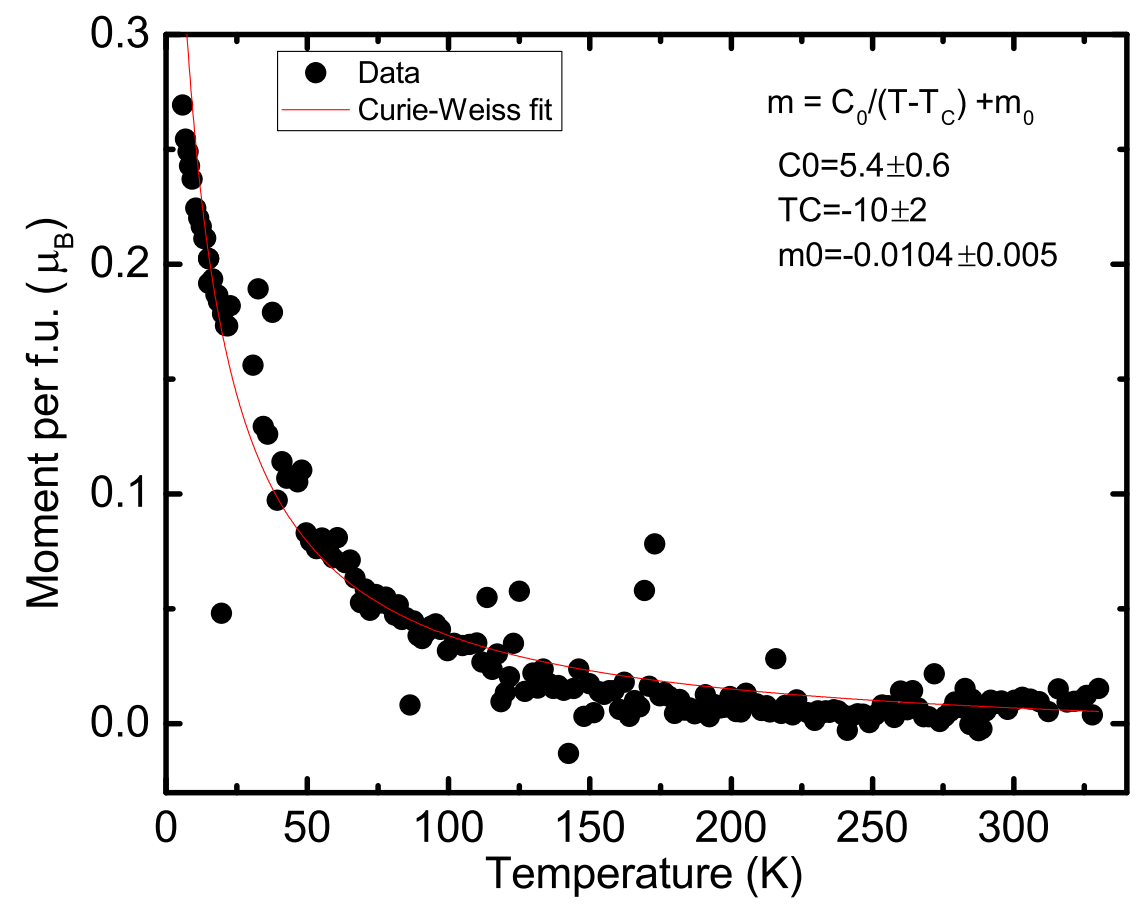

Figure E.3.14: Temperature dependence of the magnetic moment per $\mathrm{Cr}$ at $6 \mathrm{~T}$ for the asmade $\mathrm{BiCr}_{x} \mathrm{O}_{y}$ film. Also, shown in this figure is the Curie-Weiss model with $C_{0}=5.4 \pm 0.6, T_{C}=-10 \pm 2 \mathrm{~K}$.

The Figure E.3.14 shows the temperature dependence of the magnetic moment per $\mathrm{Cr}$ at $6 \mathrm{~T}$ for the as-made film. Also, shown in this figure is the Curie-Weiss 
(equation 2.1.6) model with $C_{0}=5.4 \pm 0.6$ and $T_{C}=-10 \pm 2 \mathrm{~K}$. A negative Curie-Weiss temperature suggests that there is antiferromagnetic exchange, however no long range magnetic order. From the equation D.3.1, the effective moment can be written as $P_{\text {eff }}=0.86 \times\left(C_{0}\right)^{1 / 2}$, so $P_{\text {eff }}=2.0 \pm 0.2 \mu_{B}$. This effective moment can only come from free $\mathrm{Cr}$ ions that are not magnetically ordered. $\quad P_{\text {eff }}=\mathrm{g}(\mathrm{S}(\mathrm{S}+1))^{1 / 2}$ is the theoretical effective moment. For chromium $\mathrm{Cr}^{2+}$ and $\mathrm{Cr}^{3+}, \mathrm{S}$ can be respectively $\mathrm{S}_{\mathrm{Cr}^{2+}}=2,1,0$ and $\mathrm{S}_{C r^{3+}}=3 / 2,1 / 2$. So $P_{\text {eff }}^{C r^{2+}}=4.9 \mu_{B}, 2.8 \mu_{B}, 0 \mu_{B}$ and $P_{\text {eff }}^{C r^{3+}}=3.8 \mu_{B}, 1.7 \mu_{B}$. The high measured effective moment suggests that a considerable fraction of the $\mathrm{Cr}$ magnetic ions is not magnetically ordered.

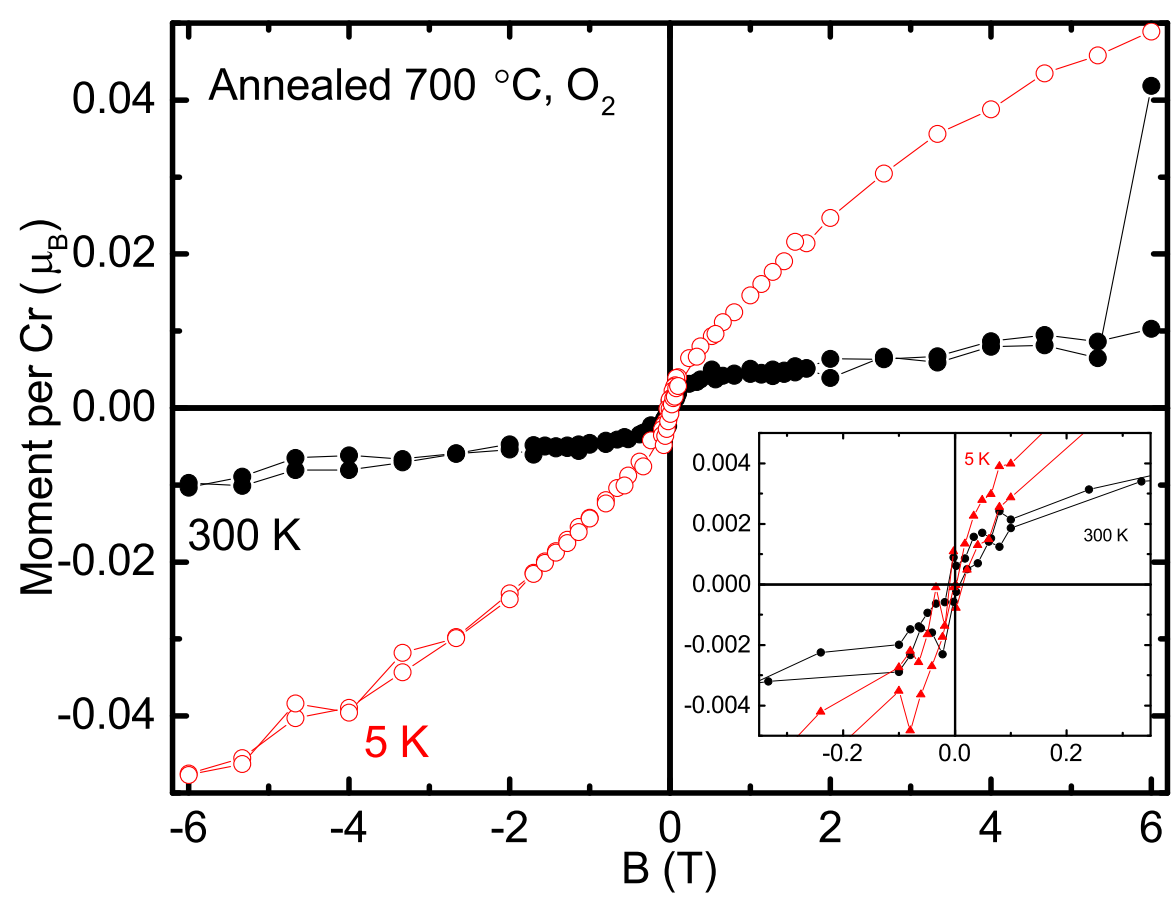

Figure E.3.15: Magnetic moment per $\mathrm{Cr}$ for the film annealed at $700{ }^{\circ} \mathrm{C}$ in oxygen. The inset shows that same data over a smaller magnetic field range.

The field loop at $300 \mathrm{~K}$ of the sample annealed at $700{ }^{\circ} \mathrm{C}$ in oxygen, Figure E.3.15, is similar to the as-made film and with similar magnetic moments at high fields. This suggests that the magnetic behaviour is due to the same magnetic phases. The ZFC-FC data plotted in Figure E.3.16 are also similar and indicate the absence of a blocking temperature in the measured temperature range.

The Figure E.3.17 shows the field loop of the film annealed at $700{ }^{\circ} \mathrm{C}$ in oxygen, then Argon. The inset shows a hysteresis that could be associated with a ferromagnetic material. The thin film annealed at $700{ }^{\circ} \mathrm{C}$ in oxygen followed by argon displays different magnetic behaviour compared to the film annealed at $700{ }^{\circ} \mathrm{C}$ only in the oxygen atmosphere. The magnetic moment is greatly enhanced: the value goes from about $\sim 0.01 \mu_{B} / C r$ at $300 \mathrm{~K}$ with oxygen annealing only, to a magnetic moment 
of $\sim 0.16 \mu_{B} / C r$ after the second annealing. At low temperature, the magnetic moment goes from $\sim 0.05 \mu_{B} / \mathrm{Cr}$ before the second annealing to $0.35 \mu_{B} / \mathrm{Cr}$ after the second annealing. The low-temperature moment is difficult to compare, as both samples annealed at $700{ }^{\circ} \mathrm{C}$ do not seem to reach saturation. They both show paramagnetic behaviour. In a low applied magnetic field (less than $0.4 \mathrm{~T}$ ), the sample annealed only in oxygen does not display a clear hysteresis loop. However, after annealing in argon both the ambient temperature and the low temperature field loop displays an identical hysteresis loop, consistent with a ferromagnetic phase [McElfresh, 1994]. From the $\mathrm{XRD}$ and Raman analysis, $\mathrm{CrO}_{2}$ is the only ferromagnetic phase present in the thin film. $\mathrm{CrO}_{2}$ has a reported magnetic saturation moment of $1.85 \mu_{B} / \mathrm{Cr}$ [Srivastava et al., 2008]. In the first analysis, the saturated magnetic moment at $300 \mathrm{~K}$ is $\sim 0.17 \mu_{B} / C r$, which means that $\mathrm{CrO}_{2}$ is present at less than $10 \%$ of the magnetic ordering, assuming no other magnetic phases are in the film.

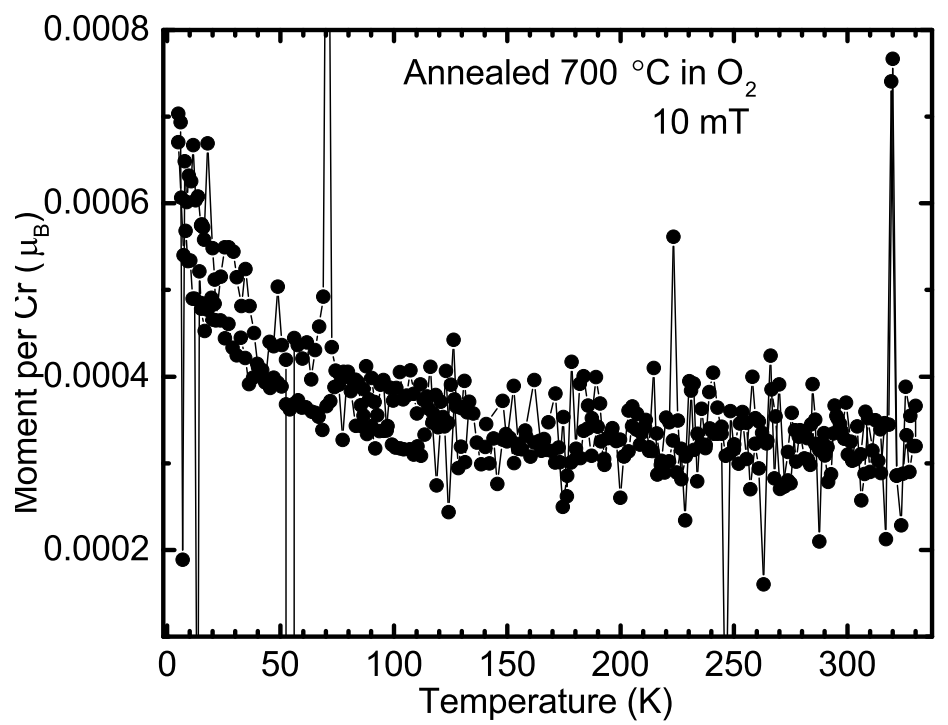

Figure E.3.16: ZFC-FC of the film annealed at $700{ }^{\circ} \mathrm{C}$ in oxygen.

The $5 \mathrm{~K}$ field loop shown in Figure E.3.17 does not saturate and continues to increase in a nearly linear fashion. This may suggest the presence of an antiferromagnetic phase and as shown later there is also some paramagnetic phases from the unordered Cr moments.

The ZFC-FC curves for the film annealed at $700{ }^{\circ} \mathrm{C}$ in $\mathrm{O}_{2}$, then $\mathrm{Ar}$ (Figure E.3.18) shows a hysteresis between the ZFC and FC curves, which was not seen when the film was only annealing in oxygen (Figure E.3.16). The ZFC-FC (Figure E.3.18) displays potentially 4 transitions. The first transition is the splitting around $\sim 270 \mathrm{~K}$. The second transition is around $\sim 225 \mathrm{~K}$ where the ZFC curve is bending. The third potential transition is around $100 \mathrm{~K}$; this transition has a small effect on the FC curve, as the linearity of the curve between $340 \mathrm{~K}$ and $100 \mathrm{~K}$ does not continue below $100 \mathrm{~K}$. The FC seems in- 
dependent of temperature below $100 \mathrm{~K}$. This transition could arise from spin-disorder [Martinez et al., 1998]. The last transition is around $\sim 22 \mathrm{~K}$, in the ZFC. These transitions have a square shape, a succession of straight lines with little noise. The fact that these transitions are abrupt could be linked to the magnetic domain size. The blocking temperature is dependent on the particles' volume (equation 2.1.6). From the anisotropy value of the different chromium oxides, a blocking temperature of $\sim 22 \mathrm{~K}$ implies a particle size of about $5 \pm 2 \mathrm{~nm}$. A blocking temperature up to $250 \mathrm{~K}$ would mean, for chromium oxide, a nanoparticle of about $10 \pm 3 \mathrm{~nm}$. This method of particle size estimation is carried out on the $\mathrm{CrO}_{2}$, with an anisotropy constant of $2.3 \times 10^{4} \mathrm{~J} \cdot \mathrm{m}^{-3}$ [Cheng et al., 2002]. According to the magnetic data, $\mathrm{CrO}_{2}$ is the main magnetic contributor, and the other chromium oxides should have a similar size. The size distribution could explain the ZFC transition at $270 \mathrm{~K}$ and $22 \mathrm{~K}$. The particle sizes estimated using the blocking temperature are similar to the values calculated with the Sheerer equation in the XRD analysis, about $5 \pm 3 \mathrm{~nm}$.

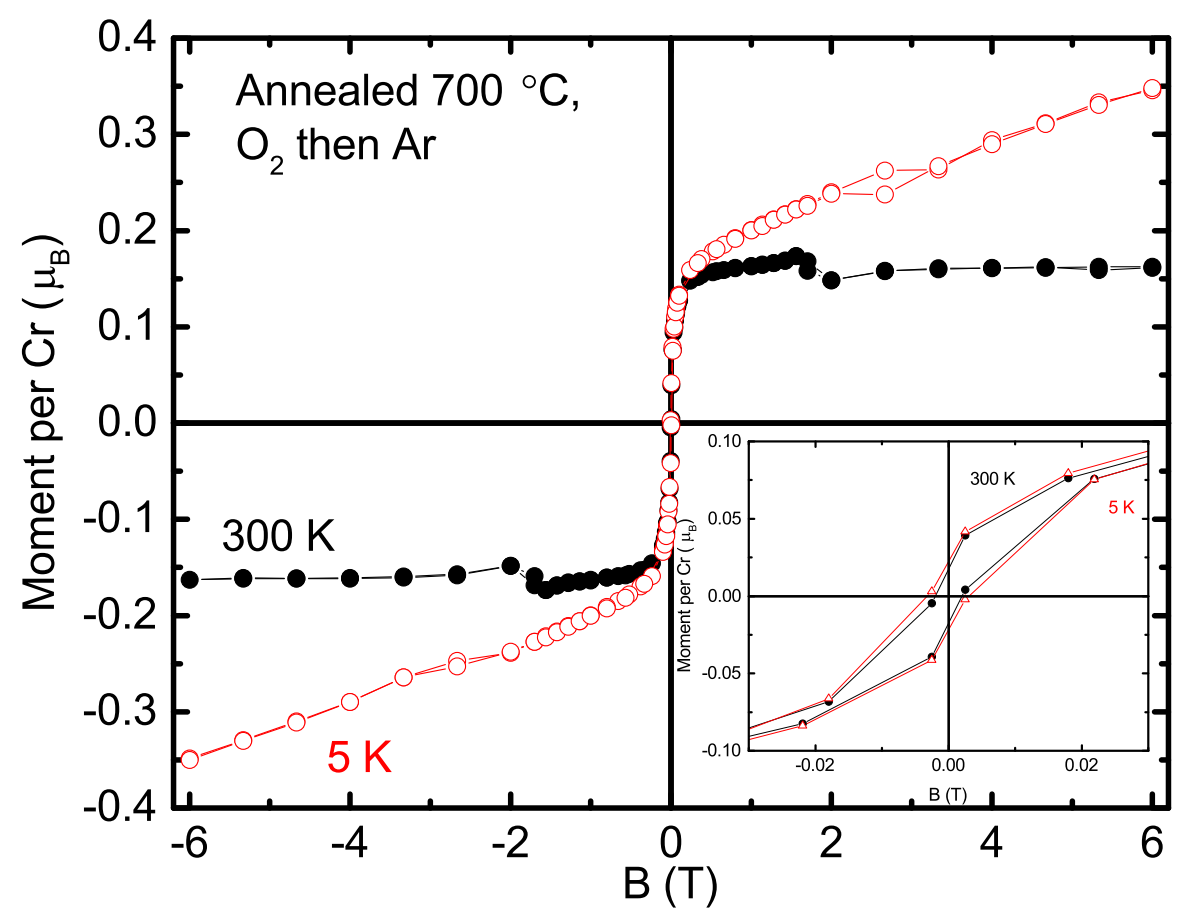

Figure E.3.17: Field loop of the film annealed at $700{ }^{\circ} \mathrm{C}$ in $\mathrm{O}_{2}$, then Ar. The inset shows a hysteresis that could be associated with a ferromagnetic phase.

Figure E.3.19 shows the temperature dependence of the film annealed at $700{ }^{\circ} \mathrm{C}$ in $\mathrm{O}_{2}$, then Ar. Also, shown in this figure is the Curie-Weiss model with $C_{0}=3.9 \pm 0.5$ and $T_{C}=-13 \pm 3 \mathrm{~K}$. A negative Curie temperature indicates antiferromagnetic interactions. The Curie-Weiss temperature after annealing at $700{ }^{\circ} \mathrm{C}$ in $\mathrm{O}_{2}$ and $\mathrm{Ar}$ is close the Curie temperature of the as-made film. The effective moment is also similar, from $C_{0}=3.9 \pm 0.5$ and equation D.3.1, $P_{\text {eff }}=1.7 \pm 0.2 \mu_{B}$ while the as-made film effective mo-

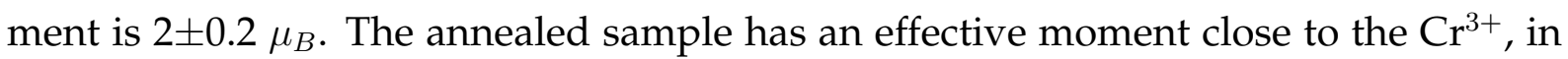




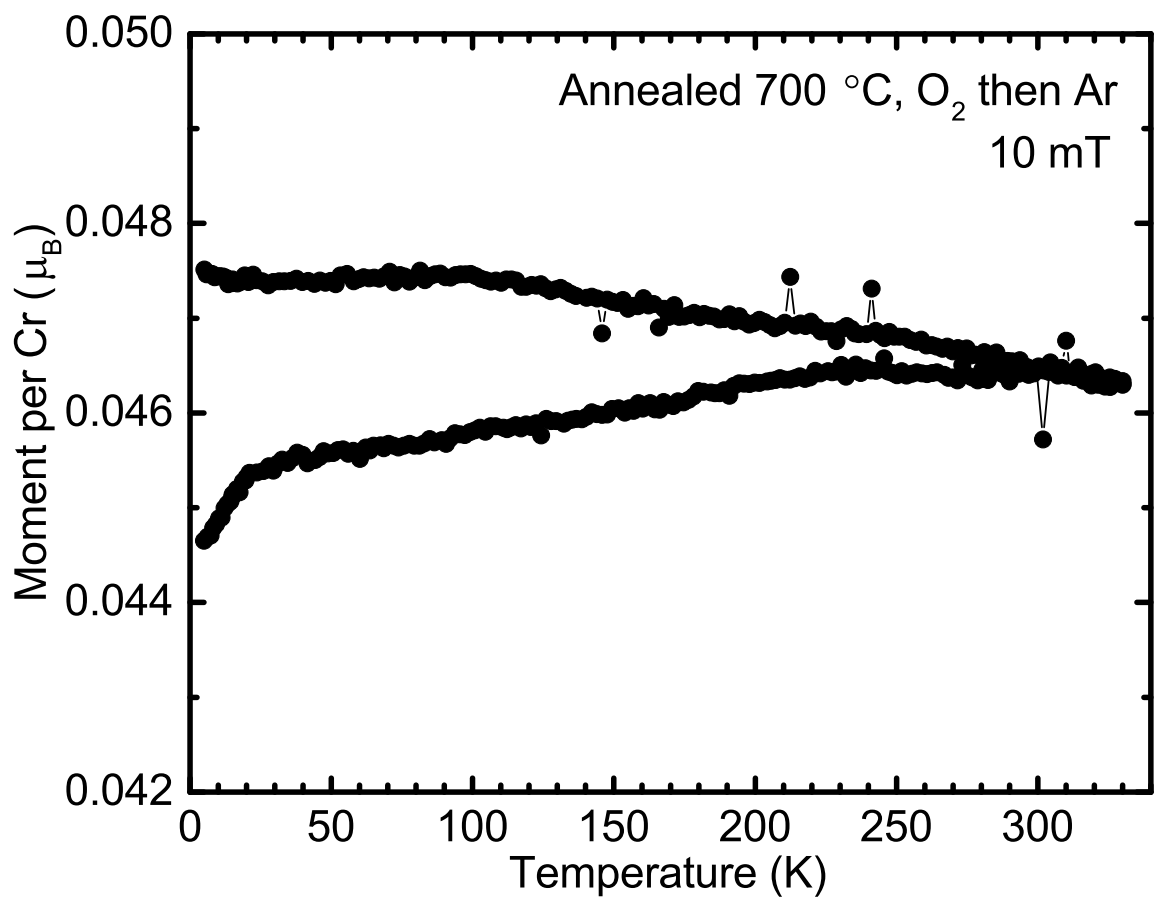

Figure E.3.18: ZFC-FC of the film annealed at $700{ }^{\circ} \mathrm{C}$ in $\mathrm{O}_{2}$, then Ar. The ZFC and FC seem to merge around $280 \mathrm{~K}$.

the low spin configuration. This results shows the limited effect annealing had, nanostructured is different, however the magnetic phases are similar in every film regardless of the annealing conditions.

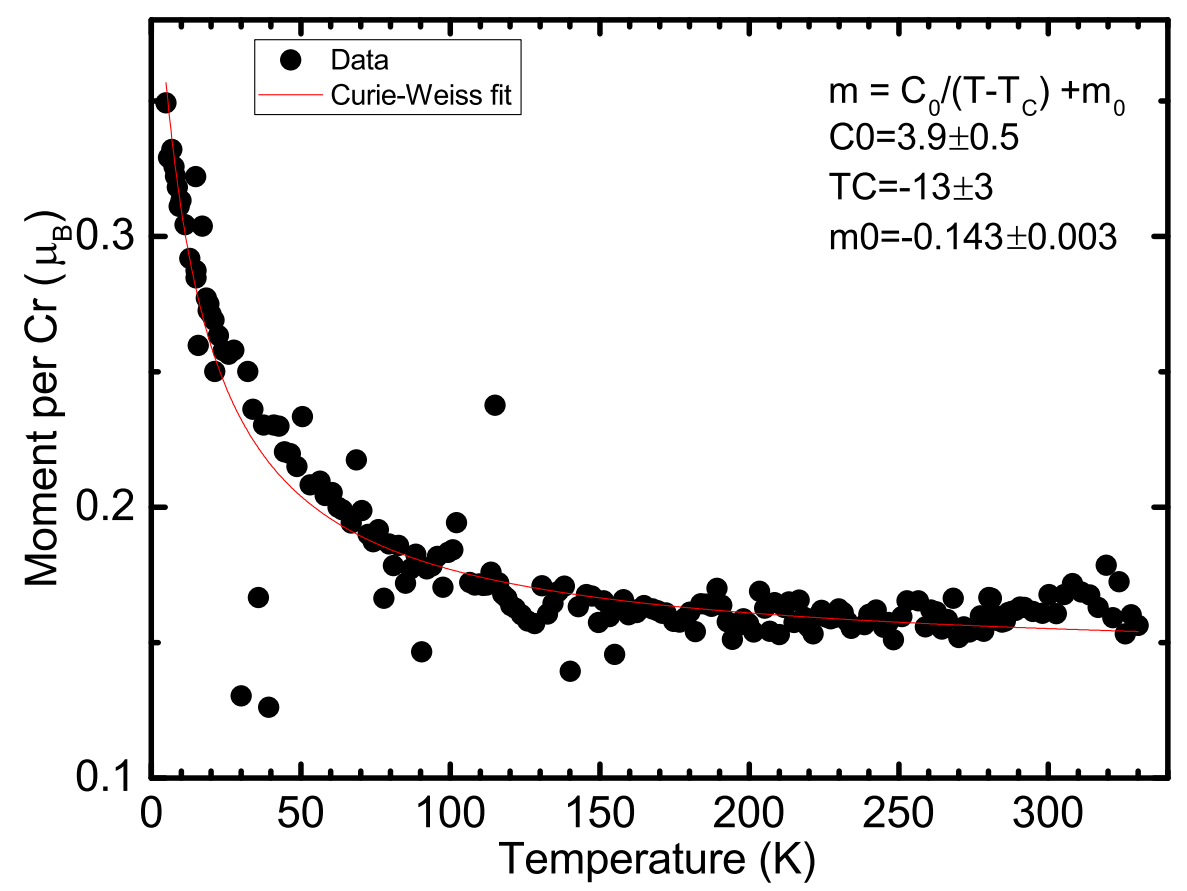

Figure E.3.19: Temperature dependence of the film annealed at $700{ }^{\circ} \mathrm{C}$ in $\mathrm{O}_{2}$, then Ar. Also, shown in this figure is the Curie-Weiss model with $C_{0}=3.9 \pm 0.5$ and $T_{C}=-13 \pm 3 \mathrm{~K}$. 


\section{E.4 Conclusion}

In conclusion, $\mathrm{BiCr}_{x} \mathrm{O}_{y}$ thin films have been made by ion beam sputtering at ambient temperature and then annealing in an oxygen atmosphere at temperatures up to $700{ }^{\circ} \mathrm{C}$. One sample was subsequently annealed in an argon atmosphere. The as-made thin film contained bismuth nanoparticles with an average diameter of $16 \pm 0.3 \mathrm{~nm}$. Annealing the sample in an oxygen atmosphere leads to $\mathrm{BiCr}_{x} \mathrm{O}_{y}$ with chromium oxides and bismuth oxide phases. There was no evidence for the formation of $\mathrm{BiCrO}_{3}$. Annealing the $\mathrm{BiCr}_{x} \mathrm{O}_{y}$ film at and below $700{ }^{\circ} \mathrm{C}$ did not lead to a significant enhancement of the saturation moment per $\mathrm{Cr}$. The high field moment was small and could be due to a small ferromagnetic $\mathrm{CrO}_{2}$ fraction or from a fraction of antiferromagnetic chromium oxide phases. The effective moment for the as-made film was large and indicates that there is a significant fraction of $\mathrm{Cr}$ moment that did not magnetically order. The Curie-Weiss temperature is negative and shows that there is antiferromagnetic exchange between the $\mathrm{Cr}$ moments without long range magnetic order.

Annealing the thin film at $700{ }^{\circ} \mathrm{C}$ in an oxygen atmosphere followed by argon annealing leads to a large enhancement of the magnetic moment and hysteresis is evident in the magnetic field loops. There is also hysteresis in the ZFC-FC data taken at $10 \mathrm{mT}$. This hysteresis is likely to be due to a fraction of ferromagnetic $\mathrm{CrO}_{2}$ that has a Curie temperature of $391 \mathrm{~K}$. The effective moment per $\mathrm{Cr}$ and the Curie-Weiss temperatures are the same within errors as those measured in the as-made film and suggests that a similar fraction of $\mathrm{Cr}$ moments have not magnetically ordered.

To summarise, ion beam sputtering followed by annealing is a process that can be used to create nanostructured $\mathrm{BiCr}_{x} \mathrm{O}_{y}$ that contains chromium oxides and bismuth oxides. There is not enough evidence to support the formation of a pure $\mathrm{BiCrO}_{3}$ film. In an attempt to investigate a different synthesis method, the annealing temperature was increased up to $700{ }^{\circ} \mathrm{C}$ in oxygen followed by argon. There was no evidence for $\mathrm{BiCrO}_{3}$ after this process although the magnetic properties did change. Sputtering on heated substrates or perhaps investigate a different method such as pulsed laser deposition, in future work, would be useful to optimise the synthesis technique and potentially form $\mathrm{BiCrO}_{3}$ nanoparticles. A piezoelectric measurement would also be useful to undertake to analyse the phase fraction of $\mathrm{BiCrO}_{3}$ in these films. Piezoelectric measurement can confirm the potential for MeRAM device.

In future work, to study $\mathrm{BiCrO}_{3}$ : structural, magnetic and electric properties, the synthesis of $\mathrm{BiCrO}_{3}$ and the optimisation of the synthesis conditions is required. The 
other millstone of $\mathrm{BiCrO}_{3}$ would be to experimentally confirm the multiferroic properties.

To study the structural and composition of $\mathrm{BiCrO}_{3}$ thin film, the synthesis technique requires further investigation. From the research presented, ion beam sputtering with the current condition of annealing, annealing furnace oven at temperature up to $700{ }^{\circ} \mathrm{C}$, does not form the stoichiometric film. Other synthesis technique should be investigated. Although in the spirit of optimising the current technique, annealing at higher temperature and higher pressure should be investigated as those conditions were reported in the literature for chemical synthesis. This might remove some of the impurities through evaporation or provide mobility to the deposited ions to form a crystal. To improve knowledge of the film synthesis, a comparison between a sputtered a chromium oxide film and the $\mathrm{BiCr}_{x} \mathrm{O}_{y}$ films would be interesting to analyse.

As $\mathrm{BiCrO}_{3}$ research for multiferroic properties continue and in order to confirm the multiferroic properties of $\mathrm{BiCrO}_{3}$, a detailed analysis of the piezoelectric response is needed. 


\section{Bibliography}

Abraham, D. W., Frank, M. M., and Guha, S. (2005). Absence of magnetism in hafnium oxide films. Applied Physics Letters, 87(25):252502.

Agbo, P., Nweke, F., Nwofe, P., and Ukwu, C. (1832). Effects of Concentration on the Properties of Zn-doped Cadmium Sulphide Thin Films. International Journal of Science and Research, 3(11):2014.

Aigner, W., Niesar, S., Mehmedovic, E., Opel, M., Wagner, F. E., Wiggers, H., and Stutzmann, M. (2013). Separation of semiconducting and ferromagnetic $\mathrm{FeSi}_{2}$ nanoparticles by magnetic filtering. Journal of Applied Physics, 114(13):134308.

Alexander, L., Büttgen, N., Nath, R., Mahajan, A., and Loidl, A. (2007). ${ }^{7}$ Li NMR studies on the triangular lattice system $\mathrm{LiCrO}_{2}$. Physical Review B, 76(6):064429.

Ali, M. Y., Hung, W., and Yongqi, F. (2010). A review of focused ion beam sputtering. International journal of precision engineering and manufacturing, 11(1):157-170.

Amable, A. K. S., Godsway, B. K., Nyaaba, R. A., and Manson, E. N. (2017). A Theoretical Study of Stopping Power and Range For Low Energy $(<3.0 \mathrm{MeV})$ Protons In Aluminium, Germanium, Lead, Gold and Copper Solid Materials. Open Science Journal, 2(2).

Andersen, H. and Bay, H. (1974). Nonlinear effects in heavy-ion sputtering. Journal of Applied Physics, 45(2):953-954.

Antic, B., Perovic, M., Kremenovic, A., Blanusa, J., Spasojevic, V., Vulic, P., Bessais, L., and Bozin, E. S. (2013). An integrated study of thermal treatment effects on the microstructure and magnetic properties of $\mathrm{Zn}$-ferrite nanoparticles. Journal of Physics: Condensed Matter, 25(8):086001.

Aragón, R. (1992). Magnetization and exchange in nonstoichiometric magnetite. Physical Review B, 46(9):5328-5333.

Arima, T.-h. (2007). Ferroelectricity induced by proper-screw type magnetic order. Journal of the Physical Society of Japan, 76(7):073702.

Arushanov, E., Behr, G., and Schumann, J. (2004). Magnetic properties of $\beta$-FeSi ${ }_{2}$ single crystals. Thin solid films, 461(1):148-151. 
Ashraf, R., Riaz, S., Akhtar, M. S., and Naseem, S. (2014). Study of structural and electronic properties of iron oxide by ADF. In Proc. The 2014 World Congress on Advances in Civil, Environmental and Materials Research (ACEM'14).

Awan, M. and Bhatti, A. (2011). Synthesis and multiferroic properties of BFO ceramics by melt-phase sintering. Journal of materials engineering and performance, 20(2):283288.

Bajpai, O., Kamdi, J., Selvakumar, M., Ram, S., Khastgir, D., and Chattopadhyay, S. (2014). Effect of surface modification of $\mathrm{BiFeO}_{3}$ on the dielectric, ferroelectric, magneto-dielectric properties of polyvinylacetate $/ \mathrm{BiFeO}_{3}$ nanocomposites. express Polymer Letters, 8(9).

Baram, M. and Kaplan, W. (2008). Quantitative HRTEM analysis of FIB prepared specimens. Journal of microscopy, 232(3):395-405.

Basu, S., Martin, L., Chu, Y., Gajek, M., Ramesh, R., Rai, R., Xu, X., and Musfeldt, J. (2008). Photoconductivity in $\mathrm{BiFeO}_{3}$ thin films. Applied Physics Letters, 92(9):091905.

Baumann, H. and Bethge, K. (1974). PIG ion source with end extraction for multiply charged ions. Nuclear Instruments and Methods, 122:517-525.

Belik, A. A., Tsujii, N., Suzuki, H., and Takayama-Muromachi, E. (2007). Magnetic properties of bulk $\mathrm{BiCrO}_{3}$ studied with dc and ac magnetization and specific heat. Inorganic chemistry, 46(21):8746-8751.

Bergerhoff, G., Hundt, R., Sievers, R., and Brown, I. (1983). The inorganic crystal structure data base. Journal of chemical information and computer sciences, 23(2):66-69. DOI: $10.1021 /$ ci00038a003.

Berling, D., Gewinner, G., Hanf, M., Hricovini, K., Hong, S., Loegel, B., Mehdaoui, A., Pirri, C., Tuilier, M., and Wetzel, P. (1999). Magnetic properties of epitaxial $\mathrm{Fe}\left(\mathrm{Si}_{1-x} \mathrm{Fe}_{x}\right)$ films grown on $\mathrm{Si}$ (111). Journal of magnetism and magnetic materials, 191(3):331-338.

Bhowmik, R. and Saravanan, A. (2010). Surface magnetism, Morin transition, and magnetic dynamics in antiferromagnetic $\alpha-\mathrm{Fe}_{2} \mathrm{O}_{3}$ (hematite) nanograins. Journal of Applied Physics, 107(5):053916.

Bi, L., Taussig, A. R., Kim, H.-S., Wang, L., Dionne, G. F., Bono, D., Persson, K., Ceder, G., and Ross, C. (2008). Structural, magnetic, and optical properties of $\mathrm{BiFeO}_{3}$ and $\mathrm{Bi}_{2} \mathrm{FeMnO}_{6}$ epitaxial thin films: an experimental and first-principles study. Physical Review B, 78(10):104106. 
Bian, Z., Zhu, J., Wang, S., Cao, Y., Qian, X., and Li, H. (2008). Self-assembly of active $\mathrm{Bi}_{2} \mathrm{O}_{3} / \mathrm{TiO}_{2}$ visible photocatalyst with ordered mesoporous structure and highly crystallized anatase. The Journal of Physical Chemistry C, 112(16):6258-6262.

Biersack, J., Berg, S., and Nender, C. (1991). T-DYN Monte Carlo simulations applied to ion assisted thin film processes. Nuclear Instruments and Methods in Physics Research Section B: Beam Interactions with Materials and Atoms, 59:21-27.

Binder, K. and Young, A. P. (1986). Spin glasses: Experimental facts, theoretical concepts, and open questions. Reviews of Modern physics, 58(4):801.

Bloch, F. (1930). Zur theorie des ferromagnetismus. Zeitschrift für Physik, 61(3-4):206219.

Boettcher, S. (2005). Extremal optimization for Sherrington-Kirkpatrick spin glasses. The European Physical Journal B-Condensed Matter and Complex Systems, 46(4):501-505.

Büngener, R., Pamler, W., and Gösele, U. (2003). Diffusion of Sr, Bi, and Ta in amorphous $\mathrm{SiO}_{2}$. Materials Science in Semiconductor Processing, 6(1):43-48.

Canepa, P., Schofield, E., Chadwick, A. V., and Alfredsson, M. (2011). Comparison of a calculated and measured XANES spectrum of $\alpha-\mathrm{Fe}_{2} \mathrm{O}_{3}$. Physical Chemistry Chemical Physics, 13(28):12826-12834.

Carter, C. B. and Williams, D. B. (2016). Transmission electron microscopy, Diffraction, Imaging and Spectrometry.

Carter, G. (2001). The physics and applications of ion beam erosion. Journal of Physics D: Applied Physics, 34(3):R1.

Castro, M., Cuerno, R., Vázquez, L., and Gago, R. (2005). Self-organized ordering of nanostructures produced by ion-beam sputtering. Physical review letters, 94(1):016102.

Catalan, G. and Scott, J. F. (2009). Physics and applications of bismuth ferrite. Advanced Materials, 21(24):2463-2485.

Chakrabarti, K., Das, K., Sarkar, B., and De, S. (2011). Magnetic and dielectric properties of Eu-doped $\mathrm{BiFeO}_{3}$ nanoparticles by acetic acid-assisted sol-gel method. Journal of Applied Physics, 110(10):103905.

Chen, B., Li, M., Liu, Y., Zuo, Z., Zhuge, F., Zhan, Q.-F., and Li, R.-W. (2011). Effect of top electrodes on photovoltaic properties of polycrystalline $\mathrm{BiFeO}_{3}$ based thin film capacitors. Nanotechnology, 22(19):195201. 
Chen, J., Huang, D., Tanaka, A., Chang, C., Chung, S., Wu, W., and Chen, C. (2004). Magnetic circular dichroism in Fe $2 \mathrm{p}$ resonant photoemission of magnetite. Physical Review B, 69(8):085107.

Chen, J., Sorensen, C., Klabunde, K., and Hadjipanayis, G. (1995). Enhanced magnetization of nanoscale colloidal cobalt particles. Physical Review B, 51(17):11527.

Chen, X., Zhang, H., Wang, T., Wang, F., and Shi, W. (2012). Optical and photoluminescence properties of $\mathrm{BiFeO}_{3}$ thin films grown on ITO-coated glass substrates by chemical solution deposition. physica status solidi (a), 209(8):1456-1460.

Chen, X., Zhu, W., Liu, W., Tan, O., and Yao, X. (2001). SPM studies on surface charge and local piezo-response of ferroelectric thin films. Ferroelectrics, 252(1):201-208.

Chen, Y., Lee, P., Tsai, C., Neeleshwar, S., Wang, C., Ho, J., and Hamdeh, H. (2007). Chemical disorder-induced magnetism in $\mathrm{FeSi}_{2}$ nanoparticles. Applied Physics Letters, 91(25):251907.

Cheng, R., Liu, Z., Bo, X., Adenwalla, S., Yuan, L., Liou, S.-H., and Dowben, P. (2002). Magnetic anisotropy in epitaxial $\mathrm{CrO}_{2}$ (100) thin films. Materials Letters, 56(3):295299.

Cheng, Z., Feng, L., and Jiang, L. (2008). Tunable adhesive superhydrophobic surfaces for superparamagnetic microdroplets. Advanced functional materials, 18(20):32193225.

Cheong, S.-W. and Mostovoy, M. (2007). Multiferroics: a magnetic twist for ferroelectricity. Nature materials, 6(1):13-20.

Chi, Y. S., Lee, J. K., Lee, S.-g., and Choi, I. S. (2004). Control of wettability by anion exchange on $\mathrm{Si} / \mathrm{SiO}_{2}$ surfaces. Langmuir, 20(8):3024-3027.

Chirita, M., Grozescu, I., Taubert, L., Radulescu, H., Princz, E., Stefanovits-Bányai, É., Caramalau, C., Bulgariu, L., Macoveanu, M., and Muntean, C. (2009). $\mathrm{Fe}_{2} \mathrm{O}_{3}-$ nanoparticles, physical properties and their photochemical and photoelectrochemical applications. Chem. Bull, 54(68):1-8.

Choi, K. J., Biegalski, M., Li, Y., Sharan, A., Schubert, J., Uecker, R., Reiche, P., Chen, Y., Pan, X., Gopalan, V., and Chen, L. (2004). Enhancement of ferroelectricity in strained $\mathrm{BaTiO}_{3}$ thin films. Science, 306(5698):1005-1009.

Chu, Y.-H., Cruz, M. P., Yang, C.-H., Martin, L. W., Yang, P.-L., Zhang, J.-X., Lee, K., $\mathrm{Yu}, \mathrm{P}$., Chen, L.-Q., and Ramesh, R. (2007a). Domain control in multiferroic $\mathrm{BiFeO}_{3}$ through substrate vicinality. Advanced materials, 19(18):2662-2666. 
Chu, Y.-H., Martin, L. W., Holcomb, M. B., and Ramesh, R. (2007b). Controlling magnetism with multiferroics. Materials Today, 10(10):16-23.

Chu, Y.-H., Zhan, Q., Martin, L. W., Cruz, M. P., Yang, P.-L., Pabst, G. W., Zavaliche, F., Yang, S.-Y., Zhang, J.-X., Chen, L.-Q., and Schlom, D. (2006). Nanoscale domain control in multiferroic $\mathrm{BiFeO}_{3}$ thin films. Advanced Materials, 18(17):2307-2311.

Coey, J. and Khalafalla, D. (1972). Superparamagnetic $\gamma-\mathrm{Fe}_{2} \mathrm{O}_{3}$. Physica status solidi (a), 11(1):229-241.

Cojocaru, S., Naddeo, A., and Citro, R. (2014). Modification of the Bloch law in ferromagnetic nanostructures. EPL (Europhysics Letters), 106(1):17001.

Colin, C. V., Pérez, A. G., Bordet, P., Goujon, C., and Dariend D282(tters.iiD282Llmetry)]TJ-6 
Davis, E. and Mott, N. (1970). Conduction in non-crystalline systems V. Conductivity, optical absorption and photoconductivity in amorphous semiconductors. Philosophical Magazine, 22(179):0903-0922.

De Faria, D., Venâncio Silva, S., and De Oliveira, M. (1997). Raman microspectroscopy of some iron oxides and oxyhydroxides. Journal of Raman spectroscopy, 28(11):873878.

Dedkov, Y. S., Rüdiger, U., and Güntherodt, G. (2002). Evidence for the half-metallic ferromagnetic state of $\mathrm{Fe}_{3} \mathrm{O}_{4}$ by spin-resolved photoelectron spectroscopy. Physical Review B, 65(6):064417.

Della Volpe, C., Brugnara, M., Maniglio, D., Siboni, S., and Wangdu, T. (2006). About the possibility of experimentally measuring an equilibrium contact angle and its theoretical and practical. Contact Angle Wettabil. Adhes., 4:79.

Deng, Y., Blöte, H. W., and Nightingale, M. (2005). Surface and bulk transitions in three-dimensional O(n) models. Physical Review E, 72(1):016128.

Deoli, N. T., Phinney, L. C., and Weathers, D. L. (2014). Ion fluence dependence of the total sputtering yield and differential angular sputtering yield of bismuth due to $50 \mathrm{keV}$ argon ion irradiation. Nuclear Instruments and Methods in Physics Research Section B: Beam Interactions with Materials and Atoms, 332:286-289.

Devi, D. A., Smitha, B., Sridhar, S., and Aminabhavi, T. (2005). Pervaporation separation of isopropanol-water mixtures through crosslinked chitosan membranes. Journal of membrane science, 262(1):91-99.

Dhanalakshmi, B., Pratap, K., Rao, B. P., and Rao, P. S. (2016). Effects of Mn doping on structural, dielectric and multiferroic properties of $\mathrm{BiFeO}_{3}$ nanoceramics. Journal of Alloys and Compounds, 676:193-201.

Dhir, G., Uniyal, P., and Verma, N. (2015). Sol-gel synthesized $\mathrm{BiFeO}_{3}$ nanoparticles: Enhanced magnetoelelctric coupling with reduced particle size. Journal of Magnetism and Magnetic Materials, 394:372-378.

Dho, J. and Blamire, M. (2009). Controlling the exchange bias in multiferroic $\mathrm{BiFeO}_{3}$ and NiFe bilayers. Journal of Applied Physics, 106(7):3914.

Dho, J., Qi, X., Kim, H., MacManus-Driscoll, J. L., and Blamire, M. G. (2006). Large electric polarization and exchange bias in multiferroic $\mathrm{BiFeO}_{3}$. Advanced Materials, 18(11):1445-1448. 
Dimitrov, D., Unruh, K., Hadjipanayis, G., Papaefthymiou, V., and Simopoulos, A. (1999). Ferrimagnetism and defect clusters in $\mathrm{Fe}_{1-x} \mathrm{O}$ films. Physical Review B, 59(22):14499.

DiPietro, R., Johnson, H., Bennett, S., Nummy, T., Lewis, L., and Heiman, D. (2010). Determining magnetic nanoparticle size distributions from thermomagnetic measurements. Applied Physics Letters, 96(22):222506.

Domingo, N., Williamson, B., Gómez-Segura, J., Gerbier, P., Ruiz-Molina, D., Amabilino, D., Veciana, J., and Tejada, J. (2004). Magnetism of isolated $\mathrm{Mn}_{12}$ singlemolecule magnets detected by magnetic circular dichroism: Observation of spin tunneling with a magneto-optical technique. Physical Review B, 69(5):052405.

Doolittle, L. R. (1985). Algorithms for the rapid simulation of Rutherford backscattering spectra. Nuclear Instruments and Methods in Physics Research Section B: Beam Interactions with Materials and Atoms, 9(3):344-351.

Du, Y., Cheng, Z., Dou, S., and Wang, X. (2011). Tunable morphology and magnetic properties of $\mathrm{Bi}_{2} \mathrm{Fe}_{4} \mathrm{O}_{9}$ nanocrystal synthesized by hydrothermal method. Journal of nanoscience and nanotechnology, 11(3):2691-2695.

Duffy, T. S. (2009). Crystal geometry formula. Princeton University Department of Geoscience, Class note. [Online; accessed 20-April-2016].

Ederer, C. and Spaldin, N. A. (2005a). Influence of strain and oxygen vacancies on the magnetoelectric properties of multiferroic bismuth ferrite. Physical Review B, 71(22):224103.

Ederer, C. and Spaldin, N. A. (2005b). Weak ferromagnetism and magnetoelectric coupling in bismuth ferrite. Physical Review B, 71(6):060401.

Engström, C., Berlind, T., Birch, J., Hultman, L., Ivanov, I., Kirkpatrick, S., and Rohde, S. (2000). Design, plasma studies, and ion assisted thin film growth in an unbalanced dual target magnetron sputtering system with a solenoid coil. Vacuum, 56(2):107-113.

Ensikat, H. J., Ditsche-Kuru, P., Neinhuis, C., and Barthlott, W. (2011). Superhydrophobicity in perfection: the outstanding properties of the lotus leaf. Beilstein journal of nanotechnology, 2:152.

Ermrich, M. and Opper, D. (2013). XRD for the Analyst: Getting Acquainted with the Principles. PANalytical $2^{\text {nd }}$ revised edition, Netherland. 9789080908604.

Fan, W., Ignatiev, A., and Wu, N. (1990). Growth of bismuth on the Si (100) surface: AES and LEED study. Surface Science, 235(2-3):169-174. 
Feder, R., Frost, F., Neumann, H., Bundesmann, C., and Rauschenbach, B. (2013). Systematic investigations of low energy Ar ion beam sputtering of Si and Ag. Nuclear Instruments and Methods in Physics Research Section B: Beam Interactions with Materials and Atoms, 317:137-142.

Fennie, C. J. and Rabe, K. M. (2005). Ferroelectric transition in $\mathrm{YMnO}_{3}$ from first principles. Physical Review B, 72(10):100103.

Fitzpatrick, M., Fry, A., Holdway, P., Kandil, F., Shackleton, J., and Suominen, L. (2005). Determination of residual stresses by $\mathrm{X}$-ray diffraction.

Forrester, M. and Kusmartsev, F. (2014). The nano-mechanics and magnetic properties of high moment synthetic antiferromagnetic particles. physica status solidi (a), 211(4):884-889.

Frost, F., Ziberi, B., Höche, T., and Rauschenbach, B. (2004). The shape and ordering of self-organized nanostructures by ion sputtering. Nuclear Instruments and Methods in Physics Research Section B: Beam Interactions with Materials and Atoms, 216:9-19.

Fruth, V., Popa, M., Berger, D., Ramer, R., Gartner, M., Ciulei, A., and Zaharescu, M. (2005). Deposition and characterisation of bismuth oxide thin films. Journal of the European Ceramic Society, 25(12):2171-2174.

Fruth, V., Tenea, E., Gartner, M., Anastasescu, M., Berger, D., Ramer, R., and Zaharescu, M. (2007). Preparation of $\mathrm{BiFeO}_{3}$ films by wet chemical method and their characterization. Journal of the European Ceramic Society, 27(2):937-940.

$\mathrm{Fu}$, Y. and Du, H. (2003). Effects of film composition and annealing on residual stress evolution for shape memory TiNi film. Materials Science and Engineering: A, 342(1):236-244.

Fujii, M., Hayashi, S., and Yamamoto, K. (1991). Growth of Ge microcrystals in $\mathrm{SiO}_{2}$ thin film matrices: a Raman and electron microscopic study. Japanese Journal of Applied Physics, 30(4R):687.

Fultz, B. and Howe, J. M. (2012). Transmission electron microscopy and diffractometry of materials. Springer Science \& Business Media.

Gailly, P., Petermann, C., Tihon, P., and Fleury-Frenette, K. (2012). Ripple topography and roughness evolution on surface of polycrystalline gold and silver thin films under low energy Ar-ion beam sputtering. Applied Surface Science, 258(19):7717-7725. 
Gajek, M., Bibes, M., Fusil, S., Bouzehouane, K., Fontcuberta, J., Barthelemy, A., and Fert, A. (2007). Tunnel junctions with multiferroic barriers. Nature materials, 6(4):296302.

Geprägs, S., Opel, M., Goennenwein, S., and Gross, R. (2007). Multiferroic materials based on artificial thin film heterostructures. Philosophical Magazine Letters, 87(34):141-154.

Giannuzzi, L. A., Drown, J. L., Brown, S. R., Irwin, R. B., and Stevie, F. A. (1998). Applications of the FIB lift-out technique for TEM specimen preparation. Microscopy research and technique, 41(4):285-290.

Goldman, A. (2012). Handbook of modern ferromagnetic materials, volume 505. Springer Science \& Business Media. ISBN 0-412-14661-4.

Goodhew, P. (2001). Electron Diffraction in the Transmission Electron Microscope. Journal of Microscopy, 204(3):263-264.

Goujon, C., Darie, C., Bacia, M., Klein, H., Ortega, L., and Bordet, P. (2008). High pressure synthesis of $\mathrm{BiCrO}_{3}$, a candidate for multiferroism. In Journal of Physics: Conference Series, volume 121, page 022009. IOP Publishing.

Goveas, L. R., Anuradha, K., Bhagyashree, K., and Bhat, S. (2015). Comparative study of magnetic ordering in bulk and nanoparticles of $\mathrm{Sm}_{0.65} \mathrm{Ca}_{0.35} \mathrm{MnO}_{3}$ : Magnetization and electron magnetic resonance measurements. Journal of Applied Physics, 117(17):17E111.

Goya, G., Berquó, T. S., Fonseca, F. C., and Morales, M. (2003). Static and dynamic magnetic properties of spherical magnetite nanoparticles. Journal of Applied Physics, 94(5):3520-3528.

Griffiths, D. J. and College, R. (1999). Introduction to electrodynamics, volume 3. prentice Hall Upper Saddle River, NJ. ISBN 0-13-805326-X.

Guerreroa, H., Rosa, G., Morales, M., del Monte, F., Moreno, E., Levy, D., del Real, R. P., Belenguer, T., and Serna, C. (1997). Faraday rotation in magnetic- $-\mathrm{Fe}_{2} \mathrm{O}_{3} / \mathrm{SiO}_{2}$ nanocomposites. measurements, 2:0.

Gujar, T., Shinde, V., Lokhande, C., Mane, R., and Han, S.-H. (2005). Bismuth oxide thin films prepared by chemical bath deposition (CBD) method: annealing effect. Applied surface science, 250(1):161-167.

Haas, C. (1968). Spin-disorder scattering and magnetoresistance of magnetic semiconductors. Physical Review, 168(2):531. 
Hall, J. and Hook, H. (1991). Solid state physics (2nd ed.). Chichester: Wiley. ISBN: 978-0-471-92805-8.

Han, S., Lee, Y., Kim, H., Kim, G.-h., Lee, J., Yoon, J.-H., and Kim, G. (1997). Polymer surface modification by plasma source ion implantation. Surface and Coatings Technology, 93(2-3):261-264.

Hase, M., Pomjakushin, V. Y., Keller, L., Dönni, A., Sakai, O., Yang, T., Cong, R., Lin, J., Ozawa, K., and Kitazawa, H. (2011). Spiral magnetic structure in spin-5/2 frustrated trimerized chains in $\mathrm{SrMn}_{3} \mathrm{P}_{4} \mathrm{O}_{14}$. Physical Review B, 84(18):184435.

Hattori, A., Hattori, K., and Daimon, H. (2007). $\beta-\mathrm{FeSi}_{2}$ thin film grown on a Si (111) surface with ferromagnetic interface. In Journal of Physics: Conference Series, volume 61, page 404. IOP Publishing.

Hayes, R., Ahmed, A., Edge, T., and Zhang, H. (2014). Core-shell particles: Preparation, fundamentals and applications in high performance liquid chromatography. Journal of Chromatography A, 1357:36-52.

Hendriksen, P. V., Linderoth, S., and Lindgård, P.-A. (1993). Finite-size modifications of the magnetic properties of clusters. Physical Review B, 48(10):7259.

Heron, J., Schlom, D., and Ramesh, R. (2014). Electric field control of magnetism using $\mathrm{BiFeO}_{3}$-based heterostructures. Applied Physics Reviews, 1(2):021303.

Hill, N. A., Bättig, P., and Daul, C. (2002). First principles search for multiferroism in $\mathrm{BiCrO}_{3}$. The Journal of Physical Chemistry B, 106(13):3383-3388.

Hoffmann, T., Thielen, P., Becker, P., Bohatỳ, L., and Fiebig, M. (2011). Time-resolved imaging of magnetoelectric switching in multiferroic $\mathrm{MnWO}_{4}$. Physical Review B, 84(18):184404.

Hull, D. and Bacon, D. J. (2001). Introduction to dislocations. Butterworth-Heinemann.

Ibach, H. and Lueth, H. (2009). Solid state physics. An introduction to principles of Materials Science. Springer Science \& Business Media, Fourth Edition Springer Science edition. ISBN 978-3-540-93804-0.

Ihlefeld, J., Podraza, N., Liu, Z., Rai, R., Xu, X., Heeg, T., Chen, Y., Li, J., Collins, R., Musfeldt, J., and Pan, X. (2008). Optical band gap of $\mathrm{BiFeO}_{3}$ grown by molecularbeam epitaxy. Applied physics letters, 92(14):142908-142908.

Ikeda, N., Ohsumi, H., Ohwada, K., Ishii, K., Inami, T., Kakurai, K., Murakami, Y., Yoshii, K., Mori, S., Horibe, Y., and Kitô, H. (2005). Ferroelectricity from iron valence ordering in the charge-frustrated system $\mathrm{LuFe}_{2} \mathrm{O}_{4}$. Nature, 436(7054):1136-1138. 
Iliev, M., Litvinchuk, A., Hadjiev, V., Gospodinov, M., Skumryev, V., and Ressouche, E. (2010). Phonon and magnon scattering of antiferromagnetic $\mathrm{Bi}_{2} \mathrm{Fe}_{4} \mathrm{O}_{9}$. Physical Review B, 81(2):024302.

Irshad, Z., Shah, S., Rafiq, M., and Hasan, M. (2015). First principles study of structural, electronic and magnetic properties of ferromagnetic $\mathrm{Bi}_{2} \mathrm{Fe}_{4} \mathrm{O}_{9}$. Journal of Alloys and Compounds, 624:131-136.

Ishiguro, T., Ishizawa, N., Mizutani, N., Kato, M., Tanaka, K., and Marumo, F. (1983). Charge-density distribution in crystals of $\mathrm{CuAlO}_{2}$ with d-s hybridization. Acta Crystallographica Section B: Structural Science, 39(5):564-569.

Jain, A., Ong, S. P., Hautier, G., Chen, W., Richards, W. D., Dacek, S., Cholia, S., Gunter, D., Skinner, D., Ceder, G., and Persson, K. (2013). Commentary: The Materials Project: A materials genome approach to accelerating materials innovation. APL Materials, 1(1):011002. DOI: 10.1063/1.4812323.

Jallad, K. and Ben-Amotz, D. (2001). Chemical imaging of iron oxides and oxyhydroxides using near infrared Raman imaging microscopy. Materials science and technology, 17(11):1479-1486.

Jeng, H.-T. and Guo, G. (2002). First-principles investigations of the electronic structure and magnetocrystalline anisotropy in strained magnetite $\mathrm{Fe}_{3} \mathrm{O}_{4}$. Physical review $B$, 65(9):094429.

Jia, D.-C., Xu, J.-H., Ke, H., Wang, W., and Zhou, Y. (2009). Structure and multiferroic properties of $\mathrm{BiFeO}_{3}$ powders. Journal of the European Ceramic Society, 29(14):30993103.

Jiles, D. C. (2001). Introduction to the electronic properties of materials. CRC Press. ISBN 0-7487-6042-3.

Johan, M. R., Suan, M. S. M., Hawari, N. L., and Ching, H. A. (2011). Annealing effects on the properties of copper oxide thin films prepared by chemical deposition. Int. J. Electrochem. Sci, 6:6094-6104.

Johnson, R., Chapon, L., Khalyavin, D., Manuel, P., Radaelli, P., and Martin, C. (2012). Giant improper ferroelectricity in the ferroaxial magnet $\mathrm{CaMn}_{7} \mathrm{O}_{12}$. Physical review letters, 108(6):067201.

Johnson, R. D., Nair, S., Chapon, L. C., Bombardi, A., Vecchini, C., Prabhakaran, D., Boothroyd, A. T., and Radaelli, P. G. (2011). $\mathrm{Cu}_{3} \mathrm{Nb}_{2} \mathrm{O}_{8}$ : A Multiferroic with Chiral Coupling to the Crystal Structure. Phys. Rev. Lett., 107:137205. 
Joy, P., Kumar, P. A., and Date, S. (1998). The relationship between field-cooled and zero-field-cooled susceptibilities of some ordered magnetic systems. Journal of Physics: Condensed Matter, 10(48):11049.

Jubb, A. M. and Allen, H. C. (2010). Vibrational spectroscopic characterization of hematite, maghemite, and magnetite thin films produced by vapor deposition. ACS Applied Materials \& Interfaces, 2(10):2804-2812.

Jun, Y.-K., Moon, W.-T., Chang, C.-M., Kim, H.-S., Ryu, H. S., Kim, J. W., Kim, K. H., and Hong, S.-H. (2005). Effects of Nb-doping on electric and magnetic properties in multi-ferroic $\mathrm{BiFeO}_{3}$ ceramics. Solid state communications, 135(1):133-137.

Kales, M. L. (1953). Modes in wave guides containing ferrites. Journal of Applied Physics, 24(5):604-608.

Kaplan, T. A. and Mahanti, S. D. (2011). Canted-spin-caused electric dipoles: A local symmetry theory. Phys. Rev. B, 83:174432.

Katsura, H., Nagaosa, N., and Balatsky, A. V. (2005). Spin current and magnetoelectric effect in noncollinear magnets. Physical review letters, 95(5):057205.

Keffer, F. (1962). Moriya Interaction and the Problem of the Spin Arrangements in $\beta$ MnS. Physical Review, 126(3):896.

Kennedy, J., Leveneur, J., Takeda, Y., Williams, G., Kupke, S., Mitchell, D., Markwitz, A., and Metson, J. (2012). Evolution of the structure and magneto-optical properties of ion beam synthesized iron nanoclusters. Journal of Materials Science, 47(3):11271134.

Kennedy, J., Markwitz, A., Trodahl, H., Ruck, B., Durbin, S., and Gao, W. (2007). Ion beam analysis of amorphous and nanocrystalline group III-V nitride and $\mathrm{ZnO}$ thin films. Journal of electronic materials, 36(4):472-482.

Kézsmárki, I., Kida, N., Murakawa, H., Bordács, S., Onose, Y., and Tokura, Y. (2011). Enhanced directional dichroism of terahertz light in resonance with magnetic excitations of the multiferroic $\mathrm{Ba}_{2} \mathrm{CoGe}_{2} \mathrm{O}_{7}$ oxide compound. Physical review letters, 106(5):057403.

Khomskii, D. (2009). Classifying multiferroics: Mechanisms and effects. Physics, 2.

Kim, D. H., Lee, H. N., Varela, M., and Christen, H. M. (2006a). Antiferroelectricity in multiferroic $\mathrm{BiCrO}_{3}$ epitaxial films. Applied physics letters, 89(16):2904. 
Kim, J. K., Kim, S. S., Kim, W.-J., Bhalla, A. S., and Guo, R. (2006b). Enhanced ferroelectric properties of $\mathrm{Cr}$-doped $\mathrm{BiFeO}_{3}$ thin films grown by chemical solution deposition. Applied physics letters, 88(13):2901.

Kindler, B., Ackermann, D., Gembalies-Datz, D., Hartmann, W., Heßberger, F., Hofmann, S., Klemm, J., Lommel, B., Mann, R., and Steiner, J. (2003). Chemical Compound Targets for SHIP-Production, Properties, and Application. In AIP Conference Proceedings, volume 680, pages 781-784. IOP INSTITUTE OF PHYSICS PUBLISHING LTD.

Kirkpatrick, S. and Sherrington, D. (1978). Infinite-ranged models of spin-glasses. Physical Review B, 17(11):4384.

Kittel, C. (2005). Introduction to Solid State Physics $8^{\text {th }}$ ed. Wiley.

Kodama, R. H., Berkowitz, A. E., McNiff Jr, E., and Foner, S. (1996). Surface spin disorder in $\mathrm{NiFe}_{2} \mathrm{O}_{4}$ nanoparticles. Physical Review Letters, 77(2):394.

Kondo, V. K. M. V. M., Singh, V. S. K., and Ishiwara, V. H. (2007). New ferroelectric material for embedded FRAM LSIs. Fujitsu Sci. Tech. J, 43(4):502-507.

Kralj, S., Makovec, D., Čampelj, S., and Drofenik, M. (2010). Producing ultra-thin silica coatings on iron-oxide nanoparticles to improve their surface reactivity. Journal of Magnetism and Magnetic Materials, 322(13):1847-1853.

Kulal, P., Dubal, D., Lokhande, C., and Fulari, V. (2011). Chemical synthesis of $\mathrm{Fe}_{2} \mathrm{O}_{3}$ thin films for supercapacitor application. Journal of Alloys and Compounds, 509(5):2567-2571.

Kumar, M. M., Palkar, V., Srinivas, K., and Suryanarayana, S. (2000). Ferroelectricity in a pure $\mathrm{BiFeO}_{3}$ ceramic. Applied Physics Letters, 76(19):2764-2766.

Kumar, N., Kaushal, A., Bhardwaj, C., and Kaur, D. (2010). Effect of La doping on structural, optical and magnetic properties of $\mathrm{BiFeO}_{3}$ thin films deposited by pulsed laser deposition technique. Optoelectr. Adv. Mater, 4:1497-502.

Kumari, L., Lin, J.-H., and Ma, Y.-R. (2007). One-dimensional $\mathrm{Bi}_{2} \mathrm{O}_{3}$ nanohooks: synthesis, characterization and optical properties. Journal of Physics: Condensed Matter, 19(40):406204.

Lacroix, B., Paumier, F., and Gaboriaud, R. J. (2011). Crystal defects and related stress in $\mathrm{Y}_{2} \mathrm{O}_{3}$ thin films: origin, modeling, and consequence on the stability of the C-type structure. Physical Review B, 84(1):014104. 
Landau, L. D., Bell, J., Kearsley, M., Pitaevskii, L., Lifshitz, E., and Sykes, J. (1984). Electrodynamics of continuous media, volume 8. elsevier.

Lawes, G., Harris, A., Kimura, T., Rogado, N., Cava, R., Aharony, A., Entin-Wohlman, O., Yildirim, T., Kenzelmann, M., Broholm, C., and Ramirez, A. (2005). Magnetically Driven Ferroelectric Order in $\mathrm{Ni}_{3} \mathrm{~V}_{2} \mathrm{O}_{8}$. Physical review letters, 95(8):087205.

Le Ru, E. and Etchegoin, P. (2008). Principles of Surface-Enhanced Raman Spectroscopy: and related plasmonic effects. Elsevier.

Lee, C.-B., Jung, S.-B., Shin, Y.-E., and Shur, C.-C. (2001). The effect of Bi concentration on wettability of $\mathrm{Cu}$ substrate by Sn-Bi solders. Materials Transactions, 42(5):751-755.

Lee, S. U., Kim, S. S., Park, M. H., Kim, J. W., Jo, H. K., and Kim, W.-J. (2007). Effects of co-substitution on the electrical properties of $\mathrm{BiFeO}_{3}$ thin films prepared by chemical solution deposition. Applied Surface Science, 254(5):1493-1497.

Levy, R. A. (1968). Principles of solid state physics. Academic Press. ISBN 978-0124457508.

Leycuras, C., Le Gall, H., Guillot, M., and Marchand, A. (1984). Magnetic susceptibility and Verdet constant in rare earth trifluorides. Journal of Applied Physics, 55(6):21612163.

Li, Z., Wu, S., Shao, F., Liu, Q., Ji, Y., Meng, K., Xu, X., Wu, Y., Miao, J., and Jiang, Y. (2016). A novel multiferroic/full-heusler $\mathrm{BiFeO}_{3} / \mathrm{Co}_{2} \mathrm{FeAl}_{0.5} \mathrm{Si}_{0.5}$ heterostructure: Structural, ferroelectric and magnetic properties. Journal of Alloys and Compounds, 660:125-130.

Liang, S., Islam, R., Smith, D. J., Bennett, P., O’Brien, J., and Taylor, B. (2006). Magnetic iron silicide nanowires on Si (110). Applied physics letters, 88(11):113111.

Lieb, E., Schultz, T., and Mattis, D. (1961). Two soluble models of an antiferromagnetic chain. Annals of Physics, 16(3):407-466.

Liu, K., Fan, H., Ren, P., and Yang, C. (2011). Structural, electronic and optical properties of $\mathrm{BiFeO}_{3}$ studied by first-principles. Journal of Alloys and Compounds, 509(5):1901-1905.

Liu, Z., Wu, B., Yin, D., Zhu, Y., and Wang, L. (2012). Enhanced photocatalytic activity in Al-substituted $\mathrm{Bi}_{2} \mathrm{Fe}_{4} \mathrm{O}_{9}$ submicrocrystals. Journal of Materials Science, 47(19):67776783.

Lopez-Santiago, A., Gangopadhyay, P., Thomas, J., Norwood, R. A., Persoons, A., and Peyghambarian, N. (2009). Faraday rotation in magnetite-polymethylmethacrylate 
core-shell nanocomposites with high optical quality. Applied Physics Letters, 95(14):143302.

Lu, A.-H., Salabas, E. e., and Schüth, F. (2007). Magnetic nanoparticles: synthesis, protection, functionalization, and application. Angewandte Chemie International Edition, 46(8):1222-1244.

Machala, L., Zboril, R., and Gedanken, A. (2007). Amorphous iron (III) oxide a review. The Journal of Physical Chemistry B, 111(16):4003-4018.

Malozemoff, A. (1987). Random-field model of exchange anisotropy at rough ferromagnetic-antiferromagnetic interfaces. Physical review B, 35(7):3679.

Malozemoff, A. (1988). Heisenberg-to-Ising crossover in a random-field model with uniaxial anisotropy. Physical Review B, 37(13):7673.

Manukyan, K. V., Chen, Y.-S., Rouvimov, S., Li, P., Li, X., Dong, S., Liu, X., Furdyna, J. K., Orlov, A., Bernstein, G. H., et al. (2014). Ultrasmall $\alpha-\mathrm{Fe}_{2} \mathrm{O}_{3}$ superparamagnetic nanoparticles with high magnetization prepared by template-assisted combustion process. The Journal of Physical Chemistry C, 118(29):16264-16271.

Markwitz, A., Bachmann, M., Baumann, H., Bethge, K., Krimmel, E., and Misaelides, P. (1992). Hydrogen profiles of thin PVD silicon nitride films using elastic recoil detection analysis. Nuclear Instruments and Methods in Physics Research Section B: Beam Interactions with Materials and Atoms, 68(1-4):218-222.

Markwitz, A. and Kennedy, J. (2005). Ion beam physics at GNS Science. New Zealand Science Review, 62:129.

Martin, L. W., Chu, Y.-H., Zhan, Q., Ramesh, R., Han, S.-J., Wang, S. X., Warusawithana, M., and Schlom, D. G. (2007). Room temperature exchange bias and spin valves based on $\mathrm{BiFeO}_{3} / \mathrm{SrRuO}_{3} / \mathrm{SrTiO}_{3} / \mathrm{Si}$ (001) heterostructures. Applied Physics Letters, 91(17):172513.

Martin, T., Merlin, R., Huffman, D., and Cardona, M. (1977). Resonant two magnon Raman scattering in $\alpha-\mathrm{Fe}_{2} \mathrm{O}_{3}$. Solid State Communications, 22(9):565-567.

Martinez, B., Obradors, X., Balcells, L., Rouanet, A., and Monty, C. (1998). Low temperature surface spin-glass transition in $\gamma-\mathrm{Fe}_{2} \mathrm{O}_{3}$ nanoparticles. Physical Review Letters, 80(1):181.

Martinez, B., Roig, A., Obradors, X., Molins, E., Rouanet, A., and Monty, C. (1996). Magnetic properties of $\gamma-\mathrm{Fe}_{2} \mathrm{O}_{3}$ nanoparticles obtained by vaporization condensation in a solar furnace. Journal of applied physics, 79(5):2580-2586. 
Massey, M., Baier, U., Merlin, R., and Weber, W. (1990). Effects of pressure and isotopic substitution on the Raman spectrum of $\alpha-\mathrm{Fe}_{2} \mathrm{O}_{3}$ : identification of two-magnon scattering. Physical Review B, 41(11):7822.

Matsunami, N., Yamamura, Y., Itoh, N., Tawara, H., and Kawamura, T. (1987). Energy dependence of ion-induced sputtering yields of monatomic solids in the low energy region. Unknown.

Mayer, M. (1997). SIMNRA user's guide.

Mazumder, R., Devi, P. S., Bhattacharya, D., Choudhury, P., Sen, A., and Raja, M. (2007). Ferromagnetism in nanoscale $\mathrm{BiFeO}_{3}$. Appl. Phys. Lett.,, 91.

McElfresh, M. (1994). Fundamentals of magnetism and magnetic measurements Featuring Quantum Design's magnetic property measurement system.

Meiklejohn, W. H. and Bean, C. P. (1957). New magnetic anisotropy. Physical Review, 105(3):904.

Meldrum, A., Boatner, L., and White, C. (2001). Nanocomposites formed by ion implantation: Recent developments and future opportunities. Nuclear Instruments and Methods in Physics Research Section B: Beam Interactions with Materials and Atoms, 178(1):7-16.

Meng, X., Qin, G., Li, S., Wen, X., Ren, Y., Pei, W., and Zuo, L. (2011). Enhanced photoelectrochemical activity for $\mathrm{Cu}$ and $\mathrm{Ti}$ doped hematite: The first principles calculations. Applied Physics Letters, 98(11):112104.

Mocherla, P. S., Karthik, C., Ubic, R., Rao, M. R., and Sudakar, C. (2014). Effect of microstrain on the magnetic properties of $\mathrm{BiFeO}_{3}$ nanoparticles. Applied Physics Letters, 105(13):132409.

Moriya, T. (1960). Anisotropic superexchange interaction and weak ferromagnetism. Physical Review, 120(1):91.

Mostovoy, M. (2006). Ferroelectricity in Spiral Magnets. Phys. Rev. Lett., 96:067601.

Mukherjee, A., Basu, S., Manna, P., Yusuf, S., and Pal, M. (2014). Enhancement of multiferroic properties of nanocrystalline $\mathrm{BiFeO}_{3}$ powder by Gd-doping. Journal of Alloys and Compounds, 598:142-150.

Müller, R., Hergt, R., Dutz, S., Zeisberger, M., and Gawalek, W. (2006). Nanocrystalline iron oxide and $\mathrm{Ba}$ ferrite particles in the superparamagnetism-ferromagnetism transition range with ferrofluid applications. Journal of Physics: Condensed Matter, 18(38):S2527. 
Mumtaz, A., Maaz, K., Janjua, B., Hasanain, S., and Bertino, M. F. (2007). Exchange bias and vertical shift in $\mathrm{CoFe}_{2} \mathrm{O}_{4}$ nanoparticles. Journal of magnetism and magnetic materials, 313(2):266-272.

Murakami, M., Fujino, S., Lim, S., Long, C., Salamanca-Riba, L., Wuttig, M., Takeuchi, I., Nagarajan, V., and Varatharajan, A. (2006). Fabrication of multiferroic epitaxial $\mathrm{BiCrO}_{3}$ thin films. Applied physics letters, 88(15):152902-152902.

Murmu, P., Kennedy, J., Ruck, B., Williams, G., Markwitz, A., Rubanov, S., and Suvorova, A. (2012). Effect of annealing on the structural, electrical and magnetic properties of Gd-implanted ZnO thin films. Journal of Materials Science, 47(3):1119-1126.

Myers, S. (1974). Ion-backscattering study of $\mathrm{LiOH}-$ to- $\mathrm{Li}_{2} \mathrm{O}$ conversion on a $\mathrm{LiH}$ substrate. Journal of Applied Physics, 45(10):4320-4329.

Nadeem, K., Krenn, H., Traussnig, T., Würschum, R., Szabo, D., and Letofsky-Papst, I. (2011). Effect of dipolar and exchange interactions on magnetic blocking of maghemite nanoparticles. Journal of Magnetism and Magnetic Materials, 323(15):19982004.

Naganuma, H. (2011). Multifunctional Characteristics of B-site Substituted BiFeO $\mathrm{B}_{3}$ Films. in Ferroelectrics-physical effects edited by Mickaël Lallart. INTECH Open Access Publisher. [Online; accessed 19-October-2014], "http : / / www . intechopen.com/", 978-953307-453-5.

Naganuma, H., Kovacs, A., Hirata, A., Hirotsu, Y., and Okamura, S. (2007). Structural analysis of polycrystalline $\mathrm{BiFeO}_{3}$ films by transmission electron microscopy. Materials transactions, 48(9):2370-2373.

Nanosurf (2015). Nanosurf FlexAFM version 5 operating instructions for C3000 control software version 3.5.

Nastasi, M., Mayer, J., and Hirvonen, J. K. (2004). Ion-solid interactions: fundamentals and applications. Cambridge University Press.

Neaton, J., Ederer, C., Waghmare, U., Spaldin, N., and Rabe, K. (2005). Firstprinciples study of spontaneous polarization in multiferroic $\mathrm{BiFeO}_{3}$. Physical Review $B, 71(1): 014113$.

Nečas, D. and Klapetek, P. (2012). Gwyddion: an open-source software for SPM data analysis. Open Physics, 10(1):181-188.

Néel, L. (1949). Preuves expérimentales du ferromagnétisme et de l'antiferromagnétisme. In Annales de l'institut Fourier, volume 1, pages 163-183. 
Neogy, R. K. (2012). Heat transport and related thermal Properties in Nanofluids and Nanostructured Materials. PhD thesis, SN Bose National Centre for Basic Sciences.

Newnham, R. and Roy, R. (1921). Structural characterization of solids. The chemical structure of solids, treatise on solid state chemistry, 1:437-533.

Niitaka, S., Azuma, M., Takano, M., Nishibori, E., Takata, M., and Sakata, M. (2004). Crystal structure and dielectric and magnetic properties of $\mathrm{BiCrO}_{3}$ as a ferroelectromagnet. Solid State Ionics, 172(1):557-559.

Nishimori, H. (2001). Statistical physics of spin glasses and information processing: an introduction, volume 111. Clarendon Press.

Nogués, J. and Schuller, I. K. (1999). Exchange bias. Journal of Magnetism and Magnetic Materials, 192(2):203-232.

Northrop Grumman (2016). Terbium Gallium Garnet. [Online; accessed 19-September2016].

Nyhus, P., Cooper, S., and Fisk, Z. (1995). Electronic Raman scattering across the unconventional charge gap in FeSi. Physical Review B, 51(21):15626.

Ortega, D., Velez-Fort, E., Garcia, D., García, R., Litran, R., Barrera-Solano, C., Ramírezdel Solar, M., and Domínguez, M. (2010). Size and surface effects in the magnetic properties of maghemite and magnetite coated nanoparticles. Philosophical Transactions of the Royal Society of London A: Mathematical, Physical and Engineering Sciences, 368(1927):4407-4418.

Ortiz-Quiñonez, J. L., Díaz, D., Zumeta-Dubé, I., Arriola-Santamaría, H., Betancourt, I., Santiago-Jacinto, P., and Nava-Etzana, N. (2013). Easy synthesis of high-purity $\mathrm{BiFeO}_{3}$ nanoparticles: new insights derived from the structural, optical, and magnetic characterization. Inorganic chemistry, 52(18):10306-10317.

Ostrovskaya, L., Perevertailo, V., Ralchenko, V., Saveliev, A., and Zhuravlev, V. (2007). Wettability of nanocrystalline diamond films. Diamond and related materials, 16(12):2109-2113.

Park, T.-J., Papaefthymiou, G. C., Viescas, A. J., Moodenbaugh, A. R., and Wong, S. S. (2007). Size-dependent magnetic properties of single-crystalline multiferroic $\mathrm{BiFeO}_{3}$ nanoparticles. Nano letters, 7(3):766-772.

Patterson, A. (1939). The Scherrer formula for X-ray particle size determination. Physical review, 56(10):978. 
Pisarev, R., Moskvin, A., Kalashnikova, A., and Rasing, T. (2009). Charge transfer transitions in multiferroic $\mathrm{BiFeO}_{3}$ and related ferrite insulators. Physical Review $B$, $79(23): 235128$.

Ponosov, Y. S., Shorikov, A. O., Streltsov, S., Lukoyanov, A. V., Shchegolikhina, N., Prekul, A., and Anisimov, V. I. (2016). Raman scattering by electron and phonon excitations in FeSi. JETP letters, 103(5):316-320.

Popa, M., Crespo, D., Calderon-Moreno, J. M., Preda, S., and Fruth, V. (2007). Synthesis and structural characterization of single-phase $\mathrm{BiFeO}_{3}$ powders from a polymeric precursor. Journal of the American Ceramic Society, 90(9):2723-2727.

Prakash, T., Williams, G., Kennedy, J., and Rubanov, S. (2016a). $\mathrm{Ni}_{1-x} \mathrm{Fe}_{x}$ nanoparticles made by low energy dual ion implantation into $\mathrm{SiO}_{2}$. Materials Research Express, 3(12):126102.

Prakash, T., Williams, G. V., Kennedy, J., and Rubanov, S. (2016b). Formation of magnetic nanoparticles by low energy dual implantation of $\mathrm{Ni}$ and $\mathrm{Fe}$ into $\mathrm{SiO}_{2}$. Journal of Alloys and Compounds, 667:255-261.

Prashanthi, K., Gupta, M., Tsui, Y., and Thundat, T. (2013). Effect of annealing atmosphere on microstructural and photoluminescence characteristics of multiferroic $\mathrm{BiFeO}_{3}$ thin films prepared by pulsed laser deposition technique. Applied Physics A, 110(4):903-907.

Prati, E. (2003). Propagation in gyroelectromagnetic guiding systems. Journal of electromagnetic waves and applications, 17(8):1177-1196.

Priya, S., Islam, R., Dong, S., and Viehland, D. (2007). Recent advancements in magnetoelectric particulate and laminate composites. Journal of Electroceramics, 19(1):149166.

Puri, S., McMahon, P. L., and Yamamoto, Y. (2014). Single-shot quantum nondemolition measurement of a quantum-dot electron spin using cavity exciton-polaritons. Physical Review B, 90(15):155421.

Qi, X., Dho, J., Blamire, M., Jia, Q., Lee, J.-S., Foltyn, S., and MacManus-Driscoll, J. L. (2004). Epitaxial growth of $\mathrm{BiFeO}_{3}$ thin films by LPE and sol-gel methods. Journal of magnetism and magnetic materials, 283(2):415-421.

Qiu, Y., Liu, D., Yang, J., and Yang, S. (2006). Controlled synthesis of bismuth oxide nanowires by an oxidative metal vapor transport deposition technique. Advanced materials, 18(19):2604-2608. 
Raghavender, A., Hong, N. H., Park, C., Jung, M.-H., Lee, K. J., and Lee, D. (2011). Thickness dependent magnetic properties of $\mathrm{BiFeO}_{3}$ thin films prepared by pulsed laser deposition. Materials Letters, 65(17):2786-2788.

Ramachandran, B., Dixit, A., Naik, R., Lawes, G., and Rao, M. R. (2010). Charge transfer and electronic transitions in polycrystalline $\mathrm{BiFeO}_{3}$. Physical Review B, 82(1):012102.

Ramesh, R. and Spaldin, N. A. (2007). Multiferroics: progress and prospects in thin films. Nature materials, 6(1):21-29.

Reddy, V. A., Pathak, N., and Nath, R. (2012). Particle size dependent magnetic properties and phase transitions in multiferroic $\mathrm{BiFeO}_{3}$ nano-particles. Journal of Alloys and Compounds, 543:206-212.

Renkert, S. (2014). Conductive atomic force microscopy measurements on organic nanocrystals. Master thesis, University of Albert-Ludwigs Freiburg, Department of Mathematik and Physik, Freiburg, Germany.

Ressouche, E., Simonet, V., Canals, B., Gospodinov, M., and Skumryev, V. (2009). Magnetic frustration in an iron-based Cairo pentagonal lattice. Physical review letters, 103(26):267204.

Ribeiro, J., Perez-Mato, J., and Vieira, L. (2016). Landau model for the multiferroic delafossite antiferromagnets. Journal of Magnetism and Magnetic Materials, 416:15-24.

Rieker, T., Hanprasopwattana, A., Datye, A., and Hubbard, P. (1999). Particle size distribution inferred from small-angle $X$-ray scattering and transmission electron microscopy. Langmuir, 15(2):638-641.

Rivière, J. C. and Myhra, S. (2009). Handbook of surface and interface analysis: methods for problem-solving. CRC press. ISBN: 0-8247-0080-5.

Rohlf, J. W. (1994). Modern Physics from A to Z. John Wiley and Sons. ISBN: 9780471572701.

Rojac, T., Bencan, A., Malic, B., Tutuncu, G., Jones, J. L., Daniels, J. E., and Damjanovic, D. (2014). $\mathrm{BiFeO}_{3}$ ceramics: processing, electrical, and electromechanical properties. Journal of the American Ceramic Society, 97(7):1993-2011.

Rout, D., Moon, K.-S., and Kang, S.-J. L. (2009). Temperature-dependent Raman scattering studies of polycrystalline $\mathrm{BiFeO}_{3}$ bulk ceramics. Journal of Raman Spectroscopy, 40(6):618-626. 
SAED (2016). Interdisciplinary centre for electron microscopy cime, Selectedarea diffraction. [Online; accessed 16-September-2016].

Salazar-Pérez, A., Camacho-López, M., Morales-Luckie, R., Sánchez-Mendieta, V., Ureña-Núñez, F., and Arenas-Alatorre, J. (2005). Structural evolution of $\mathrm{Bi}_{2} \mathrm{O}_{3}$ prepared by thermal oxidation of bismuth nano-particles. Superficies y vacío, 18(3):4-8.

Sando, D., Agbelele, A., Rahmedov, D., Liu, J., Rovillain, P., Toulouse, C., Infante, I., Pyatakov, A., Fusil, S., Jacquet, E., and Carrétéro, C. (2013). Crafting the magnonic and spintronic response of $\mathrm{BiFeO}_{3}$ films by epitaxial strain. Nature materials, 12(7):641646.

Sasaki, M., Jönsson, P., Takayama, H., and Mamiya, H. (2005). Aging and memory effects in superparamagnets and superspin glasses. Physical Review B, 71(10):104405.

Scherrer, P. (1918). Gottinger Nachuchter Gesseel.

Schiemer, J. A., Withers, R. L., Liu, Y., and Carpenter, M. A. (2013). Ca-doping of $\mathrm{BiFeO}_{3}$ : the role of strain in determining coupling between ferroelectric displacements, magnetic moments, octahedral tilting, and oxygen-vacancy ordering. Chemistry of Materials, 25(21):4436-4446.

Schneider, C. A., Rasband, W. S., and Eliceiri, K. W. (2012). NIH Image to ImageJ: 25 years of image analysis. Nature methods, 9(7):671-675.

Schrettle, F., Kant, C., Lunkenheimer, P., Mayr, F., Deisenhofer, J., and Loidl, A. (2012). Wüstite: electric, thermodynamic and optical properties of FeO. The European Physical Journal B-Condensed Matter and Complex Systems, 85(5):1-12.

Schwinger, J. (1969). A magnetic model of matter. Science, 165(3895):757-761.

Scott, J. (2007). Data storage: Multiferroic memories. Nature materials, 6(4):256-257.

Seah, M. (1981). Pure element sputtering yields using 500-1000 eV argon ions. Thin Solid Films, 81(3):279-287.

Seidel, J., Martin, L. W., He, Q., Zhan, Q., Chu, Y.-H., Rother, A., Hawkridge, M., Maksymovych, P., Yu, P., Gajek, M., and Balke, N. (2009). Conduction at domain walls in oxide multiferroics. Nature materials, 8(3):229-234.

Selbach, S. M., Einarsrud, M.-A., and Grande, T. (2008). On the thermodynamic stability of $\mathrm{BiFeO}_{3}$. Chemistry of Materials, 21(1):169-173.

Sergienko, I. A. and Dagotto, E. (2006). Role of the Dzyaloshinskii-Moriya interaction in multiferroic perovskites. Physical Review B, 73(9):094434. 
Sherrington, D. and Kirkpatrick, S. (1975). Solvable model of a spin-glass. Physical review letters, 35(26):1792.

Shi, J., Gider, S., Babcock, K., and Awschalom, D. (1996). Magnetic clusters in molecular beams, metals, and semiconductors. Science, 271(5251):937.

Shim, S.-H. and Duffy, T. S. (2002). Raman spectroscopy of $\mathrm{Fe}_{2} \mathrm{O}_{3}$ to $62 \mathrm{GPa}$. American Mineralogist, 87(2-3):318-326.

Sigmund, P. (2004). Stopping of heavy ions: a theoretical approach, volume 204. Springer Science \& Business Media. ISBN: 3-540-22273-1.

Simões, A., Gonzalez, A., Cavalcante, L., Riccardi, C., Longo, E., and Varela, J. A. (2007). Ferroelectric characteristics of $\mathrm{BiFeO}_{3}$ thin films prepared via a simple chemical solution deposition. Journal of applied physics, 101(7):074108.

Siratori, K. and Kino, Y. (1980). A note on the magnetic anisotropy of $\mathrm{Fe}_{3} \mathrm{O}_{4}$. Journal of Magnetism and Magnetic Materials, 20(1):87-90.

Sosnowska, I., Neumaier, T. P., and Steichele, E. (1982). Spiral magnetic ordering in bismuth ferrite. Journal of Physics C: Solid State Physics, 15(23):4835.

Spaldin, N. A., Cheong, S.-W., and Ramesh, R. (2010). Multiferroics: past, present, and future. Phys. Today, 63(10):38-43.

Srivastava, C. and Aiyar, R. (1987). Spin wave stiffness constants in some ferrimagnetics. Journal of Physics C: Solid State Physics, 20(8):1119-1128.

Srivastava, V., Rajagopalan, M., and Sanyal, S. (2008). First principles study of structural, magnetic and electronic properties of half-metallic $\mathrm{CrO}_{2}$ under pressure. The European Physical Journal B-Condensed Matter and Complex Systems, 61(2):131-139.

Steele, J. and Lewis, R. (2014). In situ micro-Raman studies of laser-induced bismuth oxidation reveals metastability of $\beta-\mathrm{Bi}_{2} \mathrm{O}_{3}$ microislands. Optical Materials Express, $4(10): 2133-2142$.

Stephens, P. (1974). Magnetic circular dichroism. Annual Review of Physical Chemistry, 25(1):201-232.

Tadic, M., Kralj, S., Jagodic, M., Hanzel, D., and Makovec, D. (2014). Magnetic properties of novel superparamagnetic iron oxide nanoclusters and their peculiarity under annealing treatment. Applied Surface Science, 322:255-264. 
Tan, B., Sow, C., Koh, T., Chin, K., Wee, A., and Ong, C. (2005). Fabrication of sizetunable gold nanoparticles array with nanosphere lithography, reactive ion etching, and thermal annealing. The Journal of Physical Chemistry B, 109(22):11100-11109.

Tang, C.-J., Jaing, C.-C., Wu, K., and Lee, C.-C. (2009). Residual stress of gradedindex-like films deposited by radio frequency ion-beam sputtering. Thin Solid Films, 517(5):1746-1749.

Tang, X.-L., ZHANG, H.-w., Hua, S., ZHONG, Z.-y., and JING, Y.-l. (2006). Structure and magnetic properties of amorphous and polycrystalline $\mathrm{Fe}_{3} \mathrm{O}_{4}$ thin films. Transactions of Nonferrous Metals Society of China, 16:s249-s252.

Tauc, J., Grigorovici, R., and Vancu, A. (1966). Optical properties and electronic structure of amorphous germanium. physica status solidi (b), 15(2):627-637.

Tepper, T., Ilievski, F., Ross, C., Zaman, T., Ram, R., Sung, S., and Stadler, B. (2003). Magneto-optical properties of iron oxide films. Journal of applied physics, 93(10):69486950.

Tian, Y., Stroppa, A., Chai, Y., Yan, L., Wang, S., Barone, P., Picozzi, S., and Sun, Y. (2014). Cross coupling between electric and magnetic orders in a multiferroic metalorganic framework. Scientific reports, 4 .

Tooth, B., Brugger, J., Ciobanu, C., and Liu, W. (2008). Modeling of gold scavenging by bismuth melts coexisting with hydrothermal fluids. Geology, 36(10):815-818.

Townsend, P. D., Chandler, P., and Zhang, L. (2006). Optical effects of ion implantation, volume 13. Cambridge University Press. ISBN: 9780521394307.

Townsend, P. D., Kelly, J. C., and Hartley, N. E. W. (1976). Ion implantation, sputtering and their applications. Academic Press, New York, United-States. ISBN 0-12-696950-7.

Valbusa, U., Boragno, C., and de Mongeot, F. B. (2002). Nanostructuring surfaces by ion sputtering. Journal of Physics: Condensed Matter, 14(35):8153.

Van Diepen, A. and Popma, T. J. (1978). Temperature dependence of the hyperfine field in amorphous $\mathrm{Fe}_{2} \mathrm{O}_{3}$. Solid State Communications, 27(2):121-125.

Vargas, J., Nunes, W., Socolovsky, L., Knobel, M., and Zanchet, D. (2005). Effect of dipolar interaction observed in iron-based nanoparticles. Physical Review B, 72(18):184428.

Viefhaus, H., Hennesen, K., Lucas, M., Müller-Lorenz, E. M., and Grabke, H.-J. (1994). Ion sputter rates and yields for iron-, chromium-and aluminium oxide layers. Surface and interface analysis, 21(9):665-672. 
Vijayasundaram, S., Suresh, G., Mondal, R., and Kanagadurai, R. (2016). Substitutiondriven enhanced magnetic and ferroelectric properties of $\mathrm{BiFeO}_{3}$ nanoparticles. Journal of Alloys and Compounds, 658:726-731.

Wang, D.-J. and Wu, S.-T. (1994). The influence of oxidation on the wettability of aluminum on sapphire. Acta metallurgica et materialia, 42(12):4029-4034.

Wang, H., Zheng, Y., Cai, M.-Q., Huang, H., and Chan, H. L. (2009a). First-principles study on the electronic and optical properties of $\mathrm{BiFeO}_{3}$. Solid State Communications, 149(15):641-644.

Wang, J., Neaton, J., Zheng, H., Nagarajan, V., Ogale, S., Liu, B., Viehland, D., Vaithyanathan, V., Schlom, D., Waghmare, U., et al. (2003). Epitaxial $\mathrm{BiFeO}_{3}$ multiferroic thin film heterostructures. science, 299(5613):1719-1722.

Wang, K., Alzate, J., and Amiri, P. K. (2013). Low-power non-volatile spintronic memory: STT-RAM and beyond. Journal of Physics D: Applied Physics, 46(7):074003.

Wang, K., Liu, J.-M., and Ren, Z. (2009b). Multiferroicity: the coupling between magnetic and polarization orders. Advances in Physics, 58(4):321-448.

Wang, Y., Jiang, Q.-h., He, H.-c., and Nan, C.-W. (2006). Multiferroic $\mathrm{BiFeO}_{3}$ thin films prepared via a simple sol-gel method. Applied Physics Letters, 88(14):142503.

Wang, Y. and Nan, C.-W. (2006). Enhanced ferroelectricity in Ti-doped multiferroic $\mathrm{BiFeO}_{3}$ thin films. Applied Physics Letters, 89(5):2903.

Wang, Y. and Nastasi, M. (2009). Handbook of Modern Ion beam materials analysis. second edition, Materials research Society, Warrenddale, PA.

Wannier, G. (1950). Antiferromagnetism. the triangular ising net. Physical Review, 79(2):357.

Watanabe, H., Yamamoto, H., and Ito, K.-i. (1963). Neutron diffraction study of the intermetallic compound FeSi. Journal of the Physical Society of Japan, 18(7):995-999.

Webster, J. G. and Eren, H. (2014). Measurement, Instrumentation, and Sensors Handbook: Spatial, Mechanical, Thermal, and Radiation Measurement, volume 1. CRC press.

Webster, T. J., Ergun, C., Doremus, R. H., Siegel, R. W., and Bizios, R. (2001). Enhanced osteoclast-like cell functions on nanophase ceramics. Biomaterials, 22(11):1327-1333.

Wei, D. T., Kaufman, H. R., and Lee, C.-C. (1995). Ion beam sputtering. Thin Films for Optical Systems,](Flory, FR, ed.), page 133. 
Weinberger, P. (2008). John Kerr and his effects found in 1877 and 1878. Philosophical Magazine Letters, 88(12):897-907.

Wen, X., Chen, Z., Liu, E., Lin, X., and Chen, C. (2016). Effect of Ba and Mn doping on microstructure and multiferroic properties of $\mathrm{BiFeO}_{3}$ ceramics. Journal of Alloys and Compounds, 678:511-517.

Wenk, H.-R. and Bulakh, A. (2004). Minerals: their constitution and origin. Cambridge University Press.

Wiesendanger, R. and Meyer, E. (2009). Noncontact atomic force microscopy. Springer.

Winpenny, R. (2012). Molecular Cluster Magnets, volume 3. World Scientific.

Wu, S., Cybart, S. A., Yu, P., Rossell, M., Zhang, J., Ramesh, R., and Dynes, R. (2010). Reversible electric control of exchange bias in a multiferroic field-effect device. $\mathrm{Na}$ ture materials, 9(9):756-761.

Wu, W., Wu, Z., Yu, T., Jiang, C., and Kim, W.-S. (2015). Recent progress on magnetic iron oxide nanoparticles: synthesis, surface functional strategies and biomedical applications. Science and technology of advanced materials, 16(2):023501.

Würschum, R., Herth, S., and Brossmann, U. (2003). Diffusion in nanocrystalline metals and alloys-A status report. Advanced Engineering Materials, 5(5):365-372.

Wylie-van Eerd, B., Damjanovic, D., Klein, N., Setter, N., and Trodahl, J. (2010). Structural complexity of $\left(\mathrm{Na}_{0.5} \mathrm{Bi}_{0.5}\right) \mathrm{TiO}_{3}-\mathrm{BaTiO}_{3}$ as revealed by Raman spectroscopy. Physical Review B, 82(10):104112.

Xiao, X., Zhu, J., Li, Y., Luo, W., Yu, B., Fan, L., Ren, F., Liu, C., and Jiang, C. (2007). Greatly reduced leakage current in $\mathrm{BiFeO}_{3}$ thin film by oxygen ion implantation. Journal of Physics D: Applied Physics, 40(18):5775.

Yan, J., Hu, G., Chen, X., Wu, W., and Yang, C. (2008). Ferroelectric properties, morphologies, and leakage currents of $\mathrm{Bi}_{0.97} \mathrm{La}_{0.03} \mathrm{FeO}_{3}$ thin films deposited on indium tin oxide/glass substrates. Journal of Applied Physics, 104(7):076103.

Yang, C., Wang, G. W. G., Lin, L. L. L., Tasch, A. F., Banerjee, S., et al. (2002). Quantum mechanical model of electronic stopping power for ions in a free electron gas. In Proceedings of the 14th International Conference on Ion Implantation Technology. 2002., pages 556-559. IEEE.

Yang, H., Wang, Y., Wang, H., and Jia, Q. (2010a). Oxygen concentration and its effect on the leakage current in $\mathrm{BiFeO}_{3}$ thin films. Applied Physics Letters, 96(1):012909. 
Yang, S., Martin, L., Byrnes, S., Conry, T., Basu, S., Paran, D., Reichertz, L., Ihlefeld, J., Adamo, C., Melville, A., and Chu, Y. (2009). Photovoltaic effects in $\mathrm{BiFeO}_{3}$. Applied Physics Letters, 95(6):062909.

Yang, S., Seidel, J., Byrnes, S., Shafer, P., Yang, C.-H., Rossell, M., Yu, P., Chu, Y.-H., Scott, J., Ager, J., and Martin, L. (2010b). Above-bandgap voltages from ferroelectric photovoltaic devices. Nature nanotechnology, 5(2):143-147.

Yoshitake, T., Nagamoto, T., and Nagayama, K. (2001). Microstructure of $\beta-\mathrm{FeSi}_{2}$ thin films prepared by pulsed laser deposition. Thin Solid Films, 381(2):236-243.

Zavaliche, F., Yang, S., Zhao, T., Chu, Y., Cruz, M., Eom, C., and Ramesh, R. (2006). Multiferroic $\mathrm{BiFeO}_{3}$ films: domain structure and polarization dynamics. Phase transitions, 79(12):991-1017.

Zayat, M., del Monte, F., Morales, M., Rosa, G., Guerrero, H., Serna, C. J., and Levy, D. (2003). Highly Transparent $\gamma-\mathrm{Fe}_{2} \mathrm{O}_{3} /$ Vycor-Glass Magnetic Nanocomposites Exhibiting Faraday Rotation. Advanced Materials, 15(21):1809-1812.

ZHANG, G.-j., CHENG, J.-r., Rui, C., YU, S.-w., and MENG, Z.-y. (2006). Preparation of $\mathrm{BiFeO}_{3}$ thin films by pulsed laser deposition method. Transactions of Nonferrous Metals Society of China, 16:s123-s125.

Zhang, L., Wang, W., Yang, J., Chen, Z., Zhang, W., Zhou, L., and Liu, S. (2006). Sonochemical synthesis of nanocrystallite $\mathrm{Bi}_{2} \mathrm{O}_{3}$ as a visible-light-driven photocatalyst. Applied Catalysis A: General, 308:105-110.

Zhao, T., Scholl, A., Zavaliche, F., Lee, K., Barry, M., Doran, A., Cruz, M., Chu, Y., Ederer, C., Spaldin, N., and Das, R. (2006). Electrical control of antiferromagnetic domains in multiferroic $\mathrm{BiFeO}_{3}$ films at room temperature. Nature materials, 5(10):823829.

Ziegler, J. (1998). RBS/ERD simulation problems: Stopping powers, nuclear reactions and detector resolution. Nuclear Instruments and Methods in Physics Research Section B: Beam Interactions with Materials and Atoms, 136:141-146. 